\title{
Zur Wirksamkeit von Trainings bei Kindern mit Aufmerksamkeitsstörungen
}

\author{
Dissertation \\ zur Erlangung des Doktorgrades \\ der Mathematisch-Naturwissenschaftlichen Fakultäten \\ der Georg-August-Universität zu Göttingen
}

\author{
vorgelegt von \\ Thomas Dreisörner \\ aus Verl
}

Göttingen 2004 


\section{7}

Referent: Prof. Dr. Marcus Hasselhorn

Korreferent: Prof. Dr. Roland Brünken

Tag der mündlichen Prüfungen: 04.11.2004 


\section{Danksagung}

An dieser Stelle möchte ich allen meinen Dank aussprechen, die mich bei dieser Arbeit unterstützt haben.

Zunächst möchte ich allen teilnehmenden Familien, den Eltern und insbesondere den Kindern danken, die an den verschiedenen Trainings teilgenommen haben. Kindern mit Hyperkinetischen Störungen fällt das Durchhalten über mehrere Monate nicht immer leicht. Vielen Dank, dass ihr trotzdem nicht aufgegeben habt.

Ein großer Dank geht an Prof. Marcus Hasselhorn, der mich in meinem Bestreben unterstützt hat, das praktische Feld von Therapien wissenschaftlich zu beforschen. Er gab mir wertvolle Denkanstöße und motivierte mich auch in schwierigen Zeiten durchzuhalten.

Vielen Dank auch an Prof. Roland Brünken, der mich bei methodischen Fragen unterstützte.

Gedankt sei den Therapeutinnen Jana Fuge, Julia Sykulla, Verena Kalinke, Antje Gülsdorf, Kirsten Priebsch, Claudia Mähler, Katrin Merettig sowie den Diplomandinnen Marion Mock, Pia Müller-Scherzberg, Katrin Bartels und Fenn Felstehausen, die die Trainingsprogramme mit mir durchführten. Nur durch ihre Hilfe konnte diese Arbeit in diesem Umfang geleistet werden.

Danke an meine Kolleginnen und Kollegen der Abteilung Pädagogische Psychologie und Entwicklungspsychologie, die mir bei Forschungsfragen weitergeholfen haben. Tina Seufert und Dietmar Grube gaben mir wertvolle methodische Anregungen und verbesserten die Lesbarkeit der Arbeit.

Stephanie Schreblowski wusste meine Rechtschreibfehler zu korrigieren, Thorgund RehBergen half das Literaturverzeichnis zu vervollständigen.

Mein besonderer Dank gilt meiner Familie und meinen Freunden, die mich liebevoll unterstützten. 


\section{Inhaltsverzeichnis}

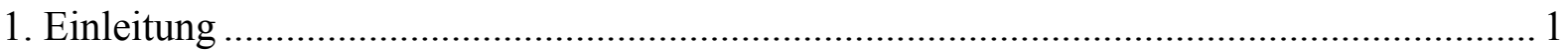

2. Theoretischer und empirischer Hintergrund .............................................................. 4

2.1 Aufmerksamkeit und Konzentration - Definition der wesentlichen Aspekte der beiden Konstrukte.............................................................................................. 4

2.2 Symptomatik, Epidemiologie und Verlauf hyperkinetischer Störungen.................... 6

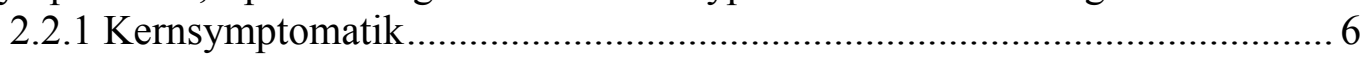

2.2.2 Diagnose und Diagnostik ....................................................................... 7

2.2.3 Fehldiagnosen, assoziierte Probleme und komorbide Störungen ................ 11

2.2.4 Prävalenz und Verlauf............................................................................ 13

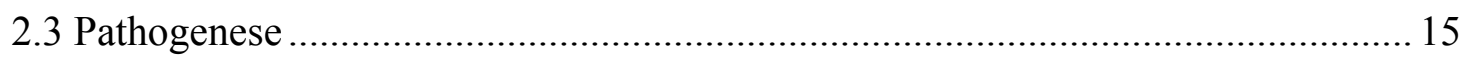

2.3.1 Neurobiologische Faktoren der Ätiologie.................................................. 15

2.3.2 Neuropsychologische Modelle der Ätiologie ............................................... 17

2.3.3 Aufrechterhaltung; Biopsychosoziales Modell.......................................... 20

2.4 Zentrale Aspekte bei der Diagnostik von Aufmerksamkeitsstörungen ................... 20

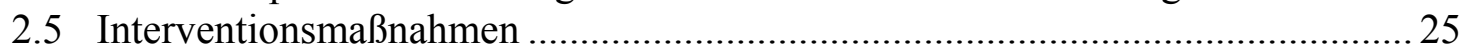

2.5.1 Pharmakologische Behandlung ............................................................ 25

2.5.2 Kognitiv-behaviorale Verfahren ........................................................... 28

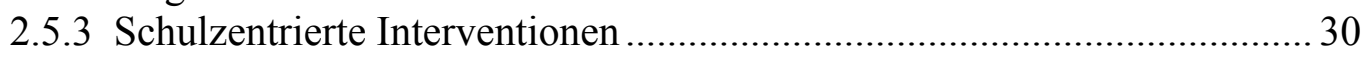

2.5.4 Elterntrainings und familienzentrierte Interventionen .............................. 32

2.5.5 Wirksamkeit multimodaler Interventionen ............................................... 34

2.6 Behandlungsrealitäten im deutschsprachigen Raum .............................................. 36

2.7 Sind Gruppentrainings möglich und/oder nötig? ................................................... 41

2.8 Evaluation klinisch-psychologischer Interventionen ........................................... 42

3. Fragestellungen der vorliegenden Evaluationsstudien ......................................................52



3.1.1 Aufbau und Ziele ............................................................................... 54

3.1.2 Wirksamkeitsuntersuchung..................................................................... 60

3.2 Therapieprogramm für Kinder mit hyperkinetischem und oppositionellem

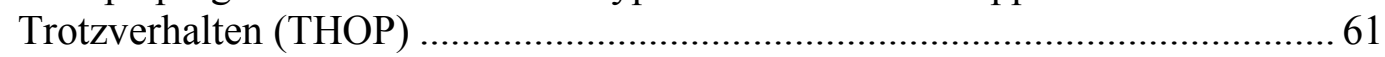

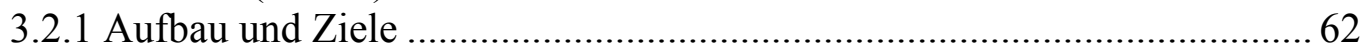

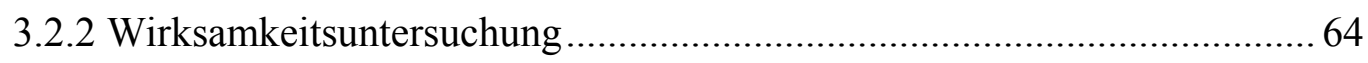

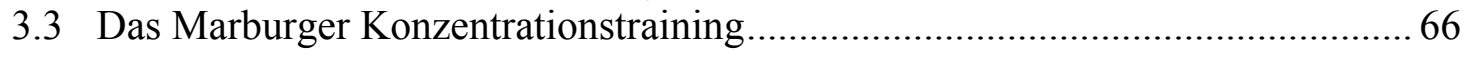



3.3.2 Wirksamkeitsuntersuchung ...................................................................... 69

3.4 Psychologische Hypothesen ( $\mathrm{PHn}$ ) ……………….......................................... 70

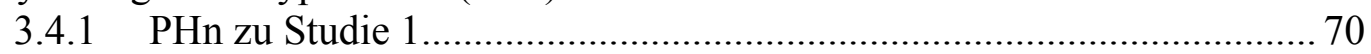

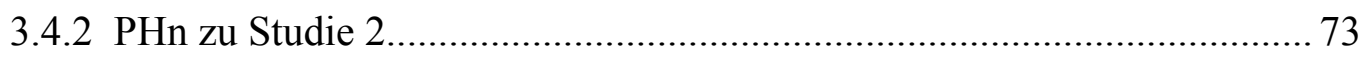



3.4.4 PHn zu Studie 4.................................................................................. 75

3.4.5 PHn zur Vergleichbarkeit der Programme (Studien 1 - 4) ........................ 77

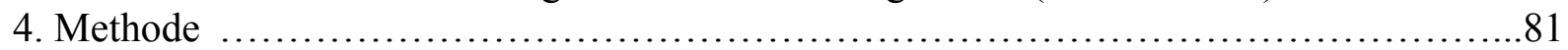

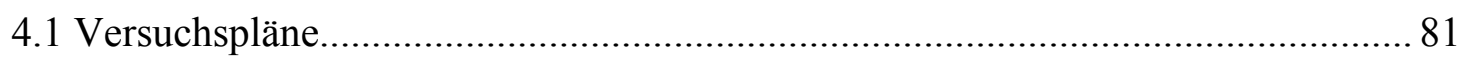

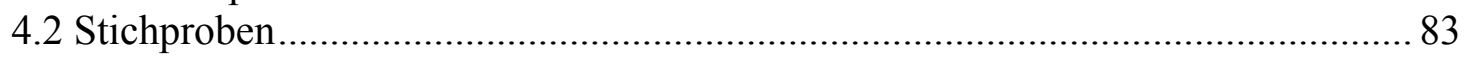

4.3 Untersuchungsverfahren und Konkretisierung der abhängigen Variablen ............. 88

4.4 Ableitung psychologischer Vorhersagen und statistischer Hypothesen;



4.4.1 Psychologische Vorhersagen (PVn), Statistische Hypothesen (SHn) und Testplanung zu Studie 1: Wirksamkeit des Basistrainings nach Lauth und Schlottke ... 
4.4.2 Psychologischen Vorhersagen (PVn), Statistische Hypothesen (SHn) und Testplanung zu Studie 2: Wirksamkeit der Kombination von Basistraining und Strategietraining nach Lauth und Schlottke

4.4.3 Psychologischen Vorhersagen (PVn), Statistische Hypothesen (SHn) und Testplanung zu Studie 3: Wirksamkeit des THOP.

4.4.4 Psychologische Vorhersagen (PVn), Statistische Hypothesen (SHn) und Testplanung zu Studie 4: Wirksamkeit des Marburger

Konzentrationstrainings

4.4.5 Psychologischen Vorhersagen (PVn), Statistische Hypothesen (SHn) und Testplanung zur vergleichenden Evaluation der Trainings aus den Studien $1-4$.

4.5 Durchführung der testpsychologischen Untersuchungen und der Trainings ....... 103

4.5.1 Durchführung des Trainings nach Lauth und Schlottke ........................ 104

4.5.2 Durchführung des THOP als Gruppentraining ...................................... 106

4.5.3 Durchführung des Marburger Konzentrationstrainings ......................... 108

5. Ergebnisse

5.1 Ergebnisse der Studie 1 ..................................................................... 113

5.1.1 Isolierte Evaluation des Basistrainings nach Lauth- und Schlottke........ 113

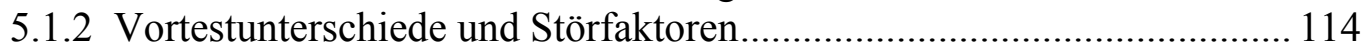

5.1.3 Veränderungen innerhalb der Versuchsgruppen.................................... 115

5.1.4 Veränderungen zwischen den Versuchsgruppen .................................. 116

5.1.5 Effektgrößen der Interaktionseffekte ................................................ 116

5.1.6 Moderatoreffekte (Alter, Intelligenz, Medikation) ............................... 117

5.2 Ergebnisse der Studie 2 (Isolierte Evaluation) ...................................................... 118

5.2.1 Isolierte Evaluation des Lauth- und Schlottke-Trainings (Basis- und

Strategietraining; 15 Sitzungen) ...................................................... 118

5.2.2 Vortestunterschiede der LS-Gruppe Basis- und Strategietraining

(15 Sitzungen) vs. Wartegruppe ......................................................... 118

5.2.3 Veränderungen innerhalb der Versuchsgruppen..................................... 119

5.2.4 Veränderungen zwischen den Versuchsgruppen ................................... 119

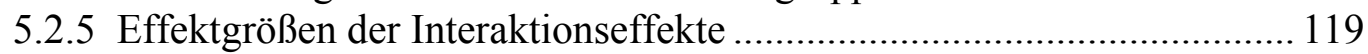

5.2.6 Moderatoreffekte (Alter, Intelligenz, Medikation) ................................ 120

5.3 Ergebnisse der Studie 3 (Isolierte Evaluation) .................................................. 120



5.3.2 Vortestunterschiede und Störfaktoren.................................................. 120

5.3.3 Veränderungen innerhalb der Versuchsgruppen................................... 121

5.3.4 Veränderungen zwischen den Versuchsgruppen ................................... 122

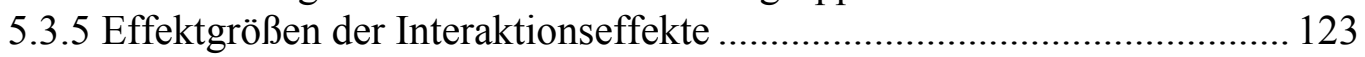

5.3.6 Moderatoreffekte (Alter, Intelligenz, Medikation) ................................ 123

5.3.7 Veränderungen der Eltern-Kind-Interaktion.......................................... 124

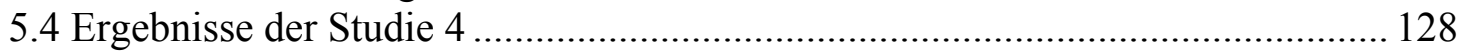

5.4.1 Isolierte Evaluation des Marburger Konzentrationstrainings .............. 128

5.4.2 Vortestunterschiede und Störfaktoren................................................... 128

5.4.3 Veränderungen innerhalb der Versuchsgruppen.................................. 128

5.4.4 Veränderungen zwischen den Versuchsgruppen ................................... 129

5.4.5 Effektgrößen der Interaktionseffekte .................................................. 129

5.4.6 Moderatoreffekte (Alter, Intelligenz, Medikation) ............................... 129

5.5 Vergleichende Evaluation der Studien 1 - 4 .................................................... 130

5.5.1 Vergleich der Gruppen Lauth und Schlottke 10 Sitzungen mit

LS 15 Sitzungen 
5.5.1.1 Vortestunterschiede und Störfaktoren LS 10 Sitzungen vs.

LS 15 Sitzungen

5.5.1.2 Veränderungen zwischen den Versuchsgruppen LS 10 Sitzungen vs. LS 15 Sitzungen.

5.5.1.3 Effektgrößen der Interaktionseffekte LS 10 Sitzungen vs.

LS 15 Sitzungen

5.5.2 Vergleich des Kombinierten Basis- und Strategietrainings nach Lauth und

Schlottke (15 Sitzungen) mit dem THOP

5.5.2.1 Vortestunterschiede und Störfaktoren LS 15 Sitzungen vs. THOP ...... 132

5.5.2.2 Veränderungen zwischen den Versuchsgruppen LS 15 Sitzungen vs. THOP

5.5.2.3 Effektgrößen der Interaktionseffekte

5.5.3 Vergleich des Kombinierten Basis- und Strategietrainings nach Lauth und

Schlottke 15 Sitzungen mit dem Marburger Konzentrationstraining für die AVn TAP und d2 .................................................................... 134

5.5.3.1 Vortestunterschiede und Störfaktoren LS 15 Sitzungen vs. MK ......... 135

5.5.3.2 Veränderungen zwischen den Versuchsgruppen LS15 vs. MK............ 135

5.5.3.3 Effektgrößen der Interaktionseffekte LS15 vs. MK.............................. 135

5.5.4 Vergleich der THOP-Gruppen mit dem Marburger Konzentrationstraining

für die AVn TAP und d2 .................................................................. 136

5.5.4.1 Vortestunterschiede und Störfaktoren THOP vs. MK ......................... 136

5.5.4.2 Veränderungen zwischen den Versuchsgruppen ................................ 136

5.5.4.3 Effektgrößen der Interaktionseffekte THOP vs. MK ............................ 136

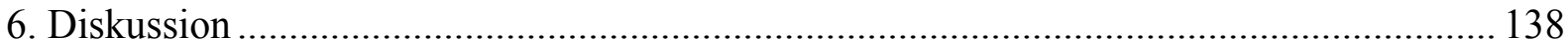

6.1 Bewertung der Ergebnisse der isolierten Evaluation der Studien 1 bis 4 ................ 139

6.1.1 Isolierte Evaluation des Basistrainings nach Lauth und Schlottke

(Studie 1)

6.1.2 Isolierte Evaluation des um Komponenten des Strategietrainings

erweiterten Basistrainings nach Lauth und Schlottke (Studie 2) ............ 145

6.1.3 Isolierte Evaluation des THOP (Studie 3)............................................. 149

6.1.4 Isolierte Evaluation des Marburger Konzentrationstrainings (Studie 4) . 161

6.2 Bewertung der Ergebnisse der vergleichenden Evaluation ................................. 164



6.4 Schlussfolgerungen, Fazit, Ausblick ……......................................................... 182

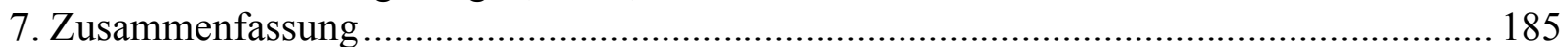

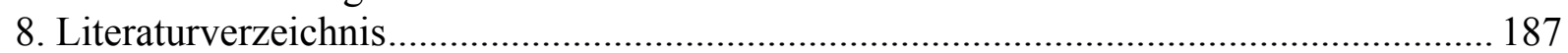






\section{Einleitung}

Aufmerksamkeitsstörungen und Hyperaktivität bei Kindern führen bei den Betroffenen, deren Angehörigen und Lehrern ${ }^{1}$ häufig zu großen Belastungen. Neben Fachleuten, die sich mit dem Thema auseinandersetzen, findet es auch in den Medien häufig Beachtung. In Beiträgen von Zeitschriften wie der Spiegel („Kinder mit Knacks“, Blech \& Thimm, 2002) oder der Stern („Die Not der kleinen Nervensägen“, Rohde, 2002) wird über Kinder berichtet, die sich nicht gut konzentrieren können und die mit ihrer Unruhe ihrem Umfeld viel Kummer bereiten. Nicht selten führen die Auffälligkeiten dazu, dass die Kinder zudem von ihren Alterskameraden abgelehnt werden. Bei einem extremen Ausmaß dieses Verhaltens spricht man vom Hyperkinetischen Syndrom, einer Hyperkinetischen Störung bzw. dem Aufmerksamkeits-Defizit-Hyperaktivitätssyndrom (ADHS).

Hyperkinetische Störungen nach ICD-10 (Dilling, Mombour \& Schmidt, 1991) bzw. Aufmerksamkeitsdefizit-/ Hyperaktivitätsstörungen (engl. Attention-Deficit Hyperactivity Disorder: ADHD) nach DSM-IV (Saß, Wittchen \& Zaudig, 1996) gehören zusammen mit oppositionellen Verhaltensstörungen zu den häufigsten Vorstellungsanlässen in kinder- und jugendpsychiatrischen Einrichtungen (Döpfner, Schürmann \& Frölich, 1998; Pitzer \& Schmidt, 1999). Die Symptome können situationsabhängig unterschiedlich stark ausgeprägt sein sowie kombiniert oder einzeln auftreten. Hyperkinetische Störungen äußern sich in der Regel in Beeinträchtigungen mehrerer Funktions- und Lebensbereiche, da neben der Grundproblematik häufig auch kognitive, emotionale, soziale sowie schulische Auffälligkeiten bestehen.

Entgegen der Annahme einer spontanen Remission der hyperkinetischen Problematik mit zunehmenden Alter sind Hyperkinetische Störungen als ein chronisches, oft bis ins Erwachsenenalter persistierendes Störungsbild $\mathrm{zu}$ betrachten, das ein erhebliches Entwicklungsrisiko für die betroffenen Kinder darstellt. Daraus entsteht neben dem Bedarf nach einer validen Diagnostik die Notwendigkeit nach effektiver Beratung und Behandlung, um den Betroffenen helfen zu können. Im Hinblick auf eine stetige Verbesserung therapeutischer Angebote und die Entwicklung multimodaler Therapieprogramme werden zunehmend auch im Zusammenhang mit der Behandlung kinder- und jugendpsychiatrischer Störungen Wirksamkeitsüberprüfungen von Interventionsmaßnahmen gefordert. Döpfner

\footnotetext{
${ }^{1}$ Um der besseren Lesbarkeit willen wird in der vorliegenden Arbeit auf die Verwendung zusätzlich weiblicher Personenbezeichnungen verzichtet. Die männliche Form ist jedoch durchweg geschlechtsneutral zu verstehen.
} 
(1999, S. 153) führt dazu aus: „Alle, die Therapie betreiben, müssen sich die Frage gefallen lassen, wie wirksam ihre Behandlungsmethode ist...".

Die vorliegende Dissertation soll einen Beitrag zur Beantwortung der Frage geben, welche psychotherapeutischen Behandlungsmaßnahmen dem Kind bei der Bewältigung der Störung effektiv helfen können. Durch die Therapieforschung soll den Forderungen von Betroffenen und Leistungsträgern Rechnung getragen werden, dass nur solche Interventionen erfolgen, die durch objektivierbare empirische Ergebnisse gestützt werden. Häufig werden Therapiemaßnahmen durchgeführt, von deren Wirksamkeit die Anbieter zwar subjektiv überzeugt sind, die aber nicht systematisch untersucht wurden (Döpfner und Lehmkuhl, 2003).

Den Forschungsergebnissen der letzten Jahrzehnte wurde von mehreren Fachgesellschaften Rechnung getragen. So empfiehlt z.B. die Deutsche Gesellschaft für Kinder- und Jugendpsychiatrie und -psychotherapie im Jahr 2000 in ihren Leitlinien zur Diagnostik und Therapie für Hyperkinetische Störungen eine multimodale Therapie, die neben der Aufklärung und Beratung der Eltern pharmakologische und kognitivverhaltenstherapeutische Maßnahmen in der Familie und/oder der Schule umfassen sollte.

In dieser Untersuchung sollen im deutschen Sprachraum verbreitete kognitivverhaltenstherapeutische Programme evaluiert werden, die bislang vorwiegend von den Programmautoren selbst untersucht worden sind. Lauth und Schlottke (1997) versuchen mit ihrem Training für Kinder mit Aufmerksamkeitsstörungen grundlegende Fertigkeiten für konzentriertes Arbeiten zu trainieren. Im Rahmen dieser Dissertation wird in Studie 1 überprüft, ob durch das Basistraining nach Lauth und Schlottke substanzielle Verbesserungen der Aufmerksamkeitsleistungen bei Kindern mit Hyperkinetischen Störungen erzielt werden.

In Studie 2 wurde das Basistraining mit Komponenten des Strategietrainings nach Lauth und Schlottke kombiniert, da sich nach Auswertung der Ergebnisse aus Studie 1 herausstellte, dass das Training nicht zu zufriedenstellenden Ergebnissen führte.

In Studie 3 wird eine Evaluation des „Therapieprogramms für Kinder mit hyperkinetischem und oppositionellem Trotzverhalten“ (im folgenden: THOP) durchgeführt, das von einer Forschungsgruppe um Döpfner, Schürmann und Frölich (1998) entwickelt wurde. Es kombiniert verschiedene verhaltenstherapeutische und kognitive Methoden miteinander und bezieht sowohl das Kind als auch die Eltern aktiv in die Behandlung mit ein. Bislang liegt dieses Programm nur als Einzeltraining vor. Da in vielen Einrichtungen nur 
wenige Therapeuten arbeiten, können häufig nur wenige Kinder behandelt werden. Es sollte deshalb überprüft werden, ob das THOP auch unter Gruppenbedingungen wirksam ist.

Die beiden zuvor genannten Programme haben den Nachteil, dass sie relativ umfangreich sind und viele Behandlungsstunden erfordern. Mit dem Marburger Konzentrationstraining von Krowatschek (1994), das in Studie 4 evaluiert wird, liegt ein Programm vor, das mit wenigen Behandlungseinheiten versucht deutliche Verbesserungen zu erzielen.

Die Durchführung der Trainings zu den Studien 1 bis 3 fand unter praxisnahen Bedingungen als ambulantes Therapieangebot in der Ambulanz der Kinder- und Jugendpsychiatrie in Mühlhausen, der Poliklinischen Institutsambulanz des Psychologischen Instituts der Universität Göttingen sowie in einem Sozialpädiatrischen Zentrum in Reifenstein statt.

Das Marburger Konzentrationstraining sowie ein Pseudoparalleltraing wurden in zwei Kinder- und Jugendpsychiatrischen Praxen in Kassel und Hannoversch-Münden durchgeführt.

Nach der isolierten Evaluation, bei der jeweils die Wirksamkeit der Trainings im Vergleich zu einer Wartegruppe beurteilt wird, erfolgt in einem weiteren Schritt der Vergleich der Trainings miteinander, um ggf. Aspekte der differenziellen Wirksamkeit der Trainings aufzudecken. 


\section{Theoretischer und empirischer Hintergrund}

\subsection{Aufmerksamkeit und Konzentration - Definition der wesentlichen Aspekte der beiden Konstrukte}

Freyberg (1989, zitiert nach Westhoff \& Hagemeister, 2001) geht von einer etymologischen Analyse der Begriffe Aufmerksamkeit und Konzentration aus. Demnach bezieht sich Aufmerksamkeit auf das Wahrnehmen und Konzentration auf das Arbeiten. Lauth (2001, S. 577) definiert die Begriffe wie folgt: „Mit Konzentration bezeichnet man die Bündelung der geistigen Anstrengung auf einen Inhaltsbereich, mit Aufmerksamkeit meint man hingegen die grundlegendere Bereitschaft, Reize auszuwählen, aufzunehmen und angemessen zu verarbeiten“.

In der wissenschaftlichen Analyse des Begriffes Aufmerksamkeit wird betont, dass von den vielen Einzelinformationen, die ein Mensch verarbeitet, nur ein Teil bewusst wird. Konzentration kann sich auf einen Zustand beziehen, also was eine Person zu einem bestimmten Zeitpunkt gerade an Leistung erbringen kann. Konzentration kann sich aber auch auf eine Persönlichkeitsdisposition beziehen. Es lässt sich also zwischen dem momentanen Zustand und einem generellen Merkmal bei Menschen unterscheiden (Westhoff \& Hagemeister, 2001).

Neben der automatisierten Verarbeitung von Informationen steht die kontrollierte Ausführung von Handlungen. Während selektive Aufmerksamkeit häufig unbewusst und automatisch geschieht, wird eine kontrollierte Entscheidung in der Regel mit Absicht herbeigeführt. Posner und Rafael (1987, S. 185f.) sprechen hier von „effortful concentration”.

Kleber (1978, S. 395) definiert Konzentration als „Prozeß willentlich gesteuerter Aufmerksamkeit (...), der bei der gezielten Bearbeitung von Problemen auftritt.“ Die Entwicklung unwillkürlicher durch diskrepante, vom regulären Wahrnehmungsverlauf abweichende Aufmerksamkeitsfunktionen wird von Rollett (2001) als Vorläuferfunktionen der konzentrierten Aufmerksamkeitszuwendung benannt. Nach Posner (1982, zitiert nach Schöttke \& Wiedl, 2001) kann Aufmerksamkeit auf drei Ebenen analysiert werden: (1) der experimentalpsychologischen Verhaltensebene, (2) dem subjektiven Erleben von Aufmerksamkeit bzw. Aufmerksamkeitsstörungen sowie (3) der neuronalen Struktur. Aus experimentalpsychologischer Sicht unterscheiden Schöttke und Wiedl (2001, S. 560) folgende Aspekte von Aufmerksamkeit: 
- Aktivierung bzw. Wachheit

- Selektive Aufmerksamkeit

- Vigilanz und Daueraufmerksamkeit

- Geteilte oder verteilte Aufmerksamkeit

- Kontrollaufmerksamkeit.

Durch geeignete Testverfahren (z.B. mit der Testbatterie zur Aufmerksamkeitsprüfung, Zimmermann \& Fimm, 1993) kann überprüft werden, ob es bei diesen verschiedenen Aufmerksamkeitsfunktionen Probleme gibt.

Aus neurophysiologischer Sicht ist der Grad der Aktivierung ausschlaggebend für Konzentrationsleistungen (Heilmann \& Valenstein, 1985). Störungen der Aktivierung können als unspezifische Einflussgröße die Aufmerksamkeitsstörungen bedingen. Es kann sich sowohl zu geringe wie auch $\mathrm{zu}$ hohe Aktivierung ungünstig auswirken. So führen starke Gefühls- und Erregungszustände zu einer Verminderung von Kontrolle. Für eine Vielzahl von Aufgaben des täglichen Lebens benötigen wir ein mittleres Niveau der Erregung (Eisert, 1981). Der richtige Grad der Aktivierung ist eine Bedingung für konzentriertes Arbeiten. Atkinson, Atkinson, Smith, Bem und Hilgard (1990) heben zwei Hauptfunktionen der Aufmerksamkeit hervor: zum einen die Selektion und zum anderen die Kombination von Reizen. Bei der Selektion der Aufmerksamkeit wird ein bedeutungsvoller Stimulus ausgewählt, irrelevante Stimuli müssen ausgeblendet werden. Die Kombination von Reizen zu einem neuen Ganzen führt zum Aufbau komplexerer kognitiver Strukturen, was eine Voraussetzung für effektive Informationsverarbeitungsprozesse und auch für die Handlungskontrolle von Bedeutung ist. Nach Rollett (2001) ist eine weitere wichtige Komponente der Aufmerksamkeit die Kontrolle. Diese stellt eine Metakomponente der Aufmerksamkeit dar, mit der Handlungs- und Denkprozesse überprüft und korrigiert werden.

$\mathrm{Zu}$ berücksichtigen ist, ob eine Person eine neue Aufgabe lösen muss oder ob es sich um Routineaufgaben handelt. Gehen Kinder an Aufgaben mit der Erwartung heran, dass es für sie keiner großen Anstrengung bedarf umzu einer Lösung zu gelangen („,Das ist ja kinderleicht!"), laufen sie Gefahr Fehler zu machen, weil sie Handlungsroutinen einsetzen, die zwar für bestimmte Aufgaben, aber nicht für jede Aufgabe geeignet sind. Nach Sternberg (1985) sind bei Problemlösungen folgende Strategien bedeutsam: (1) Selektives Enkodieren. Hierbei müssen wichtige von unwichtigen Problemaspekten unterschieden werden, (2) Selektives Kombinieren. Hier geht es um die Integration isolierter Informationen in einen Gesamtzusammenhang und (3) Selektives Vergleichen. Hier müssen neue Erkenntnisse mit 
alten Erklärungsmustern abgeglichen werden und neue Informationsstrukturen gebildet werden. Neben einer gut strukturierten Wissensbasis benötigen Problemlöser auch ein gut funktionierendes kognitives Verarbeitungssystem.

\subsection{Symptomatik, Epidemiologie und Verlauf hyperkinetischer Störungen}

\subsubsection{Kernsymptomatik}

Ein durchgehendes Muster von Hyperaktivität, Unaufmerksamkeit und Impulsivität wird heute als das Hauptmerkmal Hyperkinetischer Störungen gesehen, welches im Vergleich zu Kindern gleichen Entwicklungsstandes häufiger und stärker auftritt (Döpfner, Schürmann \& Frölich, 1998).

Die Unaufmerksamkeit bzw. Störung der Aufmerksamkeit zeigt sich vor allem darin, dass Aufgaben frühzeitig abgebrochen und somit nicht beendet werden. Dies tritt vor allem bei Beschäftigungen auf, die fremdbestimmt sind und die einen kognitiven Einsatz verlangen (Döpfner, 2000). Barkley (2002) weist darauf hin, dass es Kindern mit ADHS besonders schwer fällt, die Aufmerksamkeit über einen längeren Zeitraum aufrecht zu erhalten, und die Konzentrationsfähigkeit eines Zehnjährigen mit ADHS mit der eines siebenjährigen Kindes ohne ADHS vergleichbar wäre. Lauth und Schlottke (1997) beschreiben eine mangelhafte Aufmerksamkeitsorientierung und schlechte Zielgerichtetheit des Verhaltens. Diese wirkt sich auf die Fähigkeit aus, Aufgaben zu organisieren und bei der Lösung das Ziel im Auge zu behalten. Oft „verlieren sie den Faden“ und brechen eine begonnene Tätigkeit ab, weil neue Reize sie ablenken. Kindern mit ADHS fällt es nicht schwerer, Informationen zu filtern, d.h. wesentliche von unwesentlichen Dingen zu unterscheiden. Es fehlt ihnen jedoch an Ausdauer, dies über einen längeren Zeitraum zu tun (vgl. Barkley, 2002). Die Kinder verlieren schnell das Interesse, brechen Aufgaben vorzeitig ab und wechseln wiederholt von einer Tätigkeit zu einer anderen (Döpfner, Frölich \& Lehmkuhl, 2000). Dies passiert insbesondere bei Tätigkeiten, die längerfristig geistige Anstrengung erfordern und nur geringen Verstärkungswert haben (wie z.B. Hausaufgaben).

Hyperaktives Verhalten bezeichnet eine exzessive motorische Unruhe mit mangelhaft regulierten, überschießenden und desorganisierten Bewegungen (Döpfner, Frölich \& Lehmkuhl, 2000). Das Kind hat Schwierigkeiten ruhig zu spielen, klettert und läuft häufig herum, redet viel und verhält sich „wie aufgezogen“. Besonders deutlich zeigt sich dieses Verhalten in Situationen, die ein hohes Maß an Verhaltenskontrolle verlangen (Grosse \& Skrodzki, 2001). 
Barkley (2002) erweitert das Kriterium der Hyperaktivität mit dem der Hyperreaktivität. Demnach lassen sich Kinder mit ADHS mehr durch Dinge in ihrer Umgebung beeinflussen als andere Kinder. Demnach wäre das höhere Aktivitätsniveau eine Folgeerscheinung des impulsiven Reagierens auf äußere Stimuli.

Impulsivität zeigt sich in vorschnellen und unüberlegten Reaktionen. Die Kinder sind ungeduldig, unfähig abzuwarten und Bedürfnisse aufzuschieben und neigen zu gefährlichen und unbedachten Handlungen (z.B. Kletterpartien, leichtsinniges Verhalten im Straßenverkehr). Döpfner, Schürmann und Frölich (1998) grenzen kognitive Impulsivität (plötzliches Handeln ohne hinreichend zu überlegen) von motivationaler Impulsivität (Unfähigkeit, Dinge abzuwarten und Bedürfnisse aufzuschieben) ab.

Motivationale Faktoren scheinen die Ausprägung der Symptomatik zu beeinflussen, da in Situationen, die mit unmittelbarer Verstärkung oder Bestrafung einhergehen, sowie im Einzelkontakt die Symptome deutlich abnehmen. Barkley (1998) berichtet außerdem von weniger auffälligem Verhalten der ADHD-Kinder gegenüber ihren Vätern und geringerer Symptomausprägung in den Vormittagsstunden.

\subsubsection{Diagnose und Diagnostik}

In Deutschland ist für die ambulante und stationäre Versorgung die Kodierung der Diagnosen nach ICD-10 vorgeschrieben, so dass sie auch in dieser Studie verwendet wurde. In der Darstellung wird auf die Forschungskriterien Bezug genommen (Dilling, Mombour \& Schmidt, 1994). Die Klassifikationssysteme ICD-10 und DSM-IV (Saß, Wittchen \& Zaudig, 1996) setzen voraus, dass die Beeinträchtigung durch die Symptome in mindestens zwei Lebensbereichen (z.B. in der Schule und zu Hause) auftreten muss (vgl. Grosse \& Skrodzki, 2001, Dilling et al., 1991).

In den letzten Jahren wurde eine Unterteilung der Hyperkinetischen Störungen in verschiedene Subgruppen angestrebt. Es stellte sich die Frage, ob es sich um zwei verschiedene Ausprägungsformen einer Störung handelt oder ob es zwei voneinander unabhängige Störungen sind (De Quirós, Kinsbourne, Palmer \& Tocci Rufo, 1994). In mehreren Studien konnten Hinweise auf die empirische Validität von Subtypen gefunden werden (z.B. Barkley, DuPaul \& McMurray, 1990; Cantwell \& Baker, 1992; Graetz, Sawyer, Hazell, Arney \& Baghurst, 2001). Dieser Frage wird im DSM-IV und ICD-10 Rechnung getragen, sodass verschiedene Subtypen diagnostiziert werden können. Im DSM-IV werden je nach Ausprägung der Kernsymptomatik Subtypen gebildet. Bei dem vorwiegend hyperaktiv-impulsiven Typ steht die Störung der Daueraufmerksamkeit, der Impulskontrolle, 
der Ablenkung und der verminderten Anstrengungsbereitschaft im Vordergrund. Bei dem vorwiegend unaufmerksamem Typ, häufig auch als Träumertyp bezeichnet, werden Tagträumereien, eher lethargisches Verhalten und Schwierigkeiten bei der Aufmerksamkeitsfokussierung beschrieben (vgl. Barkley, 1997).

Im ICD-10 werden oben genannte Verhaltensauffälligkeiten unter der Kategorie der Hyperkinetischen Störungen (F90) zusammengefasst. Die Diagnose einer einfachen Aktivitäts- und Aufmerksamkeitsstörung (F90.0) wird gestellt, wenn sowohl ausgeprägte Unaufmerksamkeit als auch Überaktivität und Impulsivität erfüllt sind. Daneben wird festgelegt, dass die Symptome mindestens sechs Monate lang in einem mit dem Entwicklungsstand des Kindes nicht zu vereinbarenden und unangemessenen Ausmaß vorliegen, die Störung bereits vor dem Alter von sieben Jahren aufgetreten ist, sich eine Beeinträchtigung durch diese Symptome in zwei oder mehr Lebensbereichen zeigt und deutliche Hinweise auf klinisch bedeutsame Beeinträchtigungen in sozialen, schulischen oder beruflichen Funktionsbereichen bestehen. Als Ausschlussdiagnosen werden das Vorliegen einer tiefgreifenden Entwicklungsstörung, einer depressiven bzw. manischen Episode oder eine Angststörung angegeben. In Tabelle 1 sind die Diagnosekriterien aufgelistet. 
Tabelle 1

Diagnosekriterien der Klassifikationssysteme

\begin{tabular}{|c|c|}
\hline $\begin{array}{l}\text { Aufmerksamkeitsdefizit-/ Hyper- } \\
\text { Aktivitätsstörung nach DSM-IV }\end{array}$ & $\begin{array}{c}\text { Hyperkinetischen Störung } \\
\text { Nach ICD-10 } \\
\text { (Forschungsdiagnose) }\end{array}$ \\
\hline \multicolumn{2}{|c|}{$\begin{array}{l}\text { Im Vergleich zum Entwicklungsstand des Kindes abnormes Ausmaß an: } \\
\text { 1. Unaufmerksamkeit } \\
>\quad \text { Beachtet häufig Einzelheiten nicht oder macht Flüchtigkeitsfehler } \\
>\text { hat Schwierigkeiten, längere Zeit die Aufmerksamkeit bei Aufgaben oder Spielen aufrechtzuerhalten } \\
>\text { scheint häufig nicht zuzuhören, wenn er/ sie angesprochen wird } \\
>\text { führt häufig Anweisungen anderer nicht vollständig durch und kann Aufgaben nicht zu Ende bringen } \\
\text { (nicht aufgrund oppositionellen Verhaltens oder Verständnisschwierigkeiten) } \\
>\text { hat häufig Schwierigkeiten, Aufgaben und Aktivitäten zu organisieren } \\
>\text { vermeidet häufig, hat eine Abneigung gegen oder beschäftigt sich häufig nur widerwillig mit } \\
\text { Aufgaben, die länger andauernde geistige Anstrengung erfordern } \\
>\text { verliert häufig Gegenstände, die für Aufgaben oder Aktivitäten benötigt werden (z.B. Stifte, Bücher) } \\
>\text { lässt sich durch äußere Reize leicht ablenken } \\
>\text { ist bei Alltagsaktivitäten oft vergesslich }\end{array}$} \\
\hline $\begin{array}{l}\text { 3. Impulsivität } \\
\text { platzt häufig mit den Antworten heraus } \\
>\text { kann nur schwer warten, bis er/ sie an c } \\
>\text { unterbricht und stört andere häufig }\end{array}$ & $\begin{array}{l}\text { Ade gestellt ist } \\
\text { Spielen) }\end{array}$ \\
\hline
\end{tabular}

A. Mindestens sechs der Symptome von Unaufmerksamkeit (1) oder mindestens sechs der Symptome von Hyperaktivität und Impulsivität (2 und 3) müssen während der letzten sechs Monate beständig vorhanden gewesen sein.

B. Einige Symptome treten bereits vor dem Alter von sieben Jahren auf.

C. Beeinträchtigungen durch die Symptome zeigen sich in zwei oder mehr Bereichen (z.B. Schule, zu Hause)

D. Die Symptome müssen sich in klinisch bedeutsamen Beeinträchtigungen der sozialen, schulischen oder beruflichen Funktionsfähigkeit manifestieren.

E. Die Symptome treten nicht ausschließlich im Verlauf einer Tiefgreifenden Entwicklungsstörung, Schizophrenie oder einer anderen psychotischen Störung auf und können auch nicht durch eine andere psychische Störung (z.B. Affektive Störung, Angststörung) besser erklärt werden.
A. Mindestens sechs der Symptome von

Unaufmerksamkeit (1) und mindestens drei der Symptome von Überaktivität $u$ nd mindestens eines der Symptome von Impulsivität müssen mindestens sechs Monate lang erfüllt sein.

B. Störungsbeginn liegt vor dem siebten Lebensjahr.

C. Die Kriterien sollten in mehr als einer Situation erfüllt sein (zu Hause, Schule, Klinik).

D. Die Symptome verursachen deutliches Leiden oder Beeinträchtigung der sozialen, schulischen oder beruflichen Funktionsfähigkeit.

E. Die Störung erfüllt nicht die Kriterien für eine tiefgreifende Entwicklungsstörung, manische Episode, depressive Episode oder Angststörung. 
Sind neben der Diagnose einer Hyperkinetischen Störung auch die Kriterien für eine Störung des Sozialverhaltens erfüllt, wird die Kombinationsdiagnose der Hyperkinetischen Störung des Sozialverhaltens (F90.1) vergeben. Daneben sieht das ICD-10 zwei weitere Kategorien vor: die sonstige Hyperkinetische Störung (F90.8) und nicht näher bezeichnete Hyperkinetische Störungen (F90.9), die gewählt werden, wenn einige Kriterien nicht voll erfüllt sind. Weiterhin hat der Diagnostiker die Möglichkeit auch das Vorliegen einer Aufmerksamkeitsstörung ohne Hyperaktivität unter der Kategorie sonstige näher bezeichnete verhaltens- und emotionale Störungen mit Beginn in der Kindheit und Jugend (F98.8) zu kodieren. Beträgt der Intelligenzquotient eines Kindes weniger als 50 und treten hyperkinetische Symptome und Stereotypen auf, wird die Diagnose einer hyperkinetischen Störung mit Intelligenzminderung und Bewegungsstereotypen (F84.4) gestellt (Mickley \& Pisarsky, 2003).

Differentialdiagnostisch ist neben den oben genannten Ausschlussdiagnosen eine Beurteilung weiterer Faktoren nötig. Die Auffälligkeiten sollen nicht Ausdruck altersangemessener Verhaltensweisen bei aktiven Kindern, einer oppositionellen Verhaltensstörung oder Angststörung und nicht die Folge schulischer Unter- oder Überforderung, einer Intelligenzminderung oder chaotischer psychosozialer Bedingungen sein (Döpfner, 2000). Auszuschließen sind auch durch Medikamente oder neurologische Störungen (z.B. Schädel-Hirn-Trauma) bedingte hyperkinetische Symptome, tiefgreifende Entwicklungsstörungen, emotionale Störungen wie Angststörungen oder agitierte Depressionen und affektive Störungen (Knölker, Mattejat \& Schulte-Markwort, 2000). Die nur aufmerksamkeitsgestörten Kindern zeigten eher internalisierende Störungen (Angst-, Zwangs- oder Affektstörungen) und unterscheiden sich damit von den impulsiv-hyperaktiven, die zu externalisierenden Störungen neigen ((Hyperkinetische) Störung des Sozialverhaltens). Es sind eher die Impulskontrolldefizite, welche mit aggressiv-deviantem Verhalten einhergehen als die Hyperaktivität (Cantwell \& Baker, 1992; Halperin et al., 1990; Newcorn, 2001). Aufmerksamkeitsgestörte Kinder ohne Hyperaktivität fallen eher durch sozialen Rückzug und insgesamt langsamere kognitive Verarbeitung auf (Hynd, Lorys, SemrudClikeman, Nieves, Huettner \& Lahey, 1991), wohingegen vorwiegend hyperaktive Kinder im sozialen Umgang öfter abgelehnt werden und zu mehr Flüchtigkeitsfehlern neigen (Cantwell \& Baker, 1992). 


\subsubsection{Fehldiagnosen, assoziierte Probleme und komorbide Störungen}

Ein großes Problem beim Phänomen der hyperkinetischen Verhaltensstörungen stellen Fehldiagnosen dar. Häufig werden Therapiemaßnahmen initiiert, weil die Diagnose nicht korrekt gestellt wurde. Folgende Phänomene führen am häufigsten zur Fehldiagnose einer Hyperkinetischen Störung (Döpfner, 2000):

- Altersgemäße Verhaltensweisen bei aktiven Kindern,

- Durch Medikamente oder durch neurologische Störungen bedingte hyperkinetische Symptomatik,

- Hyperkinetische Symptome bei Intelligenzminderung,

- Hyperkinetische Symptome bei schulischer Überforderung,

- Hyperkinetische Symptome bei schulischer Unterforderung,

- Hyperkinetische Symptome als Folge chaotischer psychosozialer Bedingungen,

- Oppositionelle Verhaltensweisen,

- Psychomotorische Erregung und Konzentrationsstörungen bei affektiven Störungen und Angststörungen,

- Autismus, Schizophrenie, Manie.

Differentialdiagnostische Erwägungen müssen diesen Punkten Rechnung tragen. Es gilt zu entscheiden, ob bei Vorliegen der zuvor genannten Aspekte komorbid eine weitere Störung vorliegt oder ob eine übergeordnete Störung alle vorliegenden Symptome besser erklären kann.

Hyperkinetische Störungen sind häufig von weiteren psychischen Störungen begleitet, die den Verlauf erheblich beeinflussen und für die Entwicklung des Kindes zusätzliche Risikofaktoren darstellen (Barkley, 1998; Döpfner, Frölich \& Lehmkuhl, 2000; Taylor et al., 1998). Die zugrunde liegenden Mechanismen für die überzufällige Häufung sind bislang noch weitgehend ungeklärt (Tannock, 1998). Aufgrund der hohen Komorbiditätsrate ist es notwendig, dass bei der Entwicklung und Durchführung von Interventionsmaßnahmen neben der Kernsymptomatik begleitende Beeinträchtigungen und Probleme mitberücksichtigt werden. Eine besonders hohe Überschneidung besteht zwischen Hyperkinetischen Störungen und Störungen des Sozialverhaltens. Die Eigenständigkeit der Diagnosen wurde deshalb lange Zeit in Frage gestellt. In empirischen Untersuchungen konnte jedoch die Validität der Diagnose „Hyperkinetische Störungen“ gestützt werden. Es sind „reine“ Fälle beider diagnostischen Kategorien möglich, die durch unterschiedliche klinische Merkmale, 
Entstehungswege und Verläufe gekennzeichnet sind (vgl. Barkley, 1998; Fergusson, Horwood \& Lynskey, 1993; Halperin et al., 1993). In einer Zwillingsstudie fanden sich Hinweise, dass Aufmerksamkeitsstörungen und die Hyperkinetische Störung des Sozialverhaltens eine ähnliche genetische Herkunft teilen (Thapar, Harrington \& McGuffin, 2001). Zwischen beiden Störungen bestehen Überlappungen zwischen 30\% und 90\% (Hinshaw, 1987), womit eine eindeutige Diagnose oft erschwert ist.

10-25\% dieser Kinder weisen Teilleistungsstörungen auf (Döpfner, 2000). Sie wiederholen häufiger eine Klasse und werden vermehrt in Sonderschulen überwiesen (Faraone et al., 1993; Mannuzza \& Klein, 2000). Überzufällig häufig werden auch umschriebene Entwicklungsstörungen der Sprache bei diesen Kindern festgestellt (ebd.). Hinzu kommen eine Reihe kognitiver Defizite, u.a. beim Problemlösen, dem Behalten komplexer verbaler und nonverbaler Informationen, der visuell-räumlichen Merkfähigkeit und der verbalen bzw. behavioralen Flüssigkeit (Barkley, 1997). Etwa die Hälfte aller Kinder mit einer Hyperkinetischen Störung hat deutliche Probleme in sozialen Beziehungen mit Gleichaltrigen, da sie sich anderen Kindern gegenüber oft penetrant, bestimmend und kritisierend verhalten (Guevremont \& Dumas, 1994). Sie unterbrechen die Aktivitäten anderer, verletzen Regeln und zeigen oftmals wenig empathisches oder sogar aggressives Verhalten. Konflikte in der Familie sind ebenfalls häufig. Die Interaktionen zwischen Eltern und Kindern sind oft negativ, bestrafend und kontrollierend (Johnston, 1996; Saile, Röding \& Friedrich-Löffler, 1999). Eine Vielzahl der Kinder mit Hyperkinetischen Störungen ist in Leistungssituationen mit wiederholtem Versagen konfrontiert oder stößt immer wieder auf Ablehnung in sozialen Situationen. Dies kann sowohl die Ausprägung von Angststörungen wie auch von affektiven Störungen begünstigen (vgl. Krowatschek, 2003).

Etwa $25 \%$ der Kinder, die die Kriterien für die Diagnose einer Hyperkinetischen Störung erfüllen, haben eine komorbide Angststörung. Affektive Störungen, v.a.Depressionen sind bei 10-40 \% der Kinder mit hyperkinetischer Störung anzutreffen (Barkley, 1998; Döpfner, 2000).

Bei etwa einem Drittel der Kinder mit HKS liegt eine komorbide Ticstörung vor. Kinder und Jugendliche mit Tourette-Störung weisen in 60-70 \% der Fälle gleichzeitig eine Hyperkinetische Störung auf (Döpfner, 2000; Eisert, 1998). Ebenfalls gehäuft anzutreffen ist eine Enuresis. 


\subsubsection{Prävalenz und Verlauf}

\section{Prävalenz}

Im Schulalter werden psychiatrische Diagnosen bei Jungen im Vergleich zu Mädchen etwa 2-3 mal so häufig gestellt. Neben Störungen des Sozialverhaltens gehören Hyperkinetische Störungen $\mathrm{zu}$ den häufigsten Verhaltensstörungen im Grundschulalter (Döpfner, Frölich \& Lehmkuhl, 2000). Die Prävalenz wird in den westlichen Kulturen auf etwa 3-5 \% der Grundschüler geschätzt (Baumgaertel, Wolraich \& Dietrich, 1995; Brühl, Döpfner \& Lehmkuhl, 2000; Saß, Wittchen \& Zaudig, 1996). Hierbei zeigt sich ein bedeutsamer Unterschied hinsichtlich der Geschlechterverteilung. Die Störung betrifft primär Jungen. So findet sich beim vorherrschend unaufmerksamen Typ ein Verhältnis von Jungen zu Mädchen von 2 zu 1, während beim hyperaktiv-impulsiven Typ sogar ein Verhältnis von 5 zu 1 besteht (Grosse \& Skrodzki, 2001). Das Verhältnis von Jungen zu Mädchen wird in repräsentativen Stichproben als etwa 4 zul und in klinischen Stichproben sogar als etwa 9 zu 1 angegeben (Scahill \& Schwab-Stone, 2000). Der Unterschied könnte auf die erhöhte Rate komorbider expansiver Verhaltensstörungen bei Jungen zurückzuführen sein, die häufiger zu einer Inanspruchnahme kinder- und jugendpsychiatrischer Einrichtungen führen, weil bei den Eltern und den Lehrern ein erhöhter Leidensdruck entsteht.

\section{Verlauf}

Hyperkinetische Symptome fallen zumeist schon im Kleinkind- und Vorschulalter als schwierige Temperamentsmerkmale (hohes Aktivitätsniveau, mangelnde Ausdauer, forderndes Verhalten, Erregbarkeit und Gereiztheit, oppositionelle oder aggressive Verhaltenstendenzen, mangelnde soziale Integrierbarkeit, Schlaf- und Essprobleme) auf. Treten zu diesen negativen Temperamentsmerkmalen ungünstige familiäre Bedingungen hinzu (psychische Störungen der Eltern, Störungen der Interaktion zwischen Kind und Bezugspersonen, Eheprobleme, niedrige Bildung der Eltern, unvollständige Familien), so ist das Risiko für die Entwicklung und Persistenz psychischer Störungen deutlich erhöht (Barkley, 1998).

Mit Eintritt in die Schule gestalten sich neue Probleme für die Kinder, besonders im Lern- und Leistungsbereich. Es kommt nun zu einer Doppelbelastung, die einerseits aus sich verstärkenden Symptomen der Störung bestehen und andererseits aus dadurch bedingten Schwierigkeiten beim Lernen. Neben diesen Problemen kommen zwischenmenschliche Konflikte nicht nur in der Schule hinzu. Z.B. sind die Geschwister von aufmerksamkeitsgestörten Kindern oft eifersüchtig, weil ihren beeinträchtigten Brüdern und 
Schwestern mehr Aufmerksamkeit durch die Eltern geschenkt wird (Barkley, 1998). Hinzu treten abweisendes, nicht akzeptierendes und konfliktreiches Verhalten Gleichaltriger, wodurch sich die Einbindung aufmerksamkeitsgestörter Kinder in soziale Aktivitäten, wie Vereine, oft sehr schwierig bis unmöglich gestaltet.

Im Jugendalter bis hin zum Erwachsenenalter findet sich immer noch eine beträchtliche Stabilität der Symptome, wobei sich eine Symptomverschiebung weg von hyperaktiven $\mathrm{zu}$ mehr unaufmerksamen und impulsiven Verhaltensweisen erkennen lässt (Döpfner, Frölich \& Lehmkuhl, 2000; Steinhausen, 2000). Biederman, Faraone, Taylor, Sienna, Williamson und Fine (1998) konnten eine hohe Kontinuität von Hyperkinetischen Störungen im Kindes- und Jugendalter nachweisen. Zwischen hyperkinetischen Kindern und Jugendlichen bestand eine hohe Übereinstimmung hinsichtlich der klinischen Merkmale, komorbiden Störungen, psychosozialen Faktoren und verschiedenen Funktionsmerkmalen. Schwierige Problemfelder und häufiger Anlass für die Inanspruchnahme professioneller Hilfen sind im Jugendalter vor allem aggressives und dissoziales Verhalten, Schulleistungsprobleme, Schwierigkeiten mit Gleichaltrigen und Konflikte im familiären Zusammenleben sowie Selbstwertprobleme, die mit der hyperkinetischen Symptomatik einhergehen oder sich daraus ergeben (Döpfner, 2000; Steinhausen, 2000). Antisoziales Verhalten manifestieren hauptsächlich die hyperkinetischen Jugendlichen, die schon im Kindesalter durch komorbide Störungen des Sozialverhaltens auffielen. Dissoziale Verhaltensauffälligkeiten im Zusammenhang mit Hyperkinetischen Störungen gehen mit einem erhöhten Risiko für Substanzmissbrauch als Jugendliche (Fergusson, Lynskey \& Horwood, 1993) sowie für antisoziale Auffälligkeiten im Erwachsenenalter einher (Barkley, 1998; Steinhausen, 2000).

Aufmerksamkeitsdefizit-/ Hyperaktivitätsstörungen persistieren bei bis zu $80 \%$ der Fälle bis in die Adoleszenz und zeigen bei etwa der Hälfte der betroffenen Personen Auswirkungen bis ins Erwachsenenalter (Barkley, 1998; Steinhausen, 2000). Die störungsspezifischen Symptome unterliegen jedoch gewissen Entwicklungsveränderungen. Während der Adoleszenz werden Symptome ausgeprägter motorischer Aktivität seltener, Aufmerksamkeitsdefizite und Impulsivität bestehen dagegen fort (Döpfner, 2000).

Bei Erwachsenen mit Hyperkinetischen Störungen in der Vorgeschichte sind häufiger internalisierende Symptome (Ängste, Depressionen, Selbstwertprobleme), eine geringere akademische Bildung und ein niedrigerer sozioökonomischer Status, beeinträchtigte soziale Beziehungen und Interaktionen sowie vermehrt Gesetzesüberschreitungen und Gerichtsverhandlungen anzutreffen (Barkley, 1998). Eine insgesamt relativ kleine Gruppe 
hyperkinetisch auffälliger Kinder (15-30 \%) zeigt dissoziale und delinquente Entwicklungen und manifestiert im Erwachsenenalter eine Antisoziale Persönlichkeitsstörung (Döpfner, 2000; Herrero, Hechtman \& Weiss, 1994; Schmidt, Esser \& Moll, 1991). Diese Gruppe weist zumeist auch erhöhte Raten von Substanzmissbrauch auf (Barkley, 1998; Steinhausen, 2000).

Als Risikofaktoren für einen prognostisch ungünstigen Verlauf der Störung konnten eine geringe Intelligenz, aggressives und oppositionelles Verhalten im Kindesalter, schlechte Beziehungen zu Gleichaltrigen, emotionale Instabilität, chronische Konflikte, schlechte Familienkohäsion und elterliche psychische, insbesondere mütterliche Störungen ermittelt werden (Döpfner, Frölich \& Lehmkuhl, 2000). Daraus lässt sich folgern, dass eine frühzeitige Identifizierung von Risikofaktoren einer Chronifizierung entgegen wirken kann. Bei der Behandlung muss stets abgewogen werden, ob eine alleinige Interventionsmaßnahme beim Kind ausreichend ist oder ob die Eltern ebenfalls in die Behandlung mit einbezogen werden müssen.

\subsection{Pathogenese}

\subsubsection{Neurobiologische Faktoren der Ätiologie}

Mittels EEG-Ableitungen, pharmakologischen Studien und bildgebenden Verfahren (u.a. CT, MRT und PET) konnten sowohl strukturelle Auffälligkeiten (z.B. verringerte Volumina von Gehirnzentren) als auch funktionelle Auffälligkeiten, wie veränderte Gehirnaktivitätsmuster, verminderte cerebrale Metabolisierungsprozesse sowie Störungen in mehreren Neurotransmittersystemen, vor allem dem dopaminergen und noradrenergen System, festgestellt werden (Barkley, 1998; Biederman \& Spencer, 2000; Döpfner, 2000; Giedd, Blumenthal, Molloy \& Castellanos, 2001; Hechtman, 1994; Krause, Dresel \& Krause, 2000; Levy \& Swanson, 2001; Rapoport et al., 2001; Rothenberger \& Moll, 1998; Rubia et al. 1999; Steinhausen, 2000; Tannock, 1998; Trott \& Badura, 2001).

Diese Veränderungen scheinen die Basis für eine dysfunktionale Informationsverarbeitung zu sein, da sie auf neuropsychologischer Ebene mit Störungen der kognitiven und affektiven Selbstregulation, der Flexibilität im Denken, der Reaktionshemmung sowie der Planung und Organisation von Verhaltensweisen korreliert sind (Döpfner, Frölich \& Lehmkuhl, 2000). Funktionen des präfrontalen Kortex stehen somit im Mittelpunkt derzeitiger Forschungen zu biologischen Korrelaten Hyperkinetischer Störungen. Erfahrungen mit dem Wirkmechanismus von Psychostimulanzien, die am Katecholaminhaushalt angreifen, legen hier ebenfalls einen Zusammenhang zu Grunde (Faraone \& Biederman, 1998). Untersuchungen mittels bildgebender Verfahren fanden einen 
reduzierten Glukosemetabolismus (Zametkin et al., 1993), einen verringerten Blutfluss im Frontalhirn (Lou, Henrickson \& Bruhn, 1984) mit einer tendenziellen Verbesserung nach Methylphenidatgabe sowie insgesamt eine Verlangsamung der frontalen Aktivation (Armstrong, Hayes \& Martin, 2001; Baving, 1999; Chabot, di Michele, Prichep \& John, 2001). Ferner fand sich eine geringere Adrenalinaktivität aufmerksamkeitsgestörter Kinder während der Bearbeitung kognitiver Tests (Anderson et al., 2000).

Die neurobiologischen Modelle gehen davon aus, dass Hyperkinetische Störungen aufgrund einer biologischen Vulnerabilität entstehen können, die sich auf neurologischer Ebene in biochemischen Funktionsstörungen und einer Abweichung in der Entwicklung zentralnervöser Strukturen zeigt. Die Entwicklung und Steuerung neuropsychologischer Funktionen ist dadurch beeinträchtigt, was letztlich in den Verhaltens-, emotionalen und kognitiven Auffälligkeiten der Kinder sichtbar wird.

\section{Genetische Faktoren}

Basierend auf den Ergebnissen von Familien-, Adoptions- und Zwillingsstudien werden genetische Faktoren als primäre Ursache des Störungsbildes postuliert (vgl. Barkley, 1998; Döpfner, 2000; Hechtman, 1994; Tannock, 1998). In Familienstudien konnten eine familiäre Häufung von HKS sowie eine generell höhere Rate psychischer Störungen (v.a. Störungen des Sozialverhaltens, Substanzmissbrauch und affektive Störungen) bei Eltern und Verwandten von hyperkinetisch auffälligen Kindern nachgewiesen werden. Letzteres trifft insbesondere für Verwandte von Kindern mit HKS und zusätzlichen Störungen des Sozialverhaltens zu. Adoptionsstudien ergaben, dass Kindern mit HKS hinsichtlich hyperkinetischer Merkmale ihren biologischen Eltern mehr ähneln als ihren Adoptiveltern. Die Bedeutung erblicher Faktoren wird auch durch Zwillingsstudien gestützt, die bei eineiigen Zwillingen wesentlich höhere Konkordanzraten als bei zweieiigen Zwillingen aufzeigten. In Zwillingsstudien konnten im Mittel $80 \%$ der Varianz durch genetische Faktoren erklärt werden (Barkley, 1998; Biederman et al., 1992; Biederman et al., 1995; Tannock, 1998). Bis heute sind jedoch weder die Art des familiären Erbganges noch die beteiligten Gene eindeutig identifiziert (ebd.). Neuere Überlegungen weisen auf eine möglicherweise heterogene genetische Grundlage für verschiedene Phänotypen der Störung hin (Faraone, 2000; Todd, 2000). 
Schädigungen des ZNS; weitere Faktoren

Hyperkinetische Verhaltensauffälligkeiten können auch durch erworbene Hirnschädigungen ausgelöst oder verstärkt werden. Schädigungen des ZNS können durch prä-, peri- oder postnatale Risiken wie Schwangerschafts- oder Geburtskomplikationen, Verletzungen, Infektionen oder Exposition gegenüber toxischen Substanzen (Blei, Alkohol, Nikotin) bedingt sein (Barkley, 1998; Döpfner, 2000). Lange Zeit wurden frühkindliche Hirnschädigungen (Konzept der Minimalen Cerebralen Dysfunktion - MCD) als Hauptursache für Aufmerksamkeits- und Hyperaktivitätsstörungen betrachtet. Allerdings lassen sich bei der Mehrzahl hyperkinetisch auffälliger Kinder keine Hinweise auf Hirnschädigungen in der Vorgeschichte feststellen. Andererseits sind derartige Risikofaktoren aber durchaus auch bei Kindern ohne hyperkinetische Symptome zu finden (Steinhausen, 2000). Hirnschädigungen stellen demzufolge keinen spezifischen Ätiologiefaktor für HKS dar, sondern sind eher als organischer Risikofaktor für die Entwicklung psychischer Störungen anzusehen. Von Barkley (1998) werden die Ergebnisse der Studien zu neurologischen Auffälligkeiten dahingehend zusammengefasst, dass Abweichungen in der Entwicklung der Entwicklung des frontalen-striatalen Cortex mit großer Wahrscheinlichkeit der Entstehung hyperkinetischen Verhaltens zugrunde liegt.

Feingold (1975) brachte einen weiteren ätiologischen Ansatz in die Diskussion. Er vermutete, dass Allergien und Nahrungsmittelunverträglichkeiten einen Einfluss auf die hyperkinetische Symptomatik haben. Diese seien als allergische Reaktion auf bestimmte synthetische Nahrungsmittel anzusehen. Kontrollierte Studien konnten bei einzelnen Kindern die Verringerung der Symptomatik durch diätetische Maßnahmen aufzeigen, bei der Mehrzahl der Kinder hatten diese jedoch keine Einfluss (vgl. Conners, 1980).

Geteilte Umweltfaktoren (psychosoziale Faktoren wie soziale Klasse, familiärer Bildungsstatus, Wohnverhältnisse und Eltern-Kind-Interaktionen) erklären nur bis zu $13 \%$ der interindividuellen Unterschiede (Barkley, 1998). Ihr Einfluss auf die Genese der Störung ist also als gering zu betrachten.

\subsubsection{Neuropsychologische Modelle der Ätiologie}

Neuropsychologische Modelle versuchen dem Phänomen Rechnung zu tragen, dass Kinder mit Hyperkinetischen Störungen im Bereich exekutiver Funktionen häufig Probleme aufweisen. Dies betrifft nach Döpfner (2000) die Phänomene „Selbstregulation, Flexibilität 
im Denken, Reaktionshemmung, Planen und Organisieren von Verhalten sowie dessen Sequenzierung“. Um eine funktionsfähige Informationsverarbeitung zu gewährleisten, ist es notwendig, Prozesse höherer Ordnung wie Selbstregulation und Hemmung zu regulieren (Barkley, 1997).

Für diese Studie sind die Modelle von Douglas (1980) und Barkley $(1989,1997)$ von Relevanz und sollen deshalb kurz erläutert werden. Douglas (1980) stellte ein Modell vor, welches die hyperkinetische Symptomatik als Störung der Selbstregulation begreift. Dem Kind gelingt es demnach nicht, sich auf der physiologischen Ebene, der Verhaltens- und der kognitiven Ebene an situative Anforderungen anzupassen. Dies führt zu der Schwierigkeit, den kognitiven Erregungszustand an die unterschiedlichen Anforderungen anzugleichen und damit zu einer verminderten Aufmerksamkeitsleistung, impulsiven Verhalten sowie dem permanenten Suchen nach stimulierenden Reizen. Daraus leitet sich dann auch die motorische Unruhe ab. Douglas weist darauf hin, dass diese Selbstregulationsstörung sich negativ auf das planvolle Vorgehen bei der Aufgabenbearbeitung, die metakognitive Entwicklung sowie die Bildung kognitiver Schemata höherer Ordnung auswirkt.

Von Barkley (1989) wurde dieses Modell kritisiert, da die postulierten basalen Defizite situationsabhängig variieren und die Entstehung aggressiven und hyperkinetischen Verhaltens von Douglas nicht hinreichend beschrieben wird. Barkley definiert in seinem Modell der Störung des regelgeleiteten Verhaltens drei basale Störungen in der Beziehung $\mathrm{zu}$ Umweltereignissen und dem Verhalten. Demnach führt eine verminderte Verhaltenskontrolle durch diskriminative Reize dazu, dass eine Reizkontrolle nur bei massiven Verstärkerbedingungen gelingt, während partielle oder verzögerte Verstärkungen zu Unaufmerksamkeit und Impulsivität führen. Die reduzierte Verhaltenskontrolle durch Regeln hat zur Folge, dass eine verminderte situationsangepasste Verhaltenssteuerung und Beeinträchtigungen in der Befolgung von Regeln entstehen. Das bedeutet, dass sich die Kinder mit Hyperkinetischen Störungen nur dann an Regeln halten, wenn sie besonders deutlich gemacht werden. Durch das häufige und schnelle Wechseln von Spielmaterialien oder die Unterbrechung von Aufgaben kommt es zu Beeinträchtigungen beim Problemlösen und zielgerichtetem Verhalten sowie zu Verhaltensweisen, die häufige und unmittelbare Verstärkungen maximieren. Dies hat wiederum Auswirkungen auf verschiedene Lebensbereiche wie verminderte Schulleistungen, Zunahme an familiären Konflikten und eine geringe Akzeptanz von Gleichaltrigen, weil sich die betroffenen Kinder über gängige Normen hinwegsetzen bzw. es nicht schaffen, diese umzusetzen. 
In seinem einflussreichen Modell von 1997 verbindet Barkley Theorien über neuropsychologische Funktionen des präfrontalen Kortex und den Erkenntnissen neurobiologischer Studien. Barkley definiert einen exekutiven Steuerungsprozess, den er mit dem Begriff der Verhaltenshemmung beschreibt. Er wird durch drei neuropsychologische Prozesse initialisiert, die Barkley folgendermaßen definiert: (a) Hemmung eines dominanten Handlungsimpulses, (b) Unterbrechung einer laufenden Handlung und (c) Hemmung interferierender Handlungstendenzen. Durch diese Prozesse soll eine Verzögerung zwischen einem Reiz (Ereignis), welches ein Kind wahrnimmt, und dessen Reaktion darauf entstehen. Das Kind hat dadurch die Möglichkeit eine zur Situation passende Reaktion zu generieren.

Durch die exekutiven Funktionen ist das Kind in der Lage sein Verhalten zu planen und zu kontrollieren. Zu den exekutiven Funktionen zählt Barkley das Arbeitsgedächtnis, die Selbstregulation von Affekten, Motivation und Erregung, die Internalisierung und Automation von Sprache sowie die Analyse und Entwicklung von Handlungssequenzen. Sie haben direkten Einfluss auf motorische Prozesse, z.B. aufgabenirrelevante Reaktionen zu unterdrücken, aufgabenrelevante Reaktionen aufrecht zu erhalten und flexibel reagieren zu können.

Das Arbeitsgedächtnis ist bei den Kindern mit HKS nicht altersentsprechend entwickelt. Aufgrund dessen haben die Kinder Schwierigkeiten damit Handlungen zu planen und zu organisieren. Aus Erfahrungen der Vergangenheit zu lernen und für die Zukunft zu planen, gelingt nur bedingt. Sie können ihre Erlebnisse nur schwer mit aktuellen Ereignissen abgleichen und neigen demnach dazu immer wieder die gleichen Fehler zu begehen bzw. anscheinend nicht aus ihnen zu lernen.

Die Aufmerksamkeitsstörung erklärt Barkley als sekundäre Störung. Während die interne Selbstkontrolle aufgrund der Beeinträchtigung in der Verhaltenshemmung und den exekutiven Funktionen bei Kindern mit einer Hyperkinetischen Störung beeinträchtigt ist, kann Aufmerksamkeit mit Hilfe externer Kontrolle (Verstärkungen, Bestrafungen) beeinflusst werden. Dies erklärt Befunde, die zeigen, dass sich diese Kinder von gesunden Kontrollkindern häufig nur dann in ihren Leistungen unterscheiden, wenn sofortige und wiederkehrende Verstärkungen ausbleiben und längere Verhaltensketten bei verzögerter Verstärkung erwartet werden (Slusarek, Velling, Bunk \& Eggers, 2001).

Da Defizite in exekutiven Funktionen jedoch nicht ausschließlich bei dieser Störung auftreten (Pennington \& Ozonoff, 1996), müssen zukünftige Studien zeigen, welches Defizitprofil exekutiver Funktionen speziell für diese Kinder kennzeichnend ist. Unklar ist bis heute u.a. auch die genaue Bedeutung der Beziehung zwischen der Verhaltenshemmung und 
den exekutiven Funktionen zueinander und ob exekutive Funktionen beeinflussbar sind (Barkley, 1997).

\subsubsection{Aufrechterhaltung; Biopsychosoziales Modell}

Döpfner (2000) integriert den Ansatz der Störung des regelgeleiteten Verhaltens in das biopsychosoziale Modell zur Entstehung von Aufmerksamkeitsstörungen. Er geht von einer genetischen Disposition aus, welche eine Störung im Dopaminstoffwechsel bewirkt. Der Einfluss allergischer Reaktionen oder erworbener Hirnschädigungen ist in Einzelfällen nachweisbar, generalisierbare Befunde stehen jedoch aus. Die Störungen der Selbstregulation auf der neurophysiologischen Ebene führen zu den oben beschriebenen Störungen der Exekutivfunktionen, welche sich auf der Symptomebene als die Kernsymptome Hyperaktivität, Unaufmerksamkeit und Impulsivität darstellen. Diese bedingen eine Zunahme negativer Interaktionen zwischen dem Kind und seinen Bezugspersonen bzw. Peers und führen bei einem Teil der Kinder zu komorbiden Symptomen. Nach dem heutigen Wissenstand spielen psychosoziale Faktoren nur eine sekundäre Rolle bei der Entstehung der Aufmerksamkeitsstörung. Sie tragen jedoch entscheidend zur Aufrechterhaltung, Verlauf und Schweregradentwicklung der Störung bei und müssen mit berücksichtigt werden. Im Zusammenhang mit HKS wurden gehäuft auftretende negative Interaktionen zwischen dem Kind und seinen Bezugspersonen (Eltern, insbesondere der Mutter; Lehrern, Gleichaltrigen), verschiedene familiäre und schulische Belastungsfaktoren (z.B. familiäre Instabilität, elterliche Psychopathie, niedrige soziale Schicht, große Klassen) sowie unangemessenes Erziehungsverhalten (Inkonsistenz, mangelndes Interesse am Kind, übermäßige Bestrafung) als ungünstige psychosoziale Bedingungen ausgemacht (Döpfner, 2000; Steinhausen, 2000).

\subsection{Zentrale Aspekte bei der Diagnostik von Aufmerksamkeitsstörungen}

Pitzer und Schmidt (1999) fordern, dass psychische Auffälligkeiten oder Krankheiten sorgfältig definiert sein müssen. In der Literatur wird kontrovers diskutiert, ob eine kategoriale oder eine dimensionale Einteilung von Störungen sinnvoll ist. Psychische Probleme können dimensional über Symptomlisten definiert werden. Es wird dabei ein cutoff-Wert festgesetzt, um die klinische Relevanz der Auffälligkeit zu beurteilen. Problematisch ist hier jedoch, dass es bei psychischen Störungen in der Regel keinen natürlichen Grenzwert gibt, von dem ab ein Zusammenaufteten von Symptomen eine zweifelsfreie Diagnose ermöglicht (Detzner \& Schmidt, 1988). Kategorial lassen sich psychiatrische Diagnosen über die Klassifikationssysteme ICD-10 und DSM-IV stellen. Dies sollte durch einen erfahrenen 
Kliniker geschehen. Problematisch wird hier allerdings die niedrige Reliabilität der Diagnosen gesehen (Remschmidt, Schmidt \& Goebel, 1983).

Die Informationen können durch verschiedene Quellen gewonnen werden: durch Untersuchungen des Kindes selbst, Befragungen von Eltern und Lehrern. Nicht selten kommt es zu unterschiedlichen Einschätzungen. Bei expansiven Störungen können in der Regel Eltern und Lehrer gut Auskunft geben, bei emotionalen Störungen können die Kinder und Jugendlichen sich selbst am besten einschätzen (Pitzer \& Schmidt, 1999). Ein Abgleich der verschiedenen Informationen kann dem Kliniker helfen zu beurteilen, wie problematisch das Verhalten vom Kind selbst und seiner Umwelt eingestuft wird bzw. in welchen Lebensbereichen das problematische Verhalten überhaupt gezeigt wird.

Heubrock und Petermann (2001) beschreiben vier Ebenen, auf denen sich im diagnostischen Prozess Informationen gewinnen lassen können:

1. Eine ausführliche Exploration der Bezugspersonen und des Kindes sollte stets an den Anfang einer Untersuchung gestellt werden. Im Sinne einer Verhaltensanalyse sollte das Auftreten und auch Nichtauftreten der Symptomatik in bestimmten Situationen eruiert werden.

2. Direkte Verhaltensbeobachtungen des Kindes im natürlichen Umfeld (Schule, Elternhaus) helfen das Ausmaß der Störung im Lebensalltag einzuschätzen. Kritisch anzumerken ist jedoch, dass dies in vielen Institutionen aus zeitlichen Gründen kaum $\mathrm{zu}$ realisieren sein wird.

3. Direktes Beobachten und Registrieren der motorischen Aktivität. Hierzu können z.B. Aktimeter für die direkte Erfassung der motorischen Aktivitäten bzw. Ratingskalen für die Beobachtung eingesetzt werden.

4. Einsatz psychometrischer Verfahren (Paper-Pencil-Tests und computergestützte Verfahren).

Fragebögen sind ökonomisch und als Screeninginstrumente gut geeignet. Bei halbstandardisierten Interviews können systematisch psychiatrische Krankheitsbilder abgefragt werden, auffällige Bereiche dann genauer exploriert werden. Diese Interviews bieten sich als Ergänzung zu Fragebogenverfahren an. Die Verhaltensbeobachtung und der Einsatz von Testverfahren können das Vorliegen der Aufmerksamkeitsstörung diagnostisch untermauern. Diese Informationen sind von den Angaben der Bezugspersonen unabhängig und damit möglicherweise objektiver. Verhaltensbeobachtungen sind selten standardisiert, können aber z.B. durch Videoaufnahmen valider gestaltet werden. Hier entsteht allerdings ein sehr hoher Zeitaufwand. Durch psychometrische Untersuchungsverfahren können einzelne 
Komponenten der Aufmerksamkeit gezielt untersucht werden, die im Alltag miteinander konfundiert sind. Den Papier-Bleistift-Tests liegen üblicherweise monotone, kognitiv meist anspruchslose Aufgaben zugrunde. Die computergestützte Testdiagnostik hat den Vorteil, dass verschiedene Aspekte von Aufmerksamkeitsleistungen differenzierter und genauer gemessen werden können. Außerdem können mehrere Sinnesmodalitäten einbezogen werden (vgl. Barkley, 1998; Döpfner, 2000; Eisert, 1998; Taylor et al., 1998). Döpfner (2000) weist darauf hin, dass eine Aufmerksamkeitsbeeinträchtigung bei spannenden, das Kind interessierenden Tätigkeiten oder in einer für es neuen Situation oft nicht zu beobachten ist. Dies ist für den Diagnostiker wichtig, der in einer Untersuchungssituation mit dem Kind allein das fraglich problematische Verhalten evtl. nicht beobachten kann, weil das Kind das aufmerksamkeitsgestörte Verhalten in der Untersuchungssituation gar nicht zeigt. Der Diagnostiker ist bei der Beurteilung des Verhaltens also besonders auf die Einschätzung Dritter (Eltern, Lehrer, Erzieher) angewiesen. $\mathrm{Zu}$ bedenken ist, dass bei vielen Kindern mit hyperkinetischen Störungen die problematischen Verhaltensweisen erst im Gruppenkontext auftreten. Für eine valide Diagnostik wären deshalb neben Konzentrationstests zusätzlich Unterrichtsbeobachtungen hilfreich.

Mit der Störung der Aufmerksamkeit gehen häufig schulische Leistungsprobleme einher. Da eine hyperkinetische Symptomatik jedoch auch bei schulischer Überforderung beobachtet wird, ist eine Intelligenzdiagnostik sowie Überprüfung fraglicher Teilleistungsschwächen unabdingbar. Krowatschek (2003) empfiehlt außerdem die Überprüfung der emotionalen Situation des Kindes mittels geeigneter Testverfahren.

Da die ADHS als Hauptmerkmal die Störung der Aufmerksamkeit enthält, sollte diese mit geeigneten Verfahren untersucht werden. Hierbei muss zwischen den einzelnen Aufmerksamkeitsbereichen Alertness, Vigilanz, selektive, geteilte Aufmerksamkeit und Daueraufmerksamkeit unterschieden werden (vgl. Hildebrandt \& Fecht, 2003).

Einen guten Überblick über den Diagnostikprozess gibt die Abbildung 1 (nach Heubrock \& Petermann, 2001). Dabei gehören auch die beiden letzten Phasen zum Diagnostikprozess, da sich bei der Motivationsanalyse und Vereinbarung der Therapieziele sowie der Durchführung der Intervention häufig noch weitere Aspekte ergeben, die diagnostisch verwertbar sind und anhand derer das gesamte Vorgehen angepasst werden muss. Mit der Bedingungsanalyse werden Faktoren identifiziert, die zur Aufrechterhaltung oder Verschärfung der Probleme beitragen. Dies könnte z.B. ein Erziehungsstil der Eltern sein, der dazu führt, dass bestimmte Verhaltensweisen der Kinder verstärkt werden. Häufig bekommen Kinder bei störendem Verhalten Aufmerksamkeit durch die Eltern, welche in 
Form von Schimpfen zwar negativ getönt ist. Negative Aufmerksamkeit ist vielen Kindern jedoch lieber als gar keine Aufmerksamkeit.

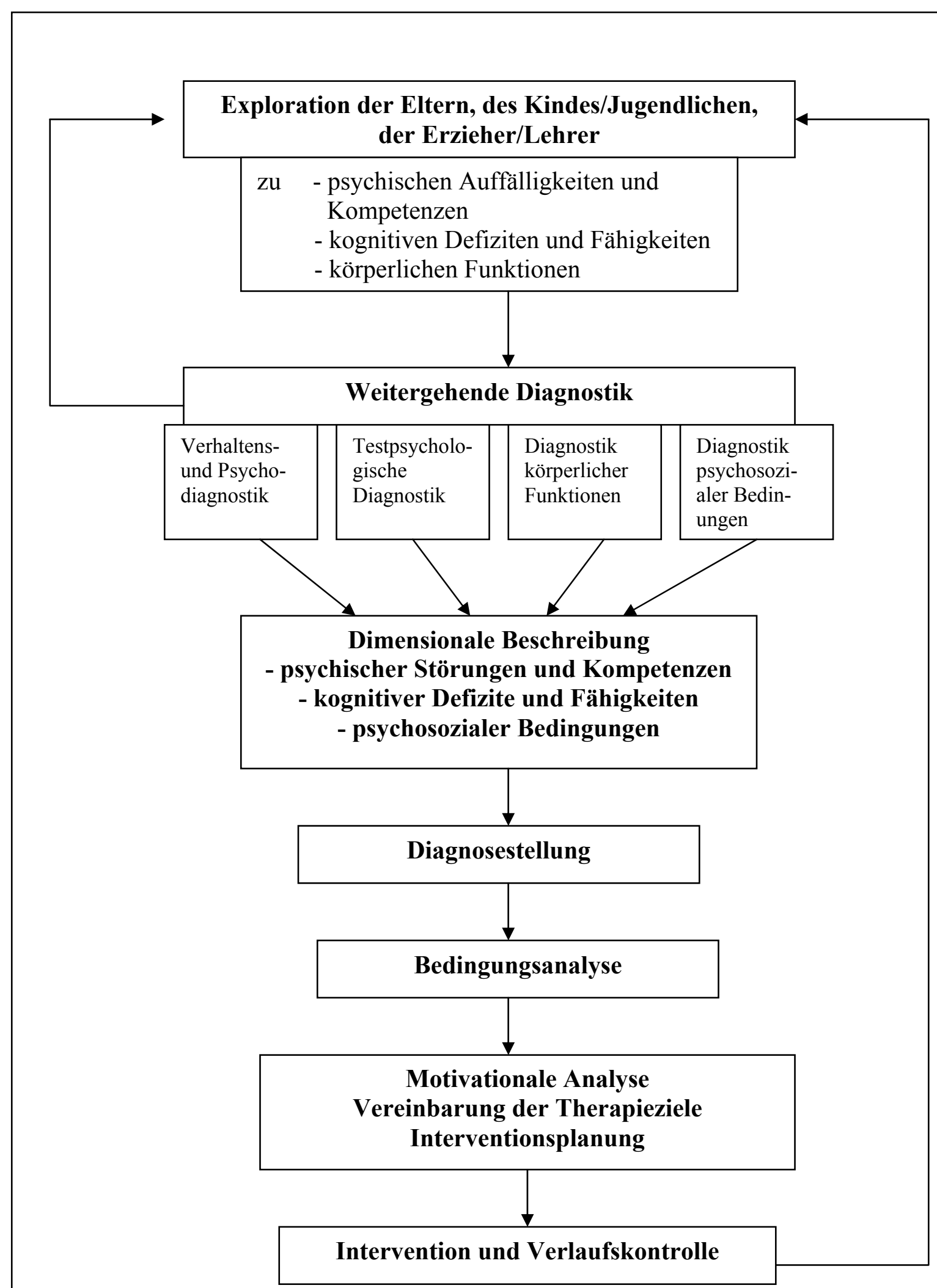

Abbildung 1: Phasen des diagnostischen Prozesses (nach Heubrock und Petermann, 2001) 
Westhoff und Hagemeister (2001, S. 523) definieren Konzentrationstests wie folgt:

Die Leistung eines hirngesunden Probanden (X) erzielt durch (mündliche oder manuelle) Reaktion auf mehr oder weniger einfache (Bilder alltäglicher Gegenstände; abstrakte Zeichen; Zahlen, Buchstaben, andere) Reize, die er klar und eindeutig wahrnehmen kann und auf die er eine einfach zu erinnernde Regel anzuwenden hat, indem er absichtsvoll Teilhandlungen so schnell wie möglich bei (sehr niedriger bis sehr hoher) Geübtheit in der Ausübung dieses Tests korrekt koordiniert in eine (sehr niedrige bis sehr hohe) Geschwindigkeit konzentrierten Handelns und einen (sehr niedrigen bis sehr hohen) Anteil an Konzentrationsfehlern.

Das Konzentrationsvermögen eines Kindes kann durch verschiedene Aspekte beeinflusst werden (vgl. Westhoff \& Hagemeister, 2001): (1) Wahrnehmung, (2) Gedächtnis (z.B. Erinnerungseffekte), (3) Lernen, (4) Lösen von Problemen, (5) Motivation, (6) Strategiebildung und (7) Geübtheit (z.B. Retesteffekte).

Durch Wiederholungsmessungen kommt es zu Temposteigerungen (vgl. z.B. Brickenkamp, 1994; Düker \& Lienert, 1965), die bei der Bewertung der Testergebnisse der wiederholten Messung entsprechend berücksichtigt werden müssen. Westhoff und Hagemeister (2001) konnten einen Übungstransfer sogar in ein anderes Medium nachweisen (also Verbesserung in PC-Tests nachdem Papier-Bleistift-Aufgaben geübt wurden). Sie fanden diese Verbesserung aber nur für die Tempo- nicht für die Sorgfaltsleistung.

Die in Konzentrationstests gestellten Aufgaben sollten den Kindern vertraut sein, damit durch die Überprüfung nicht z.B. Vorerfahrenheit statt Konzentrationsfähigkeit gemessen wird. Es muss sichergestellt sein, dass die Kinder die Aufgabe des Tests gut verstanden haben.

Als Maße für die Konzentration sind solche der Bearbeitungsgeschwindigkeit (speed) zu betrachten und solche der Lösungsgüte, also der Fehlerrate (power). Soll ein Kind nur auf einen bestimmten Reiz antworten, so spricht man von „simple reaction“. Muss das Kind zwischen mehreren Alternativen auswählen, spricht man von ,choice reaction“.

Neben den mittleren Reaktionsgeschwindigkeiten und der Fehlerrate interessiert außerdem die Streuung der Reaktionen, d.h. wie variabel oder gleichförmig ein Kind gearbeitet hat. Ein gleichförmiges Muster spricht für regelmäßige Antworten und für eine Fähigkeit die Konzentration dauerhaft zu steuern ohne dass diese Schwankungen unterliegt. Der Verlauf in manchen Konzentrationstests vermag Auskunft darüber zu geben, wie ein 
Kind auf kontinuierliche Anforderungen reagiert. Hier ist z.B. denkbar, dass ein Kind bei einem Durchstreichtest am Anfang sehr gute Tempo- und Sorgfaltsleistungen erzielt, dann aber nach einer gewissen Zeit Leistungseinbußen hat, was auf Störungen der Daueraufmerksamkeit hindeutet.

Notwendig wäre es auch, dass Konzentrationstests über einen längeren Zeitraum durchgeführt werden, weil bei manchen Probanden die Schwierigkeiten erst nach einigen Minuten der Beanspruchung auftreten. Diese Anforderung wird von vielen Konzentrationstests nicht erfüllt.

\subsection{Interventionsmaßnahmen}

\subsubsection{Pharmakologische Behandlung}

Die pharmakologische Behandlung ist eine der besonders wirksamen Methoden zur Beeinflussung hyperkinetischer Verhaltensprobleme. Psychostimulanzien sind dabei die Medikamente der ersten Wahl. Gut $70 \%$ der behandelten Kinder sprechen auf diese Medikamente gut an (so genannte Responder). Die Medikamente mit dem Wirkstoff Methylphenidat (Handelsnamen z.B. Ritalin ${ }^{\circledR}$ oder Medikinet $^{\circledR}$ ) sowie D-Amphetamin und Pemolin wirken dopaminantagonistisch. Aufgrund seiner kurzen Halbwertszeit und den sehr guten Effekten auf die klinische Hauptsymptomatik wird Methylphenidat sehr häufig verschrieben. Die Wirkung tritt nach 30 bis 45 Minuten ein und bleibt zwei bis vier Stunden auf hohem Niveau in seiner Wirksamkeit konstant. Es eignet sich deshalb besonders gut für den Einsatz während der Schulzeit. Trizyklische Antidepressiva, MAO-Hemmer und Antihypertensiva gelten nur als Mittel der zweiten Wahl, da sie in ihrem Wirkungsspektrum weniger erfolgreich sind und mehr Nebenwirkungen zeigen (Döpfner, Frölich \& Lehmkuhl, 2000).

In mehreren Meta-Analysen wurde die Wirksamkeit pharmakologischer Behandlungen bestätigt (z.B. Baving \& Schmidt, 2001; Connor, Glatt, Lopez, Jackson \& Melloni, 2002; Purdie, Hattie \& Carroll, 2002; Spencer, Biederman, Wilens, Harding, O’Donnell \& Griffin, 1996). Durch die medikamentöse Behandlung lässt sich eine Verminderung der Kernsymptomatik und oppositioneller Verhaltensweisen erzielen. Die Effekte sind in der Schule meistens ausgeprägter als im häuslichen Bereich. In geringerem Ausmaße werden durch Psychostimulanzien soziale Beziehungen verbessert. Der Einfluss auf kognitive Variablen, emotionales Empfinden und schulische Leistungen wird als noch geringer eingestuft. Die Effekte weisen eine starke inter- als auch intraindividuelle Variabilität auf. Sie sind abhängig vom Alter, der Dosis, der Dauer, der vorherrschenden 
Symptomatik, komorbider Störungen und den Messvariablen (Denney \& Rapport, 1999; The MTA Cooperative Group, 1999b).

Diese Medikamente sollten erst ab sechs Jahren verabreicht werden (Mutschler, 1991). Bei Vorschulkindern ist eine sorgfältige Abgrenzung von anderen psychischen Störungen oder Temperamentsschwankungen nicht immer möglich, weshalb hier besondere Vorsicht geboten ist.

Mit dem Einsatz von Medikamenten können verschiedene Nachteile verbunden sein. Problematisch ist, dass sich die nachgewiesene Wirksamkeit in der Regel vor allem auf Kurzzeiteffekte bezieht. Belege für die Langzeitwirksamkeit über mehrere Jahre liegen noch nicht vor (Döpfner, Frölich \& Lehmkuhl, 2000; Livingston, Mears, Marshall, Gray \& Haak, 1996). In Katamnesestudien konnte gezeigt werden, dass sich die Kinder zwar hinsichtlich der Kernsymptomatik verbessern, aber bzgl. ihres Problemverhaltens noch immer deutlich von ihren Alterskameraden abweichen (Pelham, Wheeler \& Chronis, 1998; Wells et al., 2000). Verbesserungen lassen sich in strukturierten Eltern-Kind-Interaktionen belegen, nicht aber in alltäglichen Eltern-Kind-Interaktionen (Barkley, Karlsson, Strzelecki \& Murphy, 1985). Teilweise sind die Probleme in Familien mit Kindern mit HKS unabhängig (oder zusätzlich) zur HKS so groß (z.B. Alkoholismus des Vaters oder Depressionen der Mutter), als dass zu erwarten wäre, dass eine pharmakologische Behandlung diese Probleme lösen könnte (Barkley, 1998; Johnston, 1996; Pelham et al., 1998).

Effekte über Zeiträume von ein bis zwei Jahren sind nur dann belegbar, wenn Stimulanzien kontinuierlich eingenommen wurden (Gillberg et al., 1997; The MTA Cooperative Group, 1999a).

Eine isolierte pharmakologische Behandlung sollte kritisch betrachtet werden. Wenn auch die Ergebnisse der MTA-Studie (The MTA-Group, 1999a) für die isolierte medikamentöse Therapie annähernd gleiche Ergebnisse in der Reduktion der Kernsymptomatik Unaufmerksamkeit, Hyperaktivität und Impulsivität aufweisen wie eine Kombinationsbehandlung von medikamentöser mit psychosozialer Therapie, stellt sich die Frage nach der langfristigen Wirksamkeit und der Akzeptanz. Viele Eltern lehnen aufgrund von Nebenwirkungen und vermuteten Langzeitschäden des Methylphenidats eine isolierte pharmakologische Behandlung ab (vgl. Rothenberger, 2002). Als Nebenwirkungen werden Appetitminderung und ein damit verbundener Gewichtsverlust, Einschlafstörungen, medikamentös bedingte Ticstörungen, Ängstlichkeit und Weinerlichkeit bzw. Traurigkeit angegeben (vgl. Ryffel, 2003). Langfristig beobachtet werden Gewichtsverlust, Wachstumsverlangsamung und leichte kardiovaskuläre Veränderungen (Barkley, 1998). 
Auszuschließen und kontraindiziert ist eine Stimulanzientherapie bei bestehender psychotischer Erkrankung, Tic-Störungen und Tourette-Syndrom, Angstzuständen oder schweren kardiovaskulären Vorerkrankungen (Döpfner, Frölich \& Lehmkuhl, 2000).

Kontrovers diskutiert wird derzeit noch die Behandlung bei einem bestehenden zerebralen Anfallsleiden oder Hinweisen darauf (Barkley, 1998) mit einer Tendenz zur Befürwortung.

Aufgrund der großen Anzahl von möglichen Nebeneffekten sollte nicht nur eine gründliche Aufklärung des Kindes und der Eltern erfolgen, sondern die Medikation in kontinuierlichen Abständen kontrolliert werden. Einmal jährlich sollte ein Auslassversuch durchgeführt werden.

Etwa $30 \%$ der betroffenen Kinder scheinen von den Medikamenten nicht zu profitieren, ihnen müssen daher alternative Verfahren angeboten werden (Döpfner, Frölich \& Lehmkuhl, 2000).

Kritisch zu sehen sind im Zusammenhang mit der Stimulanzienbehandlung bei HKS die oftmals mangelnde Compliance der Beteiligten, die „Gefährdung der körperlichen und seelischen Entwicklung von Kindern“ durch Hochdosistherapie mit Methylphenidat (Höger, Goering \& Schacher-Gums, 1998, S. 19) sowie der ungerechtfertigte Einsatz ohne ausreichende Indikation. In den letzten Jahren ist es zu einem deutlichen Anstieg der Verordnungsmenge von Methylphenidat gekommen. Von 1991 bis 1999 hat sich die Menge verzwanzigfacht. Angold, Erkanli, Egger und Costello (2000) berichten dazu, dass dreiviertel der behandelten Kinder die Diagnosekriterien für eine ADHD nicht erfüllen. Die Menge der verordneten Tagesdosen wird nicht so sehr als Problem gesehen, es bekommen jedoch viele Kinder das Medikament, für die es gar nicht geeignet ist, andere Kinder jedoch, die es bräuchten, bekommen es aus unterschiedlichen Gründen nicht verschrieben.

Barkley (1998) fasst die Befunde zur Stimulanzientherapie dahingehend zusammen, dass sie effektiv und sicher in der Behandlung der hyperkinetischen Symptomatik sind. Da die Kinder aber durch Medikamente nichts hinzulernen, sind weitere Therapieformen notwendig, um die übrigen sozialen, psychologischen und physischen Probleme in den Griff zu bekommen, damit die Kinder kurz- und langfristig ihre Chancen verbessern sich an die Anforderungen der Umwelt anzupassen. Nach Döpfner (2000, S. 168) ist das „Nichtbeachten medikamentöser Interventionsmöglichkeiten ... ein Kunstfehler, wenn alternative Therapien sich nicht als erfolgreich erweisen“. 
Der Einsatz und die Wirksamkeit weiterer Maßnahmen werden häufig erst durch die medikamentöse Behandlung ermöglicht bzw. gesteigert, weil sie dem Kind hilft angemessener auf die Umwelt zu achten und zu reagieren.

\subsubsection{Kognitiv-behaviorale Verfahren}

Kognitiv-behaviorale Verfahren werden als Therapiemethoden oder als Trainings bezeichnet. In der Literatur ist der Begriff Training schlecht definiert. Nach Klauer (2001) läßt sich der Begriff wie folgt fassen: „Training ist eine wiederholt ausgeführte Tätigkeit, die die Ausführung der Tätigkeit faktisch verbessert, oder sie ist eine Handlung, die darauf ausgerichtet ist, die Fertigkeit oder Fähigkeit zur Ausübung der Tätigkeit zu verbessern.“

Selbstinstruktions- und Selbstmanagementmethoden gehören $\mathrm{zu}$ den kognitiven Verfahren, mit denen versucht wird Defizite in den Bereichen der Selbstregulation und exekutiver Funktionen positiv zu beeinflussen. Weitere Techniken, die hier einzuordnen sind, sind Ärgerkontrolltrainings, Selbstverstärkungslernen, Problemlösetrainings, soziale Kompetenztrainings und Modelllernen (Döpfner, Schürmann \& Frölich, 1998).

Im Rahmen von Selbstinstruktionstrainings sollen die Kinder handlungsanleitendes „Zu-sich-selbst-sprechen“ erlernen, welches sie in die Lage versetzen soll, bei Problemen innezuhalten und reflektiert Lösungen zu entwickeln. Das Kind erfährt am Modell des Therapeuten, wie man mit Problemen umgehen kann. Der Therapeut demonstriert und verbalisiert die für eine angemessene Aufgabenbearbeitung notwendigen Problemlöseschritte: Problem erkennen, verschiedene Lösungen überlegen und abwägen, Erproben einer Lösung, Ergebniskontrolle und Fehlerkorrektur sowie sich selbst bestätigen bei Erfolg. In ihrer klassischen Arbeit von 1971 konnten Meichenbaum und Goodman mit Hilfe der Instruktion bei Lernenden das Ziel erreichen, während einer Aufgabenlösung zu sich selbst zu sprechen. Hierbei werden die Kinder dazu angehalten zunächst laut zu sprechen und nach und nach zum stummen Verbalisieren (internal speech) überzugehen. Dieses Verfahren ist bei einem impulsiven, unüberlegten Problemlösestil erfolgsversprechend.

Als umfangreichstes Training im deutschsprachigen Raum, welches auf dem Selbstinstruktionsansatz aufbaut, kann das Training von Lauth und Schlottke (1997) eingestuft werden. Dieses Training wurde im Rahmen dieser Studie mit einer Reihe von Kindern durchgeführt. Die Grundzüge des Trainings sowie deren Wirksamkeitsuntersuchungen werden in den Kapiteln 3.1.1 und 3.1.2 dargestellt.

Die Arbeitsgruppe um Lauth kommt in eigenen Studien $\mathrm{zu}$ einer positiven Wirksamkeitseinschätzung ihres Trainings (s.u., Kap. 3.1.2). Die positiven Ergebnisse des 
Trainings von Lauth und Schlottke werden von Döpfner, Frölich und Lehmkuhl (2000) auf den gleichzeitigen Einsatz von operanten Methoden und Elternarbeit zurückgeführt. Andere Autoren kommen zu weniger optimistischen Einschätzungen hinsichtlich der Wirksamkeit von Selbstinstruktionsprogrammen. In mehreren Studien konnte die Wirksamkeit der Selbstinstruktionstrainings empirisch nicht hinreichend belegt werden (vgl. hierzu Abikoff, 1991; Döpfner, Schürmann \& Frölich 1998; Döpfner \& Lehmkuhl, 2002; Mackowiak \& Hungerige, 2001). In Reviews wurden der Mangel an Aufgabentransfer, fehlende Belege für Langzeitwirkungen und der Transfer von Selbstinstruktionen auf den schulischen Alltag bemängelt (Mackowiak \& Hungerige, 2001). In einer Metaanalyse ermittelte Saile (1996) nur eine Effektstärke von $\mathrm{d}=0.36$, was als substanzieller, jedoch als nur kleiner Effekt einzustufen ist.

Unter Selbstmanagement-Methoden werden Selbstbeobachtung und Selbstverstärkung zusammengefasst. Die Kinder sollen hierbei lernen die eigenen Verhaltensprobleme wahrzunehmen und sich in bestimmten Situationen durch Befolgen bestimmter Regeln angemessener zu verhalten. Außerdem wird den Kindern vermittelt, sich nach erfolgreicher Situationsbewältigung selbst positiv zu verstärken (Döpfner, 2000).

Eine Kombination mit anderen Methoden (z.B. Selbstinstruktionstechniken und Kontingenzmanagement) erbringt deutlichere Verbesserungen als SelbstmanagementMethoden allein (Döpfner, Frölich und Lehmkuhl, 2000; Purdie, Hattie \& Carroll, 2002). Durch ein sechswöchiges kombiniertes Selbstinstruktions- und Selbstmanagementtraining konnten Frölich, Döpfner, Berner und Lehmkuhl (2002) signifikante positive Veränderungen in mehreren Bereichen erzielen (Kernsymptomatik, Sozialverhalten, Konfliktsituationen im Elternhaus, Hausaufgabenprobleme und weitere individuelle Probleme zu Hause). Die Autoren erklären den Erfolg durch alltagsnahe und problemorientierte Übungsinhalte. Dabei wurden das Alter und die kognitiven Leistungsfähigkeit der Kinder berücksichtigt und die Übungen zu Hause bzw. in der Schule durchgeführt bzw. fortgesetzt.

Als zentralen Faktor für die Effektivität kognitiver Interventionen wertet Abikoff (1987) die Anpassung der Behandlung an die individuellen Schwierigkeiten des Kindes, was eine ausführliche Diagnostik und Problemanalyse voraussetzt. Die Individualisierung der Behandlung wird auch von anderen Autoren und nicht nur im Zusammenhang mit kognitiven Interventionsansätzen als wesentlich herausgestellt (z.B. Döpfner, 2000; Waschbusch, Kipp \& Pelham, 1998).

Insgesamt muss festgehalten werden, dass sich kognitive Verfahren als alleinige Interventionen nicht als ausreichend für die Behandlung von Kindern mit Hyperkinetischer 
Störung erwiesen haben. Werden sie jedoch mit anderen Verfahren kombiniert, können sie klinisch effektiv sein. Im Kapitel 2.6 wird ein Überblick der in Deutschland einschlägigen Programme kognitiv-behavioraler Verfahren gegeben.

\subsubsection{Schulzentrierte Interventionen}

Lehrer wissen oft relativ wenig über das Störungsbild der Hyperkinetischen Störungen. In ihrer Ausbildung werden ihnen nur unzureichende Kenntnisse vermittelt, gleichzeitig haben sie aber ein hohes Interesse an einer Weiterbildung und sind in ihrer Arbeit auch regelmäßig mit dem Problem konfrontiert. Der Kontakt mit den behandelnden Ärzten und Therapeuten ist oft spärlich. Zum Teil wird er von den Eltern nicht gewünscht, zum Teil scheitert es an Zeitgründen, manchmal fehlt das Interesse und die Bereitschaft sich auszutauschen.

Als wirksam werden von Lehrern vor allem zwei Maßnahmen beurteilt: zum einen Münzverstärkungsprogramme und zum anderen Verstärkerentzugstechniken (DuPaul, Guevremont \& Barkley, 1992; Kelley, 1990; Pfiffner \& O’Leary, 1993; Rapport, Murphy \& Bailey, 1982).

Neben der Beteiligung der Lehrer bei der medikamentösen Einstellung (sofern dies vorgesehen ist) sollte mindestens ein regelmäßiger Austausch zwischen Lehrern, Eltern und Therapeuten über Fortschritte und Rückschritte erfolgen. Absprachen über Konsequenzen bei Regelübertretungen bewirken eine Vereinheitlichung des therapeutischen Vorgehens und ermöglichen dem Kind Konsequenzen seines Verhaltens besser vorherzusagen.

Die Interventionen in der Schule zielen dabei auf die Verminderung problematischer Verhaltensweisen in konkreten Situationen ab. Hauptsächlich werden operante Methoden (positive Verstärkung: z.B. Lob, Zuwendung, Token-Systeme; negative Konsequenzen z.B. Verstärkerentzug, time-out) eingesetzt (Döpfner, 2000). Dabei scheint ein unmittelbarer, konsequenter und bei positiven Konsequenzen auch häufiger Einsatz bei ADHD-Kindern sehr wichtig zu sein (Barkley, 1998). Hilfreich ist es außerdem kurze Anweisungen zu geben sowie Instruktionen und Verhaltensregeln extern $\mathrm{zu}$ repräsentieren und überschaubare Aufgabeneinheiten mit dem Kind zu bearbeiten. Außerdem sollte das Kind in der Nähe des Lehrers sitzen, damit dieser sofort auf störendes Verhalten reagieren kann (Barkley, 1998). Der Einbezug der Lehrer ist von Bedeutung, weil er die therapeutischen Bemühungen wirksam unterstützen kann. Bei Nichteinbezug können Widerstände erzeugt werden, die die Fortschritte der Behandlung behindern können. 
Sergeant (2000) fasst in seinem Modell verschiedene Ebenen aktuell favorisierter Theorien zusammen und gibt Empfehlungen, wie diese im Unterricht handlungsleitend sein können:

1. Es sollte ein angemessenes Aktivierungsniveau im Unterricht erzeugt werden, damit die Kinder nicht unter- oder überreizt werden (Theorie: Defizite zentralnervöser Aktivierung);

2. Der motorische Bewegungsdrang des Kindes soll durch einen Wechsel von Bewegung und Ruhe gesteuert werden (Theorie: Defizite in der Informationsverarbeitung);

3. Es soll den Kindern eine Verzögerung zwischen Reizstimulus und Reaktion ermöglicht werden, damit das eigene Handeln ausreichend kontrolliert und problemlösendes Denken nicht behindert wird. Schließlich ist die Fähigkeit zur Analyse und Resynthese von Informationen behindert (Theorie: Defizite bei exekutiven Handlungsfunktionen).

Lehrer befinden sich in diesem Kontext in einem Dilemma. Sie müssen einerseits den curricularen Anforderungen gerecht werden, andererseits auf die individuellen Besonderheiten der Kinder mit hyperkinetischer Problematik eingehen. Frölich, Döpfner, Biegert und Lehmkuhl (2002) empfehlen deshalb Maßnahmen einzusetzen, die allen Kindern der Schulklasse entgegen kommen. Diese Maßnahmen sollen die Ausdauer- und Konzentrationsfähigkeit verbessern sowie die Ablenkbarkeit vermindern. Komplexe Aufgaben sollen reflektierter bearbeitet werden können, die motorische Unruhe sinnvoll kanalisiert werden (vgl. ebd., S. 500f.).

Werden Lehrpersonen gebeten, bei der Behandlung von Kindern mit HKS mitzuwirken, entgegnen sie nicht selten, dass sie eine Sonderbehandlung von bestimmten Schülern nicht vertreten können und dass sie sich durch den Mehraufwand an Arbeit überfordert fühlen. Ihnen stünden auch zu wenige Verstärkermöglichkeiten zur Verfügung. Die Maßnahmen müssen deshalb nach Praktikabilität ausgewählt werden. Bewährt hat sich der Einbezug des Elternhauses. Stehen der Schule keine finanziellen Ressourcen zur Verfügung, kann dieses an die Eltern delegiert werden. Über Tagesbeurteilungen wird den Eltern jeweils das Verhalten ihres Kindes rückgemeldet.

Eine weitere Variante der Verstärkung kann das so genannte „peer teaching“ darstellen. Dabei wird die gesamte Klasse für ein gewünschtes Verhalten belohnt. Dies kann der sozialen Isolation von Schülern entgegen wirken. Bei einer Modifikation dieses Vorgehen werden Paare von einem verhaltensauffälligen und einem verhaltensunauffälligen Schüler gebildet. Sie erhalten gemeinsame Aufgaben, z.B. sich an die Hausaufgaben zu erinnern. 


\subsubsection{Elterntrainings und familienzentrierte Interventionen}

Kinder mit hyperkinetischen Verhaltensweisen zeigen ihr Problemverhalten in unterschiedlichen Kontexten. Typischerweise tritt das Verhalten in strukturierten Situationen stärker zutage. Aus diesem Grund werden Eltern von den Lehrpersonen der Grundschule auf das Verhalten der Kinder aufmerksam gemacht. Aber nicht nur in der Schule tritt das hyperkinetische Verhalten auf, sondern auch innerhalb der Familie. Dies kann z.B. geschehen, wenn Kinder die Anweisungen ihrer Eltern nicht befolgen, sich bei den Hausaufgaben selbst ablenken, die Eltern im Gespräch unterbrechen, beim Essen nicht stillsitzen können. Bereits im Grundschulalter treten die genannten Probleme innerhalb der Familie bei Kindern mit Hyperkinetischen Problemen signifikant häufiger auf als bei Kindern ohne HKS (Döpfner, Schürmann \& Frölich, 1998). Geringes Wissen der Eltern im Umgang mit den Verhaltensauffälligkeiten führt häufig zu negativen und kontrollierenden Interaktionen mit dem Kind. Die Eltern fühlen sich hilf- und machtlos, zweifeln an ihrer Erziehungskompetenz (nicht selten zurecht). Es entstehen Frust- und Stressreaktionen (Barkley, 1998; Döpfner, Frölich \& Lehmkuhl, 2000). In der Folge erhöhen sich oft noch die familiären Konflikte. Das Klima in der Familie verschlechtert sich und die Eltern können selbst psychische Probleme entwickeln (Wells et al., 2000). Es werden in diesen Trainings Verhaltensänderungen beim Kind und den Eltern angestrebt.

Der Verlauf der Störung bei den Kindern wird durch die zuvor genannten Faktoren ungünstig beeinflusst. Deshalb ist ein Ansatz zur Veränderung der negativen Eltern-KindInteraktionen und der elterlichen Störungen besonders vielversprechend, um einer Chronifizierung oder Verschlimmerung der Probleme entgegenzutreten.

Besonders wichtig sind familienzentrierte Interventionen bei den Kindern, für die eine Pharmakotherapie nicht in Betracht kommt, weil die Medikamente nicht wirken (Nonresponder) oder die Eltern eine medikamentöse Behandlung ablehnen.

Kazdin (1997) und Warnke, Beck und Hemminger (2001) konnten nachweisen, dass Elterntrainings dazu beitragen können oppositionelles und hyperkinetisches Verhalten zu vermindern und die Beziehung zu Gleichaltrigen zu verbessern.

Neben der Vermittlung und Einübung operanter Methoden (positive Verstärkung, Verstärkerentzug, Auszeit) werden in Elterntrainings auch psychoedukative Maßnahmen (Vermittlung von störungsrelevantem Wissen) sowie kognitive Interventionen, mit Hilfe derer die Eltern beispielsweise lernen sollen mit negativen Gedanken und Gefühlen besser umzugehen, eingesetzt (Barkley, 1998; Eisert, 1998). Da sich die Kinder kaum über den 
Zusammenhang von Verhalten und dessen Konsequenzen bewusst sind, sollten von den Eltern externe Stimuli, Grenzen und Konsequenzen so eingesetzt werden, dass sie Störungen in der Verhaltensregulation vermindern (Barkley, 1997, 1998; Döpfner, Frölich \& Lehmkuhl, 2000).

Neben der effektiven Beeinflussung von kindlichen Verhaltensweisen werden ein positiveres Familienklima sowie eine verbesserte Eltern-Kind-Interaktion angestrebt. In der Regel finden wöchentliche Sitzungen statt. Der Umfang dieser Trainings liegt meistens bei sechs bis zwölf Wochen (Barkley, 1998). Den Eltern wird aufgetragen, die in den Sitzungen erarbeiteten Inhalte zwischen den Sitzungen im Sinne von Hausaufgaben einzusetzen. Dadurch soll ein Transfer auf den häuslichen Alltag erreicht werden. Durch Leitfäden und Arbeitspapieren wird dies unterstützt.

Als einer der ersten hat Barkley $(1987,1997)$ ein zehnstufiges Elterntraining speziell zur Behandlung Hyperkinetischer und Oppositioneller Störungen entwickelt. Es ist von vielen Forschern in Evaluationsstudien ähnlich oder abgewandelt angewendet worden und war Ausgangspunkt für die Entwicklung weiterer Programme, u.a. auch für das in dieser Arbeit untersuchte Programm THOP von Döpfner, Schürmann und Frölich (1998).

Die vorliegenden Studien, in denen die Effektivität von Elterntrainings überprüft wurde, konnten Reduktionen oppositioneller und aggressiver Verhaltensweisen belegen. Die Kernsymptomatik ließ sich allerdings durch den Einsatz von Kontingenzmanagement nur geringfügig beeinflussen (Anastopoulos, Shelton, DuPaul \& Guevremont, 1993; Dubey, O’Leary \& Kaufman, 1983; Erhardt \& Baker, 1990; Horn, Ialongo, Greenberg, Packard \& Smith-Winberry, 1990; Pisterman et al., 1989).

In einigen Studien konnte auch eine positive Beeinflussung elterlichen Stresses, der Wahrnehmung des Kindes durch die Eltern und ihrer Problemlöse- und Erziehungskompetenzen sowie eine Verbesserung des Wissens über lerntheoretische Zusammenhänge nachgewiesen werden (Anastopoulos et al., 1993; Pisterman et al., 1989). In der Metaanalyse von Saile (1996) wird für die Elterntrainings bei hyperkinetischen Kindern insgesamt eine mittlere Effektstärke von $d=0.49$ angegeben.

Durch die Verbindung mit anderen Methoden können die Effekte noch gesteigert werden. So konnten Horn et al. (1990) z.B. die Überlegenheit einer Kombination von Elterntraining und Selbstinstruktionstraining gegenüber beiden unimodalen Behandlungsformen bei der Verminderung hyperkinetischen Verhaltens in der Familie belegen. Wenn Eltern und Kinder gemeinsam in die Therapie einbezogen werden, ist es möglich, deren Sichtweisen miteinander abzugleichen und dort anzusetzen, wo in der Familie 
die Probleme am größten sind. Den Eltern wird vermittelt, wie sie eigenes Verhalten modifizieren und ihre Kinder bei der Anwendung verhaltenstherapeutischer Maßnahmen, z.B. Selbstinstruktionen, unterstützen können (Braswell \& Bloomquist, 1991). Das in Studie 3 eingesetzte THOP gehört $\mathrm{zu}$ solchen familienzentrierten Interventionen. Es wird in den Kapiteln 3.1 und 3.2 genauer dargestellt.

Problematisch im Zusammenhang mit Elterntrainings ist die oft mangelnde Kooperation und Compliance der Eltern. Dabei zeigen Abbrecher häufig solche Merkmale (psychische Störungen der Eltern, familiäre Belastungsfaktoren), die mit einer ungünstigen Prognose für die Kinder einhergehen und daher therapeutischer Unterstützung eigentlich besonders bedürfen (Döpfner, 2000; Eisert, 2000). Kazdin (1990) berichtet von einer Abbrecherquote von $25 \%$ bei kindzentrierten und familienzentrierten Interventionen. Dabei sind die Abbrecher eher schwerer gestört, die Mütter klagen mehr über Stress in der Beziehung zum Kind und hinsichtlich der eigenen Lebenszufriedenheit. Sie sind außerdem häufiger psychisch auffällig. Personen mit niedrigem Bildungsgrad sowie allein erziehende Eltern haben zudem größere Probleme bei der Umsetzung der therapeutischen Maßnahmen.

\subsubsection{Wirksamkeit multimodaler Interventionen}

Saile (1996, S. 203) fasst die Ergebnisse seiner Metaanalyse dahingehend zusammen, dass beim Vergleich mit placebo- oder unbehandelten Kontrollgruppen ,psychologische Behandlung und Medikation als bedeutsamste Interventionsansätze bei Aktivitäts- und Aufmerksamkeitsstörungen in Bezug auf die generelle Effektivität durchaus gleichwertig“ sind. Im direkten Vergleich scheint jedoch die Stimulanzientherapie psychologischen Interventionen zumindest bei kurzzeitiger Behandlung und v.a. hinsichtlich der Verminderung der hyperkinetischen Kernsymptome überlegen zu sein (Döpfner, 2000; Eisert, 1998; National Institutes of Health, 2000; Pelham et al., 1993). Nachdem die Wirksamkeit von pharmakologischer Behandlung und psychotherapeutischen Interventionen zunächst in Einzelstudien überprüft wurde, erhoffte man sich durch Kombination der Interventionen, dass sich die unterschiedlichen Effekte aufaddieren, um dadurch eine verbesserte Wirksamkeit zu erlangen. Die Kombination von Stimulanzienbehandlung und kognitivverhaltenstherapeutischen Interventionen beim Kind erweist sich gegenüber ausschließlicher Pharmakotherapie jedoch nicht als effektiver (Eisert, 2000; Döpfner, 1997).

Die Verbindung von medikamentöser Intervention und behavioralen Verfahren scheint dagegen erfolgsversprechender zu sein. In einer Studie von Pelham und Mitarbeitern (1993) war die Kombination von Verhaltensmodifikation (u.a. positive Verstärkung, Feedback, timeout und Verstärkerentzug) und Stimulanzientherapie alleiniger Verhaltensmodifikation 
hinsichtlich der Verbesserung des Verhaltens in der Schule überlegen. Die Kombinationsbehandlung erbrachte allerdings gegenüber ausschließlicher Stimulanzientherapie keinen zusätzlichen Nutzen. Die Ergebnisse der Studie sind auf die Kurzzeiteffekte der Interventionen begrenzt. HKS sind jedoch als chronisches Störungsbild zu verstehen, „die durch kurzzeitige pharmako- oder psychotherapeutische Interventionen im Allgemeinen dauerhaft nicht zu beeinflussen sind“ (Döpfner, 2000, S. 178).

Die wichtigste Studie zur Überprüfung der Wirksamkeit der multimodalen Behandlung ist die Multimodal Treatment Study of Children with ADHD (MTA) des National Institute of Mental Health (NIMH) (1999, zitiert nach Jensen, 2000, 2001; Newcorn, 2000; Vitiello, 2001), in der 579 Grundschulkinder 14 Monate lang mit einer von vier Therapiestrategien (1. medikamentöse Behandlung einschließlich Beratung (medical management), 2. Verhaltenstherapie, 3. eine Kombination aus 1. und 2. und 4. Standardtherapie (Community Care) behandelt wurden. In allen vier Interventionsformen haben sich deutliche Prä-Post-Veränderungen nachweisen lassen. In der Studie konnten keine Unterschiede in der Wirksamkeit medikamentöser und multimodaler Behandlungen festgestellt werden, wohl aber eine Überlegenheit gegenüber verhaltenstherapeutischen Maßnahmen im Hinblick auf die Kernsymptomatik. Die kombinierte Intervention zeigte jedoch bezüglich der Kernsymptome keinen über die Pharmakotherapie hinausgehenden Nutzen. Soziale Kompetenzen, emotionale Probleme und die Eltern-Kind-Interaktion wurden durch Verhaltenstherapie und Medikation gleichermaßen erfolgreich behandelt. Das kombinierte Vorgehen sowie die behaviorale Intervention waren im Elternurteil mit höherer Zufriedenheit verbunden als die alleinige Pharmakotherapie, und nur weniger als ein Viertel der Kindern, die alleinige Verhaltenstherapie erfahren hatten, mussten zusätzlich medikamentös behandelt werden. Wenn ein kombiniertes Maß aus allen erhobenen Verhaltensmaßen erstellt wird, ist die Kombinationsbehandlung einer alleinigen medikamentösen Behandlung leicht überlegen (Döpfner \& Lehmkuhl, 2002). Langzeitergebnisse der Studie stehen noch aus.

Döpfner, Breuer, und Lehmkuhl (2000) untersuchten in der Kölner Multimodalen Therapiestudie (Cologne Multimodal Intervention Study, COMIS) 75 hyperkinetische Kinder, die entweder zu Beginn eine Verhaltenstherapie erhielten oder medikamentös eingestellt wurden. Im weiteren Verlauf wurden diese Interventionsformen kombiniert und die Wirksamkeit überprüft. Als Hauptergebnis fand sich, dass alleinige Verhaltenstherapie einer Kombination mit Stimulanzienbehandlung unterlegen war, die multimodale Behandlung führte zu einer stärkeren Verminderung der schulischen Auffälligkeiten. 
Über alle Interventionsformen hinweg lassen sich immer wieder große interindividuelle Variabilitäten messen (Döpfner, 2000; Whalen \& Henker, 1991). Die Forscher sind sich deshalb darin einig, dass eine differenzielle und sukzessive Therapiestrategie, die einem multimodalen Behandlungsansatz folgt, für jedes Kind die beste Lösung darstellt (Döpfner, Frölich \& Lehmkuhl, 2000; Greene \& Ablon, 2001; Waschbusch, Kipp \& Pelham, 1998). Dies wurde insbesondere in der Kölner Studie COMIS realisiert.

\subsection{Behandlungsrealitäten im deutschsprachigen Raum}

In Anlehnung an Witte (2002) lassen sich Programme zur Aufmerksamkeitsförderung in außerschulische und schulische Trainingsprogramme gliedern. Bei schulischen Programmen wird mit der ganzen Klasse etwas gemacht, bei außerschulischen Programmen werden nur die aufmerksamkeitsgestörten Kinder behandelt. Letztere sind in der Regel aufwändiger in der Durchführung. Unterschieden werden können die Programme außerdem danach, ob sie aufgabenbezogene Fähigkeiten oder eher kognitive Strategien trainieren. Bei einigen außerschulischen Trainings ist zusätzlich Elternarbeit vorgesehen. Tabelle 2 gibt einen Überblick über Programme zur Aufmerksamkeitsförderung.

Tabelle 2: Schulische und außerschulische Trainingsprogramme

\section{Schulische Trainingsprogramme}

Messer (1992) Konzentrations- und Aufmerksamkeitsübungen für die Grundschule

Krowatschek (1994b) Entspannung in der Schule

Krowatschek (1996) Überaktive Kinder im Unterricht

Schöll (1997) Förderung von Aufmerksamkeit in der Grundschule

Affeldt et al. (1998) Kinder konzentrieren sich. KIKO

Kieler Aufmerksamkeitstraining (1996-1999) ～(nicht publiziert) 


\section{Außerschulische Trainingsprogramme}

Wagner (1976)

Petermann (1994)

Lauth und Schlottke (1993) Training mit aufmerksamkeitsgestörten Kindern

Krowatschek (1994a)

Döpfner et al. (1997)

Ettrich (1998)

Klein-Hessling und Lohaus
Aufmerksamkeitstraining von impulsiven Kindern

Die Kapitän-Nemo-Geschichten Marburger Konzentrationstraining Therapieprogramm für Kinder mit hyperkinetischem und oppositionellem Problemverhalten (THOP) Konzentrationstrainingsprogramm für Kinder Bleib locker

Hierbei sind manche Programme gezielt für Kinder mit einer Aufmerksamkeitsstörung indiziert (z.B. das THOP oder das Training mit aufmerksamkeitsgestörten Kindern von Lauth und Schlottke), manche Trainings richten sich an Kinder mit Aufmerksamkeitsschwächen (z.B. das Marburger Konzentrationstraining). Einige der Programme haben Kinder als Zielgruppe, die verschiedene psychische Probleme haben. Ein Indikationsbereich kann dabei der von Aufmerksamkeitsproblemen sein (z.B. die „Kapitän-Nemo-Geschichten“ oder das Programm „Bleib locker“).

Im Folgenden werden die einzelnen Trainings vorgestellt:

Für das von Messer (1992) vorgestellte Programm Konzentrations- und Aufmerksamkeitsübungen für die Grundschule, bei dem Kopiervorlagen mit z.T. schulischen (z.B. ein Text mit Geheimschrift) bzw. außerschulischen Inhalten (z.B. Puzzle erkennen) bearbeitet werden, fehlt sowohl die theoretische Grundlage als auch eine empirische Evaluation.

Krowatschek (1994b) versucht bei Kindern der ersten bis zur sechsten Schulklasse Entspannung im Unterricht systematisch anzuleiten (Entspannung in der Schule). Über Autogenes Training (Schultz, 1973) und kindgemäße Geschichten (Phantasiereisen mit dem Zauberteppich) soll Erholung erzielt und Unruhephasen sowie psychosomatische Beschwerden verhindert werden. Entspannungsverfahren sind für viele psychische Störungen durch Evaluationen als wirksam eingestuft worden. Auch bei aufmerksamkeitsgestörten Kindern ist es gerechtfertigt sie einzusetzen. Krowatschek empfiehlt gesamte Schulklassen in 
die Entspannungsübungen einzubeziehen. Ggf. sollen einfache Tokenprogramme einen störungsfreien Verlauf absichern helfen. Dieses Programm ist nicht evaluiert.

Für hyperaktive 9- bis 16jährige Kinder hat Krowatschek (1996) ein weiteres Programm (Überaktive Kinder im Unterricht) vorgestellt. Trainingsziel ist es, das in der Regel negative Selbstkonzept der Kinder in ein positives Selbstkonzept zu verändern. Dies soll gelingen, indem über kognitive Interventionsmethoden die Selbstwahrnehmung und Selbstakzeptanz verbessert wird. Die Kinder sollen in die Lage versetzt werden sowohl die eigenen und auch die Gefühle anderer differenzierter wahrnehmen zu können. Es werden Aspekte der Rational-Emotiven Therapie von Ellis (1979) eingebunden, um irrationale Überzeugungen und damit verbundene (unangemessene) Gefühle zu korrigieren. Geeignet sei das Programm vor allem als präventive Maßnahme in Schulklassen, eingesetzt über ein Zeitraum von zwei Schuljahren, es könne aber auch als therapeutisches Programm mit einer Gruppengröße von drei bis sechs Kindern durchgeführt werden. Eine Evaluation dieses Programms liegt nicht vor.

Schöll (1997) konzipierte das Programm Förderung von Aufmerksamkeit in der Grundschule unter einem präventiven Aspekt für schulische Zwecke. Den Kindern wird Wissen über Aufmerksamkeit bzw. Unaufmerksamkeit vermittelt. Sie sollen lernen Aufgabenvariablen (z.B. die Auswirkungen flüchtigen Arbeitens), Personvariablen (z.B. die Auswirkungen ablenkender Gedanken), Strategievariablen vor einer Arbeit (z.B. Arbeitsanweisungen verstehen), während einer Arbeit (z.B. die momentane Überwachung der Aufmerksamkeit) oder nach einer Arbeit (z.B. Fehler kontrollieren) und Situationsvariablen (z.B. Auswirkungen von Lärm) einzuschätzen.

Über das Bearbeiten vorgefertigter Arbeitsblätter werden Strategien eingeübt. Bei den eingesetzten Methoden handelt es sich im Wesentlichen um Selbstkontrolltechniken aus der kognitiven Verhaltensmodifikation (Selbstinstruktionen, Selbsteinschätzungen und Metagespräche). Die zehn Trainingseinheiten sollen unmittelbar in den Grundschulunterricht eingebunden werden. Es ist im Unterschied $\mathrm{zu}$ allen bisherigen Trainings mittels Experimental- und Kontrollgruppenvergleich evaluiert worden. Nach Abschluss des Trainings hat sich bei den Kindern zumindest die Leistungsgüte und die Aufmerksamkeitsspanne erhöht. 
Das Programm KIKO: Kinder konzentrieren sich (Affeldt et al., 1998) folgt einem metakognitiven Ansatz. Die Materialsammlung ist eher pädagogisch. Eine Evaluation des Trainings wurde nicht durchgeführt.

Zum Kieler Aufmerksamkeitstraining liegen noch keine Publikationen vor. In diesem Programm wird mit Signalkarten gearbeitet, auf denen jeweils eine Maus (analog der „Sendung mit der Maus“) mit unterschiedlichen Selbstinstruktionen abgebildet sind, die den trainierten Kindern helfen sollen, schulische Aufgaben kontrollierter zu bearbeiten. Den Trainingsphasen sind Entspannungsübungen aus dem Autogenen Training vorgeschaltet, um die Aufmerksamkeit in den sich anschließenden Anforderungssituationen zu erleichtern.

\section{Außerschulische Trainingsprogramme}

Die außerschulischen Trainingsprogramme sind in der Regel besser evaluiert als die schulischen Programme. Sie werden einzeln oder in Kleingruppen eingesetzt. Ihr methodischer Aufwand ist oft höher. Die theoretischen Konzepte sind meistens besser evaluiert.

Im deutschsprachigen Raum hat Wagner (1976) bereits sehr früh ihr Aufmerksamkeitstraining mit impulsiven Kindern vorgelegt. Sie übte mit den Kindern Reaktionsverzögerung und sorgfältiges Arbeiten ein. Mit dem Vorbild einer erwachsenen Person wurde das innere Sprechen zur Verbesserung der Selbstkontrolle erlernt. Sie benutzte Montessori-Material und Übungen aus dem Frostig-Wahrnehmungs-Trainingsprogramm. Schon Wagner beteiligte Mütter bei dem Programm, um den Therapieerfolg zu erhöhen. Die Ergebnisse zeigten damals deutliche Verbesserungen in der Leistungsgüte, weniger in der Leistungsmenge.

Petermann (1994) verbindet mit den Kapitän-Nemo-Geschichten Autogenes Training und imaginative Strategien. Dieses für fünf- bis zwölfjährige Kinder entwickelte Training enthält vierzehn Entspannungsgeschichten, bei denen die Schwere-, Wärme- und Ruheanleitung des Autogenen Trainings mit Bildern einer Unterwasserwelt verknüpft werden. Petermann nennt ihre Abwandlung „bildgetragene Kurzentspannung“. Durch dieses Verfahren soll (unter anderem) bei aufmerksamkeitsgestörten Kindern eine Verminderung der Aktivität und eine verbesserte Selektivität der Aufmerksamkeit erreicht werden.

Die Wirksamkeit von Entspannungsverfahren konnte für verschiedene Störungsbilder nachgewiesen werden. Auch für die Anwendung bei hyperkinetischen Kindern werden sie als 
geeignet eingestuft. Merod (2001) stellt die Bedeutung von Eigenkompetenz und Selbstkontrolle bei der Anwendung von Entspannungsverfahren heraus. Kinder mit hyperkinetischen Störungen sind zu Entspannungsmethoden nicht leicht zu motivieren, da ihnen entspanntes Verhalten eher unvertraut ist. Eine gute Vorbereitung und kindgerechte Durchführung ist sehr wichtig, weil es möglicherweise dem Selbstbild insbesondere von älteren Kindern widerspricht entspannt zu sein (vielen Kindern ist es heutzutage besonders wichtig „,cool“ zu sein). Eine gute Chance für den Einsatz von Entspannungsverfahren könnte gerade darin bestehen, den Kindern $\mathrm{zu}$ vermitteln, dass es „cool“ ist, ein Entspannungsverfahren zu beherrschen.

Beim Konzentrationstrainingsprogramm für Kinder (KTP) von Ettrich (1998) wird über 20 Tage mit Kindern erarbeitet, wie sie mit Hilfe von Suchbildern, Labyrinthaufgaben, Durchstreich- und Ergänzungsübungen usw. ihre Konzentrationsfähigkeit verbessern können. Vorläufer dieses Trainings wurden von Barchmann, Ettrich, Kinze und Reschke (1986 und 1988) veröffentlicht. Das Programm kann im Rahmen von Kleingruppen mit drei bis fünf Kindern durchgeführt werden. Lerntheoretische Maßnahmen, wie z.B. aversive Kontrolle, Entzug positiver Verstärker, Tokensysteme oder Kognitives Modellieren kennzeichnen das Programm. Die Autorin beschreibt eine Verringerung der Fehlerzahl in einem Konzentrationstest.

Als Bestandteil einer Gesamtbehandlungsstrategie kann z.B. auch das Stressbewältigungstraining Bleib locker von Klein-Hessling und Lohaus (1998) eingesetzt werden. Hier geht es um die Erhöhung von Selbstwirksamkeit und um angemessene Bewältigungsstrategien, über die viele Kinder mit hyperkinetischer Problematik nicht verfügen.

Daneben gibt es noch Trainings, die im Rahmen von Rehabilitationsmaßnahmen entwickelt und eingesetzt wurden. Hier werden Aufmerksamkeitskomponenten (z.B. Geteilte Aufmerksamkeit, Daueraufmerksamkeit) trainiert. In der Regel liegen nur Einzelfallberichte vor (vgl. Lauth, 2001).

Die Programme Training mit aufmerksamkeitsgestörten Kindern von Lauth und Schlottke (1993, 1997), das Marburger Konzentrationstraining von Krowatschek (1994) und das Therapieprogramm für Kinder mit hyperkinetischem und oppositionellem 
Problemverhalten (THOP) von Döpfner et al. (1997) werden ausführlich in den Kapiteln 3.1 bis 3.3 dargestellt, da sie jene Programme sind, die vom Verfasser hinsichtlich ihrer Wirksamkeit evaluiert werden.

\subsection{Sind Gruppentrainings möglich und/oder nötig?}

Der Frage, ob Gruppentrainings möglich und/oder nötig sind, soll in diesem Kapitel nachgegangen werden. Es gibt mehrere Argumente, die für die Durchführung von Gruppentherapien sprechen. Die Forderung nach effektiven Behandlungsmethoden muss mit der Forderung nach ökonomischer Umsetzung verknüpft werden. Wenn ein Behandlungsbedarf definiert ist, sollte sich das Angebot an den Bedürfnissen der Gesellschaft messen lassen. Da die zeitlichen Ressourcen von Therapeuten begrenzt sind, ist es notwendig, solche Behandlungen durchzuführen, die möglichst vielen Kindern zugute kommen. Die Behandlung in Gruppen trägt diesem Faktor Rechnung. Gruppentherapeutische Verfahren wurden auch deshalb entwickelt, weil durch die individuumzentrierten Ansätze die Symptomatik nicht immer hinreichend gebessert wurde. Dies war insbesondere bei Defiziten der sozialen Kompetenz und der Integration in soziale Gruppen der Fall.

Ein zweiter Vorteil durch Gruppentherapien ist eine verbesserte Diagnostik. Nach Axline (1947; 1980) wird durch die Gruppensituation bei schüchternen und „beifallshungrigen“ Kindern vieles sichtbar. Insbesondere der letztgenannte Aspekt ist bei Kindern mit hyperkinetischen Problemen häufig zu beobachten. Die Kinder haben in einer Gruppe die Möglichkeit sich mit anderen zu vergleichen und einzuschätzen. In der Gruppe lernen die Kinder zu warten, sich durchzusetzen und auf andere Rücksicht zu nehmen. An anderen kann sich das Kind orientieren und auch für diese ein Vorbild sein.

Ein weiterer Zugewinn bei Gruppentherapien entsteht durch motivationale Faktoren. Die Kinder haben ggf. mehr Spaß, wenn sie mit anderen Kindern zusammen sind und können die Erfahrung machen, dass sie nicht ausgegrenzt zu werden, wenn sie mit anderen zusammen kommen, die ähnliche Probleme haben wie sie selbst.

Ein vierter Vorteil entsteht durch den sozialen Kontext. Durch die Gruppensituation bieten sich folgende Möglichkeiten: (1) die Gruppenmitglieder können als Interaktionspartner für soziale Erfahrungen genutzt werden, (2) die Gruppe kann ein Medium für emotionale Erfahrungen sein (,wie fühle ich mich, was erlebe ich, wenn ich mit anderen zusammen bin“) und (3) die Gruppe kann Übungsfeld sein, in dem pädagogische und verhaltenstherapeutische Maßnahmen eingesetzt werden. Im (strukturierten) Gruppensetting bietet sich für Kinder die 
Möglichkeit, Strategien in einem realitätsnahen aber geschützten Erfahrungsfeld einzuüben (Braswell \& Bloomquist, 1991).

Schwierigkeiten bei der Durchführung von Gruppentherapien können sein: (1) nicht alle Kinder lassen sich in einer Gruppe gut führen, weil sie so starke Verhaltensauffälligkeiten zeigen, dass die übrigen Gruppenmitglieder darunter leiden und konzentriertes Arbeiten nicht möglich ist, (2) ein Einzeltraining lässt sich besser auf den Einzelnen zuschneiden (bei der Zusammenstellung und Umsetzung der Trainingsinhalte ist ein möglichst großer Bereich der Überschneidung der Interessen bzw. Probleme der Einzelnen notwendig, um die Motivation zur Mitarbeit aufrecht zu erhalten) und (3) in einer Gruppe können manche Kinder anderen auch ein negatives Vorbild sein.

Die Ausgangsfrage lässt sich wie folgt beantworten: Gruppentherapien sind näher an realen Lebenssituationen als Einzeltrainings. Die Beziehungen $\mathrm{zu}$ peers haben für Kinder und Jugendliche einen hohen Stellenwert. Es ist bekannt, dass Kinder mit hyperkinetischen Störungen oft Probleme mit Gleichaltrigen haben. Deshalb liegt es nahe, dies in einem möglichst realen Übungsfeld zu trainieren (Braswell \& Bloomquist, 1991). Hier können die Kinder lernen die Perspektive von anderen zu übernehmen. Kinder und Jugendliche mit expansiven Störungen profitieren von zu viel Freiraum in Therapien eher wenig. Für sie ist es günstiger strukturierte Verfahren zur Vermittlung spezifischer sozialer Funktionen und anderer Funktionsbereiche einzusetzen. Bei der Durchführung ist darauf zu achten, dass sich die Gruppenmitglieder nicht gegenseitig ein negatives Vorbild bieten (Lehmkuhl \& Lehmkuhl, 2002). Es wird deshalb auf eine ausreichende Steuerung durch den Therapeuten zu achten sein. Abzuwägen ist deshalb, ob ein Therapeut allein ausreichend ist oder ob ein CoTherapeut notwenig ist, um die verschiedenen Gruppenmitglieder im Blick zu behalten.

Grundsätzlich kann man sagen, dass Gruppentherapien möglich und nötig sind, die beschriebenen Schwierigkeiten müssen jedoch bei der Umsetzung der Therapien beachtet werden.

\subsection{Evaluation klinisch-psychologischer Interventionen}

Die Evaluation von psychologischen Interventionsmaßnahmen steht in Deutschland erst in den Anfängen. Damit solche Maßnahmen wissenschaftlichen Kriterien standhalten können, muss deren Wirksamkeit nachgewiesen werden. Es gibt hier ein berechtigtes Interesse von Seiten des Gesundheitssystems und der Gesellschaft, dass im klinischen wie im 
pädagogisch-psychologischen Bereich nur solche Programme vertrieben werden, die nachgewiesenermaßen wirksam sind. Hier spielen ökonomische Überlegungen eine Rolle, aber auch ethische Gesichtspunkte müssen beachtet werden, weil viele der mit den Programmen behandelten Klienten einen hohen Leidensdruck haben.

Da viele der sich auf dem Markt befindlichen Programme ihre Wirksamkeit nicht bewiesen haben, ist eine Überprüfung für diese Programme unerlässlich. Angesichts leerer Kassen haben die Behandler, Kostenträger und natürlich die Betroffenen ein Recht auf Interventionen, die dafür sorgen, dass die Probleme der Klienten wirksam vermindert oder beseitigt werden. Die Behandlung von Kindern mit Hyperkinetischen Störungen hat eine hohe gesellschaftliche Relevanz, da von etwa 3-5 \% betroffenen Kindern ausgegangen werden kann.

Den wissenschaftlichen Anforderungen, welche diese Evaluationen genügen sollten, stehen Anforderungen der Praxis gegenüber, die ethischen Gesichtspunkten Rechnung tragen müssen. Nicht alles, was unter Forschungsgesichtspunkten zu wünschen ist, kann in der Praxis umgesetzt werden. Auf die Kontrolle von Störvariablen muss zuweilen verzichtet werden (Hager, Patry \& Brezing, 2000).

Hager und Hasselhorn (2000, S. 44) grenzen Wirkungen und Wirksamkeit von Interventionsmaßnahmen voneinander ab. Sie sprechen von Wirksamkeit „,.. wenn beobachtbaren Performanzen oder Leistungen durch eine Interventionsmaßnahme beeinflusst werden, und zwar in der von den Vertreter(inne)n des Programms behaupteten Weise ...“. Wirkungen sind die ,... theoretisch angenommenen Ursachen der beobachtbaren Verhaltensänderungen ....".

Die Wirkung von Interventionsmaßnahmen zu erklären ist für die Etablierung eines Programms keine notwendige Bedingung. Gelingt sie jedoch, sind Anpassungen des Programms an den Einzelfall oder an bestimmte Teilgruppen einer Population möglich.

Welche Aussagen können nach Evaluationen von Interventionsmaßnahmen getroffen werden? Kann die Evaluation unter einem hohen wissenschaftlichen Standard durchgeführt werden, können Störvariablen konstant und Fehlerquellen so weit wie möglich ausgeschaltet werden und fällt das Ergebnis positiv aus, so spricht dies für die Wirksamkeit dieses Programms. Damit mögliche Störfaktoren über unterschiedliche Bedingungen möglichst gleich bleibend gehalten werden, sollte die Zuweisung zu verschiedenen Interventionsgruppen oder zu Wartegruppen über eine Randomisierung erfolgen.

Kann die Wirksamkeit nicht bestätigt werden, könnte dies daran liegen, dass bei der Durchführung des Programms Fehler gemacht wurden. Es wäre auch möglich, dass sich 
gewünschte Wirkungen erst nach einer gewissen Zeit einstellen. Besonders wichtig wäre der Nachweis längerfristiger Veränderungen.

Aber welche Kriterien müssen erfüllt sein, damit einem Programm eine hinreichende Wirksamkeit bescheinigt werden kann? Kriteriumsmaße müssen definiert werden, die in der Lage sind, die Wirkungen zu erfassen. Jedoch herrscht bzgl. der Hyperkinetischen Störungen keine Einigkeit unter den Forschergruppen, welche Kriterien Veränderungsprozesse gut abbilden.

Ergibt die Untersuchung, dass ein Programm nicht wirksam ist, so ist das für Therapeuten, Klienten und nicht zuletzt die Programmautoren bedauerlich, bedeutet aber dennoch einen Erkenntnisgewinn: Weitere Verbesserungen an den Programmen werden angestoßen, neue Programme werden entwickelt oder andere (aber wirksamere Methoden) können dann zum Einsatz kommen.

Nach Campbell und Fiske (1959) muss sowohl der Nachweis konvergenter wie diskriminanter Validität erbracht werden. Konvergente Validität bedeutet, dass das Training wirklich jene Leistungen fördert, die es fördern soll. Unter diskriminanter Validität versteht man den Nachweis, dass das Training nicht zusätzlich Bereiche fördert, die es eigentlich gar nicht fördern sollte. Sollte bei der Überprüfung der diskriminanten Validität sich herausstellen, dass eine allgemeinere Fähigkeit gefördert wird, so müsste der Indikationsbereich des Trainings überdacht werden.

Klauer nennt in Anlehnung an Belmont und Butterfield (1977, zitiert nach Klauer, 2001) und Donaldson (1981, zitiert nach Klauer, 2001) fünf Kriterien, welche bei einer Trainingsevaluation berücksichtigt werden müssen: (1) die Effektstärke, (2) die Effektdauer, (3) den Effekttransfer, (4) Leistungsverbesserungen dürfen nicht durch fähigkeitsexterne Änderungen (z.B. Motivationsverbesserung) erklärt werden können und (5) das Training darf die Validität des Testes nicht verändern.

Folgende Effekte können zu einer Leistungssteigerung in Testverfahren führen, die mit dem Training nichts zu tun haben:

- Testwiederholungseffekt (Vertrautheit mit dem Verfahren und den Aufgaben dieses Typs). Es macht hier scheinbar auch kaum einen Unterschied, ob ein Parallel- oder der gleiche Test eingesetzt wird. Durch eine Sichtung von insgesamt 303 Metaanalysen konnten Lipsey und Wilson (1993) nachweisen, dass in Versuchsplänen ohne 
Kontrollgruppe eine mittlere Effektstärke von 0,76 erzielt wurde. In Versuchsplänen mit mindestens einer Kontrollgruppe betrug die mittlere Effektstärke 0,47. Zur Prüfung des Wiederholungseffekts ist eine Kontrollgruppe deshalb unerlässlich.

- Hawthorneeffekt (das Wissen, dass eine Intervention stattfindet, führt zu einer Leistungssteigerung).

- Placeboeffekt (ein Präparat ohne Wirkstoff, welches einem anderen Präparat mit Wirkstoff ähnlich sieht und eine Wirkung erzielt, obwohl kein Wirkstoff enthalten ist). Bei Testverfahren könnte sich dies in einer stärkeren Motivation nach Trainings auswirken.

- Zuwendungseffekt (Kinder bekommen besondere Zuwendung, die sie ohne die Maßnahme nicht bekommen hätten. Allein durch die Zuwendung verbessert sich das Verhalten). Adair, Sharpe und Huynh (1990) fanden eine mittlere Stärke des Placebooder ähnlicher Effekte von 0,34 Standardabweichungen. Dush, Hirt und Schroeder (1989) kamen in einer Metaanalyse zu Effekten von nur 0,1 Standardabweichungen. Um diese Effekte zu kontrollieren, wäre es sinnvoll mit der Kontrollgruppe ein Training durchzuführen, welches sich von den spezifischen Trainingsinhalten abhebt (sogenanntes Dummy-Treatment, vgl. Klauer, 2001).

Hager und Hasselhorn (2000, S. 42) beschreiben vier Funktionen, die psychologische Interventionsmaßnahmen haben können: Entfaltung (allgemeine Förderung), Prävention, kurative Funktion und Rehabilitation. Die in dieser Arbeit evaluierten Programme versprechen kurative Wirkungen, also Verbesserung oder Beseitigung von Defiziten oder Störungen im emotionalen oder Verhaltensbereich. Angestrebt wird der Aufbau von erwünschten bzw. der Abbau von unerwünschten Verhaltensweisen.

Bei den Interventionsmaßnahmen lassen sich Veränderungen auf verschiedenen Ebenen beschreiben, dem intrapersonellen und dem interpersonellen System (Baumann \& Perrez, 1998). Verbesserungen des intrapersonellen Systems betreffen z.B. verbesserte Konzentrationsleistungen, beim interpersonellen System sind es z.B. harmonischere Interaktionen innerhalb von Familien oder verbesserte Freundschaftsbeziehungen zu peers.

Damit Interventionen zu substanziellen Verbesserungen führen können, ist es notwendig, dass die Betroffenen bereit sind, an einer Verbesserung zu arbeiten. Kinder und auch Erwachsene, die von einer Behandlungsmethode nicht überzeugt sind, werden kaum bereit sein, sich stark in der Therapie zu engagieren, mit anderen Worten, sie werden sich 
nicht „compliant“ verhalten. Bevor eine Interventionsmaßnahme in Gang gebracht wird, müsste beurteilt werden, wie viel die Klienten für eine Veränderung der Probleme investieren wollen. Gerade Kinder mit Hyperkinetischen Störungen lassen diese „Einsicht“, dass Veränderungen notwendig sind, oft vermissen. Es ist häufig so, dass vor allem ihr Umfeld unter der Störung leidet. Betroffen sind insbesondere die Eltern und die unterrichtenden Lehrer. Da die Familien in der Regel schon verschiedene Dinge versucht haben, aber nicht erfolgreich waren, benötigen sie die Unterstützung von außen, z.B. durch die Interventionsmaßnahmen. Wichtiger Bestandteil dieser Interventionen ist insbesondere bei solchen Familien, welche skeptisch sind, eine gute Aufklärung über Ziele und Durchführungsmodalitäten der Intervention. Da Kinder oft nicht so sehr überzeugt sind, dass sich an ihren Verhaltensweisen etwas verändern müsste, ist es notwendig über die Eltern an einer Veränderung des Problemverhaltens zu arbeiten. Da es bestimmte Fertigkeiten sind, die die Kinder nicht beherrschen (in gewisser Weise auch die Erwachsenen nicht, z.B. wirksame Aufforderungen geben), müssen diese eingeübt werden. Nur über ein bestimmtes Problem in den Sitzungen zu reden reicht nicht aus. Das neue Verhalten muss auch zwischen den Sitzungen eingeübt werden.

Der Erfolg einer psychologischen Interventionsmaßnahme lässt nicht unabdingbar auf das zugrunde liegende theoretische Wirkmodell schließen. Das Wirkmodell bezieht sich auf die intendierten Programmwirkungen. Aber ist der Erfolg einer Maßnahme wirklich auf das Programm mit seinen intendierten Zielen zurückzuführen? Es stellt sich stets die Frage: Sind es reine Programmwirkungen, die den Erfolg einer Maßnahme bewirkten? Viele unspezifische Effekte sind therapeutisch ebenfalls wirksam und müssen hinreichend kontrolliert werden.

Neben den programmgebundenen Wirkungen müssen interventionsgebundene Wirkungen (es hat „,irgend etwas“, nämlich eine Maßnahme von Fachleuten stattgefunden) und externe Wirkungen betrachtet werden.

Da die Wirksamkeit eines Programms durch irgendetwas zustande gekommen sein muss, kann davon ausgegangen werden, dass auch eine Wirkung zugrunde liegt. Nicht immer gelingt es den Forschern jedoch diese Wirkungen $\mathrm{zu}$ identifizieren, weil geeignete Messinstrumente häufig nicht zur Verfügung stehen oder es an angemessenen Wirkhypothesen mangelt.

Im Verlauf einer Störung kann es während und kurz nach einer Maßnahme auch zu einer kurzzeitigen Verschlechterung von Leistungen kommen und zwar dadurch bedingt, dass 
dem Kind Strategien vermittelt werden mit seinen Defiziten anders umzugehen, wodurch es zunächst zu einer Verschlechterung und erst später zu einer durchgreifenden Verbesserung kommt. Die durch ein Training vermittelten Strategien beanspruchen Ressourcen des Kurzzeitgedächtnisses und müssen vom Kind erst soweit beherrscht und automatisiert werden, dass es den Zugewinn an Leistung, welches es durch die neue Strategie erlangt hat, nicht gleich wieder verliert.

Bei jeder Intervention spielen neben spezifischen Wirkungen, welche durch das Programm hervorgerufen werden sollen, auch programmunspezifische Wirkungen eine Rolle. Jede Intervention ist eine soziale Situation. Gerade aufmerksamkeitsgestörte Kinder haben hier oft ein Nachholbedürfnis, weil sich ihr soziales Miteinander mit Erwachsenen und Gleichaltrigen oft als wenig erfreulich für sie gestaltet (weil die Interaktion negativ getönt ist) oder aber sich gar nicht gestaltet (weil die Kinder nicht beachtet werden). Die soziale Zuwendung ist Teil der interventionsgebundenen Wirkung (ebd. S. 46f.). Zu den unspezifischen Faktoren gehören auch Retesteffekte, die nicht fälschlicherweise dem Programm zugeschrieben werden dürfen. Vorsicht ist auch immer dann geboten, wenn die Person, welche die Intervention durchführt, auch die testende Person ist. Allzu leicht können Testergebnisse im Sinne einer bestimmten (favorisierten) Hypothese verfälscht werden. Abhilfe schaffen können standardisierte Testverfahren, die weniger „Gestaltungsspielraum“ lassen, also eine hohe Durchführungsobjektivität besitzen.

Als externe Wirkungen können Reifungseffekte in Betracht gezogen werden. Insbesondere Kinder machen im Rahmen ihrer natürlichen Entwicklung Fortschritte, die fälschlicherweise einem durchgeführten Programm zugeschrieben werden können. Dies lässt sich jedoch über Kontrollgruppendesigns kontrollieren. Beim Kontrollgruppendesign besteht die Gefahr, dass die Effekte des Trainings unterschätzt werden, wenn in der Vergleichsgruppe (unvorhergesehen) Maßnahmen umgesetzt werden, die dem Training ähnlich sind und dazu führen, dass die Vergleichsgruppe nicht mehr das ist, was sie sein sollte. Dies könnte z.B. dann passieren, wenn in einer Schule in einer Wartegruppe untrainierte Kinder von ihren Lehrern besonders gut vorbereiteten Unterricht bekommen und damit Maßnahmen erfahren, die sie nicht bekommen hätten, wenn an der Schule keine Trainingsgruppe vorhanden wäre.

Was können Kinder eigentlich in Trainingsprogrammen lernen? Zum einen sollen Kompetenzen aufgebaut werden (Perrez, 1998; Lauth, 1998; Schulte, 1993). Darunter gefasst werden z.B. Fähigkeiten und Fertigkeiten, die ein Kind im Rahmen der 
Interventionsmaßnahme erlernen soll. Vom Begriff Kompetenz lässt sich der Begriff Performanz abgrenzen. Performanz ist die tatsächliche aktuelle Anstrengungsbereitschaft und die erbrachte Leistung, die eine Person in einer bestimmten Situation (z.B. bei einem Leistungstest) zeigt. Kompetenz ist das, was eine Person grundsätzlich zur Verfügung hat. Das Konstrukt Performanz ist dem Konstrukt Kompetenz untergeordnet. Letzteres ist der Person dauerhaft verfügbar. Zeigt ein Proband in einer Testsituation eine Leistung nicht, so heißt das nicht, das er den Test überhaupt nicht bewältigen kann, sondern zunächst einmal, dass er ihn in der jetzigen Situation nicht bewältigt. Eine Leistungserfassung über Verhaltensbeobachtung (z.B. im Unterricht) oder über Ratingverfahren kann ggf. valider sein. Hager und Hasselhorn (2000, S. 51) halten folgende Nachweise für Interventionsverfahren für notwenig:

- Zeitlicher Transfer bzw. zeitliche Generalisierung,

- Anforderungstransfer bzw. -generalisierung und

- Situationstransfer bzw. -generalisierung.

Grundsätzlich sind es eher die langfristigen Veränderungen, die man sich von Interventionen erhofft. Jedoch sollte man hinsichtlich der Effektdauer eines Trainings keine übertriebenen Erwartungen hegen. Werden die erworbenen Kompetenzen nicht weiterhin eingeübt, werden die Effekte verblassen. Hier kann eine Analogie zu Sportlern hergestellt werden, bei denen ebenfalls eingeübte Bewegungsabläufe (z.B. bei einem Hochspringer) nicht mehr so sicher durchgeführt werden können, wenn er die spezifischen Bewegungsabläufe nicht weitertrainiert (Belmont \& Butterfield, 1977).

Überprüft werden sollten die erworbenen Kompetenzen nicht durch Aufgaben, die sehr nahe an den Inhalten sind. Eine verbesserte Leistung bei Testaufgaben, die bereits während der Intervention permanent trainiert worden sind, ist nicht verwunderlich, sondern trivial.

Bei komplexen Verhaltensweisen sollte zwischen Nah- und Fernzielen differenziert werden. Für manche Probanden ist es hilfreich, komplexes Verhalten in Verhaltensketten aufzuspalten, um das Gesamtziel erreichen zu können. Für die Evaluation könnte dies bedeuten, dass manche Kinder sich erst nach und nach in der Erreichung von Teilzielen verbessern. Eine alleinige Bewertung des Endergebnisses in einem Test wird der Leistung des Kindes dann möglicherweise nicht gerecht. 
Häufig müssen Menschen komplizierte Aufgaben lösen, z.B. eine schwierige Mathematikaufgabe bearbeiten. Eine erfolgreiche Bearbeitung solcher Aufgaben setzt oft voraus, dass die Personen eine Wissensbasis geschaffen haben, die es ihnen ermöglicht, durch Einbezug der Wissensstrukturen zu einer Lösung zu gelangen. Diese Aufgabentypen lassen sich durch einfache Reiz-Reaktions-Tests nicht abbilden. Fehler bei diesen Aufgaben können darin begründet sein, dass eine ausreichende Wissensbasis nicht vorhanden ist oder keine Kontrollprozesse eingesetzt werden. Fehler können auch bei den Prozessabläufen passieren, z.B. wenn bestimmte Arbeitsschritte nicht korrekt umgesetzt werden, weil sie zu früh abgebrochen werden (Kraak, 1991). Dies ist bei aufmerksamkeitsgestörten Kindern häufig anzutreffen.

Von jedem Programm ist zu fordern, dass die Programmautoren explizit formulieren, was sie mit ihrem Programm erreichen wollen. Es sollte definiert werden, für wen ein Programm geeignet ist und für wen nicht.

Neben der Erreichung der programmimmanenten Ziele können weitere Maße zur Beurteilung eines Programms herangezogen werden. Hierzu gehört z.B. die Zufriedenheit der Klienten mit einem Programm. Je höher diese ausfällt, desto größer wird die „,compliance“ ausfallen, also die Bereitschaft der Klienten sich auf die Maßnahmen des Programms einzulassen. Zufriedenheit darf aber nicht zum Selbstzweck werden. Ein Programm, bei dem die Klienten während der Durchführung des Programms mit selbigen zufrieden waren, am Ende aber nicht substanziell profitieren, hat eher den Charakter eines Beschäftigungsprogramms, erwirbt sich damit aber noch nicht eine wissenschaftlich solide Untermauerung. Diese kann ein Programm nur dadurch erwerben, dass es auch das hält, was es verspricht, nämlich die Erreichung der programmimmanenten Ziele.

Ein weiteres fakultatives Ziel nach Hager und Hasselhorn (2000) ist die Akzeptanz von Seiten der Anwender (Trainer). Hier gilt: je kürzer und motivierender (aber gleichermaßen erfolgreich), desto besser.

Die Programme sollte bei Evaluationen fair überprüft werden. Die von den Programmautoren formulierten Ziele sollten auf einer Ebene erfasst werden, welche durch das Training bearbeitet wurde. Es müssen also solche Merkmale bei den untersuchten Kindern beleuchtet werden, die sich aus den Zielen des Trainings und deren Umsetzung ableiten lassen. 
Bei den hier untersuchten Interventionsverfahren handelt es sich um Trainings bzw. Therapiemaßnahmen, deren Ziel es ist längerfristige Wirkungen im Kompetenzbereich zu erzielen. Durch Situations- und Anforderungstransfer soll eine Umsetzung der gelernten Inhalte in Alltagssituationen erfolgen (Hager \& Hasselhorn, 2000, S. 67).

Wie lässt sich der Erfolg einer Interventionsmaßnahme messen? In Frage kommen theoriespezifische, störungsspezifische oder allgemeine externe Erfolgsmaße. Theoriespezifische Erfolgsmaße werden aus der zugrunde liegenden Theorie abgeleitet. Störungsspezifische Maße orientieren sich an den Kriterien von ICD-10 (Saß, Wittchen \& Zaudig, 1996) und DSM-IV (Dilling, Mombour \& Schmidt, 1994). Allgemeine externe Maße könnten z.B. Häufigkeit von Arztbesuchen oder Noten in der Schule sein.

Der Erfolg einer Maßnahme kann daran gemessen werden, inwieweit das anvisierte Ziel erreicht wurde oder über das Ausmaß der Veränderung im Vorher-Nachher-Vergleich. Letzterer Ansatz vergleicht also Differenzwerte. Es muss jedoch angegeben werden, was eine bestimmte Differenz bedeutet, um beurteilen zu können, ob eine Veränderung für ein behandeltes Kind überhaupt Relevanz besitzt, bei aufmerksamkeitsgestörten Kindern also klinisch bedeutsam ist.

Es ist wichtig, sich Gedanken zu machen, von wem die Daten gewonnen werden sollen. In Frage kommen natürlich die behandelten Personen, darüber hinaus wichtige Bezugspersonen, z.B. die Eltern, unabhängige Beurteiler und die Intervenierenden selbst.

Hager und Hasselhorn (2000, S. 74ff) formulieren folgende Gütekriterien, die psychologische Interventionsmaßnahmen erfüllen sollten:

1. Ethische Legitimierbarkeit

2. Theoretische Fundierung

3. Wirksamkeit

4. Fehlen von negativen oder schädlichen Nebenwirkungen

5. Bewährung in der Praxis

6. Verlässlichkeit der Wirksamkeit unter Standard-Randbedingungen

7. Robustheit (und Verlässlichkeit) unter verschiedenen Randbedingungen

8. Wirtschaftlichkeit relativ zu den Zielen 
9. Routinisierbarkeit und Adaptabilität

10. Akzeptanz des Programms durch die Behandler und Zufriedenheit mit dem Programm durch die Klienten

Die Ergebnisse von Evaluationen werden üblicherweise mittels statistischer Analysen, insbesondere mit der Überprüfung auf statistische Signifikanz getestet. Für die statistischen Analysen werden folgende Elemente im Rahmen einer Testplanung bestimmt oder vorgegeben: Stichprobengröße, Effektgröße, Signifikanzniveau und Teststärke.

Der Begriff der Effektgröße beschreibt die Intensität einer Wirkung. „Je größer der statistische Effekt unter sonst gleichen Bedingungen ausfällt, desto größer ist auch die Intensität der Wirkung.“ (Hager, 2000, S. 161).

Die Wirksamkeit bei einem Programm oder auch dessen Nichtwirksamkeit lässt sich zunächst einmal auf der Gruppenebene betrachten. Ergänzend sollte immer auch eine Einzelfallbetrachtung durchgeführt werden, da es sein kann, dass bestimmte Probanden durch eine Maßnahme überhaupt nicht oder aber besonders gut profitieren. Aus der Einzelfallbetrachtung können wertvolle Hinweise für die differenzielle Wirksamkeit abgeleitet werden.

Geprüft werden sollte auch, ob bei wirksamen, multimodalen aber kostenintensiven Verfahren die Behandlungslänge verkürzt werden kann und dann noch immer zu gleich guten Ergebnissen führt (Steigerung der Effizienz) (vgl. Scheithauer \& Petermann, 2000).

Dies ist insbesondere unter dem Gesichtspunkt wichtig, dass bei Studien mit Kindern kein Zusammenhang zwischen einer größeren Anzahl von Therapiestunden bzw. längerer Dauer der Intervention und dem Therapieerfolg gefunden werden konnte (Salzer, Bickman \& Lambert, 1999). 


\section{Fragestellungen der vorliegenden Evaluationsstudien}

Ausgangspunkt für die vorliegende Dissertation war die Frage, ob die unter Laborbedingungen bewährten aktuellen Interventionsansätze auch unter suboptimalen Therapiebedingungen wirksam sind. Nicht selten werden Therapieprogramme an Universitäten und Forschungseinrichtungen entwickelt, denen über Doktoranden und Diplomanden gute personelle Ressourcen zur Verfügung stehen und die überdies auch mit einer guten finanziellen und technischen Ausstattung aufwarten können. Studien, die die Wirksamkeit psychotherapeutischer Interventionen im Kindesalter außerhalb eines Forschungssettings bestimmen, sind eher selten (Scheithauer \& Petermann, 2000).

Allgemeines Ziel der Dissertation ist es eine Wirksamkeitsevaluation von gruppenbasierten Interventionsmaßnahmen für aufmerksamkeitsgestörte Kinder durchzuführen.

In der Ambulanz der Kinder- und Jugendpsychiatrie Mühlhausen/Thüringen wurde von Eltern, Lehrern und Kinderärzten nach der Diagnostik von Kindern mit der Fragestellung einer Aufmerksamkeitsstörung gefordert, dass es nicht bei der Diagnostik bleiben solle, sondern dass neben medikamentöser Behandlung auch eine psychotherapeutische Behandlung erfolgen müsse, um den betroffenen Familien zu helfen. Ende 1999 wurde das Training für Kinder mit Aufmerksamkeitsstörungen von Lauth und Schlottke (1997) als ein gängiges Verfahren ausgewählt. In einer ersten Studie wurde dieses Programm als Basistraining durchgeführt und im Rahmen einer vom Verfasser betreuten Diplomarbeit von Mock (2001) evaluiert. Bei dieser ersten Studie wurde nur das Basistraining eingesetzt. Die Ergebnisse waren eher enttäuschend, weshalb in einer zweiten Studie neben dem Basistraining auch das Strategietraining dieses Programms mit den Kindern durchgeführt wurde (Müller-Scherzberg, 2002). Das Lauth- und Schlottke-Programm ist eher für Kinder geeignet, die „nur“ Aufmerksamkeitsstörungen haben. Die hyperaktiven und impulsiven Kinder, welche oft zusätzlich unter sozialen Auffälligkeiten leiden, konnten durch dieses Programm nicht erreicht werden. Es war notwendig, Elemente bei der Intervention einzubeziehen, die diesen Umstand berücksichtigen. Ein Programm, welches sich ausdrücklich auch auf diese Zielgruppe bezieht, ist das Therapieprogramm für Kinder mit hyperkinetischem und oppositionellem Trotzverhalten (THOP). Es wurde für eine dritte Studie ausgewählt und verändert. Das THOP ist eigentlich als Programm im Einzelsetting konzipiert. Um eine Vergleichbarkeit der Rahmenbedingungen mit dem zuvor genannten Programm zu erreichen, 
wurde es als Gruppenverfahren implementiert. Bei dieser Studie hat maßgeblich K. Bartels im Rahmen ihrer Diplom-Arbeit mitgewirkt (Bartels, 2003).

Für eine vierte Studie wurde ein weiteres Programm ausgewählt, das Marburger Konzentrationstraining von Krowatschek (1994a). Dieses Verfahren richtet sich nicht ausdrücklich an Kinder mit Aufmerksamkeitsstörungen, versucht aber ebenfalls Aufmerksamkeitsprobleme zu vermindern. Es ist schulnäher und wird von Krowatschek selbst zum Teil in relativ großen Gruppen durchgeführt. Dieses Training wurde in der vierten Studie in kleineren Gruppen eingesetzt und mit einem Pseudo-Training verglichen. Unspezifische Effekte der Intervention wurden somit kontrolliert. Die Durchführung dieser Studie oblag F. Felstehausen im Rahmen einer weiteren vom Verfasser betreuten DiplomArbeit.

Alle vier vorliegenden Studien haben primär zum Ziel zu überprüfen, ob auch unter für Praktiker möglichst realistischen Bedingungen die von den Programmautoren postulierten Effekte erzielt werden. Das Eltern-Kind-Programm des THOP soll im Rahmen einer summativen, globalen Evaluation hinsichtlich seiner Wirksamkeit evaluiert werden, um die Frage zu klären, ob das Programm auch unter veränderten Randbedingungen wirksam ist.

Aus der Sicht des Gesundheitssystems lässt sich feststellen, dass die derzeitige Versorgungslage in Deutschland zur Behandlung psychisch kranker Kinder insgesamt ungenügend und eine bedarfsgerechte Versorgung zur Behandlung Hyperkinetischer Störungen derzeit noch nicht flächendeckend gewährleistet ist (Bundesministerium für Gesundheit, 2002). Aus Kostengründen wäre es wünschenswert, wenn Gruppentherapien ihre Wirksamkeit unter Beweis stellen würden.

Hager und Hasselhorn (2000) fordern, dass Interventionsmaßnahmen bestimmte Gütemerkmale aufweisen sollten, um den Anspruch zu erfüllen erfolgreich einsetzbar zu sein. Für diese Studien sind insbesondere die Forderung nach einer Bewährung in der Praxis sowie der Nachweis der Robustheit/Verlässlichkeit unter verschiedenen Randbedingungen von Bedeutung. Die vorliegenden Untersuchungen befinden sich im Übergangsbereich von Laborforschung und praxisbegleitender Feldforschung, da das Vorgehen einerseits so weit wie möglich standardisiert wurde, sich andererseits aber z.B. Auswahl und Zuteilung der Probanden zu den einzelnen Versuchsgruppen an den praktischen Gegebenheiten und Ressourcen der jeweiligen Ambulanzen sowie an ethischen Grundsätzen orientierten. 
Die Frage, was Programme leisten sollen, die zur Behandlung von Kindern mit Aufmerksamkeitsstörungen entwickelt wurden, lässt sich nicht einfach beantworten, da sie unterschiedliche Zielsetzungen verfolgen. Einige Interventionsmaßnahmen verfolgen explizit das Ziel die Kernsymptomatik von Kindern mit Aufmerksamkeitsstörungen zu reduzieren oder zu beseitigen. Andere versuchen sowohl dieses Ziel zu erreichen aber auch z.B. die Interaktion innerhalb der Familie zu verbessern. Für manche Therapieprogramme sind Kinder mit hyperkinetischen Störungen nur Teil der anvisierten Zielgruppe.

Um eine bessere Vergleichbarkeit der untersuchten Programme für die durchgeführten Studien zu erreichen, wurden ausschließlich außerschulische Therapiemaßnahmen ausgewählt, und hier vornehmlich solche Trainings, die überwiegend zur Behandlung von Kindern mit Aufmerksamkeitsstörungen entwickelt wurden und in Deutschland nach Einschätzung des Autors eine weite Verbreitung gefunden haben.

Bei der Auswahl der Programme wurde darauf geachtet, dass sie kindgerecht gestaltet sind, damit die Kinder bei der Intervention gern mitmachen. Dies wirkt sich günstig auf die Therapiemotivation aus. Durch die Evaluation sollen neben einer Verbesserung in Teilmerkmalen der Aufmerksamkeit (Testdiagnostik), diese Verbesserungen auch den relevanten Bezugspersonen auffallen, d.h. in Fremdeinschätzungen durch Eltern und Lehrer sollten die Kinder nach dem Training in Aktivitäten des täglichen Lebens weniger auffällig beurteilt werden. Die Effekte müssen also eine gewisse „Alltagstauglichkeit" aufweisen. Darüber hinaus sollen die erzielten Effekte stabil sein, d.h. das, was die Kinder in den Trainings erlernt haben, sollen sie dauerhaft anwenden und nicht nach kurzer Zeit in ihre alten Strategien (sofern Strategien überhaupt eingesetzt wurden) zurückfallen. Die Stabilität der Effekte konnte in dem hier durchgeführten Design jedoch nicht überprüft werden (s.u.).

Im Folgenden werden die verschiedenen Trainings und die dazu publizierten Wirksamkeitsstudien beschrieben.

\subsection{Das Training nach Lauth und Schlottke}

\subsubsection{Aufbau und Ziele}

Das Training liegt seit 1993 in publizierter Form vor. Lauth und Schlottke (1997) verstehen Aufmerksamkeit als zielgerichtetes und stetiges Verhalten. Sie ist demnach keine vorhandene Fähigkeit, sondern wird durch eine Abfolge von Handlungen (z.B. Aufgabe verstehen - relevante Informationen erkennen - Lösungsmöglichkeiten entdecken - ständige 
Kontrolle des eigenen Vorgehens (Monitoring)) „aktuell“ (S. 12) hergestellt. Aufmerksamkeitsstörungen können diesem Ansatz zufolge dadurch entstehen, dass grundlegende Fertigkeiten (z.B. visuelle Reize diskriminieren), Wissen und Vorerfahrungen sowie metakognitive Fähigkeiten der Handlungssteuerung nur unzureichend vorhanden sind oder eingesetzt werden können.

Die Therapie baut auf dem Selbstinstruktionsansatz auf. Durch Kombination mit verhaltenstherapeutischen Grundtechniken (Feedback, positive und negative Verstärkung) und Übungsanteilen soll eine Verbesserung der „Fähigkeit des Kindes, eigenständig, selbstreflexiv und situationsangemessen zu handeln“ (Lauth \& Linderkamp, 2000, S. 136), erreicht werden.

Ausgehend von einem multifaktoriellen Störungsmodell entwickelt sich die Hyperkinetische Störung durch ein komplexes Zusammenspiel biologischer, psychischer und sozialer Bedingungen. Die Entstehung und Aufrechterhaltung von Hyperkinetischen Störungen wird in diesem Modell auf verschiedenen Ebenen erklärt (vgl. Abbildung 2).

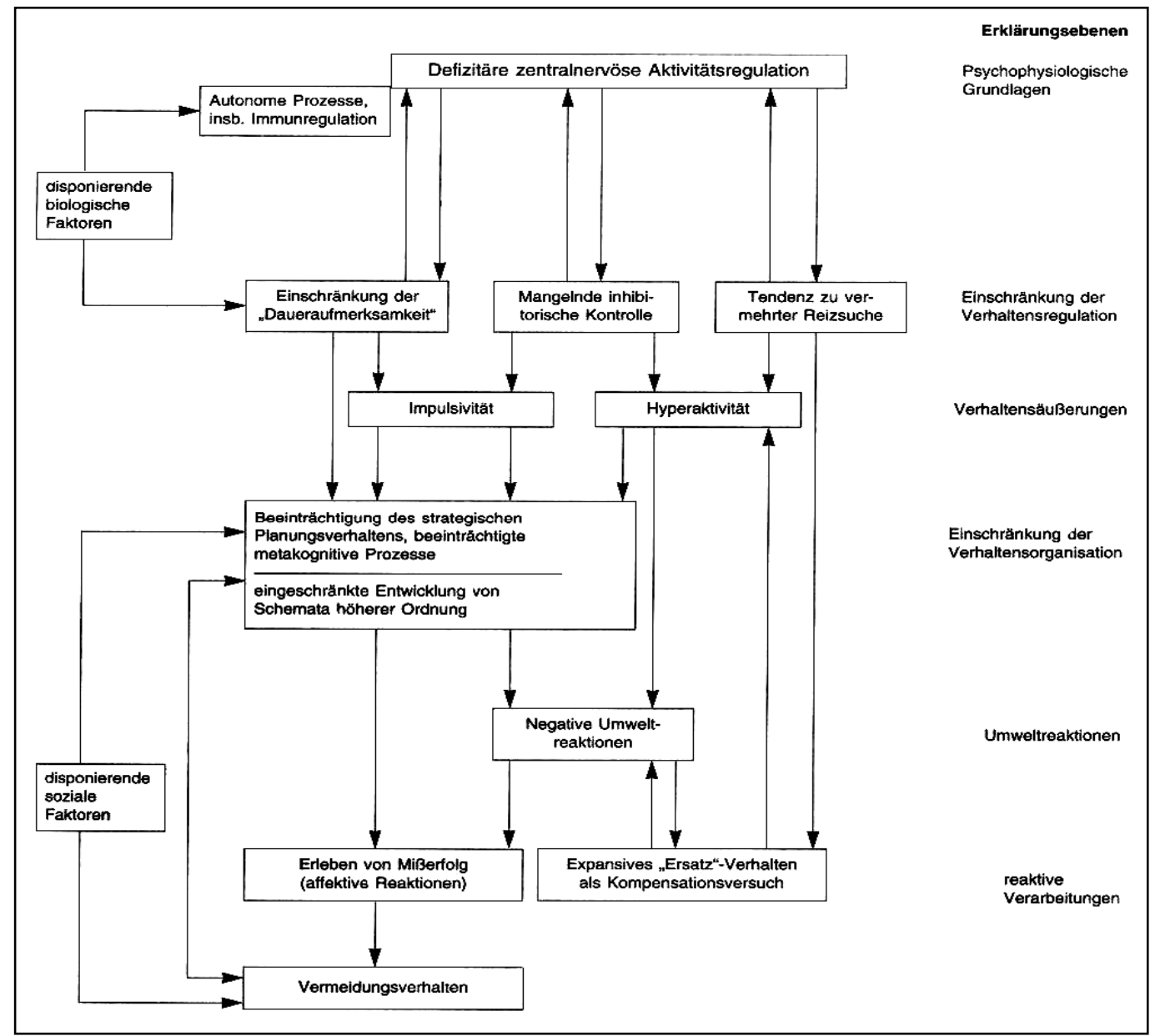

Abbildung 2: Psychophysiologisch-behaviorales Modell zur Entstehung und Aufrechterhaltung von Aufmerksamkeitsstörungen (Lauth \& Schlottke, 1997, modifiziert nach Roth, Schlottke \& Klepel, 1992) 
Störungsebene I: Als Ausgangspunkt und grundlegender Entstehungsfaktor wird eine defizitäre zentralnervöse Aktivierungsregulation (Über- oder Untererregung) auf der psychophysiologischen Ebene angenommen, die auf eine unspezifische biologische Vulnerabilität zurückgeführt wird.

Störungsebene II: Die Unfähigkeit, die eigene Aktiviertheit auf die jeweilige Situation auszurichten, führt auf der Ebene der Verhaltensregulation unmittelbar zu verringerten Selbststeuerungsfähigkeiten, die sich als Einschränkung der Daueraufmerksamkeit, als mangelnde inhibitorische Kontrolle sowie Tendenz zur vermehrten Reizsuche äußern. Auf der Verhaltensebene kann dies als Impulsivität, motorische Unruhe und leicht ablenkbares, trödelndes Verhalten direkt beobachtet werden.

Störungsebene III: Als Folge dieser Beeinträchtigungen entwickeln sich Defizite in der Verhaltensorganisation, die sich in Form mangelnder Ausbildung und Anwendung von Problemlösestrategien, eingeschränkter Wissenssysteme und unzureichender metakognitiver Prozesse zeigen.

Störungsebene IV: Durch bestimmte disponierende Umweltbedingungen (z.B. unstrukturiertes familiäres Umfeld) und negative Umweltreaktionen (Zurückweisung, Bestrafung, Missbilligung), die häufig zu ungünstigen reaktiven Verarbeitungsweisen der betroffenen Kinder (Wutausbrüche, Trotzverhalten, „Kaspern“, sozialer Rückzug, negatives Selbstbild) führen, wird die Gesamtproblematik weiter verschärft. Psychosoziale Faktoren (negative Umweltreaktionen, ungünstige Verarbeitungsweisen der vorhandenen Beeinträchtigungen, soziales Umfeld) werden folglich nicht als ursächliche, sondern als verstärkende und aufrechterhaltende Bedingungen betrachtet.

Das Therapiekonzept des Programmes von Lauth und Schlottke setzt in erster Linie auf den Ebenen der Verhaltensregulation, der Verhaltensorganisation und den verhaltensorganisierenden Wissensanteilen an. Es werden außerdem Veränderungen der Umweltreaktionen und -bedingungen durch die Zusammenarbeit mit wichtigen Bezugspersonen der Kinder (Eltern, Lehrer) angestrebt.

Lauth und Schlottke (1997) empfehlen einen individuellen Therapieeinstieg je nach Ausprägung des Störungsgrades. Wenn ein Kind bestimmte Fertigkeiten erworben hat, soll mit den Inhalten eines anderen Therapiebausteins weiter gearbeitet werden. Wenn ein Kind nur langsam vorankommt, sollen Therapiesitzungen wiederholt werden. 
Das Programm beinhaltet verschiedene Bausteine, die im Folgenden kurz erläutert werden sollen. Im Basistraining werden dem Kind Grundfertigkeiten vermittelt (z.B. genau hinschauen, genau zuhören, Wahrgenommenes wiedergeben) sowie Reaktionskontrolle durch allmählich verinnerlichte Reaktionsverzögerung aufgebaut. Zur Vermittlung der Reaktionsverzögerung wird eine Stop-Signalkarte eingeführt, die den Kindern zunächst real (vor Augen) und später imaginativ als Hinweis zur Reaktionskontrolle „Stop - Aufpassen, Nachdenken und Überprüfen“ dienen soll. Mit dem Basistraining wird überdies der Aufbau verbaler Handlungsregulation eingeleitet. Die Kinder sollen lernen, ihre Handlungen durch verbale Selbstanweisungen $\mathrm{zu}$ steuern. Als therapeutische Verfahren werden operante Verstärkung, Diskussionen und Gespräche mit den Kindern, Modellierungen und Demonstrationen durch den Trainer/Therapeuten sowie Übungs- und Spielphasen eingesetzt. Ziel des Basistrainings ist die Verbesserung der Verhaltensregulation.

Der Ablauf des Basistrainings sieht folgende Schritte vor:

1. Ableitung förderlicher Vorgehensweisen: Ziele und Aufbau des Trainings sowie Hintergrundwissen über die Störung werden mit den Kindern anhand von standardisierten Videobeispielen erarbeitet.

2. Genau hinsehen und das Gesehene so genau wie möglich beschreiben.

3. Genau hinhören, d.h. Informationen trotz Hintergrund- und Störgeräuschen wahrnehmen.

4. Genau zuhören, d.h. Gehörtes aufnehmen, speichern und wiedergeben.

5. Wahrgenommenes genau wiedergeben: Aufnahme verbaler Informationen von anderen Personen und eigene Umsetzung dieser Informationen.

6. Reaktionsverzögerung: Erlernen einer überlegten Handlungsdurchführung und Bekanntgabe des Ergebnisses nach (verzögerter) Überprüfung der Ergebnisse.

7. Selbständige Reaktionsverzögerung zur Festigung des „Überprüfens“ sowie Üben des Transfers in den Alltag.

8. Differenzierte Reaktionsverzögerung, d.h. Lösungen sollen prozessbezogen überprüft werden.

9. Prüfprozesse: Übertragung der Reaktionsverzögerung und des Überprüfens auf schulische Inhalte durch verinnerlichte Selbstanweisung (verbale Handlungsregulation).

10. Ablenkung: Anwendung der bis hierher erworbenen Kompetenzen unter ablenkenden Bedingungen. 
Laut Trainingsmanual ist das Basistraining nur dann indiziert, wenn sich bei einem Kind Schwierigkeiten bezüglich der Grundfertigkeiten andeuten bzw. seine Selbststeuerungsfertigkeiten unzureichend sind.

Mit dem Strategietraining werden der Erwerb und die Umsetzung von Problemlösestrategien angestrebt. Dazu werden Signalkarten eingesetzt, die einzelne Problemlöseschritte (z.B. Analyse der Anforderung, Planung des Vorgehens, Überprüfung des eigenen Handelns) veranschaulichen. Die Kinder sollen handlungssteuernde Selbstinstruktionen erlernen. Schwerpunktmäßig wird damit an den Einschränkungen der Verhaltensorganisation angesetzt. Als Therapietechniken kommen Methoden des Selbstinstruktionstrainings (Modellierung förderlichen Vorgehens durch den Therapeuten und Anleitung zur verbalen Selbstanweisung, die allmählich in ein „inneres Sprechen“ überführt werden soll), Übungsphasen und verhaltenstherapeutische Grundtechniken (Rückmeldung, soziale Verstärkung und Selbstverstärkung) zur Anwendung.

Der Ablauf des Strategietrainings sieht folgende Schritte vor:

1. Erklärung der Ziele und der Struktur des erweiterten Trainings:

- (vertiefte) Vermittlung von Wissen über die Aufmerksamkeitsstörung anhand von Beispielen,

- Einführung spezieller Signalkarten,

- Modelldemonstration anhand der Signalkartenanwendung,

- Übungsphase der Kinder an eigenen Aufgaben.

2. Flexibler Einsatz des „Lauten Denkens“ und kooperative Aufgabenlösung: Übertragung der Therapieinhalte auf andere Lebensbereiche (Schule, Hausaufgaben).

3. Flexibilisierung der bisherigen Bearbeitungsstruktur: Strategien festigen und lernen, diese auf inhaltsähnliche Probleme zu übertragen.

4. Übertragung der Bearbeitungsstruktur: Bisheriges Lösungsvorgehen verallgemeinern und auf neue Problemstellungen übertragen.

5. Übertragung auf abstrakte Probleme: Gedankliche Modelldemonstration wird gefördert durch die Anwendung verschiedener Analyseschritte zur Lösungsfindung.

Weitere Therapiebausteine, die eingesetzt werden können, sind Einheiten zur Wissensvermittlung sowie Einheiten zur Vermittlung sozialer Kompetenzen. Der Baustein der Wissensvermittlung fokussiert auf die Übertragung erlernter Trainingsinhalte (v.a. des Strategietrainings) auf schulische Aufgaben. Es werden außerdem Lernstrategien vermittelt. 
Mit dem Baustein „Vermittlung sozialer Kompetenzen“ wird versucht die Selbststeuerungsfähigkeit des Kindes zu erhöhen, die Wahrnehmung und Verarbeitung sozialer Informationen zu verbessern, die Verfügbarkeit sozialer Fertigkeiten zu erreichen und das Selbstvertrauen zu erhöhen.

Die Therapiebausteine bestehen aus einzelnen Trainingseinheiten (Basistraining: 13 Einheiten; Strategietraining: 12 Einheiten). Es ist vorgesehen, dass die Einheiten adaptiv durchlaufen werden, d.h. zu einer neuen, komplexeren Einheit wird erst übergegangen, wenn das Zielverhalten der vorhergehenden Sitzungen von dem Kind übernommen wurde. Die einzelnen Trainingseinheiten weisen dabei eine identische Struktur auf (einleitende Bemerkungen und Feststellung der jeweiligen Sitzungsziele; Modelldemonstration oder gemeinsame Ableitung förderlicher Vorgehensweisen; Übungen der Kinder zur Umsetzung des angestrebten Vorgehens; spielerischer Ausklang, in dem die Sitzungsinhalte nochmals auf spielerische Weise aufgegriffen werden, ggf. Entspannungsübungen).

Neben der Elternanleitung wird außerdem eine Zusammenarbeit mit Lehrern angestrebt. In der Elternanleitung wird zunächst über die Diagnose Aufmerksamkeitsstörungen (Besonderheiten der Kinder, Bedingungsfaktoren und Auswirkungen) und die Therapie (Ziele, Vorgehen) informiert, um die Zusammenarbeit und die Akzeptanz der Intervention zu fördern und damit die Übertragung der Trainingsinhalte auf das Alltagsverhalten der Kinder zu ermöglichen. Die Eltern sollen überdies Fähigkeiten erwerben, mit denen sie ihre Kinder im Alltag unterstützen können (z.B. durch veränderte Erziehungsmaßnahmen oder alternative Interpretationen konfliktreiche Situationen anders zu gestalten). Die jeweiligen Inhalte der Elternanleitungen sind an den Stand der gerade mit den Kindern durchgeführten Therapie orientiert. Die Zusammenarbeit mit den Lehrern zielt auf ein angemessenes Störungsverständnis und die Unterstützung der therapeutischen Bemühungen sowie der Kinder im Schulalltag ab. Günstig ist es eine Unterstützung der Therapie durch den Lehrer (z.B. durch besondere Zuwendung bei erwünschtem Verhalten des Schülers) sowie auch eine Verbesserung der Lernfähigkeit des Kindes anzustreben, in dem „prozessorientierte Hilfen“ gegeben werden (z.B. Modelldemonstrationen).

Laut Programmautoren ist es möglich, das Trainingsprogramm als Einzel- und Gruppentraining durchzuführen. Das Training in der Gruppe hat nach Meinung von Lauth und Schlottke (1997) den Vorteil, alltagsnähere Therapiebedingungen zu schaffen und soziale Lernprozesse des Kindes zu ermöglichen. In einer Gruppe sollten jedoch nur maximal drei 
Kinder zusammengefasst werden. Dabei sollten die Kinder in einer Gruppe hinsichtlich ihrer Leistungsfähigkeit und ihrer Störungsschwerpunkte homogen sein.

Ein wesentliches Element bei der Durchführung des Trainings ist die Verstärkung der Kinder mit Büroklammern, welche sie für erwünschtes Verhalten bekommen. Pro Sitzung können die Kinder sich bis zu fünf Klammern verdienen. Wenn sie 15 Klammern gesammelt haben, können sie diese gegen ein kleines Geschenk aus einer „Schatzkiste“ eintauschen. Dieses Token-System erweist sich bei der Verstärkung der Kinder für gewünschtes Verhalten als sehr wirksam.

Durch das Manual ist dem Anwender eine gut durchschaubare Anleitung an die Hand gegeben, die auf der einen Seite genaue Durchführungsrichtlinien zur erleichterten Anwendung bietet, auf der anderen Seite aber auch eine gewisse flexible Therapiegestaltung zulässt.

\subsubsection{Wirksamkeitsuntersuchung}

Das Trainingsprogramm liegt mittlerweile in der fünften Auflage vor. Es hat im deutschsprachigen Raum eine weite Verbreitung gefunden. Es liegen insgesamt 11 Studien zu diesem Programm vor, die in der Mehrzahl von den Autoren selbst durchgeführt wurden (vgl. Lauth \& Linderkamp, 2000; Lauth \& Schlottke, 1997). In einer kontrollierten Studie (Schlottke, 1984, zitiert nach Lauth \& Schlottke, 2002), in der Kinder mit einer hyperkinetischen Störung einer Therapiegruppe (Basis- oder Strategietraining mit begleitender Elternanleitung) oder einer Wartegruppe (ohne Behandlung) zugewiesen wurden, wurde im Eltern- und Lehrerurteil eine Verbesserung des Alltagsverhaltens der trainierten Kinder festgestellt. In der Mehrzahl der Studien führte die Intervention sogar zu Störungsfreiheit. Der Effekt war in Nachuntersuchungen zum Teil noch nach sechs Jahren nachzuweisen (Linderkamp, 2002). Es waren aber vor allem die Eltern, welche ihre Kinder dann als störungsfrei einschäzten, von den Lehrern wurde keine Störungsfreiheit beobachtet. Die Lehrer berichteten zum Teil, dass die Intervention keine Auswirkungen auf das Schulund Unterrichtsverhalten hatte (Kausch, 2002).

Beck und Mock (1995, 1996) konnten in ihrer Studie keine verbesserte Aufmerksamkeitsleistung feststellen, was von Lauth und Schlottke (2003) so erklärt wird, dass die Gruppen nicht klinisch auffällig gewesen seien, die Kinder starr dem Basistraining zugeordnet wurden und die Gruppen inhomogen waren.

Im Alltagsverhalten treten Verbesserungen ein, besonders bei den Hausaufgaben und bei den Mahlzeiten (Lauth \& Fellner, 1998; Schlottke, 1984). 
Lauth (1996) führte in einer Studie das Strategietraining (ohne und mit Elternanleitung) durch und verglich die Ergebnisse mit einer unbehandelten Kontrollgruppe, die das gleiche Material bearbeitete. Die Intervention wurde zweimal pro Woche in Gruppen von zwei bis vier Kindern durchgeführt. Einschließlich Datenerhebung umfasste die Intervention einen Zeitraum von fünf Monaten. In der Eltern- und Lehrerbefragung wurden nach dem Training in den Interventionsgruppen Verbesserungen im Aufmerksamkeits- und Unterrichtsverhalten berichtet: Verbesserte Problemlösereflexivität, das Vorgehen war besser organisiert, und sie erreichten bei Problemlöseaufgaben mehr richtige Lösungen, die Sorgfalt und Bedachtheit im Unterricht wurde von den Lehrern als höher eingestuft.

Die Durchführungspraktikabilität kann als hoch eingestuft werden. Lauth und Linderkamp (1998) ließen das Training von Nicht-Psychologen nach entsprechender Einweisung und unter Supervision durchführen. Die Studentinnen bewerteten das Programm hinsichtlich Vorbereitungsaufwand, Zufriedenheit der Trainingsleiter und Therapieschwierigkeiten als zufrieden stellend.

In Einzeltherapien konnten Lauth, Naumann, Roggenkämper und Heine (1996) eine verbesserte Aufmerksamkeitsleistung sowie eine positive Beeinflussung der zentralnervösen Aktivierung nachweisen. In einer katamnestischen Untersuchung nach zwei bis drei bzw. vier bis sechs Jahren wies Linderkamp (2002) stabile Effekte in Bezug auf die Störungssymptomatik nach, unabhängig davon, ob die Kinder zwischendurch weitere Interventionen erhalten hatten oder nicht. Das Training hatte jedoch keinen oder nur geringen Einfluss auf oppositionelle Verhaltensstörungen und verminderte Schulleistungen, die bereits vor der Intervention bestanden. Kritisch ist aber zu bemerken, dass es sich hier nicht um eine kontrollierte Studie handelt.

\subsection{Therapieprogramm für Kinder mit hyperkinetischem und oppositionellem Trotzverhalten (THOP)}

Beim Therapieprogramm für Kinder mit hyperkinetischem und oppositionellem Trotzverhalten (THOP) von Döpfner, Schürmann und Frölich (1997) handelt sich um ein manualisiertes verhaltenstherapeutisches Eltern-Kind-Programm, das auch zur Durchführung als schulzentrierte Intervention geeignet ist. Die Programmautoren orientiert sich in der theoretischen Fundierung des THOP am Modell der Störung des regelgeleiteten Verhaltens von Barkley (1989) und dem Teufelskreismodell über negative Eltern-Kind-Interaktionen (Barkley, 1981). 
Die charakteristischen Inhalte und Methoden des Trainings bauen auf den Arbeiten von Barkley (1987), Forehand und McMahon (1981) und vielfältigen Studien anderer Forschungsgruppen zur Wirksamkeit verhaltenstherapeutischer Interventionen bei hyperkinetischen und oppositionellen Verhaltensstörungen auf.

\subsubsection{Aufbau und Ziele}

Das THOP wurde für Kinder zwischen 3 und 12 Jahren entwickelt und umfasst in der zweiten Auflage, welche im Rahmen dieser Studie für die Durchführung benutzt wurde, 21 Behandlungsbausteine.

(Für die dritte Auflage (Döpfner, Schürmann \& Frölich, 2002) wurde das Programm um einen Therapiebaustein zu Familienregeln erweitert; der Aufbau des Textes wurde verändert, und es wurde eine CD-ROM beigefügt, auf der die diagnostischen Materialien, Materialien zur Therapieplanung und Verlaufsgeschichte, Materialien für das Eltern-KindProgramm sowie weitere Therapieunterlagen enthalten sind).

Im Eltern-Kind-Programm sind zwei Interventionsformen miteinander verbunden. In der familienzentrierten Intervention werden die Eltern zur Durchführung spezifischer Interventionen in der Familie angeleitet. Den Eltern werden kognitive und operante Techniken (Stimuluskontrolle, Kontingenzmanagement) und Methoden vermittelt. Ein wesentliches Element stellt für viele Familien der Aufbau positiver Eltern-Kind-Interaktionen dar. Bei der kindzentrierten Intervention werden anhand von Kurzgeschichten dem Kind die Inhalte der einzelnen Behandlungsbausteine vermittelt. Die Hauptfigur der Geschichten (Wackelpeter) soll den Kindern als Bewältigungsmodell dienen. Die Geschichten behandeln typische familiäre und schulische Problemsituationen. Der Therapeut versucht mit dem Kind Bewältigungsmöglichkeiten zu entwickeln.

Als Methoden werden hier kognitive Techniken, Modelllernen und Methoden des Selbstmanagements angewendet. Angestrebt werden eine Verbesserung der Interaktion mit den Eltern sowie eine Verminderung der Verhaltensprobleme. Mit dem Spieltraining und dem Selbstinstruktionstraining, welches zunächst vom Therapeuten durchgeführt wird und in das die Eltern im weiteren Verlauf als Co-Therapeuten integriert werden, wird das Kind zur Ausdauer beim Spielen und systematischer Aufgabenbearbeitung angeleitet. Begleitend zu einer medikamentösen Therapie bzw. einer schulzentrierten Intervention können zwei weitere Bausteine durchgeführt werden.

Die Bausteine des Programms sollen aufgrund der individuellen Problemkonstellation der jeweiligen Familie zusammengestellt werden. Es müssen also nicht alle Bausteine 
bearbeitet werden, sondern nur solche, die für die Familie relevant sind. Dem Programm soll eine umfassende Diagnostik vorgeschaltet sein. Anhand der Ergebnisse der Diagnostik wird dann gemeinsam mit den Eltern eine Problemdefinition vorgenommen. Es werden insbesondere Prinzipien umgesetzt, die die Bedingungen verändern, die das Problem (besonders externale Auffälligkeiten) innerhalb der Familie aufrechterhalten.

Es werden folgende Grundprinzipien formuliert:

- Konsequenzen müssen unmittelbar auf das Verhalten folgen,

- Verbale Konsequenzen (Lob und Tadel) müssen spezifisch sein,

- Konsequenzen müssen konsistent erfolgen,

- Zuerst werden Belohnungs-, danach erst Bestrafungsmethoden vermittelt,

- Reaktionen auf mögliches Fehlverhalten sollten in jeder Situation bedacht werden.

Ausgehend von Belastungsfaktoren auf der Makroebene der Familie (z.B. Eigenschaften, Wünsche, Ziele und Belastungen der Eltern und des Kindes) wird eine Verbindung auf der Mikroebene hergestellt. Ziele des Programms ist es auf der Mikroebene die alltäglichen Belastungen der Eltern-Kind-Interaktion zu verändern. Dabei ist die Intervention auf der Mikroebene in eine Intervention bzgl. des familiären und psychosozialen Bedingungsgefüges (Makroebene) eingebettet. Positive Veränderungen auf der Mikroebene können nur dann stabil sein, wenn auf der Makroebene keine grundlegenden Faktoren den Erfolg der Maßnahmen behindern. Da Mikro- und Makroebene zirkulär miteinander verknüpft sind, muss die wechselseitige Beeinflussung dieser Ebenen beachtet werden.

Die äußere Struktur des Eltern-Kind-Programms ist wie folgt gegliedert:

1. Problemdefinition, Entwicklung eines Störungskonzeptes und Behandlungsplanung (Erwerb von Kenntnissen über das Störungsbild und mögliche Ursachen der Entstehung und Aufrechterhaltung).

2. Förderung positiver Eltern-Kind-Interaktionen und Eltern-Kind-Beziehungen (Unterbrechung dysfunktionaler Interaktionsmuster durch die Wahrnehmung positiver Eigenschaften des Kindes und positiver Reaktionen hierauf durch die Eltern).

3. Pädagogisch-therapeutische Interventionen zur Verminderung impulsivem und oppositionellem Verhalten (Erwerb und Einsatz pädagogisch-therapeutischer Interventionen bzw. spezifischer operanter Methoden durch die Eltern).

4. Spezielle operante Methoden (Vermittlung von Token- und response-cost-Systemen). 
5. Intervention bei spezifischen Verhaltensproblemen (Verbesserung von z.B. Spiel- und Beschäftigungsintensität bzw. -ausdauer, Arbeitsstil, Hausaufgabenbearbeitung, Verhalten in der Öffentlichkeit durch den Erwerb kognitiver Strategien durch das Kind und den Erwerb und Einsatz spezifischer Interventionen durch die Eltern ).

6. Stabilisierung der Effekte (erreichte Veränderungen werden durch den Erwerb von Strategien zur Lösung zukünftig auftretender Probleme durch die Eltern angepeilt).

\subsubsection{Wirksamkeitsuntersuchung}

Die Wirksamkeit des THOP wurde von den Autoren selbst im Rahmen der Kölner Multimodalen Therapiestudie (COMIS) (Döpfner, Breuer und Lehmkuhl, 2000) untersucht. Die Forscher führten die Interventionen anhand einer festgelegten Therapiestrategie durch. Nach einer initialen sechswöchigen Phase der Psychoedukation und des Beziehungsaufbaus wurden 75 Kinder je nach individuellem Behandlungsverlauf entweder medikamentös (Psychostimulanzien, $\mathrm{N}=28$ ) oder verhaltenstherapeutisch (THOP und begleitende Lehrerkontakte, $\mathrm{N}=45$ ) behandelt. Bei zwei Kindern wurde die Therapie abgebrochen. Je nach Verlauf wurde zur jeweils anderen Therapieform gewechselt. War die medikamentöse Therapie nicht erfolgreich, wurde zu einer Verhaltenstherapie gewechselt. Bei teilweisem Erfolg wurde eine zusätzliche verhaltenstherapeutische Behandlungsphase eingerichtet. Erbrachte die Stimulanzientherapie ausreichende Erfolge, blieb es bei dieser Behandlung (die gleichen Zuweisungsstrategien galten bei initial durchgeführter Verhaltenstherapie). Bisher liegen die Ergebnisse von Einzelfallstudien und Vorstudien vor, die folgendes Bild über das Eltern-Kind-Training ergeben (Döpfner \& Lehmkuhl, 2002; Döpfner, Lehmkuhl \& Schürmann, 1996; Frölich \& Döpfner, 1997):

- Die Abbrecherquote war mit $10 \%$ insgesamt sehr gering und die Zufriedenheit der Eltern sehr groß.

- Bei $72 \%$ der Kinder, die initial verhaltenstherapeutisch behandelt wurden, wurde keine zusätzliche Stimulanzientherapie durchgeführt. Demgegenüber wurden $82 \%$ der Kinder, die zunächst eine Stimulanzientherapie erhalten hatten, aufgrund klinischer Kriterien auch verhaltenstherapeutisch behandelt.

- 50 bis $60 \%$ der Kinder, die ausschließlich mit Verhaltenstherapie behandelt wurden, zeigten bei Behandlungsende nur noch minimale Verhaltensauffälligkeiten in der Familie (therapierelevante individuelle Verhaltensprobleme oder keine Störungsdiagnose mehr). 
- 55 bis $60 \%$ der Kinder zeigten bei Behandlungsende nur noch geringe Verhaltensauffälligkeiten in der Schule, so dass die DSM-IV-Kriterien nicht mehr erfüllt waren.

- Der Anteil der Kinder mit emotionalen Auffälligkeiten sank deutlich. Nach der Therapie wurden im CBCL (Achenbach, 1991) auf dieser Skala 61\% als unauffällig eingestuft ( $\mathrm{Zu}$ Beginn der Therapie waren es $35 \%$ der Kinder).

- Zusätzliche Effekte von Verhaltenstherapie nach vorausgegangener Stimulanzientherapie ließen sich nur teilweise nachweisen, während durch eine zusätzlich zur Verhaltenstherapie durchgeführte Stimulanzienbehandlung deutliche Symptomminderungen erzielt werden konnten.

- Verlaufsdaten zur Langzeitwirkung liegen bislang nicht vor.

Das Programm wird als gut erlernbar beschrieben. Besonders Therapeuten mit einer Grundausbildung in VT können es relativ schnell beherrschen. Das Programm kann auch mit Eltern mit geringerem Bildungsniveau durchgeführt werden. Die Zufriedenheit der Eltern mit dem Programm ist hoch, selbst dann, wenn nicht alle Therapieziele bzgl. der Symptomatik der Kinder erreicht wurden. Nur wenige Familien scheinen von dem Programm überhaupt nicht $\mathrm{zu}$ profitieren, es sind aber auch nur wenige Kinder zum Ende der Therapiemaßnahmen symptomfrei.

Die Motivation von Lehrkräften in Grundschulen und Erzieherinnen des Kindergartens bei dem Therapieprogramm mitzuwirken ist höher als bei Lehrkräften weiterführender Schulen. Bei Kindern mit sehr stark ausgeprägter hyperkinetischer Symptomatik reichen verhaltenstherapeutische Maßnahmen oft nicht aus, so dass mit Medikamenten behandelt werden muss.

Die Hauptprobleme bei der Durchführung des Programms werden in Widerständen in Familie, Kindergarten und Schule gesehen.

Um einen günstigen Verlauf auch nach dem Ende der Behandlung zu erreichen, wird eine kontinuierliche Nachsorge empfohlen.

Frölich (1993) konnte im Rahmen einer multimodalen Intervention die Effektivität des Elterntrainings an 18 Patienten im Alter von sechs bis zwölf Jahren belegen. Individuelle Verhaltensprobleme konnten durch Selbstinstruktion und Selbstmanagement-Techniken vermindert werden. Eine weitere Verbesserung konnte durch das Elterntraining (THOP) erreicht werden. Verbesserungen zeigten sich hinsichtlich der Kernsymptomatik, Störung des 
Sozialverhaltens, bei Konfliktsituationen zu Hause, den Hausaufgabenproblemen und bei individuellen Verhaltensproblemen. Das Verhalten in der Schule stabilisierte sich, verbesserte sich aber nicht, was auf den spezifischen Effekt des Elterntrainings innerhalb der Familie zurückgeführt wird.

Die guten Effekte dieser Studie führen Frölich, Döpfner, Berner und Lehmkuhl (2002) darauf zurück, dass die behandelten Probleme für die Kinder „lebensnah“ waren, das kognitive Entwicklungsniveau beachtet wurde und die Eltern und Lehrer aktiv einbezogen wurden. Die Kombination von Selbstmanagement und Selbstinstruktionsmethoden bei der kognitiven Verhaltenstherapie wird von den Autoren als wirksam eingestuft.

Selbstkritisch schränken Frölich et al. ein, dass in ihrer Studie keine Kontrollgruppe unauffälliger Kinder als Vergleichsgruppe gebildet wurde. Die Probandenzahl von 18 Kindern und fehlende Follow-up-Untersuchungen schränken die Aussagefähigkeit der Studie ebenfalls ein. Die Autoren erwarten Symptomrückfälle und empfehlen Auffrischungssitzungen in größeren Abständen.

\subsection{Das Marburger Konzentrationstraining}

\subsubsection{Aufbau und Ziele}

Das Marburger Konzentrationstraining wurde für Kinder des ersten bis sechsten Schuljahres konzipiert. Der Autor benennt nicht explizit Kinder mit der klinischen Diagnose einer Aufmerksamkeitsstörung als Zielgruppe für dieses Verfahren. Das Training sei geeignet für Kinder mit einem impulsiven Arbeitsstil, welcher durch planloses, oberflächliches und zu schnelles Vorgehen gekennzeichnet ist. Dieses steht in Zusammenhang mit der kognitiven Steuerung der Aufmerksamkeit. Das Kind soll lernen, seine Aufmerksamkeit zu zentrieren. Ziel des Trainings ist es den Arbeitsstil bzw. kognitiven Stil des Kindes zu verändern und somit einen reflexiven Arbeitsstil zu fördern (vgl. Krowatschek, 1994a).

Das Training besteht aus drei Bereichen: den Trainingsstunden mit dem Kind, dem häuslichen Übungsprogramm und der Elternarbeit. Die folgende Abbildung verdeutlicht den Gesamtablauf: 


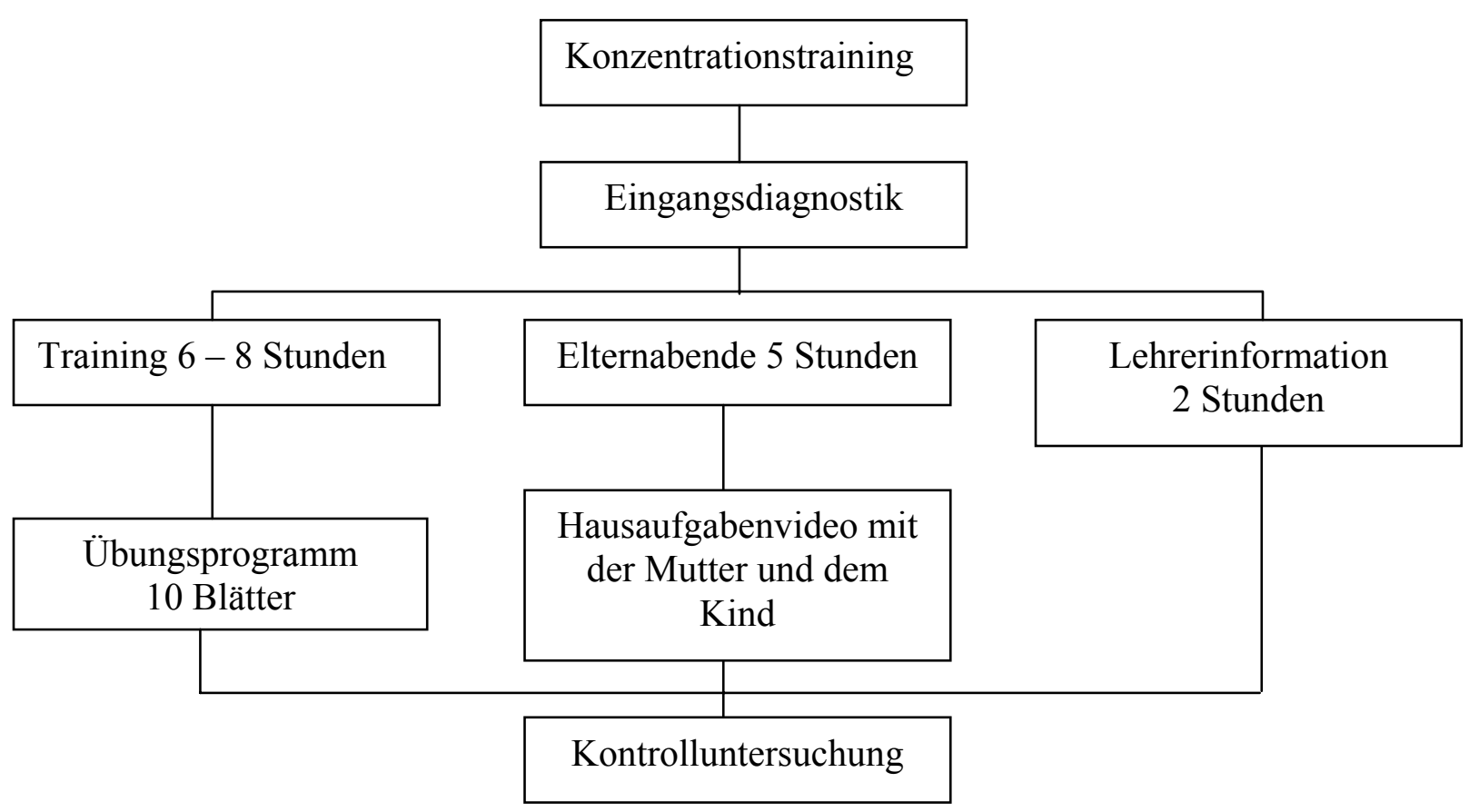

Abbildung 3

Aufbau des Konzentrationstrainings (Krowatschek, 1994a)

Die Grundlage für das Marburger Konzentrationstraining bildet die Methode der verbalen Selbstinstruktion nach Meichenbaum und Goodman (1971). Durch diese Methode sollen die Kinder ihre Selbstregulationsfähigkeiten und reflexiven Problemlösestrategien verbessern. Kinder mit Konzentrationsstörungen weisen neben einem Defizit in der kognitiven Steuerung auch deutlich weniger aufgabenbezogene Selbstinstruktionen auf (vgl. Krowatschek, 1994a). Durch das Selbstinstruktionstraining soll systematisches Handeln, eine Reaktionsverzögerung und inneres Sprechen erreicht werden. Der Therapeut dient als Modell, welcher zunächst durch das eigene laute Denken dem Kind den Problemlöseprozess über Selbstanweisungen verdeutlicht. Im Verlauf der Trainings sollen die Kinder die Selbstanweisungen über fünf Stufen bis hin zum lautlosen Sprechen (,innerer Monolog“) erlernen.

Für das Marburger Konzentrationstraining werden vom Autor folgende Ziele genannt:

- Erhöhung der Selbststeuerung, der Selbständigkeit und der Selbstakzeptanz des Kindes

- Verbesserung der Motivation durch erfolgreiches Bearbeiten von Aufgaben und durch angemessenes Umgehen mit Fehlern

- Veränderung der Eltern-Kind- und Lehrer-Kind-Interaktion (Krowatschek, 1994a). 
Im Folgenden wird der Ablauf des Marburger Konzentrationstrainings dargestellt: Krowatschek hat das Training für Gruppen bis zu acht Kindern konzipiert. Es sollte idealerweise jedoch mit drei bis fünf Kindern pro Gruppe durchgeführt werden. Es ist außerdem möglich das Training als Einzelförderung durchzuführen. Es eignet sich auch zur Förderung weniger begabter bzw. entwicklungsverzögerter Kinder.

Die Trainingsstunden sollten einmal wöchentlich durchgeführt werden und für eine Gruppe von drei Kindern mindestens sechs Stunden umfassen. Eine Trainingsstunde selbst besteht aus drei verschiedenen Bereichen. Zu Beginn wird eine Entspannungsübung durchgeführt, welche mit einer Rückmeldung der Erfahrungen abgeschlossen wird. Im Anschluss folgen zwei bis vier Arbeitsphasen, für welche Arbeitsblätter und Bildvorlagen genutzt werden. Den Abschluss der Stunde bildet eine Spielphase.

In der ersten Stunde führt der Trainer die Aufgabenbearbeitung als Modell vor, indem er laut zu sich selbst spricht. Anschließend führt das Kind mit Instruktionen, die es vom Trainer erhält, die Aufgabe durch. In den folgenden Stunden wird die Instruktion vom Kind erst laut, dann flüsternd und abschließend lediglich gedanklich selbst durchgeführt.

Zusätzlich zum wöchentlichen Training in der Gruppe ist ein häusliches Übungsprogramm vorgesehen. Die Kinder erhalten Aufgabenblätter mit der Methode der Selbstinstruktion, welche sie zu Hause bearbeiten sollen. Die Materialien sind jenen in der Stunde verwandten sehr ähnlich. Dadurch soll die Methode der Selbstinstruktion intensiviert werden. Die Eltern sollen vor allem zu Beginn ihre Kinder dabei unterstützen.

Begleitend werden Elternabende durchgeführt, auf denen über Konzentrationsstörungen informiert wird. Sie sollen aber auch dem Austausch der Eltern untereinander dienen. Im Manual wird die Durchführung der Elternabende nur sehr kurz erwähnt. Die Ausgestaltung der Elternabende ist nicht transparent.

Zwischen dem ersten und zweiten Elternabend soll ein Video aufgenommen werden, was die Elternteil-Kind-Interaktion in einer Hausaufgabensituation zeigt. Auf dem dritten Elternabend sollen positive Interaktionen zwischen Kind und Eltern gezeigt und diskutiert werden.

Den Eltern sollen folgende Themenbereiche nahe gebracht werden: (1) Einführung in Aufbau und Methode des Konzentrationstrainings, (2) Was ist Konzentration?, (3) Besprechung der Hausaufgaben, (4) Hemmende und fördernde Faktoren der Konzentration, (5) Vorstellung von Fördermaterialien. 
Wie die über zwei Stunden durchzuführende Lehrerinformationen durchgeführt werden soll, wird nicht erläutert.

Detaillierter werden Entspannungsübungen beschrieben. Insbesondere durch den Einsatz des Autogenen Trainings komme es meist sehr schnell zu einer besseren Konzentrationsfähigkeit (Krowatschek, 1994a). Der ruhige und entspannte Zustand schaffe durch den Abbau physiologischer Erregung die notwendige Ruhe, um das Selbstinstruktionstraining durchzuführen. Als Modifikationsmethoden soll Folgendes eingesetzt werden: (1) Positive Verstärkung, (2) Differenzierte Aufmerksamkeit (positive Aufmerksamkeit und Ignorieren), (3) Lernen am Modell, (4) Selbsteinschätzung, (5) Timeout, (6) Token-Verstärkerprogramme, (7) Response-Cost-Methoden und (8) Selbstkontrolle.

\subsubsection{Wirksamkeitsuntersuchung}

Bislang wurde die Wirksamkeit des Marburger Konzentrationstrainings nur in unveröffentlichten Diplomarbeiten im Fachbereich Psychologie der Philipps-Universität Marburg untersucht (Krowatschek, 2001). In dem Trainingsmanual finden sich nur wenige Hinweise auf empirische Studien. Die Wirksamkeit des Trainings ist demnach nicht hinreichend belegt. Bei Veröffentlichung des Trainings wurde dieses an über 300 Kindern erprobt. Der Autor berichtet, die trainierten Kinder hätten ihren Arbeitsstil verbessert, motiviert bei den Stunden mitgearbeitet, und ihre Selbstakzeptanz sei nach dem Training höher gewesen (Krowatschek, 1994a). Die Eltern hätten ebenfalls gern an dem Training teilgenommen. Ihr Verhalten bei den Hausaufgaben und im Umgang mit den Kindern sei verändert. Auch Lehrer berichteten von Verbesserungen. Diese wurden jedoch nicht näher beschrieben. Es werden keine Testverfahren oder Operationalisierungen der Überprüfungen benannt. In einer neueren Veröffentlichung von Krowatschek (2003) berichtet er von einer Studie, in der eine Vorläuferversion des Marburger Konzentrationstrainings eingesetzt wurde. Smid (1992, zitiert nach Krowatschek, 2003) konnte ein reflexiveres und weniger impulsives Verhalten in einem Aufmerksamkeitsbelastungstest nachweisen. Die Eltern gaben einen Rückgang emotionaler Auffälligkeiten an.

Domsch (2002, zitiert nach Krowatschek, 2003) stellte in Befragungen nach Durchführung des Trainings fest, dass das elterliche Stresserleben abgenommen hat. Krowatschek (1996, zitiert nach Krowatschek, 2003) wertete Videoaufnahmen von Hausaufgabensituationen aus und stellte eine verbesserte Interaktion fest.

Das Marburger Konzentrationstraining wurde von Beck (1998) in einer Vergleichsstudie mit dem Lauth- und Schlottke-Programm als „Quasi-Alternativtraining“ im 
Rahmen schulischer Alltagsbedingungen eingesetzt. Beck schränkt die Aussagefähigkeit seiner Untersuchung dahingehend ein, dass eine Stichprobe ausgewählt wurde, die in der Zielgruppe des Marburger Konzentrationstrainings als Extremgruppe gewertet werden muss. Auf die Elternarbeit wurde in dieser Studie verzichtet. Das Marburger Konzentrationstraining führte zu nur sehr geringen Wirkungen (Es trat lediglich eine Verbesserung des Konzentrationsleistungswertes im Test d2 auf (Brickenkamp, 1994)). Der Wartekontrollgruppe war das Training im Prä-Post-Vergleich nicht überlegen.

Witte (2002) konnte jedoch in einer Vergleichsstudie die Wirksamkeit des Marburger Konzentrationstrainings in Vergleich mit einer Kontrollgruppe nachweisen. Es wurden Verbesserungen in den Parametern Konzentrationsleistung (d2), kognitive Leistungs- und Verarbeitungsgeschwindigkeit (Zahlen-Verbindungs-Test, Oswald \& Roth, 1978) und visuellmotorische Koordination (Zahlen-Symbol-Test des Hamburg-Wechsler-Intelligenztests für Erwachsene HAWIE, Wechsler, 1982) erzielt. Ein Transfer auf schulische Inhalte wurde nicht erreicht. Witte konnte zeigen, dass ein videogestütztes Selbstinstruktionstraining dem Marburger Konzentrationstraining überlegen ist.

\subsection{Psychologische Hypothesen (PHn)}

\subsubsection{PHn zu Studie 1}

Die Hauptfrage der ersten Studie lautet: Führt das Basistraining nach Lauth und Schlottke (1997) im Rahmen der regulären klinisch-psychologischen Versorgung einer Kinder- und Jugendpsychiatrie $\mathrm{zu}$ der theoretisch postulierten Verbesserung der Verhaltensregulation und damit zu einem verbesserten Aufmerksamkeitsverhalten der Kinder? Forschungsbefunde aus Studien der Programmautoren lassen dies erwarten. Es wird deshalb folgende psychologische Hypothese formuliert:

PH 1.1: Das Basistraining nach Lauth und Schlottke führt unter den praktischen Rahmenbedingungen einer kinder- und jugendpsychiatrischen Ambulanz zu Verbesserungen der Aufmerksamkeitsleistung sowie zu einer Verminderung hyperkinetischer Verhaltenssymptome.

Die PH 1 bezieht sich auf die Wirksamkeit der Intervention. Es geht um angestrebte Verbesserungen von beobachtbaren Leistungs- und Verhaltensaspekten. Jeder Wirksamkeit liegt eine bestimmte, nicht-beobachtbare Wirkung zugrunde. Nach Hager und Hasselhorn (2000, S. 44) geht „der Begriff ,Wirkungen“ auf theoretisch angenommene Ursachen der 
beobachtbaren Verhaltensänderungen“" zurück. Aus theoretischer Perspektive ist es interessant der Frage nachzugehen, wie sich die beobachtbaren Sachverhalte erklären lassen (Hager \& Hasselhorn, 1995).

Es soll deshalb untersucht werden, ob, gemäß den Annahmen neuerer Enstehungsmodelle (Barkley, 1997), die vermutete Wirksamkeit des Trainings durch Veränderungen im Bereich der inhibitorischen Kontrolle erklärbar ist. Nach Barkley fällt es den aufmerksamkeitsgestörten Kindern schwer einen dominanten Handlungsimpuls zu unterdrücken. Interferierende Handlungstendenzen zu hemmen und eine laufende Handlung zu unterbrechen, gelingt ihnen kaum. Es wird erwartet, dass durch das Basistraining nach Lauth und Schlottke eine Verbesserung erzielt wird. Die Ursache der gestörten Verhaltenshemmung und damit den Ursprung von ADHD sieht Barkley (1998) in Entwicklungs-, Struktur- und Funktionsabweichungen des präfrontalen Kortex und damit in Verbindung stehender Gehirnregionen. Die defizitären Hemmungsprozesse und die dadurch beeinträchtigten exekutiven Funktionen resultieren entsprechend dem Modell in mangelnder Selbstregulation und unzulänglicher motorischer Verhaltenskontrolle: Beispielsweise können irrelevante Reaktionen nicht unterdrückt werden, das Verhalten ist wenig zielgerichtet und wird nur unzureichend durch internale Informationen kontrolliert. Bei ADHD scheint also nicht die Informationsverarbeitung an sich gestört zu sein (Wahrnehmung, Selektion und Verarbeitung von Informationen). Vielmehr ergeben sich infolge der Störungen auf höherer Ebene (mangelnde Verhaltenshemmung, exekutiven Funktionsdefizite) Schwierigkeiten auf der „Output“-Seite hinsichtlich der motorischen Kontrolle des Verhaltens (Barkley, 1998). Die entsprechende psychologische Hypothese (Wirkungshypothese) lautet:

PH 1.2: Das Basistraining nach Lauth und Schlottke führt zu positiven Veränderungen auf der Ebene der kognitiven Verhaltenshemmung. Den Kindern gelingt es durch das Training besser vorherrschende Handlungsimpulse $\mathrm{zu}$ unterdrücken und Interferenzen besser zu kontrollieren.

Die aktuelle Verbesserung von Leistungen hinsichtlich geübter Aufgaben durch ein Training ist zunächst ein erster Schritt in die richtige Richtung. Um den Kindern mit Aufmerksamkeitsproblemen aber die notwendigen Voraussetzungen zu vermitteln, damit sie sich in ihrem Leben gut zurechtfinden, ist es notwendig, dass sie das Gelernte flexibel einsetzen und auf neue Inhalte übertragen können. Im Rahmen einer dritten Fragestellung soll deshalb dem Aspekt möglicher Transfereffekte nachgegangen werden. Betrachtet man das 
Arbeitsgedächtnis als kognitives System, welches anfallende Informationen zu organisieren und festzuhalten vermag und damit für die Bewältigung kognitiver Anforderungen von wesentlicher Bedeutung ist, ist zu erwarten, dass Kinder mit Aufmerksamkeitsdefiziten in der Organisation und Verarbeitung von Reizen beeinträchtigt sind. Den aktuell diskutierten Arbeitsgedächtnismodellen ist gemeinsam, dass sie darunter ein kognitives System verstehen, in dem Informationen zeitweilig gespeichert und miteinander verknüpft werden können. Baddeley geht davon aus, dass das Arbeitsgedächtnis aus unterschiedlichen modalitätsspezifischen Hilfssystemen (der „phonologischen Schleife“ und dem „visuellräumlichen Notizblock“) besteht, denen die „zentrale Exekutive“ als übergeordnete Einheit vorangestellt ist (Baddeley, 1986; Hasselhorn \& Grube, 2003). Sollte durch die Interventionsmaßnahme eine Verbesserung der Fähigkeit zur Strukturierung von Informationen erreicht werden, ist zu erwarten, dass die Kinder auch bei solchen Aufgaben bessere Leistungen erzielen können, die nicht direkt die Konzentrationsfähigkeit überprüfen, bei denen aber die zentrale Exekutive beteiligt ist. Die zentrale Exekutive stellt die Kontrollinstanz der beiden Subsysteme zur Verarbeitung auditiorisch-verbaler (phonologische Schleife) und visuell-räumlicher Information (visuell-räumlicher Notizblock) dar (Baddeley, 1997; Hasselhorn \& Schumann-Hengsteler, 1998). Es ist anzunehmen, dass eine positive Beeinflussung von Hemmungs- und Aufmerksamkeitsprozessen die Regulations- und Kontrollfunktionen der Zentralen Exekutive verbessern kann, was sich wiederum günstig auf die Funktionstüchtigkeit der Hilfssysteme auswirken sollte. Diese Überlegung führt zu einer weiteren psychologischen Hypothese, speziell für das der Sprachverarbeitung dienende Hilfssystem:

PH 1.3a: Das Basistraining nach Lauth und Schlottke führt $\mathrm{zu}$ einer Steigerung der funktionellen Nutzung des phonologischen Arbeitsgedächtnisses.

Häufig leiden Kinder mit HKS unter sozialen und emotionalen Auffälligkeiten, welche sich auf die Aufmerksamkeitsdefizite und die hyperkinetischen Regulationsdefizite zurückführen lassen. Die Verminderung hyperkinetischer Symptome sollte sich deshalb auch günstig auf diese Bereiche auswirken. Im Rahmen der Transferfrage soll deshalb die folgende weitere Hypothese überprüft werden:

PH 1.3b: Das Basistraining nach Lauth und Schlottke führt zu einer Verbesserung der psychosozialen Anpassung der Kinder. 


\subsubsection{PHn zu Studie 2}

Das Basistraining allein ist für manche Kinder nicht ausreichend, da sie beim Bearbeiten von Aufgaben Schwierigkeiten damit haben, ihre Arbeitsweise zu strukturieren. Mit dem Strategietraining werden der Erwerb und die Umsetzung von Problemlösestrategien angestrebt. Es ist zu erwarten, dass Kinder, welche sowohl das Basistraining als auch das Strategietraining durchlaufen, zu verbesserten Aufmerksamkeitsleistungen befähigt sind, und sich die hyperkinetische Symptomatik vermindert.

Die bei Studie 1 genannten Hypothesen 1.1 bis $1.3 \mathrm{~b}$ können sinngemäß auf Studie 2 übertragen werden. Da das Training mehr Stunden umfasst und qualitativ umfangreicher ist, sollten die Effekte jeweils etwas stärker zu Tage treten.

PH 2.1: Das durch Komponenten des Strategietrainings erweiterte Basistraining führt im Rahmen der praktischen Durchführungsbedingungen in einer kinder- und jugendpsychiatrischen Ambulanz zu einem Abbau der Aufmerksamkeitsdefizite und zu einer Verminderung der hyperkinetischen Symptomatik.

PH 2.2: Das durch Komponenten des Strategietrainings erweiterte Basistraining führt zu positiven Veränderungen auf der Ebene der kognitiven Verhaltenshemmung. Den Kindern gelingt es durch das Training besser vorherrschender Handlungsimpulse zu unterdrücken und Interferenzen besser zu kontrollieren.

PH 2.3a: Das durch Komponenten des Strategietrainings erweiterte Basistraining führt zu einer Steigerung der funktionellen Nutzung des phonologischen Arbeitsgedächtnisses.

PH 2.3b: Das durch Komponenten des Strategietrainings erweiterte Basistraining führt zu einer Verbesserung der psychosoziale Anpassung der Kinder.

\subsubsection{PHn zu Studie 3}

Einige der aufmerksamkeitsgestörten Kinder sind durch ihre Symptomatik so schwer beeinträchtigt, dass ihnen mit einem Training, das nur Aufmerksamkeitsfunktionen trainiert, nicht wirksam geholfen werden kann. Dies gilt insbesondere für solche Kinder, die auch Probleme im Sozialverhalten haben. Das Therapieprogramm für Kinder mit hyperkinetischem 
und oppositionellem Problemverhalten (THOP) ist als Einzeltraining für diese Kinder konzipiert. Es erfüllt eine Reihe von Anforderungen, die sich aus den Erkenntnissen zur Symptomatik, dem Verlauf, komorbider Auffälligkeiten und der Ätiologie und Aufrechterhaltung der Hyperkinetischen Störung ergeben. Das THOP integriert z.B. verschiedene erfolgreiche kognitive und behaviorale Interventionsmethoden, es berücksichtigt die individuelle Situation des Kindes und der Eltern und erlaubt aufgrund seines Bausteinsystems eine sukzessive und differenzielle Anpassung der Interventionsmethoden an den Einzelfall.

In dieser Studie sollte das THOP hinsichtlich seines Anwendungsspektrums genauer überprüft werden. Die vorliegende Untersuchung hat primär zum Ziel das Eltern-KindProgramm des THOP im Rahmen einer summativen, globalen Evaluation hinsichtlich seiner Wirksamkeit zu evaluieren, um die Frage zu klären, ob das Programm auch unter veränderten Randbedingungen wirksam ist. Diese umfassen insbesondere die Adaptation des Trainings für eine Durchführung in Gruppen (sowohl für die Eltern als auch für die Kinder) unter praxisnahen Bedingungen in Ambulanzen der Kinder- und Jugendpsychiatrie. Die Umsetzung des Trainings in diesem Setting beruht auf den Überlegungen, welche in Kapitel 2.7 erwähnt wurden und durch folgende Punkte ergänzt werden können:

- Eltern fühlen sich entlastet, wenn sie erfahren, dass auch andere Eltern Schwierigkeiten im Umgang mit dem Verhalten ihrer Kinder haben. Es kann ein Austausch zwischen den Familien stattfinden, der die Interventionsvorschläge des Therapeuten erweitert. Gegenseitige soziale Unterstützung kann bei der Bewältigung der individuellen Probleme helfen.

- Die Compliance wird möglicherweise dadurch erhöht, dass es für die Eltern leichter sein kann, Vorschläge von einer Nichtfachperson anzunehmen.

- Es entsteht ein gewisser sozialer Druck durch die anderen Gruppenmitglieder, wenn in den Therapiesitzungen die Umsetzung der Hausaufgaben besprochen wird. Dadurch kann sich die Motivation erhöhen die aufgetragenen Hausaufgaben durchzuführen.

Die guten Erfolge des THOP im Einzeltraining sowie die Vorteile, die Gruppen im Rahmen therapeutischer Interventionen bieten, lassen die Annahme zu, dass das THOP auch als Gruppenprogramm wirksam sein könnte. Diese Wirksamkeit soll in der vorliegenden Arbeit daran gemessen werden, inwiefern die Maßnahmen zu einer Verminderung der Störungssymptomatik führen. Die folgende Wirksamkeitshypothese wird daher formuliert: 
PH 3.1: Das THOP führt zu einer Verminderung der Störungssymptomatik, wenn es in Form einer Gruppenbehandlung im Rahmen einer kinder- und jugendpsychiatrischen Ambulanz durchgeführt wird.

In vielen Studien wurde nachgewiesen, dass Kinder mit Hyperkinetischen Störungen oft auch oppositionelle und aggressive Verhaltensweisen zeigen. Da das THOP explizit auch für Kinder mit oppositionellen und aggressiven Störungen geeignet ist, soll der Frage nachgegangen werden, ob sich diese Verhaltensweisen durch das THOP als Gruppentraining vermindern lassen. Als zweite Wirksamkeitshypothese wird daher formuliert:

PH 3.2: Das THOP als Gruppenprogramm führt zu einer Verminderung von oppositionellen und aggressiven Verhaltensweisen.

Kinder mit hyperkinetischen Verhaltensweisen sowie aggressiven Störungen entwickeln als Folge der Symptomatik häufig emotionale Störungen, weil sie sich von anderen Kindern und von Erwachsenen unverstanden fühlen und ihre Bedürfnisse nicht befriedigt werden. Sie erleben sich häufig als Außenseiter. Es ist zu erwarten, dass durch das THOP das emotionale Wohlbefinden verbessert werden kann. Daraus folgt die dritte Wirksamkeitshypothese:

PH 3.3: Das THOP als Gruppenprogramm führt zu einer Verminderung emotionaler Beeinträchtigungen.

\subsubsection{PHn zu Studie 4}

In Deutschland hat sowohl das THOP als auch das „Training mit aufmerksamkeitsgestörten Kindern" von Lauth und Schlottke eine weite Verbreitung gefunden. Beiden Programmen mit ihren unterschiedlichen Schwerpunkten und Zielsetzungen ist gemein, dass sie sehr zeitaufwändig sind. Es stellte sich die Frage, ob sich durch die Durchführung des Marburger Konzentrationstrainings mit nur $5-8$ Sitzungen ebenfalls eine Verbesserung der Aufmerksamkeitsleistung erzielen lässt.

In den Vorüberlegungen für diese Arbeit wurde die Elternarbeit, welche mit der Erstellung eines Videos verknüpft ist, aus dieser Studie herausgenommen. Dies geschah nicht aus der Erkenntnis heraus, dass eine solche nicht notwendig oder förderlich wäre, sondern aus 
rein pragmatischen Gesichtspunkten. Eine Erweiterung auf die Elternarbeit war aus organisatorischen Gründen bei dieser Studie nicht möglich.

Kinder mit der Aufmerksamkeitsstörungen weisen in zwei Bereichen der Aufmerksamkeit Defizite auf: Sie sind zum einen häufig nicht in der Lage, ihre Aufmerksamkeit langfristig auf eine Aufgabe auszurichten (reduzierte Daueraufmerksamkeit), und zum anderen ist die selektive Aufmerksamkeit eingeschränkt, die Kinder können nur eingeschränkt aufgabenrelevante Reize fokussieren und gleichzeitig irrelevante Reize ignorieren. Im Marburger Konzentrationstraining wird mittels Selbstinstruktionstraining eine Förderung des reflexiven Arbeitsstils angestrebt, d.h. das Kind soll durch das innere Sprechen die Fähigkeit trainieren, systematisch und überlegt zu handeln. Durch das Training sollte sich also insbesondere der Bereich der Unaufmerksamkeit verbessern lassen. Es wird erwartet, dass die Kinder weniger Flüchtigkeitsfehler machen, längerfristig aufmerksam sein können, Anweisungen vollständig ausführen, Aufgaben planvoll bearbeiten und sich nicht durch unwesentliche Reize ablenken lassen.

Dies führt für die isolierte Evaluation zu folgender Hypothese:

PH 4.1: Das Marburger Konzentrationsprogramm führt bei Kindern mit Aufmerksamkeitsstörungen zu einer verbesserten Konzentrationsleistung, wenn es im Rahmen eines Gruppentrainings in einer Kinder- und Jugendpsychiatrischen Praxis durchgeführt wird

Eine Veränderung der Fähigkeit zur Aufgabenbearbeitung in der Trainingssituation oder ähnlich konzipierter Testaufgaben muss von dem trainierten Kind auf andere Situationen übertragen werden können. Hierzu sollen Transferleistungen untersucht werden, welche durch Selbstinstruktion zum Verinnerlichen metakognitiver Fertigkeiten erreicht werden sollen. Für Kinder mit Aufmerksamkeitsstörungen ist vor allem die schulbezogene Konzentrationsfähigkeit von besonderer Bedeutung. Schon in der Grundschulzeit fallen viele Kinder mit dieser Diagnose durch Lern- und Leistungsschwierigkeiten und damit verbundenen Klassenwiederholungen oder Umschulungen auf. Nach einer Untersuchung von Cantwell und Baker (1992) liegen bis zu 80 \% der Kinder mindestens zwei Noten unter dem Klassendurchschnitt. Die Auswirkungen einer Aufmerksamkeitsstörung können also einen 
beträchtlichen Einfluss auf die schulische Laufbahn und somit auch den weiteren Lebensentwurf nehmen.

PH 4.2: Durch das Marburger Konzentrationstraining übertragen die trainierten Kinder die erarbeiteten Selbstanweisungen auch auf schulische Belange.

In der vorliegenden Studie wurde aus zeitlichen Gründen auf die Durchführung von Elternabenden verzichtet. Trotzdem wird davon ausgegangen, dass sich die Eltern-Kindsowie die Lehrer-Kind-Interaktion verändern. Kinder mit Aufmerksamkeitsstörungen entwickeln zu einem hohen Prozentsatz emotionale Auffälligkeiten. Der hohe Anteil an komorbiden Störungen lässt sich aus der Tatsache ableiten, dass diese Kinder in einem hohen Ausmaß mit negativen Rückmeldungen, Ablehnungen und Misserfolgen konfrontiert sind. Döpfner (2000) weist auf die Zunahme negativer Interaktionen zwischen dem Kind und seinen Bezugspersonen sowohl im häuslichen wie auch im schulischen Umfeld hin. In einem Teufelskreis kommt es in der Regel zu einer Steigerung der Symptome, welche wiederum mit Sanktionen belegt werden usw.

Es wird erwartet, dass sich durch die erfolgreiche Bearbeitung von Aufgaben in der Trainingssituation auch die Selbstakzeptanz des Kindes sowie seine Motivation verbessern, was zu einer Veränderung in den Interaktionen führen wird. Viele Forscher berichten, dass die Tatsache an sich, dass das Kind an einem Training teilnimmt, schon zu einer Veränderung in der Interaktion führt. Die Kinder werden positiver und genauer betrachtet, es entsteht der Eindruck, dass es nun endlich zu einer Veränderung der oftmals unerfreulichen Lage kommt. Diese Haltung von Seiten der Eltern und Lehrer entlastet das Kind und lenkt den Blick auf seine Ressourcen.

Es wird demnach die Hypothese formuliert, dass sich auch ohne den ausdrücklichen Einbezug der Eltern und Lehrer eine positive Veränderung in der Interaktion ergeben wird.

PH 4.3: Das Marburger Konzentrationstraining führt zu einer positiven Veränderung der Eltern-Kind- und Lehrer-Kind-Interaktion

\subsubsection{PHn zur Vergleichbarkeit der Programme (Studien 1 - 4)}

Neben der Frage nach der Wirksamkeit des jeweiligen Programms soll auch der Frage der vergleichenden Wirksamkeit der Trainings untereinander nachgegangen werden. Hierbei interessiert die Frage, ob die Programme sich in ihrer Wirksamkeit unterscheiden. 
In den Studien 1 und 2 wurde jeweils das Training nach Lauth und Schlottke durchgeführt. In der Studie 2 umfasste das Training einen größeren Stundenumfang und mehr thematische Inhalte. Eine Erweiterung der trainierten Inhalte des Basistrainings nach Lauth und Schlottke um Elemente des Strategietrainings sollte zu einer weiteren Verminderung der Störungssymptomatik führen, da die Kinder ausgiebiger und differenzierter geschult werden.

PH 5.1: Das Training nach Lauth und Schlottke führt zu einer stärkeren Reduzierung der Symptomatik, wenn die Kinder sowohl mit dem Basis- als auch dem Strategietraining behandelt werden als wenn sie nur das Basistraining erhalten.

Sowohl das Training nach Lauth und Schlottke, als auch das THOP sowie das Marburger Konzentrationstraining (im weiteren Sinne) wurden zur Behandlung von Kindern mit Hyperkinetischer Störung entwickelt. Die Programme verfolgen zum Teil gleiche, zum Teil aber auch unterschiedliche Ziele und setzen auch unterschiedliche Schwerpunkte, mit welchen Strategien diese Ziele erreicht werden sollen.

Während das THOP über die Veränderung elterlichen Verhaltens die Eltern-KindInteraktion und spezifische Verhaltensprobleme des Kindes zu beeinflussen versucht und dabei vor allem Methoden des Kontingenzmanagements eingesetzt werden, verstehen sich das Programm von Lauth und Schlottke (1997) sowie das Marburger Konzentrationstraining als Entwicklungsintervention, die direkt die eigenständige Entwicklung selbstregulatorischer Fähigkeiten eines Kindes fördern und damit ebenfalls eine Verbesserung der Symptomatik herbeiführen will. Erreicht werden sollen diese Ziele primär über Selbstinstruktionstechniken und kognitives Modellieren. Eine begleitende Elternberatung soll die Akzeptanz der Interventionen fördern, das Wissen über die Störung und die Therapie verbessern und Fähigkeiten vermitteln, wie die Eltern ihre Kinder im Alltag unterstützen können.

Die relative Wirksamkeit wird wie in der isolierten Evaluation daran gemessen, inwiefern die Trainings eine Verminderung der Störungssymptomatik erreichen können. Sowohl das Training nach Lauth und Schlottke als auch das Marburger Konzentrationstraining verfolgen über den Ansatz der Selbstinstruktion insbesondere eine Verbesserung von Aufmerksamkeitsfähigkeiten. Sie sollten sich in der Wirksamkeit, die nach dem Training überprüft wird, nicht signifikant unterscheiden. 
PH 5.2: Das Training nach Lauth und Schlottke führt in gleichem Maße zu einer Reduzierung der Symptomatik wie das Marburger Konzentrationstraining.

Da sowohl mit dem Training nach Lauth und Schlottke als auch dem Marburger Konzentrationstraining insbesondere das Aufmerksamkeitsverhalten trainiert wird, sollte diese Interventionsformen dem THOP in diesem Bereich überlegen sein. Es lässt sich deshalb folgende Überlegenheitshypothese formulieren:

PH 5.3: Das Training nach Lauth und Schlottke sowie das Marburger Konzentrationstraining führen $\mathrm{zu}$ einer stärkeren Verminderung von Aufmerksamkeitsproblemen als das THOP als Gruppenprogramm.

Da das THOP als familienzentrierte Intervention einen umfassenderen therapeutischen Ansatz darstellt und kognitiven Verfahren insgesamt eine geringere Wirksamkeit als behavioralen Verfahren bescheinigt wird (vgl. Saile, 1996), kann angenommen werden, dass das THOP einem Training, das den Ansatz von Lauth und Schlottke (1997) sowie dem Marburger Konzentrationstraining zugrunde legt, in bestimmten Bereichen überlegen ist. Dies sollte sich insbesondere bei hyperaktiven und impulsiven Verhaltensweisen sowie bei den externalisierenden Störungen zeigen.

Entsprechend der Psychologischen Hypothese 3.2 sollen die Programme auch hinsichtlich ihrer Wirkung auf oppositionelle und aggressive Verhaltensweisen beurteilt werden. Während im THOP diese Verhaltenprobleme neben den Beeinträchtigungen, die die Hyperkinetische Störung ausmachten, ebenfalls explizit Gegenstand der Interventionen sein können, werden ihnen in den Elternsitzungen des Trainings nach Lauth und Schlottke sowie dem Marburger Konzentrationstraining nur verhältnismäßig wenig Aufmerksamkeit gewidmet. Da sich gerade Elterntrainings besonders gut eignen, solche externalisierenden Verhaltensauffälligkeiten zu vermindern (Kazdin, 1997) wird erwartet, dass das THOP zu einer stärkeren Reduktion dieser Verhaltensauffälligkeiten führt. Aufgrund des Schwerpunktes, den das THOP im familiären Rahmen setzt, ist jedoch anzunehmen, dass sich die Überlegenheit des THOP nur im häuslichen Bereich zeigt, während in schulischen Situationen die Wirkungen des THOP nicht unbedingt größer als die des Trainings nach Lauth und Schlottke sowie des Marburger Konzentrationstrainings sein müssen, jedoch auch nicht geringer ausfallen sollten. 
PH 5.4: Das THOP als Gruppenprogramm führt zu einer stärkeren Verminderung von oppositionellen und aggressiven Verhaltensweisen im häuslichen Bereich als das Vergleichsprogramm nach Lauth und Schlottke sowie das Marburger Konzentrationstraining. Außerdem sollte es mindestens im gleichen Maße wie die Vergleichsprogramme zu einer Verminderung von oppositionellen und aggressiven Verhaltensweisen im schulischen Bereich führen.

Hinsichtlich des emotionalen Befindens wird aus Sicht des THOP eine NichtUnterlegenheitshypothese formuliert, da in keinem der Programme explizite Aussagen hierzu gemacht werden:

PH 5.5: Das THOP als Gruppenprogramm führt mindestens im gleichen Maße wie die Vergleichsprogramme zu einer Verminderung emotionaler Beeinträchtigungen. 


\section{Methode}

Es wurden isolierte Evaluationen durchgeführt, d.h. die spezifischen Interventionen wurden jeweils mit einer Wartekontrollgruppe verglichen. Unbehandelte Kontroll- oder Wartegruppen sollen Behandlungseffekte von spontanen Remissionen unterscheiden.

Die Wirkungen der verschiedenen Interventionen wurden später einander gegenübergestellt. Bei dieser Form der vergleichenden Evaluation werden die Therapieziele konstant gehalten, während die Randbedingungen divergieren.

Die randomisierte Zuteilung von Probanden zu Behandlungs- und Kontrollgruppen erfolgt, um sicher $\mathrm{zu}$ stellen, dass nicht konfundierende Faktoren (Eigenschaften der Probanden oder systematische andere Fehler bei der Zuteilung zu den jeweiligen Gruppen) für eine Veränderung verantwortlich sind. Die in der Psychotherapieforschung geforderte Randomisierung konnte in den Studien nur zum Teil umgesetzt werden, weil die verschiedenen Interventionen nicht parallel sondern nacheinander durchgeführt wurden. Dies war wegen eingeschränkter Ressourcen (Therapeuten) nicht anders zu realisieren.

Um eine reliable Durchführung sicherzustellen, wurden die Therapieprogramme so weit wie möglich nach den Angaben der Therapiemanuale umgesetzt. Einschränkend muss aber bemerkt werden, dass Hyperkinetische Kinder eine starre Durchführung einer Therapiesitzung nicht immer zulassen. Bei Störungen durch die Kinder wird der Therapeut häufig flexibel reagieren müssen.

\subsection{Versuchspläne}

Bei allen durchgeführten vier Studien wurden Prätest-Posttest-Kontrollgruppendesigns realisiert. Die Daten der isolierten und der vergleichenden Evaluation werden in getrennten Versuchsplänen dargestellt und später auch getrennt berechnet. Für die isolierte Evaluation ergibt sich jeweilig ein 2 (Gruppe) $x \quad 2$ (Zeit) faktorieller Versuchsplan mit Messwiederholung, wobei eine Wartegruppe (WG) als Vergleichsgruppe fungiert. Bei den Studien 1, 2 und 3 handelt es sich um eine reine Wartekontrollgruppe. Bei Studie 4 wurde mit der Kontrollgruppe ein Pseudotraining durchgeführt. 


\section{Zu Studie 1}

Tabelle 3

Versuchsplan mit zwei Erhebungszeitpunkten (isolierte Evaluation)

\begin{tabular}{l|ccc}
\hline & Vortest & Intervention & Nachtest \\
\hline Wartegruppe (WG) & Erfassung der AV & Keine Intervention & $\begin{array}{c}\text { Wiederholte Erfassung } \\
\text { der AV }\end{array}$ \\
$\begin{array}{l}\text { Trainingsgruppe (Lauth }+ \\
\text { Schlottke) (LS) }\end{array}$ & Erfassung der AV & 10 Sitzungen Basistraining & $\begin{array}{c}\text { Wiederholte Erfassung } \\
\text { der AV }\end{array}$ \\
\hline Anmerkungen. AV: abhängige Variablen. & & \\
\hline
\end{tabular}

\section{Zu Studie 2}

Tabelle 4

Versuchsplan mit zwei Erhebungszeitpunkten (isolierte Evaluation)

\begin{tabular}{l|ccc}
\hline & Vortest & Intervention & Nachtest \\
\hline Wartegruppe (WG) & Erfassung der AV & Keine Intervention & $\begin{array}{c}\text { Wiederholte Erfassung } \\
\text { der AV }\end{array}$ \\
$\begin{array}{l}\text { Trainingsgruppe (Lauth + } \\
\text { Schlottke) (LS) }\end{array}$ & Erfassung der AV & $\begin{array}{c}\text { 10 Sitzungen Basistraining + } \\
5 \text { Sitzungen Strategietraining }\end{array}$ & $\begin{array}{c}\text { Wiederholte Erfassung } \\
\text { der AV }\end{array}$ \\
\hline Anmerkungen. AV: abhängige Variablen. & & \\
\hline
\end{tabular}

\section{Zu Studie 3}

Tabelle 5

Versuchsplan mit zwei Erhebungszeitpunkten (isolierte Evaluation)

\begin{tabular}{l|ccc}
\hline & Vortest & Intervention & Nachtest \\
\hline Wartegruppe (WG) & Erfassung der AV & Keine Intervention & $\begin{array}{c}\text { Wiederholte Erfassung } \\
\text { der AV }\end{array}$ \\
Trainingsgruppe (THOP) & Erfassung der AV & $\begin{array}{c}15 \text { Trainingssitzungen in } \\
\text { Gruppen }\end{array}$ & $\begin{array}{c}\text { Wiederholte Erfassung } \\
\text { der AV }\end{array}$ \\
\hline Anmerkungen. AV: abhängige Variablen. & & \\
\hline
\end{tabular}




\section{Zu Studie 4}

Tabelle 6

Versuchsplan mit zwei Erhebungszeitpunkten (isolierte Evaluation)

\begin{tabular}{l|ccc}
\hline & Vortest & Intervention & Nachtest \\
\hline Wartegruppe (WG) & Erfassung der AV & $\begin{array}{c}\text { Trainingssitzungen in } \\
\text { Gruppen mit dem } \\
\text { Computerspiel „Neues von } \\
\text { Pettersson und Findus“ }\end{array}$ & $\begin{array}{c}\text { Wiederholte Erfassung } \\
\text { der AV }\end{array}$ \\
$\begin{array}{l}\text { Trainingsgruppe (Marburger } \\
\text { Konzentrationstraining) }\end{array}$ & Erfassung der AV & 5 Trainingssitzungen in & Giederholte Erfassung \\
der AV
\end{tabular}

Anmerkungen. AV: abhängige Variablen.

\section{Zu den Studien 1 - 4}

Tabelle 7

Versuchsplan mit zwei Erhebungszeitpunkten (vergleichende Evaluation)

\begin{tabular}{|c|c|c|c|}
\hline & Vortest & Intervention & Nachtest \\
\hline Trainingsgruppe (LS) & Erfassung der AV & 10 Sitzungen Basistraining & $\begin{array}{c}\text { Wiederholte Erfassung } \\
\text { der AV }\end{array}$ \\
\hline Trainingsgruppe (LS) & Erfassung der AV & $\begin{array}{l}10 \text { Sitzungen Basistraining }+ \\
5 \text { Sitzungen Strategietraining }\end{array}$ & $\begin{array}{c}\text { Wiederholte Erfassung } \\
\text { der AV }\end{array}$ \\
\hline Trainingsgruppe (THOP) & Erfassung der AV & 15 Trainingssitzungen in Gruppen & $\begin{array}{c}\text { Wiederholte Erfassung } \\
\text { der AV }\end{array}$ \\
\hline $\begin{array}{l}\text { Trainingsgruppe (Marburger } \\
\text { Konzentrationstraining) }\end{array}$ & Erfassung der AV & 5 Trainingssitzungen in Gruppen & $\begin{array}{c}\text { Wiederholte Erfassung } \\
\text { der AV }\end{array}$ \\
\hline
\end{tabular}

\subsection{Stichproben}

Die Stichprobe zu den Studien 1 - 3 wurde aus der Gruppe von Kindern gewonnen, die in einem Zeitraum von viereinhalb Jahren (1998-2003) in der Poliklinik der Kinder- und Jugendpsychiatrie Mühlhausen, der Poliklinischen Institutsambulanz des Psychologischen Instituts der Universität Göttingen sowie im Sozialpädiatrischen Zentrum in Reifenstein mit Verdacht auf eine Hyperkinetische Störung vorstellig wurden.

Alle Kinder nahmen an einer umfangreichen diagnostischen Untersuchung teil, die ein klinisches Interview mit den Eltern, Fremdbeurteilungsbögen zum Verhalten und Erleben der 
Kinder (ausgefüllt von Eltern und Klassenlehrer), eine Intelligenzdiagnostik (HAWIK-III (Tewes, Rossmann \& Schallberger, 1999), K-ABC (Melchers \& Preuß, 1994), CFT-20 (Weiß, 1997)), Aufmerksamkeits- und Konzentrationstests und je nach individueller Fragestellung weitere Verfahren (z.B. Lese- und Rechtschreibtests) umfasste.

In die Studien wurden Kinder eingeschlossen, die aufgrund der Ergebnisse der Untersuchung eine Diagnose aus dem Spektrum der Hyperkinetischen Störungen nach ICD10 (F90) bzw. die Diagnose einer Aufmerksamkeitsstörung ohne Hyperaktivität (F98.8) erhalten hatten oder für die der dringende Verdacht bestand, dass sie unter einer solchen Störung leiden würden. Um ein in der Praxis möglichst realistisches Vorgehen umzusetzen, wurden auch Kinder, die komorbide Störungen innerhalb oder außerhalb der F90-Kategorie aufwiesen (z.B. Hyperkinetische Störung des Sozialverhaltens oder auch umschriebene Entwicklungsstörung schulischer Fertigkeiten) eingeschlossen. Als Ausschlusskriterien galten schwere neurologische Schädigungen, ein Lebensalter unter 7 bzw. über 13 Jahren und ein Intelligenzquotient von $\leq 75$. Erhielten die Kinder bereits vor dem Training eine pharmakologische Behandlung, wurde diese über das Training bzw. die Wartezeit hinweg beibehalten.

Insgesamt wurden fünf Gruppen mit dem Training nach Lauth und Schlottke und acht mit dem THOP behandelt. Es wird davon ausgegangen, dass die Interventionen vergleichbar sind und die Daten zu Gesamtgruppen aggregiert werden können, weil versucht wurde, die Behandlung der Gruppen jeweils anhand des Manuals durchzuführen und die Inhalte überwiegend gleich waren. Zwischen der Eingangsuntersuchung und dem Beginn des Trainings lagen im Schnitt sechs bis acht Wochen. Zwischen Vor- und Nachtest für die Therapie- und Wartegruppen lagen etwa sechs Monate.

Von 115 Kindern, die zunächst in die Studien 1 - 3 einbezogenen wurden, konnten 96 Datensätze für die Auswertung verwendet werden. Beim THOP brachen sechs Kinder und beim Training nach Lauth und Schlottke 11 Kinder bereits nach ein oder zwei Sitzungen die Behandlung ab, ein Kind musste wegen unzuverlässiger Teilnahme an den Sitzungen aus der Auswertung ausgeschlossen werden. Als Gründe gaben die Eltern an, dass sie die Intervention für sich nicht für geeignet hielten, dass ein Elternteil die Behandlung ablehnte (in der Mehrzahl waren dies die Väter der betroffenen Kinder), dass ihnen die Fortschritte nicht schnell genug gingen oder dass aus Mangel an Zeit bzw. durch einen zu hohen 
organisatorischen Aufwand die Trainingstermine nicht hätten wahrgenommen werden können. In der Wartegruppe gab es vier Kinder, deren Daten aufgrund fehlender Nachtestwerte nicht berücksichtig werden konnten. In Relation zu den Gesamtgruppengrößen der verschiedenen Gruppen ist die Abbrecherquote relativ gleich groß. Es ist ersichtlich, dass Abbrüche nicht zwangsläufig allein dem Training selbst angelastet werden können, sondern wie bspw. in der Wartegruppe auch auf einem generellen Mangel an Engagement oder auf Organisationshindernissen beruhen können. Tabelle 8 enthält einige informative Beschreibungsmerkmale über die 96 Kinder in den vier Gruppen. Die Kinder der THOPGruppe weisen im Durchschnitt acht IQ-Punkte mehr auf als die der Wartegruppe $\left(t_{\mathrm{IQ}}(45)=\right.$ -2.39; $p=0.02$ ). Einige der THOP-Gruppen wurden in Göttingen durchgeführt wurden, während alle Kinder der Wartegruppe aus Mühlhausen und Umgebung kamen. Der Unterschied könnte dadurch bedingt sein, dass in Göttingen aufgrund der Universität der Anteil an Menschen mit hohem Bildungsgrad sehr groß ist und daher auch die Kinder entsprechende intellektuelle Fähigkeiten besitzen. Die Gruppen unterscheiden sich außerdem bezüglich der Medikation. In der THOP-Gruppe wurden doppelt so viele Kinder begleitend medikamentös behandelt (vorrangig Ritalin) wie in der Lauth-Schlottke-Gruppe (15 Sitzungen). In der Wartegruppe und ebenso in der Studie 1 (Lauth und Schlottke, 10 Sitzungen) galt dies nur für je ein Kind. Die Heterogenität wurde zugunsten der Realisierung einer möglichst großen Stichprobe in Kauf genommen.

Tabelle 8

Gruppengröße, mittleres Alter, Intelligenzniveau und gleichzeitige medikamentöse Behandlung je Untersuchungsgruppe (Studien 1-3)

\begin{tabular}{lcccc}
\hline & LS (10 Sitzungen) & LS (15 Sitzungen) & THOP & WG \\
\hline Alter & $\mathrm{N}=15$ & $\mathrm{~N}=34$ & $\mathrm{~N}=31$ & $\mathrm{~N}=16$ \\
Intelligenz & $10 ; 5(1.3)$ & $9 ; 3(1.4)$ & $9 ; 4(1.5)$ & $9 ; 9(1.3)$ \\
Geschlecht & $90.9(11.0)$ & $96.6(9.9)$ & $98.6(11.5)$ & $90.8(8.0)$ \\
Medikation & $14 / 1$ & $33 / 1$ & $30 / 1$ & $13 / 3$ \\
\hline
\end{tabular}

Anmerkungen. LS: Trainingsgruppe nach Lauth und Schlottke, THOP: THOP-Trainingsgruppe, WG: Wartegruppe. Alter (Mittelwert in Jahren; Monaten, in Klammern Standardabweichungen), Intelligenz (Mittelwert, in Klammern Standardabweichungen), Geschlecht (Jungen/ Mädchen), Medikation (Methylphenidat; ja/nein)

Tabelle 9 verdeutlicht, dass sich die Stichproben in den Versuchsgruppen hinsichtlich der Diagnosen unterscheiden. Für das Training nach Lauth und Schlottke (15 Sitzungen) wurden vorrangig Kinder mit einer Aufmerksamkeitsstörung ohne Hyperaktivität ausgewählt, 
da für diese Gruppe positive Vorerfahrungen einiger Mitarbeiter in Mühlhausen mit dem Programm (Studie 1 Basistraining 10 Sitzungen) bestanden. Das THOP wurde erst zu einem Zeitpunkt angeboten, als aus Mangel an wirksamen Behandlungsmaßnahmen für Kinder mit stark ausgeprägter Hyperkinetischer Störung eine Alternative gesucht wurde. Es befinden sich daher insgesamt mehr Kinder in der THOP-Gruppe, die die Kriterien einer einfachen Aktivitäts- und Aufmerksamkeitsstörung erfüllen, und solche, die zusätzlich noch eine Störung des Sozialverhaltens aufweisen.

Tabelle 9

Diagnose und sozialer Status je Untersuchungsgruppe

\begin{tabular}{|c|c|c|c|c|}
\hline & $\begin{array}{c}\text { LS } \\
(10 \\
\text { Sitz. }) \\
\end{array}$ & $\begin{array}{c}\text { LS } \\
(15 \\
\text { Sitz.) } \\
\end{array}$ & THOP & WG \\
\hline \multicolumn{5}{|l|}{ Diagnose } \\
\hline Einfache Aktivitäts- und Aufmerksamkeitsstörung (F90.0) & 2 & 5 & 14 & 5 \\
\hline Aufmerksamkeitsstörung ohne Hyperaktivität (F98.8) & 3 & 12 & 2 & 5 \\
\hline Hyperkinetische Störung des Sozialverhaltens (F90.1) & 2 & 1 & 7 & 1 \\
\hline $\begin{array}{l}\text { Einfache Aktivitäts- und Aufmerksamkeitsstörung (F90.0) und sonstige } \\
\text { Störungen }\end{array}$ & 3 & 7 & 3 & 3 \\
\hline $\begin{array}{l}\text { Aufmerksamkeitsstörung ohne Hyperaktivität (F98.8) und umschriebene } \\
\text { Entwicklungsstörungen schulischer Fertigkeiten (F81...) }\end{array}$ & 3 & 4 & 1 & 1 \\
\hline Aufmerksamkeitsstörung ohne Hyperaktivität (F98.8) und sonstige Störungen & 2 & 3 & 1 & 1 \\
\hline Nicht näher bezeichnete Hyperkinetische Störung (F90.9) & 0 & 2 & 3 & 0 \\
\hline \multicolumn{5}{|l|}{ Sozialer Status (Elternteil mit höchster Qualifikation) } \\
\hline Angelernte Berufe & 1 & 4 & 4 & 0 \\
\hline Facharbeiter, Handwerker, Angestellte, Beamte im einfachen Dienst & 10 & 18 & 11 & 13 \\
\hline Mittlere Angestellte, Beamte im mittleren Dienst & 2 & 3 & 8 & 1 \\
\hline Höher qualifizierte Angestellte, Beamte im gehobenen Dienst & 2 & 3 & 4 & 1 \\
\hline Leitende Angestellte, Beamte im höheren Dienst & 0 & 2 & 1 & 0 \\
\hline Selbständige (Handwerker, Landwirte, Gewerbetreibende) & 0 & 4 & 2 & 1 \\
\hline Keine Angaben & 0 & 0 & 1 & 0 \\
\hline
\end{tabular}

Die Stichprobe zu Studie 4 wurde aus dem Patientenkreis von Kinder- und Jugendpsychiatrischen Praxen von zwei Kasseler Kinder- und Jugendpsychiatern und einer Kinder- und Jugendpsychiatrischen Praxis in Hannoversch Münden gezogen. Die Störungsbilder bei diesen Kindern sind etwas homogener als in den Studien 1 - 3. Alle Kinder hatten die klinische Diagnose einer einfachen Aktivitäts- und Aufmerksamkeitsstörung (F 
90.0) oder der nicht näher bezeichneten Verhaltens- und emotionalen Störung mit Beginn in der Kindheit und Jugend - Aufmerksamkeitsstörung ohne Hyperaktivität (F 98.8) - erhalten bzw. bestand der dringende Verdacht, dass sie unter einer solchen Störung leiden würden. Über Komorbiditäten und sozialen Status liegen leider keine genauen Angaben vor, da dies von den Kinder- und Jugendpsychiatern nicht mitgeteilt wurde. Das Alter der Kinder lag zwischen 7 und 12 Jahren. Es wurden in diese Studie nur Kinder aufgenommen, die zumindest einen durchschnittlichen Intelligenzquotient aufwiesen.

Alle Eltern und Kinder erklärten sich mit der Teilnahme an einer dieser Gruppen einverstanden und wussten, dass untersucht werden sollte, ob ein bestimmtes Trainingsprogramm für Kinder mit Aufmerksamkeitsstörungen wirksam ist oder ein Computerprogramm die gleichen bzw. ähnliche Effekte zeigt. Es wurden fünf Gruppen à drei Kinder mit dem Marburger Konzentrationstraining und fünf Gruppen à drei Kinder mit dem Spiel „Petterson und Findus“ (GAMOP Samproduktion AB, 1998) behandelt.

Die Kinder wurden den einzelnen Gruppen zufällig zugeteilt, nur in einem Fall musste ein Kind aus der Trainingsgruppe mit einem Kind der Kontrollgruppe tauschen, da die Mutter die Teilnahme am Entspannungstraining, das Bestandteil des Marburger Konzentrationstrainings ist, aus religiösen Gründen ablehnte. Einigen der Kinder waren im Rahmen von ergotherapeutischen Behandlungen Elemente aus Konzentrationstrainings bekannt. Tabelle 10 gibt Auskunft über die Zugangsdaten der Probanden.

Tabelle 10

Ausgangsmerkmale der Kinder in der Trainings- und Kontrollgruppe

\begin{tabular}{|c|c|c|}
\hline & $\begin{array}{l}\text { Marburger Konzentrationstraining } \\
\qquad \mathrm{N}=15\end{array}$ & $\begin{array}{c}\text { Kontrollgruppe „Pettersson und Findus“ } \\
\qquad \mathrm{N}=15\end{array}$ \\
\hline Alter & $10 ; 4(1.0)$ & $9 ; 9(0.1)$ \\
\hline Intelligenz & $105.2(11.2)$ & $96.43(7.67)$ \\
\hline Geschlecht & $12 / 3$ & $11 / 4$ \\
\hline Medikation & $9 / 6$ & $2 / 13$ \\
\hline \multicolumn{3}{|c|}{$\begin{array}{l}\text { Anmerkungen. } \\
\text { Stichprobengröße (Jungen/ Mädchen), Alter (Mittelwert in Jahren; Monaten, in Klammern Standardabweichungen), } \\
\text { Intelligenz (Mittelwert, in Klammern Standardabweichungen). Medikation (Methylphenidat; ja/nein) }\end{array}$} \\
\hline
\end{tabular}

Die Kinder der Trainings- und Kontrollgruppe unterscheiden sich nicht bedeutsam hinsichtlich des Alters und der Zusammensetzung von Jungen und Mädchen in den Gruppen. Die Kinder der Trainingsgruppe weisen jedoch einen höheren IQ auf als die Kinder in der Kontrollgruppe $\left(t_{\mathrm{IQ}}(29)=-1.93 ; p=0.06\right)$. Ein noch bedeutsamerer Unterschied ergibt sich 
hinsichtlich der medikamentösen Behandlung. In der Trainingsgruppe wurden über die Hälfte der Kinder mit Ritalin ${ }^{\circledR}$ oder Medikinet $^{\circledR}$ behandelt $\left(\mathrm{t}_{\mathrm{IQ}}(29)=2.92 ; p<0.01\right)$.

\subsection{Untersuchungsverfahren und Konkretisierung der abhängigen Variablen}

Um die Wirkungen der verschiedenen Interventionen bewerten zu können wurden in den Messungen vor und nach den Trainings sowohl objektive Maße in Form von Tests als auch eine subjektive Beurteilung von Eltern und Lehrern erhoben. Dies ist deshalb sinnvoll, weil die Deckung zwischen verschiedenen subjektiven Beurteilungen nicht besonders hoch und die Übereinstimmung zwischen subjektiven und objektiven Maßen gering ist (Földényi, Imhof \& Steinhausen, 2000; Lehmkuhl \& Döpfner, 2000). Bei Hyperkinetischen Störungen kommt eine starke Situationsabhängigkeit der Störungssymptomatik hinzu.

Durch die unterschiedlichen Kriteriumsmaße können Wirksamkeitsprofile analysiert werden. Psychometrische Verfahren gestatten die gesonderte Erfassung einzelner Aufmerksamkeitskomponenten, welche in komplexen Beurteilungen durch Eltern und Lehrer nicht voneinander getrennt werden können. In den Studien 1 - 3 werden folgende Verfahren eingesetzt:

- Fremdbeurteilungsbogen-Hyperkinetische Störungen (FBB-HKS) (Döpfner \& Lehmkuhl, 2000),

- (Fragebogen zur Einschätzung hyperkinetischen und aufmerksamkeitsgestörten Verhaltens nach DSM-IV) (vgl. Anhang D),

- Elternfragebogen über das Verhalten von Kindern und Jugendlichen (CBCL/4-18) (Arbeitsgruppe Deutsche Child Behavior Checklist, Achenbach, 1993),

- Lehrerfragebogen über das Verhalten von Kindern und Jugendlichen (TRF) (Arbeitsgruppe Deutsche Child Behavior Checklist, Achenbach, 1993),

- Testbatterie zur Aufmerksamkeitsprüfung (TAP) (Zimmermann \& Fimm, 1993),

- Aufmerksamkeits- und Belastungstest d2 (Brickenkamp, 1994),

- Mottier-Test (abgedruckt in Welte, 1981).

In Studie 4 werden folgende Verfahren eingesetzt:

- FBB-HKS (Fremdbeurteilungsbogen-Hyperkinetische Störungen) (Döpfner \& Lehmkuhl, 2000),

- Elternfragebogen über das Verhalten von Kindern und Jugendlichen (CBCL 4-18 / Child Behavior Checklist 4-18) (Arbeitsgruppe Deutsche Child Behavior Checklist, Achenbach, 1993), 
- Lehrerfragebogen über das Verhalten von Kindern und Jugendlichen (TRF) (Arbeitsgruppe Deutsche Child Behavior Checklist, Achenbach, 1993),

- Testbatterie zur Aufmerksamkeitsprüfung (TAP) (Zimmermann \& Fimm, 1993),

- Aufmerksamkeits-Belastungstest d2 (Brickenkamp, 1994),

- Zahlensymboltest aus dem HAWIK-III (Tewes, Rossmann \& Schallberger, 1999),

- CFT 20 (Weiß, 1997),

- Testbatterie zur Prüfung der Konzentrationsfähigkeit (TPK) (Kurth \& Büttner, 1999).

Die in den vier Studien eingesetzten Instrumente werden im Folgenden beschrieben. Der Fremdbeurteilungsbogen-Hyperkinetische Störungen (FBB-HKS) ist Bestandteil des Diagnostik-Systems für psychische Störungen im Kindes- und Jugendalter nach ICD-10 und DSM-IV (DISYPS-KJ) von Döpfner und Lehmkuhl (2000). Hierbei erfolgt die klinische Beurteilung mittels Diagnose-Checklisten. Eltern, Lehrer und Kinder können anhand von Ratingskalen einschätzen, wie zutreffend die Beschreibung ist und wie problematisch das Verhalten erlebt wird. Der FBB-HKS erfasst im ersten Teil in 20 Items die 18 Symptomkriterien (Aufmerksamkeitsstörungen/Unaufmerksamkeit (9 Items), Überaktivität (7 Items) und Impulsivität (4 Items)) der beiden Klassifikationssysteme. Die Items werden im Hinblick auf den Schweregrad sowie die subjektiv empfundene Problemstärke anhand vierstufiger Skalen von Bezugspersonen des Kindes (hier Eltern und Lehrer) beurteilt.

Für die vorliegende Arbeit wurde sowohl eine kategoriale als auch dimensionale Auswertung jeweils für ein Eltern- und ein Lehrerurteil hinsichtlich des Schweregrades vorgenommen. Bei der kategorialen Auswertung werden diejenigen Symptomkriterien summiert, die als „erfüllt" gelten, was dann der Fall ist, wenn sie vom Beurteiler als „weitgehend“ bzw. „besonders zutreffend“ beurteilt wurden. Die dimensionale Auswertung sieht die Berechnung eines Kennwertes (arithmetisches Mittel) getrennt für jede der drei Symptomgruppen vor (Range: 0.0-3.0). Döpfner und Lehmkuhl (2000) sowie Brühl, Döpfner und Lehmkuhl (2000) ermittelten befriedigende Reliabilitäts- und Validitätswerte für Elternund Lehrerurteile bei diesem Verfahren.

Da zu Beginn der Studie das DISYPS noch nicht veröffentlicht worden war, wurde bei einigen Kindern der Fragebogen zur Einschätzung hyperkinetischen und aufmerksamkeitsgestörten Verhaltens nach DSM-IV zur Einschätzung der Kernsymptomatik verwendet, der von Mitarbeitern der Kinder- und Jugendpsychiatrie Mühlhausen entwickelt wurde. Der Fragebogen besteht aus 18 Items, die basierend auf den 
Kriterien des DSM-IV typische Verhaltensweisen hyperkinetisch auffälliger Kindern beschreiben (je 9 Items, die sich auf die Dimensionen Hyperaktivität-Impulsivitätsstörung und Aufmerksamkeitsstörung beziehen). Für jedes Item ist der Ausprägungsgrad anhand einer Skala von 0-7 (,0“ bedeutet, dass sich das Verhalten überhaupt nicht zeigt, „7“, dass sich entsprechendes Verhalten immer oder fast immer bzw. in sehr starkem Maße zeigt) zu beurteilen. Zusätzlich ist anhand einer 7stufigen Ratingskala einzuschätzen, ob und ggf. wie stark das Kind durch die zuvor beschriebenen Verhaltensweisen in den Lebensbereichen „familiäres Zusammenleben bzw. Heimgruppe“, „Schule“ und „Umgang mit Gleichaltrigen“ beeinträchtigt ist (,0“- überhaupt nicht bis , $7^{“}$ - extrem stark).

Als kritisch, im Sinne des Vorliegens einer hyperkinetischen Problematik, gilt ein Item dann, wenn es auf der Skala mit $\geq 5$ bewertet wurde. Entsprechend den diagnostischen Kriterien des DSM-IV ist von einer Aufmerksamkeitsdefizit- und/oder Hyperaktivitätsstörung auszugehen, wenn mindestens sechs Items, die sich auf Aufmerksamkeitsprobleme beziehen und/ oder mindestens sechs Items, die hyperaktives, impulsives Verhalten beschreiben, als kritisch eingeschätzt werden und Beeinträchtigungen in mehreren Bereichen vorliegen. Durch eine lineare Transformation wurden die Werte bzgl. des Schweregrades in das Antwortformat des FBB-HKS überführt, um eine einheitliche Datenbasis zu erstellen. Die Kennwerte konnten dagegen übernommen werden, da sie an der Anzahl der Items relativiert sind.

Der standardisierte Elternfragebogen über das Verhalten von Kindern und Jugendlichen (CBCL 4-18) ist die deutschsprachige Version der von Achenbach (1991) entwickelten Child Behavior Checklist (CBCL). Die CBCL wird als Screening-Verfahren zur Erfassung von Kompetenzen und psychischen Auffälligkeiten von Kindern und Jugendlichen eingesetzt. In der vorliegenden Studie wird der zweite Teil des Fragebogens genutzt, der aus 120 Items besteht, in denen Verhaltensauffälligkeiten, emotionale Auffälligkeiten und körperliche Beschwerden beschrieben werden. Das kindliche Verhalten innerhalb der letzten sechs Monate soll bezogen auf jedes Item anhand einer dreistufigen Skala $(0=$ nicht zutreffend, 1 = etwas oder manchmal zutreffend, 2 = genau oder häufig zutreffend) beurteilt werden. Aus den Items werden acht Syndromskalen gebildet, die basierend auf Faktorenanalysen zu drei Gruppen zusammengefasst sind (Externalisierende Störungen mit den Subskalen: Dissoziales Verhalten und Aggressives Verhalten; Internalisierende Störungen mit den Subskalen: Sozialer Rückzug, Körperliche Beschwerden und Angst/Depressivität; Gemischte Störungen mit den Subskalen: Soziale Probleme, 
Schizoid/Zwanghaft und Aufmerksamkeitsstörungen). Die Items (mit Ausnahme von zwei Items, die wegen mangelnder Differenzierungsfähigkeit nicht aufgenommen werden) werden zu einem Gesamtwert verrechnet. Für die übergeordneten Skalen Externalisierende und Internalisierende Störungen sowie den Gesamtwert gilt ein T-Wert $\geq 63$ als klinisch auffällig. Die Syndromskalen liegen mit einen T-Wert $\geq 70$ im klinisch auffälligen Bereich. Die Skalen Internalisierende Störungen, Externalisierende Störungen als auch der Gesamtwert, sowie die Syndromskalen aggressives Verhalten, delinquentes Verhalten, Aufmerksamkeitsstörung und Angst/Depression weisen gute bis sehr gute Reliabilitäten auf (Döpfner, Schmeck, Berner, Lehmkuhl und Poustka, 1994).

In den hier durchgeführten Studien wurden die T-Werte der übergeordneten Skalen „externalisierende Störungen“ und „Gesamtauffälligkeit“ sowie der T-Wert der Syndromskala „Aufmerksamkeitsstörungen“ genutzt. Für die zwei übergeordneten Skalen wurden sehr gute interne Konsistenzen (.93 und .94) und für die Syndromskala eine befriedigende Konsistenz (.76) in einer Studie von Döpfner, Schmeck, Berner, Lehmkuhl und Poustka (1994) ermittelt, die sie an einer klinischen Stichprobe deutschsprachiger Kinder durchführten.

\section{Der Lehrerfragebogen über das Verhalten von Kindern und Jugendlichen (TRF $\left.{ }^{\mathbf{2}}\right)$}

wurde in Anlehnung an den Elternfragebogen entwickelt (Arbeitsgruppe Deutsche Child Behavior Checklist, 1993). 95 der 120 Problem-Items im zweiten Teil des Fragebogens sind mit dem Elternfragebogen völlig identisch, während 25 Items des Elternfragebogens durch Fragen über schulspezifisches Verhalten ausgetauscht wurden. Beurteilungszeitraum, Antwortalternativen, übergeordnete und Syndromskalen sind identisch mit denen des Elternfragebogens. Im Lehrerfragebogen gehen allerdings alle 120 Items in den Gesamtauffälligkeitswert mit ein. Als abhängige Variablen wurden in den hier durchgeführten Studien dieselben Indizes wie im Elternfragebogen verwendet. Die faktorielle Validität und Reliabilität des TRF wurden von Döpfner, Berner und Lehmkuhl (1997) in einer Untersuchung an einer klinischen Stichprobe deutschsprachiger Kinder untersucht. Für die hier genannten drei Skalen wurden sehr gute interne Konsistenzen ermittelt (.96 für die übergeordneten Skalen und .91 für die Skala Aufmerksamkeitsstörungen).

Die Testbatterie zur Aufmersamkeitsprüfung (TAP) ist ein computergestütztes Verfahren, das von Zimmermann und Fimm (1993) zur Diagnostik von

\footnotetext{
${ }^{2}$ Teacher's Report Form
} 
Aufmerksamkeitsstörungen bei Erwachsenen entwickelt wurde, zunehmend aber auch zur klinischen Untersuchung von Kindern eingesetzt wird (Földenyi, Imhof \& Steinhausen, 2000). Die TAP besteht insgesamt aus 12 Untertests, in denen die Probanden per Tastendruck einfache Reaktionen auf visuelle und/oder akustische Reize geben sollen.

Földényi, Imhof und Steinhausen (2000) belegten die klinische Validität der TAP für die Gruppe von Kindern mit Hyperkinetischen Störungen. Sie zeigten mehr Fehler und Auslassungen und eine erhöhte intraindividuelle Streuung der Reaktionszeiten in verschiedenen Subtests gegenüber gesunden Kontrollkindern. Die mittleren Reaktionszeiten unterschieden dagegen nicht zwischen den Gruppen.

Die Testleistung eines Probanden kann bezüglich der Leistungsgüte und der Leistungsgeschwindigkeit beurteilt werden, für die das Programm automatisiert verschiedene Kennwerte liefert. Parameter der Leistungsgüte sind:

- die Anzahl richtiger Reaktionen (das sind alle Reaktionen, die nicht falsch und innerhalb des zulässigen Zeitspanne erfolgen);

- die Anzahl falscher Reaktionen (das sind Reaktionen auf nicht-kritische Reize bzw. Reaktionen, bei denen eine falsche Taste gedrückt wird, wenn ein kritischer Reiz erscheint; sie wird als Zeichen einer mangelnden Reaktionshemmung betrachtet);

- die Anzahl von Auslassungen (fehlende Reaktionen auf kritische Reize; sie stellt einen Indikator für Unaufmerksamkeit/ gestörte selektive Aufmerksamkeit dar).

Wichtige Parameter der Leistungsgeschwindigkeit sind:

- der Median der Reaktionszeiten, der, wegen der häufig schiefen Verteilung von Reaktionszeiten, als angemessenster Kennwert für die mittlere Reaktionszeit gilt. Die Größe des Medians wird im Vergleich zum arithmetischen Mittel nicht von Ausreißern beeinflusst. Ein erhöhter Median gilt, sofern er sich über alle Verfahren nachweisen lässt, als Maß für eine allgemeine Verlangsamung.

- die Standardabweichung der Reaktionszeiten als Maß für die Regelmäßigkeit (Variabilität) der Antworten. Je geringer sie ist, desto konstanter wurde die Aufmerksamkeit aufrechterhalten.

Im Rahmen der vorliegenden Evaluationsstudie wurden die Subtests Geteilte Aufmerksamkeit, Go/Nogo, Inkompatibilität sowie der Untertest Visuelles Scanning eingesetzt. In Anlehnung an die Untersuchung von Földenyi et al. werden in dieser eigenen Studie die Kennwerte Fehler, Auslassungen und Standardabweichung der Reaktionszeiten als Kriterien der TAP-Testleistungen herangezogen. Es handelt sich bei diesen Variablen 
allerdings um Rohwerte, mit denen keine individuelle Vergleichbarkeit der Daten möglich ist. Jedoch wurden die Rohwerte den normierten Daten vorgezogen, da diese bisher nur in wenig differenzierter Form für den gegebenen Altersbereich vorliegen (vgl. Földényi, TagwerkerNeuenschwander, Giovanoli, Schallberger \& Steinhausen, 1999; Kunert, Derichs \& Irle, 1996).

Die Durchführungs- wie auch Auswertungsobjektivität ist durch die standardisierte Instruktion und die Auswertung gewährleistet. Die Probanden erhalten bis auf den Untertest Visuelles Scanning die Möglichkeit Vorversuche durchzuführen, bis sie die Instruktionen eindeutig verstanden haben. Es ist jedoch häufig notwendig, den Kindern die Instruktionen mit eigenen Worten zu erklären. Die Split-Half-Reliabilität wird für die einzelnen Untertests mit Werten von .78 bis .95 angegeben (Zimmermann \& Fimm, 1993). Die faktorielle Validität der TAP wurde für Erwachsenenstichproben anhand von Faktorenanalysen belegt (Zimmermann \& Fimm, 1993). Für Kinderstichproben ist die Befundlage diesbezüglich uneinheitlich. In der Studie von Kunert, Derichs und Irle (1996) erhielt keiner der untersuchten Subtest eine reine faktorielle Ladung. Die Autoren schlussfolgerten, dass eine Differenzierung von Aufmerksamkeitsfunktionen erst im Erwachsenenalter erreicht wird. Dem widersprechen jedoch die faktorenanalytischen Ergebnisse der Arbeitsgruppe um Földenyi (1999), die andeuten, dass eine Trennung von Aufmerksamkeitskomponenten bei 610jährigen Kindern möglich ist.

Die einzelnen Testaufgaben, Durchführungsbedingungen sowie die damit zu messenden Konstrukte werden im Folgenden kurz beschrieben.

Der Subtest Geteilte Aufmerksamkeit stellt eine „dual-task“ Aufgabe dar, bei der gleichzeitig zwei Reizdarbietungen aus verschiedenen Informationskanälen (optisch/akustisch) zu bearbeiten sind. Der erste Teil der Aufgabe besteht darin, auf eine bestimmte Konstellation optischer Reize (vier Kreuze, die ein kleines Quadrat bilden) per Tastendruck zu reagieren, während simultan auf Unregelmäßigkeiten in einer Folge hoher und tiefer Töne zu achten ist. Ist der gleiche Ton zweimal hintereinander zu hören, soll ebenfalls so schnell wie möglich auf die Taste gedrückt werden. Für die vorliegende Untersuchung wurde die Testbedingung C mit 100 optischen und 200 akustischen Darbietungen ausgewählt (Bedingung: Quadrate und Töne). Diese Anforderung stellt eine sehr komplexe Alltagsleistung dar, in der mehrere Dinge gleichzeitig bewältigt werden müssen. Störungen in 
diesem Bereich deuten auf eine eingeschränkte Verarbeitungskapazität des kognitiven Systems, auf eine allgemeine Verlangsamung oder Probleme bei der Koordination verschiedener Anforderungen hin (Zimmermann \& Fimm, 1993).

Mit dem Subtest Go/Nogo wird die Fähigkeit zur Reaktionsunterdrückung bzw. Reaktionsselektion auf nicht adäquate, irrelevante Reizdarbietungen geprüft. Es wird in einer einfachen „Go/Nogo“-Bedingung die Unterscheidung von zwei Reizen (,+,“ oder „, $\mathrm{X}^{\text {“) }}$ gefordert, wobei durch den Patienten nur auf einen $\left(, \mathrm{x}^{“}\right)$ von beiden reagiert werden darf und die Antwort auf den nicht-kritischen Stimulus (,,+“) unterdrückt werden muss. Schnelle Reaktionszeiten mit einer großen Fehlerzahl deuten auf Defizite im Bereich der selektiven Aufmerksamkeit hin, die durch gestörte Impulshemmung bzw. mangelnde motorische Modulationsfähigkeit bedingt werden und insbesondere durch Schädigungen im präfrontalen Kortex determiniert sein sollen (Zimmermann \& Fimm, 1993). Für die vorliegende Untersuchung wurde bei diesem Subtest die erste Testbedingung mit 50 Darbietungen ausgewählt.

Der Subtest Inkompatibilität untersucht die Fähigkeit zur Fokussierung der Aufmerksamkeit, die die Zurückweisung irrelevanter, u.U. automatisch verarbeiteter Reizaspekte und damit Reaktionshemmung voraussetzt. Eine Beeinträchtigung dieser Fähigkeit führt zu erhöhter Interferenzneigung, die bei Personen mit frontalen Läsionen angenommen wird (Zimmermann \& Fimm, 1993). Die Interferenzkontrolle wird bei diesem Subtest durch eine Reiz-Reaktionsinkompatibilität geprüft. Es werden links oder rechts von einem Fixationspunkt nach links bzw. rechts gerichtete Pfeile präsentiert. Weist der Pfeil nach rechts, soll die rechte Taste gedrückt werden, zeigt er nach links, ist die linke Taste zu drücken. Dabei ist es unerheblich, auf welcher Seite der Pfeil zu sehen ist. Der Test besteht aus einer kompatiblen (Seite des Reizes und Richtung des Pfeils stimmen überein) und einer inkompatiblen Reizbedingung (Seite der Reizpräsentation und Richtung des Pfeils sind unterschiedlich). Somit muss die Aufmerksamkeit auf die Pfeilrichtung fokussiert und die Erscheinungsseite unterdrückt werden. Hiermit wird die Fähigkeit zur Zurückweisung irrelevanter und u.U. automatisch verarbeiteter Reizaspekte überprüft. Der Subtest wurde in der vorliegenden Untersuchung gemäß den Voreinstellungen (60 Darbietungen) durchgeführt.

Beim Subtest Visuelles Scanning soll der Proband eine 5x5-Matrix mit Quadraten, die jeweils nach einer Seite offen sind, nach einem kritischen Reiz absuchen (Quadrat, welches oben offen ist). Über zwei separate Tasten soll angegeben werden, ob der kritischer Reiz vorhanden ist (kritische Trials) oder nicht (nicht-kritische Trials). Mit diesem Subtest wird die Fähigkeit zum visuellen Abtasten des Gesichtsfeldes geprüft. Zwar setzt die angemessene 
Bewältigung der Aufgabe die Funktionstüchtigkeit mehrerer Teilfunktionen voraus (u.a. Blickbewegungen, Systematik des Scannings), jedoch lassen sich durch die Länge des Tests auch Hinweise auf eine möglicherweise reduzierte Daueraufmerksamkeit finden. Für die vorliegende Untersuchung wurden 50 Darbietungen ausgewählt.

Der Aufmerksamkeits-Belastungs-Test ,d2 ${ }^{6}$ ist ein Durchstreichtest, welcher eine auf visuelle Reize bezogene Konzentrationsleistung erfordert, die aus der Koordination von Antriebs- und Kontrollfunktionen (Arbeitstempo und Bearbeitungsgüte) resultiert (Brickenkamp, 1994). Die Aufgabe besteht darin, aus einer Reihe ähnlicher Zeichen

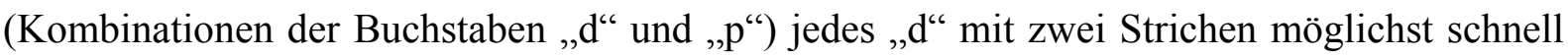
und ohne Fehler durchzustreichen. Dazu wird dem Probanden ein Testbogen vorgelegt, auf dessen Rückseite 14 Zeilen mit jeweils 47 Zeichen zu bearbeiten sind. Die Bearbeitungszeit ist in der Standard-Durchführung auf 20 Sekunden pro Testzeile begrenzt. Anhand einer Übungszeile wird vor Testbeginn geprüft, ob das Kind die Aufgabe verstanden hat. Die Handanweisung enthält standardisierte Instruktionen, wodurch die Durchführungsobjektivität gewährleistet ist. Die Auswertung erfolgt mit Hilfe von Schablonen und ist bei Beachtung der Auswertungsregeln ebenfalls hoch objektiv. Der d2 liefert Kennwerte des Bearbeitungstempos (Gesamtzahl aller bearbeiteten Zeichen: GZ; fehlerkorrigierte Leistungsmenge: GZ - F) und der Leistungsgüte (Summe der Auslassungsfehler [relevante Zeichen werden nicht durchgestrichen] und Verwechslungsfehler [irrelevante Zeichen werden versehentlich durchgestrichen]: F1 und F2; Fehlerprozentwert als Fehleranteil innerhalb des bearbeiteten Testteils: F \%). Je geringer der Fehleranteil ist, umso höher ist die Leistungsgüte, also die Genauigkeit und Sorgfalt der Testbearbeitung. Aus der Summe aller richtig durchgestrichenen Zeichen, abzüglich der Verwechslungsfehler, lässt sich als Kriterium der Gesamtleistung der Konzentrationsleistungswert KL ermitteln. Der KL ist normal verteilt, hoch reliabel $(\mathrm{r}>.90)$, relativ stabil $(\mathrm{r}>.70)$ und verfälschungsresistent, da er durch instruktionswidriges Verhalten (Überspringen von Testteilen, beliebiges Durchstreichen aller Zeichen) nicht erhöht werden kann (ebd.). Als abhängige Maße werden in der eigenen Studie die Anzahl der Fehler (F1 + F2) und der KL-Wert herangezogen.

Der MOTTIER-Test (abgedruckt bei Welte, 1981) ist ein Verfahren, bei dem es jeweils sechs zwei-, drei-, vier-, fünf- und sechssilbigen Kunstwörter nach einmaligem Hören nachgesprochen werden müssen. Für jedes richtig nachgesprochene Wort wird ein Punkt vergeben. Durch Aufsummieren der Punkte wird ein Gesamtwert (max. 30 Punkte) gebildet, 
der mit einer Alters- bzw. Schulklassennorm verglichen werden kann. Nach Hasselhorn, Tiffin-Richards, Woerner, Banaschewski und Rothenberger (2000) dient der Test der Überprüfung der auditiven Merkfähigkeit und eignet sich gut zur Erfassung der Funktionstüchtigkeit des phonologischen Arbeitsgedächtnisses. Der Einsatz dieses Instrumentes innerhalb dieser Arbeit ergibt sich aus Beobachtungen aufmerksamkeitsgestörter Kinder, die in diesem Test regelmäßig schlechter abschneiden als andere Kinder. Es wird vermutet, dass Funktionen der Zentralen Exekutive, welche abhängig von Aufmerksamkeitsund Hemmungsprozessen in diesem Bereich sind, auffällig werden können.

Der Zahlensymboltest (ZST) aus dem HAWIK-III (Tewes, Rossmann \& Schallberger, 1999) wurde in Studie 4 eingesetzt, da er neben der visuo-motorischen Koordination auch die Konzentration und die Arbeitsgeschwindigkeit erfasst. Hierbei müssen die Probanden Zeichen, welche den Zahlen eins bis neun zugeordnet sind, in die freien Felder unter den Zahlen eintragen. Hierfür ist eine Zeitdauer von zwei Minuten vorgegeben. Die Auswertung erfolgt mittels einer Schablone. Die innere Konsistenz des Untertests wird mit $r=$ .85 angegeben. In einer separaten Studie konnten die Testautoren bei einer Gruppe von 41 Kindern mit den Diagnosen einfache Aktivitäts- und Aufmerksamkeitsstörung sowie Hyperkinetische Störung des Sozialverhaltens für diesen Subtest ein signifikant schwächeres Ergebnis eruieren.

Der Cultural Fair Test 20 (CFT 20) (Weiß, 1997) wurde zur Überprüfung der Lernund Leistungsmöglichkeiten in Studie 4 eingesetzt. Der CFT 20 stellt die Weiterentwicklung des Culture Fair Test Test-Scale 2 von Cattell (1960) dar. Cattel unterscheidet zwischen der Genereal Fluid Ability, der Fähigkeit, Relationen unabhängig von der Sinneswahrnehmung zu erkennen, und der Crystallized General Ability, welche als erlernte Fertigkeit beschrieben wird. Im CFT 20 wird vor allem die Fluid Ability abgebildet, der Einsatz der Ergänzungstests Wortschatz und Zahlenfolgen kann eingeschränkt auch die Crystallized General Ability erfassen. In dieser Untersuchung wurde jedoch nur die Langform des CFT 20 ohne Ergänzungstests verwendet. Der Test wird zur Diagnostik im Altersbereich von 8;7 Jahren bis 70 Jahren eingesetzt. Er kommt in der Schulpsychologie bzw. Bildungsberatung, in der Erziehungsberatung sowie in der Forschung zur Anwendung. Mit ihm wird das allgemeine intellektuelle Niveau (Grundintelligenz) anhand des logischen schlussfolgernden Denkens erfasst. Diese kann umschrieben werden als Fähigkeit, figurale Beziehungen und formallogische Denkprobleme mit unterschiedlichem Komplexitätsgrad zu erkennen und innerhalb einer bestimmten Zeit zu verarbeiten. Der CFT 20 gehört zu der Gruppe der sprachfreien 
Tests und besteht aus zwei gleichartig aufgebauten Testteilen mit je vier Untertests (Reihenfortsetzen, Klassifikationen, Matrizen und topologische Schlussfolgerungen), welche jeweils in der Form A und B erschienen sind. Jeder Testteil besteht aus 46 sprachfreien Items, welche zeitabhängig bearbeitet werden müssen. Hierbei muss der Proband seine Antworten auf einem Antwortbogen markieren. Im ersten Teil stehen den Probanden für die Bearbeitung der Subtests I und II vier Minuten und für die Subtests III und IV drei Minuten zur Verfügung. Im zweiten Teil wird die Bearbeitungsdauer für alle Subtests auf drei Minuten beschränkt. Die Auswertung erfolgt mittels Schablonen, es können sowohl altersspezifische Werte als auch Schulstandardwerte ermittelt werden. Hierbei werden sowohl der Intelligenzquotient, wie auch T-Werte und Prozentränge für die beiden Teile und den Gesamttest angegeben. Die Halbierungszuverlässigkeit wird für die Testteile mit $r=.90 \mathrm{bzw}$. $\mathrm{r}=.91$ sowie für den Gesamttest mit $\mathrm{r}=.95$ angegeben (Baving \& Schmidt, 2000).

Für Kinder mit Aufmerksamkeitsstörungen kann erwartet werden, dass sie in den beiden Testhälften ihre Aufmerksamkeit nicht in gleichem Maße aufrechterhalten können, d.h. dass die Ergebnisse der ersten Testhälfte besser als die der zweiten sind.

Die Testbatterie zur Prüfung der Konzentrationsfähigkeit (TPK) (Kurth \& Büttner, 1999) stellt ein Verfahren dar, welches die bei Tätigkeiten des Schulalltags notwendige Konzentration erfassen will. Die TPK kann als Gruppentest durchgeführt werden und dauert ungefähr 45 Minuten. In diesem Testverfahren schreiben die Probanden zunächst einen Text ab, wofür eine Zeitdauer von 10 Minuten vorgegeben ist. Nach jeweils zwei Minuten erfolgt die Instruktion: „Eine Reihe freilassen“, damit die Schwankung innerhalb der Bearbeitungszeit errechnet werden kann. Für diesen Test werden sowohl die geschriebenen Silben ausgezählt (TSL) wie auch die Fehler addiert. Diese werden dann in Relation zu der Gesamtmenge gestellt, woraus sich der TF \%-Wert ergibt. Als zweite Aufgabe bekommen die Probanden eine Geschichte vorgelesen, von der sie sich möglichst viele Tiere merken sollen, die im Anschluss aufgeschrieben werden. Hierbei wird die Anzahl richtig reproduzierter Tiere (TI) vermerkt. Im dritten Teil werden einfache Additions- und Subtraktionsrechnungen durchgeführt, für welche wiederum 10 Minuten Zeit zur Verfügung stehen. Auch hier wird jeweils nach 2 Minuten eine Markierung unter die zuletzt gerechnete Aufgabe angebracht, um die Schwankung berechnen zu können. Mit den Messwerten TSL und RL (Rechenleistung) wird die Leistungsmenge erfasst, die Fehlerzahl als Prozentsatz der Leistungsmenge dient als Kriterium für die Genauigkeit.

Für die einzelnen Variablen können den Rohwerten C-Werte zugeordnet werden. Diese werden dann bis auf die Schwankungsprozentwerte TS \% und RS \%, welche eine 
niedrige Validität erreichten, addiert und können in den Konzentrationswert (KW), welcher in die IQ-Skala transformiert wurde, umgeformt werden.

Die innere Konsistenz mit vergleichbaren Untertests liegt bei $r=.95$, die Retestreliabilität des Gesamttests nach zwei Monaten bei $r=.80$.

\subsection{Ableitung psychologischer Vorhersagen und statistischer Hypothesen; Testplanung}

Aus den in Abschnitt 3.4 aufgestellten psychologischen Hypothesen wird unter Berücksichtigung der Versuchspläne $\mathrm{zu}$ jeder erhobenen abhängigen Variablen eine psychologische Vorhersage abgeleitet und in testbare statistische Hypothesen umgesetzt. Dies wird wie im Abschnitt 3.4 für die jeweiligen Studien separat aufgelistet. Die Tabelle 11 und die entsprechenden Tabellen im Anhang C.2, C.3 und C.4 enthalten eine Übersicht über die den jeweiligen psychologischen Hypothesen der verschiedenen Studien zugeordneten Operationalisierungen.

Das Vorgehen wird an dieser Stelle exemplarisch wegen der besseren Lesbarkeit nur für Studie 1 dargestellt. Für die anderen Studien wird dieser Punkt im Anhang C beschrieben.

\subsubsection{Psychologische Vorhersagen (PVn), Statistische Hypothesen (SHn) und Testplanung zu Studie 1: Wirksamkeit des Basistrainings nach Lauth und Schlottke}

In Tabelle 11 werden die Psychologischen Hypothesen und die zugehörigen Operationalisierungen dargestellt.

Tabelle 11

Operationalisierungen der psychologischen Hypothesen

PH 1.1: Das Basistraining nach Lauth und Schlottke führt unter den praktischen Rahmenbedingungen einer kinder- und jugendpsychiatrischen Ambulanz zu Verbesserungen der Aufmerksamkeitsleistung sowie zu einer Verminderung hyperkinetischer Verhaltenssymptome.

\begin{tabular}{l|ll}
\hline \multicolumn{1}{c|}{ Untersuchungsverfahren } & \multicolumn{1}{c}{ Kriteriumsmaße (AV) } \\
\hline TAP & - & Standardabweichung \\
\multirow{2}{*}{ Geteilte Aufmerksamkeit } & - & Anzahl Fehler \\
& - & Anzahl Auslassungen \\
\hline \multirow{2}{*}{ Visuelles Scanning } & - & Standardabweichung kritische Trials \\
& - & Standardabweichung nicht-kritische Trials \\
\hline
\end{tabular}




\begin{tabular}{l|l}
\hline & - Anzahl Auslassungen \\
\hline Test d2 & $-\quad$ Konzentrationsleistungswert \\
\hline FBB-HKS: Elternurteil & $\begin{array}{l}\text { Jeweils für die Bereiche Unaufmerksamkeit, Hyperaktivität und } \\
\text { Impulsivität: } \\
-\quad \text { Anzahl erfüllter Kriterien }\end{array}$ \\
FBB-HKS: Lehrerurteil & $-\quad$ Kennwert \\
\hline CBCL & $-\quad$ Jeweils T-Wert der Skala Aufmerksamkeitsstörungen \\
TRF &
\end{tabular}

PH 1.2: Das Basistraining nach Lauth und Schlottke führt zu positiven Veränderungen auf der Ebene der kognitiven Verhaltenshemmung. Den Kindern gelingt es durch das Training besser vorherrschende Handlungsimpulse zu unterdrücken und Interferenzen besser zu kontrollieren.

\begin{tabular}{|c|c|}
\hline Untersuchungsverfahren & Kriteriumsmaße (AV) \\
\hline Go/ Nogo & $\begin{array}{ll}- & \text { Standardabweichung } \\
- & \text { Anzahl Fehler } \\
- & \text { Anzahl Auslassungen }\end{array}$ \\
\hline Inkompatibilität & $\begin{array}{ll}- & \text { Standardabweichung } \\
- & \text { Anzahl Fehler }\end{array}$ \\
\hline
\end{tabular}

PH 1.3a: Das Basistraining nach Lauth und Schlottke führt zu einer Steigerung der funktionellen Nutzung des phonologischen Arbeitsgedächtnisses.

\begin{tabular}{l|l}
\hline \multicolumn{1}{c|}{ Untersuchungsverfahren } & \multicolumn{1}{c}{ Kriteriumsmaße (AV) } \\
\hline Mottier-Test & $-\quad$ Gesamtnachsprechleistung \\
\hline
\end{tabular}

PH 1.3b: Das Basistraining nach Lauth und Schlottke führt zu einer Verbesserung der psychosoziale Anpassung der Kinder.

\begin{tabular}{|c|c|}
\hline Untersuchungsverfahren & Kriteriumsmaße (AV) \\
\hline $\begin{array}{l}\text { CBCL } \\
\text { TRF }\end{array}$ & $\begin{array}{ll}\text { - } & \text { Jeweils T-Wert der Skala Externalisierende Störungen } \\
\text { - } & \text { Jeweils T-Wert der Gesamtskala }\end{array}$ \\
\hline
\end{tabular}

Anmerkungen. PH: psychologische Hypothese, AV: abhängige Variable, FBB-HKS: Fremdbeurteilungsbogen

Hyperkinetische Störungen, CBCL: Child Behavior Checklist, TRF: Teacher's Report Form, TAP: Testbatterie zur Aufmerksamkeitsprüfung.

Gemäß den Ansprüchen des Trainings nach Lauth und Schlottke und bisheriger Evaluationsuntersuchungen werden in der Trainingsgruppe in allen AVn Verbesserungen vom Vor- zum Nachtest erwartet. In der Wartegruppe sollten sich hingegen keine (bzw. wegen z.B. Spontanremission, möglicher Reifungs- oder Retesteffekten lediglich geringfügige) Verbesserungen zeigen (vgl. Hager, 2000). Je nach Definition der AV äußern sich positive Veränderungen vom Vor- zum Nachtest in einem Absinken oder einem Ansteigen der entsprechenden Kennwerte. 
Zur Überprüfung der PH 1 wurden mehrere abhängige Maße (AVn) herangezogen (s.o.). $\mathrm{Zu}$ erwarten ist für jede dieser $\mathrm{AVn}$, mit Ausnahme des Konzentrationsleistungswertes im d2-Test, ein Absinken der Kennwerte vom Vor- zum Nachtest. Daraus ergibt sich folgende empirische Vorhersage, die exemplarisch für die AV Auslassungen im TAP-Untertest „Geteilte Aufmerksamkeit“ dargestellt wird.

PV 1.1a: In der Trainingsgruppe (TG) nimmt die Anzahl der Auslassungen im TAPUntertest „Geteilte Aufmerksamkeit“ vom Vor- zum Nachtest deutlicher ab als in der Wartegruppe (WG), d.h. die Differenz der Auslassungen vom Vor- vs. Nachtest ist in der TG größer als in der WG.

Die PVn werden in statistische Hypothesen ( $\mathrm{SHn}$ ) überführt, die sich auf die Mittelwerte der AVn beziehen. Die SHn werden nur dann geprüft, wenn im Falle eines erwarteten Absinkens der Kennwerte numerisch geringere Nachtest- im Vergleich zu Vortestwerten in der TG bzw. im Falle erwarteter Leistungszuwächse numerisch höhere Nachtest- im Vergleich zu Vortestwerten feststellbar sind. Exemplarisch für die AV Auslassungen im TAP-Untertest „Geteilte Aufmerksamkeit“ lautet die statistische Interaktionshypothese (SH):

SH 1.1a: $\quad\left[\mu_{\mathrm{TG}, \mathrm{V}}(\mathrm{A}-\mathrm{GA})-\mu_{\mathrm{TG}, \mathrm{N}}(\mathrm{A}-\mathrm{GA})\right]-\left[\mu_{\mathrm{WG}, \mathrm{V}}(\mathrm{A}-\mathrm{GA})-\mu_{\mathrm{WG}, \mathrm{N}}(\mathrm{A}-\mathrm{GA})\right]>0$

Anmerkung: A-GA steht für die AV: Anzahl der Auslassungen im TAP-Untertest „Geteilte Aufmerksamkeit“, $\mu_{\mathrm{TG}}$ bzw. $\mu_{\mathrm{WG}}$ zeigt an, dass der Mittelwert für die Trainingsgruppe bzw. die Wartegruppe betrachtet wird, $\mathrm{V}$ steht für Vortest und $\mathrm{N}$ für Nachtest

Die Beträge in der Gleichung müssen einen positiven Wert annehmen, da davon ausgegangen wird, dass sich weder die Trainingskinder noch die Kontrollkinder von der Vorzur Nachtestung verschlechtern.

Für die anderen abhängigen Maße sind die empirische Vorhersage und die statistische Hypothese (SH) analog aufzustellen.

Eine Ausnahme bildet, wie bereits erwähnt, der Konzentrationsleistungswert (KL) des d2-Tests. Hier wird vom Vor- zum Nachtest ein Leistungszuwachs, also ein Ansteigen des KL-Wertes erwartet. Als psychologische Vorhersage ergibt sich deshalb: 
PV 1.1b: In der Trainingsgruppe steigt der KL-Wert vom Vor- zum Nachtest deutlicher an als in der Wartegruppe, d.h. die Differenz der KL-Werte von Vor- vs. Nachtest ist in der TG größer als in der WG.

Als statistische Interaktionshypothese ergibt sich dann:

SH 1.1b: $\quad\left[\mu_{\mathrm{TG}, \mathrm{N}}(\mathrm{KL})-\mu_{\mathrm{TG}, \mathrm{V}}(\mathrm{KL})\right]-\left[\mu_{\mathrm{WG}, \mathrm{N}}(\mathrm{KL})-\mu_{\mathrm{WG}, \mathrm{V}}(\mathrm{KL})\right]>0$

Anmerkung: KL steht für den Konzentrationsleistungswert des d2-Test, $\mu_{\mathrm{TG}}$ bzW. $\mu_{\mathrm{WG}}$ zeigt an, dass der Mittelwert für die Trainingsgruppe bzw. die Wartegruppe betrachtet wird, V (Vortest) und N (Nachtest) beziehen sich auf den Erhebungszeitpunkt.

Zur Überprüfung der PH 1.2 werden ebenfalls verschiedene abhängige Maße (s.o.) eingesetzt, für die wieder jeweils eine PV formuliert wird. Wenn das Basistraining die theoretisch angenommenen Wirkungen zeigt, wird für jede dieser AVn ein Absinken der Kennwerte vom Vor- zum Nachtest erwartet. Die psychologische Vorhersage und statistische Hypothese sind daher analog zu der PV 1.1a und SH 1.1a zu formulieren, weswegen von einer nochmaligen Darstellung abgesehen wird.

Als Prüfinstanz der PH 1.3a wird die AV „Nachsprechleistung“ im Mottier-Test eingesetzt. Wenn das Training Transfer auf die funktionelle Nutzung des Arbeitsgedächtnisses zeigt, erwarten wir einen Leistungszuwachs, d.h. ein Ansteigen der Nachsprechleistung vom Vor- zum Nachtest. Die psychologische Vorhersage und die statistische Hypothese entsprechen dann formal der PV 1.1b und SH 1.1b.

Zur Überprüfung der PH 1.3b werden der T-Wert Gesamtskala und der T-Wert der Skala Externalisierende Störungen der CBCL und der TRF als AVn herangezogen. Wenn die Trainingsmaßnahme Transfer auf die psychosoziale Anpassung zeigt, erwarten wir bei diesen AVn ein Absinken der entsprechenden Kennwerte vom Vor- zum Nachtest. Für jede abhängige Variable werden die psychologischen Vorhersagen und die statistische Hypothese dann wieder analog zu der PV 1.1a und SH 1.1a abgeleitet.

Für die Prüfung der psychologischen Hypothesen 1.1 bis $1.3 \mathrm{~b}$ findet in Anlehnung an die Überlegungen von Hager (1992) eine separate Prüfung für alle Operationalisierungen 
statt. Mit diesem univariaten Vorgehen kann das Problem einer Kumulation der Fehlerwahrscheinlichkeiten bei mehreren abhängigen Variablen umgangen werden.

Bei der Ableitung (und Testung) von Vorhersagen wird auf Überlegungen von Hager (1995) zurückgegriffen. Hiernach kann der Nachweis eines erwartungskonformen Ausgangs der Intragruppenvergleiche als Voraussetzung für eine sinnvolle Prüfung des Vergleiches zwischen den Versuchsgruppen angesehen werden, und die Tests innerhalb der Versuchsgruppen werden von denjenigen zwischen den Versuchsgruppen getrennt bzw. gehen diesen voraus. Gelangt man aufgrund der durchgeführten Tests über die Intragruppenvergleiche $\mathrm{zu}$ der Entscheidung, dass keine erwartungskonformen Veränderungen stattgefunden haben, wird auf den Test über den Intergruppenvergleich verzichtet.

\section{Testplanung zu Studie 1}

Ein Aspekt von größerer Bedeutung im Rahmen der Versuchsplanung betrifft die Kontrolle der Determinanten von Signifikanztests. Bei gegebener Stichprobengröße und geschätzter Effektgröße ist es möglich, sowohl das Signifikanzniveau $\alpha$ als auch die Wahrscheinlichkeit $\beta$ für falsche Entscheidungen simultan $\mathrm{zu}$ kontrollieren (vgl. Hager, 1987). Die Testplanung wird im Folgenden für Studie 1 für den jeweils interessierenden Interaktionskontrast der isolierten Evaluation anhand der Hagerschen Testplanungsstrategie 2 vorgenommen. Für die Studien 2-4 und die vergleichenden Evaluationen werden die entsprechenden Werte jeweils nach Darstellung der Psychologischen Vorhersagen und Statistischen Hypothesen kurz beschrieben.

Da in den durchgeführten Studien die Stichprobengröße $n$ festgelegt ist, wird mit Hilfe einer Schätzung für die Effektgröße $\delta$ und die mittlere Korrelation $\rho$ über alle Paare von Wertereihen sowie der Setzung des Signifikanzniveaus $\alpha$ auf den allgemein akzeptierten Wert von 0.05 der Wert für die Irrtumswahrscheinlich $\beta$ berechnet. Die Formel für den Fall einer intraindividuellen Bedingungsvariation mit zwei Versuchsbedingungen wurde Hager (1999) entnommen und entsprechend umgeformt. Als Schätzung für die Effektgröße d wird der Wert 0.5 eingesetzt, der sich an den Ergebnissen vergleichbarer Trainingsstudien orientiert. Als akzeptabler Schätzwert für die mittlere Korrelation über alle Paare von Wertereihen kann nach Hasselhorn und Hager (1996) ein Wert von .6 angenommen werden. Bei einer Stichprobengröße von maximal 15 (für Studie 1) errechnet sich für den Interaktionskontrast (unter Verwendung der Verteilungsfunktion der Standardnormalverteilung in Bortz, 1993) in der isolierten Evaluation für die Fehlerwahrscheinlichkeit $\beta$ ein Wert von .55. Über den 
Ausdruck 1- $\beta$ kann damit auch die Teststärke eines Signifikanztests bestimmt werden. Für den Interaktionskontrast liegt sie bei einem Wert von 0.45. (Durch drop-out aus unterschiedlichen Gründen wurden die Zellen zum Teil kleiner; der Minimalwert liegt bei 9 bei den Fremdbeurteilungsbögen. Die Fehlerwahrscheinlichkeit $\beta$ erhöht sich hier auf den Wert von .68).

4.4.2 Psychologischen Vorhersagen (PVn), Statistische Hypothesen (SHn) und Testplanung zu Studie 2: Wirksamkeit der Kombination von Basistraining und Strategietraining nach Lauth und Schlottke

Der Leser vergleiche hierzu bitte Anhang C.1.

4.4.3 Psychologischen Vorhersagen (PVn), Statistische Hypothesen (SHn) und Testplanung zu Studie 3: Wirksamkeit des THOP

Der Leser vergleiche hierzu bitte Anhang C.2.

4.4.4 Psychologische Vorhersagen (PVn), Statistische Hypothesen (SHn) und Testplanung zu Studie 4: Wirksamkeit des Marburger Konzentrationstrainings

Der Leser vergleiche hierzu bitte Anhang C.3.

4.4.5 Psychologischen Vorhersagen (PVn), Statistische Hypothesen (SHn) und Testplanung zur vergleichenden Evaluation der Trainings aus den Studien 1 - 4

Der Leser vergleiche hierzu bitte Anhang C.4.

\subsection{Durchführung der testpsychologischen Untersuchungen und der Trainings}

Die Testerhebungen und Trainingsgruppen der Lauth und Schlottke-Gruppen sowie der THOP-Gruppe fanden zwischen 1998 und 2003 statt. Die Durchführung der Gruppen erfolgte nacheinander, begonnen wurde mit Trainingsgruppen des Basistrainings nach Lauth und Schlottke (Studie 1), es folgten die Gruppen mit der Kombination aus Basis- und Strategietraining nach Lauth und Schlottke (Studie 2) und schließlich die THOP-Gruppen (Studie 3).

Die Kinder aus Studie 4 wurden im Jahr 2002 untersucht und mit dem Marburger Konzentrationstraining behandelt. 
Die Eltern und das Kind kamen im Rahmen der regulären Ambulanzsprechstunden zunächst zu einem Einzelgespräch. Es wurden Termine vereinbart, bei denen die testpsychologischen Untersuchungen durchgeführt wurden, in deren Verlauf auch die für diese Untersuchung relevanten Variablen erhoben wurden. Dann erfolgten ein Auswertungsgespräch und die Absprache über die Teilnahme an einem Training. Auf den Beginn des Trainings mussten die Familien zum Teil einige Monate warten, weil es einige Zeit dauerte, bis so viele Kinder rekrutiert waren, dass mit einer Interventionsgruppe begonnen werden konnte. Etwa zwei bis vier Wochen nach dem Training erfolgten die Nachtestung und ein individuelles Abschlussgespräch mit der Familie. Bei den Kindern der Wartegruppe erfolgte die Nachtestung etwa sieben bis acht Monate nach der Vortestung. Der Abstand zwischen Vor- und Nachtestung war somit für Warte- und Interventionsgruppe in etwa identisch. Die Eltern gaben jeweils ihr Einverständnis dafür, dass die erhobenen Testbefunde zum Zwecke einer wissenschaftlichen Untersuchung in anonymisierter Form verwendet werden dürfen. Die Datenerhebungen erfolgten durch die in den Ambulanzen tätigen Diplom-Psychologen oder durch Diplomandinnen im Rahmen der Durchführung der Studien.

Die Rücklaufquote bei den Fragebögen TRF, CBCL und FBB-HKS weist Lücken auf. Von mehreren Eltern kamen die Unterlagen nicht zurück, und in einigen Fällen haben die Eltern darum gebeten, von einer Verhaltenseinsschätzung durch die Lehrer abzusehen.

\subsubsection{Durchführung des Trainings nach Lauth und Schlottke}

Die Intervention wurde von den psychologischen Fachkräften der KJP Mühlhausen sowie Diplomandinnen, unterstützt durch Praktikantinnen der Einrichtung, geleistet. Die Grundlage der Intervention für Studie 1 bildete das Basistraining von Lauth und Schlottke (1997). Das Aufmerksamkeitstraining fand in der Ambulanz der KJP Mühlhausen statt und umfasste insgesamt 10 Trainingssitzungen und drei begleitende Elternsitzungen. Das Training fand alle 14 Tage nachmittags statt und dauerte pro Sitzung ca. 90 Minuten. Die Gruppengröße lag zwischen sechs und neun Kindern. Die Gesamtgruppe wurde bei einigen Teilaufgaben innerhalb der Trainingssitzungen weiter in Kleingruppen aufgeteilt, so dass im weiteren Verlauf nur drei bis max. fünf Kinder von einem Trainingsleiter betreut wurden.

In zwei begleitenden Elternsitzungen wurden organisatorische Fragen geklärt und den Eltern Störungswissen vermittelt. Ihnen wurden konkrete Hilfestellungen für die Kinder im 
Alltag aufgezeigt, aber auch die Möglichkeit zum Erfahrungsaustausch untereinander gegeben.

Die einzelnen Sitzungen mit den Kindern verfolgten entsprechend den Vorgaben im Handbuch unterschiedliche Schwerpunkte. Zunächst stand die Vermittlung von Störungswissen und der Ableitung förderlicher Vorgehensweisen für das eigene Handeln im Vordergrund. In den folgenden vier Sitzungen wurden grundlegende Fertigkeiten eingeübt. In den restlichen Einheiten wurde die Ausbildung von Selbststeuerungsfertigkeiten (Reaktionsverzögerung, Prüfprozesse, verbale Handlungsregulation und Umgang mit Ablenkung) trainiert. Der Ablauf jeder Sitzung gestaltete sich dabei nach der im Manual vorgegebenen Struktur (Einleitung, Demonstration, Übung, Spielzeit), unter Anwendung der bereits dargelegten therapeutischen Techniken (vgl. Kap. 3.1).

Um die Kinder für das Training zu motivieren, wurde mit operanter Verstärkung gearbeitet. Für erwünschtes Verhalten (z.B. angemessene Bearbeitung der Aufgaben unter Einhaltung erlernter Schritte) erhielten die Kinder positive Verstärker (Büroklammern), die, sobald eine bestimmte Anzahl gesammelt war, gegen ein kleines Spielzeug aus der „Kramkiste“ eingetauscht werden konnten. Bei Verstößen gegen zuvor vereinbarte Verhaltensregeln musste jeweils ein Verstärker zurückgegeben werden (response-costSystem). Die Grundlagen des Verstärkerprogramms wurden den Kindern zu Beginn der Intervention genau erklärt und in jeder Trainingssitzung wiederholt.

Das inhaltliche Vorgehen war für die Trainingsgruppen im Wesentlichen identisch. Geringfügige Abweichungen resultierten aus altersbedingt unterschiedlichen Kenntnissen der Kinder. Die Teilnahme am Training unterlag krankheitsbedingt leichten Unregelmäßigkeiten: drei Kinder nahmen an allen zehn Trainingssitzungen, sechs Kinder an neun Terminen und fünf Kinder an acht Sitzungen teil. Ein Kind war an nur sieben Terminen anwesend. An Ferien- und Feiertagen fand kein Training statt.

Die Rekrutierung, Testung und Durchführung der Intervention unterlag in Studie 2 ähnlichen Modalitäten wie in Studie 1. Das Aufmerksamkeitstraining umfasste in Studie 215 Sitzungen und wurde wöchentlich an einem bestimmten Nachmittag durchgeführt. Der eigentliche Ablauf der einzelnen Trainingseinheiten unter Verwendung verschiedener kognitiv-verhaltenstherapeutischer Grundtechniken, wie beispielsweise der operanten Verstärkung, wurde bereits an anderer Stelle eingehend vorgestellt (vgl. Kapitel 3.1). 
Einzelne Abwandlungen des vorgegebenen Manuals sollen an dieser Stelle in vollständiger Form zusammengefasst werden, da sie die Grundüberlegungen vor Beginn des Trainings bildeten, mit dem Ziel, die Wirksamkeit der Behandlungsmaßnahme zu verbessern.

1. Das Basistraining wurde von 13 Sitzungen auf 10 reduziert. Dafür wurde das gesamte Training auf 15 durch Einbeziehung von 5 Einheiten aus dem Strategietraining aufgestockt.

2. Ein wöchentlicher Durchführungsrhythmus soll gegen Vergessenseffekte der vermittelten Inhalte helfen, wie dies bei vorherigen Trainings, die in 14tägigen Abstand durchgeführt wurden, zu beobachten war.

3. Die Gruppenzusammensetzung wurde maßgeblich hinsichtlich Alter (mindestens 8 Jahre und höchstens 12 Jahre), intellektueller Voraussetzungen der Kinder (IQ mindestens 80) und Diagnose (vorwiegend Aufmerksamkeitsstörung ohne Hyperaktivität) homogenisiert.

Innerhalb der 15 Einheiten war eine sehr zufriedenstellende Teilnahme der Kinder zu beobachten. Fünf Kinder waren immer anwesend, acht fehlten nur einmal oder zweimal und zwei Kinder konnten an drei bzw. vier Trainingssitzungen krankheitsbedingt nicht teilnehmen.

\subsubsection{Durchführung des THOP als Gruppentraining}

In Studie 3 wurde das THOP als Gruppentraining durchgeführt. Es umfasste insgesamt 15 wöchentliche Sitzungen à 90 Minuten, die jeweils an Nachmittagen stattfanden. Jede Gruppe bestand zu Beginn aus vier bis fünf Familien. An den Elternsitzungen nahm in der Regel nur die Mutter teil. Die Kindergruppe traf sich parallel mit einer Co-Therapeutin. Für die jeweils letzten 15 Minuten kamen Kinder und Eltern zusammen und berichteten sich gegenseitig, was in der jeweiligen Sitzung besprochen wurde.

Die Durchführung der Elternsitzungen orientierte sich an dem im Manual des THOP angegebenen allgemeinen Aufbau und wurde vom Autor oder anderen Psychologischen Psychotherapeutinnen durchgeführt. $\mathrm{Zu}$ Anfang jeder Sitzung wurde über Ergebnisse und Umsetzung der Hausaufgaben seit dem letzten Kontakt gesprochen. Dabei wurden Schwierigkeiten, Vorbehalte und positive Erfahrungen mit den Hausaufgaben genau analysiert, in der Gruppe diskutiert und ggf. Modifikationen erarbeitet. Es folgte dann entweder die Vertiefung eines bereits begonnenen Therapiebausteins oder die Einführung eines weiteren. Die Inhalte des Bausteins wurden den Eltern vermittelt, die Ziele und 
wichtigsten Schritte für die Durchführung des Therapiebausteins in der Familie erarbeitet. Danach wurden die konkreten Durchführungsmodalitäten je Familie festgelegt und Therapieaufgaben für die Familie vereinbart. Als Orientierungshilfe bekamen die Eltern in der Regel einen Elternleitfaden mit nach Hause. Die Kindergruppen wurden zum Teil von ausgebildeten bzw. sich in der Ausbildung zur Psychologischen Psychotherapeutin befindlichen Diplompsychologinnen und in einem Fall von einer Diplomandin geleitet. Um die Kinder zu motivieren, wurde auch in dieser Studie mit einem Token-System gearbeitet (analog dem Vorgehen in Studie 1 und 2). Die ersten 10 bis 15 Minuten wurde dafür genutzt den Kindern die Möglichkeit zu geben über Geschehnisse seit dem letzten Treffen zu berichten. Dann wurde in den meisten Sitzungen eine dem jeweiligen Baustein zugeordnete Geschichte vom „Wackelpeter“ vorgelesen und mit den Kindern erörtert. Mit den Kindern wurde besprochen, was ihnen gut bzw. weniger gut gefallen habe, was hinsichtlich der Situationen und Erlebnisse des Wackelpeters bei ihnen ähnlich bzw. anders sei. Je nach Baustein erfolgte dann die Bearbeitung spezieller Vorlagen, wie z.B. der „Wunschliste“ über Verhalten, das sich ändern soll oder die Erstellung eines „Was-ist-schön-Tagebuchs“. In den Sitzungen zum Selbstinstruktions- und Selbstmanagementtraining wurde keine Geschichte vorgelesen, sondern anhand eines Leitfadens im Manual mit den Kindern die einzelnen Arbeitsschritte erarbeitet und anhand von Übungsmaterial angewendet. Dieses Übungsmaterial wurde z.B. aus Rätselheften zusammengestellt. Das Thema der Stunde wurde mit der Besprechung der Therapiehausaufgaben abgeschlossen, indem den Kindern bspw. die Handhabung und Regeln des Punkteplans erläutert wurden und sie von der Therapeutin darin bestärkt wurden, die Hausaufgaben und Interventionen in der Familie umzusetzen. In den letzten 15 bis 20 Minuten war jeweils eine Spielzeit vorgesehen, in der die Kinder gemeinsam oder alleine etwas spielen, malen oder sich erzählen konnten. Ziel der Spielzeit war neben dem belohnenden Charakter v.a.die Motivation für die Teilnahme an dem Training zu erhalten.

Die Art der eingesetzten Bausteine sowie die dafür aufgewendete Zeit unterschieden sich von Gruppe zu Gruppe geringfügig. Wie auch im Falle einer Einzelbehandlung wurde versucht, den Therapieplan so gut wie möglich den Problemschwerpunkten, die in den jeweiligen Gruppen vorherrschten, anzupassen. Die Therapiebausteine 1-9, 11-12, 16b+c, 17 und 20 wurden immer durchgeführt, die Bausteine 10, 13-15 und 18-19 gelegentlich. Teilweise wurden die Inhalte zweier Bausteine zusammengefasst und in einer Sitzung behandelt, bei Bedarf wurde aber auch für ein Thema mehr als eine Sitzung Zeit genommen. 


\subsubsection{Durchführung des Marburger Konzentrationstrainings}

Die Studie 4 wurde anhand einer anderen Stichprobe durchgeführt. Die Untersuchungen und Durchführung des Marburger Konzentrationstrainings für alle zehn Gruppen fanden innerhalb von 7 Monaten im Jahr 2002 statt. Sowohl die Untersuchungen als auch die Trainings wurden von Felstehausen (2003) im Rahmen ihrer Diplomarbeit durchgeführt und ausgewertet. Die Kooperation der um Mithilfe gebetenen Lehrpersonen in dieser Studie war nicht zufrieden stellend, was dazu führte, dass der Rücklauf der Fragebögen insbesondere nach dem Training sehr schwach verlief. Trotz telefonischer Nachfragen wurden kaum Fragebögen zurückgeschickt. Auch von Seiten der Eltern war der Rücklauf sehr eingeschränkt.

Die Voruntersuchungen fanden im Einzelkontakt in den jeweiligen Kinder- und Jugendpsychiatrischen Praxen statt. Das Marburger Konzentrationstraining fand über einen Zeitraum von fünf Wochen einmal wöchentlich in Gruppen von drei Kindern statt. Auf die begleitenden Elternsitzungen wurde im Rahmen dieser Untersuchung verzichtet. Das Training wurde immer am selben Wochentag nachmittags durchgeführt und dauerte pro Gruppe eine Stunde.

Der Ablauf des Marburger Konzentrationstrainings orientierte sich an der vorgegebenen Struktur des Manuals, welche für jede Sitzung beibehalten wurde. Es begann mit einem Entspannungsverfahren, dem eine Arbeitsphase folgte. Den Schluss der Stunde bildete eine Spielphase. Abweichend von der Empfehlung Krowatscheks mit einer Fantasiereise auf einem Zauberteppich $\mathrm{zu}$ beginnen, wurde in dieser Untersuchung als Entspannungsverfahren die Kapitän-Nemo-Geschichten (Petermann, \& Petermann, 1994) ausgewählt, da sie sich besonders als Vorbereitung für ein anschließendes kognitivbehaviorales Verfahren anbieten. Die Autoren weisen darauf hin, dass durch dieses Entspannungsverfahren eine Aktivitätsreduktion sowie eine Strukturierung der Imaginationen der Kinder erfolgen. Dadurch wird eine gesteigerte selektive Aufmerksamkeit erzielt, welche eine gute Voraussetzung für nachfolgende psychologische Interventionen bietet. Die Durchführung der Entspannung nahm immer die erste Viertelstunde der Sitzungen ein. Im Anschluss wurden nach den Vorgaben die einzelnen Stunden durchgeführt und zum Abschluss immer ein Spiel gespielt, was sich an KIM-Spielen, welche die Sinneswahrnehmung trainieren, orientiert.

Um den Kindern die Inhalte zu verdeutlichen dient der Trainer den Kindern als Modell und führt eine Aufgabe durch, während er laut zu sich selbst spricht. Dabei ist darauf zu 
achten, dass auch Fehler gemacht werden, die dementsprechend verbalisiert werden. Im Anschluss führt das Kind die gleiche Aufgabe durch und wird durch die Instruktion des Trainers geleitet. Über die Methode der Selbstinstruktion (orientiert an den Vorgaben, wie sie Meichenbaum \& Goodman (1971) vorgesehen haben) demonstriert der Trainer den Kindern, wie sie selbst Aufgaben lösen können. Über eine Aufgabenanalyse (,Was soll ich tun? Was ist meine Aufgabe?“), eine Materialanalyse (,Was weiß ich schon? Ich kann mir einen Plan machen.“) und eine Zielanalyse (,Was will ich erreichen? Bin ich auf dem richtigen Weg?“) werden die Kinder angeleitet. Es wird immer wieder betont, wie wichtig es ist, langsam vorzugehen („Ich kann mir ruhig Zeit lassen.“). Die Bildung von Teilzielen erweist sich vor allem bei komplexen Anforderungen als unumgänglich („Bis hierher ist es schon richtig. Was ist der nächste Schritt?“). Wenn Probleme bei der Aufgabenbearbeitung auftreten, sollte der Trainer sich fragen: „Warum habe ich jetzt Schwierigkeiten? Was stört mich im Moment?“.

Bedeutsam ist die Bewältigung von Misserfolg und Frustration, da Kinder mit Aufmerksamkeitsstörungen häufig eine niedrige Frustrationstoleranz aufweisen: „Fehler kann man verbessern, das ist nicht schlimm. Auch wenn mir am Anfang ein Fehler unterlaufen ist, habe ich die Aufgabe doch noch richtig zu Ende geführt.“ Auch sollte eine Bewertung und Selbstbestätigung erfolgen (,Das habe ich gut gemacht“").

Die Kinder werden in den Stunden dazu angeleitet, die Selbstinstruktionen zunächst nachzuahmen, dann selber laut, später flüsternd zu sagen. Schließlich sollen sie die Instruktionen nur noch denken.

Die Kinder der Kontrollgruppe kamen ebenfalls über einen Zeitraum von fünf Wochen einmal wöchentlich in die Praxis, um an einer Computergruppe mit drei Kindern teilzunehmen. Es wurde jedem Kind ein Computer zur Verfügung gestellt, auf dem das Computerprogramm „Neues von Pettersson und Findus“ (GAMOP, 1998) installiert war. Hierbei handelt es sich um eine spezielle Kindersoftware, welche bei vielen Kindern sehr beliebt ist und von Fachleuten als pädagogisch wertvoll eingstuft wird. Das Kind kann zwischen ganz unterschiedlichen Aufgaben wählen. Angeboten werden Spiele, welche psychomotorische Geschicklichkeit erfordern, Rechenaufgaben darstellen, komplexe Aufgaben, welche spielerisch dargestellt werden, und Spiele, in denen das Kind künstlerisch tätig werden kann. Alle diese Aufgaben müssen in einem Garten erledigt werden, das Kind läuft vertreten durch den Kater Findus durch diesen Garten und erhält bei erfolgreicher Bearbeitung Gewinne, welche im Gartenschuppen abzuholen sind. Hierbei gewinnt das Kind entweder Gartenwerkzeuge oder Samen diverser, teilweise skurriler Pflanzen. Diese können 
dann in die Beete eingepflanzt werden und wachsen, wenn sie dementsprechend mit Wasser und Unkrautpflege versorgt werden. Wenn das Kind eine Reihe von Aufgaben erfolgreich gelöst hat, bekommt es einen Hinweis auf den Verbleib des verschollenen Gärtners, der sich in der Unterwelt befindet. Auch hier müssen wieder diverse Aufgaben gelöst werden, um durch dieses Labyrinth zu gelangen.

Die Kinder speicherten ihren Spielstand am Ende der Stunde ab und spielten in der nächsten Woche weiter, so dass es einigen Kindern gelang, bis einen fortgeschrittenen Level („Unterwelt“) zu erreichen. Von Seiten des Trainers wurden die Kinder unterstützt, es wurde bei Instruktionsschwierigkeiten geholfen und systematisches Vorgehen positiv verstärkt. Obwohl jedes Kind seinen eigenen Computer hatte, ergab sich in allen Gruppen nach kurzer Zeit eine Zusammenarbeit unter den Kindern.

Sowohl in der Trainingsgruppe wie auch in der Kontrollgruppe fehlte jeweils ein Kind einmal, so dass von diesen nur vier Trainingstermine absolviert wurden.

Bei der Nachuntersuchung wurden die Fragebögen für Eltern und Lehrer mit der Bitte ausgehändigt, sie bei dem abschließenden Elterngesprächen ausgefüllt wieder abzugeben. Dieses Vorgehen hatte leider nur einen sehr eingeschränkten Erfolg, da wie bereits erwähnt der Rücklauf der Fragebögen in der Nachuntersuchung sehr schlecht war. 


\section{Ergebnisse}

Aufgrund der niedrigen Rücklaufquote der Fragebögen im Nachtest wies die Rohdatenmatrix z.T. größere Lücken auf, weswegen einige Fälle nicht berücksichtigt werden konnten. Fehlende Werte wurden derart behandelt, dass der Fall für die jeweilige Analyse(n) ausgeschlossen wurde. Daraus ergeben sich für die verschiedenen Analysen variierende Stichprobenumfänge, die entsprechend kenntlich gemacht werden.

Zur Auswertung der Daten wurden für die verschiedenen AVn zunächst wichtige deskriptive Kennwerte der verschiedenen Trainingsgruppen und der Wartegruppen ermittelt. Den Beginn der Studie markierten die Durchführung einer Trainingsgruppe, mit der das Basistraining nach Lauth und Schlottke durchgeführt werden sollte sowie die Zusammenstellung einer Kontrollgruppe. Für das kombinierte Basis- und Strategietraining nach Lauth und Schlottke und das THOP wurde die gleiche Kontrollgruppe zum Vergleich genutzt. Weitere Wartekontrollgruppen zusammenzustellen erschien aus ethischen Gründen nicht gerechtfertigt, sobald den Familien ein Trainigsangebot gemacht werden konnte. Aus methodischen Gründen ist diese Vorgehensweise gerechtfertigt, da die Prüfung der verschiedenen Trainings mit der gleichen Kontrollgruppe nicht zu einer Fehlerkumulation führt, da die Prüfinstanzen für die Gruppenvergleiche, welche für die Beurteilung der Psychologischen Hypothesen notwendig sind, voneinander unabhängig sind.

Die Überprüfung der gerichtet formulierten Interaktionshypothesen erfolgte dann anhand 2 (Gruppen: TG/ WG) $\times 2$ (Messzeitpunkte: Vor-/Nachtest)-faktorieller Varianzanalysen mit Messwiederholung. Die 2-faktorielle Varianzanalyse mit Messwiederholung prüft mit F-Tests drei voneinander unabhängige Nullhypothesen, die sich auf die Haupteffekte des Gruppenfaktors und des Messwiederholungsfaktors sowie auf die Interaktion der beiden Faktoren beziehen. Es wird erwartet, dass die beiden Haupteffekte statistisch signifikant werden. Bei Gültigkeit der aufgestellten statistischen Alternativhypothesen wird außerdem ein signifikanter Interaktionseffekt erwartet. Die Interaktionseffekte stehen im Zentrum des Interesses, weil nur sie über gruppenspezifische Veränderungen vom Vor- zum Nachtest informieren. Für alle inferenzstatistischen Prüfungen wurde das Signifikanzniveau auf $5 \%$ festgesetzt.

Für die Prüfung der psychologischen Hypothesen findet in Anlehnung an die Überlegungen von Hager (1992) eine separate Prüfung für alle Operationalisierungen statt. 
Mit dem gewählten univariaten Vorgehen kann so das Problem einer Kumulation der Fehlerwahrscheinlichkeiten bei mehreren abhängigen Variablen umgangen werden.

Des Weiteren wird bei der Ableitung (und Testung) von Vorhersagen ebenfalls auf Überlegungen von Hager (1995) zurückgegriffen. Hiernach kann der Nachweis eines erwartungskonformen Ausgangs der Intragruppenvergleiche als Voraussetzung für eine sinnvolle Prüfung des Vergleiches zwischen den Versuchsgruppen angesehen werden, und die Tests innerhalb der Versuchsgruppen werden von denjenigen zwischen den Versuchsgruppen getrennt bzw. gehen diesen voraus. Gelangt man aufgrund der durchgeführten Tests über die Intragruppenvergleiche $\mathrm{zu}$ der Entscheidung, dass keine erwartungskonformen Veränderungen stattgefunden haben, wird auf den Test über den Intergruppenvergleich verzichtet. Die Voraussetzungen sind jedoch dann nicht mehr Bestandteil der zu prüfenden psychologischen Hypothese, und dieser wird dann jeweils nur eine direkt testbare statistische Hypothese zugeordnet, wodurch keine Kumulierung der Fehlerwahrscheinlichkeiten auftritt.

Hager (2000, S. 209) geht auf die Forderung nach multivariaten Analysen ein, wenn verschiedene Kriterienmaße eingesetzt werden. Er sagt hierzu: „Die Verwendung mehrerer Kriteriumsmaße muss also nicht notwendigerweise dazu führen, multivariate Analysen einzusetzen“. Er begründet dies damit, dass jeder einzelne Test valide durchführbar ist (unabhängig von der Variableninterkorrelation), wenn die ihm zugrunde liegenden Daten voneinander unabhängig sind. Dies trifft für diese Untersuchung sowohl für die einzelnen Variablen als auch für die verschiedenen Trainings zu. Es wird jeweils eine isolierte Evaluation der Trainings und später eine vergleichende Evaluation durchgeführt.

Da für die Intragruppenvergleiche nur jeweils zwei Tests zur Prüfung verwendet werden, wäre eine möglicherweise auftretende Kumulation der Fehlerwahrscheinlichkeiten nur sehr gering, so dass sie an dieser Stelle vernachlässigt wird.

Damit die Ergebnisse für den Leser zu den verschiedenen Studien 1-4 der isolierten Evaluation für den Leser besser nachvollziehbar und vergleichbar sind, folgt die Darstellung jeweils folgendem Ablauf:

a) In einer Tabelle werden für die Wartegruppe und die Interventionsgruppe die Prä- und Posttestwerte der Abhängigen Variablen dargestellt. Durch Fettdruck und hellblauen Hintergrund der Zelle werden Wertepaare hervorgehoben, bei denen der VortestNachtest-Unterschied innerhalb der Versuchsgruppen zu signifikanten Ergebnissen $(\mathrm{p}<.05)$ führt. Falls ein signifikanter Interaktionseffekt auftritt, der hypothesenkonträr ist, wird dies mit einem orangefarbenen Hintergrund der Zelle dargestellt. In den 
rechten Spalten wird dargestellt, ob sich ein signifikanter Interaktionseffekt ergeben hat. Angegeben ist der F-Wert, der p-Wert, die mittlere Fehlerquadratsumme und die Entscheidung, ob sich die Psychologische Hypothese bewährt hat oder nicht. Hypothesenkonforme und signifikante Ergebnisse sind grün unterlegt. Hypothesenkonträre und gleichzeitig signifikante Unterschiede zugunsten der Wartegruppe sind rot unterlegt. Die Tabellen finden sich im Anhang A (Tabellen A-1 bis A-8).

b) Es werden dann Vortestunterschiede und Störfaktoren dargestellt. Die Vortestunterschiede geben darüber Auskunft, ob sich die Gruppen bei den Prätests in bestimmten Merkmalen signifikant voneinander unterscheiden. Diesem Faktor kommt eine besondere Bedeutung $\mathrm{zu}$, weil keine echte Randomisierung erfolgte. Störfaktoren können z.B. das Alter oder der Einfluss von Medikamenten sein. Dies wird ebenfalls geprüft.

c) Im nächsten Schritt wird die Veränderung innerhalb der Versuchsgruppen beschrieben.

d) Es folgt die Darstellung der Veränderung zwischen den Versuchsgruppen für die Variablen, für die sich bedeutsame Vortest-Nachtest-Unterschiede innerhalb der jeweiligen Trainingsgruppe gezeigt haben.

e) Anschließend werden die Effektgrößen für die signifikanten Interaktionseffekte beschrieben,

f) die Beschreibung von Moderatoreffekten (Alter, Intelligenz, Medikation) bildet den Abschluss der Ergebnisdarstellung.

(Die Ergebnisse der Haupteffekte Gruppe und Messzeitpunkt werden im Anhang B dargestellt)

Da die Stichprobe zu Studie 4 eine andere ist als jene, aus der die übrigen Trainingskinder rekrutiert wurden, soll der Vergleich des Marburger Konzentrationstrainings mit dem Training nach Lauth und Schlottke und dem THOP als Gruppenprogramm explorativ erfolgen.

\subsection{Ergebnisse der Studie 1}

\subsubsection{Isolierte Evaluation des Basistrainings nach Lauth- und Schlottke}

Das Hauptaugenmerk bei der Auswertung der Daten liegt auf der Überprüfung von Gruppenunterschieden. Verbessern sich die Kinder der Lauth- und Schlottke-Gruppe im 
Mittel signifikant stärker als die Kinder der Wartegruppe, spricht dies für die Wirksamkeit des Gruppentrainings. Grundlage für diese, wie auch für alle anderen Berechnungen stellen im Wesentlichen die gruppenspezifischen Mittelwerte und Standardabweichungen der erhobenen Variablen dar, die in Tabelle A-1 (Anhang A) im Überblick zusammengefasst sind. In der Tabelle sind alle AVn jeweils mit den Vor- und Nachtestwerten für die Gruppen dargestellt. Der Interaktionseffekt gibt Auskunft darüber, ob sich die Gruppenunterschiede vom Vor- zum Nachtest im Vergleich zueinander signifikant unterscheiden.

\subsubsection{Vortestunterschiede und Störfaktoren}

Auf statistischem Wege wird zunächst überprüft, ob die Unterschiede in den Mittelwerten der Vortestwerte zwischen Warte- und Therapiegruppe nach Lauth und Schlottke so groß sind, dass man von signifikant verschiedenen Ausgangsniveaus ausgehen muss. Durch einen zweiseitigen t-Test für unabhängige Stichproben ergab sich, dass die Kinder in den beiden Gruppen trotz der fehlenden Randomisierung bei den meisten Variablen kaum abweichende Vortestwerte aufwiesen. Signifikante Unterschiede ergaben sich in Bezug auf die Impulsivitätswerte im Lehrerurteil des FBB-HKS $\left[t_{\text {Anzahl der Kriterien Impulsivität }}(23)=2.70\right.$, $\left.p=.01) ; t_{\text {Kennwert Impulsivität }}(23)=2,44, p=.02\right]$. Das bedeutet, dass die Kinder der Wartegruppe im Mittel größere Impulsivitätswerte im Lehrerurteil aufwiesen. Die übrigen Variablen unterschieden sich nicht signifikant voneinander. Das bedeutet, dass insgesamt nur geringfügige Unterschiede in den Ausgangsdaten der Kinder vorliegen.

Für die durchgeführten Studien wurden neben den bereits genannten Kriteriumsmaßen weitere Daten über die Kinder aufgenommen, die im Rahmen der Vortestung erfasst wurden. Hierzu gehörten das Alter, das Intelligenzniveau, der soziale Status der Eltern, eine bereits vorhandene Medikamenteneinnahme sowie die Art der klinischen Diagnose. Anhand dieser Merkmale wurden deskriptive Stichprobenkennwerte gewonnen. Diese Maße können außerdem dazu dienen, ihren Einfluss als potentielle Störfaktoren zu überprüfen. Aufgrund der fehlenden Randomisierung ist damit zu rechnen, dass sich bestimmte Merkmale der Kinder ungleich auf die beiden Gruppen verteilt haben und somit als Störfaktoren mit dem Treatment (Training vs. Warten) konfundiert sind. Aus der Tatsache einer Variablenkonfundierung folgt jedoch, dass die interne Validität der Untersuchung eingeschränkt ist und keine hinreichend eindeutigen Aussagen darüber zulässt, ob die gemessenen Effekte auch tatsächlich auf die Variation des gewählten Treatments zurückzuführen sind. 
Für die nominalskalierten Merkmale Medikation, sozialer Status, klinische Diagnose und Geschlecht wurde zur Überprüfung der Verteilung ein Chi-Quadrat-Test über relative Häufigkeiten und für die intervallskalierten Merkmale Alter und Intelligenz ein zweiseitiger Zweistichproben-t-Test für unabhängige Stichproben durchgeführt.

Bei der Überprüfung der potentiellen Störfaktoren Alter, Diagnose, Medikamenteneinnahme, sozialer Status der Eltern und Intelligenz wurde in keiner der Variablen ein systematischer Effekt nachgewiesen. Die Gruppen unterscheiden sich in diesen Variablen nicht bedeutsam voneinander.

\subsubsection{Veränderungen innerhalb der Versuchsgruppen}

Nach Hager (2000) sind Vergleiche innerhalb der Gruppen eine notwendige Voraussetzung für die Interpretierbarkeit der Vergleiche zwischen den Gruppen. Signifikante Unterschiede zwischen den Gruppen können nicht zwingend als Beleg für die Wirksamkeit eines Programms gewertet werden, da sie auch dann auftreten können, wenn Veränderungen innerhalb der Gruppen nicht vorhersagekonform ausfallen oder nicht die erwarteten Verbesserungen auftreten. Für die Veränderungen innerhalb der Gruppen wurden jeweils einseitige t-Tests für abhängige Stichproben berechnet, da erwartet wurde, dass es vom Vorzum Nachtest zu einer Verbesserung kommt. Variablen, für die diese Berechnung zu einem statistisch signifikanten Ergebnis führte, sind in der Tabelle Anhang A-1 hervorgehoben.

Es wird deutlich, dass sich in der Wartegruppe signifikante Verbesserungen auf fast allen Variablen der psychometrischen Aufmerksamkeitstests zeigen, jedoch nur bei einer Variablen der Fremdeinschätzungen (Kennwert im Bereich Impulsivität im Lehrerurteil). Im Wartezeitraum haben sich im Mittel also keine Verbesserungen in alltagsbezogenen Verhaltensweisen eingestellt. Die Verbesserungen bei den psychometrischen Verfahren stellen in diesem Fall Reifungs- und Retest-Effekte dar.

In der Lauth- und Schlottke-Gruppe (LS-Gruppe) zeigen sich bei 20 der 35 Variablen signifikante Veränderungen. Hierunter fallen ebenso wie in der Wartegruppe fast alle Kriterien der psychometrischen Verfahren. In der LS-Gruppe fällt außerdem der Nachsprechwert im Mottiertest höher aus. Hinsichtlich der Fremdbeurteilungen lassen sich mehr Veränderungen im Eltern- als im Lehrerurteil feststellen. Nach Angabe der Eltern haben sich Verbesserungen sowohl im Aufmerksamkeitsverhalten als auch in Bezug auf die motorische Unruhe und externale Verhaltensauffälligkeiten im Alltag ergeben. Im Lehrerurteil des FBB-HKS haben sich nur die Werte für den Bereich Hyperaktivität der LS- 
Gruppe deutlich verbessert. In der Wartegruppe beurteilen die Lehrer die Kinder als weniger impulsiv.

\subsubsection{Veränderungen zwischen den Versuchsgruppen}

Die Signifikanzprüfung zwischen den Gruppen erfolgte über eine zweifaktorielle Varianzanalyse mit Messwiederholung für jene Variablen, bei denen sich in der Trainingsgruppe die Kinder vom Vor- zum Nachtest signifikant verbessert haben. Für drei Variablen ließ sich ein signifikanter Interaktionseffekt nachweisen. Die trainierten Kinder machten weniger Fehler im Subtest Inkompatibilität der TAP $[F(1,27)=5.49 ; p=.02 ; M Q F$ =43.83], während die Wartegruppe gleich bleibende Werte zeigte.

Signifikant bessere Leistungen erzielten die trainierten Kinder außerdem im MottierTest bei den vier-, fünf- und sechssilbigen Wörtern $[F(1,27)=14.01 ; p<.01 ; M Q F=1.75]$ sowie in der Gesamtnachsprechleistung $[F(1,27)=11.55 ; p<.01 ; M Q F=4.42]$, die Wartegruppe hingegen verbesserte sich nicht.

Die Variablen, bei denen Vortestunterschiede identifiziert wurden, wurden nach Berechnung der Interaktionseffekte zusätzlich als Kovariate eingeführt. Dies führte in keinem Fall dazu, dass bei den o.g. Variablen die Signifikanz nicht erreicht wurde. Die Vortestunterschiede hatten also keinen maßgeblichen Einfluss auf die Ergebnisse der Kinder.

\subsubsection{Effektgrößen der Interaktionseffekte}

Ergänzend zu den Signifikanztests erfolgt eine Betrachtung der Effektgrößen. Diese stellen eine statistische Operationalisierung der Wirkungsintensität dar und haben den Vorteil, dass sie unabhängig sind von allen Wahrscheinlichkeitsangaben und vor allem gänzlich unabhängig von der betrachteten Stichprobengröße (Hager, 2000). Nach Hager (1987) kann gerade bei kleinen Stichproben eine hohe $B$-Wahrscheinlichkeit dazu führen, dass der Signifikanztest zugunsten der Nullhypothese ausfällt. Bei Untersuchungen wie der vorliegenden Studie, welche sich nur auf eine Stichprobengröße von $n=31$ bezieht, kann das Vorliegen eines großen Effektes zumindest ein Hinweis für die Wirksamkeit einzelner Bereiche des Trainings sein. In Anlehnung an Cohen (1988) wird ein Effekt von $d=0.20$ als klein, von $\mathrm{d}=0.50$ als mittel und von $\mathrm{d}=0.80$ als groß betrachtet. 
Die Berechnung der Effektgrößen erfolgte auf der Grundlage der Ausführungen von Ray und Shadish (1996) mit Methode 11 („Between-groups $t$-test on change scores“; Anhang S. 1325). Die Ergebnisse wurden in der Tabelle A-1 aufgenommen.

Die Effektgrößen fallen bei den Subtests der TAP überwiegend klein oder mittel aus, insbesondere beim FBB-HKS der Elterneinschätzung fallen mehrere Effekte mittelgroß aus. Die Kinder werden als weniger hyperaktiv und impulsiv erlebt. In der Einschätzung durch die Lehrer treten keine Effekte auf.

Im Mottiertest (Gesamtleistung) wird ein Effekt groß. Er fällt zugunsten der trainierten Kinder aus. Ein mittlerer Effekt zugunsten der trainierten Kinder wird auch bei den vier- bis sechssilbigen Wörtern aufgedeckt.

Das signifikante Ergebnis im Subtest Inkompatibilität (Fehler) deckt einen mittleren Effekt zugunsten der trainierten Kinder auf.

\subsubsection{Moderatoreffekte (Alter, Intelligenz, Medikation)}

Es wurden ergänzend zu den Tests zur Überprüfung der Interaktionseffekte weitere Variablen untersucht, welche ggf. Moderatorfunktionen beim Basistraining nach Lauth und Schlottke haben könnten. Durch Mediansplit für die Variablen Intelligenz und Alter wurden die Kinder, welche das Basistraining durchlaufen haben, in zwei Gruppen geteilt. Die gefundenen Effekte müssen jedoch mit Vorsicht interpretiert werden, da die durch Mediansplit gebildeten Gruppen relativ klein sind. Für das Alter konnte in keiner der Variablen ein signifikanter Effekt aufgedeckt werden.

Die Höhe der Intelligenz scheint nur eine geringe Rolle zu spielen, die Befunde sind nicht eindeutig $\mathrm{zu}$ interpretieren. In einer Variablen der TAP erzielen die weniger intelligenten Kinder bessere Ergebnisse $\left[t_{\text {Fehler Inkompatibilität }}(4,501)=3.61 ; p=0.02 ; \mathrm{n}_{1}=5, \mathrm{n}_{2}=\right.$ $5]$.

Die intelligenteren Kinder werden von den Eltern als weniger impulsiv eingeschätzt $\left[t_{\text {Summenwert Impulsivität FBB-HKS Eltern }}(7)=-4.60 ; p<0.01 ; \mathrm{n}_{1}=5, \mathrm{n}_{2}=4\right]$.

Die Kontrolle, ob eine medikamentöse Behandlung die Ergebnisse des Trainings beeinflusst hat, entfiel hier, da nur ein Kind mit Ritalin behandelt wurde.

Moderatorvariablen konnten für diese Studie nicht eindeutig identifiziert werden. 


\subsection{Ergebnisse der Studie 2 (Isolierte Evaluation)}

\subsubsection{Isolierte Evaluation des Lauth- und Schlottke-Trainings (Basis- und Strategietraining; 15 Sitzungen)}

In Tabelle A-2 werden die wesentlichen Daten in einer Übersicht dargestellt. Es gibt in dieser Studie insgesamt 34 Kinder in der Trainingsbedingung, zu denen Daten vorliegen. Die Kinder wurden mit dem Basistraining (10 Sitzungen) sowie dem Strategietraining (5 Sitzungen) behandelt. Die Wartegruppe entspricht der aus Studie 1.

\subsubsection{Vortestunterschiede der LS-Gruppe Basis- und Strategietraining (15 Sitzungen) vs. Wartegruppe}

Beim Vergleich der Prätestwerte der Wartegruppe mit der Gesamtgruppe gibt es in mehreren Variablen Unterschiede in den Eingangsvoraussetzungen der Gruppen. Die trainierten Kinder sind etwas intelligenter $\left[t_{\mathrm{IQ}}(48)=-2.02, p=.04\right]$.

Bei der TAP gab es bei drei AVn Vortestunterschiede: Signifikante Unterschiede ergaben sich in Bezug auf die Auslassungen im Subtest Go/Nogo, den Fehlern bei kritischen Reizen sowie den Auslassungen bei nicht-kritischen Reizen im Visuellen Scanning $\left[t_{\text {Auslassungen Go/Nogo }}(17,29)=2.25, p=.03\right) ; t_{\text {Fehler krit. Reize VS }}(42)=-2.52, p=.01 ; t_{\text {Auslassungen nicht- }}$ krit. Reize VS $(18.39)=3.62, p=.02]$. Beim Go/Nogo und beim Visuellen Scanning machen die trainierten Kinder weniger Fehler.

Beim Konzentrations- und Belastungstest d2 unterscheiden sich die Prätestwerte deutlich voneinander, wiederum zugunsten der trainierten Kinder $\left[t_{\text {Konzentrationsleistungswert d2 }}(34)\right.$ $\left.=-2.28, p=.03) ; t_{\text {Fehler d2}}(12.85)=2.39, p=.03\right]$.

In den Fremdbeurteilungsbögen von Eltern und Lehrer werden die Wartegruppenkinder als etwas auffälliger beurteilt: $\left[\left(t_{\mathrm{FBB}} \mathrm{HKS}\right.\right.$ Rohwert Hyperaktivität Eltern $(43)=2.14$, $\left.p=.03) ; t_{\mathrm{FBB}-\mathrm{HKS} \text { Kennwert Hyperaktivität Eltern }}(43)=2.14, p=.03\right) ; t_{\mathrm{FBB}-\mathrm{HKS} \text { Kennwert Hyperaktivität Lehrer }}(41)$ $\left.=2.28, p=.02) ; t_{\mathrm{FBB}-\mathrm{HKS} \text { Rohwert Impulsivität Lehrer }}(41)=3.50, p<.01\right) ; t_{\mathrm{FBB}-\mathrm{HKS} \text { Kennwert Impulsivität }}$ Lehrer $(41)=3.95, p<.01)]$.

Die übrigen Variablen unterschieden sich nicht signifikant voneinander.

Über die Gründe für die Vortestunterschiede kann hier nur spekuliert werden. Die Unterschiede sind vermutlich auf die nicht randomisierte Zuteilung $\mathrm{zu}$ den Gruppen zurückzuführen. Die trainierten Kinder sind insgesamt weniger stark durch die hyperkinetische Symptomatik beeinträchtig. 


\subsubsection{Veränderungen innerhalb der Versuchsgruppen}

Die Veränderungen der Wartegruppe wurden bereits oben beschrieben (s. S. 116). In der Lauth- und Schlottke-Gruppe zeigen sich bei 20 der 35 Variablen signifikante Veränderungen. Hierunter fallen ebenso wie in der Wartegruppe fast alle Kriterien der psychometrischen Verfahren. In der LS-Gruppe fällt außerdem der Nachsprechwert im Mottiertest höher aus. Hinsichtlich der Fremdbeurteilungen lassen sich mehr Veränderungen im Eltern- als im Lehrerurteil feststellen. Nach Angabe der Eltern haben sich Verbesserungen sowohl im Aufmerksamkeitsverhalten (CBCL) als auch hinsichtlich externaler Verhaltensauffälligkeiten und der Gesamtbelastung ergeben. Im Lehrerurteil des FBB-HKS haben sich die Werte für den Bereich Hyperaktivität und Impulsivität der LS-Gruppe deutlich verbessert. In der Wartegruppe beurteilten die Lehrer die Kinder als weniger impulsiv.

\subsubsection{Veränderungen zwischen den Versuchsgruppen}

Die Signifikanzprüfung zwischen den Gruppen erfolgte wiederum über eine zweifaktorielle Varianzanalyse mit Messwiederholung. Für vier Variablen ließ sich ein signifikanter Interaktionseffekt nachweisen. Die trainierten Kinder machten weniger Fehler im Subtest Visuelles Scanning (kritische Trials) der TAP $[F(1,36)=6.85 ; p<.01 ; M Q F=$ 8.43], die Wartekinder verbesserten sich nur geringfügig. Die Nachsprechleistung beim Mottier-Test ist sowohl bei den zwei- und dreisilbigen, bei den vier-, fünf- und sechssilbigen Kunstwörtern als auch in der Gesamtleistung bei den trainierten Kindern im Vergleich zu jenen der Wartegruppe signifikant besser $[F(1,45)=5.14 ; p<.01 ; M Q F=4.99 ; F(1,45)=$ $5.14 ; p<.01 ; M Q F=1.44 ; F(1,45)=9.16 ; p<.01 ; M Q F=3.15]$.

Die Variablen, bei denen Vortestunterschiede identifiziert wurden, wurden nach Berechnung der Interaktionseffekte zusätzlich als Kovariate eingeführt. Dies führte lediglich in zwei von dreizehn Fällen (Kovariate: Auslassungsfehler beim Visuellen Scanning der TAP und Konzentrationsleistungswert d2) bei zwei- und dreisilbigen Wörtern des Mottiertests dazu, dass bei den o.g. Variablenkein signifikantes Ergebnis erzielt wurde. Die Vortestunterschiede sind nicht statistisch bedeutsam.

\subsubsection{Effektgrößen der Interaktionseffekte}

Die Interaktionseffekte wurden insbesondere beim Mottiertest zugunsten der trainierten Kinder signifikant. Die Effekte fallen für die zwei- und dreisilbigen Wörter groß, für die vier- bis sechssilbigen Wörter klein und für die Gesamtleistung mittelmäßig aus. Ein 
großer Effekt wird in der Fremdbeurteilung durch die Lehrer (Summenwert Unaufmerksamkeit) aufgedeckt. Er ist jedoch nicht statistisch bedeutsam.

\subsubsection{Moderatoreffekte (Alter, Intelligenz, Medikation)}

Bei der Überprüfung der Moderatorvariablen konnten einige Faktoren identifiziert werden, die für einige Abhängige Variablen Moderatorfunktionen besitzen. Durch Mediansplit für die Variablen Intelligenz und Alter wurden die Kinder, welche das kombinierte Basis- und Strategietraining durchlaufen haben, in zwei Gruppen geteilt. Kontrolliert wurde außerdem der Einfluss, den eine medikamentöse Behandlung auf die Ergebnisse des Trainings hatte.

Die weniger intelligenten Kindern machten im Subtest Visuelles Scanning (kritische Reize) weniger Fehler $\left[t(21)=2.36, p=0.02 ; \mathrm{n}_{1}=14, \mathrm{n}_{2}=13\right]$. Im Konzentrations- und Belastungstest $\mathrm{d} 2$ machten die jüngeren Kinder signifikant weniger Fehler als die älteren Kinder $\left[t(20)=2.60 ; p=0.01 ; \mathrm{n}_{1}=8, \mathrm{n}_{2}=14\right]$. Ein weiterer Moderatoreffekt liegt in der Variable Medikation. Die gleichzeitig mit Ritalin behandelten Kinder werden von den Lehrern als weniger hyperaktiv beurteilt $\left[t_{\text {Hyperaktivität Summenwert }}(24)=-2.22 ; p=0.03\right.$; $\left.t_{\text {Hyperaktivität Kennwert }}(24)=-2.53 ; p=0.02 ; \mathrm{n}_{1}=7, \mathrm{n}_{2}=19\right]$.

\subsection{Ergebnisse der Studie 3 (Isolierte Evaluation)}

\subsubsection{Isolierte Evaluation des THOP}

Anhand Tabelle A-3 werden im Vergleich zur Wartegruppe die wesentlichen Daten der THOP-Gruppe in einer Übersicht dargestellt.

\subsubsection{Vortestunterschiede und Störfaktoren}

Zwischen der Wartegruppe und den mit dem THOP behandelten Kindern gab es in den Prätests lediglich beim Visuellen Scanning in einigen Parametern Unterschiede: $\left[t_{\text {Standardabweichung krit. Reize VS }}(45)=3.40, p<.01 ; t_{\text {Standardabweichung nicht-krit. Reize VS }}(45)=2.11, p=\right.$ $\left.0.04 ; t_{\text {Fehler krit. Reize VS }}(45)=-2.43, p=.02\right]$.

Für die nominalskalierten Merkmale Medikation, sozialer Status, klinische Diagnose und Geschlecht wurde zur Überprüfung der Verteilung ein Chi-Quadrat-Test über relative Häufigkeiten und für die intervallskalierten Merkmale Alter und Intelligenz ein zweiseitiger Zweistichproben-t-Test für unabhängige Stichproben durchgeführt. Signifikante Effekte ergaben sich für die Merkmale Medikation $\left[\chi^{2}(1, \mathrm{~N}=47)_{\text {Medikation }}=8.34, p<.01\right]$ und Intelligenz $[t(45)=-2.39, p=.02]$. Wie auch in Tabelle 8 ersichtlich, liegen die 
Intelligenzleistungen der THOP-Gruppenkinder im Mittel etwa acht IQ-Punkte über denen der Wartegruppe, und es gibt hier anteilig deutlich mehr Kinder, die während des Untersuchungszeitraums auch medikamentös behandelt wurden. Für diese beiden Variablen gilt also, dass im Falle eines substanziellen Zusammenhanges mit den abhängigen Variablen potentiell gemessene Unterschiede zwischen Warte- und Kontrollgruppe durch diese Merkmale erklärt werden können, die damit nicht dem Training anzurechnen wären, bzw. dass sie zu Wirkungen führen, die denen des Trainings entgegenstehen und damit möglicherweise tatsächlich vorhandene Wirkungen des THOP verdecken. Um die Aussagekraft der Daten zu erhöhen, sollten also die Merkmale Medikation und Intelligenz nachträglich kontrolliert werden. Eine Möglichkeit besteht darin, diese Kontrollvariablen als weitere gruppierende Faktoren in das Versuchsdesign aufzunehmen. Hierfür hätten sowohl die Warte- als auch die THOP-Gruppe hinsichtlich der beiden interessierenden Variablen in zwei Untergruppen eingeteilt werden müssen, wobei gleichzeitig auf eine Homogenität hinsichtlich weiterer Störfaktoren zu achten gewesen wäre. Da eine weitere Untergruppierung der Stichproben jedoch zu sehr kleinen Teilgruppen (insbesondere bei der Wartegruppe) geführt hätte, wurde diese Analyse nicht durchgeführt. Es wurden stattdessen ebenso wie bei den Lauth- und Schlottke-Gruppen diese Variablen kontrolliert, indem sie als Kovariaten eingeführt und berechnet wurden.

Der Einfluss der Variablen Medikation und Intelligenz auf die Interaktionseffekte der AVn wird bei der Berechnung von Moderatorvariablen geprüft und dargestellt (vgl. Kap. 5.3.6).

\subsubsection{Veränderungen innerhalb der Versuchsgruppen}

Die Veränderungen der Wartegruppe wurden bereits oben beschrieben (s. S. 116). In der THOP-Gruppe zeigen sich bei 27 der 35 Variablen signifikante Veränderungen. Hierunter fallen ebenso wie in der Wartegruppe fast alle Kriterien der psychometrischen Verfahren. In der THOP-Gruppe fällt außerdem der Nachsprechwert im Mottiertest höher aus. Hinsichtlich der Fremdbeurteilungen lassen sich mehr Veränderungen im Eltern- als im Lehrerurteil feststellen. Nach Angabe der Eltern haben sich Verbesserungen in den Bereichen Aufmerksamkeit, Hyperaktivität und Impulsivität ergeben. Sowohl im Aufmerksamkeitsverhalten (CBCL) als auch hinsichtlich externaler Verhaltensauffälligkeiten und der Gesamtbelastung wurde ein Rückgang der Verhaltensauffälligkeiten konstatiert. Im Lehrerurteil des FBB-HKS und TRF haben sich die Werte für den Bereich Aufmerksamkeit 
und der Gesamtbelastung verbessert. In der Wartegruppe beurteilen die Lehrer die Kinder als weniger impulsiv.

\subsubsection{Veränderungen zwischen den Versuchsgruppen}

Die Signifikanzprüfung zwischen den Gruppen erfolgte wiederum über eine zweifaktorielle Varianzanalyse mit Messwiederholung. Für drei Variablen ließ sich ein signifikanter Interaktionseffekt nachweisen, bei zwei Variablen wird die Signifikanz knapp verfehlt. In einer Variable verbessern sich die Wartegruppenkinder stärker als die THOPKinder.

Die trainierten Kinder machten weniger Fehler im Subtest Visuelles Scanning (nichtkritische Trials) der TAP $[F(1,44)=4.38 ; p<.01 ; M Q F=13.83]$. Beim Subtest Visuelles Scanning (kritische Trials) wird bzgl. der Auslassungsfehler die Signifikanz knapp verfehlt $[F(1,44)=3.49 ; p=.06 ; M Q F=8.96]$. Dieses Resultat wird jedoch nicht mehr signifikant, wenn bestimmte Parameter als Kovariaten eingegeben werden. Der Fehlertyp hängt direkt mit dem Fehlertyp beim Visuellen Scanning (kritische Trials) zusammen. Wenn sich die Kinder besonders darum bemüht haben bei den kritischen Trials wenig Fehler zu machen, geht dies offensichtlich zu Lasten der Fehler bei nicht-kritischen Trials $[F(1,43)=1.04 ; p=.31 ; M Q F$ $=12.44]$. Wenn die Kovariaten Alter, Intelligenz und Intelligenz eingegeben werden, wird die Signifikanz jeweils knapp verfehlt.

Die Nachsprechleistung beim Mottier-Test ist bei den zwei- und dreisilbigen Kunstwörtern signifikant besser $[F(1,43)=6.14 ; p<.01 ; M Q F=1.59]$. Dieser Effekt verfehlt die Signifikanz, wenn die Medikation als Kovariate kontrolliert wird $[F(1,44)=3.38 ; p=.07$; $M Q F=1.60]$. Bei der Gesamtleistung wird die Signifikanz knapp verfehlt $[F(1,43)=3.31 ; p$ $=.07 ; M Q F=4.70]$. Die Signifikanz wird noch deutlicher verfehlt, wenn das Alter, die Intelligenz und die Medikation als Kovariate kontrolliert werden.

Die THOP-Kinder verbesserten im Konzentrationstest d2 ihren Konzentrationsleistungswert signifikant stärker als die Wartegruppenkinder $[F(1,26)=5.04 ; p$ $<.01 ; M Q F=75.01]$. Bei Kontrolle von Intelligenz sowie der Medikation als Kovariaten wird die Signifikanz verfehlt $\left[F_{\mathrm{d} 2} \mathrm{KL}(\mathrm{IQ})(1,25)=1.62 ; p=.21 ; M Q F=67.07\right] ;\left[F_{d 2 K L}\right.$ (Medikation) $(1,25)=4.38 ; p=.07 ; M Q F=77.78]$.

Die Wartegruppenkinder reagierten regelmäßiger beim Subtest Visuelles Scanning (Standardabweichung; kritische Trials) der TAP $[F(1,44)=8.33 ; p<.01 ; M Q F=790434]$. Dieses erwartungskonträre Ergebnis ist vermutlich auf die Vortestunterschiede zwischen den Gruppen zurückzuführen. Die Schwankungen der Antworten waren bei den Kontrollkindern 
beim Prätest viel stärker als bei den Trainingskindern. Wenn der Wert für die Schwankungsbreite bei den nicht-kritischen Trials als Kovariate eingegeben wird, verschwindet der signifikante Effekt $[F(1,43)=1.82 ; p=.18 ; M Q F=926257]$.

\subsubsection{Effektgrößen der Interaktionseffekte}

Die Effekte fallen in der Fremdeinschätzung durch die Eltern fast durchweg klein aus. Alle Interaktionen fallen zugunsten der THOP-Gruppe aus, wurden jedoch ausnahmslos nicht signifikant. Ein großer Effekt fällt zugunsten der THOP-Kinder bei den zwei- und dreisilbigen Wörtern des Mottiertests aus, der auch signifikant war. Der signifikante Test bei den Auslassungsfehlern (nicht-kritische Trials) des Visuellen Scannings der TAP führt zu einem mittelgroßen Effekt zugunsten der THOP-Gruppe. Der ebenfalls signifikante Test bei der Standardabweichung des Visuellen Scannings (kritische Reize) fällt mit einem großen Effekt zugunsten der Wartekinder aus.

\subsubsection{Moderatoreffekte (Alter, Intelligenz, Medikation)}

Die statistischen Analysen konnten nur geringe Belege für die Wirksamkeit des THOP-Gruppentrainings erbringen. Die Veränderungen innerhalb der THOP-Gruppe zeigen jedoch an, dass sich die Kinder in den meisten Variablen deutlich verbessert hatten. Interessant erscheint es nun zu untersuchen, ob das Training bei bestimmten Kindern besser gewirkt hat. Auch für das THOP werden Variablen gesucht, die Moderatorfunktionen besitzen. Dies wurde für die Variablen Alter, Intelligenz und Medikation untersucht, indem die Kinder der THOP-Gruppe jeweils anhand des Medians in zwei Untergruppen eingeteilt wurden. Durch die Variable Medikation wurde die Gruppe in Kinder mit und ohne begleitende medikamentöse Behandlung eingeteilt. Da keine spezifischen Vorhersagen geprüft werden sollten, wurden zweiseitige t-Tests für unabhängige Stichproben über die Veränderungswerte (Differenz aus Vortestwert minus Nachtestwert) berechnet.

Zwischen den Kindern mit höherem und niedrigerem IQ ergab sich nur ein signifikanter Gruppenunterschied. Nach den Lehrereinschätzungen zeigten die Kinder mit höherem IQ nach dem Training signifikant weniger externalisierendes Verhalten als die Kinder mit niedrigerem IQ $\left[t(18)=-2.90, p<.01 ; \mathrm{n}_{1}=11, \mathrm{n}_{2}=9\right]$.

Bezüglich des Alters gab es ebenfalls nur einen signifikanten Unterschied. Im Aufmerksamkeitsverhalten - gemessen an der Anzahl erfüllter Kriterien im Elternurteil verbesserten sich die älteren Kinder stärker $\left[t(27)=-2.05, p=.05 ; \mathrm{n}_{1}=15, \mathrm{n}_{2}=14\right]$. 
Beim Vergleich von Kindern mit und ohne Medikation ergaben sich mehrere signifikante Effekte. Die Kinder, die während des Trainings auch medikamentös behandelt wurden, zeigten sowohl nach Auskunft der Eltern $\left[t(28)=-2.32, p=.02 ; \mathrm{n}_{1}=15, \mathrm{n}_{2}=15\right]$ als auch der Lehrer $\left[t(20)=-2.59, p=.02 ; \mathrm{n}_{1}=10, \mathrm{n}_{2}=12\right]$ deutlichere Verminderungen in externalisierenden Verhaltensweisen. In der Familie traten nach dem Training bei diesen Kindern auch weniger hyperaktive Verhaltensweisen - gemessen an der Anzahl erfüllter Kriterien im FBB-HKS - auf $\left[t(28)=-2.34, p=.02 ; \mathrm{n}_{1}=15, \mathrm{n}_{2}=15\right]$, während in der Schule größere Verminderungen in der Anzahl impulsiver Reaktionen $\left[t(21)=-3.61, p<.01 ; \mathrm{n}_{1}=11\right.$, $\left.\mathrm{n}_{2}=12\right]$ sowie in dessen Intensität $\left[t(21)=-3.12, \mathrm{p}<.01 ; \mathrm{n}_{1}=11, \mathrm{n}_{2}=12\right]$ berichtet wurden. Die Gesamtbelastung ist nach Einschätzung der Eltern im CBCL 4-18 bei den mit Ritalin behandelten Kindern deutlicher zurückgegangen $\left[t(28)=-2.06 ; p=0.05 ; \mathrm{n}_{1}=15, \mathrm{n}_{2}=15\right]$.

Insgesamt lassen sich anhand dieser Analyse nur wenige Hinweise auf alters- und intelligenzbedingte Effekte finden. Mit der Fraktionierung der Gruppen am Median erfolgte jedoch bei den ersten beiden Variablen eine relativ willkürliche Einteilung, so dass die Ergebnisse nicht eindeutig interpretierbar sind. Die Ergebnisse zur moderierenden Funktion der medikamentösen Behandlung sind dagegen aussagekräftiger und weisen darauf hin, dass offenbar die Kinder mit simultaner medikamentöser Behandlung mehr vom Training profitiert haben.

\subsubsection{Veränderungen der Eltern-Kind-Interaktion}

Während der mehrjährigen Datenerhebung wurde deutlich, dass sich einerseits nur geringfügige Veränderungen auf den Kriteriumsmaßen abzeichneten, andererseits in den Elterngesprächen sehr positive Rückmeldungen gegeben wurden. Der Verfasser konzipierte daraufhin einen eigenen Fragebogen, der weitere Wirkungen des THOP erfassen sollte. Dieser Interaktionsfragebogen besteht aus 27 Fragen, die von den Eltern anhand von Ratingskalen beantworten werden sollen, wobei jeweils drei Fragen zu einem Thema zusammengehören (s. Anhang E.1). Erfragt wird die allgemeine Belastung von Kindern und Eltern, Aspekte der Eltern-Kind-Interaktion sowie Wissen und Einsatz von Fertigkeiten zum Umgang mit Verhaltensauffälligkeiten, wie sie jeweils (rückblickend) vor und nach dem durchgeführten Training erlebt wurden. Als Beispiel sei Item 1 genannt: 1a: „Wie stark sahen Sie Ihr Kind vor der Behandlung durch die Verhaltensprobleme belastet?“, 1b: „Wie stark sehen Sie Ihr Kind derzeit durch die Verhaltensprobleme belastet?“, 1c: „Wie stark hat sich die Belastung Ihres Kindes durch die Verhaltensprobleme im Vergleich zur Zeit vor der Behandlung verändert?“. 
Die Beantwortung der ersten beiden Fragen erfolgt jeweils auf einer zehnstufigen Skala von 0 bis 100, wobei höhere Werte für Verbesserungen stehen (Bezeichnung je nach Frage etwas unterschiedlich, z.B. 0: ,gelingt gar nicht“" und 100: „gelingt sehr gut“). Das dritte Item je Thema erfragt direkt das Ausmaß der subjektiv wahrgenommenen Veränderungen und wird anhand einer fünfstufigen Skala beurteilt (Bezeichnung je nach Frage etwas unterschiedlich, z.B. „gelingt viel besser“ - „gelingt besser“ - „gleich“ - „gelingt schlechter" - ,gelingt viel schlechter“). Den einzelnen Stufen dieser Skala wurden Werte so zugeordnet, dass sich Verbesserungen in höheren Werten ausdrücken (vgl. Anhang E.2). In Tabelle 12 sind Mittelwerte und Standardabweichungen für die jeweils ersten beiden Fragen je Thema aufgeführt.

Tabelle 12

Mittelwerte und Standardabweichungen der Items des Interaktionsfragebogens

\begin{tabular}{|c|c|c|c|c|}
\hline & & & $\begin{array}{r}\text {...vor der } \\
\text { Behandlung... }\end{array}$ & $\begin{array}{r}\text {...nach der } \\
\text { Behandlung... }\end{array}$ \\
\hline \multicolumn{5}{|c|}{ Fragen je Thema } \\
\hline & \multirow{2}{*}{ Wie stark sahen/ sehen Sie Ihr Kind ... belastet? } & M & 66.82 & 42.27 \\
\hline & & SD & 18.87 & 23.29 \\
\hline & \multirow{2}{*}{ Wie stark sahen/ sehen Sie sich als Mutter/ Vater ... belastet? } & $\mathrm{M}$ & 76.36 & 48.64 \\
\hline & & SD & 15.60 & 19.59 \\
\hline \multirow[t]{2}{*}{3.} & \multirow{2}{*}{$\begin{array}{l}\text { Wie gut gelang/ gelingt es Ihnen ..., die Verhaltensauffälligkeiten } \\
\text { Ihres Kindes positiv zu beeinflussen? }\end{array}$} & $\mathrm{M}$ & 37.27 & 60.00 \\
\hline & & SD & 12.79 & 16.33 \\
\hline & \multirow{2}{*}{$\begin{array}{l}\text { Wenn mein Kind verhaltensauffällig war/ ist, wusste/ weiß ich ... } \\
\text { nicht, was ich tun soll. }\end{array}$} & M & 60.45 & 39.55 \\
\hline & & SD & 20.35 & 21.49 \\
\hline & \multirow{2}{*}{$\begin{array}{l}\text { Wenn mein Kind ... nicht das tat/ tut, was ich von ihm erwarte, } \\
\text { setze ich Konsequenzen ein. }\end{array}$} & $\mathrm{M}$ & 46.36 & 74.55 \\
\hline & & SD & 21.94 & 17.92 \\
\hline & \multirow{2}{*}{$\begin{array}{l}\text { Durch meine Erziehungsmaßnahmen erreichte/ erreiche ich ... } \\
\text { bei meinem Kind, was ich will. }\end{array}$} & $\mathrm{M}$ & 42.73 & 67.05 \\
\hline & & SD & 18.31 & 17.77 \\
\hline & \multirow{2}{*}{ Ich sah/ sehe ... viele positive Dinge an meinem Kind. } & $\mathrm{M}$ & 58.18 & 75.00 \\
\hline & & SD & 25.00 & 17.11 \\
\hline & \multirow{2}{*}{ Mein Kind hielt/ hält sich ... an Familienregeln. } & $\mathrm{M}$ & 40.00 & 62.73 \\
\hline & & $\mathrm{SD}$ & 18.52 & 17.23 \\
\hline & \multirow{2}{*}{$\begin{array}{l}\text { Bei Verhaltensproblemen meines Kindes wusste ich/ weiß ich ..., } \\
\text { was ich tun konnte/ kann, um sie zu bewältigen. }\end{array}$} & M & 40.91 & 70.45 \\
\hline & & SD & 15.40 & 16.18 \\
\hline
\end{tabular}

Anmerkungen. $\mathrm{M}=$ Mittelwert, $\mathrm{SD}=$ Standardabweichung; $\mathrm{N}=22$.

Da das Verfahren bisher keinerlei Reliabilitäts- und Validitätsuntersuchungen unterzogen wurde, wurde es hier als informelles Verfahren eingesetzt und nur den Eltern der THOP-Trainingsgruppe nach Beendigung des Trainings vorgelegt. Es erfasst deren subjektiv wahrgenommenen Verbesserungen in der Eltern-Kind-Interaktion und in ihrer Interaktionsbzw. Erziehungskompetenz.

Zur Schätzung der internen Konsistenz des Fragebogens wurde Cronbachs Alpha berechnet. Mit einem Wert von .80 liegt eine befriedigende interne Konsistenz vor. Die 
Korrelationen zwischen den Werten für die Situation vor dem Training und den Veränderungsitems sind bis auf zwei Ausnahmen (Items 3 und 6) signifikant und zeigen an, dass Eltern, bei denen vor dem Training größere Schwierigkeiten/Unkenntnis/Belastungen bestanden haben, die Veränderungen um so größer ausfallen.

Entsprechend der programmspezifischen Ziele des THOP konnte erwartet werden, dass sich durch das Training sowohl die alltägliche Eltern-Kind-Interaktion als auch die Interaktions- bzw. Erziehungskompetenz der Eltern verbessern würde. Für alle Fragen wurde über einen einseitigen t-Test für abhängige Stichproben geprüft, ob durch das Training eine deutliche Verbesserung in der elterlichen Wahrnehmung von der Situation vor und nach dem Training erzielt werden konnte. Alle Veränderungen erreichten das vorgegebene Signifikanzniveau. Die Eltern sahen im Mittel ihr Kind und sich selbst als weniger belastet an und gaben an, dass sie sich nun besser in der Lage fühlten mit Verhaltensproblemen ihres Kindes umgehen zu können.

Diese Ergebnisse lassen vermuten, dass das THOP zwar in der Lage ist, eine Veränderung in der Wahrnehmung der Verhaltensauffälligkeiten durch die Eltern sowie eine Verbesserung des Umgangs mit diesen Problemen $\mathrm{zu}$ erzielen, jedoch nur ein geringes Veränderungspotential im Hinblick auf die Kernsymptomatik besitzt. Dies mag daran liegen, dass die Eltern die im Training erlernten Strategien nicht adäquat oder gar nicht eingesetzt haben oder die Interventionen nicht effektiv genug waren. Letztere Annahme erscheint jedoch aufgrund der Vielzahl an Befunden zur Effektivität operanter Methoden als auch der Korrelationen zwischen den Veränderungen in den Elternurteilen in Bezug auf die Kernsymptomatik sowie weiterer Verhaltensauffälligkeiten (hier: Differenz Nachtestwert minus Vortestwert) und den Veränderungen, wie sie über die Interaktionsitems erfasst wurden (hier: Differenz Item nach der Behandlung minus Item vor der Behandlung), als eher unwahrscheinlich (vgl. Tabelle 13). 
Tabelle 13

Korrelationen der Veränderungswerte im Interaktionsfragebogen mit den Veränderungswerten der Erfolgsmaße der Elternbeurteilungen in der THOP-Gruppe

\begin{tabular}{|c|c|c|c|c|c|c|c|c|c|c|}
\hline \multirow{2}{*}{\multicolumn{2}{|c|}{ Veränderung in Bezug auf: }} & \multicolumn{9}{|c|}{ Veränderung in Bezug auf: } \\
\hline & & UES & UEK & HES & HEK & IES & IEK & $\mathrm{CA}$ & CEx & CGes \\
\hline & Belastung des Kindes ${ }^{\mathrm{a}}$ & .23 & .03 & .42 & .19 & -.21 & -.15 & $-.46^{*}$ & $-.51^{*}$ & $-.53 *$ \\
\hline 2. & Belastung der Eltern ${ }^{\mathrm{a}}$ & .18 & .06 & $.44 *$ & .25 & -.14 & -.03 & -.36 & $-.44 *$ & -.48 \\
\hline 3. & $\begin{array}{l}\text { Positive Beeinflussung von } \\
\text { Verhaltensauffälligkeiten }\end{array}$ & .12 & .25 & -.18 & -.13 & -.02 & -.08 & .31 & $.46^{*}$ & $.50^{*}$ \\
\hline 4. & $\begin{array}{l}\text { Unwissen, was zu tun war bei } \\
\text { Verhaltensauffälligkeiten }^{\mathrm{a}}\end{array}$ & $-.42 *$ & $-.49 *$ & -.07 & -.03 & -.07 & -.02 & -.14 & -.22 & -.19 \\
\hline 5. & Einsatz von Konsequenzen ${ }^{\mathrm{b}}$ & -.15 & .08 & -.26 & -.27 & .03 & -.18 & .19 & $.41^{*}$ & -.35 \\
\hline 6. & Effektivität von Erziehungsmaßnahmen ${ }^{\mathrm{b}}$ & -.02 & .14 & -.14 & -.09 & .18 & .07 & .34 & $.49 *$ & $.50 *$ \\
\hline 7. & Sehen vieler positiver Dinge am Kind ${ }^{\mathrm{b}}$ & .33 & .40 & -.13 & -.09 & .21 & .22 & .30 & .40 & $.42 *$ \\
\hline 8. & Einhalten von Familienregeln ${ }^{\mathrm{b}}$ & .05 & .21 & -.18 & -.15 & .14 & -.03 & .37 & $.47^{*}$ & $.48^{*}$ \\
\hline & $\begin{array}{l}\text { Wissen um Handlungsmöglichkeiten zur } \\
\text { Bewältigung von Verhaltensproblemen }\end{array}$ & .02 & .16 & -.26 & -.18 & .05 & -.04 & .33 & $.43^{*}$ & $.46^{*}$ \\
\hline
\end{tabular}

Anmerkungen. $\mathrm{N}=22$, Kennwert (K) im Elternurteil (E)

U: Unaufmerksamkeitsdimension im FBB-HKS (Fremdbeurteilungsbogen Hyperkinetische Störungen)

H: Hyperaktivitätsdimension im FBB-HKS

I: Impulsivitätsdimension im FBB-HKS

S: Anzahl erfüllter Kriterien

CA: Skala „Aufmerksamkeitsstörungen“ der Child Behavior Checklist (CBCL)

CEx: Skala „externalisierende Störungen“ der CBCL

SGes: „Gesamtskala“ der CBCL.

(Anwendungsbeispiel: CA: Wert der Skala Aufmerksamkeitsstörungen in der CBCL)

${ }^{a}$ Hinweis zur Interpretation der Korrelationen: Die Items sind so formuliert, dass sich Verbesserungen in einer Verringerung des Wertes ausdrücken, so dass die zugrunde liegenden Veränderungswerte negativ sind.

${ }^{\mathrm{b}}$ Hinweis zur Interpretation der Korrelationen: Die Items sind so formuliert, dass sich Verbesserungen in einer Vergrößerung des Wertes ausdrücken, so dass die zugrunde liegenden Veränderungswerte positiv sind. $* p<.05, * * p<.01$.

Wird als Kriteriumsleistung die Veränderung auf der Skala Aufmerksamkeitsstörungen der CBCL herangezogen, so sind mit stärkeren Verbesserungen in der Interaktion und der Interaktionskompetenz der Eltern bzw. mit stärkeren Verringerungen der Belastungen auch größere Verringerungen der Kernsymptomatik verbunden. Dieser Zusammenhang ist für oppositionelle und aggressive Verhaltensweisen sogar noch größer und auch für emotionale und soziale Verhaltensbeeinträchtigungen substanziell (CBCL 4-18). Dagegen liegen mit den Daten des FBB-HKS diesem Resultatsmuster z.T. widersprechende Befunde vor, die intuitiv auch nur schwer nachzuvollziehen sind (z.B. gehen größere Verringerungen der Belastung der Eltern mit geringeren Verbesserungen hinsichtlich unaufmerksamen und hyperaktiven Verhaltens einher). 


\subsection{Ergebnisse der Studie 4}

\subsubsection{Isolierte Evaluation des Marburger Konzentrationstrainings}

Anhand Tabelle A-4 werden im Vergleich zur Wartegruppe die wesentlichen Daten der Trainingsgruppe in einer Übersicht dargestellt. In der Kontrollgruppe „Petterson und Findus“ liegt nur von einem Kind ein Vor- und Nachtestwerte der Fragebögen TRF und FBBHKS (Lehrer) vor. Dies wird deshalb nicht in einer Tabelle dargestellt. Gleiches gilt für den Lehrerfragebogen in der Gruppe Marburger Konzentrationstraining.

\subsubsection{Vortestunterschiede und Störfaktoren}

Unterschiede in den Prätestwerten gibt es lediglich bzgl. der Medikation. Deutlich mehr Kinder des Marburger Konzentrationstrainings wurden medikamentös behandelt als die Kontrollgruppenkinder $\left[\left(t_{\text {Medikation }}(24.94)=2.92, p<.01\right]\right.$. Bei der Betrachtung der Ausgangsmerkmale der Trainings- und Kontrollgruppe werden Unterschiede zwischen den Gruppen bezüglich des IQs, ermittelt mit dem CFT 20, deutlich. So erzielten die Kinder der Trainingsgruppe im CFT-20 einen durchschnittlichen IQ von 105,2, die Kinder in der Kontrollgruppe erreichten eine durchschnittlichen IQ von 96,43. Um einen möglichen Einfluss des Intelligenzquotienten auf die Wirksamkeitsprüfung auszuschließen, wurde diese Variable mit einem t-Test für unabhängige Stichproben auf Signifikanz überprüft, um sie gegebenenfalls als Kovariate zu betrachten. Die Signifikanz wird jedoch knapp verfehlt $\left[t_{\mathrm{IQ}}(28)=1.93, p=.06\right]$.

\subsubsection{Veränderungen innerhalb der Versuchsgruppen}

Die Veränderungen vom Vor- zum Nachtest sind der A-4 zu entnehmen. In der Tabelle wurden die CBCL- und FBB-HKS-Werte aufgenommen. Aufgrund des niedrigen Rücklaufs ist es aber nicht sinnvoll sie statistisch auszuwerten. In der Gruppe „Petterson und Findus" traten in 10 der 31 AVn signifikante Verbesserungen vom Vor- zum Nachtest ein, in der Gruppe „Marburger Konzentrationstraining“ war dies bei 12 AVn der Fall. Die Kinder der Kontrollgruppe verbesserten sich beim CFT 20, im Zahlen-Symbol-Test des HAWIK-III, einigen Variablen des TPK (Merkleistung für Tiere und Konzentrationsleistungswert), verbesserter Konzentrationsleistungswert im d2 und geringere Leistungsschwankungen in einigen Untertests der TAP. Bemerkenswert ist eine größere Differenz der Leistungen des Teil 1 minus Teil 2 im CFT 20, was als Verschlechterung zu werten ist, da die Kinder weniger gleichmäßig antworten. 
Die Kinder des Marburger Konzentrationstrainings verbesserten sich in der Regelmäßigkeit der Beantwortung der Items beim Visuellen Scanning, beim CFT 20, im Zahlen-Symbol-Test des HAWIK-III, im Konzentrationsleistungswert und bei der Fehleranzahl im d2, sowie in vier von sechs Subtests der TPK (u.a. verbesserte Rechenleistung und mehr bearbeitete Silben).

Aufgrund des geringen Rücklaufs der Fragebögen von Eltern und Lehrer lassen sich die Fremdbeurteilungswerte nicht interpretieren.

\subsubsection{Veränderungen zwischen den Versuchsgruppen}

Die Signifikanzprüfung zwischen den Gruppen erfolgte wiederum über eine zweifaktorielle Varianzanalyse mit Messwiederholung. In einer Variable ist ein deutlicher Leistungszuwachs bei den trainierten Kindern festzustellen. Im TPK bearbeiten die mit dem Marburger Konzentrationstraining trainierten Kinder deutlich mehr Silben als die Kontrollgruppenkinder $[F(1,27)=7.17 ; p<.01 ; M Q F=212.30]$. Die Signifikanz wird allerdings verfehlt, wenn die Intelligenz als Kovariate eingegeben wird $[F(1,26)=3.44 ; p=$ $.07 ; M Q F=204.31]$.

\subsubsection{Effektgrößen der Interaktionseffekte}

Der signifikante Interaktionseffekt fällt zugunsten des Marburger Konzentrationstrainings aus. Die bearbeitete Silbenzahl im TPK ist zwar signifikant verbessert, der Effekt ist aber nur klein. Es traten überdies noch vier kleine Effekte zugunsten des Marburger Konzentrationstrainings auf, und zwar bei der Standardabweichung des Visuellen Scanning (kritische Reize), den Fehlern im d2, den bearbeiteten Zeichen im ZahlenSymbol-Test des HAWIK-III sowie bei der Rechenleistung im TPK.

\subsubsection{Moderatoreffekte (Alter, Intelligenz, Medikation)}

Bei der Beurteilung der Modeartoreffekte dieser Studie ist wieder einschränkend zu berücksichtigen, dass die Stichprobe durch den Mediansplit sehr klein wird und die Ergebnisse deshalb nur vorsichtig interpretiert werden können.

Auch hier wurde überprüft, ob sich die Messwerte vor und nach dem Training für die gesplitteten Gruppen hinsichtlich des Alters, der Intelligenz und einer gleichzeitigen medikamentösen Behandlung unterscheiden. Die älteren Kinder konnten nach dem Training in der TPK mehr Silben schreiben $\left[t(13)=2.36 ; p=0.03 ; \mathrm{n}_{1}=8, \mathrm{n}_{2}=7\right]$ und machten weniger Fehler $\left[t(13)=-2.95 ; p=0.01 ; \mathrm{n}_{1}=8, \mathrm{n}_{2}=7\right]$. Weniger intelligente Kinder konnten 
sich bei der TPK nach dem Training mehr Tiere merken $\left[t(13)=-3.45 ; p<0.01 ; \mathrm{n}_{1}=7, \mathrm{n}_{2}=\right.$ 8]. Bzgl. der Medikation sind die Befunde noch weniger eindeutig. In einer Variablen verbessern sich die nicht mit Medikamenten behandelten Kinder stärker (Fehler im d2: $t(13)$ $=-2.30 ; p=0.04 ; \mathrm{n}_{1}=6, \mathrm{n}_{2}=9$ ), in einer anderen Variable verbesserten sich die mit Ritalin behandelten Kinder stärker. Ihr Differenzwert vom ersten Halbtest zum zweiten Halbtest im CFT-20 verringert sich stärker $\left[t(13)=-2.28 ; p=0.04 ; \mathrm{n}_{1}=9, \mathrm{n}_{2}=6\right]$.

\subsection{Vergleichende Evaluation der Studien 1 - 4}

Die AVn der Studien 1 bis 3 sind gleich. Hier liegen sowohl Ergebnisse von psychometrischen Tests als auch Fremdbeurteilungsbögen der Eltern und Lehrer vor. In Studie 4 wurden zum Teil andere psychometrische Verfahren benutzt, insbesondere war aber der Rücklauf der Fragebögen durch Eltern und Lehrer so gering, dass eine vergleichende Evaluation für diese Variablen nicht möglich ist.

Für statistische Berechnungen ausreichende Datensätze aus allen Therapiegruppen liegen nur für die Testbatterie zur Aufmerksamkeitsprüfung und den Konzentrations- und Belastungstest d2 vor. Diese AVn werden mit der Lauth- und Schlottke-Gruppe und der THOP-Gruppe verglichen.

Die Veränderungen innerhalb der Versuchsgruppen wurden bereits für die jeweilige Therapiegruppe bei der isolierten Evaluation dargestellt. Der Leser vergleiche hierzu die Kapitel 5.1.3, 5.2.3., 5.3.3 und 5.4.3.

\subsubsection{Vergleich der Gruppen Lauth und Schlottke 10 Sitzungen mit LS 15 Sitzungen}

In Tabelle A-5 wird die Lauth und Schlottke-Gruppe 10 Sitzungen mit der LS-Gruppe 15 Sitzungen gegenüber gestellt.

\subsubsection{Vortestunterschiede und Störfaktoren LS 10 Sitzungen vs. LS 15 Sitzungen}

Beim Vergleich der Prätestwerte der Baistrainingsgruppe nach Lauth und Schlottke (LS10) mit der Gruppe, welche Basis- und Strategietraining (LS15) erhielt, gibt es in den Vortestwerten in mehreren Variablen Unterschiede in den Eingangsvoraussetzungen der Gruppen. Die Kinder der LS15-Gruppe sind 15 Monate jünger $\left[t_{\text {Alter }}(47)=2.94, p<.01\right]$. Bei der TAP gab es signifikante Vortestunterschieden in Bezug auf die Fehler bei kritischen Reizen sowie den Auslassungen bei nicht-kritischen Reizen im Visuellen Scanning $\left[t_{\text {Fehler krit }}\right.$


Visuellen Scanning machten die LS15-Kinder mehr Auslassungsfehler bei den kritischen 
Reizen, die LS10-Kinder machten mehr Fehler bei den nicht-kritischen Reizen. Beim Konzentrations- und Belastungstest $\mathrm{d} 2$ unterscheiden sich die Prätestwerte deutlich zugunsten

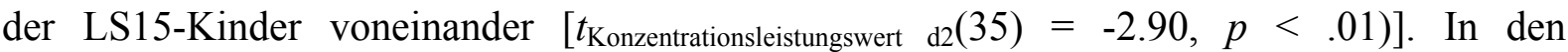
Fremdbeurteilungsbögen der Lehrer werden die LS15-Kinder als etwas auffälliger beurteilt: $\left[\left(t_{\text {FBB-HKS Rohwert Aufmerksamkeit Lehrer }}(40)=-2.74, p<.01\right) ; t_{\text {FBB-HKS Kennwert Aufmerksamkeit Lehrer }}(40)=\right.$ -2.79, $p<.01)$ ]. Die übrigen Variablen der intervallskalierten AVn unterschieden sich nicht signifikant voneinander. Für die nominalskalierten Merkmale Medikation, sozialer Status, klinische Diagnose und Geschlecht ergaben sich keine signifikanten Unterschiede.

\subsubsection{Veränderungen zwischen den Versuchsgruppen LS 10 Sitzungen vs. LS 15 Sitzungen}

Die Signifikanzprüfung zwischen den Gruppen erfolgte wiederum über eine zweifaktorielle Varianzanalyse mit Messwiederholung, sofern in wenigstens einer der Trainingsgruppen eine signifikante Verbesserung vom Vor- zum Nachtest eingetreten ist. Für sechs Variablen ließ sich ein signifikanter Interaktionseffekt nachweisen, davon allerdings hypothesenkonträr für fünf Variablen zugunsten der Gruppe, welche nur das Basistraining (LS10) absolviert hat, bei einer anderen Variablen wird das Signifikanzkriterium knapp verfehlt. In einer Variablen verbessern sich die Kinder, welche das kombinierte Training nach Lauth und Schlottke (LS15) erhalten haben.

Die LS10-Kinder machten weniger Auslassungsfehler im Subtest Go/Nogo der TAP, gleichzeitig verschlechterten sich die LS15-Kinder von der Vor- zur Nachtestung $[F(1,43)=$ 10.48; $p<.01 ; M Q F=13.07]$. Bei diesem Untertest reagierten die LS10-Kinder nach dem Training außerdem deutlich regelmäßiger $[F(1,43)=4.06 ; p=.05 ; M Q F=13471]$.

Die LS10-Kinder verbesserten im Konzentrationstest d2 ihren Konzentrationsleistungswert tendenziell stärker $[F(1,33)=3.40 ; p=.07 ; M Q F=79.04]$ als die LS15-Kinder und machten signifikant weniger Fehler $[F(1,33)=7.45 ; p=.01 ; M Q F=$ 241.16]. Wenn das Alter und der Fremdbeurteilungswert der Lehrereinschätzung für Aufmerksamkeit als Kovariate kontrolliert werden, wird die Signifikanz beim Konzentrationsleistungswert noch deutlicher verfehlt. Bezüglich der Fehlerzahl wird die Signifikanz dann verfehlt, wenn der Konzentrationsleistungswert des d2 als Kovariate eingegeben wird $[F(1,33)=2.04 ; p=.16 ; M Q F=199.29]$. Diese beiden Variablen sind bei der Testbearbeitung voneinander abhängig. Schnelles Bearbeiten führt in der Regel zu mehr Fehlern, sehr sorgfältiges Arbeiten geht zu Lasten der Tempoleistung. 
In den Fremdbeurteilungsbögen der Eltern werden die LS10-Kinder als weniger impulsiv beurteilt: $\left[\left(F_{\mathrm{FBB}-\mathrm{HKS}}\right.\right.$ Rohwert Impulsivität Eltern $\left.(39)=8.11, p<.01 ; M Q F=1.19\right) ; t_{\mathrm{FBB}-\mathrm{HKS}}$ Kennwert Impulsivität Eltern $(39)=8.84, p<.01 ; M Q F=0.31)]$.

Den einzigen hypothesenkonformen Interaktionseffekt zugunsten der LS15-Gruppe gab es bei den Auslassungsfehlern der kritischen Reize beim Visuellen Scanning der TAP $\left[F_{\text {Fehler krit Reize VS }}(35)=11.07, p<.01 ; M Q F=7.00\right]$. Dieser Effekt bleibt auch dann signifikant, wenn die Variablen als Kovariaten überprüft werden, in denen sich die Gruppen bei den Vortests unterschieden.

\subsubsection{Effektgrößen der Interaktionseffekte LS 10 Sitzungen vs. LS 15 Sitzungen}

Beim Vergleich des Basistrainings mit dem kombinierten Training (Basis- und Strategietraining) gibt es sechs signifikante Interaktionseffekte zugunsten des Basistrainings, bei zwei Variablen wird die Signifikanz knapp verfehlt. Es treten davon fünf große Effekte zugunsten des Basistrainings auf (Auslassungsfehler Go/Nogo der TAP, Fehler beim d 2 sowie in der Elterneinschätzung die Anzahl der Kriterien für Hyperaktivität, Anzahl der Kriterien für Impulsivität und dessen Kennwert). Ein kleiner Effekt zugunsten des Basistrainings fällt bei der Verbesserung des Konzentrationsleistungswertes im d2 auf, der Kennwert Hyperaktivität im Elternurteil fällt mittelgroß aus. Auch im Lehrerurteil schneiden die Kinder des Basistrainings hinsichtlich Unaufmerksamkeit und Hyperaktivität besser ab. Lediglich in einem Interaktionseffekt fällt ein großer Effekt zugunsten des kombinierten Trainings aus (Auslassungsfehler Visuelles Scanning (kritische Trials)).

\subsubsection{Vergleich des Kombinierten Basis- und Strategietrainings nach Lauth und Schlottke (15 Sitzungen) mit dem THOP}

Zunächst werden wieder die beiden Trainingsgruppen einander gegenübergestellt (Tabelle A-6).

\subsubsection{Vortestunterschiede und Störfaktoren LS 15 Sitzungen vs. THOP}

Beim Vergleich der Prätestwerte der Gesamtgruppe, welche mit dem Training nach Lauth und Schlottke behandelt wurde, und dem THOP als Gruppentraining gibt es bei einigen Variablen signifikante Gruppenunterschiede. Unterschiede in den Prätestwerten gibt es bzgl. der Medikation. Deutlich mehr Kinder der THOP-Gruppe wurden medikamentös behandelt als jene der Lauth- und Schlottke-Gruppe $\left[t_{\text {Medikation }}(57.49)=2.41, p=.02\right]$. In der TAP unterscheiden sich die Prätestwerte bei drei Variablen, den Fehlern beim Subtest Inkompatibilität, der Standardabweichung bei den kritischen Reizen des Visuellen Scannings 
und den Fehlern beim Visuellen Scanning (nicht-kritische Reize): [( $t_{\text {Standardabweichung krit. Reize }}$ $\mathrm{vS}(45)=3.40, p<.01) ;\left(t_{\text {Standardabweichung nicht-krit. Reize VS }}(57)=-2.43, p=0.02\right) ;\left(t_{\text {Fehler nicht-krit. }}\right.$ Reize VS $\left.(37.42)=3.74, p<.01) ;\left(t_{\text {Fehler Inkompatibilität }}(59)=2.02, p=.04\right)\right]$.

Bei den Fremdbeurteilungsbögen gibt es Unterschiede in der Beurteilung der Hyperaktivität und Impulsivität in der Eltern- und Lehrereinschätzung (FBB-HKS) sowie im Elternfragebogen $\mathrm{CBCL}$ für den Bereich der Externalisierenden Störungen und der Gesamtskala: $\left[\left(t_{\mathrm{FBB}-\mathrm{HKS} \text { Rohwert Hyperaktivität Eltern }}(60)=2.22, p=.03\right) ;\left(t_{\mathrm{FBB}-\mathrm{HKS} \text { Kennwert Hyperaktivität }}\right.\right.$ Eltern $(60)=2.21, p=.03) ;\left(t_{\mathrm{FBB}-\mathrm{HKS} \text { Rohwert Impulsivität Eltern }}(60)=3.07, p<.01\right) ;\left(t_{\mathrm{FBB}-\mathrm{HKS} \text { Kennwert }}\right.$ Impulsivität Eltern $(60)=3.23, p<.01) ;\left(t_{\mathrm{FBB}-\mathrm{HKS} \text { Rohwert Impulsivität Lehrer }}(54)=2.86, p<.01\right) ;\left(t_{\mathrm{FBB}-\mathrm{HKS}}\right.$ Kennwert Impulsivität Lehrer $(54)=3.28, p<.01) ;\left(t_{\mathrm{CBCL}}\right.$ Externalisierende Störungen $\left.(62)=3.23, p<.01\right)$; $\left.\left(t_{\text {CBCL Gesamtskala }}(55)=2.49, p=.01\right)\right]$.

In allen genannten Variablen schneiden die THOP-Kinder schlechter in den Prätestwerten ab als die Kinder der Lauth- und Schlottke-Gruppe, d.h. für die THOP-Kinder bestanden zu Beginn des Trainings schlechtere Grundvoraussetzungen bzw. waren sie durch die HKS-Symptomatik stärker beeinträchtigt.

\subsubsection{Veränderungen zwischen den Versuchsgruppen LS 15 Sitzungen vs. THOP}

Die Signifikanzprüfung zwischen den Gruppen erfolgte auch hier über eine zweifaktorielle Varianzanalyse mit Messwiederholung. Für drei Variablen ließ sich ein signifikanter Interaktionseffekt nachweisen, in zwei Variablen wird das Signifikanzkriterium knapp verfehlt. Nur in einer Variablen fällt der Interaktionseffekt zugunsten der Kinder aus, welche das kombinierte Training nach Lauth und Schlottke (LS15) erhalten haben.

Die THOP-Kinder machten weniger Auslassungsfehler im Subtest Go/Nogo der TAP, gleichzeitig verschlechterten sich die LS15-Kinder von der Vor- zur Nachtestung $[F(1,60)=$ 9.32; $p<.01 ; M Q F=8.30]$. Dieser Effekt bleibt auch nach der Kontrolle von Störvariablen stabil.

Im Subtest Visuelles Scanning machten die THOP-Kinder weniger Fehler bei nichtkritischen Reizen beim Visuellen Scanning der TAP $\left[F_{\text {Fehler nicht-krit Reize Vs }}(52)=4.17, p=.04\right.$; $M Q F=8.87]$. Werden die Werte der Einschätzungen der Eltern und Lehrer im FBB-HKS und TRF als Kovariate eingegeben, wird die Signifikanz verfehlt.

Die THOP-Kinder verbesserten im Konzentrationstest d2 ihren Konzentrationsleistungswert signifikant stärker $[F(1,34)=14.16 ; p<.01 ; M Q F=52.04]$ als die LS15-Kinder und machten tendenziell weniger Fehler $[F(1,34)=3.74 ; p=.06 ; M Q F=$ 250.13]. Dieser Effekt bleibt auch nach Kontrolle der AVn als Kovariaten signifikant. 
In den Fremdbeurteilungsbögen der Eltern werden die THOP-Kinder tendenziell als weniger impulsiv beurteilt: $\left[\left(F_{\text {FBB-HKS Rohwert Impulsivität Eltern }}(56)=3.34, p=.06 ; M Q F=0.91\right]\right.$.

Den einzigen hypothesenkonformen Interaktionseffekt zugunsten der LS15-Gruppe gab es in der Nachsprechleistung bei vier-, fünf- und sechssilbigen Wörtern beim MottierTest $\left[F_{\text {Mottier 4-, 5- und 6silbige Wörter }}(35)=4.90, p=.04 ; M Q F=3.16\right]$. Bei Kontrolle der Kovariate Impulsivität der Lehrereinschätzung wird die Signifikanz verfehlt $\left[F_{\text {Mottier vier-, fünf- und sechssilbige }}\right.$ Wörter (Impulsivität FBB-HKS Lehrer $(1,47)=3.11, p=.08 ; M Q F=3.49]$.

\subsubsection{Effektgrößen der Interaktionseffekte}

Insgesamt sechs Variablen werden signifikant oder verfehlen die Signifikanz nur knapp. Es tritt ein großer Effekt zugunsten der THOP-Gruppe auf bei den Auslassungsfehlern im Go/Nogo. Drei mittelgroße Effekte traten zugunsten des THOP auf (Konzentrationsleistungswert und Fehler im d2 sowie Fehler bei nicht-kritischen Reizen im Visuellen Scannings der TAP). Auch die kleinen Effekte fallen überwiegend zugunsten des THOP aus. Hier sind es vor allem die Elterneinschätzungen zu Unaufmerksamkeit, Hyperaktivität und Impulsivität.

Zugunsten der Lauth- und Schlottke-Gruppe fallen die Ergebnisse des Mottiertests aus. Die Effekte sind klein, bei den vier- bis sechssilbigen Wörtern wird der Test signifikant. Überdies werden die LS-Kinder von den Lehrern als etwas weniger unaufmerksam eingeschätzt (kleiner Effekt).

Zusammengefasst lässt sich hier anhand der Effektgrößen eine Überlegenheit des THOP gegenüber dem kombinierten Basis- und Strategietraining nach Lauth und Schlottke feststellen.

\subsubsection{Vergleich des Kombinierten Basis- und Strategietrainings nach Lauth und}

\section{Schlottke 15 Sitzungen mit dem Marburger Konzentrationstraining für die AVn}

TAP und d2

Die Kennwerte der beiden Therapiegruppen werden in Tabelle A-7 dargestellt. 


\subsubsection{Vortestunterschiede und Störfaktoren LS 15 Sitzungen vs. MK}

In der mit dem Marburger Konzentrationstraining trainierten Gruppe wurden signifikant mehr Kinder mit Methylphenidat behandelt $\left[\chi^{2}(1, \mathrm{~N}=49)_{\text {Medikation }}=7.35, p<.01\right]$. Außerdem sind relativ mehr weibliche Kinder in der Marburger Konzentrationstrainingsgruppe $\left[\chi^{2}(1\right.$, $\left.\mathrm{N}=49)_{\text {Geschlecht }}=4.04, p=.04\right]$.

Die Kinder des Marburger Konzentrationstrainings schneiden im Intelligenztest signifikant besser $\mathrm{ab}\left[t_{\mathrm{IQ}}(47)=2,69, p=0.01\right]$. Hier ist aber zu berücksichtigen, dass die Kinder des Marburger Konzentrationstrainings mit dem CFT 20 untersucht wurden, die LS15Kinder mit der Kaufman ABC oder dem HAWIK-III. Erfahrungsgemäß werden die Kinder durch den CFT 20 in der Intelligenzleistung häufig um bis zu 10 Punkte überschätzt. Weitere Unterschiede in den Vortestwerten gibt es in der Schwankung der Antworten beim Visuellen Scanning (nicht kritische Reize) [ $\left.t_{\text {Standardabweichung nicht-krit. Reize VS }}(40)=-2.38, p=0.02\right]$ (die MKKinder antworten regelmäßiger) und in der Einschätzung der Hyperaktivität und Impulsivität durch die Eltern $\left[\left(t_{\mathrm{FBB}-H K S}\right.\right.$ Kennwert Hyperaktivität Eltern $\left.(41)=2.59, p=.01\right) ;\left(t_{\mathrm{FBB}-\mathrm{HKS} \text { Kennwert Impulsivität }}\right.$ Eltern $(15.00)=2.43, p=.02)]$. Die MK-Kinder werden als hyperaktiver und impulsiver beurteilt.

\subsubsection{Veränderungen zwischen den Versuchsgruppen LS15 vs. MK}

Die Kinder des Marburger Konzentrationstrainings verbesserten im Konzentrationstest d2 ihren Konzentrationsleistungswert signifikant stärker $[F(1,34)=8.01 ; p<.01 ; M Q F=$ 66.91] als die LS15-Kinder und machten signifikant weniger Fehler $[F(1,34)=5.13 ; p=.03$; $M Q F=33.72]$. In den Subtests der TAP unterscheiden sich die Ergebnisse der Gruppen nicht voneinander. Die Kontrolle der Intelligenz als Kovariate führte dazu, dass der Interaktionseffekt bei den Fehlern des d2 nicht mehr signifikant wurde $[F(1,34)=3.43$; $p=$ $.07 ; M Q F=34.55]$

\subsubsection{Effektgrößen der Interaktionseffekte LS15 vs. MK}

Beim Vergleich der Effektgrößen gibt es einen großen Effekt zugunsten des Marburger Konzentrationstrainings (Auslassungsfehler Go/Nogo TAP). Im Test d2 gibt es signifikante Unterschiede zugunsten des Marburger Konzentrationstrainings. Die Effekte sind klein (Konzentrationsleistungswert) und mittelgroß (Fehler). Der Wert Fehler im Subtest Go/Nogo verfehlt die Signifikanz nur kanpp. Er fällt als mittelgroßer Effekt zugunsten der LS-Gruppe aus. Insgesamt sind die mittleren und kleinen Effekte in etwa zu gleichen Teilen zugunsten beider Trainings verteilt. Aufgrund des zu geringen Rücklaufs der Fragebögen der 
Kinder des Marburger Konzentrationstrainings können die Fremdurteile nicht miteinander verglichen werden.

\subsubsection{Vergleich der THOP-Gruppen mit dem Marburger Konzentrationstraining für die AVn TAP und d2}

In Tabelle A-8 sind die wesentlichen Werte der Gruppenvergleiche zusammengestellt.

\subsubsection{Vortestunterschiede und Störfaktoren THOP vs. MK}

Die nominalskalierten Werte für Geschlecht und Medikation unterscheiden sich in diesen beiden Gruppen nicht voneinander.

Unterschiede in den Vortestwerten gibt es in der Schwankung der Antworten beim Visuellen Scanning (nicht kritische Reize) [ $t_{\text {Standardabweichung nicht-krit. Reize vs }}(43)=-2.12 ; p=$

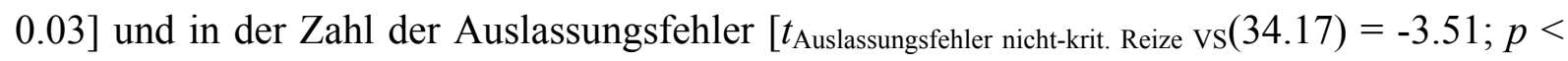
0.01)] (die MK-Kinder antworten regelmäßiger und machen weniger Fehler) und in der Einschätzung der Gesamtbelastung und hinsichtlich Externalisierter Störungen durch die Lehrer $\left[\left(t_{\text {TRF-Gesamt }}(34)=-2.68, p=.01\right) ;\left(t_{\text {TRF-Externalisierende Störungen }}(34)=-2.89, p<.01\right)\right]$. Die MK-Kinder werden zu Beginn des Trainings von den Lehrern als insgesamt weniger belastet wahrgenommen und zeigen weniger externalisierte Auffälligkeiten.

\subsubsection{Veränderungen zwischen den Versuchsgruppen}

Die THOP-Kinder verbesserten sich in den Subtests der TAP in mehreren Subtests tendenziell, in einem Subtest signifikant. Sie machen weniger Fehler in den Subtests Go/Nogo $[F(1,44)=2.84 ; p=.09]$ und Geteilte Aufmerksamkeit; $[F(1,42)=3.60 ; p=.06 ; M Q F=$ 19.40]. Im Subtest Inkompatibilität ist die Leistung der THOP-Kinder weniger schwankend $[F(1,44)=3.65 ; p=.06 ; M Q F=13148]$. Ein signifikanter Effekt zeigt sich bei den Fehlern der nicht-kritischen Trials der TAP $[F(1,40)=4.08 ; p<.01 ; M Q F=16.76]$. Der einzige Unterschied zugunsten der Kinder des Marburger Konzentratonstrainings zeigt sich bei der Regelmäßigkeit der Antworten bei den kritischen Trials des Visuellen Scannings $[F(1,40)=$ 3.46; $p=.07 ; M Q F=1162602]$. Im Konzentrationstest d2 unterscheiden sich die Gruppen nicht voneinander.

\subsubsection{Effektgrößen der Interaktionseffekte THOP vs. MK}

Beim Vergleich dieser beiden Gruppen miteinander wurde das Ergebnis einer Variablen signifikant, vier Ergebnisse verfehlen die Signifikanz knapp. Ein großer Effekt fällt zugunsten des THOP beim signifikanten Ergebnis der Fehler bei kritischen Trials des 
Visuellen Scannings auf. Mittlere und kleine Effekte treten zugunsten des THOP auf bei den Fehlern im Go/Nogo, den Fehlern in der Geteilten Aufmerksamkeit, Fehlern der Inkompatibilität, den Fehlern bei kritischen Reizen des Visuellen Scannings sowie den Fehlern im d2.

Ein großer Effekt zugunsten des Marburger Konzentrationstrainings fällt bei der Standardabweichung der kritischen Trials des Visuellen Scannings sowie der Standardabweichung des Tests Go/Nogo auf. Die MK-Kinder reagieren hier regelmäßiger.

Aufgrund des zu geringen Rücklaufs der Fragebögen der Kinder des Marburger Konzentrationstrainings können die Fremdurteile nicht miteinander verglichen werden.

Insgesamt läßt sich feststellen, dass der überwiegende Teil der Vergleiche zugunsten der THOP-Gruppe ausfällt. 


\section{Diskussion}

Mit der vorliegenden Dissertation werden kognitiv-verhaltenstherapeutische Interventionen evaluiert, die bei Kindern mit HKS unter den praktischen Bedingungen einer kinder- und jugendpsychiatrischen Ambulanz bzw. Praxis durchgeführt wurden. Die Untersuchung wurde von verschiedenen psychologischen Hypothesen (PHn) geleitet. Die Bewertung der Ergebnisse erfolgt zunächst anhand der Psychologischen Hypothesen der isolierten Evaluationen der vier Studien. In der vergleichenden Evaluation werden dann die verschiedenen Trainings miteinander verglichen. Die Durchführung der Trainings unterlag den Einschränkungen der Praxis. Auf damit verbundene Einschränkungen der Untersuchungen geht der Verfasser in Kapitel 6.3 ein. Den Abschluss der Diskussion bilden ein Fazit und ein Ausblick in Kapitel 6.4.

Den Ursprung der vorliegenden Untersuchung bildete eine in Deutschland häufig in der medizinisch-psychotherapeutischen Versorgung in Erscheinung tretende Situation: Täglich werden in den kinder- und jugendpsychiatrischen Ambulanzen und Beratungsstellen Kinder mit dem Verdacht auf eine Hyperkinetische Störung vorgestellt und einer je nach Einrichtung unterschiedlich praktizierten psychodiagnostischen Untersuchung unterzogen. Es werden steigende Mengen der Verordnung von Methylphenidat in Deutschland registriert, die ein Anwachsen hyperkinetischer Auffälligkeiten bei Kindern suggerieren. Lange Wartezeiten vor den Türen der Kinderpsychiatrien und den Praxen niedergelassener Kinder- und Jugendpsychiater bzw. Kinder- und Jugendlichenpsychotherapeuten auf einen Untersuchungstermin und noch längere Wartezeiten auf einen Behandlungsplatz (sofern nicht Medikamente verschrieben werden) sind Kennzeichen eines bedauerlichen Versorgungsstatus. Da es nur relativ wenige Therapieplätze bei Psychotherapeuten gibt, lag die Überlegung nahe über gruppentherapeutische Angebote mehr Kinder psychotherapeutisch versorgen $\mathrm{zu}$ können. Gruppentherapeutische Behandlungen haben sich bereits in verschiedenen klinisch-psychiatrischen Bereichen als erfolgreiche Interventionsmethode bewährt (Fiedler, 1996). Da viele Kinder mit hyperkinetischen Störungen insbesondere auch im Gruppenkontext problematisches Verhalten zeigen, lag der Gedanke nahe, eine solche Form der Therapie auch für diese Kinder und ihre Eltern anzubieten. Da viele Kinder das Problemverhalten im Einzelsetting mit einem Erwachsen gar nicht zeigen, ist es sinnvoll dies in einem Gruppensetting zu behandeln. Innerhalb dieser Gruppen treten naturgemäß auch solche Probleme auf, die dem Kind in seinen übrigen Lebensfeldern Schwierigkeiten machen. 
Unter therapeutischer Anleitung kann versucht werden sie zu lösen bzw. den Kindern Handlungsstrategien zu vermitteln, die sozialverträglich sind.

Durch die Interventionsmaßnahmen könnten sich Versorgungslücken schließen sowie Behandlungskosten reduzieren lassen. Diese beiden Ziele sind jedoch nur dann zu erreichen, wenn durch die Gruppenbehandlung tatsächlich auch eine effektive Therapie zum Einsatz kommt. Um dies zu prüfen wurde die vorliegende Evaluationsstudie durchgeführt. Da die Therapien im Rahmen der regulären ambulanten Versorgung durchgeführt wurden, haben die Ergebnisse unmittelbare Implikationen hinsichtlich des Nutzens der verschiedenen Interventionsformen in der gesundheitlichen Versorgung in Deutschland.

\subsection{Bewertung der Ergebnisse der isolierten Evaluation der Studien 1 bis 4}

\subsubsection{Isolierte Evaluation des Basistrainings nach Lauth und Schlottke (Studie 1)}

Die erste Hypothese, der zu Folge das Basistraining nach Lauth und Schlottke unter den praktischen Rahmenbedingungen einer kinder- und jugendpsychiatrischen Ambulanz zu Verbesserungen der Aufmerksamkeitsleistung sowie zu einer Verminderung hyperkinetischer Verhaltenssymptome führt, hat sich nicht bewährt. Die Therapiegruppe unterscheidet sich weder in den Testmaßen zur Erfassung der Aufmerksamkeitsleistung noch in den Verhaltensbeurteilung durch Eltern und Lehrer von der Wartegruppe. In beiden Untersuchungsgruppen traten Verbesserungen von der Vor- zur Nachtestung ein, die Gruppenunterschiede zwischen den Gruppen in den abhängigen Variablen waren aber nicht signifikant. Da die Verbesserung auch ohne Intervention in der Wartegruppe eingetreten ist, muss davon ausgegangen werden, dass sie aufgrund von Reifungs- oder Retesteffekten zustande gekommen ist. Dieses Resultat ist angesichts der Ergebnisse der Therapieforschung nicht überraschend. Es stützt die verbreitete Ansicht, dass die Wirksamkeit kognitivverhaltenstherapeutischer Interventionen bei Kindern mit Hyperkinetischen Störungen nicht hinreichend belegt ist (Abikoff, 1987; Saile, 1996).

Das Ausbleiben deutlich positiver Effekte des Trainings könnte mit der relativ kurzen Interventionsdauer von nur 10 Sitzungen zusammenhängen. Bei der vergleichenden Evaluation wird dieser Frage weiter nachgegangen. Problematisch aus Sicht des Verfassers ist die mangelnde Verankerung der therapeutischen Inhalte in das Alltagsleben der Kinder. So konnten sich manche Kinder nicht an die Inhalte der vergangenen Sitzung erinnern, wenn sie nach einer Woche danach befragt wurden. Es ist davon auszugehen, dass die vermittelten Strategien weder zu Hause noch in der Schule zum Einsatz gekommen sind, wenn sich die Kinder bereits nach einer Woche nicht mehr daran erinnern können. Eine engere Verzahnung 
mit schulischen Inhalten hätte hier möglicherweise Abhilfe schaffen können. Aufgrund der zeitlichen Befristung des Trainings hatten die Kinder nicht ausreichend Gelegenheit die erlernten Fertigkeiten in ihrem natürlichen Umfeld einzuüben. Die Intensität scheint für die behandelten Kinder nicht ausreichend zu sein. Der Erfolg kognitiver Interventionsansätze misst sich an der Übertragung der erlernten Fertigkeiten auf das Alltagsverhalten, was aber der Unterstützung durch das soziale Umfeld (Eltern, Schule) der Kinder bedarf. Aus diesem Grund wurden ergänzend zum Training der Kinder den Eltern einige Sitzungen zur Anleitung und Vertiefung der erlernten Fertigkeiten angeboten. Die Teilnahme an diesen Veranstaltungen war aber nicht zufriedenstellend. Die Lehrer bzw. Schule wurde in das Training nicht mit einbezogen. Die mangelnde Wirksamkeit der Intervention könnte deshalb auch dadurch zu erklären sein, dass die Trainingsinhalte von wichtigen Bezugspersonen nicht aufgegriffen wurden und das Training damit zu wenig in den Alltag der Kinder integriert war. Für nachfolgende Durchführungen ist dringend zu empfehlen, eine engere Koordination mit Elternhaus und Schule anzustreben. Der intensive Einbezug der Eltern ist auch deshalb von großer Bedeutung, weil das Ausmaß psychosozialer Belastungen (z.B. gestörte familiäre Beziehungen, mangelnde pädagogische Fähigkeiten) mit dem Erfolg von Therapiemaßnahmen bei Kindern mit Hyperkinetischen Störungen zusammenhängt (Laumann \& Poustka, 1991). Häufig wird die Zusammenarbeit mit Eltern (und auch mit Lehrern) durch deren mangelnde Bereitschaft erschwert, sich bezüglich solcher Interventionen $\mathrm{zu}$ engagieren. Dies zeigte sich in dieser Studie z.B. schon am geringen Rücklauf der Fragebögen nach dem Training.

Ein weiterer Punkt, der zu den eher schwachen Ergebnissen des Trainings geführt haben könnte, ist die Nichtbeachtung der Forderung der Programmautoren, zum einen die Zuweisung $\mathrm{zu}$ den einzelnen Therapiebausteinen entsprechend den individuellen Störungsschwerpunkten vorzunehmen, zum anderen möglichst homogene Gruppen zusammenzustellen. Unter den praktischen Randbedingungen, unter denen diese Studie durchgeführt wurde, war diese Forderung aber kaum zu erfüllen. Die Durchführung als Gruppenverfahren war einem Einzelsetting möglicherweise deshalb unterlegen, weil in einer Einzelintervention die Inhalte genauer auf den jeweiligen Patienten zugeschnitten werden können. Da die therapeutischen Ressourcen für die Behandlung von Kindern mit psychiatrischen Problemen jedoch insgesamt gering sind, ist es notwendig, sich mit den Möglichkeiten zur Optimierung von Gruppentrainings auseinanderzusetzen.

Auffällig sind zudem die hohen Zuwächse, die sich in der Wartegruppe ergeben haben. Zwar fielen bis auf eine Ausnahme keine der Indizes der Fremdbeurteilungsbögen 
signifikant aus, jedoch scheint dies eher ein Problem der Stichprobengröße zu sein. Die Familien der Wartegruppe haben ebenso wie die Familien der beiden Trainingsgruppen nach der Vortestung ein Feedback über die Diagnose sowie einige Empfehlungen bekommen. Diese Psychoedukation umfasste in der Regel die Erläuterung eines Erklärungsmodells über Entstehung und Aufrechterhaltung der Störung, eine Aufklärung über therapeutische Behandlungsmöglichkeiten, elementare Hilfen zur Strukturierung von Abläufen (z.B. Leitfaden für Hausaufgabenprobleme), Vorschläge für Übungen und Spiele, die das Konzentrationsvermögen der Kinder fördern sowie das Aufzeigen von Möglichkeiten, wie im Rahmen von Alltagstätigkeiten Konzentrations- und Aufmerksamkeitsverhalten geübt werden kann. Häufig wurden darüber hinaus den Eltern Buchempfehlungen gegeben. Somit haben auch bei den Familien der Wartegruppe therapeutische Interventionen stattgefunden. Es ist nicht auszuschließen, dass sich die Eltern nach diesen Informationen in eigener Regie noch weitere Hilfen bzw. Informationen geholt haben. 4 der 16 Familien haben zwischen der Vorund Nachtestung sogar noch eine zusätzliche Beratung erhalten.

Ein weiterer wichtiger Gesichtspunkt im Hinblick auf die Wartegruppe könnte der jeweilige Messzeitpunkt sein. Eltern suchen in der Regel dann eine Beratungsstelle auf, wenn sich eine krisenhafte Zuspitzung zu Hause oder in der Schule ergeben hat. Unter dem Einfluss einer solchen angespannten Stresssituation könnten die Vortestwerte entsprechend hoch ausgefallen sein, so dass das wahre Störungsausmaß vielleicht überschätzt wurde. Der Nachtestzeitpunkt wurde in der Wartegruppe zufällig gewählt, und es ist denkbar, dass zu jener Zeit in der Familie gerade keine großen Probleme herrschten, und die Testscores deshalb so gering ausfielen. Interessant wäre zu untersuchen, ob in einer Follow-up-Erhebung nach einigen Monaten bei den Kindern der Wartegruppe noch immer derart positive Ergebnisse festgestellt werden können.

Mit der zweiten Hypothese sollte überprüft werden, ob es durch das Training zu positiven Veränderungen auf der Ebene der kognitiven Verhaltenshemmung kommt. Es sollte überprüft werden, ob die trainierten Kinder vorherrschende Handlungsimpulse besser unterdrücken und Interferenzen besser kontrollieren können. Sowohl beim Go/Nogo als auch beim Subtest Inkompatibilität spielt die Unterdrückung irrelevanter Reize eine wesentliche Rolle. Hier wird einer der Interaktionskontraste signifikant. Die trainierten Kinder vermögen es nach dem Training besser Handlungsinterferenzen (hier beim Inkompatibilitätstest der TAP) zu unterdrücken.

Eine graphische Veranschaulichung des Ergebnisses gibt die Abbildung 4. 


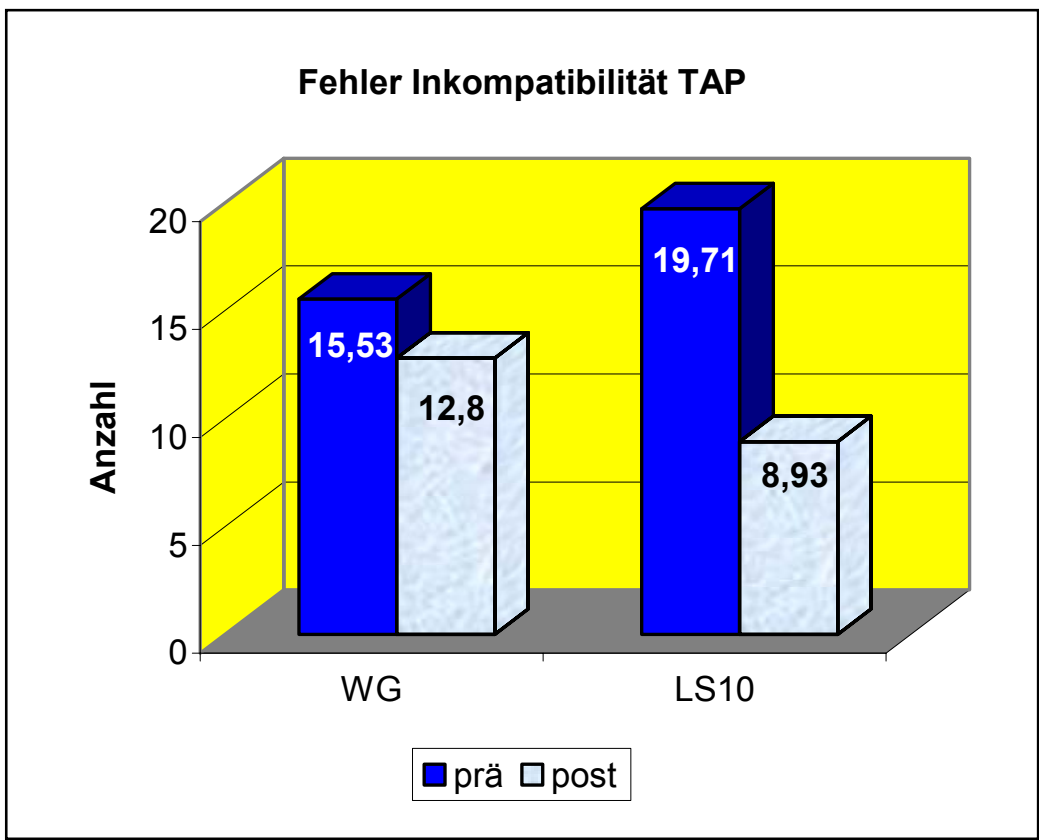

\begin{tabular}{|c|c|c|c|c|}
\hline & & $\mathrm{n}$ & $\mathrm{M}$ & $\mathrm{SD}$ \\
\hline \multirow{2}{*}{ WG } & prä & \multirow{2}{*}{15} & 15.53 & 9.12 \\
\cline { 2 - 3 } \cline { 4 - 5 } & post & & 12.80 & 8.03 \\
\hline \multirow{2}{*}{ LS10 } & prä & \multirow{2}{*}{14} & 19.71 & 13.10 \\
\cline { 2 - 3 } \cline { 5 - 5 } & post & & 8.93 & 8.65 \\
\hline
\end{tabular}

Anmerkungen:

WG: Wartegruppe

LS10: Lauth und Schlottke 10 Sitzungen

n: $\quad$ Anzahl der Kinder pro

Gruppe

M: $\quad$ Mittelwert

SD: Standardabweichung

prä: Vortest

post: Nachtest

Abbildung 4

Darstellung der Fehler im Subtest „Inkompatibilität“ der Testbatterie zur Aufmerksamkeitsprüfung TAP im Prä-Post-Vergleich der Wartegruppe und dem Basistraining nach Lauth und Schlottke (10 Sitzungen)

Die Verbesserungen in dem anderen Subtest, der dieser Hypothese zugeordnet wurde (Go/Nogo), weisen tendenziell in die hypothesenkonforme Richtung, verfehlen aber das Signifikanzniveau. Diese Hypothese findet also eine gewisse Bestätigung, die Effekte sind klein oder mittelgroß. Durch das Training scheinen kognitive Hemmungsdefizite positiv beeinflusst werden zu können.

Da nach dem kognitiven Erklärungsmodell von Barkley (1997) Hemmungsprozesse eine wesentliche Voraussetzung für die Verhaltensregulation darstellen, sollte eine positive Beeinflussung von Hemmungsprozessen auch $\mathrm{zu}$ verbesserten selbstregulatorischen Fähigkeiten auf der Verhaltensebene führen, was in dieser Studie jedoch nicht nachgewiesen werden konnte. Die Kinder vermochten es demnach nicht, die positiven Effekte der Hemmungskontrolle auch auf der Verhaltensebene umzusetzen.

Unter einer dritten Fragestellung wurden Auswirkungen der Intervention auf Bereiche außerhalb des Trainingskontextes gesucht. Auch wenn für die generelle Wirksamkeit des Basistrainings unter den hier durchgeführten Bedingungen keine Belege gefunden werden konnten, kann das Training dennoch zu Transfereffekten geführt haben (vgl. Hager, 1995). Entsprechend der PH 1.3a wurde erwartet, dass das Basistraining zu einer Steigerung der funktionellen Nutzung des phonologischen Arbeitsgedächtnisses führt. 
Die beiden folgenden Grafiken veranschaulichen die Ergebnisse

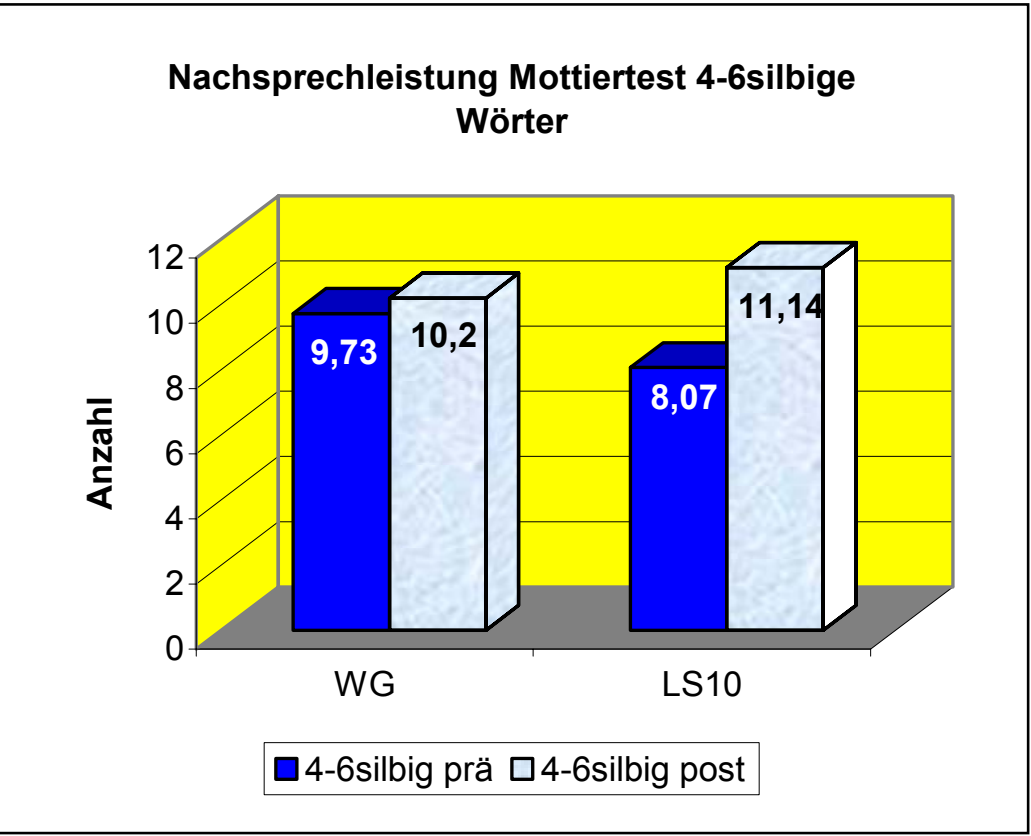

\begin{tabular}{|c|c|c|c|c|}
\hline & & $\mathrm{n}$ & $\mathrm{M}$ & $\mathrm{SD}$ \\
\hline \multirow{2}{*}{ WG } & prä & \multirow{2}{*}{15} & 9.73 & 3.17 \\
\cline { 2 - 3 } \cline { 4 - 5 } & post & & 10.20 & 3.86 \\
\hline \multirow{2}{*}{ LS10 } & prä & \multirow{2}{*}{14} & 8.07 & 3.41 \\
\cline { 2 - 3 } \cline { 5 - 5 } & post & & 11.14 & 3.13 \\
\hline
\end{tabular}

Anmerkungen:

WG: Wartegruppe

LS10: Lauth und Schlottke 10 Sitzungen

n: Anzahl der Kinder pro Gruppe

M: $\quad$ Mittelwert

SD: $\quad$ Standardabweichung

prä: Vortest

post: Nachtest

\section{Abbildung 5}

Darstellung der Nachsprechleistung bei 4-6silbigen Wörtern im Mottier-Test im Prä-PostVergleich der Wartegruppe und dem Basistraining nach Lauth und Schlottke (10 Sitzungen)

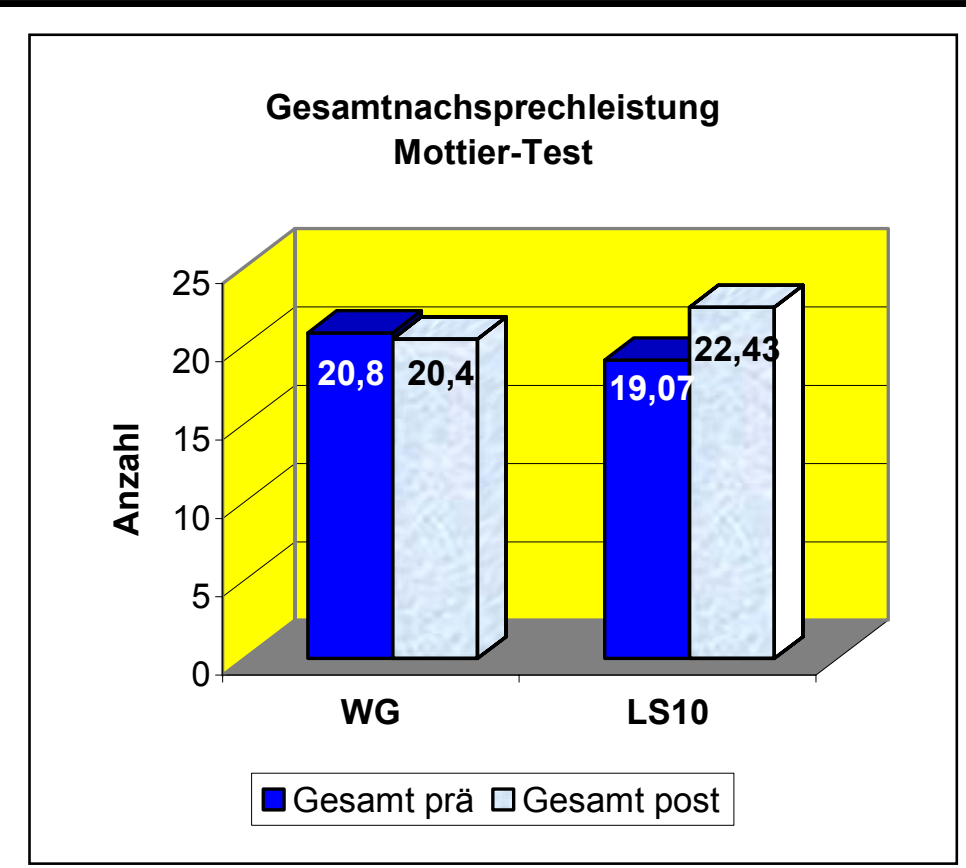

\begin{tabular}{|c|c|c|c|c|}
\hline & & $\mathrm{n}$ & $\mathrm{M}$ & $\mathrm{SD}$ \\
\hline \multirow{2}{*}{ WG } & prä & \multirow{2}{*}{15} & 20.80 & 3.97 \\
\cline { 2 - 3 } \cline { 5 - 5 } & post & & 20.40 & 5.01 \\
\hline \multirow{2}{*}{ LS10 } & prä & \multirow{2}{*}{14} & 19.07 & 4.01 \\
\cline { 2 - 2 } & post & & 22.43 & 3.11 \\
\hline \multicolumn{2}{|c|}{$A$} & & \multicolumn{2}{|c}{} \\
\hline
\end{tabular}

Anmerkungen:

WG: Wartegruppe

LS10: Lauth und Schlottke 10 Sitzungen

n: $\quad$ Anzahl der Kinder pro

Gruppe

M: $\quad$ Mittelwert

SD: $\quad$ Standardabweichung

prä: Vortest

post: Nachtest

\section{Abbildung 6}

Darstellung der Gesamtnachsprechleistung im Mottier-Test im Prä-Post-Vergleich der Wartegruppe und dem Basistraining nach Lauth und Schlottke (10 Sitzungen) 
Die PH 1.3a hat sich bewährt. Sowohl in der Gesamtnachsprechleistung im Mottiertest als auch bei den vier-, fünf- und sechssilbigen Wörtern verbessern sich die trainierten Kinder signifikant. Die Effekte sind groß bzw. mittelgroß. Da die funktionelle Nutzung des Arbeitsgedächtnisses von Hemmungsprozessen abhängig ist, steht der positive Transfer möglicherweise im Zusammenhang $\mathrm{zu}$ den erreichten Verbesserungen der Hemmungskontrolle. Denkbar ist, dass sich die im Training vermittelten Fertigkeiten („Genau zuhören“ und „Wahrgenommenes genau wiedergeben“) sich an dieser Stelle förderlich ausgewirkt haben. Denkbar ist auch, dass die trainierten Kinder sich nun besser auf die Bedingungen bei dieser Leistungsanforderung einlassen können, sich also konzentriert einer akustischen Reizquelle zuzuwenden und die Reize adäquat zu verarbeiten. Es kann nicht erwartet werden, dass die akustische Differenzierungsfähigkeit durch das Training verbessert worden ist, jedoch die Kompetenz, wie sich die Kinder auf die Anforderung einlassen, deutlich verbessert wurde.

In einer weiteren Transferhypothese wurden positive Auswirkungen des Basistrainings auf die psychosoziale Anpassung vermutet (PH 1.3b). Die trainierten Kinder erzielen zwar tendenziell vom Vor- zum Nachtest in vielen Items der Fremdbeurteilungsbögen (FBB-HKS und CBCL 4-18) der Elterneinschätzung eine Verbesserung, diese wird aber bei keiner der AVn signifikant.

In der Lehrereinschätzung ändert sich vom Vor- zum Nachtest sowohl in der Wartewie auch in der Trainingsgruppe praktisch nichts, so dass von einem Transfer in den Schulalltag nicht ausgegangen werden kann. Um auch in diesem Lebensbereich Effekte zu erzielen ist ein Austausch mit den Lehrern notwendig. Die trainierten Inhalte sind von dem, was in der Schule von den Kindern verlangt wird, offensichtlich zu weit entfernt.

Als Fazit zu dieser Untersuchung kann gezogen werden, dass die Wirksamkeit des Basistrainings für Kinder mit Hyperkinetischen Störungen unter den spezifischen, praxisnahen Durchführungsbedingungen nicht hinreichend belegt ist. Die Ergebnisse deuten aber auf Verbesserungen hinsichtlich der Hemmungskontrolle und einer verbesserten Nutzung des phonologischen Arbeitsgedächtnisses hin. Dies bedeutet nicht, dass das Training für die Behandlung von Kindern mit Hyperkinetischen Störungen generell ungeeignet ist, aber eben nicht unter den hier durchgeführten Bedingungen. 


\subsubsection{Isolierte Evaluation des um Komponenten des Strategietrainings erweiterten Basistrainings nach Lauth und Schlottke (Studie 2)}

In Studie 2 wurde ein intensiveres Training durchgeführt als in Studie 1. Hier wurden neben dem Basistraining zusätzlich über fünf Sitzungen Elemente des Strategietrainings nach Lauth und Schlottke vermittelt. Erwartet wurde, dass durch das intensivere Training auch stärkere Effekte erzielt werden.

Beim Vergleich der Vor- und Nachtestwerte verbessern sich die trainierten Kinder in deutlich mehr Variablen als die Wartekinder. Sie verbessern sich im Gegensatz zu Studie 1 auch in den Lehrereinschätzungen.

Die Variablen, welche zur Überprüfung der generellen Wirksamkeit des kombinierten Basis- und Strategietraining eingesetzt wurden, werden beim Vergleich der Veränderungen zwischen den Versuchsgruppen mit einer Ausnahme nicht signifikant. Lediglich in der Variablen Visuelles Scanning reagieren die trainierten Kinder sorgfältiger. Abbildung 7 veranschaulicht dieses Ergebnis.



Abbildung 7

Darstellung der Fehler im Subtest „Visuelles Scanning“ (kritische Reize) der Testbatterie zur Aufmerksamkeitsprüfung TAP im Prä-Post-Vergleich der Wartegruppe und dem kombinierten Basis- und Strategietraining nach Lauth und Schlottke (15 Sitzungen)

Insgesamt lässt sich zur Bewertung der PH 2.1 sagen, dass auch das kombinierte Basis- und Strategietraining keine substanziellen Verbesserungen der 
Aufmerksamkeitsleistungen bewirkt, wenn es im Rahmen der praktischen Durchführungsbedingungen in einer kinder- und jugendpsychiatrischen Ambulanz durchgeführt wird. Die Erweiterung des Basistrainings um Komponenten des Strategietrainings erzielt keine weiteren Verbesserungen.

Interessant ist hier die Verbesserung in den Urteilen der Lehrer in den Fragebögen FBB-HKS und TRF. Beim Strategietraining werden Inhalte vermittelt, die sich an schulischen Fragestellungen orientieren. Die Veränderungen scheinen von den Kindern in ersten Ansätzen auch im schulischen Kontext gezeigt zu werden, der Interaktionseffekt in den jeweiligen Variablen ist jedoch nicht signifikant.

Mit der zweiten Hypothese dieser Studie sollte geprüft werden, ob sich durch das kombinierte Basis- und Strategietraining positive Veränderungen auf der Ebene der kognitiven Verhaltenshemmung zeigen und es den Kindern durch das Training gelingt, vorherrschende Handlungsimpulse besser $\mathrm{zu}$ unterdrücken und Interferenzen besser $\mathrm{zu}$ kontrollieren. Im Gegensatz zu Studie 1 lässt sich dies für das kombinierte Training nicht nachweisen.

Entsprechend der PH 2.3a wurde erwartet, dass das Basistraining zu einer Steigerung der funktionellen Nutzung des phonologischen Arbeitsgedächtnisses führt. Wie schon in Studie 1 hat sich die PH 2.3a bewährt.

Die graphische Veranschaulichung der Ergebnisse erfolgt anhand Abbildung 8. 


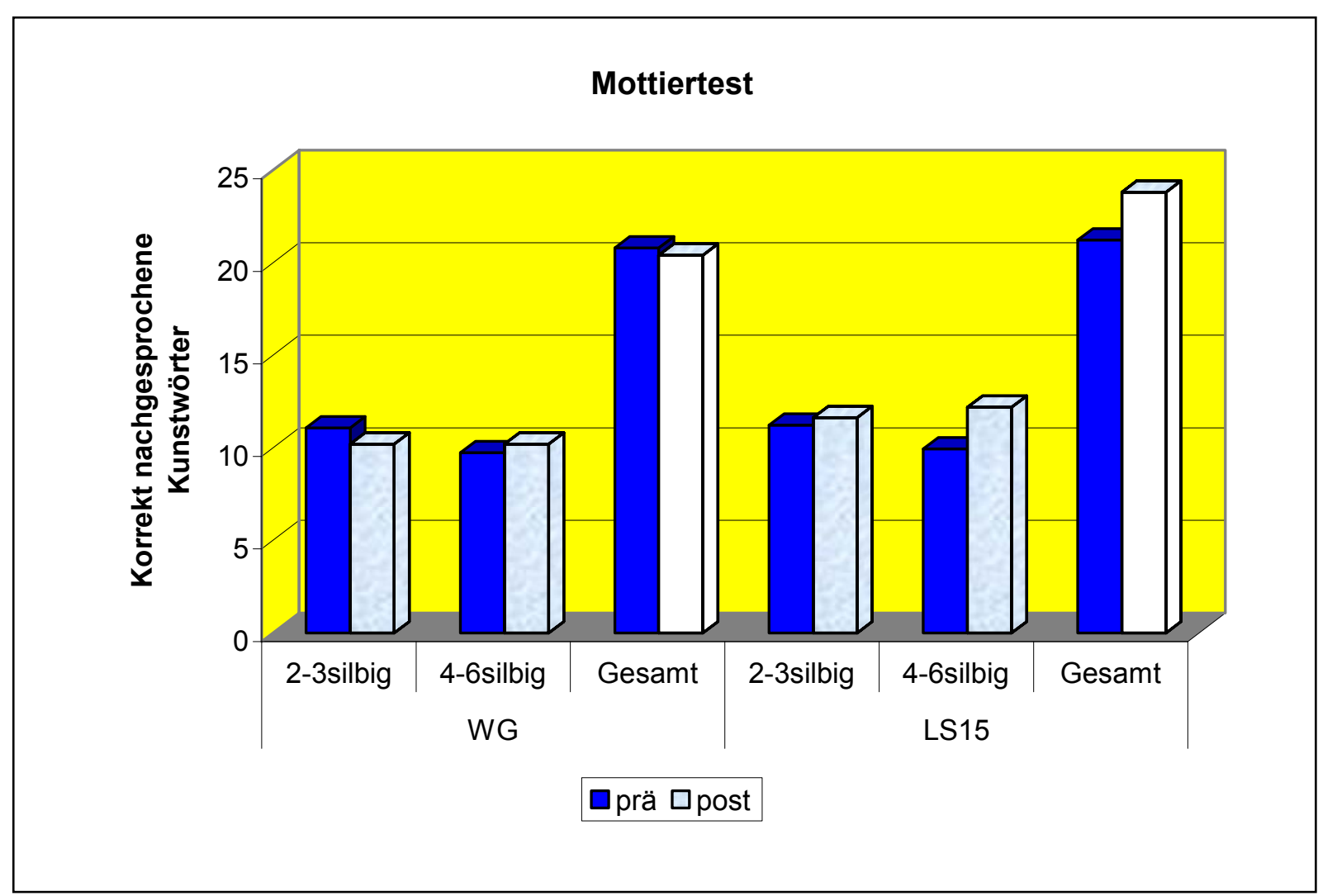

\begin{tabular}{|c|c|c|c|c|c|}
\hline & Wörter & & $\mathrm{N}$ & M & SD \\
\hline \multirow{2}{*}{ WG } & \multirow{4}{*}{ 2- und 3silbig } & prä & \multirow{2}{*}{15} & 11.07 & 1.53 \\
\hline & & post & & 10.20 & 2.21 \\
\hline \multirow{2}{*}{ LS15 } & & prä & \multirow{2}{*}{32} & 11.28 & 1.02 \\
\hline & & post & & 11.63 & 0.79 \\
\hline \multirow{2}{*}{ WG } & \multirow{4}{*}{ 4-, 5- und 6silbig } & prä & \multirow{2}{*}{15} & 9.73 & 3.17 \\
\hline & & post & & 10.20 & 2.21 \\
\hline \multirow{2}{*}{ LS15 } & & prä & \multirow{2}{*}{32} & 9.94 & 4.44 \\
\hline & & post & & 12.19 & 3.41 \\
\hline \multirow{2}{*}{ WG } & \multirow{4}{*}{ Gesamt } & prä & \multirow{2}{*}{15} & 20.80 & 3.97 \\
\hline & & post & & 20.40 & 5.01 \\
\hline \multirow{2}{*}{ LS 15} & & prä & \multirow{2}{*}{32} & 21.22 & 4.96 \\
\hline & & post & & 23.81 & 3.89 \\
\hline
\end{tabular}

Anmerkungen:

WG: Wartegruppe

LS15: Lauth und Schlottke 15 Sitzungen

n: $\quad$ Anzahl der Kinder pro Gruppe

M: $\quad$ Mittelwert

SD: Standardabweichung

2- und 3silbig: Korrekt nachgesprochene 2und 3silbige Kunstwörter

4-, 5- und 6silbig:

Korrekt nachgesprochene 4-, 5- und 6silbige Kunstwörter

Gesamt: Alle korrekt nachgesprochenen Kunstwörter

Abbildung 8

Darstellung der Nachsprechleistung im Mottier-Test im Prä-Post-Vergleich der Wartegruppe und dem kombinierten Basis- und Strategietraining nach Lauth und Schlottke (15 Sitzungen) 
Sowohl in der Gesamtnachsprechleistung im Mottiertest, bei den 2- und 3silbigen als auch vier-, fünf- und sechssilbigen Wörtern verbessern sich die trainierten Kinder signifikant. Die Effekte sind groß (zwei- und dreisilbig), klein (vier-, fünf- und sechssilbige Wörter) und mittelgroß (Gesamtleistung). Da die mit dem kombinierten Basis- und Strategietraining trainierten Kinder keine Verbesserung der Hemmungskontrolle erzielten, ist für diese Kinder eher davon auszugehen, dass sie hinsichtlich des „Sicheinlassens“ auf die Anforderung dieses Tests Fortschritte gemacht haben.

In der Transferhypothese $2.3 \mathrm{~b}$, nach der die trainierten Kinder die gelernten Inhalte auch in anderen Lebensbereichen einsetzen, was dann zu einer verbesserten psychosozialen Anpassung führen sollte, erzielen die trainierten Kinder im Prä-Postvergleich auf einer Vielzahl von Variablen sowohl im Elternurteil (CBCL 4-18) als auch im Lehrerurteil (FBBHKS und TRF) signifikante Verbesserungen. Die Interaktionseffekte werden im Gruppenvergleich indes nicht signifikant. Die größere Nähe der trainierten Inhalte zu dem, was in der Schule von den Kindern verlangt wird, führt hier zu Verbesserungen. Da aber wie auch in Studie 1 die Lehrer nicht in die Therapie einbezogen wurden, konnten sich diese naturgemäß auch nicht an einer Vertiefung der Inhalte in schulischen Alltagssituationen einbringen.

Als Fazit zu dieser Studie kann gezogen werden, dass die Wirksamkeit des erweiterten Trainings für Kinder mit Hyperkinetischen Störungen unter den spezifischen, praxisnahen Durchführungsbedingungen ebenfalls nicht hinreichend belegt ist. Die Ergebnisse der Studie belegen eine verbesserte Nutzung des phonologischen Arbeitsgedächtnisses. Dies wurde jedoch bereits durch das Basistraining erreicht. Die Erweiterung des Trainings um Komponenten aus dem Strategietraining scheint für die behandelten Kinder keinen weiteren Nutzen mit sich zu bringen.

Eine Einschränkung für die schwachen Untersuchungsergebnisse ist wie schon in Studie 1 darin zu sehen, dass die Nichtbeachtung der Forderung der Programmautoren zum einen die Zuweisung zu den einzelnen Therapiebausteinen entsprechend den individuellen Störungsschwerpunkten vorzunehmen, zum anderen möglichst homogene Gruppen zusammenzustellen auch hier nicht realisiert wurde. 
Der Vergleich der Wirksamkeit des Basistrainings mit dem durch Komponenten des Strategietrainings erweiterten Basistrainings wird bei der vergleichenden Evaluation beschrieben.

\subsubsection{Isolierte Evaluation des THOP (Studie 3)}

Für das THOP konnten in der Durchführung als Einzeltherapie für hyperkinetisch auffällige Kinder im Rahmen der Kölner Multimodalen Therapiestudie (Döpfner \& Lehmkuhl, 2002; Döpfner, Lehmkuhl \& Schürmann, 1996) gute Erfolge verzeichnet werden. Da viele betroffene Kinder ihre hyperkinetischen Auffälligkeiten nur in der Gruppe zeigen, lag es nahe, dass das THOP auch in einer modifizierten Form als Gruppenprogramm effektiv sein könnte. Häufig werden kognitive Verfahren nicht im natürlichen Lebensumfeld des Kindes durchgeführt. Störungen des Sozialverhaltens zeigen sich im Einzelkontakt eines Kindes mit einem Erwachsenen evtl. überhaupt nicht und können dann in der Therapie auch nur schwerlich bearbeitet werden. Im Setting eines Gruppenkontextes hingegen treten diese Probleme im Sozialverhalten naturgemäß auf und können vom Therapeuten aufgegriffen werden.

Mit dem Programm wurde versucht die Eltern-Kind-Interaktion zu verbessern und den Eltern für verschiedene umschriebene Problembereiche adäquate erzieherische Maßnahmen zu vermitteln. Gleichzeitig wurden die Kinder über die Geschichten des „Wackelpeters“ für die Schwierigkeiten in der Familie sensibilisiert sowie aufgefordert und unterstützt auf ihr eigenes Verhalten zu achten. Die mit den Eltern besprochenen Maßnahmen wurden auf jene abgestimmt, die mit den Kindern durchgeführt wurden.

Die Bewertung der Wirksamkeit wird anhand der Psychologischen Hypothesen vorgenommen. Es wurde postuliert, dass das THOP in der Durchführung als Gruppenprogramm im Rahmen einer kinder- und jugendpsychiatrischen Ambulanz zu einer Verminderung der Störungssymptomatik führt (PH 3.1).

Beim Vergleich der Vor- mit den Nachtestwerten ist in 27 der 35 Abhängigen Variablen eine signifikante Verbesserung zu verzeichnen, bei der Wartegruppe gilt dies nur für 10 Variablen.

Beim Vergleich der Veränderungen zwischen den beiden Gruppen wurden Verminderungen der Störungssymptomatik sowohl in der Wartegruppe als auch in der THOPGruppe festgestellt. Mit Ausnahme der Regelmäßigkeit, mit der die Wartegruppenkinder nach dem Training im Subtest Visuelles Scanning (kritische Trials) reagieren, fallen die anderen 
signifikanten Effekte bzw. jene, die fast signifikant wurden, zugunsten des THOP aus. Die trainierten Kinder machten weniger Auslassungsfehler im Untertest Visuelles Scanning der TAP und erreichten einen signifikant besseren Konzentrationsleistungswert im d2. Wie auch die Kinder, welche das Aufmerksamkeitstraining nach Lauth und Schlottke absolviert haben, erzielen auch die THOP-Kinder im Mottier-Test eine höhere Nachsprechleistung als die Kinder der Wartegruppe. Es gelang also durch das Training einige Aufmerksamkeitsfunktionen zu verbessern, jedoch wurde das Alltagsverhalten der Kinder zu Hause und in der Schule kaum verbessert. Die psychologische Hypothese 3.1, wonach das Training zu einer Verminderung der Störungssymptomatik führt, konnte sich damit in dieser Untersuchung tendenziell bewähren. Die Effekte sind überwiegend klein.

Die graphische Veranschaulichung der Ergebnisse geben die Abbildungen 9 bis 12 .



\begin{tabular}{|c|c|c|c|c|}
\hline & & $\mathrm{n}$ & $\mathrm{M}$ & $\mathrm{SD}$ \\
\hline \multirow{2}{*}{ WG } & prä & \multirow{3}{*}{15} & 3952.57 & 1864.41 \\
\cline { 2 - 2 } \cline { 4 - 5 } & post & & 2386.72 & 1274.29 \\
\hline \multirow{2}{*}{ THOP } & prä & \multirow{2}{*}{31} & 2472.98 & 1048.18 \\
\cline { 5 - 5 } \cline { 5 - 5 } & post & & 2049.00 & 995.37 \\
\hline
\end{tabular}

WG: Wartegruppe

THOP: Therapiegruppe

n: $\quad$ Anzahl der Kinder pro

Gruppe

M: $\quad$ Mittelwert

SD: $\quad$ Standardabweichung

prä: Vortest

post: Nachtest

Abbildung 9

Darstellung der Leistung im Subtest Visuelles Scanning (kritische Trials, Standardabweichung) im Prä-Post-Vergleich der Wartegruppe und dem THOP 


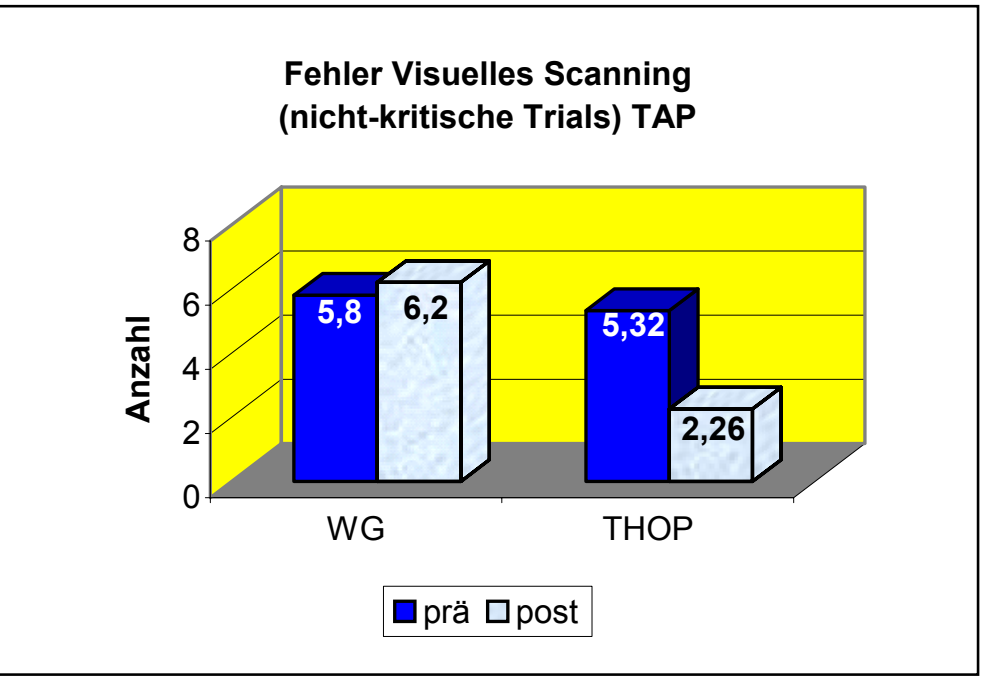

\begin{tabular}{|c|c|c|c|c|}
\hline & & $\mathrm{n}$ & $\mathrm{M}$ & $\mathrm{SD}$ \\
\hline \multirow{2}{*}{ WG } & prä & \multirow{2}{*}{15} & 5.80 & 4.50 \\
\cline { 2 - 3 } \cline { 4 - 5 } & post & & 6.20 & 4.95 \\
\hline \multirow{2}{*}{ THOP } & prä & \multirow{2}{*}{31} & 5.32 & 5.96 \\
\cline { 2 - 3 } & post & & 2.26 & 3.26 \\
\hline
\end{tabular}

WG: Wartegruppe

THOP: THOP-Gruppe

n: $\quad$ Anzahl der Kinder pro

Gruppe

M: $\quad$ Mittelwert

SD: Standardabweichung

prä: Vortest

post: Nachtest

Abbildung 10

Darstellung der Leistung im Subtest Visuelles Scanning (nicht-kritische Trials, Fehler) im

Prä-Post-Vergleich der Wartegruppe und dem THOP

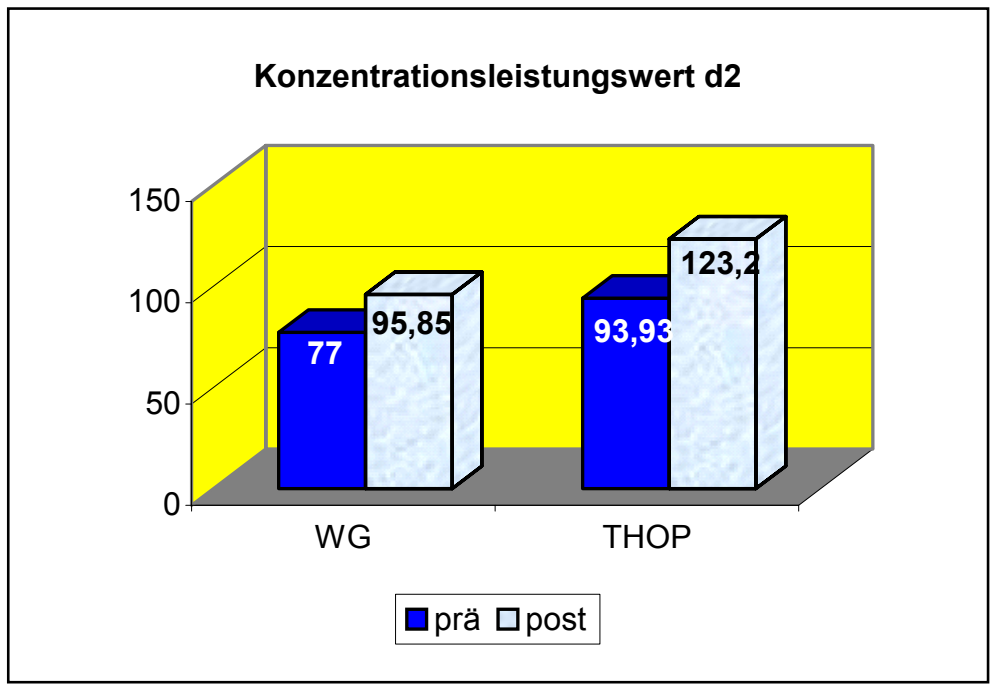

\begin{tabular}{|c|c|c|c|c|}
\hline & & $\mathrm{n}$ & $\mathrm{M}$ & $\mathrm{SD}$ \\
\hline \multirow{2}{*}{ WG } & prä & \multirow{2}{*}{13} & 77.00 & 26.59 \\
\cline { 2 - 3 } \cline { 4 - 5 } & post & & 95.85 & 25.78 \\
\hline \multirow{2}{*}{ THOP } & prä & \multirow{2}{*}{15} & 93.93 & 25.92 \\
\cline { 2 - 3 } & post & & 123.20 & 25.27 \\
\hline
\end{tabular}

WG: Wartegruppe

THOP: THOP-Gruppe

n: $\quad$ Anzahl der Kinder pro

Gruppe

M: $\quad$ Mittelwert

SD: $\quad$ Standardabweichung

prä: Vortest

post: Nachtest

\section{Abbildung 11}

Darstellung der Leistung im Konzentrations- und Belastungstest d2 (Fehler) im Prä-PostVergleich der Wartegruppe und dem THOP 


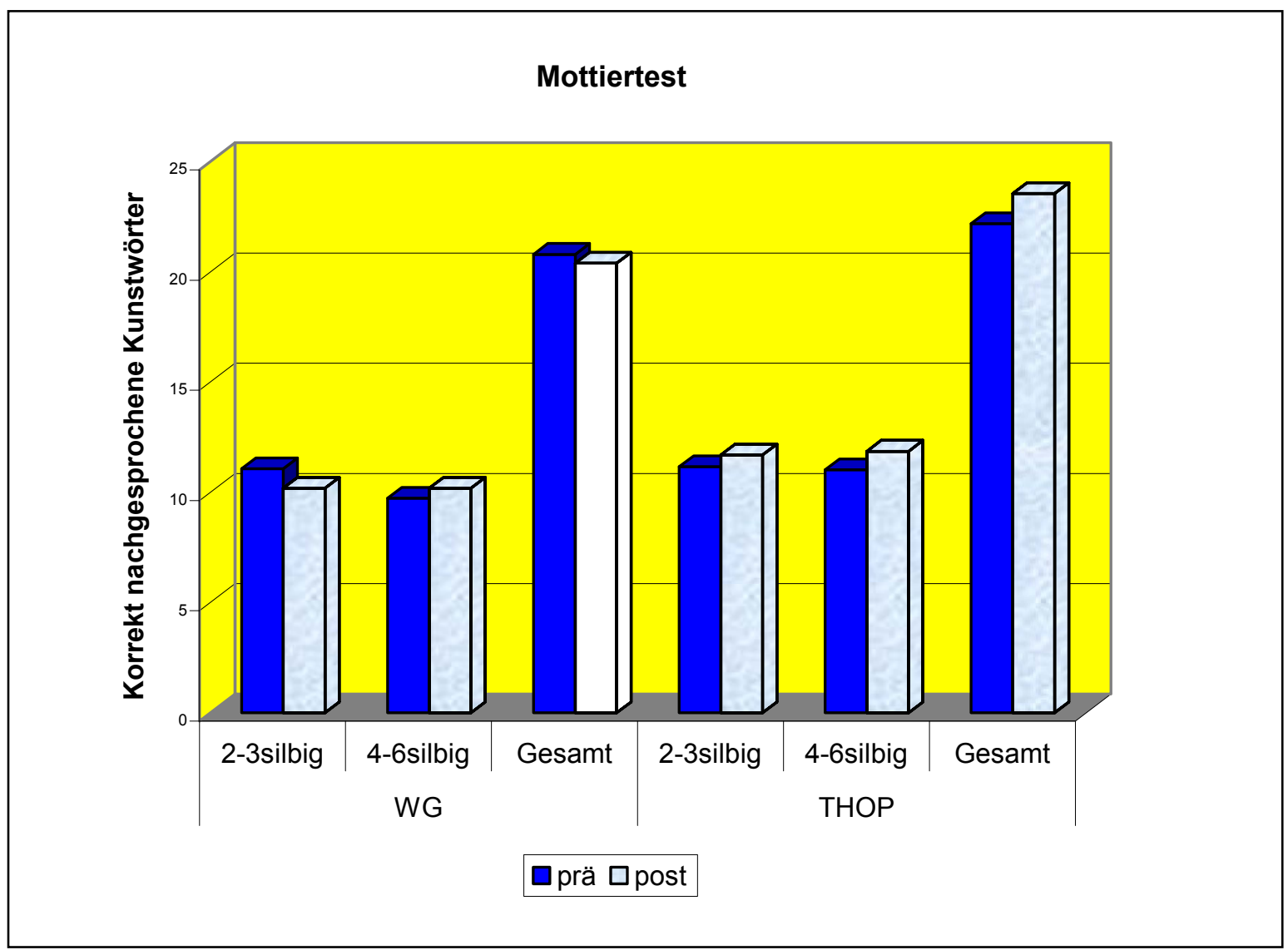

\begin{tabular}{|c|c|c|c|c|c|}
\hline & Wörter & & $\mathrm{n}$ & $\mathrm{M}$ & $\mathrm{SD}$ \\
\hline \multirow{2}{*}{ WG } & \multirow{4}{*}{ 2- und 3silbig } & prä & \multirow{2}{*}{15} & 11.07 & 1.53 \\
\hline & & post & & 10.20 & 2.21 \\
\hline \multirow{2}{*}{ THOP } & & prä & \multirow{2}{*}{30} & 11.17 & 1.02 \\
\hline & & post & & 11.70 & 0.60 \\
\hline \multirow{2}{*}{ WG } & \multirow{4}{*}{ 4-, 5- und 6silbig } & prä & \multirow{2}{*}{15} & 9.73 & 3.17 \\
\hline & & post & & 10.20 & 2.21 \\
\hline \multirow{2}{*}{ THOP } & & prä & \multirow{2}{*}{30} & 11.03 & 3.40 \\
\hline & & post & & 11.87 & 3.46 \\
\hline \multirow{2}{*}{ WG } & \multirow{4}{*}{ Gesamt } & prä & \multirow{2}{*}{15} & 20.80 & 3.97 \\
\hline & & post & & 20.40 & 5.01 \\
\hline \multirow{2}{*}{ THOP } & & prä & \multirow{2}{*}{30} & 22.20 & 4.00 \\
\hline & & post & & 23.57 & 3.79 \\
\hline
\end{tabular}

Anmerkungen:

WG: Wartegruppe

THOP: Therapiegruppe THOP

n: $\quad$ Anzahl der Kinder pro Gruppe

M: $\quad$ Mittelwert

SD: Standardabweichung

2- und 3silbig: Korrekt nachgesprochene 2und 3silbige Kunstwörter Mottiertest 4-, 5- und 6silbig:

Korrekt nachgesprochene

4-, 5- und 6silbige Kunstwörter Mottiertest

Gesamt: Alle korrekt nachgesprochenen Kunstwörter Mottiertest

\section{Abbildung 12}

Darstellung der Nachsprechleistung im Mottier-Test im Prä-Post-Vergleich der Wartegruppe und dem THOP 
Die Überprüfung der Hypothese 3.2, nach der das THOP als Gruppenprogramm zu einer Verminderung von oppositionellen und aggressiven Verhaltensweisen führt, wurde anhand von Fremdbeurteilungsmaßen vorgenommen. Es ist explizites Ziel des THOP oppositionelles und aggressives Verhalten zu verändern, weshalb hier eine Verbesserung erwartet wurde.

Im Elternurteil verbesserten sich oppositionelle und aggressive Verhaltensweisen im Mittel um sechs T-Wert-Punkte (Externalisierende Verhaltensweisen). Im Lehrerurteil sind die Verbesserungen schwächer, weisen aber tendenziell in die gleiche Richtung, konnten jedoch zufallskritisch nicht abgesichert werden. Das Item des Interaktionsfragebogens („Mein Kind hielt/hält sich an Familienregeln“) belegt eine deutliche Verbesserung und kann im weiteren Sinne als Hinweis für die Bewährung der Hypothese gewertet werden, da es häufig aggressive Verhaltensweisen sind, die die Eltern als Verletzung von Familienregeln beklagen. Die in dem THOP als Gruppentherapieverfahren nachgewiesenen positiven Effekte bei der Verminderung oppositioneller Verhaltensweisen sind ermutigend. Bereits Barkley (1990) und Forehand und McMahon (1981) konnten in diesem Bereich positive Effekte nachweisen. Die verbesserte Kenntnis bei den Eltern führt zwar noch nicht zwingend dazu, dass sich auch Verhaltensverbesserungen bei den Kindern hinsichtlich der Kernsymptomatik einstellen, es ist aber zu erwarten, dass die Eltern insgesamt entspannter mit ihrem Kind umgehen, wenn sie sich weniger hilflos fühlen. Neben der positiveren Wahrnehmung ihres Kindes erleben Eltern, die an einem Training teilgenommen haben, weniger Stress, haben ein besseres Selbstwertgefühl und weniger Eheprobleme (Anastopoulos, Shelton, DuPaul \& Guevremont, 1993).

Mit der dritten Hypothese zu dieser Studie (PH 3.3) wurde postuliert, dass das THOP als Gruppenprogramm zu einer Verminderung emotionaler Beeinträchtigungen führt. Im Elternurteil verbessern sich die THOP-Kinder um sechs T-Wertpunkte, im Lehrerurteil um 3,5 T-Wertpunkte, in letzterem erreichte der Interaktionseffekt fast Signifikanzniveau, weil sich die Wartekinder hier leicht verschlechtern. Aus den beiden Fragebogenwerten konnte deshalb nur tendenziell Bestätigung für die PH 3.3 gefunden werden. Verbesserungen lassen sich jedoch aus dem Interaktionsfragebogen ableiten, der eine Abnahme der Belastung beim Kind anzeigt. 
Da erwartungskonträr die Interaktionseffekte überwiegend nicht signifikant wurden, sollen im Folgenden einige Aspekte diskutiert werden, warum nur schwache Hinweise für die Wirksamkeit des THOP als Gruppenprogramm gefunden werden konnten.

Auf die Zuwächse bei der Wartegruppe wurde schon oben hingewiesen. Auch im Vergleich zum THOP schneiden die Wartekinder relativ gut ab. Döpfner, Frölich und Lehmkuhl (2000) haben die Aufklärung und Beratung der Eltern als ersten Punkt in ihre Leitlinien zur Therapie aufgenommen und als Basis aller weiteren Interventionen beschrieben. Wenn Eltern aufgeklärt sind, gewinnen sie häufig eine andere Sicht von ihrem Kind und der Problematik. Viele Eltern fühlen sich entlastet, wenn sie erfahren, dass die Störung bei den meisten Kindern vorwiegend durch genetische Ursachen bedingt ist und nicht etwa durch Erziehungsprobleme der Eltern. Besser informierte Eltern gehen möglicherweise auch verständnisvoller mit ihrem Kind um.

Auf Ebene der statistischen Tests wurde es den psychologischen Hypothesen aufgrund der relativ geringen Stichprobe und der in den Versuchsgruppen extrem großen Binnenstreuungen relativ erschwert sich zu bewähren. Damit die Signifikanzentscheidung positiv ausfällt, müssen bei diesen Voraussetzungen schon sehr große Effekte vorliegen. Bei den Intragruppenveränderungen haben sich in den Fremdbeurteilungsmaßen in der Wartegruppe mit einer Ausnahme keine Veränderungen ergeben, in der THOP-Gruppe wurden dagegen von den Eltern in allen drei Kernbereichen und von den Lehrern Verbesserungen im Aufmerksamkeitsverhalten und der Gesamtbelastung berichtet. Bedenkt man, dass die in der Versuchsplanung errechnete Irrtumswahrscheinlichkeit $\beta$ mit 0.41 relativ groß war, wurde die Signifikanzentscheidung gegen die Alternativhypothese zudem mit einer hohen Wahrscheinlichkeit fälschlich getroffen.

Erklärungen für die hohen Binnenstreuungen sind zum einen die Heterogenität der Diagnosen, zum anderen starke Unterschiede im Störungsgrad. Viele Kinder litten zudem unter weiteren Störungen. Dieser hohe Grad an komorbiden Störungen ist in der Praxis nicht ungewöhnlich, zu fragen ist unter solchen Bedingungen aber nach differenziellen Effekten. Um zu klären, ob manche Kinder besonders gut, andere hingegen kaum oder gar nicht von der Therapie profitiert haben, wurde anhand der vorliegenden Daten versucht, Anhaltspunkte für den moderierenden Einfluss der Merkmale Intelligenz, Alter und Medikation zu finden. Die Untersuchung ergab, dass das Alter und die Intelligenz kaum einen moderierenden Effekt haben. Kinder jedoch, die gleichzeitig zum Trainingszeitraum medikamentös behandelt 
wurden, zeigten größere Veränderungen in externalisierenden, hyperaktiven und impulsiven Verhaltensweisen als Kinder ohne Psychostimulanzientherapie. Aufgrund der insgesamt geringen Effekte ist davon auszugehen, dass letztere so gut wie gar nicht vom Training profitiert haben. Der geringe Gruppeneffekt lässt sich vielleicht dadurch erklären, dass jene Hälfte der THOP-Gruppe, die nicht medikamentös behandelt wurde, das Gesamtergebnis der Gruppe negativ beeinflusst hat. Welchen Beitrag die jeweilige Interventionsmethode an den Effekten geliefert hat, ist aufgrund der Konfundierung von medikamentöser und psychotherapeutischer Behandlung nicht festzustellen. Dieser Befund spricht gleichzeitig dafür, dass eine multimodale Behandlung einer unimodalen überlegen ist.

In den Gesprächen mit den Eltern wurde deutlich, dass manche Schwierigkeiten damit hatten, die verhaltenstherapeutischen Interventionen umzusetzen. Typischerweise sind dies einerseits Eltern mit geringem Bildungsgrad und/oder allein erziehende Eltern (Döpfner, Schürmann \& Frölich, 1998). Andererseits konnte beobachtet werden, dass auch einige Eltern mit höherem Bildungsniveau dazu neigten Interventionen ständig zu hinterfragen und lang anhaltend in der Elterngruppe wie auch zu Hause zu diskutieren. Diese Eltern hatten dann am Ende genauso wenig umgesetzt, wie die Familien, die vorgaben keine Zeit gehabt zu haben.

Ebenfalls zwei konträre Effekte ließen sich in Hinsicht auf das Alter beobachten: Die Störungssymptomatik und begleitende Beeinträchtigungen waren bei älteren Kinder stärker verfestigt. Die bei ihnen und ihren Eltern eingespielten Verhaltensweisen zu verändern erwies sich als schwierig. Jüngere Kinder vermochten sich noch nicht so gut in die Gespräche einzulassen, da es ihnen an Problemeinsicht mangelte. Viele von ihnen versuchten sich durch Kaspereien in den Mittelpunkt zu rücken. Die Analyse von Moderatoreffekten ist allerdings unter Vorbehalt zu betrachten, da durch den Mediansplit die erhaltenen Gruppen z.T. relativ klein waren.

Anhand der Stundenprotokolle ließ sich rückblickend feststellen, dass insbesondere bei Kindern mit komorbid vorhandenen aggressiven Verhaltensstörungen die Eltern den Schwerpunkt auf die Verbesserung von Wutausbrüchen, aufsässigem Verhalten oder ständige Streitereien ihrer Kinder mit ihnen und Geschwistern legten. Die Verbesserung der hyperkinetischen Symptomatik war vielen Eltern weniger wichtig. Hierfür spricht auch der Befund, dass der Wert für externalisierende Störungen mit allen Items aus dem Interaktionsfragebogen deutlich höher korreliert ist als mit den Indizes für die Kernsymptomatik. Mit Abnahme der Belastungen des Kindes und der Eltern sowie der Zunahme positiver Interaktionen und Bewältigungsmöglichkeiten war in erster Linie eine Abnahme oppositioneller und aggressiver Verhaltensweisen verbunden. 
Bei einigen Bausteinen wirkten sich geringe Rechtschreibfähigkeiten ungünstig aus. Wenn die Kinder Veränderungswünsche notieren und ein „Was-ist-schön-Tagebuch“ führen sollten, waren sie nur schwer zu motivieren. Dies könnte ein weiterer differenzieller Effekt in solchen Therapien mit Kindern sein, er wurde hier aber nicht gesondert erfasst.

Die Störungsschwere stellte einen weiteren Einflussfaktor auf die Effektivität dar. Zu Beginn des Trainings wurden von vielen Eltern und/oder Lehrern nur verhältnismäßig geringe Beeinträchtigungen im familiären bzw. schulischen Umfeld berichtet, während bei einigen Kindern extreme Schwierigkeiten bestanden. Bei erstgenannten Kindern können dadurch kaum substanzielle Veränderungen in positiver Richtung erwartet werden. Im Gegenteil kann bei Wiederholungsmessungen eine Regression zur Mitte auftreten, was bedeutet, dass jene Kinder im Nachtest eher mittlere Werte aufweisen. Da dies für relativ viele Kinder der Stichprobe zutrifft, kann je nach Reliabilität des Messverfahrens mit mehr oder minder starken Regressionen gerechnet werden, was zu einer Verzerrung der Effektivitätsschätzung für das THOP führt. Bei Kindern mit ausgeprägtem Störungsgrad könnte dagegen die Therapie nicht ausreichend gewesen sein, um ihre starken Beeinträchtigungen deutlich zu verringern, was sowohl an kindlichen als auch an elterliche Faktoren gelegen haben könnte. Die Problematik des Kindes ist unter Umständen ein Marker für gravierende Probleme der Eltern (Barkley, 1997). Bei mehreren Familien waren die äußeren Umstände so schwierig, dass mit den zur Verfügung stehenden Mitteln ein substanzieller Erfolg nicht zu erwarten war. Hierzu zählt z.B. eine allein erziehende Mutter mit vier Kindern, die aufgrund der zeitlichen Belastung einige Bausteine nicht umsetzen konnte. Bei einer Familie torpedierte der getrennt lebende Vater die Bemühungen der Mutter. Schwerwiegende Probleme der Eltern wirkten sich außerdem negativ auf die Gruppengespräche aus, so dass empfohlen wird bei Hinweisen auf starke (psychische) Belastungen der Eltern von einem Gruppensetting abzusehen oder begleitende individuelle Beratung anzubieten um dies aufzufangen.

Ein weiterer Punkt, der hinsichtlich der Wirksamkeit eine Rolle gespielt haben könnte, ist in der Auswahl der Abhängigen Variablen begründet. Mittels der hier eingesetzten Fremdbeurteilungsbögen wurde überprüft, ob die Kinder wichtige Verhaltensänderungen, die durch das Training erreicht werden sollten, auf Alltagssituationen übertragen können, während mit den objektiven Verfahren erfasst wird, inwiefern die Kinder zu einem Aufgabentransfer in der Lage sind. Für eine Bewährung der Hypothesen mussten somit relativ strenge Wirksamkeitskriterien erfüllt werden. Anhand dieser Kriterien können nach Hager und Hasselhorn (2000) die für die Beurteilung der Güte einer Interventionsmaßnahme 
wichtigen Aussagen überprüft werden, ob mit dem Training außer Performanz- auch Kompetenzveränderungen verbunden waren. Würden bei den AVn nur trainingsähnliche Anforderungen erfasst, wären Veränderungen auf der Kompetenzebene nicht zu beurteilen. Die Autoren des THOP wiesen in den von ihnen publizierten Einzelfallanalysen anhand einer so genannten individuellen Problemliste nach, bei der sich Eltern, Kind und Therapeut auf etwas drei bis vier Verhaltensprobleme einigen, die durch die Intervention vermindert werden soll. Der Erfolg der Intervention wurde auf einer sechsstufigen Antwortskala beurteilt, die subjektive Problembelastung anhand einer zehnstufigen Skala eingeschätzt. Döpfner, Schürmann und Frölich (1998) konnten im häuslichen Bereich durch die Therapie die Verhaltensprobleme fast gänzlich beseitigen. In dieser Untersuchung (Studie 3) konnten über den Interaktionsfragebogen ähnliche Effekte beschrieben werden. Die Eltern gaben in diesem Beurteilungsinstrument an, dass sich das Kind wesentlich besser an Familienregeln halten kann. Die Veränderung einzelner problematischer Verhaltensweisen darf nicht das Endziel der Therapie markieren. Das wichtigste Kriterium für einen Therapieerfolg ist eine Symptomverringerung.

Mit Vorsicht sind überdies die abhängigen Maße der Testbatterie zur Aufmerksamkeitsprüfung zu bewerten. Über die TAP liegt lediglich eine Validitätsstudie mit hyperkinetisch auffälligen Kindern (Földényi, Imhof \& Steinhausen, 2000) und nur eine weitere zur Reliabilität und Retest-Stabilität einiger Untertests des Verfahrens vor (Földényi, Giovanoli, Tagwerker-Neuenschwander, Schallberger \& Steinhausen, 2000). In letzterer wurde eine ungenügende Retest-Reliabilität nachgewiesen, die vor allem durch differenzielle Übungseffekte erklärt wurde. Gelöst werden müsste auch das Problem der starken Übungseffekte, welche bei Wiederholungen von Konzentrationstesten beobachtet werden. Für die Evaluation von Trainingsstudien erscheinen sie nur bedingt geeignet.

Der Gesamtauffälligkeitswert der CBCL 4-18 und TRF ist anscheinend kein optimales Maß gewesen um emotionale Beeinträchtigungen zu erfassen, weil sich hier neben Aspekten des emotionalen Befindens auch solche sozialer Probleme und schizoid-zwanghafter Verhaltensweisen ausdrückten. Es ist denkbar, dass die Probleme, die auf die Hyperkinetische Störung und die externalisierenden Verhaltensweisen zurückgehen, so vorherrschend waren, dass den Eltern emotionale Probleme nicht aufgefallen sind. Gleichwohl leiden viele Kinder mit Hyperkinetischen Störungen auch unter emotionalen Problemen. Das hier eingesetzte $\mathrm{Maß}$ zur Erfassung dieser Probleme ist vermutlich nicht differenziert genug. 
In dieser Studie wurde für alle Gruppen ein Umfang von 15 Sitzungen festgelegt. Dies wurde zum einen wegen der direkten Vergleichbarkeit mit dem kombinierten Basis- und Strategietraining nach Lauth und Schlottke (15 Sitzungen) ausgemacht, zum anderen war dies eine Anzahl, die die meisten Eltern gut durchhalten konnten. Viele Familien mussten für die Therapien, die am Nachmittag durchgeführt wurden, größere organisatorische Mühen auf sich nehmen, z.B. Geschwisterkinder betreuen lassen. Eine Ausdehnung des Trainings auf weitere Sitzungen wurde aus diesen Gründen nicht durchgeführt. Die Autoren schlagen jedoch für ein Einzelsetting 10 bis 40 Sitzungen vor, so dass in dieser Studie vermutlich der Umfang des Trainings zu gering veranschlagt war, um der Komplexität der durchgeführten einzelnen Bausteine gerecht werden zu können. Möglich ist durchaus, dass die vorgegebene Zeit für einige Kinder nicht ausreichend war. Dagegen spricht allerdings, dass nach Scheithauer und Petermann (2000) Studien im Kindesalter keinen Zusammenhang zwischen einer größeren Anzahl an Therapiesitzungen oder einer längeren Dauer der Intervention und der Effektivität ermittelten.

Von größerer Bedeutung scheint aber die Intensität zu sein, mit der die verschiedenen Bausteine des THOP behandelt werden und ob die Familien von der Gruppe als solcher profitieren konnten. Bei den Kindern war ein Entlastungseffekt, der dadurch entstehen sollte, dass sie mit Kindern zusammenkommen, die ähnliche Probleme wie sie haben, nicht zu erkennen. Die Gespräche innerhalb der Kindergruppen waren wenig zielorientiert. Es mussten große Bemühungen darauf verwandt werden, die Kinder zum konzentrierten Arbeiten zu bringen oder sie davon abzuhalten andere Kinder abzulenken. Trotz operanter Verstärkung war es einigen Kindern kaum möglich im Gruppenverband zu arbeiten. Auf die Frage am Ende der Behandlung, was ihnen in der Therapie am besten gefallen hat, antworteten die Kinder, dass sie Geschenke eintauschen konnten oder Spielsachen mitbringen durften. Der Leidensdruck, der durch die Umwelt induziert wird, führt nicht zwangsläufig zu einer entsprechenden Therapiemotivation bei den Kindern. Da mangelnde Problemeinsicht und -reflexion bei Kindern mit Hyperkinetischer Störung als Phänomen bekannt ist, könnte ein Ziel der Behandlung sein, ein Problembewusstsein aufzubauen. Im THOP soll dieses Ziel über die Geschichten vom Wackelpeter erreicht werden. Die meisten Kinder konnten sich aber mit dieser Figur nicht identifizieren. Da die Kinder nicht zu einer Auseinandersetzung mit ihren Schwierigkeiten motiviert werden konnten, kam die Gruppe als soziales Übungsfeld für veränderte Verhaltensmuster nicht zum Tragen. Zudem waren einige Kinder nicht an den 
Interventionen interessiert, die mit den Eltern abgesprochen worden sind (z.B. Punktepläne). Ungünstig wirkte sich in den Gruppen ein zu großer Altersunterschied aus, da die älteren Kinder bei den spielerischen Inhalten den jüngeren zu stark überlegen waren. Auch der unterschiedliche Ausprägungsgrad der hyperkinetischen Störung kann sich ungünstig auf die Gruppe auswirken. Für manche Kinder war der Grad der Komplexität der Trainingsinhalte zu hoch. Er sollte eher niedrig gehalten werden, um die Kinder nicht zu überfordern und nicht zu entmutigen.

Wenn auch die Resultate der Kindergruppen wenig ermutigend sind, so waren doch die Rückmeldungen von den Elterngruppen während und nach der Therapie überwiegend sehr positiv. Nur selten wurden Sitzungen versäumt, die Diskussionen waren meistens konstruktiv, und es kam zu einem fruchtbaren Gedankenaustausch zwischen den Eltern. Es gab aber auch Familien, in denen die Probleme so schwerwiegend waren, dass sie in der Gruppe nicht hinlänglich beraten werden konnten. Für diese Familien wäre ein individuelleres Angebot hilfreicher gewesen. Einigen Familien wurde deshalb nach der Gruppentherapie eine Fortsetzung in einem Einzelsetting angeboten.

Da innerhalb der Gruppe bestimmte Themen stark fokussiert wurden, kann am Ende der Therapie ein zunächst negativer Effekt hinsichtlich der Bewertung der Probleme dadurch entstanden sein, dass manchen Eltern einige schon bestehende Probleme erst durch die Behandlung bewusst wurden.

Einige spezifische Interventionen wurden von manchen Eltern stark hinterfragt und für sie als wenig geeignet eingestuft (z.B. Punktepläne). Dies führte dazu, dass Hausaufgaben nur halbherzig umgesetzt wurden, z.T. waren sich die Eltern bei der Umsetzung nicht sicher genug. Manche Eltern hatten Probleme damit verschiedene Maßnahmen gleichzeitig anzuwenden. Therapeuten sollten diese Probleme bei der Umsetzung der Interventionen mit einplanen. Mangelndes Engagement oder Verständnisschwierigkeiten der Eltern erfordern von Seiten des Therapeuten eine entschiedene Intervention, damit die Eltern das Gelernte im Alltag umsetzen.

Mit dem vom Verfasser entworfenen Interaktionsfragebogen konnte für die Familien, die am THOP teilgenommen hatten, erfasst werden, wie wirksam die Eltern ihre Erziehungsmaßnahmen im Vergleich von vor zu nach dem Training einschätzen. Für alle Variablen gaben die Eltern deutliche Verbesserungen an und spendeten auch in den Gesprächen nach Abschluss der Therapie ein positives Feedback. Zum Interaktionsfragebogen muss einschränkend gesagt werden, dass er kein validiertes 
Erhebungsinstrument darstellt, die Aussagen durch objektivierbare Daten (z.B. direkte systematische Verhaltensbeobachtung der Eltern-Kind-Interaktion) nicht verifiziert wurden und die zum Teil retrospektiven Daten aufgrund von Erinnerungs- und Selektionseffekten verzerrt sein können. Leider liegen keine Vergleichswerte für die Wartegruppe und für die Lauth- und Schlottke-Gruppe vor. Es ist denkbar, dass die Eltern nur aufgrund der Tatsache, dass sie an einem mehrmonatigen Training teilgenommen haben, zu einer positiven Beurteilung gekommen sind. Ohne Vergleichswerte aus den beiden anderen Gruppen sollten die festgestellten Veränderungen nicht zwangsläufig dem THOP zugesprochen werden. Zumindest kann aber aus den Ergebnissen eine hohe Zufriedenheit der Eltern mit dem Training abgeleitet werden. Das Wissen der Eltern über Hyperkinetische Störungen und verhaltensmodifizierende Maßnahmen wurde durch das Training deutlich verbessert, es mangelte aber an der Umsetzung durch die Eltern im familiären Alltag.

Auch in dieser Studie wurden die Lehrer nicht in therapeutische Maßnahmen einbezogen. Es wurden zwar einige Bausteine mit den Kindern bearbeitet, welche Strukturierungshilfen für Hausaufgaben sowie Selbstinstruktionstechniken für planvolles Arbeiten beinhaltete. Diese Themen wurden aber insgesamt nur in einem geringen Umfang mit den Kindern eingeübt. Um hier eine erfolgreiche Umsetzung im Schulalltag zu erreichen, hätten vermutlich die Lehrer einbezogen werden müssen. Besonders für kognitive Methoden wurde oft der fehlende Transfer auf den schulischen Alltag bemängelt (Mackowiak \& Hungerige, 2001). Einige Kinder wollten die im Selbstinstruktionstraining-Baustein eingeführten Signalkarten nicht einsetzen, da sie befürchteten damit von anderen Mitschülern gesehen zu werden. Hinshaw, Henker und Whalen (1984) belegen, dass Therapieinhalte dann eher von den Kindern angewandt werden, wenn sie durch Eltern oder Lehrer außerhalb der Behandlungssituation angemessen verstärkt werden.

Ein Transfer in nicht trainierte Bereiche gelingt offensichtlich nicht. Dies wird durch diese Untersuchung erneut belegt. Es ist deshalb notwendig, überall dort anzusetzen, wo die Probleme auftauchen, also in der Familie, im Unterricht usw.

Dass die Ergebnisse der kognitiven Verhaltenstherapien bei diesen Kindern eher ernüchternde Ergebnisse erbringen, wird von Frölich, Döpfner, Berner und Lehmkuhl (2002) auf verschiedene Ursachen zurückgeführt. Oft sind die in den Trainings behandelten Problemstellungen zu schulfern. Den Kindern fällt es aufgrund der Transferprobleme schwer die Trainingsinhalte auf schulische Kontexte zu übertragen. 
Als Fazit zu diesem Programm kann festgestellt werden, dass durch das THOP als Gruppenprogramm insbesondere das Interaktionsverhalten innerhalb der Familie verbessert werden kann, die Kinder weniger oppositionell und aggressiv reagieren und die Eltern sich bei der Durchführung von Erziehungsmaßnahmen kompetenter fühlen. Die Kernsymptomatik ließ sich durch das Gruppenprogramm aber nur geringfügig verbessern. Sowohl Eltern als auch Kinder werden nach Einschätzung der Eltern nach dem Training psychisch als weniger stark belastet erlebt.

6.1.4 Isolierte Evaluation des Marburger Konzentrationstrainings (Studie 4)

Ein wichtiger Aspekt bei Trainingsmaßnahmen ist die Dauer der Durchführung (Trainingsumfang bzw. -dauer) und der Fortführung der erlernten Maßnahmen nach dem Training. Ein Programm sollte solange fortgesetzt werden, bis die erlernten Inhalte verinnerlicht sind. Hier gilt es natürlich Kosten und Nutzen gegeneinander abzuwägen. Je kürzer das Training ist, desto günstiger ist die Maßnahme, aber desto fraglicher ist möglicherweise auch der Erfolg der Bemühungen.

Um neben den umfangreicheren Programmen von Lauth und Schlottke (1997), welches auf der Methode der Selbstinstruktion aufbaut, sowie dem THOP ein sehr viel kürzeres auf seine Wirksamkeit zu untersuchen, wurde in dieser Studie die Wirksamkeit des Marburger Konzentrationstrainings bei Kindern mit Aufmerksamkeitsstörungen untersucht. In der Praxis der Versorgung von Kindern mit Hyperkinetischen Störungen in Deutschland gibt es ein großes Missverhältnis von Kindern, bei denen die Diagnose einer Hyperkinetischen Störung gestellt wird, und der Möglichkeit diese Kinder nach Diagnosestellung psychotherapeutisch zu behandeln. Häufig werden langwierige Einzeltherapien oder aufwändige Therapien mit kleinen Gruppen durchgeführt.

Auch wenn Krowatschek nicht den Anspruch formuliert, dass das Marburger Konzentrationstraining explizit für Kinder mit der klinischen Diagnose einer Aufmerksamkeitsstörung als Zielgruppe konzipiert ist, hat es in Deutschland dennoch eine große Verbreitung gefunden. Mit dieser Studie wurde eine Überprüfung vorgenommen. Die Ergebnisse werden im Folgenden anhand der aufgestellten Hypothesen diskutiert.

Mit der Psychologischen Hypothese (PH 4.1) sollte überprüft werden, ob durch das Marburger Konzentrationstraining bei Kindern mit Aufmerksamkeitsstörungen eine verbesserte Konzentrationsleistung erzielt wird, wenn es im Rahmen eines Gruppentrainings in einer Kinder- und Jugendpsychiatrischen Praxis durchgeführt wird. 
Die Hypothese hat sich nicht bewährt. In keiner der zugeordneten Abhängigen Variablen der TAP und der Paper-Pencil-Verfahren konnte ein Interaktionseffekt nachgewisen werden.

Der Rücklauf der Fragebogen war insgesamt so schlecht, dass eine interferenzstatistische Auswertung nicht sinnvoll war.

Bei der Überprüfung der Transferhypothese (PH 4.2), demnach die trainierten Kinder die erarbeiteten Selbstanweisungen auch auf schulische Belange übertragen, lassen sich etwas mehr Ergebnisse zugunsten des Marburger Konzentrationstrainings nachweisen. Hasselhorn und Mähler (2000) weisen auf die Problematik der Definition des Transferbegriffes hin. In Anlehnung an Hager und Hasselhorn (2000) wird bei der psychologischen Hypothese PH 4.2 der Anforderungstransfer überprüft. Für diese wurde den Kindern ein Untersuchungsverfahren vorgegeben, welches die bei Tätigkeiten des Schulalltags aufgewandte Konzentration erfasst (vgl. Kurth \& Büttner, 1999). Die Autoren weisen ausdrücklich darauf hin, dass ihr Verfahren sich auch für die Evaluation von Trainingsprogrammen eignen würde. In vier der sechs Variablen der TPK kommt es zu signifikanten Prä-Post-Veränderungen beim Marburger Konzentrationstraining, wovon einer statistisch signifikant wird. Für die Variable TSL (Silbenanzahl innerhalb von 10 Minuten) konnte ein signifikanter Interaktionskontrast ermittelt werden. Die Kinder der Trainingsgruppe konnten durch aufmerksameres Vorgehen eine gesteigerte Abschreibleistung erbringen. Dies geht auch nicht mit einer Erhöhung der Fehleranzahl einher, so dass hier die Transferhypothese bestätigt werden kann. Dieser Effekt war aber nur bedingt stabil. Wenn die Intelligenz als Kovariate in die Berechnung einbezogen wurde, wird das Ergebnis nicht signifikant. Bei der Berechnung der Moderatorvariablen wurde zudem festgestellt, dass insbesondere die älteren Kinder nach dem Training mehr Silben schreiben konnten. Hier könnte das Resultat also durch das Alter mit beeinflusst worden sein. 


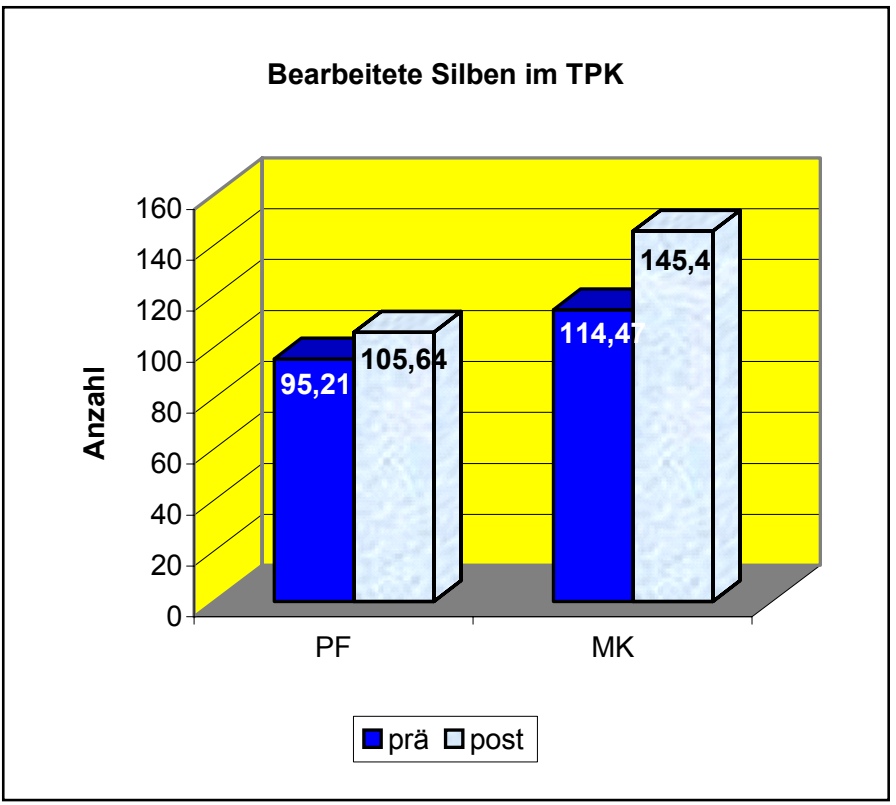

\begin{tabular}{|c|c|c|c|c|}
\hline & & $\mathrm{n}$ & $\mathrm{M}$ & $\mathrm{SD}$ \\
\hline \multirow{2}{*}{ PF } & prä & \multirow{2}{*}{14} & 95.21 & 40.32 \\
\cline { 2 - 3 } \cline { 4 - 5 } & post & & 105.64 & 46.52 \\
\hline \multirow{2}{*}{ MK } & prä & \multirow{2}{*}{15} & 114.47 & 39.45 \\
\cline { 2 - 3 } \cline { 5 - 5 } & post & & 145.40 & 50.34 \\
\hline
\end{tabular}

Anmerkung:

PF: „Petterson und

Findus"

MK: Marburger

Konzentrationstraining

n: Anzahl der Kinder pro

Gruppe

M: $\quad$ Mittelwert

SD: $\quad$ Standardabweichung

prä: Vortest

post: Nachtest

Abbildung 13

Darstellung der bearbeiteten Silben im TPK im Prä-Post-Vergleich des Marburger Konzentrationstrainings mit dem Pseudotraining „Petterson und Findus“

Die PH 4.3, nach der es durch das Training zu einer positiven Veränderung der ElternKind- und Lehrer-Kind-Interaktion kommt, kann nicht beantwortet werden, da der Rücklauf der Fragebögen dafür zu niedrig war. In den Elterngesprächen, die nach dem Training durchgeführt wurden, gaben mehrere Eltern an, dass sich das Verhältnis zu ihren Kindern verbessert habe. Hierbei gab es jedoch keinen deutlichen Unterschied zwischen den Kindern beider Gruppen. Es ist demnach zu vermuten, dass allein die Tatsache, dass die Kinder in eine Intervention eingebunden waren, zu einer Veränderung in der Eltern-Kind Interaktion geführt hat. Die Eltern der Kinder beider Gruppen bekamen in den Praxen durch die Kinder- und Jugendpsychiater einen Überblick über die Störungssymptomatik, Ätiologie und Behandlungsmöglichkeiten. Damit war bei vielen Eltern ein Abbau eigener Ängste bezogen auf Erziehungsfehler verbunden. Durch die veränderte Haltung ihrerseits entsteht häufig auch eine Veränderung in der Interaktion, da die Eltern sich ihren Kindern gegenüber vermehrt ressourcen- und weniger problemorientiert verhalten.

Kritisch einschränkend muss festgehalten werden, dass mit dem hier durchgeführten Stundenumfang von nur fünf Therapiestunden der ohnehin schon niedrig angesetzte vorgeschlagene Stundenumfang von sechs bis acht Stunden von Krowatschek noch 
unterschritten wurde. Für eine Automatisierung der Selbstinstruktionen ist dieser Umfang vermutlich zu gering.

Als Fazit zur Wirksamkeit des Marburger Konzentrationstrainings kann gezogen werden, dass sich durch relativ wenige Therapiestunden in einer Gruppe leichte Verbesserungen im schulischen Bereich erzielen lassen, obwohl in dieser Studie die Lehrer nicht explizit in die Behandlung einbezogen waren. Anhand der psychometrischen Tests konnte keine deutliche Verbesserung der Aufmerksamkeitsleistungen nachgewiesen werden. Eine Beurteilung hinsichtlich der Kernsymptomatik kann aufgrund der geringen Anzahl an vorliegenden Einschätzungen von Eltern und Lehrer nicht vorgenommen werden.

In der hier durchgeführten Studie wurde zu Beginn der Stunden mit den Kindern ein Entspannungsverfahren durchgeführt. Der Beginn des Trainings mit den Kapitän-NemoGeschichten hat sich als sinnvoll erwiesen. Die Kinder konnten durch die Entspannungsgeschichten Erregung abbauen und so die Ruhe finden, welche für die Durchführung eines Selbstinstruktionstrainings in der Gruppe notwendig ist.

Es sei an dieser Stelle auf die Metaanalyse von Saile (1996) verwiesen, welche den Selbstinstruktionstrainings mit einer Effektstärke von 0.3 nur einen kleinen Effekt zuweist. Den Entspannungsverfahren wird jedoch mit einer Effektstärke von 0.83 ein großer Effekt bescheinigt, und für Elterntrainings wird eine mittlere Effektstärke angegeben. Insofern bietet sich eine Kombination von Entspannungsverfahren und Selbstinstruktionstraining sowie Elternarbeit an. Um eine weitere Automatisierung der erlernten Inhalte zu erreichen, wäre eine intensive Zusammenarbeit mit Lehrern wünschenswert.

\subsection{Bewertung der Ergebnisse der vergleichenden Evaluation}

Mit der vergleichenden Evaluation sollte überprüft werden, wie die drei Trainings im Vergleich zueinander bewertet werden können. Nachdem die Ergebnisse der ersten Studie zur Wirksamkeit des Basistrainings nach Lauth und Schlottke relativ schlecht ausfielen, sollte überprüft werden, ob eine Erweiterung um Komponenten des Strategietrainings zu einer Verbesserung der Ergebnisse führt.

Die graphische Veranschaulichung der Ergebnisse geben die Abbildungen 14 bis 19. 


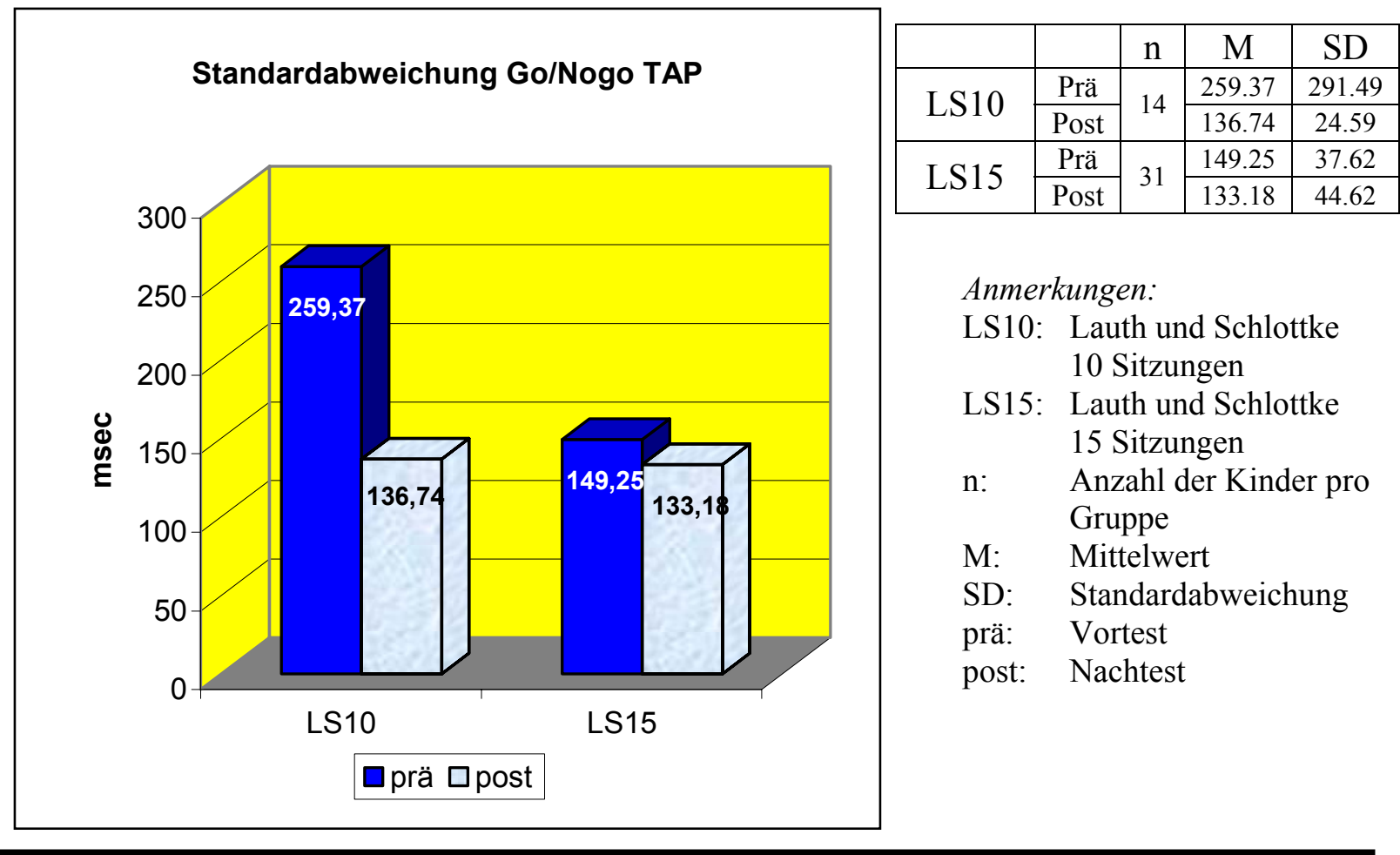

\section{Abbildung 14}

Darstellung der Leistung im Subtest Go/Nogo (Standardabweichung) im Prä-Post-Vergleich des Basistrainings und des kombinierten Basis- und Strategietrainings nach Lauth und Schlottke

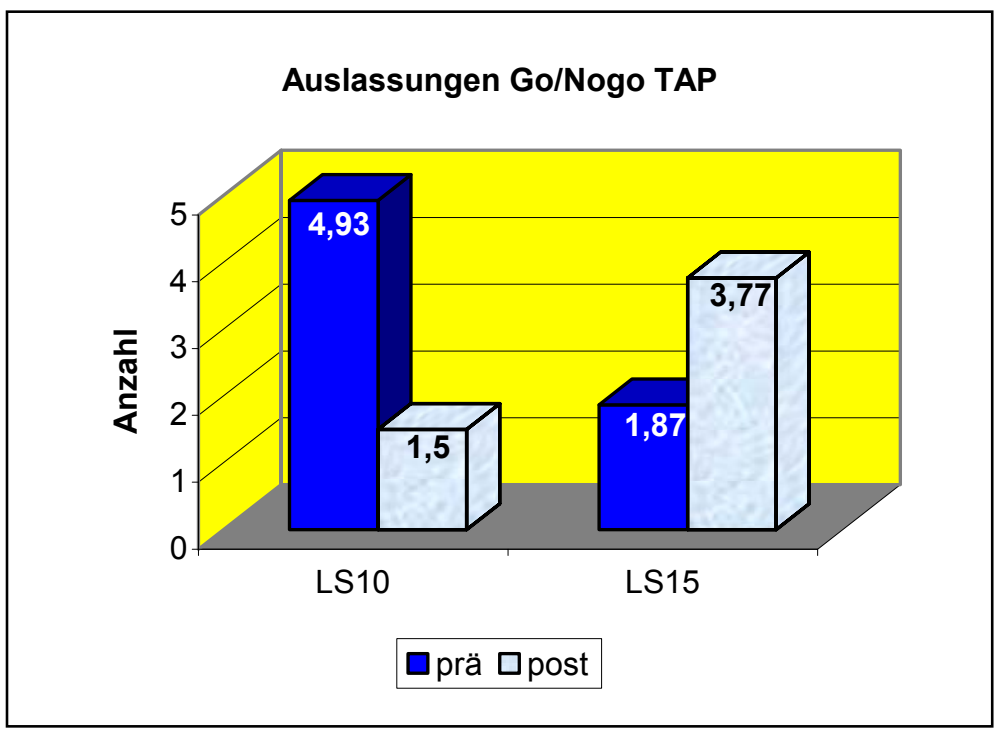

\begin{tabular}{|c|c|c|c|c|}
\hline & & $\mathrm{n}$ & $\mathrm{M}$ & $\mathrm{SD}$ \\
\hline \multirow{2}{*}{ LS10 } & Prä & & 4.93 & 6.24 \\
\cline { 2 - 3 } \cline { 4 - 5 } & Post & & 1.50 & 1.83 \\
\hline \multirow{2}{*}{ LS15 } & Prä & \multirow{2}{*}{31} & 1.87 & 2.13 \\
\cline { 2 - 5 } & Post & & 3.77 & 4.36 \\
\hline
\end{tabular}

Anmerkungen:

LS10: Lauth und Schlottke 10 Sitzungen

LS15: Lauth und Schlottke 15 Sitzungen

n: $\quad$ Anzahl der Kinder pro

Gruppe

M: $\quad$ Mittelwert

SD: $\quad$ Standardabweichung

prä: Vortest

post: Nachtest

\section{Abbildung 15}

Darstellung der Leistung im Subtest Go/Nogo (Auslassungsfehler) im Prä-Post-Vergleich des Basistrainings und des kombinierten Basis- und Strategietrainings nach Lauth und Schlottke 


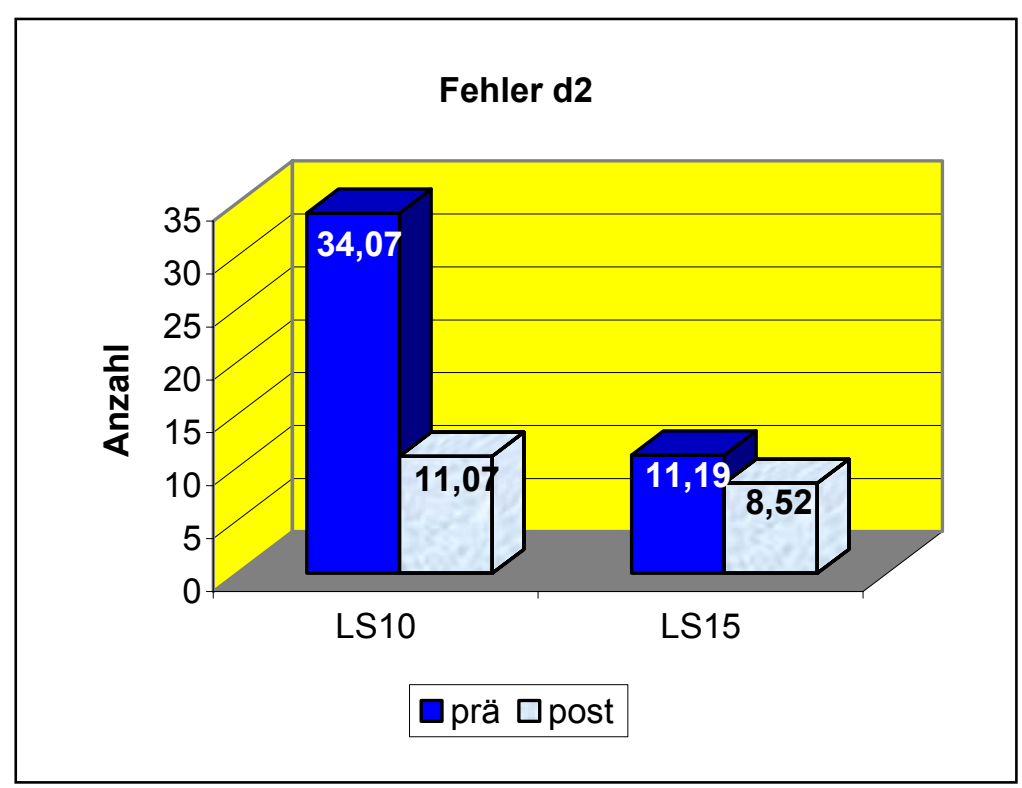

\begin{tabular}{|c|c|c|c|c|}
\hline & & $\mathrm{n}$ & $\mathrm{M}$ & $\mathrm{SD}$ \\
\hline \multirow{2}{*}{ LS10 } & Prä & & 34.07 & 41.29 \\
\cline { 2 - 3 } \cline { 4 - 4 } & Post & & 11.07 & 9.93 \\
\hline \multirow{2}{*}{ LS15 } & Prä & \multirow{2}{*}{21} & 11.19 & 8.69 \\
\cline { 2 - 5 } \cline { 4 - 5 } & Post & & 8.52 & 4.98 \\
\hline
\end{tabular}

Anmerkungen:

LS10: Lauth und Schlottke 10 Sitzungen

LS15: Lauth und Schlottke 15 Sitzungen

n: $\quad$ Anzahl der Kinder pro

Gruppe

M: $\quad$ Mittelwert

SD: $\quad$ Standardabweichung

prä: Vortest

post: Nachtest

Abbildung 16

Darstellung der Leistung im Konzentrations- und Belastungstest d2 (Fehler) im Prä-PostVergleich des Basistrainings und des kombinierten Basis- und Strategietrainings nach Lauth und Schlottke

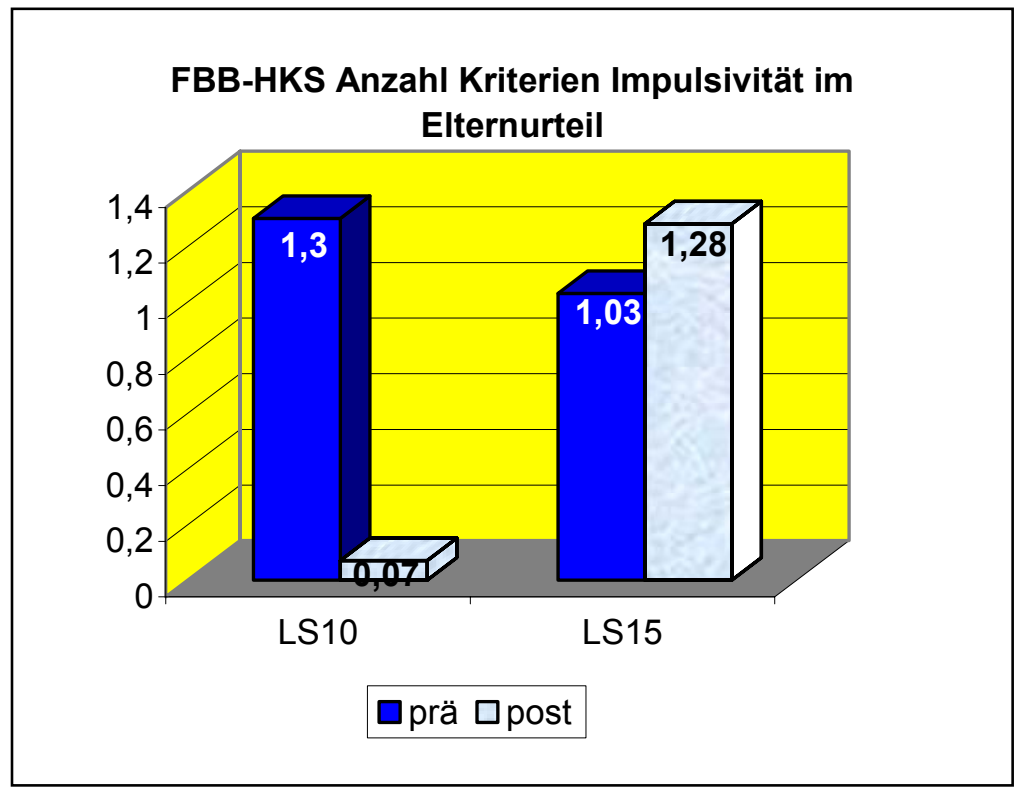

\begin{tabular}{|c|c|c|c|c|}
\hline & & $\mathrm{N}$ & $\mathrm{M}$ & $\mathrm{SD}$ \\
\hline \multirow{2}{*}{ LS10 } & Prä & \multirow{2}{*}{13} & 1.30 & 1.70 \\
\cline { 2 - 3 } \cline { 4 - 5 } & Post & & 0.07 & 0.27 \\
\hline \multirow{2}{*}{ LS15 } & Prä & \multirow{2}{*}{28} & 1.03 & 1.45 \\
\cline { 2 - 3 } \cline { 4 - 5 } & Post & & 1.28 & 1.56 \\
\hline
\end{tabular}

Anmerkungen:

LS10: Lauth und Schlottke 10 Sitzungen

LS15: Lauth und Schlottke 15 Sitzungen

n: $\quad$ Anzahl der Kinder pro Gruppe

M: $\quad$ Mittelwert

SD: $\quad$ Standardabweichung

prä: Vortest

post: Nachtest

\section{Abbildung 17}

Darstellung der Impulsivität im Elternurteil (Kriterien) im Prä-Post-Vergleich des Basistrainings und des kombinierten Basis- und Strategietrainings nach Lauth und Schlottke 


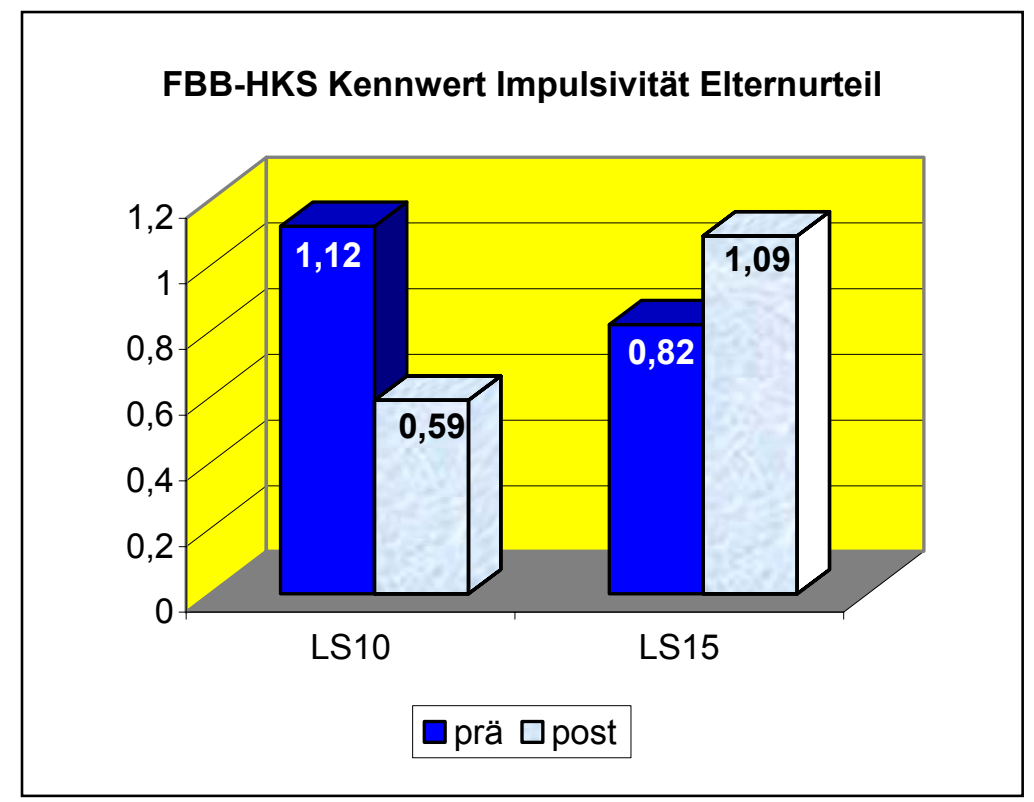

\begin{tabular}{|c|c|c|c|c|}
\hline & & $\mathrm{N}$ & $\mathrm{M}$ & $\mathrm{SD}$ \\
\hline \multirow{2}{*}{ LS10 } & Prä & & 1.12 & 0.79 \\
\cline { 2 - 4 } \cline { 4 - 5 } & Post & & 0.59 & 0.37 \\
\hline \multirow{2}{*}{ LS15 } & Prä & \multirow{2}{*}{28} & 0.82 & 0.75 \\
\cline { 2 - 5 } \cline { 4 - 5 } & Post & & 1.09 & 0.87 \\
\hline
\end{tabular}

Anmerkungen:

LS10: Lauth und Schlottke 10 Sitzungen

LS15: Lauth und Schlottke 15 Sitzungen

n: $\quad$ Anzahl der Kinder pro

Gruppe

M: $\quad$ Mittelwert

SD: $\quad$ Standardabweichung

prä: Vortest

post: Nachtest

Abbildung 18

Darstellung der Impulsivität im Elternurteil (Kennwert) im Prä-Post-Vergleich des Basistrainings und des kombinierten Basis- und Strategietrainings nach Lauth und Schlottke



Abbildung 19

Darstellung der Leistung im Subtest Visuelles Scanning (Auslassungsfehler, kritische Trials) im Prä-Post-Vergleich des Basistrainings und des kombinierten Basis- und Strategietrainings nach Lauth und Schlottke 
Die PH 5.1, nach der das Training nach Lauth und Schlottke zu einer stärkeren Reduzierung der Symptomatik führt, wenn die Kinder sowohl mit dem Basis- als auch mit dem Strategietraining behandelt werden, als wenn sie nur das Basistraining erhalten, musste verworfen werden. Hypothesenkonträr wird der Interaktionsvergleich bei mehreren Variablen zugunsten der Kinder signifikant, welche nur das Basistraining erhalten haben. Die Kinder, welche nur das Basistraining absolvierten, machten weniger Auslassungsfehler im Go/Nogo der TAP und reagierten regelmäßiger. Gleichzeitig verschlechterten sich die Kinder des kombinierten Trainings. Die erstgenannten Kinder erzielten im Konzentrationsleistungswert des d2 eine stärkere Verbesserung und machten weniger Fehler. Außerdem wurden sie von den Eltern als weniger impulsiv beurteilt.

Dieses Ergebnis ist auf den ersten Blick erstaunlich, konnte doch erwartet werden, dass Kinder, die ein umfangreicheres Training erhalten haben, auch zu besseren Ergebnissen kommen, da länger an ihren Defiziten gearbeitet wurde. Auf den zweiten Blick sind die Ergebnisse jedoch gut zu erklären, da die Abhängigen Variablen eher soche Fertigkeiten überprüfen, die im Basistraining vermittelt werden. Die zusätzlichen Effekte, die durch die Einheiten des Strategietrainings erzielt wurden, konnten sich vermutlich in den AVn nicht niederschlagen. Es wird hier jedoch auch deutlich, dass eine Erweiterung eines Trainings nicht unbedingt $\mathrm{zu}$ besseren Ergebnissen führen muß, sondern die Anwendung gelernter Fertigkeiten sogar verhindern kann, weil die Kinder mit mehreren Strategien, die sie gleichzeitig versuchen einzusetzen, überfordert sind.

Mit der PH 5.2 sollte überprüft werden, ob das Training nach Lauth und Schlottke in gleichem Maße zu einer Reduzierung führt wie das Marburger Konzentrationstraining. Zur Bewertung dieser Hypothese können leider nur die Ergebnisse der TAP und des d2 herangezogen werden, da nur sie in ausreichender Zahl vorliegen und gleichzeitig in beiden Gruppen durchgeführt wurden.

Die graphische Veranschaulichung der Ergebnisse geben die Abbildungen 20 und 21. 


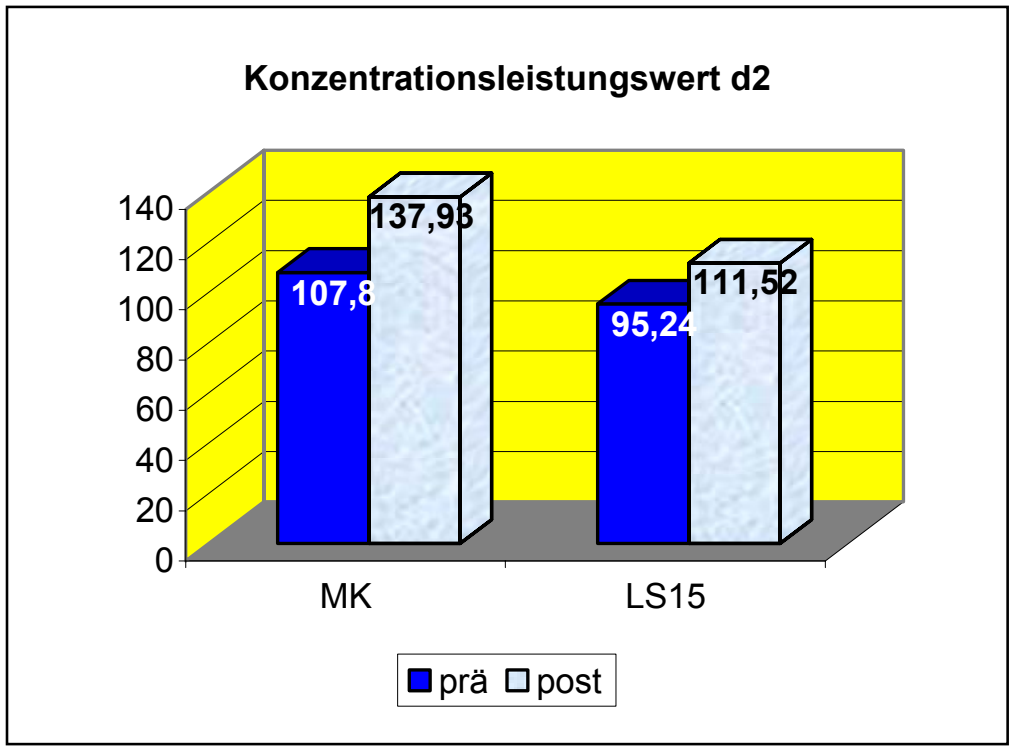

\begin{tabular}{|c|c|c|c|c|}
\hline & & $\mathrm{n}$ & $\mathrm{M}$ & $\mathrm{SD}$ \\
\hline \multirow{2}{*}{$\mathrm{MK}$} & Prä & \multirow{2}{*}{15} & 107.80 & 26.56 \\
\cline { 5 - 5 } \cline { 4 - 5 } & Post & 137.93 & 33.93 \\
\hline \multirow{2}{*}{ LS15 } & Prä & \multirow{2}{*}{21} & 95.24 & 21.93 \\
\cline { 5 - 5 } \cline { 4 - 5 } & Post & & 111.52 & 20.07 \\
\hline
\end{tabular}

Anmerkungen:

MK: Marburger

Konzentrationstraining

LS15: Lauth und Schlottke 15 Sitzungen

n: Anzahl der Kinder pro Gruppe

M: $\quad$ Mittelwert

SD: Standardabweichung

prä: Vortest

post: Nachtest

Abbildung 20

Darstellung der Leistung im Konzentrations- und Belastungstest d2 (Fehler) im Prä-PostVergleich des Marburger Konzentrationstraining und des kombinierten Basis- und Strategietrainings nach Lauth und Schlottke



\begin{tabular}{|c|c|c|c|c|}
\hline & & $\mathrm{N}$ & $\mathrm{M}$ & $\mathrm{SD}$ \\
\hline \multirow{2}{*}{$\mathrm{MK}$} & Prä & \multirow{2}{*}{15} & 14.40 & 12.11 \\
\cline { 2 - 3 } & Post & & 5.67 & 4.10 \\
\hline \multirow{2}{*}{ LS15 } & Prä & \multirow{2}{*}{21} & 11.19 & 8.69 \\
\cline { 5 - 5 } \cline { 4 - 5 } & Post & & 8.52 & 4.98 \\
\hline
\end{tabular}

Anmerkungen:

MK: $\quad$ Marburger

Konzentrationstraining

LS15: Lauth und Schlottke 15 Sitzungen

n: $\quad$ Anzahl der Kinder pro Gruppe

M: $\quad$ Mittelwert

SD: $\quad$ Standardabweichung

prä: Vortest

post: Nachtest

Abbildung 21

Darstellung der Leistung im Konzentrations- und Belastungstest d2 (Fehler) im Prä-PostVergleich des Marburger Konzentrationstrainings und des kombinierten Basis- und Strategietrainings nach Lauth und Schlottke 
In den Subtests der TAP unterscheiden sich die beiden Trainingsgruppen nicht voneinander, beim d2 hingegen erzielen die Kinder des Marburger Konzentrationstrainings deutlichere Verbesserungen als die Kinder der Lauth-Schlottke-Gruppe.

Hier können also insgesamt leichte Vorteile zugunsten des Marburger Konzentrationstrainings festgestellt werden. Da jedoch zuwenig Fragebögen der Eltern und Lehrer aus der Gruppe des Marburger Konzentrationstrainings vorlagen und bezüglich der Symptomatik deshalb kein Vergleich möglich war, sollten die bessere Leistung im d2 nicht überbewertet werden.

Sowohl mit dem Aufmerksamkeitstraining nach Lauth und Schlottke als auch dem Marburger Konzentrationstraining werden insbesondere Aufmerksamkeitsfunktionen trainiert und sollten deshalb im Vergleich zum THOP in dieser Hinsicht auch die besseren Ergebnisse erzielen (PH 5.3).

Beim Vergleich des Aufmerksamkeitstrainings nach Lauth und Schlottke mit dem THOP ließ sich diese Hypothese nicht bestätigen. Es ist sogar so, dass sich in mehreren Variablen die THOP-Kinder signifikant stärker verbesserten. Dies betraf die Anzahl der Auslassungsfehler im Go/Nogo der TAP, die Anzahl der Fehler bei nicht kritischen Trials im Visuellen Scanning der TAP, eine deutlich bessere Fehlerleistung im d2 und ein verbesserter Konzentrationsleistungswert im d2.

Lediglich in der funktionellen Nutzung des phonologischen Arbeitsgedächtnisses erzielen die Kinder der Lauth-Schlottke-Gruppe die deutlicheren Verbesserungen.

Die Abbildungen 22 - 28 illustrieren die Ergebnisse. 


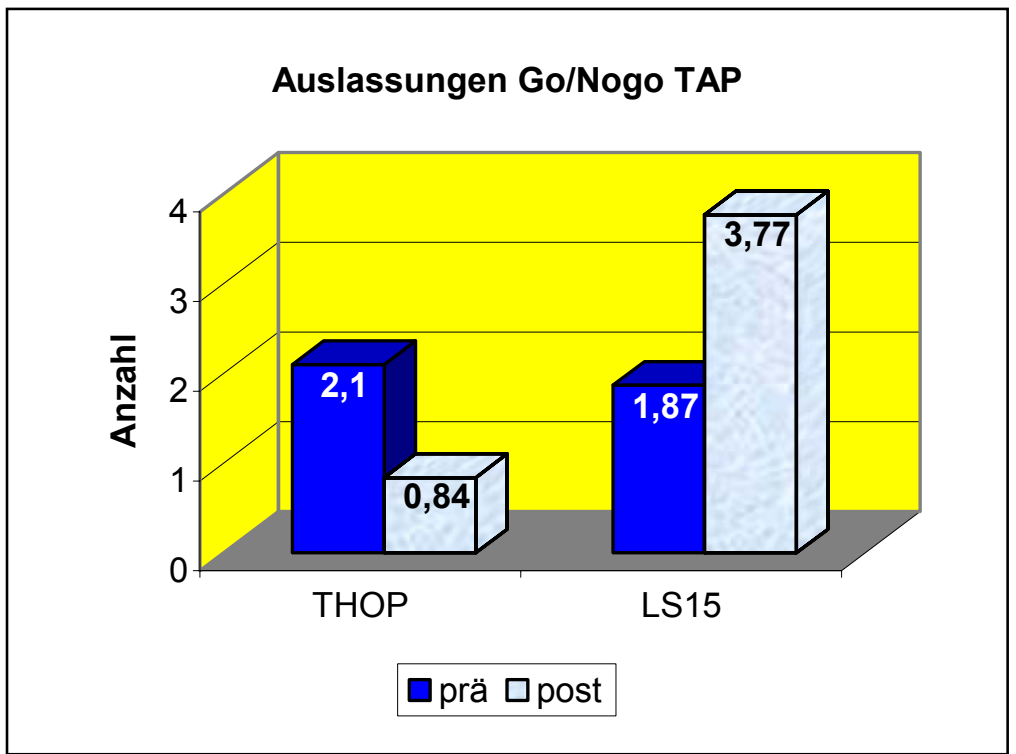

\begin{tabular}{|c|c|c|c|c|}
\hline & & $\mathrm{n}$ & $\mathrm{M}$ & $\mathrm{SD}$ \\
\hline \multirow{2}{*}{ THOP } & Prä & \multirow{2}{*}{31} & 2.10 & 2.70 \\
\cline { 2 - 4 } \cline { 4 - 5 } & Post & & 0.84 & 1.44 \\
\hline \multirow{2}{*}{ LS15 } & Prä & \multirow{2}{*}{31} & 1.87 & 2.13 \\
\cline { 2 - 5 } & Post & & 3.77 & 4.36 \\
\hline
\end{tabular}

Anmerkungen:

THOP: THOP-Gruppe

LS15: Lauth und Schlottke 15 Sitzungen

n: Anzahl der Kinder pro Gruppe

M: Mittelwert

SD: Standardabweichung prä: Vortest

post: Nachtest

Abbildung 22

Darstellung der Leistung im Subtest Go/Nogo (Auslassungsfehler) im Prä-Post-Vergleich des THOP und des kombinierten Basis- und Strategietrainings nach Lauth und Schlottke

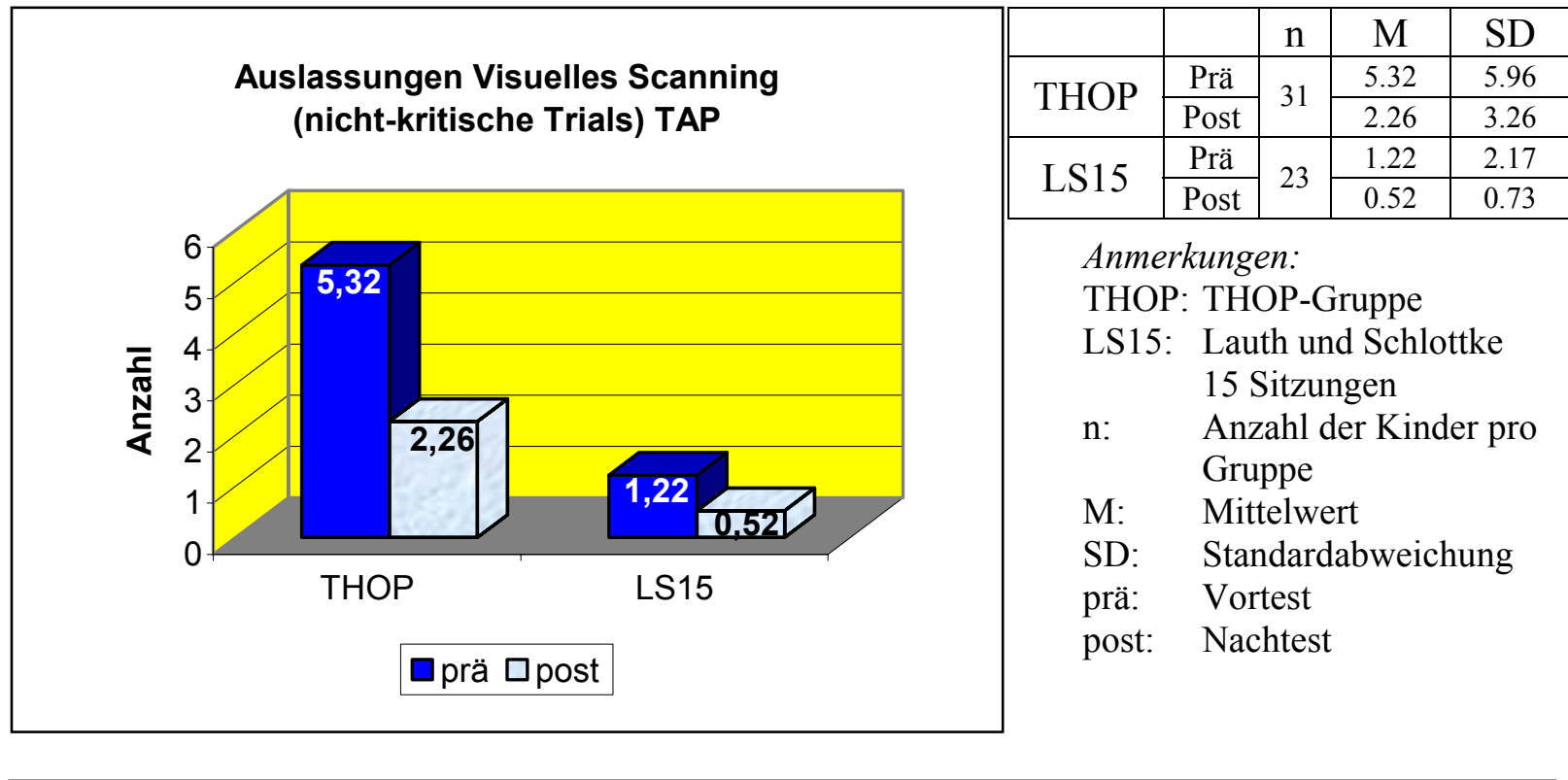

Abbildung 23

Darstellung der Leistung im Subtest Visuelles Scanning (nicht kritische Trials, Auslassungsfehler) im Prä-Post-Vergleich des THOP und des kombinierten Basis- und Strategietrainings nach Lauth und Schlottke 


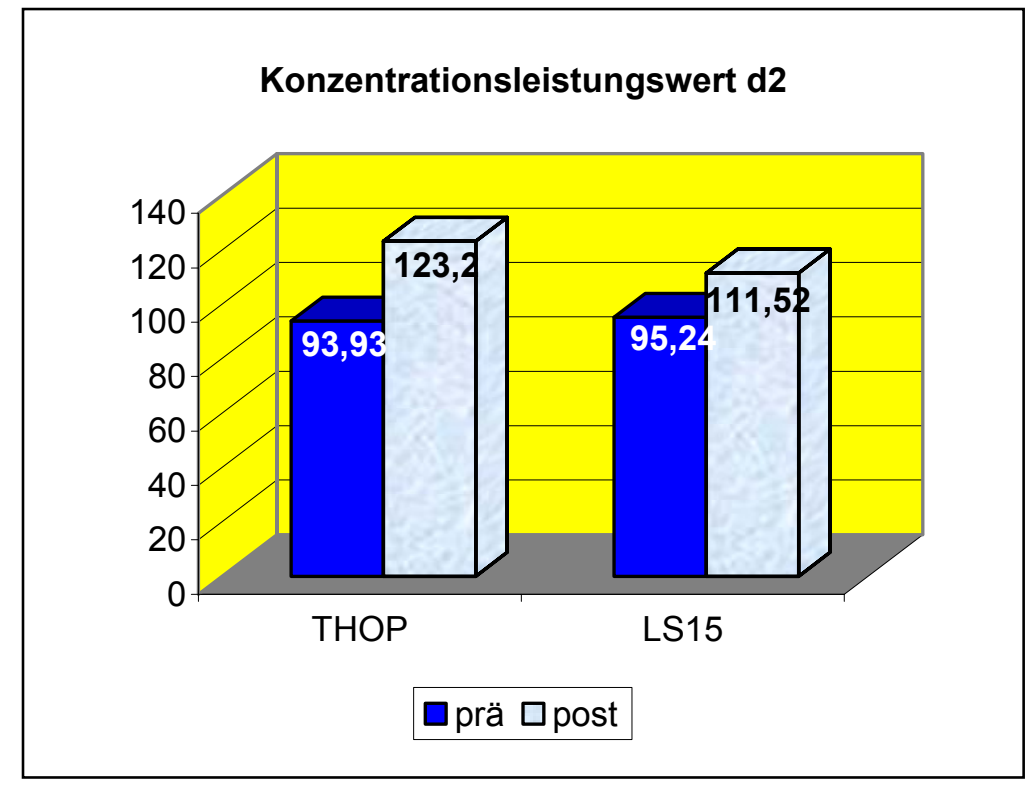

\begin{tabular}{|c|c|c|c|c|}
\hline & & $\mathrm{n}$ & $\mathrm{M}$ & $\mathrm{SD}$ \\
\hline \multirow{2}{*}{ THOP } & Prä & \multirow{2}{*}{15} & 93.93 & 25.92 \\
\cline { 2 - 3 } \cline { 4 - 5 } & Post & & 123.20 & 25.27 \\
\hline \multirow{2}{*}{ LS15 } & Prä & \multirow{2}{*}{21} & 95.24 & 21.93 \\
\cline { 2 - 3 } \cline { 4 - 5 } & Post & & 111.52 & 20.07 \\
\hline
\end{tabular}

Anmerkungen:

THOP: THOP-Trainingsgruppe

LS15: Lauth und Schlottke 15 Sitzungen

n: $\quad$ Anzahl der Kinder pro Gruppe

M: $\quad$ Mittelwert

SD: $\quad$ Standardabweichung

prä: Vortest

post: Nachtest

Abbildung 24

Darstellung der Leistung im Konzentrations- und Belastungstest d2 (Konzentrationsleistungswert) im Prä-Post-Vergleich des THOP und des kombinierten Basisund Strategietrainings nach Lauth und Schlottke

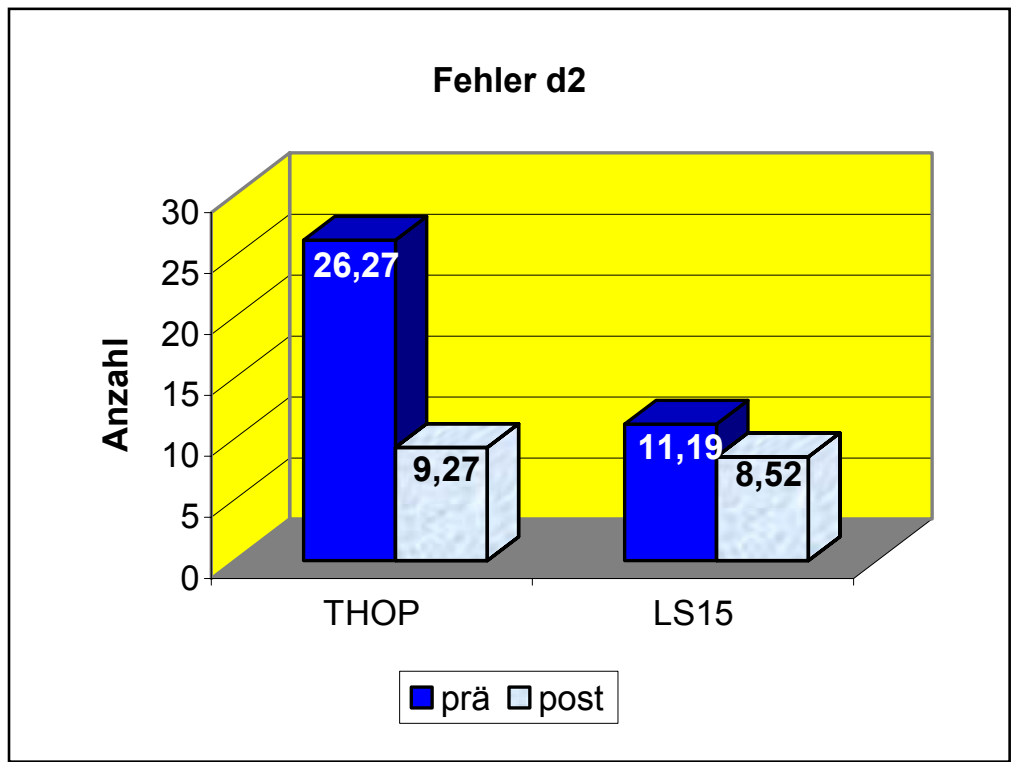

\begin{tabular}{|c|c|c|c|c|}
\hline & & $\mathrm{n}$ & $\mathrm{M}$ & $\mathrm{SD}$ \\
\hline \multirow{2}{*}{ THOP } & Prä & \multirow{2}{*}{15} & 26.27 & 37.59 \\
\cline { 2 - 3 } \cline { 4 - 5 } & Post & & 9.27 & 6.30 \\
\hline \multirow{2}{*}{ LS15 } & Prä & \multirow{2}{*}{21} & 11.19 & 8.69 \\
\cline { 2 - 3 } \cline { 4 - 5 } & Post & & 8.52 & 4.98 \\
\hline
\end{tabular}

Anmerkungen:

THOP: THOP-Trainingsgruppe

LS15: Lauth und Schlottke 15 Sitzungen

n: $\quad$ Anzahl der Kinder pro

Gruppe

M: $\quad$ Mittelwert

SD: Standardabweichung

prä: Vortest

post: Nachtest

Abbildung 25

Darstellung der Leistung im Konzentrations- und Belastungstest d2 (Fehler) im Prä-PostVergleich des THOP und des kombinierten Basis- und Strategietrainings nach Lauth und Schlottke 


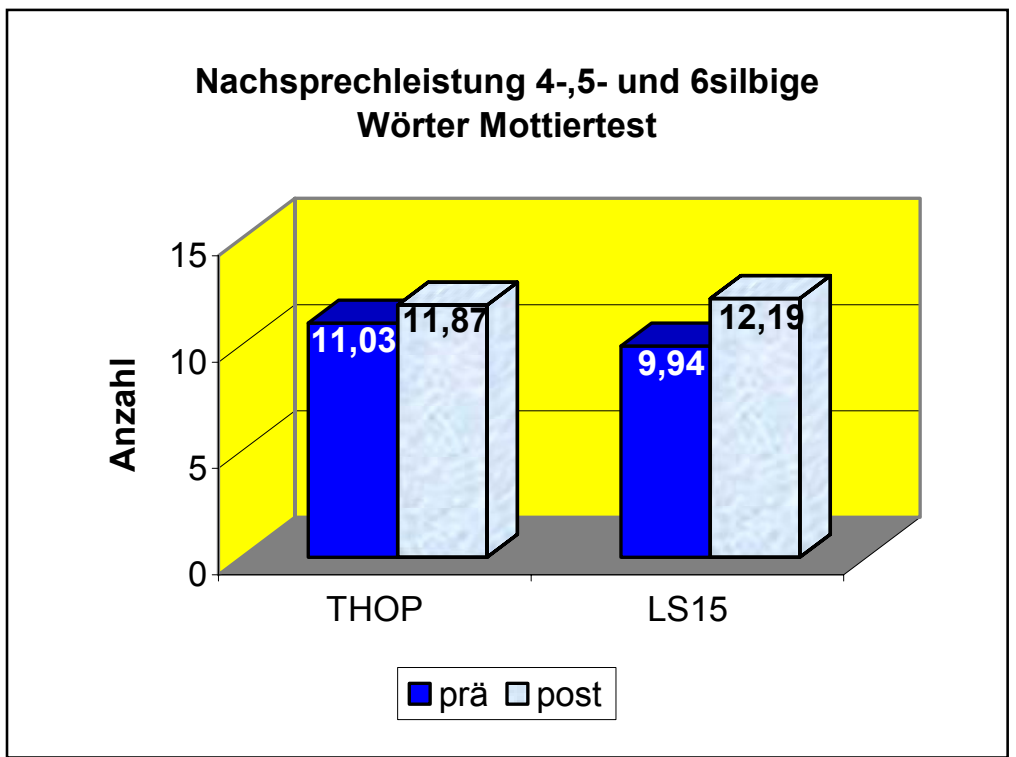

\begin{tabular}{|c|c|c|c|c|}
\hline & & $\mathrm{n}$ & $\mathrm{M}$ & $\mathrm{SD}$ \\
\hline \multirow{2}{*}{ THOP } & Prä & \multirow{2}{*}{30} & 11.03 & 3.40 \\
\cline { 2 - 4 } \cline { 4 - 5 } & Post & 11.87 & 4.90 \\
\hline \multirow{2}{*}{ LS15 } & Prä & \multirow{2}{*}{32} & 9.94 & 4.44 \\
\cline { 2 - 3 } \cline { 4 - 5 } & Post & & 12.19 & 3.41 \\
\hline
\end{tabular}

Anmerkungen:

THOP: THOP-Trainingsgruppe

LS15: Lauth und Schlottke

\section{Sitzungen}

n: Anzahl der Kinder pro

Gruppe

M: Mittelwert

SD: Standardabweichung

prä: Vortest

post: Nachtest

Abbildung 26

Darstellung der Leistung im Mottiertest (4-, 5- und 6silbige Wörter) im Prä-Post-Vergleich des THOP und des kombinierten Basis- und Strategietrainings nach Lauth und Schlottke

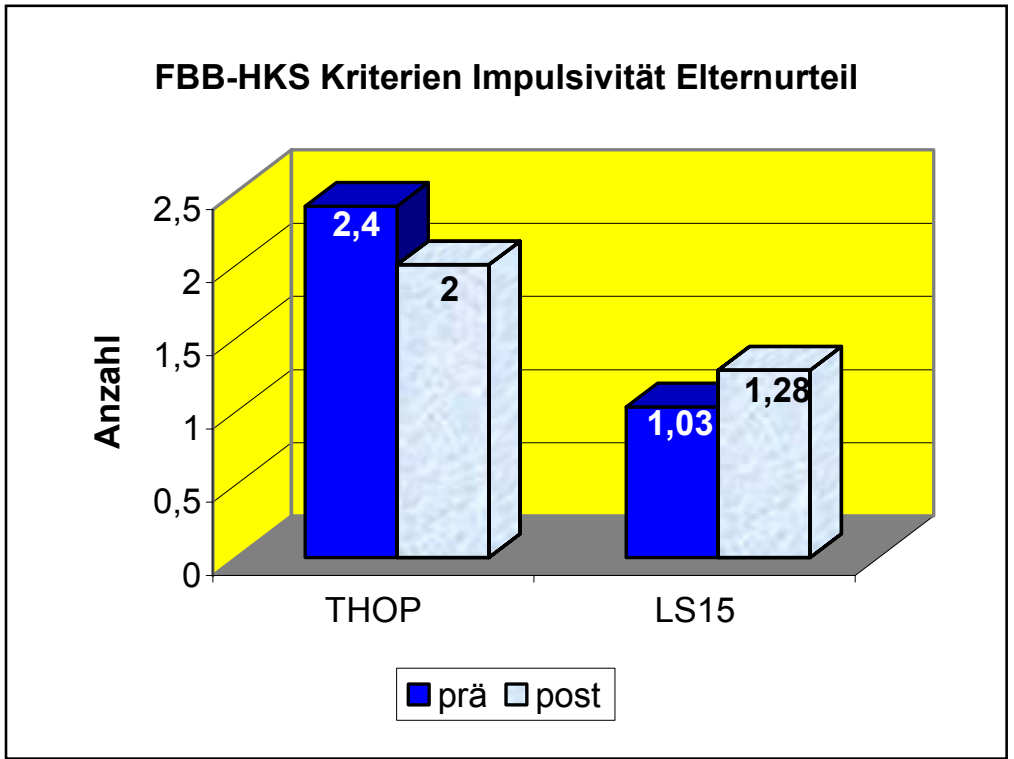

\begin{tabular}{|c|c|c|c|c|}
\hline & & $\mathrm{n}$ & $\mathrm{M}$ & $\mathrm{SD}$ \\
\hline \multirow{2}{*}{ THOP } & Prä & \multirow{2}{*}{30} & 2.40 & 1.61 \\
\cline { 2 - 3 } & Post & & 2.00 & 1.43 \\
\hline \multirow{2}{*}{ LS15 } & Prä & \multirow{2}{*}{28} & 1.03 & 1.45 \\
\cline { 2 - 5 } & Post & & 1.28 & 1.56 \\
\hline
\end{tabular}

Anmerkungen:

THOP: THOP-Trainingsgruppe

LS15: Lauth und Schlottke 15 Sitzungen

n: $\quad$ Anzahl der Kinder pro

Gruppe

M: $\quad$ Mittelwert

SD: $\quad$ Standardabweichung

prä: Vortest

post: Nachtest

Abbildung 27

Darstellung der Summenwerte für Impulsivität (Elternurteil) im Prä-Post-Vergleich des THOP und des kombinierten Basis- und Strategietrainings nach Lauth und Schlottke 




\begin{tabular}{|c|c|c|c|c|}
\hline & & $\mathrm{n}$ & $\mathrm{M}$ & $\mathrm{SD}$ \\
\hline \multirow{2}{*}{ THOP } & Prä & \multirow{2}{*}{30} & 1.61 & 0.93 \\
\cline { 4 - 5 } \cline { 4 - 5 } & Post & & 1.50 & 0.81 \\
\hline \multirow{2}{*}{ LS15 } & Prä & \multirow{2}{*}{28} & 0.82 & 0.75 \\
\cline { 2 - 5 } & Post & & 1.09 & 0.87 \\
\hline
\end{tabular}

Anmerkungen:

THOP: THOP-Trainingsgruppe

LS15: Lauth und Schlottke

5 Sitzungen

n: Anzahl der Kinder pro

Gruppe

M: $\quad$ Mittelwert

SD: Standardabweichung

prä: Vortest

post: Nachtest

Abbildung 28

Darstellung der Kennwerte für Impulsivität (Elternurteil) im Prä-Post-Vergleich des THOP und des kombinierten Basis- und Strategietrainings nach Lauth und Schlottke

Dieses Ergebnis ist erstaunlich, da im THOP im Vergleich zum Training nach Lauth und Schlottke der Anteil an aufmerksamkeitsbezogenen Aufgaben deutlich geringer ist. Erklärlich wird dieses Resultat aber dadurch, dass in der THOP-Gruppe die Eltern wesentlich stärker involviert waren. Als Mediatoren konnten sie auch zwischen den Therapiesitzungen an den Aufmerksamkeitsleistungen ihrer Kinder arbeiten. Selbst wenn das Aufmerksamkeitsverhalten in der THOP-Gruppe nicht so explizit in den Vordergrund gerückt wurde, scheint doch der Effekt, der durch die Eltern bewirkt werden kann, sich deutlich auszuwirken.

Auch beim Vergleich des THOP mit dem Marburger Konzentrationstraining können nur die psychometrischen Verfahren als Vergleichsgrundlage herangezogen werden. Im PräPost-Vergleich innerhalb der Gruppen verbessert sich die THOP-Gruppe in 12 der 14 Variablen der TAP und des d2, beim Marburger Konzentrationstraining sind es nur vier Variablen. In einer Variablen der TAP wird ein signifikanter Effekt zugunsten der THOPGruppe aufgedeckt (Fehler bei nicht-kritischen Trials des Visuellen Scannings der TAP). In drei weiteren Variablen wird die Signifikanz nur knapp verfehlt. Im d2 unterscheiden sich die beiden Interventionsgruppen nicht voneinander. $\mathrm{Zu}$ berücksichtigen ist allerdings, dass die Kinder des Marburger Konzentrationstrainings hinsichtlich externalisierender Störungen 
sowie bei zwei Variablen des Visuellen Scannings als unauffälliger beurteilt werden. Der positive Effekt bei den THOP-Kindern könnte also zum Teil auch mit einer Regression zur Mitte erklärt werden.

Die folgenden Abbildungen illustrieren die Ergebnisse.

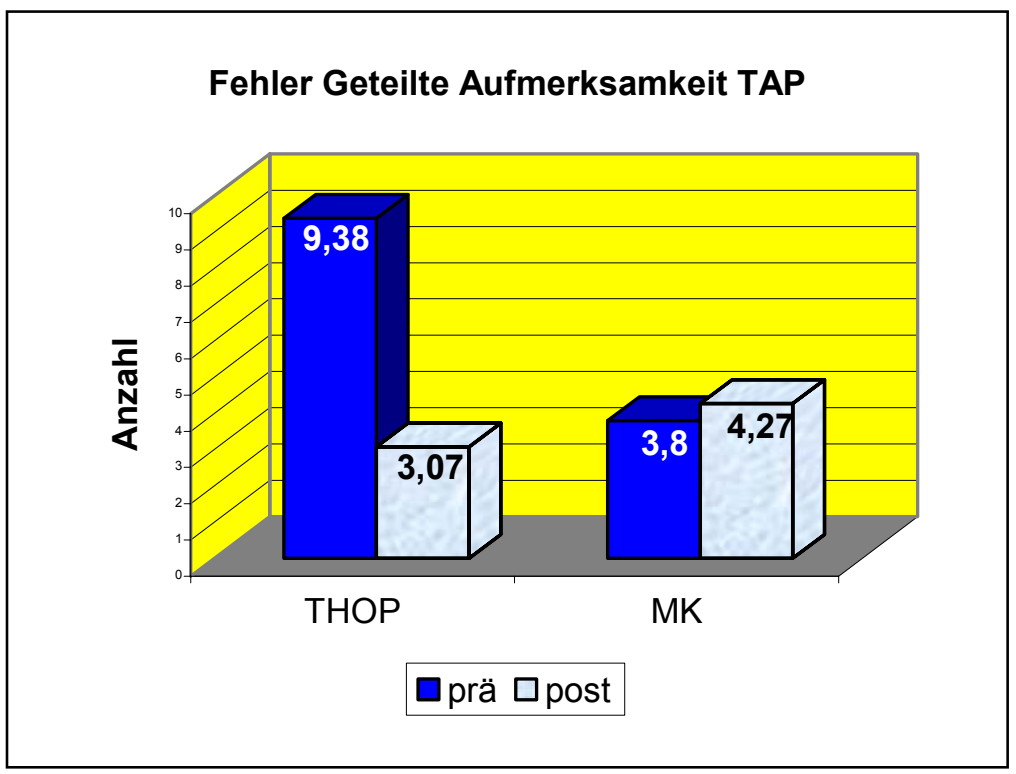

\begin{tabular}{|c|c|c|c|c|}
\hline & & $\mathrm{N}$ & $\mathrm{M}$ & $\mathrm{SD}$ \\
\hline \multirow{2}{*}{ THOP } & Prä & \multirow{2}{*}{29} & 9.38 & 13.44 \\
\cline { 2 - 3 } \cline { 4 - 5 } & Post & & 3.07 & 4.03 \\
\hline \multirow{2}{*}{ MK } & Prä & \multirow{2}{*}{15} & 3.80 & 6.04 \\
\cline { 2 - 5 } \cline { 4 - 5 } & Post & & 4.27 & 4.22 \\
\hline
\end{tabular}

Anmerkungen:

THOP: THOP-Trainingsgruppe

MK: Marburger

Konzentrationstraining

n: $\quad$ Anzahl der Kinder pro

Gruppe

M: $\quad$ Mittelwert

SD: $\quad$ Standardabweichung

prä: $\quad$ Vortest

post: Nachtest

\section{Abbildung 29}

Darstellung der Leistung im Subtest Geteilte Aufmerksamkeit (Fehler) im Prä-Post-Vergleich des THOP und des Marburger Konzentrationstrainings

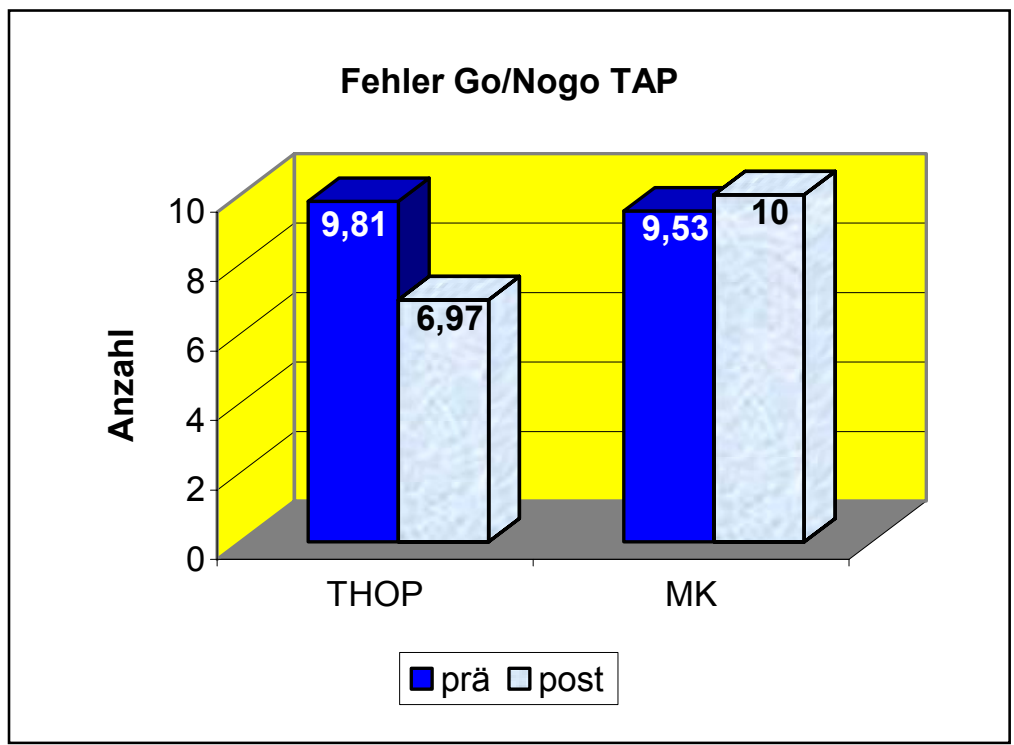

\begin{tabular}{|c|c|c|c|c|}
\hline & & $\mathrm{N}$ & $\mathrm{M}$ & $\mathrm{SD}$ \\
\hline \multirow{2}{*}{ THOP } & Prä & \multirow{2}{*}{31} & 9.81 & 5.50 \\
\cline { 2 - 4 } \cline { 4 - 5 } & Post & & 6.97 & 5.33 \\
\hline \multirow{2}{*}{ MK } & Prä & \multirow{2}{*}{15} & 9.53 & 4.79 \\
\cline { 2 - 5 } & Post & & 10.00 & 6.23 \\
\hline
\end{tabular}

Anmerkungen:

THOP: THOP-Trainingsgruppe

MK: Marburger

Konzentrationstraining

n: $\quad$ Anzahl der Kinder pro

Gruppe

M: $\quad$ Mittelwert

SD: $\quad$ Standardabweichung

prä: Vortest

post: Nachtest

Abbildung 30

Darstellung der Leistung im Subtest Go/Nogo (Fehler) im Prä-Post-Vergleich des THOP und des Marburger Konzentrationstrainings 


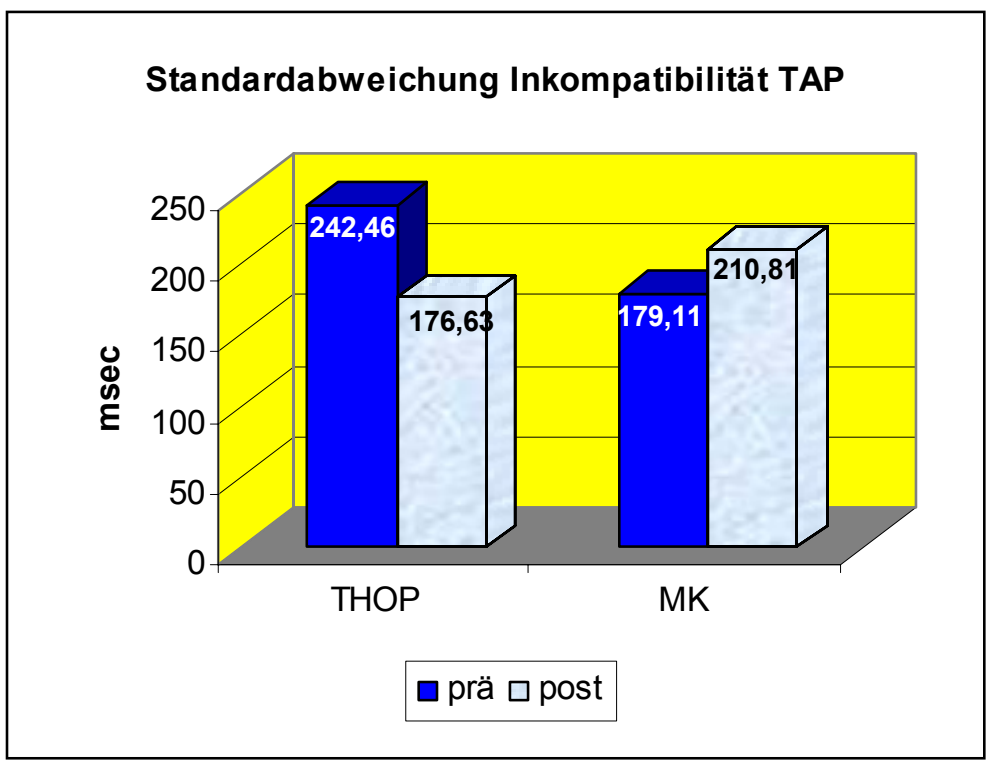

\begin{tabular}{|c|c|c|c|c|}
\hline & & $\mathrm{N}$ & $\mathrm{M}$ & $\mathrm{SD}$ \\
\hline \multirow{2}{*}{ THOP } & Prä & \multirow{2}{*}{31} & 242.46 & 123.11 \\
\cline { 2 - 3 } & Post & & 176.63 & 64.19 \\
\hline \multirow{2}{*}{ MK } & Prä & \multirow{2}{*}{15} & 179.11 & 80.37 \\
\cline { 2 - 3 } \cline { 4 - 5 } & Post & & 210.81 & 230.13 \\
\hline
\end{tabular}

Anmerkungen:

THOP: THOP-Trainingsgruppe

MK: Marburger

Konzentrationstraining

n: Anzahl der Kinder pro

Gruppe

M: $\quad$ Mittelwert

SD: $\quad$ Standardabweichung

prä: Vortest

post: Nachtest

Abbildung 31

Darstellung der Leistung im Subtest Inkompatibilität (Standardabweichung) im Prä-PostVergleich des THOP und des Marburger Konzentrationstrainings

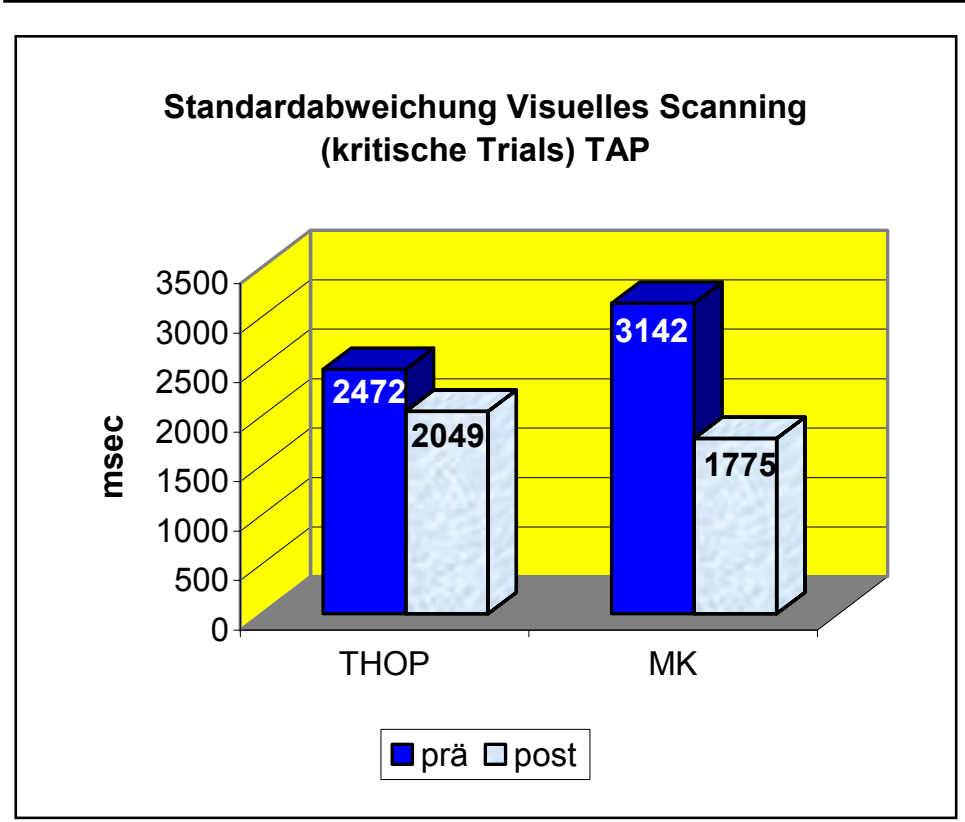

\begin{tabular}{|c|c|c|c|c|}
\hline & & $\mathrm{N}$ & $\mathrm{M}$ & $\mathrm{SD}$ \\
\hline \multirow{2}{*}{ THOP } & Prä & \multirow{2}{*}{31} & 2472.9 & 1048.1 \\
\cline { 2 - 3 } \cline { 4 - 5 } & Post & & 2049.0 & 995.3 \\
\hline \multirow{2}{*}{ MK } & Prä & \multirow{2}{*}{11} & 3142.8 & 2312.2 \\
\cline { 2 - 3 } \cline { 4 - 5 } & Post & & 1775.0 & 603.2 \\
\hline
\end{tabular}

Anmerkungen:

THOP: THOP-Trainingsgruppe

MK: Marburger

Konzentrationstraining

n: $\quad$ Anzahl der Kinder pro

Gruppe

M: $\quad$ Mittelwert

SD: Standardabweichung

prä: Vortest

post: Nachtest

\section{Abbildung 32}

Darstellung der Leistung im Subtest Visuelles Scanning (Standardabweichung, kritische Trials) im Prä-Post-Vergleich des THOP und des Marburger Konzentrationstrainings 


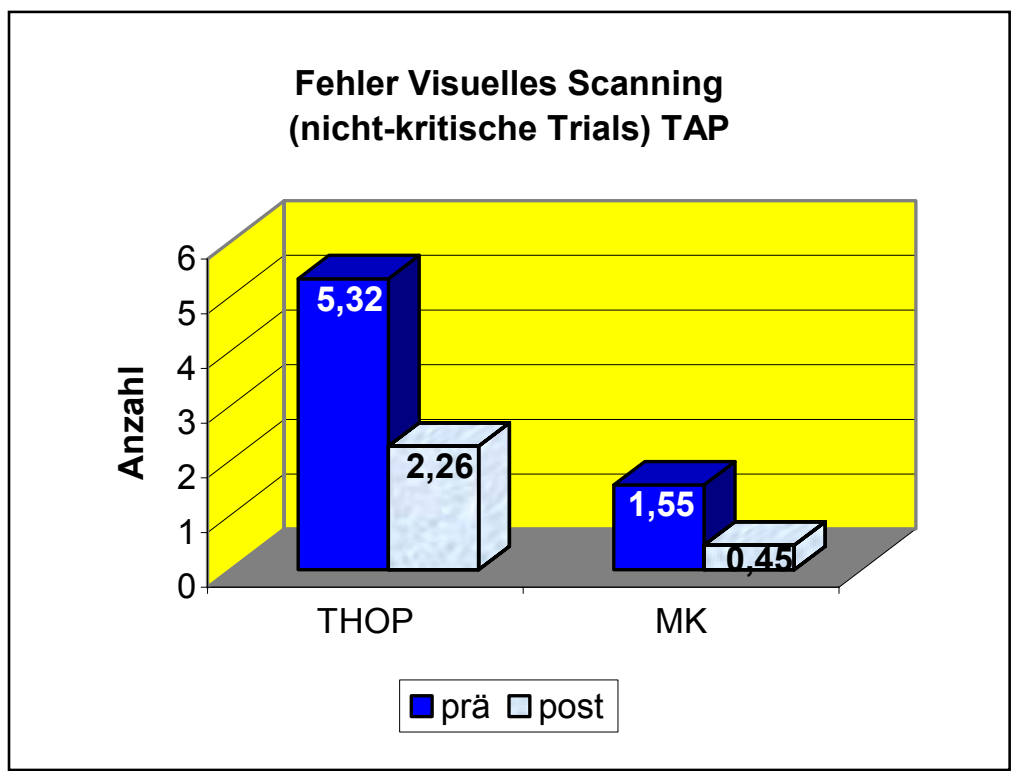

\begin{tabular}{|c|c|c|c|c|}
\hline & & $\mathrm{N}$ & $\mathrm{M}$ & $\mathrm{SD}$ \\
\hline \multirow{2}{*}{ THOP } & Prä & \multirow{2}{*}{31} & 5.32 & 5.96 \\
\cline { 2 - 4 } & Post & & 2.26 & 3.26 \\
\hline \multirow{2}{*}{$\mathrm{MK}$} & Prä & \multirow{2}{*}{11} & 1.55 & 1.13 \\
\cline { 2 - 3 } \cline { 4 - 5 } & Post & & 0.45 & 0.69 \\
\hline
\end{tabular}

Anmerkungen:

THOP: THOP-Trainingsgruppe

MK: Marburger

Konzentrationstraining

n: $\quad$ Anzahl der Kinder pro

Gruppe

M: $\quad$ Mittelwert

SD: Standardabweichung

prä: Vortest

post: Nachtest

Abbildung 33

Darstellung der Leistung im Subtest Visuelles Scanning (Fehler, nicht-kritische Trials) im Prä-Post-Vergleich des THOP und des Marburger Konzentrationstrainings

Im Wesentlichen deckt sich aber das Ergebnis mit dem Vergleich des THOP mit dem Aufmerksamkeitstraining nach Lauth und Schlottke. Das THOP schneidet auch hier im Vergleich bei den Aufmerksamkeitsleistungen besser ab. Die Erklärung dürfte auch hier darin zu finden sein, dass die Eltern zwischen den Sitzungen günstig auf die Aufmerksamkeitsprobleme ihrer Kinder eingewirkt haben.

Mit dem THOP als Gruppenprogramm wurde eine Verminderung von oppositionellen und aggressiven Verhaltensweisen angestrebt. $\mathrm{Da}$ in dem Programm in der hier durchgeführten Form die Eltern intensiv einbezogen wurden, sollte sich die Verbesserung im häuslichen Bereich zeigen, im schulischen Bereich sollten sich die Interventionsgruppen nicht voneinander unterscheiden $(\mathrm{PH}$ 5.4). $\mathrm{Da}$ für die Gruppe des Marburger Konzentrationstrainings für eine Analyse zu wenige Fragebögen von den Eltern und den Lehrern zurückgegeben wurden, kann dieser Vergleich nicht erfolgen.

Beim Vergleich der T-Werte für Externalisierende Störungen unterscheiden sich die Kinder des THOP von denen der Lauth- und Schlottke-Gruppe in der Lehrereinschätzung nicht voneinander, die Hypothese wird also bestätigt. Im schulischen Bereich scheinen keine Unterschiede vorzuliegen. Im Bereich der Familie verbessern sich sowohl die LS-Kinder als auch die THOP-Kinder, letztere verbessern sich tendenziell etwas stärker. Hier wäre es 
interessant gewesen, wenn den LS-Kindern ebenfalls der Interaktionsfragebogen vorgelegt worden wäre. Da die Therapie dieser Gruppe aber schon einige Zeit zurücklag, war dies, als der Interaktionsfragebogen erstellt wurde und vorlag, nicht mehr möglich. Eine durch das THOP erzielte deutliche Verbesserung von aggressiven und oppositionellen Verhaltensweisen im familiären Bereich konnte im Vergleich zum LS-Training nicht belegt werden.

Für die PH 5.5, nach der das THOP im Vergleich $\mathrm{zu}$ den beiden anderen Interventionsprogrammen in gleichem Maße $\mathrm{zu}$ einer Verminderung emotionaler Beeinträchtigungen führt, kann wiederum nur im Vergleich zum LS-Training durchgeführt werden, da für die Marburger Konzentrationstrainingsgruppe zu wenig Fragebögen vorliegen.

Es ergibt sich das gleiche Muster wie bei der PH 5.4. Der T-Wert der Gesamtskala der Lehrereinschätzung (TRF) verbessert sich in beiden Interventionsgruppen um ca. 3 T-Punkte. In der Elterneinschätzung (CBCL 4-18) verbessern sich die THOP-Kinder um 6 T-Punkte, die LS-Kinder nur um 3,6 T-Punkte. Der Effekt wird aber nicht signifikant. Das THOP ist dem LS-Programm hinsichtlich der Verminderung emotionaler Beeinträchtigungen also nicht unterlegen.

$\mathrm{Zu}$ bedenken ist jedoch, dass die THOP-Kinder in einer Reihe von Variablen beim Prätestvergleich der beiden Gruppen von Eltern und Lehrer vor dem Training insgesamt als stärker beeinträchtigt erlebt. Das bedeutet, dass zum einen bei den Ergebnissen eine Regression zur Mitte eine Rolle gespielt haben könnte, zum anderen, dass Verbesserungen schwerer zu erreichen sind, weil die Kinder massiver gestört und damit in den Interventionen schwerer zu erreichen waren.

Als Fazit zur vergleichenden Evaluation kann festgestellt werden, dass in der hier durchgeführten Form eines Gruppentrainings unter den praxisnahen Bedingungen einer kinder- und jugendpsychiatrischen Ambulanz das Basistraining dem kombinierten Basis- und Strategietraining nach Lauth und Schlottke vorzuziehen ist, da durch letzteres kein zusätzlicher Gewinn erzielt wird. Das Marburger Konzentrationstraining scheint dem Training nach Lauth und Schlottke in der Wirksamkeit leicht überlegen zu sein. Dieses Ergebnis sollte aber nur unter Vorbehalt bewertet werden, weil die Stichproben nicht identisch waren, die Unterschiede nur in einem Testverfahren belegt werden konnten und die Bewertung über Fremdeinschätzungen der Eltern und Lehrer nicht vorgenommen werden konnte. 
Erstaunlich gut verbesserten sich die THOP-Kinder im Vergleich zu den beiden anderen Trainings in den Aufmerksamkeitsleistungen, was auf den Einfluss der Eltern zurückgeführt werden kann. Diese arbeiteten zwischen den Sitzungen intensiver mit ihren Kindern als die Eltern der Kinder, die an den beiden anderen Trainings teilgenommen haben. Durch den Interaktionsfragebogen wurden zudem Verbesserungen der Erziehungskompetenz der Eltern sowie eine bessere Befolgung von Familienregeln durch die Kinder belegt.

\subsection{Einschränkungen der Untersuchung}

In den Grenzen der vorliegenden Evaluationsuntersuchung musste ein Kompromiss zwischen dem Ziel der Wissenschaftlichkeit, das sich in den Standards und Empfehlungen für die Evaluationsforschung ausdrückt und der praktischen Durchführbarkeit gefunden werden (Patry \& Hager, 2000). Während auf der einen Seite die Wahrung der internen Validität durch die Kontrolle von Störfaktoren als Basis für die Interpretierbarkeit der Ergebnisse im Vordergrund steht, ist auf der anderen Seite der Kontakt und Umgang mit dem Patienten und die Verfügbarkeit personeller und materieller Ressourcen maßgeblich. Mit einer Evaluationsuntersuchung, die außerhalb des Labors mit einer weniger kontrollierbaren und manipulierbaren Umwelt sowie verschiedenen einschränkenden Rahmenbedingungen umzugehen hat, sind daher zwangsläufig Validitätseinbußen verbunden (vgl. Bortz \& Döring, 1995; Rossi, Freeman \& Hofmann, 1988).

Da die Trainingsgruppen nacheinander durchgeführt wurden, konnte keine Randomisierung vorgenommen werden, was besonders problematisch ist, da nicht auszuschließen ist, dass dadurch Selektionseffekte zustande gekommen sind. Die Fremdbeurteilungsbögen CBCL 4-18, TRF und FBB-HKS wurden durch Eltern und insbesondere durch Lehrer nur lückenhaft ausgefüllt und zurückgegeben. Die Unvollständigkeit der Datenerhebung birgt das Risiko, dass die Ergebnisse durch eine nicht zufällige Verteilung der Fehlwerte verzerrt wurden.

Ein weiteres methodisches Problem hängt mit der Heterogenität des Störungsbildes zusammen. HKS-Kinder zeigen große Unterschiede hinsichtlich der beeinträchtigten Funktionen und Bereiche, was sich in der vorliegenden Studie in einer Diagnosenvielfalt in den Vergleichsgruppen widerspiegelte. Die Unterschiedlichkeit in den Gruppen ist vor allem deshalb kritisch zu betrachten, da die Stichprobengröße in der Untersuchung relativ gering war. Bei kleiner Stichprobengröße und großer Variation innerhalb der Stichprobe resultieren große Stichprobenschwankungen, so dass die vermuteten Effekte relativ groß ausfallen müssen, um nicht mehr als zufällige Abweichungen zu gelten. Damit wird es für die 
Forschungshypothesen bzw. den daraus abgeleiteten empirischen Vorhersagen im vorliegenden Fall jedoch relativ erschwert, sich zu bewähren.

Die Untersuchung weist einige gravierende Schwachstellen auf, die die Aussagefähigkeit der gefundenen Ergebnisse einschränken. Aufgrund geringer personeller Ressourcen konnte für die Studien 1 - 3 für die isolierte Evaluation nur eine Wartekontrollgruppe realisiert werden. Mit einer nicht-trainierten Kontrollgruppe sind zwar interventionsunabhängige Wirkungen wie Retesteffekte (z.B. Erinnerung oder Übung) und Effekte des zwischenzeitlichen Geschehens (z.B. Entwicklungseffekte), jedoch nicht interventionsgebundene Effekte kontrolliert worden. Diese haben möglicherweise den Interventionseffekt beeinflusst. $\mathrm{Zu}$ den interventionsgebundenen Effekten werden insbesondere Aspekte der sozialen Interaktion wie Aufmerksamkeit und Zuwendung gezählt, die den Kindern und Eltern während der mehrmonatigen Behandlung, aber nicht den Eltern der Wartegruppe zuteil wurden. Hager, Hübner und Hasselhorn (2000) konnten für den Bereich kognitiver Förderprogramme zeigen, dass der sozialen Interaktion eine große Bedeutung im Hinblick auf die Beurteilung der Programmwirksamkeit zukommt, da sie mit ihrer allgemeinen Wirkung jene des Trainings vortäuscht. Es ist denkbar, dass die Programmwirkungen des THOP (Studie 3) überschätzt wurden, da insbesondere in dieser Studie Zuwendungs- und Aufmerksamkeitseffekte eine wichtige Rolle für die therapeutische Beziehung gespielt haben mögen, die ihrerseits wichtige Einflussfaktoren für den Therapieprozess und -erfolg sind (vgl. z.B. Hoffmann, 2000).

Weitere mit einem Training verbundene Einflussfaktoren können z.B. eine allgemeine Motivationsförderung und Erfolgserwartungshaltungen der Eltern sein, die zu einer verzerrten Einschätzung der Programmwirksamkeit führen. In der vorliegenden Untersuchung können daher keine reinen Trainingseffekte, sondern nur „Bruttowirkungen im engeren Sinne“ (vgl. Hager und Hasselhorn, 2000) bestimmt werden.

Eine der wirksamsten Maßnahmen zur Kontrolle bekannter und vor allem auch unbekannter Störfaktoren ist die Randomisierung, die im vorliegenden Fall nicht eingesetzt wurde. Wenn die Familien randomisiert der Trainings- und der Wartegruppe zugewiesen worden wären, hätte man den Patienten bei vorhandener Indikation und Möglichkeit eine Therapie vorenthalten, was aus ethischen Gründen nicht vertretbar war. Die Zusammenstellung der Gruppen orientierte sich nach den Behandlungsterminen, die von den Therapeuten angeboten werden konnten und danach, wann entsprechend viele Familien bereit 
und organisatorisch in der Lage waren an einer 15-wöchigen Behandlung teilzunehmen. Wegen der geringen zur Verfügung stehenden Versuchspersonenzahl konnten bekannte potentielle Störfaktoren durch Eliminierung oder Konstanthaltung nicht in vollem Umfang kontrolliert werden. Alle Familien, bei denen die Teilnahmevoraussetzungen erfüllt waren, wurden in die Studie aufgenommen, was zu nicht-äquivalenten Gruppen geführt hat. Sowohl das Intelligenzniveau als auch die gleichzeitige Behandlung mit Psychostimulanzien waren in den Gruppen ungleichverteilt. Die Kinder der THOP-Gruppe sowie die der Gruppe, welche das kombinierte Training nach Lauth und Schlottke erhielt, waren im Schnitt acht bzw. sechs IQ-Punkte intelligenter als die Kinder der Wartegruppe und jene, die nur das Basistraining nach Lauth und Schlottke erhalten haben. Die letztgenannten Kinder waren etwas älter. Ein entscheidender Faktor entstand durch die gleichzeitige Behandlung mit Psychostimulanzien. In der LS10-Gruppe und in der Wartegruppe erhielten 6\% Psychostimulanzien, in der LS15Gruppe 20\% und in der THOP-Gruppe sogar 48\%. Durch Kontrolle dieses Faktors konnte belegt werden, dass Kinder, die gleichzeitig zum Trainingszeitraum medikamentös behandelt wurden, größere Veränderungen in externalisierenden, hyperaktiven und impulsiven Verhaltensweisen zeigten, als Kinder ohne Psychostimulanzientherapie. Dieses Ergebnis spricht für eine Kombination von verhaltenstherapeutischen Maßnahmen mit Psychostimulanzien. Erst durch die Medikamenteneffekte scheinen manche Kinder von therapeutischen Maßnahmen profitieren zu können.

In Studie 4 kam trotz Randomisierung bzgl. Intelligenz und gleichzeitiger Behandlung mit Psychostimulanzien eine vergleichbare Konstellation wie in den Studien 1-3 zustande. Die mit dem Marburger Konzentrationstraining behandelten Kinder waren intelligenter als die Kinder der Kontrollgruppe (ca. neun IQ-Punkte) und wurden häufiger medikamentös behandelt (60\% vs. 13\%). Der Effekt der Medikamente ist in dieser Studie jedoch nicht eindeutig zu interpretieren.

Ein weiterer Aspekt für die relativ geringe Wirksamkeit der Trainingsprogramme könnte darin begründet sein, dass die Nachtestung in den einzelnen Studien dieser Arbeit relativ früh erfolgte. Verzögerte Auswirkungen der Therapie zeigen sich aufgrund notwendiger Umstrukturierungsprozesse manchmal erst nach einigen Monaten. Dies ist ein so genannter ,sleeper effect“ (Bell, Lynne \& Kolvin, 1989). 


\subsection{Schlussfolgerungen, Fazit, Ausblick}

Wird nach der Durchführung einer Intervention der Erfolg oder Misserfolg dieser Maßnahme nur auf der Gruppenebene bewertet, kann es passieren, dass wesentliche Aspekte übersehen werden. Sollte ein Programm im Mittel erfolgreich sein, ist denkbar, dass einige besonders stark und andere überhaupt nicht von der Maßnahme profitiert haben.

Bei der Beurteilung der differenziellen Wirksamkeit eines Trainings können zwei Resultate entstehen: der so genannte Matthäus-Effekt oder ein kompensatorischer Effekt. Der Matthäus-Effekt (Matthäus 13, Vers 12, Die Bibel) („Denn wer etwas hat, dem wird noch mehr gegeben werden, ...“) besagt, dass diejenigen am meisten profitieren, die die besten Grundvoraussetzungen haben, die Leistungsschwächeren hingegen am wenigsten. Der kompensatorische Effekt bedeutet, dass die Leistungsschwächeren mehr profitieren als die Leistungsstärkeren. Weder der eine noch der andere Effekt ließ sich bei den verschiedenen Trainings für das Alter oder die Höhe der Intelligenz durchgängig nachweisen. Insbesondere aber durch den Einfluss von Psychostimulanzien erreichen in der THOP-Gruppe die Kinder deutliche Verbesserungen.

Einzelfallanalysen von besonders erfolgreichen Verläufen könnten weitere Aufschlüsse über die Faktoren geben, die bei diesen Kindern zum Erfolg führten.

Bei vielen Kindern mit Hyperkinetischen Störungen ist es wichtig an der Motivation zu arbeiten. Rheinberg und Fries (2001) führen aus, dass erfolgszuversichtliche Leistungsmotivation erst dann zum Erfolg führt, wenn passende Anregungsbedingungen geschaffen werden. Gerade für Kinder mit hyperkinetischen Störungen ist es notwendig, geeignete äußere Bedingungen zu schaffen, damit sie bereit sind, gelernte Kompetenzen auch wirklich einzusetzen. Der Einsatz von Signalkarten wird beispielsweise nur dann erfolgen, wenn ihnen die Nutzung dieser Karten nicht peinlich sein muss, sondern durch die Lehrpersonen gefördert wird. Diese Kinder reagieren emotional oft sehr empfindlich, sie brauchen deshalb günstige Rahmenbedingungen, damit sie innerlich bereit sind in der Therapie Gelerntes z.B. in der Schule einzusetzen. Da die Effekte der Behandlung nicht in das natürliche Umfeld des Kindes übertragen wurden, ist es notwendig, die Inhalte stark an den Bedürfnissen und Anforderungen der Kinder anzupassen. Nur dann, wenn sie für sich einen Nutzen der Behandlungsschritte erkennen, werden sie diese auch in ihrem familiären und schulischen Alltag einsetzen (Hinshaw \& Erhardt, 1991). Mähler und Hasselhorn (2001) weisen bezogen auf den Einsatz bei Gedächtnistrainings darauf hin, dass Kinder Transferleistungen nur dann erbringen werden, wenn die trainierten Inhalte für sie persönliche 
Bedeutung und funktionalen Wert haben. Geringe Zuwächse, welche Kinder nach einem Training in Leistungstests zeigen, können also durchaus durch geringe Motivation der Kinder bedingt sein. Nach eigenen Erfahrungen ist es eher die Regel, dass Kinder mit Hyperkinetischen Störungen schlecht als gut motiviert sind. Deshalb muss bei der Konzeption von Trainings unbedingt darauf geachtet werden, dass die Inhalte für die Kinder möglichst attraktiv sind, um bei ihnen eine hohe Compliance für die Therapiemaßnahmen erzielen zu können. Durch Unterstützung der Lehrer könnte den Kindern zudem die Wichtigkeit dieser Maßnahmen besser vermittelt werden. Dafür müssten allerdings zunächst einmal viele Lehrer selbst erst überzeugt werden.

Ein wichtiger Aspekt bei Trainingsmaßnahmen ist die Dauer der Durchführung (Trainingsumfang bzw. -dauer) und die Fortführung der erlernten Maßnahmen nach dem Training. Ein Programm sollte solange fortgesetzt werden, bis die erlernten Inhalte verinnerlicht sind. Hier gilt es natürlich Kosten und Nutzen gegeneinander abzuwägen. Je kürzer das Training ist, desto günstiger ist die Maßnahme aber desto fraglicher ist möglicherweise auch der Erfolg der Bemühungen. Bei vielen Trainings verblassen die Effekte nach einigen Wochen oder Monaten. Für einige Trainings (z.B. solche zum induktiven Denken) haben sich Auffrischungssitzungen als sehr effektiv erwiesen (Möller, 1999). Diese Sitzungen könnten den Therapieerfolg einer Maßnahme zu mehr Beständigkeit verhelfen. Beim Auftreten neuer Schwierigkeiten könnte außerdem schnell auf die Probleme reagiert werden. Für viele der behandelten Kinder dürfte der Behandlungszeitraum zu kurz gewesen sein. Sie leiden in der Regel bereits seit vielen Jahren unter dem Problem, bis sie sich in Therapie begeben. Erfolgreiche Therapieansätze müssen vermutlich über lange Zeiträume durchgeführt werden, um einen hinreichenden Erfolg gewährleisten zu können (Brown, 1980; Eastman \& Rasbury, 1981; Varni \& Henker, 1979). Selbst die in der MTA-Studie mit 14 Monaten schon sehr umfassende Behandlungsdauer ist vermutlich noch zu kurz angelegt.

Eltern-Kind-Programme erzielen insbesondere bei oppositionellen Kindern ermutigende Ergebnisse (Döpfner, Frölich \& Lehmkuhl, 2000; Miller \& Prinz, 1990). Auch bei hyperaktiven Kindern konnten kurzfristige Verbesserungen zu Hause festgestellt werden (Anastopoulos, Shelton, DuPaul \& Guevremont, 1993). Dies ist ein wichtiger Aspekt bei der Beurteilung der Wirksamkeit eines Verfahrens. Häufig werden nur die Kinder untersucht und die Wirksamkeit einer Intervention hinsichtlich verschiedener Verhaltensmerkmale beurteilt. Befragt man die Eltern, stellt man fest, dass Eltern-Kind-Trainings das oftmals angespannte 
Interaktionsverhalten zwischen Eltern und Kind verändern können. Eltern vermögen ihr Kind wieder positiver $\mathrm{zu}$ sehen, erleben weniger Stress, das Selbstwertgefühl steigt, Ehestreitigkeiten nehmen ab (ebd., S. 479f.). Dies war hier auch in Studie 3 durch den Interaktionsfragebogen zu belegen. Die Eltern intensiv in die Behandlung einzubeziehen erscheint auch nach dieser Studie erfolgsversprechend zu sein.

Im Vergleich zu den vorliegenden Veröffentlichungen der Programmautoren zu ihren Trainings fallen die Ergebnisse der hier durchgeführten Studien relativ dürftig aus. Dies ist jedoch nicht ungewöhnlich. Experten einer Therapieform und insbesondere Entwickler eines Verfahrens erzielen in der Regel bessere Ergebnisse als andere Anwender (Scheithauer \& Petermann, 2000).

Als Fazit zur Bewertung der Programme kann gezogen werden, dass das Aufmerksamkeitstraining nach Lauth und Schlottke nur bedingt das einhalten kann, was es verspricht. Im Vergleich zum Marburger Konzentrationstraining, welches hier mit deutlich weniger Sitzungen durchgeführt wurde, kam letzteres bei den psychometrischen Verfahren zu etwas besseren Ergebnissen. Das THOP als Gruppenprogramm hingegen vermag im Bereich der Aufmerksamkeitsfunktionen sogar eine deutlichere Leistungssteigerung zu bewirken. Außerdem verbessert sich die Interaktion in der Familie.

Bei allen hier durchgeführten Trainings wurde aus organisatorischen Gründen und begrenzten materiellen und personellen Ressourcen darauf verzichtet die Lehrer der Kinder enger einzubeziehen. 


\section{Zusammenfassung}

In der vorliegenden Dissertation wurde die Wirksamkeit von drei kognitivverhaltenstherapeutischen Programmen für Kinder mit Aufmerksamkeitsstörungen untersucht. In vier Studien wurden die Programme evaluiert. Insgesamt nahmen 126 Kinder an den Studien teil. 96 Kinder zwischen 7 und 13 Jahren, die als Patienten der Kinder- und Jugendpsychiatrie Mühlhausen und der Poliklinischen Institutsambulanz des Psychologischen Instituts der Universität Göttingen sowie des Sozialpädiatrischen Zentrums in Reifenstein vorstellig wurden, nahmen an der Untersuchung für die Studien 1 bis 3 teil. Neben psychometrischen Verfahren zur Messung verschiedener Aufmerksamkeitsfunktionen, wurden Fremdbeurteilungsmaße von Eltern und Lehrern als Erfolgsmaße verwendet und in einem Vortest-Nachtest-Design ausgewertet. 31 Kinder nahmen gemeinsam mit ihren Eltern an einem 15wöchigen THOP-Training (Therapieprogramm für Kinder mit hyperkinetischem und oppositionellem Trotzverhalten) und 34 an einem 15wöchigen Training für aufmerksamkeitsgestörte Kinder nach Lauth und Schlottke (1997) teil, 15 Kinder absolvierten das Basistraining nach Lauth und Schlottke. Beim Lauth- und Schlottke-Training wurden zwei begleitende Elternsitzungen angeboten. 16 Kinder bildeten eine Wartegruppe.

15 Kindern, aus denen fünf Gruppen gebildet wurden, kamen über einen Zeitraum von fünf Wochen einmal wöchentlich zum Marburger Konzentrationstraining in eine Kinder- und Jugendpsychiatrische Praxis. Die Kontrollgruppe setzte sich auch aus 15 Kindern zusammen, welche in Dreiergruppen einmal wöchentlich in einem Zeitraum von fünf Wochen das Computerspiel „Pettersson und Findus“ spielten.

Die Wirksamkeit des Trainings für aufmerksamkeitsgestörte Kinder nach Lauth und Schlottke (1997) unter den gegebenen praktischen Rahmenbedingungen konnte weder in Bezug auf die Aufmerksamkeitsleistung noch bezüglich der hyperkinetischen Verhaltenssymptome nachgewiesen werden. Das Training resultierte jedoch in einer Verbesserung auf der Ebene der kognitiven Hemmungskontrolle. Außerdem zeigte das Training positive Transfereffekte auf die Funktionstüchtigkeit des phonologischen Arbeitsgedächtnisses. Dabei waren die Verbesserungen durch das Basistraining stärker als durch das kombinierte Basis- und Strategietraining.

Mit dem THOP wurde eine als Einzeltraining konzipierte familienzentrierte Interventionen unter veränderten Randbedingungen (als Gruppenprogramm) untersucht, sowohl Eltern wie Kinder nahmen an insgesamt 15 Terminen teil. Es konnten tendenzielle 
Verbesserungen der Aufmerksamkeitsleistungen festgestellt werden. Insbesondere ließ sich aber die familiäre Interaktion durch das THOP als Gruppenprogramm verbessern. Die besten Erfolge erzielten Kinder, die gleichzeitig mit Methylphenidat behandelt wurden.

Bei der Studie zur Überprüfung des Marburger Konzentrationstrainings konnten in einzelnen Bereichen Verbesserungen der Aufmerksamkeitsleistungen nachgewiesen werden, diese betreffen vor allem die schulbezogene Konzentrationsfähigkeit, welche mit der Testbatterie zur Prüfung der Konzentrationsfähigkeit (TPK) erhoben wurde. Als positiv wird die Einbindung von Entspannungsverfahren erachtet, da durch diese ein Erregungsabbau erreicht wird, welche die Durchführung des Selbstinstruktionstrainings erleichtert. 


\section{Literaturverzeichnis}

Abikoff, H. (1987). Efficacy of cognitive training interventions in hyperactive children: A critical review. Clinical Psychology Review, 5, 479-512.

Abikoff, H. (1991). Cognitive training in ADHD children: Less to it than meets to the eye. Journal of Learning Disabilities, 24 (4), 205-209.

Achenbach, T. M. (1991). Manual for the Child Behavior Checklist/4-18 and 1991 profile. Burlington. University of Bermont, Department of Psychiatry.

Adair, J. G., Sharpe, D. \& Huynh, C-L. (1990). The Placebo control group: An analysis of its effectiveness in educational research. Journal of Experimental Education, 59, (1), 6786.

Affeldt, M., Deister, W., Granzner, D., Leipold, M., Leitner, W., Miller, R., Petraschke, A., Pfeiffer, C., Prenzlow, R. \& Thieme, A. (1998). KIKO: Kinder konzentrieren sich. Anregungen und Übungen für die Praxis. Hannover: Schroedel.

Anderson, G. M., Dover, M. A., Yang, B. P., Holahan, J. M., Shaywitz, S. E., Marchione, K. E., Hall, L. M., Fletcher, J. M. \& Shaywitz, B. A. (2000). Adrenomodullary function during cognitive testing in attention-deficit/ hyperactivity disorder. Journal of the American Academy of Child and Adolescent Psychiatry, 39, 635-643.

Angold, A., Erkanli, A., Egger, H. \& Costello, H. L. (2000). Stimulant treatment for children: a community perspective. Journal of the American Academy of Child and Adolescent Psychiatry, 39, 975-984.

Anastopoulos, A. D., Shelton, T. L., DuPaul, J. \& Guevremont, D. C. (1993). Parent training for attention-deficit hyperactivity disorder: Its impact on parent functioning. Journal of Abnormal Child Psychology, 21 (5), 581-596.

Arbeitsgruppe Deutsche Child Behavior Checklist (1998a). Elternfragebogen über das Verhalten von Kindern und Jugendlichen; deutsche Bearbeitung der Child Behavior Checklist (CBCL/ 4 -18). Einführung und Anleitung zur Handauswertung, 2.Auflage mit deutschen Normen, bearbeitet von Döpfner, M., Plück, J., Bölte, S., Lenz, K., Melchers, P. \& Heim, K. Köln: Arbeitsgruppe Kinder-, Jugend- und Familiendiagnostik (KJFD).

Arbeitsgruppe Deutsche Child Behavior Checklist (1993). Lehrerfragebogen über das Verhalten von Kindern und Jugendlichen; deutsche Bearbeitung der Teacher's Report Form der Child Behavior Checklist (TRF). Einführung und Anleitung zur Handauswertung, bearbeitet von Döpfner, M. \& Melchers, P. Köln: Arbeitsgruppe Kinder-, Jugend- und Familiendiagnostik (KJFD).

Armstrong, C. L., Hayes, K. M. \& Martin, R. (2001). Neurocognitive problems in attention deficit disorder: Alternative concepts and evidence for impairment in inhibition of selective attention. Annals of the New York Academy of Sciences, 931, 196-215.

Atkinson, R. L., Atkinson, R. C., Smith, E. E., Bem, D. J. \& Hilgard, E. R. (1990). Introduction to Psychology. New York: Harcourt Brace Jovanovich. 
Axline, O. M. (1947; 1980). Kinder-Spieltherapie im nicht-direktiven Verfahren. München: Reinhardt.

Baddeley, A. D. (1986). Working memory. Oxford: Oxford University Press.

Baddeley, A. D. (1997). Human Memory Theory \& Practice (rev. ed.). Psychology Press.

Barchmann, H., Ettrich, K.-U., Kinze, W. \& Reschke, K. (1986). Konzentrationstraining. Ein Therapieprogramm für Kinder von 6 bis 10. Leipzig: Universitätsdruck.

Barchmann, H., Ettrich, K.-U., Kinze, W. \& Reschke, K. (1988). Konzentrationstraining. Ein Therapieprogramm für Kinder von 6 bis 10. Leipzig: Universitätsdruck.

Barkley, R. A. (1981). Hyperactive children: A handbook for diagnosis and treatment. New York: Guilford.

Barkley, R. A. (1987). Defiant children. A clinican's manual for parent training. New York: Guilford.

Barkley, R. A. (1989). The problem of stimulus control and rulegoverned behavior in attention deficit disorder with hyperactivity. In L. M. Bloomingdale \& J. M. Swanson (Eds.), Attention deficit disorder, Volume IV (pp. 203-234). Oxford: Pergamon.

Barkley, R. A. (1990). Attention deficit hyperactivity disorder: A handbook for diagnosis and treatment. Hove East Sussex: Guilford.

Barkley, R. A. (1997). ADHD and the nature of self-control. New York: Guilford Press.

Barkley, R. A. (1998). Attention-Deficit Hyperactivity Disorder: A Handbook for Diagnosis and Treatment $\left(2^{\text {nd }}\right.$ ed.). New York: Guilford Press.

Barkley, R. A. (2002). Das große ADHS - Handbuch für Eltern. Bern: Hans Huber

Barkley, R. A., DuPaul, G. J. \& McMurray, M. B. (1990). Comprehensive evaluation of attention deficit disorder with and without hyperactivity as defined by research criteria. Journal of Consulting and Clinical Psychology, 58 (6), 775-789.

Barkley, R. A., Karlsson, J., Strzelecki, E. \& Murphy, J. V. (1985). Effects of age and ritalin dosage on the mother-child interactions of hyperactive children. Journal of Consulting and Clinical Psychology, 52, 750-758.

Bartels, K. (2003). Wirkt eine Gruppenversion des THOP bei Aufmerksamkeitsstörungen besser als ein Selbstinstruktionstraining? Unveröffentlichte Diplomarbeit. GeorgAugust-Universität, Göttingen.

Baumann, U. \& Perrez, M. (1998). Grundbegriffe - Einleitung. In U. Baumann \& Perrez (Hrsg.), Lehrbuch Klinische Psychologie - Psychotherapie. (2. Aufl.). Bern: Huber.

Baumgaertel, A., Wolraich, M. L. \& Dietrich, M. (1995). Comparison of diagnostic criteria for attention deficit disorder in a german elementary school sample. Journal of the American Academy of Child and Adolescent Psychiatry, 34 (5), 629-638. 
Baving, L. (1999). Atypical frontal brain activation in ADHD: Preschool and elementary school boys and girls. Journal of the American Academy of Child and Adolescent Psychiatry, 38, 1363-1371.

Baving, L. \& Schmidt, M. H. (2001). Evaluierte Behandlungsansätze in der Kinder- und Jugendpsychiatrie I. Zeitschrift für Kinder- und Jugendpsychiatrie und Psychotherapie, 29 (3), 189-205.

Beck, M. (1998). Therapiebaukasten oder Trainingsprogramm? Das Aufmerksamkeitstraining von Lauth \& Schlottke als schulische Intervention. In M. Beck (Hrsg.), Evaluation als Maßnahme der Qualitätssicherung: pädagogisch-psychologische Intervention auf dem Prüfstand (S. 161-172). Tübingen: DGVT-Verlag.

Beck, M. \& Mock, A. (1995). Aufmerksamkeitsförderung in der Schule. Heilpädagogische Forschung, 21 (4), 180-185.

Beck, M. \& Mock, A. (1996). Aufmerksamkeitsförderung in der Schule. In: E. Witruk, G. Friedrich, B. Sabisch, \& D. Kotz. Pädagogische Psychologie im Streit um ein neues Selbstverständnis. Bericht über die 5. Tagung der Fachgruppe Pädagogische Psychologie in der Deutschen Gesellschaft für Psychologie e.V. in Leipzig 1995. Serie Psychologie, Band 12. (S. 303-311). Landau: Verlag Empirische Pädagogik.

Bell, V., Lynne, S. \& Kolvin, L. (1989). Play group therapy: Processes and patterns and delayed effects. In M. H.Schmidt \& H. Remschmidt (eds.), Needs and prospects of child and adolescent psychiatry. Göttingen: Hogrefe und Bern: Huber.

Belmont, J. M. \& Butterfield, E. G. (1977). The instructional approach to developmental cognitive research. In R. Kail \& J. Magen (Eds.), Perspectives on the development of memory and cognition (pp. 437-481). Hillsdale, N. J.: Erlbaum.

Biederman, J., Faraone, S. V., Keenan, K., Benjamin, J., Krifcher, B., Moore, C., SprichBuckminster, S., Ugaglia, K., Jellinek, M. S., Steingard, R., Spencer, T., Norman, D., Kolodny, R., Kraus, I., Perrin, J., Keller, M. B. \& Tsuang, M. T. (1992). Further evidence for family-genetic risk factors. Archives of General Psychiatry, 49, 728-738.

Biedermann, J., Faraone, S. V., Mick, E., Spencer, T., Wilens, T., Kiely, K., Guite, J., Ablon, J. S., Reed, A. \& Warburton, R. (1995). High risk for attention deficit hyperactivity disorder among children of parents with childhood onset of the disorder: A pilot study. American Journal of Psychiatry, 152 (3), 431-435.

Biederman, J., Faraone, S. V., Taylor, A., Sienna, M., Williamson, S. \& Fine, C. (1998). Diagnostic continuity between child and adolscent ADHD: Findings from a longitudinal clinical sample. Journal of the American Academy of Child and Adolescent Psychiatry, 37 (3), 305-313.

Biederman, J. \& Spencer, T. J. (2000). Genetics of childhood disorders: XIX. ADHD, Part 3: Is ADHD a noradrenergic disorder? Journal of the American Academy of Child and Adolescent Psychiatry, 39 (10), 1330-1333.

Blech, J. \& Thimm, K. (2002, 15. Juli). Kinder mit Knacks. Der Spiegel, 29, S. 122-131. 
Bortz, J. (1993). Statistik für Sozialwissenschaftler (4. Aufl.). Berlin: Springer.

Bortz, J. \& Döring, N. (1995). Forschungsmethoden und Evaluation für Sozialwissenschaftler (2. Aufl.). Berlin: Springer.

Braswell, L. \& Bloomquist, M. L. (1991). Cognitive-behavioral therapy with ADHD children: Child, family, and school interventions. New York: Guilford Press.

Brickenkamp, R. (1994). Test d2. Aufmerksamkeits-Belastungs-Test (8. Aufl.). Göttingen: Hogrefe.

Brown, R. T. (1980). Impulsivity and psychoeducational intervention in hyperactive children. Journal of Learning Disability, 13, 249-254.

Brühl, B., Döpfner, M. \& Lehmkuhl, G. (2000). Der Fremdbeurteilungsbogen für Hyperkinetische Störungen (FBB-HKS). Prävalenz hyperkinetischer Störungen im Elternurteil und psychometrische Kriterien. Kindheit und Entwicklung, 9, 115-125.

Bundesministerium für Gesundheit (2002). Pressemitteilung: Einigung zur Diagnose und Behandlung der Aufmerksamkeitsdefizit-Hyperaktivitätsstörung erzielt [www]. Verfügbar unter: http://www.bmgs.bund.de/archiv/presse bmgs/presse $2002 / \mathrm{m} /$ 213.htm [21.01.03].

Campbell, D. T. \& Fiske, D. (1959). Convergent and diskriminant validation by the multitraitmultimethod matrix. Psychological Bulletin, 56, 81-105.

Cantwell, D. P. \& Baker, L. (1992). Attention deficit disorder with and without hyperactivity: A review and comparison of matched groups. Journal of the American Academy of Child and Adolescent Psychiatry, 31 (3), 432-438.

Cattell, R. B. (1960). Culture Fait Intelligence Test, Scale 2 (Handbuch). 3. Aufl. Champaign, III. IPAT.

Chabot, R. J., di Michele, F., Prichep, L. \& John, E. R. (2001). The clinical role of computerized EEG in the evaluation and treatment of learning and attention disorders in children and adolescents. Journal of Neuropsychiatry and Clinical Neurosciences, $13,171-186$.

Cohen, J. (1988). Statistical Power Analysis for the Behavioral Sciences $\left(2^{\text {nd }}\right.$ ed). Hillsdale, NJ: Erlbaum.

Conners, C.K. (1980). Food additives and hyperactive children. New York: Plenum

Connor, D. F., Glatt, S. J., Lopez, I. D., Jackson, D. \& Melloni, R. H. (2002). Psychopharmacology and aggression. I: A meta-analysis of stimulant effects on overt/covert aggression-related behaviors in ADHD. Journal of the American Academy of Child and Adolescent Psychiatry, 41 (3), 253-261. 
Denney, C. B. \& Rapport, M. D. (1999). Predicting methylphenidate response in children with ADHD: Theoretical, empirical, and conceptual models. Journal of the American Academy of Child and Adolescent Psychiatry, 38 (4), 393-401.

De Quirós, G. B., Kinsbourne, M., Palmer, R. L. \& Tocci Rufo, D. (1994). Attention deficit disorder in children: Three clinical variants. Developmental and Behavioral Pediatrics, 15, 311-319.

Detzner, M. \& Schmidt, M. H. (1988). Epidemiologische Methoden. In Remschmidt, H. \& Schmidt, H. M. (Hrsg.), Kinder- und Jugendpsychiatrie in Klinik und Praxis (S. 320337). Stuttgart: Thieme-Verlag.

Die Bibel (1975). Stuttgart: Deutsche Bibelstiftung.

Dilling, H., Mombour, W. \& Schmidt, M. H. (Hrsg.). (1991). Internationale Klassifikation psychischer Störungen ICD-10 Kapitel V (F). Forschungskriterien. Bern: Huber.

Döpfner, M. (1999). Ergebnisse der Therapieforschung zur Verhaltenstherapie mit Kindern und Jugendlichen. In M. Borg-Laufs (Hrsg.), Lehrbuch der Verhaltenstherapie mit Kindern und Jugendlichen (S. 153-185). Tübingen: DGVT Verlag.

Döpfner, M. (2000). Hyperkinetische Störungen. (4. vollständig überarbeitete und erweiterte Auflage). In F. Petermann (Hrsg.), Lehrbuch der Klinischen Kinderpsychologie und -psychotherapie. Göttingen: Hogrefe.

Döpfner, M., Berner, W. \& Lehmkuhl, G. (1997). Verhaltensauffälligkeiten von Schülern im Urteil der Lehrer- Reliabilität und faktorielle Validität der Teacher's Report Form der Child Behavior Checklist. Zeitschrift für Differenzielle und Diagnostische Psychologie, 18 (4), 199-214.

Döpfner, M., Breuer, D. \& Lehmkuhl, G. (2000). The Cologne Multimodal Intervention Study (COMIS): Study design and overall treatment outcomes. In M.Döpfner, J. Frölich, G. Lehmkuhl (Hrsg.), Hyperkinetische Störungen. Göttingen, Bern, Toronto, Seattle: Hogrefe.

Döpfner, M., Frölich, J. \& Lehmkuhl, G. (2000). Hyperkinetische Störungen. Göttingen: Hogrefe.

Döpfner, M. \& Lehmkuhl, G. (2000). DISYPS-KJ: Diagnostiksystem für psychische Störungen im Kindes- und Jugendalter nach ICD-10 und DSM-IV. Bern: Huber.

Döpfner, M. \& Lehmkuhl, G. (2002). Evidenzbasierte Therapie von Kindern und Jugendlichen mit Aufmerksamkeitsdefizit/Hyperaktivitätsstörung (ADHS). Praxis der Kinderpsychologie und Kinderpyschiatrie, 51, 419-440.

Döpfner, M. \& Lehmkuhl, G. (2003). Evidenzbasierte Therapie. ADHS-ReportAufmerksamkeitsdefizit- und Hyperaktivitätsstörungen, 4, 1-3.

Döpfner, M., Lehmkuhl, G. \& Schürmann, S. (1996). Das Therapieprogramm für Kinder mit hyperkinetischem und oppositionellem Problemverhalten (THOP) - Aufbau und Einzelfall-Evaluation. Zeitschrift für Kinder- und Jugendpsychiatrie, 24, 145-163. 
Döpfner, M., Schmeck, K., Berner, W., Lehmkuhl, G. \& Poustka, F. (1994). Zur Reliabilität und faktoriellen Validität der Child Behavior Checklist- eine Analyse in einer klinischen und einer Feldstichprobe. Zeitschrift für Kinder- und Jugendpsychiatrie und Psychotherapie, 22, 189-205.

Döpfner, M., Schürmann, S. \& Frölich, J. (1997). Therapieprogramm für Kinder mit hyperkinetischem und oppositionellem Problemverhalten (THOP). Weinheim: Beltz.

Döpfner, M., Schürmann, S. \& Frölich, J. (1998). Therapieprogramm für Kinder mit hyperkinetischem und oppositionellem Trotzverhalten (THOP). (2. korrigierte Aufl.). Weinheim. Psychologie Verlags Union.

Döpfner, M., Schürmann, S. \& Frölich, J. (2002). Therapieprogramm für Kinder mit hyperkinetischem und oppositionellem Trotzverhalten (THOP). (3. vollständig überarbeitete Aufl.). Weinheim. Psychologie Verlags Union.

Döpfner, M., Schürmann, S. \& Lehmkuhl, G. (1999). Wackelpeter \& Trotzkopf - Hilfen bei hyperkinetischem und oppositionellem Verhalten. Weinheim: Psychologie Verlags Union.

Douglas, V. I. (1980). Treatment and training approaches to hyperactivity: Establishing internal or external control. In C. K. Whalen \& B. Henker (Eds.), Hyperactive children. The social ecology of identification and treatment (pp. 283-318). New York: Academic Press.

Dubey, D. R., O'Leary, S. G. \& Kaufman, K. F. (1983). Training parents of hyperactive children in child management: A comparative outcome study. Journal of Abnormal Child Psychology, 11 (2), 229-246.

DuPaul, G.J., Guevremont, D. C. \& Barkley, R. A. (1992). Behavioral treatment of attentiondeficit hyperactive disorder in the classroom. Behavior Modification, 16, 204-225.

Düker, H. \& Lienert, G. A. (1965). Konzentratons-Leistungs-Test (KLT). Göttingen: Hogrefe.

Dush, D. M., Hirt, M. L. \& Schroeder, H. E. (1989). Self-statement modification in the treatment of child behavior disorders: A meta-analysis. Psychological Bulletin, 106, 97-106.

Eastman, B. G. \& Rasbury, W. C. (1981). Cognitive self-instruction for the control of impulsive classroom behavior: Ensuring the treatment package. Journal of Abnormal Child Psychology, 9, 381-387.

Eisert, H. G. (1981). Entwicklung der Vigilanzfunktionen. In H. Remschmidt \& M. Schmidt (Hrsg.), Neuropsychologie des Kindesalters (S. 110-122). Stuttgart: Enke.

Eisert, H. G. (1998). Hyperkinetische Störungen. In H. C. Steinhausen \& M. von Aster (Hrsg.), Handbuch Verhaltenstherapie und Verhaltensmedizin bei Kindern und Jugendlichen (S. 131-159). Weinheim: Beltz/ Psychologie Verlags Union. 
Eisert, H. G. (2000). Kognitiv-verhaltenstherapeutische Behandlung hyperaktiver Kinder. In H. C. Steinhausen (Hrsg.), Hyperkinetische Störungen bei Kindern, Jugendlichen und Erwachsenen (S. 158-173). Stuttgart: Kohlhammer.

Ellis, A. (1979). Praxis der rational-emotiven Therapie. München: Urban und Schwarzenberg.

Erhardt, D. \& Baker, B. L. (1990). The effects of behavioral parent training on families with young hyperactive children. Journal of Behavior Therapy and Experimental Psychiatry, 21 (2), 121-132.

Esser, G. \& Schmidt, M. H. (1987). Minimale cerebrale Dysfunktion. Stuttgart: Enke

Ettrich, C. (1998). Konzentrationstrainingsprogramm für Kinder (III: 3. und 4. Schulklassen). Göttingen: Vandenhoeck \& Ruprecht.

Faraone, S. V. (2000). Genetics of childhood disorders: XX. ADHD, Part 4: Is ADHD genetically heterogeneous? Journal of the American Academy of Child and Adolescent Psychiatry, 39 (11), 1455-1457.

Faraone, S. V. \& Biederman, J. (1998). Neurobiology of attention-deficit hyperactivity disorder. Biological Psychiatry, 44, 951-958.

Faraone, S. V., Biederman, J., Krifcher Lehmann, B. K., Spencer, T., Norman, D., Seidman, L. J., Kraus, I., Perrin, J. Chen, W. J. \& Tsuang, M. T. (1993). Intellectual performance and school failure in children with attention deficit hyperactivity disorder and in their siblings. Journal of Abnormal Psychology, 102 (4), 616-623.

Feingold, B.F. (1975). Why your child is hyperactive. New York: Random House

Fergusson, D. M., Horwood, L. J. \& Lynskey, M. T. (1993). The effects of conduct disorder and attention deficit in middle childhood on offending and scholastic ability at age 13. Journal of Child Psychology and Psychiatry, 34 (6), 899-916.

Felstehausen, F. (2003). Evaluation eines kognitiv-behavioralen Trainings bei Kindern mit Aufmerksamkeitsstörungen. Marburger Konzentrationstraining. Unveröffentlichte Diplomarbeit. Georg-August-Universität, Göttingen.

Fiedler, P. (1996). Verhaltenstherapie in und mit Gruppen. Weinheim: Psychologie Verlags Union.

Földényi, M., Imhof, K. \& Steinhausen, H.- C. (2000). Klinische Validität der computerunterstützten TAP bei Kindern mit Aufmerksamkeits/Hyperaktivitätsstörungen. Zeitschrift für Neuropsychologie, 11 (3), 154-167.

Földényi, M., Tagwerker-Neuenschwander, F., Giovanoli, A., Schallberger, U. \& Steinhausen, H.- C. (1999). Die Aufmerksamkeitsleistungen von 6-10jährigen Kindern in der TAP. Zeitschrift für Neuropsychologie, 10 (2), 87-102.

Forehand, R. \& McMahon, R. J. (1981). Helping the non-compliant child: A clinical's guide to parent training. New York: Guilford Press. 
Frölich, J. (1993). Möglichkeiten des pädagogischen Umgangs mit hyperkinetischen Kindern mit Störungen des Sozialverhaltens im Alter von 6-12 Jahren. Dissertation: Universität Bonn.

Frölich, J. \& Döpfner, M. (1997). Individualisierte Diagnostik bei Kindern mit hyperkinetischen Störungen. Praxis der Kinderpsychologie und Kinderpyschiatrie, 46 (8), 597-609.

Frölich, J., Döpfner, M., Berner, W. \& Lehmkuhl, G. (2002). Behandlungseffekte kombinierter kognitiver Verhaltenstherapie mit Elterntraining bei hyperkinetischen Kindern. Praxis der Kinderpsychologie und Kinderpsychiatrie, 51, 476-493.

Frölich, J., Döpfner, M., Biegert, H. \& Lehmkuhl, G. (2002). Praxis des pädagogischen Umgangs von Lehrern mit hyperkinetisch-aufmerksamkeitsgestörten Kindern im Schulunterricht. Praxis der Kinderpsychologie und Kinderpsychiatrie, 51, 494-506.

GAMOP Samproduktion AB (Hrsg.) (1998). Neues von Pettersson und Findus. Hamburg: Oetinger interaktiv.

Giedd, J. N., Blumenthal, J., Molloy, E. \& Castellanos, F. X. (2001). Brain imaging of attention deficit/ hyperactivity disorder. Annals of the New York Academy of Sciences, 931, 33-49.

Gillberg, C., Melander, H., von Knorring, A.-L., Janols, L.-O., Thernlund, G., Hägglöf, B., Eidevall-Wallin, L., Gustafsson, P. \& Kopp, S. (1997). Long-term stimulant treatment of children with attention-deficit hyperactivity disorder symptoms. Archives of General Psychiatry, 54, 857-864.

Graetz, B. W., Sawyer, M. S., Hazell, P. L., Arney, F. \& Baghurst, P. (2001). Validity of DSM-IV ADHD subtypes in a nationally representative sample of australien children and adolescents. Journal of the American Academy of Child and Adolescent Psychiatry, 40 (12), 1410-1417.

Greene, R. W. \& Ablon, J. S. (2001). What does the MTA study tell us about effective psychosocial treatment for ADHD? Journal of Clinical Child Psychology, 30 (1), 114121.

Grosse, K.-P. \& Skrodzki, K. (2001). Diagnostik und Therapie bei ADHS, Arbeitsgemeinschaft der wissenschaftlichen medizinischen Fachgesellschaften (AWMF) [www] verfügbar unter: http://www.awmf-online.de [20.5.04]

Guevremont, D. C. \& Dumas, M. C. (1994). Peer relationship problems and disruptive disorder. Journal of Emotional and Behavioral Disorders, 2 (3), 164-172.

Hager, W. (1987). Grundlagen einer Versuchsplanung zur Prüfung empirischer Hypothesen der Psychologie. In G. Lüer (Hrsg.), Allgemeine experimentelle Psychologie (S. 43264). Stuttgart: Gustav Fischer Verlag.

Hager, W. (1992). Jenseits von Experiment und Quasi-Experiment. Zur Struktur psychologischer Versuche und zur Ableitung von Vorhersagen. Göttingen: Hogrefe. 
Hager, W. (1995). (Hrsg.). Programme zur Förderung des Denkens bei Kindern: Konstruktion, Evaluation und Metaevaluation. Göttingen: Hogrefe.

Hager, W. (1999). Testplanung bei geplanten Kontrasten, Ableitung von Vorhersagen und Entscheidungen über psychologische Vorhersagen (Testplanung 2) (Version 11.4). Unveröffentlichtes Manuskript, Georg-August-Universität, Göttingen.

Hager, W. (2000). Zur Wirksamkeit von Interventionsprogrammen: Allgemeine Kriterien der Wirksamkeit von Programmen in einzelnen Untersuchungen. In W. Hager, J.-L. Patry \& H. Brezing (Hrsg.), Evaluation psychologischer Interventionsmaßnahmen. Standards und Kriterien: Ein Handbuch (S. 153-168). Bern: Hans Huber.

Hager, W. \& Hasselhorn, M. (1995). Konzeption und Evaluation von Programmen zur kognitiven Förderung: theoretische Überlegungen. In W. Hager (Hrsg.), Programme zur Förderung des Denkens bei Kindern: Konstruktion, Evaluation und Metaevaluation (S. 41-85). Göttingen: Hogrefe.

Hager, W. \& Hasselhorn, M. (2000). Psychologische Interventionsmaßnahmen: Was sollen sie bewirken können? In W. Hager, J.- L. Patry \& H. Brezing (Hrsg.), Evaluation psychologischer Interventionsmaßnahmen. Standards und Kriterien: Ein Handbuch (S. 41-85). Bern: Huber.

Hager, W., Hübner, S. \& Hasselhorn, M. (2000). Zur Bedeutung der sozialen Interaktion bei der Evaluation kognitiver Förderprogramme. Zeitschrift für Pädagogische Psychologie, 14 (2-3), 106-115.

Hager, W., Patry, J.-L. \& Brezing, H. (2000). Einleitung und Überblick. In W. Hager, J.-L. Patry \& H. Brezing (Hrsg.), Evaluation psychologischer Interventionsmaßnahmen. Standards und Kriterien: Ein Handbuch (S. 1-7). Bern: Hans Huber.

Halperin, J. M., Newcorn, J. H., Sharma, V., Healey, J. M., Wolf, L. E., Pascualvaca, D. M. \& Schwartz, S. (1990). Inattentive and noninattentive ADHD children: Do they constitute a unitrary group?. Journal of Abnormal Child Psychology, 18, 437-449.

Hasselhorn, M. \& Grube, D. (2003). Das Arbeitsgedächtnis: Funktionsweise, Entwicklung und Bedeutung für kognitive Leistungsstörungen. Sprache, Stimme, Gehör 27, 31-37.

Hasselhorn, M. \& Hager, W. (1996). Neuere Programme zur Denkförderung bei Kindern: Bewirken sie größere Kompetenzsteigerungen als herkömmliche Wahrnehmungsübungen? Psychologie in Erziehung und Unterricht, 43, 169-181.

Hasselhorn, M. \& Mähler, C. (2000). Transfer: Theorien, Technologien und empirische Erfassung. In W. Hager, J. L. Patry \& H. Brenzing (Hrsg.), Evaluation psychologischer Interventionsmassnahmen. Standards und Kriterien: Ein Handbuch (S. 86-101). Bern: Huber.

Hasselhorn, M. \& Schumann-Hengsteler, R. (1998). In D.H. Rost (Hrsg.), Handwörterbuch Pädagogische Psychologie (S. 14-17). Weinheim: Psychologie Verlags Union. 
Hasselhorn, M., Tiffin-Richards, M. C., Woerner, W., Banaschewski, T. \& Rothenberger, A. (2000). Spielt der phonetische Speicher des Arbeitsgedächtnisses eine bedeutsame Rolle für die Differentialdiagnose von Lese-Rechtschreib-Schwierigkeiten? Analysen zum „Kunstwörter-Nachsprechen“ bei Kindern mit LRS- und/ oder HKS-Diagnose. In Hasselhorn, M., Schneider, W. \& Marx, H. (Hrsg), Diagnostik von LeseRechtschreibschwierigkeiten. Test \& Trends, Neue Folge 1, 149-165. Jahrbuch der pädagogisch- psychologischen Diagnostik. Göttingen: Hogrefe.

Hechtman, L. (1994). Genetic and neurobiological aspects of attention deficit hyperactive disorder: a review. Journal of psychiatry and neuroscience, 19 (3), 193-201.

Heilmann, K. M. \& Valenstein, E. (Eds.) (1985). Clinical Neuropsychology. New York: Oxford University Press.

Herrero, M. A., Hechtman, L. \& Weiss, G. (1994). Antisocial disorders in hyperactive subjects from childhood to adulthood: Predictive factors and charcterization of subgroups. American Journal of Orthopsychiatry, 64, 510-529.

Heubrock, D. \& Petermann, F. (2001). Aufmerksamkeitsdiagnostik: Kompendien Psychologische Diagnostik (Band 2). Göttingen: Hogrefe.

Hildebrand, H. und Fecht, A. von (2003). Behandlung von Aufmerksamkeitsstörungen verfügbar unter: http://www.psychologie.uni-oldenburg.de/ gesundheit/ neuro/ $\underline{\text { REHAUFM2.htm }}$ [14.5.03]

Hinshaw, S. P. (1987). On the distinction between attentional deficits/ hyperactivity and conduct problems/ aggression in child psychopathology. Psychological Bulletin, 101, 443-463.

Hinshaw, S. P. \& Erhardt, D. (1991). Attention-deficit hyperactive disorder. In P.C. Kendall (Ed.), Child and adolescent therapy: Cognitive behavioral procedures (pp. 98-128). New York: Guilford Press.

Hinshaw, S., Henker, B. \& Whalen, C. (1984). Cognitive behavioural interventions for hyperactive boys: Comparative and combined effects. Journal of Consulting Clinical Psychology, 52, 739-749.

Höger, C., Goering, S. \& Schacher-Gums, G. (1998). Indikation und Risiken - Die Behandlung des Hyperkinetischen Syndroms mit Methylphenidat. Niedersächsisches Ärzteblatt, 5, $15-19$.

Hoffmann, N. (2000). Therapeutische Beziehung und Gesprächsführung. In J. Margraf (Hrsg.), Lehrbuch der Verhaltenstherapie Band 1: Grundlagen, Diagnostik, Verfahren, Rahmenbedingungen (2. Aufl., S. 363-371). Berlin: Springer.

Horn, W. F., Ialongo, N., Greenberg, G., Packard, T. \& Smith-Winberry, C. (1990). Additive effects of behavioral parent training and self-control therapy with attention deficit hyperactivity disordered children. Journal of Clinical Child Psychology, 19 (2), 98110. 
Hynd, G. W., Lorys, A. R., Semrud-Clikeman, M., Nieves, N., Huettner, M. \& Lahey, B. B. (1991). Attention deficit disorder without hyperactivity: A distinct behavioural and neurocognitive syndrome. Journal of Child Neurology, 6, 37-43.

Jensen, P. S. (2000). Current concepts and controversies in the diagnosis and treatment of attention deficit hyperactivity disorder. Current Psychiatry Reports, 2, 102-109.

Jensen, P. S. (2001). ADHD comorbidity findings from the MTA study: Comparing comorbid subgroups. Journal of the American Academy of Child and Adolescent Psychiatry, 40, 147-158.

Johnston, C. (1996). Parent characteristics and parent-child interactions in families of nonproblem children and ADHD children with higher and lower levels of oppositional-defiant behavior. Journal of Abnormal Child Psychology, 24 (1), 85-104.

Kausch, T. (2002). Evaluation kognitiv-behavioraler Interventionsmethoden zur Behandlung von aufmerksamkeitsdefizit- und hyperaktivitätsgestörten Kindern: Messung von Einzel- und Additiveffekten. Dissertation: Universität Tübingen.

Kazdin, A. E. (1990). Premature termination from treatment among children referred for antisocial behavior. Journal of Child Psychology and Psychiatry, 31, 415-425.

Kazdin, A. E. (1997). Practitionier Review: Psychosocial treatments for conduct disorder in children. Journal of Child Psychology and Psychiatry, 38 (2), 161-178.

Klauer, K. J. (2001). Trainingsforschung: Ansätze - Theorien - Ergebnisse (S. 5-66). In K. J. Klauer (Hrsg.), Handbuch kognitiver Trainings. Göttingen: Hogrefe.

Kelley, M. L. (1990). School-home notes: Promoting Children's classroom success. New York: Guilford.

Kleber, E. W. (1978). Zum Begriff Konzentration. In K. J. Klauer (Hrsg.), Handbuch der Pädagogischen Diagnostik. Bd. 1 (S. 395-401). Düsseldorf: Schwann.

Klein-Hessling, J. \& Lohaus, A. (1998). Bleib locker. Ein Stresspräventionstraining für Kinder im Grundschulalter. Göttingen: Hogrefe.

Knölker, U., Mattejat, F. und Schulte-Markwort (2000). Kinder- und Jugendpsychiatrie systematisch. Bremen: Uni-Med.

Kraak, B. (1991). Der riskante Weg von der Information zum Wissen. Göttingen: Hogrefe.

Krause, K.-H., Dresel, S. \& Krause, J. (2000). Neurobiologie der Aufmerksamkeitsdefizit-/ Hyperaktivitätsstörung. Psycho, 26, 199-208.

Krowatschek, D. (1994a). Das Marburger Konzentrationstraining (2. Auflage). Dortmund: Verlag Modernes Lernen.

Krowatschek, D. (1994b). Entspannung in der Grundschule. Dortmund: Borgmann. 
Krowatschek, D. (1996). Überaktive Kinder im Unterricht. Ein Programm zur Förderung der Selbstwahrnehmung, Strukturierung, Sensiblisierung und Selbstakzeptanz unruhiger Kinder im Unterricht und in der Gruppe. Dortmund: Borgmann.

Krowatschek, D. (2001). Alles über ADS. Ein Ratgeber für Eltern und Lehre. Walter-Verlag.

Krowatschek, D. (2003). ADS und ADHS. Diagnose und Therapie. Dortmund: Borgmann.

Kunert, H. J., Derichs, G. \& Irle, E. (1996). Entwicklung von Aufmerksamkeitsfunktionen im Kindesalter: Ergebnisse einer vorläufigen Normierung der computerunterstützten Testbatterie zur Aufmerksamkeitsprüfung (TAP) an 9- bis 12jährigen Kindern. Zeitschrift für Neuropsychologie, 7 (2), 92-113.

Kurth, E. \& Büttner, G. (1999). Testreihe zur Prüfung der Konzentrationsfähigkeit (TPK). (2., neu bearbeitete Auflage). Göttingen: Hogrefe.

Laumann, A. \& Poustka, F. (1991). Differenzielle Diagnostik und Therapieerfolg beim Hyperkinetischen Syndrom. Zeitschrift für Kinder- und Jugendpsychiatrie, 19, 231239.

Lauth, G. W. (1996). Effizienz eines metakognitiv-strategischen Trainings bei lern- und aufmerksamkeitsbeeinträchtigten Grundschülern. Zeitschrift für Klinische Psychologie, 25, 21-32.

Lauth, G. (1998). Gedächtnisstörungen: Intervention. In U. Baumann \& M. Perrez (Hrsg.), Lehrbuch Klinische Psychologie - Psychotherapie. (2. Aufl., S. 593-605). Bern: Huber.

Lauth, G. (2001). Konzentrations- und Aufmerksamkeitstrainings. In M. Borg-Laufs (Hrsg.), Lehrbuch der Verhaltenstherapie mit Kindern und Jugendlichen. Band 2: Interventionsmethoden (S. 577-603). Tübingen: DGVT Deutsche Gesellschaft für Verhaltenstherapie.

Lauth, G. W. \& Fellner, C. (1998). Evaluation eines multimodalen Therapieprogramms bei Aufmerksamkeitsdefizit-/ Hyperaktivitätsstörungen über eine differenzierte Einzelfallforschung. In Greisbach, M., Kullick, U, \& Souvignier, E. (Hrsg.), Von der Lernbehindertenpädagogik zur Praxis schulischer Förderung (S. 109-124). Lengerich: Pabst.

Lauth, G. W. \& Linderkamp, F. (1998). Durchführungspraktikabilität eines Therapieprogrammes für aufmerksamkeitsgestörte Kinder. Psychologie, Erziehung, Unterricht, 45, 81-91.

Lauth, G. W. \& Linderkamp, F. (2000). Diagnostik und Therapie bei Aufmerksamkeitsstörungen. In H.C. Steinhausen (Hrsg.), Hyperkinetische Störungen bei Kindern, Jugendlichen und Erwachsenen (S. 127-157). Stuttgart: Kohlhammer.

Lauth, G.W., Naumann, K., Roggenkämper, A. \& Heine, A. (1996). Verhaltensmedizinische Indikation und Evaluation einer kognitiv-behavioralen Therapie mit aufmerksamkeitsgestörten/ hyperaktiven Kindern. Zeitschrift für Kinder- und Jugendpsychiatrie, 24, 164-175. 
Lauth, G. W. \& Schlottke, P. F. (1993). Training mit aufmerksamkeitsgestörten Kindern. Weinheim: Psychologie Verlags Union.

Lauth, G. W. \& Schlottke, P. F. (1997). Training mit aufmerksamkeitsgestörten Kindern. 3. Auflage. Weinheim: Psychologie Verlags Union.

Lauth, G. W. \& Schlottke, P.F. (2002). Training mit aufmerksamkeitsgestörten Kindern. 5. Auflage. Weinheim: Psychologie Verlags Union.

Lauth, G. W. \& Schlottke, P.F. (2003). Training mit aufmerksamkeitsgestörten Kindern. In Petermann, F. (Hrsg.), Kinderverhaltenstherapie. Grundlagen, Anwendungen und manualisierte Trainingsprogramme. 2. Auflage. (S. 268-289). Baltmannshausen: Schneider Verlag Hohengeren.

Lehmkuhl, G. \& Döpfner, M. (2000). Hyperkinetische und aggressive Symptome bei Kindern mit hyperkinetischen Störungen im Urteil von Eltern, Lehrern und klinischen Untersuchern - zur Bedeutung eines Diagnostik-Systems. In F. Poustka (Hrsg.), Aktuelle Forschung in der Kinder- und Jugendpsychiatrie (S. 44-68). Lengerich: Pabst.

Lehmkuhl, G. \& Lehmkuhl, U. (2002). Gruppenpsychotherapie mit Kindern und Jugendlichen. In G. Lehmkuhl (Hrsg.), Theorie und Praxis individualpsychologischer Gruppenpsychotherapie. Göttingen: Vandenhoek \& Ruprecht.

Levy, F. \& Swanson, J. M. (2001). Timing, space and ADHD: The dopamine theory revisited. Australien and New Zealand Journal of Psychiatry, 35, 504-511.

Linderkamp, F. (2002). Katamnestische Untersuchung zu einem Selbstinstruktionstraining mit aufmerksamkeitsgestörten Kindern. Verhaltenstherapie und Verhaltensmedizin, 23 (1), 53-73.

Lipsey, M. W. \& Wilson, D. B. (1993). The efficacy of psychological, educational, and behavioral treatment. Confirmation from meta-analysis. American Psychologist, 48, 1181-1209.

Livingston, R. B., Mears, G., Marshall, R., Gray, R. \& Haak, R. A. (1996). Psychostimulant effects on neuropsychological, intellectual, and achievement measures for children and adolescents with attention deficit hyperactivity disorder. Applied Neuropsychology, 3$4,174-177$.

Lou, H. C., Henrickson, L. \& Bruhn, P. (1984). Striatal dysfunction in attention deficit hyperkinetic disorder. Arch Neurology, 465, 48-52.

Mackowiak, K. \& Hungerige, H. (2001). Selbstinstruktionsmethoden. In M. Borg-Laufs (Hrsg.), Lehrbuch der Verhaltenstherapie mit Kindern und Jugendlichen. Band 2: Interventionsmethoden (S. 455-484). Tübingen: DGVT-Verlag.

Mähler, C. \& Hasselhorn M. (2001). Lern- und Gedächtnistraining bei Kindern. In K. J. Klauer (Hrsg.), Handbuch kognitiver Trainings. Göttingen: Hogrefe. 
Mannuzza, S. \& Klein, R. G. (2000). Long-term prognosis in attention- deficit hyperactivity disorder. Child and Adolescent Psychiatric Clinics of North America, 9 (3), 711-726.

Meichenbaum, D.H. \& Goodman, J. (1971). Training impulsive children to talk to themselves. A means of developing self-control. Journal of Abnormal Psychology, 77, 115-126.

Melchers, P. \& Preuß, U. (1994). K-ABC: Kaufman-Assessment Battery for Children. Durchführungs- und Auswertungshandbuch. Übersetzung der K-ABC von A. S. Kaufman und N. L. Kaufman. Amsterdam, Lisse, Frankfurt am Main: Swets.

Merod, R. (2001). Entspannungsverfahren. In Borg-Laufs, M. (Hrsg.), Lehrbuch der Verhaltenstherapie mit Kindern und Jugendlichen. Band 2: Interventionsmethoden (S. 301-325). Tübingen: DGVT Deutsche Gesellschaft für Verhaltenstherapie.

Messer, E. (1992). K-A-Training. Konzentrations- und Aufmerksamkeitsübungen für die Grundschule. Stuttgart: Klett.

Mickley, M. und Pisarsky, B.C. (2003). Aufmerksamkeitsdefizit/Hyperaktivitätsstörung von der multimodalen Therapie zur systemischen Perspektive. In Forum der Kinder- und Jugendpsychiatrie und Psychotherapie, 2003, Heft 1 unter http://www.kinderpsychiater.org.forum/for103/systemische-perspektive.htm [11.6.03].

Miller, R. J. \& Prinz, G. E. (1990). The enhancement of social learning: Family interventions for childhood conduct disorder. Psychological Bulletin, 108, 291-307.

Mock, M. (2001). Evaluation einer kognitiv-verhaltenstherapeutischen Intervention bei Kindern mit hyperkinetischen Störungen. Unveröffentlichte Diplomarbeit, GeorgAugust-Universität, Göttingen.

Möller, J. (1999). Denktraining für Jugendliche: Homogenität der Trainingsgruppen und Booster-Session. Heilpädagogische Forschung, 25, S. 2-7.

Müller-Scherzberg, P. (2002). Evaluation einer kognitiv-verhaltenstherapeutischen Intervention bei Kindern mit Aufmerksamkeitsstörung. Training mit aufmerksamkeitsgestörten Kindern nach Lauth und Schlottke, 1997. Unveröffentlichte Diplomarbeit, Friedrich-Schiller-Universität, Jena.

Mutschler, E. (1991). Arzneimittelwirkungen. Lehrbuch der Pharmakologie und Toxikologie. Stuttgart: Wissenschaftliche Verlagsgesellschaft mbH.

National Institutes of Health (NIH; 2000). Consensus Development Conference Statement: Diagnosis and Treatment of Attention-Deficit/ Hyperactivity Disorder (ADHD). Journal of the American Academy of Child and Adolescent Psychiatry, 39 (2), 182193.

Newcorn, J. H. (2000). The Multimodal Treatment Study of children with attention deficit hyperactivity disorder. Current Psychiatry Reports, 2, 85-89. 
Newcorn, J. H. (2001). Symptom profiles in children with ADHD: Effects of comorbidity and gender. Journal of the Academy of Child and Adolescent Psychiatry, 40, 137-146.

Oswald, D. \& Roth, E. (1978). Der Zahlen-Verbindungs-Test (ZVT), Handanweisung. Göttingen: Hogrefe.

Patry, J.-L. \& Hager, W. (2000). Abschließende Bemerkungen: Dilemmata in der Evaluation. In W. Hager, J.-L. Patry \& H. Brezing (Hrsg.), Evaluation psychologischer Interventionsmaßnahmen. Standards und Kriterien: Ein Handbuch (S. 258-275). Bern: Hans Huber.

Pelham, W. E., Carlson, C., Sams, S. E., Vallano, G., Dixon, M. J. \& Hoza, B. (1993). Separate and combined effects of methylphenidate and behavior modification on boys with Attention Deficit - Hyperactivity Disorder in the Classroom. Journal of Consulting and Clinical Psychology, 61 (3), $506-515$.

Pelham, W. E., Wheeler, T. \& Chronis, A. (1998). Empirically supported psychosocial treatments for attention deficit hyperactivity disorder. Journal of Clinical Child Psychology, 27 (2), 190-205.

Pennington, B. F. \& Ozonoff, S. (1996). Executive functions and developmental psychopathology. Journal of Child Psychology and Psychiatry, 37 (1), 51-87.

Perrez, M. (1998). Prävention und Gesundheitsförderung. In U. Baumann \& Perrez (Hrsg.), Lehrbuch Klinische Psychologie - Psychotherapie. 2. Aufl. (S. 366-391). Bern: Huber.

Petermann, U. (1994). Materialien zu Imagiantionsverfahren für Kinder: Die Kaptitän Nemo Geschichten. In F. Petermann \& D. Vaitl (Hrsg.), Handbuch der Entspannungsverfahren, Band 2, Anwendungen (S. 305-345). Weinheim: Psychologie Verlags Union.

Petermann, U. \& Petermann, F. (1994). Entspannungsverfahren bei Kindern und Jugendlichen. In F. Petermann \& D. Vaitl. Handbuch der Entspannungsverfahren Band II: Anwendungen. 392 - 413 Weinheim: Beltz PVU

Pfiffner, L. J. \& O’Leary, S. G. (1993). School based psychological treatments. In J. L. Matson (Ed.), Handbook of Hyperactivity in Children. Chap. 12. Boston, MA: Allyn \& Bacon.

Pisterman, S., McGrath, P., Firestone, P., Goodman, J. T., Webster, I. \& Mallory, R. (1989). Outcome of parent-mediated treatment of preschoolers with attention deficit disorder with hyperactivity. Journal of Consulting and Clinical Psychology, 57 (5), 628-635.

Pitzer, M. \& Schmidt, M. (1999). Epidemiologie psychischer Störungen des Kindes- und Jugendalters. In M. Borg-Laufs (Hrsg.), Lehrbuch der Verhaltenstherapie mit Kindern und Jugendlichen. Band 1: Grundlagen (S. 123-152). Tübingen: DGVT-Verlag.

Posner, M. I. \& Rafael, R. D. (1987). Cognitive theories of attention and the rehabilitation of attentional deficits. In M. J. Meir, A. L. Benton \& L. Diller (Eds.), Neuropsychological Rehabilitation (pp. 182-201). New York: Guilford. 
Purdie, N., Hattie, J. \& Carroll, A. (2002). A review of the research on interventions for attention deficit hyperactivity disorder: What works best? Review of Educational Research, 72 (1), 61-99.

Rapoport, J. L., Castellanos, F. X., Gogate, N., Janson, K., Kohler, S. \& Nelson, P. (2001). Imaging normal and abnormal brain development: New perspectives for child psychiatry. Australien and New Zealand Journal of Psychiatry, 35, 272-281.

Rapport, M. D., Murphy, A. \& Bailey, J. S. (1982). Ritalin versus response cost in the control of hyperactive children: a within subject comparison. Journal of Applied Behavior Analysis, 15, 205-216.

Ray, J. W. \& Shadish, W. R. (1996). How interchangeable are different estimators of effect size? Journal of Consulting and Clinical Psychology, 64 (6), 1316-1325.

Remschmidt, H., Schmidt, M. H. \& Goebel, D. (1983). Erprobungs- und Reliabilitätsstudie zum multiaxialen Klassifikationsschema für psychiatrische Erkrankungen. In Remschmidt, H. \& Schmidt, M. H. (Hrsg.), Multiaxiale Diagnostik in der Kinder- und Jugendpsychiatrie (S. 43-75). Bern: Huber.

Rheinberg, F. \& Fries, S. (2001). Motivationstraining. In K. J. Klauer (Hrsg.), Handbuch kognitiver Trainings. Göttingen: Hogrefe.

Rohde, Sven (2002, 2. Januar). Die Not der kleinen Nervensägen. Stern, 2, 106-110.

Rollett, B. (2001). Die integrativen Leistungen des Gehirns und Konzentration: Theoretische Grundlagen und Interventionsprogramme. In K. J. Klauer (Hrsg.), Handbuch kognitiver Trainings. Göttingen: Hogrefe.

Rossi, P. H., Freeman, H. E. \& Hofmann, G. (1988). Programm-Evaluation. Einführung in die Methoden angewandter Sozialforschung. Stuttgart: Enke.

Roth, N., Schlottke, P. F. \& Klepel, H. (1992). Hyperaktive und aufmerksamkeitsgestörte Kinder: Erklärungsansätze, psychophysiologische Korrelate und Behandlungskonzepte. Zeitschrift für Medizinische Psychologie, 1, 77-84.

Rothenberger, A. \& Moll, G. H. (1998). Klassifikation und neurobiologischer Hintergrund des Hyperkinetischen Syndroms. In U. Franke (Hrsg.), Therapie aggressiver und hyperaktiver Kinder (3. Auflage). München: Urban \& Fischer.

Rothenberger, A. (2002). Editorial. Zeitschrift für Kinder- und Jugendpsychiatrie, 30 (3), 159-161.

Rubia, K., Overmeyer, S., Taylor, E., Brammer, M., Williams, S., Simmons, A. \& Bullmore, E.T. (1999). Hypofrontality in attention deficit hyperactivity disorder during higherorder motor control: A study with functional MRI. American Journal of Psychiatry, 156, 891-896.

Ryffel, M.H. (2003). Langzeiterfahrungen mit Stimulanzien bei ADHS: Empfehlungen für die Praxis. Forum der Kinder- und Jugendpsychiatrie und Psychotherapie, Band 1 unter: 
http://www.kinderpsychiater.org/forum/for103/langzeiterfahrungen-stimulanzienADHS.htm [30.6.03]

Saile, M. (1996). Metaanalyse zur Effektivität psychologischer Behandlung hyperaktiver Kinder. Zeitschrift für Klinische Psychologie, 25 (3), 190-207.

Saile, H., Röding, A. \& Friedrich-Löffler, A. (1999). Familienprozesse bei Aufmerksamkeitsund Hyperaktivitätsstörung. Zeitschrift für Kinder- und Jugendpsychiatrie und Psychotherapie, 27 (1), 19-26.

Salzer, M. S., Bickman, L. \& Lambert, W. (1999). Dose-effect relationship in children's psychotherapy services. Journal of Consulting and Clinical Psychology, 67, 228-238.

Saß, H., Wittchen, H.- U. \& Zaudig, M. (1996). Diagnostisches und Statistisches Manual Psychischer Störungen (3. unveränderte Aufl.). Göttingen: Hogrefe.

Scahill, L. \& Schwab-Stone, M. (2000). Epidemiology of ADHD in school-age children. Child and Adolescent Psychiatric Clinics of North America, 9 (3), 541-555.

Scheithauer, H. \& Petermann, F. (2000). Review: Die Ermittlung der Wirksamkeit und Effektivität psychotherapeutischer Interventionen: Eine internationale Bestandsaufnahme. Zeitschrift für Klinische Psychologie, Psychiatrie und Psychotherapie, 48 (3), 211-233.

Schlottke, P. F. (1984). Psychologische Behandlung von Aufmerksamkeitsstörungen bei Kindern. Tübingen: Unveröffentlichte Habilitationsschrift.

Schmidt, M. H., Esser, G. \& Moll, G. H. (1991). Der Verlauf hyperkinetischer Syndrome in klinischen und Feldstichproben. Zeitschrift für Kinder- und Jugendpsychiatrie, 19 (4), S. 240-247.

Schöll, G. (1997). Förderung von Aufmerksamkeit in der Grundschule. Ein metakognitiv orientierter Trainingsansatz. Münster: Waxmann.

Schöttke, H. \& Wiedl, K. H. (2001). Training bei Aufmerksamkeitsstörungen und visuellem Neglect. Verhaltensmedizinisch-neuropsychologische Modelle. In K. J. Klauer (Hrsg.), Handbuch kognitiver Trainings. Göttingen: Hogrefe.

Schulte, D. (1993). Wie soll der Therapieerfolg gemessen werden? Zeitschrift für Klinische Psychologie, 22, 374-393.

Schultz, J. H. (1973). Das Autogene Training. Stuttgart: Thieme Verlag.

Sergeant, J. (2000). The cognitive-energetic model: An empirical approach to attention-deficit hyperactive disorder. Neuroscience and Biobehavior, 24, 7-12.

Slusarek, M., Velling, S., Bunk, D. \& Eggers, C. (2001). Motivational effects on inhibitory control in children with ADHD. Journal of the American Academy of Child and Adolescent Psychiatry, 40 (3), 355-363. 
Spencer, T., Biederman, J., Wilens, T., Harding, M., O'Donnell, D. \& Griffin, S. (1996). Pharmacotherapy of attention-deficit hyperactivity disorder across the life cycle. Journal of the American Academy of Child and Adolescent Psychiatry, 35 (4), 409432.

Steinhausen, H.-C. (2000). Klinik und Konzepte der hyperkinetischen Störungen. In H. C. Steinhausen (Hrsg.), Hyperkinetische Störungen bei Kindern, Jugendlichen und Erwachsenen (S. 9-37). Stuttgart: Kohlhammer.

Sternberg, R. J. (1985). Beyond IQ. A triarchic theory of human intelligence. London: Cambridge University Press.

Tannock, R. (1998). Attention deficit hyperactivity disorder: Advances in cognitive, neurobiological, and genetic research. Journal of Child Psychology and Psychiatry, 39 (1), 65-99.

Taylor, E., Sergeant, J., Döpfner, M., Gunning, B., Overmeyer, S., Möbius, H.- J. \& Eisert, H.- G. (1998). Clinical guidelines for hyperkinetic disorder. Eurpean Child \& Adolescent Psychiatry, 7, $184-200$.

Tewes, U., Rossmann, P. \& Schallberger, U. (1999). Hamburg-Wechsler-Intelligenztest für Kinder (HAWIK-III). Übersetzung und Adaptation der Wechsler Intelligence Scale for Children (WISC-III) von D. Wechsler. Bern, Göttingen, Toronto, Seattle: Hans Huber.

Thapar, A., Harrington, R. \& McGuffin, P. (2001). Examining the comorbidity of ADHDrelated behaviors and conduct problems using a twin study design. British Journal of Psychiatry, 179, 224-229.

The MTA Cooperative Group (1999a). A 14-month randomized clinical trial of treatment strategies for attention-deficit/hyperactivity disorder. Archives of General Psychiatry, $56,1073-1086$.

The MTA Cooperative Group (1999b). Moderators and mediators of treatment response for children with attention-deficit/hyperactivity disorder. Archives of General Psychiatry, $56,1088-1096$.

Todd, R. D. (2000). Genetics of childhood disorders: XXI. ADHD, Part 5: A behavioral genetic perspective. Journal of the American Academy of Child and Adolescent Psychiatry, 39 (12), 1571-1573.

Trott, G.-E. \& Badura, F. (2001). Aufmerksamkeits- und Aktivitätsstörung bei Kindern. Früh erkennen - soziales Abrutschen verhindern. MMW-Schwerpunkt - Fortschritte der Medizin, 143, 32-35.

Varni, J. W. \& Henker, B. (1979). A self-regulation approach to the treatment of three hyperactive boys. Child Behavior Therapy, 1, 171-192.

Vitiello, B. (2001). Methylphenidate dosage for children with ADHD over time under controlled conditions: Lessons from the MTA. Journal of the American Academy of Child and Adolescent Psychiatry, 40, 188-196. 
Wagner, I. (1976). Aufmerksamkeitstraining mit impulsiven Kindern. Stuttgart: Ernst Klett Verlag.

Warnke, A., Beck, N. \& Hemminger, U. (2001). Elterntrainings. In M. Borg-Laufs (Hrsg.), Lehrbuch der Verhaltenstherapie mit Kindern und Jugendlichen. Band 2: Interventionsmethoden (S. 631-656). Tübingen: DGVT-Verlag.

Waschbusch, D. A., Kipp, H. L. \& Pelham, W. E. (1998). Generalization of behavioral and psychostimulant treatment of attention-deficit/hyperactivity disorder (ADHD): Discussion and examples. Behaviour Research and Therapy, 36, 675-694.

Wechsler, D. (1982). Handanweisung zum Hamburg-Wechsler-Intelligenztest für Erwachsene HAWIE. Bern: Huber.

Weiß, R. H. (1997). Grundintelligenztest Skala 2 (CFT 20). 4. überarbeitete Auflage. Göttingen: Hogrefe

Wells, K. C., Pelham, W. E., Kotkin, R. A. Hoza, B., Abikoff, H. B., Abramowitz, A., Arnold, L. E., Cantwell, D. P., Conners, C. K., Del Carmen, R., Elliott, G., Greenhill, L. L., Hechtman, L., Hibbs, E., Hinshaw, S.O., Jensen, P. S., March, J. S., Swanson, J. M. \& Schiller, E. (2000). Psychosocial treatment strategies in the MTA study: Rationale, methods, and critical issues in design and implementation. Journal of Abnormal Child Psychology, 28 (6), 483-505.

Welte, V. (1981). Der Mottier-Test, ein Prüfmittel für die Lautdifferenzierungsfähigkeit und die auditive Merkfähigkeit. Sprache - Stimme - Gehör, 5, 121-125.

Westhoff, K. \& Hagemeister, C. (2001). Konzentrationstraining. In K. J. Klauer (Hrsg.), Handbuch kognitiver Trainings. Göttingen: Hogrefe.

Whalen, C. K. \& Henker, B. (1991). Therapies for hyperactive children: comparisons, combinations and compromises. Journal of Consulting and Clinical Psychology, 59 (1), 126-137.

Witte, S. (2002). Entwicklung und Evaluation eines videogestützten Selbstinstruktionstrainings für aufmerksamkeitsgestörte Kinder. Göttingen: Cuvillier Verlag.

Zametkin, A. J., Liebenauer, L. L., Fitzgerald, G. A., King, A. C., Minkunas, D. V., Hersovitch, P., Yamada, E. M. \& Cohen, R. M. (1993). Brain metabolism in teenagers with attention-deficit hyperactivity disorder. Arch Gen Psychiatry, 50, 333-340.

Zimmermann, P. \& Fimm, B. (1993). Testbatterie zur Aufmerksamkeitsprüfung (TAP) Version 1.02c Handbuch Teil 1. Herzogenrath: Psychologische Testsysteme. 


\section{Anhang}

A Tabellarische Übersicht über die Mittelwerte und Standardabweichungen im Vor- und Nachtest, Kennwerte $(F, p, M Q F)$ des Interaktionseffektes, Bewertung der Hypothesen und Effektgrößen der Gruppenvergleiche

A-1 Vergleich der Wartegruppe mit dem Basistraining nach Lauth und Schlottke (LS10)

A-2 Vergleich der Wartegruppe mit dem kombinierten Basis- und Strategietraining nach Lauth und Schlottke (LS15)

A-3 Vergleich der Wartegruppe mit dem THOP

A-4 Vergleich des Marburger Konzentrationstrainings mit dem Pseudotraining „Petterson und Findus“

A-5 Vergleich des Basistrainings nach Lauth und Schlottke (LS10) mit dem kombinierten Basis- und Strategietraining nach Lauth und Schlottke (LS15)

A-6 Vergleich des kombinierten Basis- und Strategietraining nach Lauth und Schlottke (LS15) mit dem THOP

A-7 Vergleich des kombinierten Basis- und Strategietraining nach Lauth und Schlottke (LS15) mit dem Marburger Konzentrationstraining

A-8 Vergleich des THOP mit dem Marburger Konzentrationstraining

B Tabellarische Übersicht über die Haupteffekte Zeit und Gruppe der Gruppenvergleiche

B-1 Vergleich der Wartegruppe mit dem Basistraining nach Lauth und Schlottke (LS10)

B-2 Vergleich der Wartegruppe mit dem kombinierten Basis- und Strategietraining nach Lauth und Schlottke (LS15)

B-3 Vergleich der Wartegruppe mit dem THOP

B-4 Vergleich des Marburger Konzentrationstrainings mit dem Pseudotraining „Petterson und Findus“

B-5 Vergleich des Basistrainings nach Lauth und Schlottke (LS10) mit dem kombinierten Basis- und Strategietraining nach Lauth und Schlottke (LS15)

B-6 Vergleich des kombinierten Basis- und Strategietraining nach Lauth und Schlottke (LS15) mit dem THOP

B-7 Vergleich des kombinierten Basis- und Strategietraining nach Lauth und 
Schlottke (LS15) mit dem Marburger Konzentrationstraining

B-8 Vergleich des THOP mit dem Marburger Konzentrationstraining

C Psychologischen Vorhersagen (PVn), Statistische Hypothesen (SHn) und Testplanung

C.1 zu Studie 2: Wirksamkeit der Kombination von Basistraining und Strategietraining nach Lauth und Schlottke

C.2 zu Studie 3: Wirksamkeit des THOP

C.3 zu Studie 4: Wirksamkeit des Marburger Konzentrationstrainings

C. 4 zur vergleichenden Evaluation der Trainings aus den Studien 1 - 4

D Fragebogen zur Einschätzung hyperkinetischen und aufmerksamkeitsgestörten Verhaltens nach DSM-IV

E E.1 Interaktionsfragebogen

E.2 Kodierung für die Auswertung des Interaktionsfragebogens 


\section{Anhang A}

Tabelle A-1

Mittelwerte und Standardabweichungen (in Klammern) im Vor- und Nachtest für die AVn und Kennwerte $(F, p, M Q F)$ des Interaktionseffekts zweifaktorieller Varianzanalysen mit Messwiederholung getrennt für die Wartegruppe und die Lauth- und SchlottkeTrainingsgruppe (10 Sitzungen)

\begin{tabular}{|c|c|c|c|c|c|c|c|c|c|c|c|}
\hline \multirow[t]{2}{*}{$\begin{array}{c}\text { Abhängige } \\
\text { Variable }\end{array}$} & \multicolumn{3}{|c|}{ Wartegruppe (WG) } & \multicolumn{3}{|c|}{$\begin{array}{l}\text { Basistraining nach } \\
\text { Lauth und Schlottke } \\
10 \text { Sitzungen }\end{array}$} & \multicolumn{4}{|c|}{ Interaktionseffekt } & \multirow{2}{*}{$\begin{array}{c}\text { Effekt } \\
\text {-größe } \\
d\end{array}$} \\
\hline & $n$ & Vortest & Nachtest & $n$ & Vortest & Nachtest & $F$ & $p$ & $M Q F$ & $\begin{array}{c}\text { hypo- } \\
\text { thesen- } \\
\text { konform } \\
?\end{array}$ & \\
\hline \multicolumn{12}{|c|}{ TAP: Untertest „Geteilte Aufmerksamkeit“" } \\
\hline $\begin{array}{l}\text { Standard- } \\
\text { abweichung }\end{array}$ & 15 & $\begin{array}{c}\mathbf{3 6 4 . 0 5} \\
(100.99) \\
\end{array}$ & $\begin{array}{l}\mathbf{2 9 3 . 6 0} \\
(83.94) \\
\end{array}$ & 14 & $\begin{array}{l}331.74 \\
(74.95) \\
\end{array}$ & $\begin{array}{l}316.96 \\
(75.04) \\
\end{array}$ & - & - & - & - & \\
\hline Anzahl Fehler & 15 & $\begin{array}{c}\mathbf{8 . 8 0} \\
(7.06) \\
\end{array}$ & $\begin{array}{c}\mathbf{2 . 6 0} \\
(1.45) \\
\end{array}$ & 14 & $\begin{array}{c}\mathbf{8 . 4 3} \\
(8.38) \\
\end{array}$ & $\begin{array}{c}\mathbf{2 . 5 0} \\
(1.70) \\
\end{array}$ & 0.01 & 0.92 & 26.98 & nein & $(0.04)$ \\
\hline $\begin{array}{l}\text { Anzahl } \\
\text { Auslassungen }\end{array}$ & 15 & $\begin{array}{c}8.73 \\
(6.77)\end{array}$ & $\begin{array}{c}6.80 \\
(3.36)\end{array}$ & 14 & $\begin{array}{c}\mathbf{6 . 7 9} \\
(3.93)\end{array}$ & $\begin{array}{c}4.29 \\
(2.52)\end{array}$ & 0.11 & 0.73 & 9.97 & nein & $(0.15)$ \\
\hline \multicolumn{12}{|c|}{ TAP: Untertest ,Go/Nogo“6 } \\
\hline $\begin{array}{l}\text { Standard- } \\
\text { abweichung }\end{array}$ & 16 & $\begin{array}{l}146.28 \\
(61.36)\end{array}$ & $\begin{array}{l}148.38 \\
(52.80)\end{array}$ & 14 & $\begin{array}{l}259.37 \\
291.49 \\
\end{array}$ & $\begin{array}{l}136.74 \\
24.59\end{array}$ & - & - & - & - & - \\
\hline Anzahl Fehler & 16 & $\begin{array}{c}8.19 \\
(4.79) \\
\end{array}$ & $\begin{array}{c}\mathbf{5 . 7 5} \\
(3.13) \\
\end{array}$ & 14 & $\begin{array}{c}9.21 \\
(4.85)\end{array}$ & $\begin{array}{c}4.71 \\
(3.97) \\
\end{array}$ & 1.39 & 0.24 & 11.41 & nein & $(0.48)$ \\
\hline $\begin{array}{l}\text { Anzahl } \\
\text { Auslassungen }\end{array}$ & 16 & $\begin{array}{c}\mathbf{5 . 0 0} \\
(5.40)\end{array}$ & $\begin{array}{c}\mathbf{2 . 3 8} \\
(3.12)\end{array}$ & 14 & $\begin{array}{c}4.93 \\
(6.24)\end{array}$ & $\begin{array}{c}\mathbf{1 . 5 0} \\
(1.83)\end{array}$ & 2.41 & 0.15 & 15.52 & nein & $(0.61)$ \\
\hline \multicolumn{12}{|c|}{ TAP: Untertest „Inkompatibilität““ } \\
\hline $\begin{array}{l}\text { Standard- } \\
\text { abweichung }\end{array}$ & 15 & \begin{tabular}{|c|}
$\mathbf{3 2 3 . 8 2}$ \\
$(259.98)$
\end{tabular} & $\begin{array}{l}\mathbf{1 9 6 . 6 9} \\
(56.45)\end{array}$ & 14 & $\begin{array}{c}274.24 \\
(273.95)\end{array}$ & $\begin{array}{l}189.13 \\
(41.07)\end{array}$ & - & - & - & - & - \\
\hline Anzahl Fehler & 15 & $\begin{array}{l}15.53 \\
(9.12) \\
\end{array}$ & $\begin{array}{l}12.80 \\
(8.03) \\
\end{array}$ & 14 & $\begin{array}{c}\mathbf{1 9 . 7 1} \\
(13.10) \\
\end{array}$ & $\begin{array}{r}\mathbf{8 . 9 3} \\
(8.65) \\
\end{array}$ & 5.49 & 0.02 & 43.83 & ja & 0.78 \\
\hline \multicolumn{12}{|c|}{ TAP: Untertest „Visuelles Scanning“ } \\
\hline $\begin{array}{l}\text { Standard- } \\
\text { abweichung } \\
\text { kritische Trials } \\
\end{array}$ & 15 & $\begin{array}{c}\mathbf{3 9 5 2 . 5 7} \\
(1864.41)\end{array}$ & $\begin{array}{c}\mathbf{2 3 8 6 . 7 2} \\
(1274.29)\end{array}$ & 14 & $\begin{array}{l}\mathbf{2 8 3 3 . 1 1} \\
(1265.7)\end{array}$ & $\begin{array}{c}\mathbf{1 9 9 9 . 7 1} \\
(1174.35)\end{array}$ & 1.90 & 0.17 & 1020588 & nein & $(0.51)$ \\
\hline $\begin{array}{l}\text { Standard- } \\
\text { abweichung } \\
\text { nicht-kritische } \\
\text { Trials }\end{array}$ & 15 & $\begin{array}{c}\mathbf{3 6 8 3 . 6 4} \\
(1374.86)\end{array}$ & $\begin{array}{c}\mathbf{2 6 3 6 . 7 3} \\
(1305.89)\end{array}$ & 14 & $\begin{array}{l}3332.51 \\
(1761.5)\end{array}$ & $\begin{array}{c}2637.14 \\
(1509.64)\end{array}$ & - & - & - & - & - \\
\hline $\begin{array}{l}\text { Anzahl Fehler } \\
\text { (kritische } \\
\text { Trials) } \\
\end{array}$ & 15 & $\begin{array}{c}2.33 \\
(5.23)\end{array}$ & $\begin{array}{l}1.47 \\
(2.92)\end{array}$ & 14 & $\begin{array}{c}1.21 \\
(1.12)\end{array}$ & $\begin{array}{l}1.00 \\
(2.15)\end{array}$ & - & - & - & - & - \\
\hline $\begin{array}{l}\text { Anzahl } \\
\text { Auslassungen } \\
\text { (nicht-kritische } \\
\text { Trials) }\end{array}$ & 15 & $\begin{array}{c}5.80 \\
(4.50)\end{array}$ & $\begin{array}{c}6.20 \\
(4.95)\end{array}$ & 14 & $\begin{array}{c}5.14 \\
(3.21)\end{array}$ & $\begin{array}{c}5.36 \\
(3.95)\end{array}$ & - & - & - & - & - \\
\hline \multicolumn{12}{|l|}{ Test d2 } \\
\hline $\begin{array}{l}\text { Konzentrations- } \\
\text { leistungswert }\end{array}$ & 13 & $\begin{array}{c}\mathbf{7 7 . 0 0} \\
(26.59) \\
\end{array}$ & $\begin{aligned} \mathbf{9 5 . 8 5} \\
(25.78) \\
\end{aligned}$ & 14 & $\begin{array}{c}72.21 \\
(27.26) \\
\end{array}$ & $\begin{array}{c}96.50 \\
(19.07) \\
\end{array}$ & 0.89 & 0.35 & 111.57 & nein & $(0.20)$ \\
\hline Anzahl Fehler & 13 & $\begin{array}{c}\mathbf{3 4 . 8 5} \\
(34.98)\end{array}$ & $\begin{array}{c}\mathbf{1 2 . 1 5} \\
(14.67)\end{array}$ & 14 & $\begin{array}{c}\mathbf{3 4 . 0 7} \\
(41.29)\end{array}$ & $\begin{array}{l}\mathbf{1 1 . 0 7} \\
(9.93)\end{array}$ & 0.01 & 0.98 & 560.45 & nein & $(0.03)$ \\
\hline
\end{tabular}




\begin{tabular}{|c|c|c|c|c|c|c|c|c|c|c|c|}
\hline \multirow[t]{2}{*}{$\begin{array}{c}\text { Abhängige } \\
\text { Variable }\end{array}$} & \multicolumn{3}{|c|}{ Wartegruppe (WG) } & \multicolumn{3}{|c|}{$\begin{array}{c}\text { Basistraining nach } \\
\text { Lauth und Schlottke } \\
10 \text { Sitzungen }\end{array}$} & \multicolumn{4}{|c|}{ Interaktionseffekt } & \multirow{2}{*}{$\begin{array}{c}\text { Effekt } \\
\text {-größe } \\
d\end{array}$} \\
\hline & $n$ & Vortest & Nachtest & $n$ & Vortest & Nachtest & $F$ & $p$ & $M Q F$ & \begin{tabular}{|c|} 
hypo- \\
thesen- \\
konform \\
$?$
\end{tabular} & \\
\hline \multicolumn{12}{|l|}{ Mottier-Test } \\
\hline $\begin{array}{l}\text { Nachsprechleist } \\
\text { ung Gesamt } \\
(\max .30)\end{array}$ & 15 & $\begin{array}{l}20.80 \\
(3.97)\end{array}$ & $\begin{array}{l}20.40 \\
(5.01)\end{array}$ & 14 & $\begin{array}{l}19.07 \\
(4.01)\end{array}$ & $\begin{array}{r}\mathbf{2 2 . 4 3} \\
(3.11)\end{array}$ & 11.55 & 0.00 & 4.42 & ja & 0.89 \\
\hline $\begin{array}{l}\text { Nachsprechleist } \\
\text { ung für 2- und } \\
\text { 3-silbige } \\
\text { Kunstwörter } \\
\text { (max. 12) }\end{array}$ & 15 & $\begin{array}{l}11.07 \\
(1.53)\end{array}$ & $\begin{array}{l}10.20 \\
(2.21)\end{array}$ & 14 & $\begin{array}{l}11.00 \\
(1.24)\end{array}$ & $\begin{array}{l}11.29 \\
(1.14)\end{array}$ & - & - & - & - & - \\
\hline $\begin{array}{l}\text { Nachsprechleist } \\
\text { ung für 4-, 5- } \\
\text { und 6-silbige } \\
\text { Kunstwörter } \\
\text { (max. 18) }\end{array}$ & 15 & $\begin{array}{c}9.73 \\
(3.17)\end{array}$ & $\begin{array}{l}10.20 \\
(3.86)\end{array}$ & 14 & $\begin{array}{c}\mathbf{8 . 0 7} \\
(3.41)\end{array}$ & $\begin{array}{l}\mathbf{1 1 . 1 4} \\
(3.13)\end{array}$ & 14.01 & 0.00 & 1.75 & ja & 0.71 \\
\hline \multicolumn{12}{|c|}{ Fremdbeurteilungsbogen FBB-HKS (Eltern) } \\
\hline $\begin{array}{l}\text { Unaufmerksam- } \\
\text { keit: Anzahl an } \\
\text { Kriterien }\end{array}$ & 11 & $\begin{array}{c}4.91 \\
(3.11)\end{array}$ & $\begin{array}{c}4.45 \\
(4.06)\end{array}$ & 13 & $\begin{array}{c}\mathbf{4 . 6 9} \\
(1.75)\end{array}$ & $\begin{array}{c}3.23 \\
(2.31)\end{array}$ & 0.57 & 0.45 & 5.22 & nein & $(0.35)$ \\
\hline $\begin{array}{l}\text { Unaufmerksam- } \\
\text { keit: Kennwert }\end{array}$ & 11 & $\begin{array}{c}1.64 \\
(0.62)\end{array}$ & $\begin{array}{c}1.58 \\
(0.87)\end{array}$ & 13 & $\begin{array}{c}\mathbf{1 . 5 4} \\
(0.37)\end{array}$ & $\begin{array}{c}\mathbf{1 . 1 8} \\
(0.43)\end{array}$ & 1.07 & 0.31 & 0.24 & nein & $(0.50)$ \\
\hline $\begin{array}{l}\text { Hyperaktivität: } \\
\text { Anzahl an } \\
\text { Kriterien } \\
\end{array}$ & 11 & $\begin{array}{c}2.72 \\
(1.68)\end{array}$ & $\begin{array}{l}2.36 \\
(2.38)\end{array}$ & 13 & $\begin{array}{c}\mathbf{1 . 7 6} \\
(1.48)\end{array}$ & $\begin{array}{c}\mathbf{0 . 4 6} \\
(0.87)\end{array}$ & 1.51 & 0.22 & 1.71 & nein & $(0.50)$ \\
\hline $\begin{array}{l}\text { Hyperaktivität: } \\
\text { Kennwert }\end{array}$ & 11 & $\begin{array}{c}1.63 \\
(0.70) \\
\end{array}$ & $\begin{array}{c}1.29 \\
(0.76) \\
\end{array}$ & 13 & $\begin{array}{c}1.29 \\
(0.71) \\
\end{array}$ & $\begin{array}{c}\mathbf{0 . 6 6} \\
(0.45) \\
\end{array}$ & 1.04 & 0.31 & 0.22 & nein & $(0.37)$ \\
\hline $\begin{array}{l}\text { Impulsivität: } \\
\text { Anzahl an } \\
\text { Kriterien } \\
\end{array}$ & 11 & $\begin{array}{c}1.45 \\
(1.75)\end{array}$ & $\begin{array}{c}1.27 \\
(1.79)\end{array}$ & 13 & $\begin{array}{c}\mathbf{1 . 3 0} \\
(1.70)\end{array}$ & $\begin{array}{c}\mathbf{0 . 0 7} \\
(0.27)\end{array}$ & 1.50 & 0.23 & 5.22 & nein & $(0.63)$ \\
\hline $\begin{array}{l}\text { Impulsivität: } \\
\text { Kennwert }\end{array}$ & 11 & $\begin{array}{c}1.18 \\
(0.69) \\
\end{array}$ & $\begin{array}{c}1.15 \\
(0.78) \\
\end{array}$ & 13 & $\begin{array}{c}1.12 \\
(0.79) \\
\end{array}$ & $\begin{array}{c}\mathbf{0 . 5 9} \\
(0.37) \\
\end{array}$ & 1.88 & 0.18 & 0.38 & nein & $(0.70)$ \\
\hline \multicolumn{12}{|c|}{ Child Behavior Checklist (CBCL) } \\
\hline $\begin{array}{l}\text { T-Wert } \\
\text { Aufmerksam- } \\
\text { keitsstörungen } \\
\end{array}$ & 10 & $\begin{array}{l}64.50 \\
(6.89)\end{array}$ & $\begin{array}{l}61.60 \\
(8.63)\end{array}$ & 12 & $\begin{array}{l}\mathbf{6 8 . 3 3} \\
(8.74)\end{array}$ & $\begin{array}{c}\mathbf{6 3 . 5 8} \\
(10.95)\end{array}$ & 0.33 & 0.57 & 28.27 & nein & $(0.20)$ \\
\hline $\begin{array}{l}\text { T-Wert } \\
\text { externalisieren- } \\
\text { de Störungen } \\
\end{array}$ & 10 & $\begin{array}{c}59.50 \\
(12.04)\end{array}$ & $\begin{array}{c}59.00 \\
(12.18)\end{array}$ & 12 & $\begin{array}{l}\mathbf{6 3 . 1 7} \\
(9.23)\end{array}$ & $\begin{array}{l}\mathbf{5 8 . 6 7} \\
(8.07)\end{array}$ & 1.29 & 0.26 & 33.68 & nein & $(0.37)$ \\
\hline $\begin{array}{l}\text { T-Wert } \\
\text { Gesamtskala }\end{array}$ & 10 & $\begin{array}{l}61.00 \\
(9.46)\end{array}$ & $\begin{array}{c}57.70 \\
(10.87)\end{array}$ & 12 & $\begin{array}{l}\mathbf{6 4 . 1 7} \\
(7.07)\end{array}$ & $\begin{array}{l}\mathbf{5 9 . 2 5} \\
(7.33)\end{array}$ & 0.50 & 0.48 & 14.17 & nein & $(0.19)$ \\
\hline \multicolumn{12}{|c|}{ Fremdbeurteilungsbogen FBB-HKS (Lehrer) } \\
\hline $\begin{array}{l}\text { Unaufmerksam- } \\
\text { keit: Anzahl an } \\
\text { Kriterien }\end{array}$ & 12 & $\begin{array}{l}4.75 \\
(2.83)\end{array}$ & $\begin{array}{c}4.92 \\
(3.09)\end{array}$ & 9 & $\begin{array}{c}3.33 \\
(2.17)\end{array}$ & $\begin{array}{c}3.00 \\
(2.44)\end{array}$ & - & - & - & - & - \\
\hline $\begin{array}{l}\text { Unaufmerksam- } \\
\text { keit: Kennwert }\end{array}$ & 12 & $\begin{array}{l}1.62 \\
(.58) \\
\end{array}$ & $\begin{array}{l}1.58 \\
(.69) \\
\end{array}$ & 9 & $\begin{array}{c}1.28 \\
(0.44) \\
\end{array}$ & $\begin{array}{c}1.13 \\
(0.52) \\
\end{array}$ & - & - & - & - & - \\
\hline $\begin{array}{l}\text { Hyperaktivität: } \\
\text { Anzahl an } \\
\text { Kriterien }\end{array}$ & 12 & $\begin{array}{c}2.75 \\
(2.01)\end{array}$ & $\begin{array}{l}1.67 \\
(2.19)\end{array}$ & 9 & $\begin{array}{c}\mathbf{1 . 7 7} \\
(1.56)\end{array}$ & $\begin{array}{c}\mathbf{0 . 6 6} \\
(0.70)\end{array}$ & 0.00 & 0.97 & 2.78 & nein & $(0.00)$ \\
\hline
\end{tabular}




\begin{tabular}{|c|c|c|c|c|c|c|c|c|c|c|c|}
\hline \multirow[t]{2}{*}{$\begin{array}{c}\text { Abhängige } \\
\text { Variable }\end{array}$} & \multicolumn{3}{|c|}{ Wartegruppe (WG) } & \multicolumn{3}{|c|}{$\begin{array}{l}\text { Basistraining nach } \\
\text { Lauth und Schlottke } \\
10 \text { Sitzungen }\end{array}$} & \multicolumn{4}{|c|}{ Interaktionseffekt } & \multirow{2}{*}{$\begin{array}{c}\text { Effekt } \\
\text {-größe } \\
d\end{array}$} \\
\hline & $n$ & Vortest & Nachtest & $n$ & Vortest & Nachtest & $F$ & $p$ & $M Q F$ & $\begin{array}{c}\text { hypo- } \\
\text { thesen- } \\
\text { konform } \\
? \\
\end{array}$ & \\
\hline $\begin{array}{l}\text { Hyperaktivität: } \\
\text { Kennwert }\end{array}$ & 12 & $\begin{array}{c}1.63 \\
(0.79)\end{array}$ & $\begin{array}{l}1.10 \\
(0.88)\end{array}$ & 9 & $\begin{array}{l}1.14 \\
(0.56)\end{array}$ & $\begin{array}{c}0.77 \\
(0.52)\end{array}$ & - & - & - & - & - \\
\hline $\begin{array}{l}\text { Impulsivität: } \\
\text { Anzahl an } \\
\text { Kriterien } \\
\end{array}$ & 12 & $\begin{array}{c}2.42 \\
(1.73)\end{array}$ & $\begin{array}{c}1.67 \\
(1.83)\end{array}$ & 9 & $\begin{array}{c}1.00 \\
(1.58)\end{array}$ & $\begin{array}{c}0.66 \\
(1.00)\end{array}$ & - & - & - & - & - \\
\hline $\begin{array}{l}\text { Impulsivität: } \\
\text { Kennwert }\end{array}$ & 12 & $\begin{array}{c}\mathbf{1 . 7 0} \\
(0.94)\end{array}$ & $\begin{array}{c}\mathbf{1 . 2 0} \\
(0.89)\end{array}$ & 9 & $\begin{array}{c}1.06 \\
(0.70)\end{array}$ & $\begin{array}{c}0.86 \\
(0.51)\end{array}$ & - & - & - & - & - \\
\hline \multicolumn{12}{|c|}{ Teacher's Report Form (TRF) } \\
\hline $\begin{array}{l}\text { T-Wert } \\
\text { Aufmerksam- } \\
\text { keitsstörungen } \\
\end{array}$ & 7 & $\begin{array}{l}61.86 \\
(4.38)\end{array}$ & $\begin{array}{l}59.86 \\
(5.61)\end{array}$ & 11 & $\begin{array}{l}59.36 \\
(5.30)\end{array}$ & $\begin{array}{l}58.00 \\
(5.46)\end{array}$ & - & - & - & - & - \\
\hline $\begin{array}{l}\text { T-Wert } \\
\text { externalisieren- } \\
\text { de Störungen }\end{array}$ & 7 & $\begin{array}{l}65.00 \\
(8.15)\end{array}$ & $\begin{array}{l}65.14 \\
(8.75)\end{array}$ & 11 & $\begin{array}{l}59.09 \\
(8.94)\end{array}$ & $\begin{array}{l}59.64 \\
(8.56)\end{array}$ & - & - & - & - & - \\
\hline $\begin{array}{l}\text { T-Wert } \\
\text { Gesamtskala }\end{array}$ & 7 & $\begin{array}{l}60.71 \\
(7.16)\end{array}$ & $\begin{array}{l}61.43 \\
(5.29)\end{array}$ & 11 & $\begin{array}{l}58.73 \\
(5.52)\end{array}$ & $\begin{array}{l}59.09 \\
(6.19)\end{array}$ & - & - & - & - & - \\
\hline
\end{tabular}

Anmerkung: p: einseitige Irrtumswahrscheinlichkeit des F-Wertes; Signifikanzniveau $\alpha=0.05$; MQF: Mittleres Fehlerquadrat

n: Anzahl der Messwertpaare (Die Anzahl umfasst nur diejenigen Kinder, von denen sowohl Vor- als auch Nachtestwert der jeweiligen Variablen vorhanden waren. Auf dieser Anzahl basieren die inferenzstatistischen Auswertungen).

TAP: Testbatterie zur Aufmerksamkeitsprüfung

In Anlehnung an Cohen (1988) wird ein Effekt von 0.20 als klein, von 0.50 als mittel und von 0.80 als groß betrachtet. 


\section{Anhang A}

Tabelle A-2

Mittelwerte und Standardabweichungen (in Klammern) im Vor- und Nachtest für die AVn und Kennwerte $(F, p, M Q F)$ des Interaktionseffekts zweifaktorieller Varianzanalysen mit Messwiederholung getrennt für die Wartegruppe und das kombinierte Basis- und Strategietraining nach Lauth und Schlottke (15 Sitzungen)

\begin{tabular}{|c|c|c|c|c|c|c|c|c|c|c|c|}
\hline \multirow[t]{2}{*}{$\begin{array}{c}\text { Abhängige } \\
\text { Variable }\end{array}$} & \multicolumn{3}{|c|}{ Wartegruppe (WG) } & \multicolumn{3}{|c|}{$\begin{array}{c}\text { Basis- und } \\
\text { Strategietraining } \\
\text { Lauth und Schlottke } \\
15 \text { Sitzungen }\end{array}$} & \multicolumn{4}{|c|}{ Interaktionseffekt } & \multirow{2}{*}{$\begin{array}{c}\begin{array}{c}\text { Effekt } \\
\text {-größe }\end{array} \\
d\end{array}$} \\
\hline & $n$ & Vortest & Nachtest & $N$ & Vortest & Nachtest & $F$ & $p$ & $M Q F$ & $\begin{array}{c}\text { hypo- } \\
\text { thesen- } \\
\text { konform } \\
?\end{array}$ & \\
\hline \multicolumn{12}{|c|}{ TAP: Untertest, ,Geteilte Aufmerksamkeit"“ } \\
\hline $\begin{array}{l}\text { Standard- } \\
\text { abweichung }\end{array}$ & 15 & $\begin{array}{c}\mathbf{3 6 4 . 0 5} \\
(100.99)\end{array}$ & $\begin{array}{l}\mathbf{2 9 3 . 6 0} \\
(83.94)\end{array}$ & 29 & $\begin{array}{c}\mathbf{3 4 8 . 0 1} \\
(103.47)\end{array}$ & $\begin{array}{l}\mathbf{2 9 5 . 9 1} \\
(88.70)\end{array}$ & 0.24 & 0.62 & 6751.93 & nein & $(0.19)$ \\
\hline Anzahl Fehler & 15 & $\begin{array}{c}\mathbf{8 . 8 0} \\
(7.06) \\
\end{array}$ & $\begin{array}{c}\mathbf{2 . 6 0} \\
(1.45) \\
\end{array}$ & 29 & $\begin{array}{c}\mathbf{9 . 0 3} \\
(13.53) \\
\end{array}$ & $\begin{array}{r}2.97 \\
(3.46) \\
\end{array}$ & 0.00 & 0.97 & 65.24 & nein & $(0.00)$ \\
\hline $\begin{array}{l}\text { Anzahl } \\
\text { Auslassungen }\end{array}$ & 15 & $\begin{array}{c}8.73 \\
(6.77)\end{array}$ & $\begin{array}{c}6.80 \\
(3.36)\end{array}$ & 29 & $\begin{array}{c}8.21 \\
(6.28)\end{array}$ & $\begin{array}{c}6.34 \\
(4.59)\end{array}$ & - & - & - & - & - \\
\hline \multicolumn{12}{|c|}{ TAP: Untertest ,Go/Nogo“6 } \\
\hline $\begin{array}{l}\text { Standard- } \\
\text { abweichung }\end{array}$ & 16 & $\begin{array}{l}146.28 \\
(61.36) \\
\end{array}$ & $\begin{array}{l}148.38 \\
(52.80) \\
\end{array}$ & 31 & $\begin{array}{l}\mathbf{1 4 9 . 2 5} \\
(37.62) \\
\end{array}$ & $\begin{array}{l}\mathbf{1 3 3 . 1 8} \\
(44.62)\end{array}$ & 1.28 & 0.26 & 1351.00 & nein & $(0.38)$ \\
\hline Anzahl Fehler & 16 & $\begin{array}{c}\mathbf{8 . 1 9} \\
(4.79)\end{array}$ & $\begin{array}{c}\mathbf{5 . 7 5} \\
(3.13)\end{array}$ & 31 & $\begin{array}{c}7.84 \\
(6.02)\end{array}$ & $\begin{array}{c}4.68 \\
(5.18)\end{array}$ & 0.13 & 0.71 & 21.02 & nein & $(0.14)$ \\
\hline $\begin{array}{l}\text { Anzahl } \\
\text { Auslassungen }\end{array}$ & 16 & $\begin{array}{c}\mathbf{5 . 0 0} \\
(5.40)\end{array}$ & $\begin{array}{c}\mathbf{2 . 3 8} \\
(3.12)\end{array}$ & 31 & $\begin{array}{c}1.87 \\
(2.13)\end{array}$ & $\begin{array}{c}3.77 \\
(4.36)\end{array}$ & - & - & - & - & - \\
\hline \multicolumn{12}{|c|}{ TAP: Untertest ,Inkompatibilität ${ }^{66}$} \\
\hline $\begin{array}{l}\text { Standard- } \\
\text { abweichung }\end{array}$ & 15 & $\begin{array}{c}\text { 323.82 } \\
(259.98)\end{array}$ & $\begin{array}{l}196.69 \\
(56.45)\end{array}$ & 29 & $\begin{array}{c}227.93 \\
(163.70)\end{array}$ & $\begin{array}{l}187.49 \\
(80.30)\end{array}$ & - & - & - & - & - \\
\hline Anzahl Fehler & 15 & $\begin{array}{l}15.53 \\
(9.12) \\
\end{array}$ & $\begin{array}{l}12.80 \\
(8.03) \\
\end{array}$ & 29 & $\begin{array}{r}\mathbf{1 4 . 7 2} \\
(10.56) \\
\end{array}$ & $\begin{array}{c}8.52 \\
(6.76) \\
\end{array}$ & 1.16 & 0.28 & 51.37 & nein & $(0.36)$ \\
\hline \multicolumn{12}{|c|}{ TAP: Untertest ,Visuelles Scanning“ } \\
\hline $\begin{array}{l}\text { Standard- } \\
\text { abweichung } \\
\text { kritische Trials } \\
\end{array}$ & 15 & $\begin{array}{c}\mathbf{3 9 5 2 . 5 7} \\
(1864.41)\end{array}$ & $\begin{array}{c}\mathbf{2 3 8 6 . 7 2} \\
(1274.29)\end{array}$ & 23 & $\begin{array}{c}3577.49 \\
(1875.05)\end{array}$ & $\begin{array}{c}2887.19 \\
(1434.07)\end{array}$ & - & - & - & - & - \\
\hline $\begin{array}{l}\text { Standard- } \\
\text { abweichung } \\
\text { nicht-kritische } \\
\text { Trials }\end{array}$ & 15 & $\begin{array}{c}\mathbf{3 6 8 3 . 6 4} \\
(1374.86)\end{array}$ & $\begin{array}{c}\mathbf{2 6 3 6 . 7 3} \\
(1305.89)\end{array}$ & 23 & $\begin{array}{c}\mathbf{3 3 3 7 . 7 4} \\
(1680.22)\end{array}$ & $\begin{array}{l}\mathbf{2 5 1 0 . 1 5} \\
(905.79)\end{array}$ & 0.16 & 0.69 & 1360486 & nein & $(0.16)$ \\
\hline $\begin{array}{l}\text { Anzahl Fehler } \\
\text { (kritische } \\
\text { Trials) } \\
\end{array}$ & 15 & $\begin{array}{c}2.33 \\
(5.23)\end{array}$ & $\begin{array}{l}1.47 \\
(2.92)\end{array}$ & 23 & $\begin{array}{c}7.78 \\
(4.59)\end{array}$ & $\begin{array}{l}3.35 \\
(2.84)\end{array}$ & 6.85 & 0.01 & 8.43 & ja & 0.74 \\
\hline $\begin{array}{l}\text { Anzahl } \\
\text { Auslassungen } \\
\text { (nicht-kritische } \\
\text { Trials) }\end{array}$ & 15 & $\begin{array}{c}5.80 \\
(4.50)\end{array}$ & $\begin{array}{c}6.20 \\
(4.95)\end{array}$ & 23 & $\begin{array}{c}1.22 \\
(2.17)\end{array}$ & $\begin{array}{c}0.52 \\
(0.73)\end{array}$ & - & - & - & - & - \\
\hline \multicolumn{12}{|l|}{ Test d2 } \\
\hline $\begin{array}{l}\text { Konzentrations- } \\
\text { leistungswert }\end{array}$ & 13 & $\begin{array}{l}\mathbf{7 7 . 0 0} \\
(26.59)\end{array}$ & $\begin{array}{l}\mathbf{9 5 . 8 5} \\
(25.78)\end{array}$ & 21 & $\begin{array}{c}\mathbf{9 5 . 2 4} \\
(21.93)\end{array}$ & $\begin{array}{l}111.52 \\
(20.07)\end{array}$ & 0.53 & 0.47 & 49.59 & nein & $(0.11)$ \\
\hline Anzahl Fehler & 13 & $\begin{array}{c}\mathbf{3 4 . 8 5} \\
(34.98)\end{array}$ & $\begin{array}{c}\mathbf{1 2 . 1 5} \\
(14.67)\end{array}$ & 21 & $\begin{array}{l}11.19 \\
(8.69)\end{array}$ & $\begin{array}{c}8.52 \\
(4.98)\end{array}$ & - & - & - & - & - \\
\hline
\end{tabular}




\begin{tabular}{|c|c|c|c|c|c|c|c|c|c|c|c|}
\hline \multirow[t]{2}{*}{$\begin{array}{l}\text { Abhängige } \\
\text { Variable }\end{array}$} & \multicolumn{3}{|c|}{ Wartegruppe (WG) } & \multicolumn{3}{|c|}{$\begin{array}{c}\text { Basis- und } \\
\text { Strategietraining } \\
\text { Lauth und Schlottke } \\
15 \text { Sitzungen }\end{array}$} & \multicolumn{4}{|c|}{ Interaktionseffekt } & \multirow{2}{*}{$\begin{array}{c}\text { Effekt } \\
\text {-größe } \\
D\end{array}$} \\
\hline & $n$ & Vortest & Nachtest & $N$ & Vortest & Nachtest & $F$ & $p$ & $M Q F$ & \begin{tabular}{|c|} 
hypo- \\
thesen- \\
konform \\
?
\end{tabular} & \\
\hline \multicolumn{12}{|l|}{ Mottier-Test } \\
\hline $\begin{array}{l}\text { Nachsprechleist } \\
\text { ung Gesamt } \\
\text { (max. 30) }\end{array}$ & 15 & $\begin{array}{l}20.80 \\
(3.97)\end{array}$ & $\begin{array}{l}20.40 \\
(5.01)\end{array}$ & 32 & $\begin{array}{l}\mathbf{2 1 . 2 2} \\
(4.96)\end{array}$ & $\begin{array}{l}\mathbf{2 3 . 8 1} \\
(3.89)\end{array}$ & 9.16 & 0.00 & 4.99 & ja & 0.64 \\
\hline $\begin{array}{l}\text { Nachsprechleist } \\
\text { ung für 2- und } \\
\text { 3-silbige } \\
\text { Kunstwörter } \\
\text { (max. 12) }\end{array}$ & 15 & $\begin{array}{l}11.07 \\
(1.53)\end{array}$ & $\begin{array}{l}10.20 \\
(2.21)\end{array}$ & 32 & $\begin{array}{l}\mathbf{1 1 . 2 8} \\
(1.02)\end{array}$ & $\begin{array}{l}\mathbf{1 1 . 6 3} \\
(0.79)\end{array}$ & 5.14 & 0.02 & 1.44 & ja & 0.90 \\
\hline $\begin{array}{l}\text { Nachsprechleist } \\
\text { ung für 4-, 5- } \\
\text { und 6-silbige } \\
\text { Kunstwörter } \\
\text { (max. 18) }\end{array}$ & 15 & $\begin{array}{c}9.73 \\
(3.17)\end{array}$ & $\begin{array}{l}10.20 \\
(3.86)\end{array}$ & 32 & $\begin{array}{c}\mathbf{9 . 9 4} \\
(4.44)\end{array}$ & $\begin{array}{l}\mathbf{1 2 . 1 9} \\
(3.41)\end{array}$ & 5.15 & 0.02 & 3.15 & ja & 0.44 \\
\hline \multicolumn{12}{|c|}{ Fremdbeurteilungsbogen FBB-HKS (Eltern) } \\
\hline $\begin{array}{l}\text { Unaufmerksam- } \\
\text { keit: Anzahl an } \\
\text { Kriterien }\end{array}$ & 11 & $\begin{array}{c}4.91 \\
(3.11)\end{array}$ & $\begin{array}{c}4.45 \\
(4.06)\end{array}$ & 28 & $\begin{array}{c}5.78 \\
(2.48)\end{array}$ & $\begin{array}{c}5.57 \\
(3.07)\end{array}$ & - & - & - & - & - \\
\hline $\begin{array}{l}\text { Unaufmerksam- } \\
\text { keit: Kennwert }\end{array}$ & 11 & $\begin{array}{c}1.64 \\
(0.62)\end{array}$ & $\begin{array}{c}1.58 \\
(0.87)\end{array}$ & 28 & $\begin{array}{c}1.78 \\
(0.50)\end{array}$ & $\begin{array}{c}1.69 \\
(0.60) \\
\end{array}$ & - & - & - & - & - \\
\hline $\begin{array}{l}\text { Hyperaktivität: } \\
\text { Anzahl an } \\
\text { Kriterien }\end{array}$ & 11 & $\begin{array}{c}2.72 \\
(1.68)\end{array}$ & $\begin{array}{c}2.36 \\
(2.38)\end{array}$ & 28 & $\begin{array}{c}1.32 \\
(1.49)\end{array}$ & $\begin{array}{c}1.07 \\
(1.56)\end{array}$ & - & - & - & - & - \\
\hline $\begin{array}{l}\text { Hyperaktivität: } \\
\text { Kennwert }\end{array}$ & 11 & $\begin{array}{c}1.63 \\
(0.70)\end{array}$ & $\begin{array}{c}1.29 \\
(0.76)\end{array}$ & 28 & $\begin{array}{c}1.01 \\
(0.61)\end{array}$ & $\begin{array}{c}0.82 \\
(0.79)\end{array}$ & - & - & - & - & - \\
\hline $\begin{array}{l}\text { Impulsivität: } \\
\text { Anzahl an } \\
\text { Kriterien }\end{array}$ & 11 & $\begin{array}{c}1.45 \\
(1.75)\end{array}$ & $\begin{array}{c}1.27 \\
(1.79)\end{array}$ & 28 & $\begin{array}{c}1.03 \\
(1.45)\end{array}$ & $\begin{array}{c}1.28 \\
(1.56)\end{array}$ & - & - & - & - & - \\
\hline $\begin{array}{l}\text { Impulsivität: } \\
\text { Kennwert }\end{array}$ & 11 & $\begin{array}{c}1.18 \\
(0.69) \\
\end{array}$ & $\begin{array}{c}1.15 \\
(0.78) \\
\end{array}$ & 28 & $\begin{array}{c}0.82 \\
(0.75) \\
\end{array}$ & $\begin{array}{c}1.09 \\
(0.87) \\
\end{array}$ & - & - & - & - & - \\
\hline \multicolumn{12}{|c|}{ Child Behavior Checklist (CBCL) } \\
\hline $\begin{array}{l}\text { T-Wert } \\
\text { Aufmerksam- } \\
\text { keitsstörungen }\end{array}$ & 10 & $\begin{array}{l}64.50 \\
(6.89)\end{array}$ & $\begin{array}{l}61.60 \\
(8.63)\end{array}$ & 30 & $\begin{array}{l}\mathbf{6 5 . 4 7} \\
(8.38)\end{array}$ & $\begin{array}{c}\mathbf{6 2 . 1 7} \\
(7.28)\end{array}$ & 0.02 & 0.88 & 95.99 & nein & $(0.05)$ \\
\hline $\begin{array}{l}\text { T-Wert } \\
\text { Externalisieren- } \\
\text { de Störungen }\end{array}$ & 10 & $\begin{array}{c}59.50 \\
(12.04)\end{array}$ & $\begin{array}{c}59.00 \\
(12.18)\end{array}$ & 30 & $\begin{array}{c}\mathbf{5 5 . 0 0} \\
(11.90)\end{array}$ & $\begin{array}{c}\mathbf{5 1 . 7 7} \\
(11.04)\end{array}$ & 0.80 & 0.37 & 34.73 & nein & $(0.24)$ \\
\hline $\begin{array}{l}\text { T-Wert } \\
\text { Gesamtskala }\end{array}$ & 10 & $\begin{array}{l}61.00 \\
(9.46)\end{array}$ & $\begin{array}{l}57.70 \\
(10.87)\end{array}$ & 30 & $\begin{array}{l}\mathbf{5 9 . 3 0} \\
(8.55)\end{array}$ & $\begin{array}{l}\mathbf{5 5 . 7 0} \\
(9.06)\end{array}$ & 0.01 & 0.89 & 19.07 & nein & $(0.02)$ \\
\hline \multicolumn{12}{|c|}{ Fremdbeurteilungsbogen FBB-HKS (Lehrer) } \\
\hline $\begin{array}{l}\text { Unaufmerksam- } \\
\text { keit: Anzahl an } \\
\text { Kriterien }\end{array}$ & 12 & $\begin{array}{c}4.75 \\
(2.83)\end{array}$ & $\begin{array}{c}4.92 \\
(3.09)\end{array}$ & 26 & $\begin{array}{c}\mathbf{6 . 0 3} \\
(2.44)\end{array}$ & $\begin{array}{c}\mathbf{4 . 0 3} \\
(2.58)\end{array}$ & 2.46 & 0.19 & 7.82 & nein & $(0.82)$ \\
\hline $\begin{array}{l}\text { Unaufmerksam- } \\
\text { keit: Kennwert }\end{array}$ & 12 & $\begin{array}{l}1.62 \\
(.58) \\
\end{array}$ & $\begin{array}{l}1.58 \\
(.69) \\
\end{array}$ & 26 & $\begin{array}{c}\mathbf{1 . 8 3} \\
(0.55) \\
\end{array}$ & $\begin{array}{c}\mathbf{1 . 4 3} \\
(0.61) \\
\end{array}$ & 1.40 & 0.24 & 0.36 & nein & $(0.59)$ \\
\hline $\begin{array}{l}\text { Hyperaktivität: } \\
\text { Anzahl an } \\
\text { Kriterien } \\
\end{array}$ & 12 & $\begin{array}{c}2.75 \\
(2.01)\end{array}$ & $\begin{array}{l}1.67 \\
(2.19)\end{array}$ & 26 & $\begin{array}{c}\mathbf{1 . 5 0} \\
(1.47)\end{array}$ & $\begin{array}{c}\mathbf{0 . 6 9} \\
(1.15)\end{array}$ & 0.12 & 0.72 & 2.51 & nein & $(0.17)$ \\
\hline $\begin{array}{l}\text { Hyperaktivität: } \\
\text { Kennwert }\end{array}$ & 12 & $\begin{array}{c}1.63 \\
(0.79)\end{array}$ & $\begin{array}{c}1.10 \\
(0.88)\end{array}$ & 26 & $\begin{array}{c}\mathbf{0 . 9 7} \\
(0.71)\end{array}$ & $\begin{array}{c}\mathbf{0 . 5 4} \\
(0.60)\end{array}$ & 0.06 & 0.79 & 0.48 & nein & $(0.12)$ \\
\hline
\end{tabular}




\begin{tabular}{|c|c|c|c|c|c|c|c|c|c|c|c|}
\hline \multirow[t]{2}{*}{$\begin{array}{c}\text { Abhängige } \\
\text { Variable }\end{array}$} & \multicolumn{3}{|c|}{ Wartegruppe (WG) } & \multicolumn{3}{|c|}{$\begin{array}{c}\text { Basis- und } \\
\text { Strategietraining } \\
\text { Lauth und Schlottke } \\
15 \text { Sitzungen }\end{array}$} & \multicolumn{4}{|c|}{ Interaktionseffekt } & \multirow{2}{*}{$\begin{array}{c}\begin{array}{c}\text { Effekt } \\
\text {-größe }\end{array} \\
D\end{array}$} \\
\hline & $n$ & Vortest & Nachtest & $N$ & Vortest & Nachtest & $F$ & $p$ & $M Q F$ & $\begin{array}{c}\text { hypo- } \\
\text { thesen- } \\
\text { konform } \\
?\end{array}$ & \\
\hline $\begin{array}{l}\text { Impulsivität: } \\
\text { Anzahl an } \\
\text { Kriterien } \\
\end{array}$ & 12 & $\begin{array}{c}2.42 \\
(1.73)\end{array}$ & $\begin{array}{c}1.67 \\
(1.83)\end{array}$ & 26 & $\begin{array}{c}0.80 \\
(1.29)\end{array}$ & $\begin{array}{c}0.69 \\
(1.37)\end{array}$ & - & - & - & - & - \\
\hline $\begin{array}{l}\text { Impulsivität: } \\
\text { Kennwert }\end{array}$ & 12 & $\begin{array}{c}\mathbf{1 . 7 0} \\
(0.94)\end{array}$ & $\begin{array}{r}1.20 \\
(0.89) \\
\end{array}$ & 26 & $\begin{array}{c}0.72 \\
(0.67)\end{array}$ & $\begin{array}{c}0.65 \\
(0.85)\end{array}$ & - & - & - & - & - \\
\hline \multicolumn{12}{|c|}{ Teacher's Report Form (TRF) } \\
\hline $\begin{array}{l}\text { T-Wert } \\
\text { Aufmerksam- } \\
\text { keitsstörungen } \\
\end{array}$ & 7 & $\begin{array}{l}61.86 \\
(4.38)\end{array}$ & $\begin{array}{l}59.86 \\
(5.61)\end{array}$ & 25 & $\begin{array}{l}\mathbf{5 9 . 2 1} \\
(5.49)\end{array}$ & $\begin{array}{l}\mathbf{5 7 . 0 8} \\
(4.32)\end{array}$ & 0.00 & 0.95 & 37.91 & nein & $(0.00)$ \\
\hline $\begin{array}{l}\text { T-Wert } \\
\text { externalisieren- } \\
\text { de Störungen }\end{array}$ & 7 & $\begin{array}{l}65.00 \\
(8.15)\end{array}$ & $\begin{array}{l}65.14 \\
(8.75)\end{array}$ & 25 & $\begin{array}{c}55.04 \\
(10.67)\end{array}$ & $\begin{array}{l}53.42 \\
(9.60)\end{array}$ & - & - & - & - & - \\
\hline $\begin{array}{l}\text { T-Wert } \\
\text { Gesamtskala }\end{array}$ & 7 & $\begin{array}{l}60.71 \\
(7.16)\end{array}$ & $\begin{array}{l}61.43 \\
(5.29)\end{array}$ & 25 & $\begin{array}{l}\mathbf{5 8 . 7 6} \\
(6.80)\end{array}$ & $\begin{array}{l}\mathbf{5 5 . 9 2} \\
(5.77)\end{array}$ & 2.48 & 0.12 & 13.91 & nein & $(0.49)$ \\
\hline
\end{tabular}

Anmerkung: p: einseitige Irrtumswahrscheinlichkeit des F-Wertes;

Signifikanzniveau $\alpha=0.05$;

MQF: Mittleres Fehlerquadrat

n: Anzahl der Messwertpaare (Die Anzahl umfasst nur diejenigen Kinder, von denen sowohl Vor- als auch

Nachtestwert der jeweiligen Variablen vorhanden waren. Auf dieser Anzahl basieren die inferenzstatistischen Auswertungen).

TAP: Testbatterie zur Aufmerksamkeitsprüfung

In Anlehnung an Cohen (1988) wird ein Effekt von 0.20 als klein, von 0.50 als mittel und von 0.80 als groß betrachtet. 


\section{Anhang A}

Tabelle A-3

Mittelwerte und Standardabweichungen (in Klammern) im Vor- und Nachtest für die AVn und Kennwerte $(F, p, M Q F)$ des Interaktionseffekts zweifaktorieller Varianzanalysen mit Messwiederholung getrennt für die Wartegruppe und die Gruppe THOP als Gruppentraining

\begin{tabular}{|c|c|c|c|c|c|c|c|c|c|c|c|}
\hline \multirow{2}{*}{$\begin{array}{c}\text { Abhängige } \\
\text { Variable }\end{array}$} & \multicolumn{3}{|c|}{ Wartegruppe (WG) } & \multicolumn{3}{|c|}{ Trainingsgruppe THOP } & \multicolumn{4}{|c|}{ Interaktionseffekt } & \multirow{2}{*}{$\begin{array}{c}\begin{array}{c}\text { Effekt } \\
\text {-größe }\end{array} \\
D\end{array}$} \\
\hline & $n$ & Vortest & Nachtest & $N$ & Vortest & Nachtest & $F$ & $p$ & $M Q F$ & $\begin{array}{l}\text { hypo- } \\
\text { thesen- } \\
\text { konform } \\
\text { ? }\end{array}$ & \\
\hline \multicolumn{12}{|c|}{ TAP: Untertest „Geteilte Aufmerksamkeit“6 } \\
\hline $\begin{array}{l}\text { Standard- } \\
\text { abweichung }\end{array}$ & 15 & $\begin{array}{c}\mathbf{3 6 4 . 0 5} \\
(100.99)\end{array}$ & $\begin{array}{l}\mathbf{2 9 3 . 6 0} \\
(83.94)\end{array}$ & 29 & $\begin{array}{c}\mathbf{3 3 7 . 8 2} \\
(116.48)\end{array}$ & $\begin{array}{l}\mathbf{2 9 5 . 8 6} \\
(95.81)\end{array}$ & 0.52 & 0.47 & 7669.33 & nein & $(0,28)$ \\
\hline Anzahl Fehler & 15 & $\begin{array}{c}\mathbf{8 . 8 0} \\
(7.06)\end{array}$ & $\begin{array}{c}\mathbf{2 . 6 0} \\
(1.45)\end{array}$ & 29 & $\begin{array}{c}\mathbf{9 . 3 8} \\
(13.44)\end{array}$ & $\begin{array}{c}\mathbf{3 . 0 7} \\
(4.03)\end{array}$ & 0.01 & 0.97 & 65.74 & nein & $(0,04)$ \\
\hline $\begin{array}{l}\text { Anzahl } \\
\text { Auslassungen }\end{array}$ & 15 & $\begin{array}{c}8.73 \\
(6.77)\end{array}$ & $\begin{array}{c}6.80 \\
(3.36)\end{array}$ & 29 & $\begin{array}{c}7.10 \\
(5.02)\end{array}$ & $\begin{array}{c}5.28 \\
(4.71)\end{array}$ & - & - & - & - & - \\
\hline \multicolumn{12}{|c|}{ TAP: Untertest ,Go/Nogo ${ }^{66}$} \\
\hline $\begin{array}{l}\text { Standard- } \\
\text { abweichung }\end{array}$ & 16 & $\begin{array}{l}146.28 \\
(61.36) \\
\end{array}$ & \begin{tabular}{r|}
148.38 \\
$(52.80)$ \\
\end{tabular} & 31 & $\begin{array}{l}140.54 \\
(42.28) \\
\end{array}$ & $\begin{array}{r}125.59 \\
(42.09) \\
\end{array}$ & - & - & - & - & - \\
\hline Anzahl Fehler & 16 & $\begin{array}{c}8.19 \\
(4.79) \\
\end{array}$ & $\begin{array}{c}\mathbf{5 . 7 5} \\
(3.13) \\
\end{array}$ & 31 & $\begin{array}{c}9.81 \\
(5.50) \\
\end{array}$ & $\begin{array}{r}\mathbf{6 . 9 7} \\
(5.33) \\
\end{array}$ & 0.05 & 0.81 & 15.02 & nein & $(0,08)$ \\
\hline $\begin{array}{l}\text { Anzahl } \\
\text { Auslassungen }\end{array}$ & 16 & $\begin{array}{c}\mathbf{5 . 0 0} \\
(5.40)\end{array}$ & $\begin{array}{c}\mathbf{2 . 3 8} \\
(3.12)\end{array}$ & 31 & $\begin{array}{l}2.10 \\
(2.70)\end{array}$ & $\begin{array}{c}\mathbf{0 . 8 4} \\
(1.44)\end{array}$ & 1.19 & 0.28 & 8.24 & nein & $(0,45)$ \\
\hline \multicolumn{12}{|c|}{ TAP: Untertest „,Inkompatibilität" } \\
\hline $\begin{array}{l}\text { Standard- } \\
\text { abweichung }\end{array}$ & 15 & $\begin{array}{c}\mathbf{3 2 3 . 8 2} \\
(259.98)\end{array}$ & $\begin{array}{l}196.69 \\
(56.45)\end{array}$ & 31 & $\begin{array}{c}\mathbf{2 4 2 . 4 6} \\
(123.11)\end{array}$ & $\begin{array}{l}\mathbf{1 7 6 . 6 3} \\
(64.19)\end{array}$ & 1.40 & 0.24 & 13532.9 & nein & $(0,39)$ \\
\hline Anzahl Fehler & 15 & $\begin{array}{l}15.53 \\
(9.12) \\
\end{array}$ & $\begin{array}{l}12.80 \\
(8.03) \\
\end{array}$ & 31 & $\begin{array}{r}\mathbf{2 0 . 4 2} \\
(12.05) \\
\end{array}$ & $\begin{array}{c}\mathbf{1 3 . 5 5} \\
(10.00) \\
\end{array}$ & 1.75 & 0.19 & 49.34 & nein & $(0,38)$ \\
\hline \multicolumn{12}{|c|}{ TAP: Untertest „Visuelles Scanning“6 } \\
\hline $\begin{array}{l}\text { Standard- } \\
\text { abweichung } \\
\text { kritische Trials }\end{array}$ & 15 & \begin{tabular}{c|}
$\mathbf{3 9 5 2 . 5 7}$ \\
$(1864.41)$
\end{tabular} & $\begin{array}{c}\mathbf{2 3 8 6 . 7 2} \\
(1274.29)\end{array}$ & 31 & $\begin{array}{c}\mathbf{2 4 7 2 . 9 8} \\
(1048.18)\end{array}$ & $\begin{array}{l}\mathbf{2 0 4 9 . 0 0} \\
(995.37)\end{array}$ & 8.33 & 0.00 & 790434 & nein & $(0,90)$ \\
\hline $\begin{array}{l}\text { Standard- } \\
\text { abweichung } \\
\text { nicht-kritische } \\
\text { Trials }\end{array}$ & 15 & $\begin{array}{c}\mathbf{3 6 8 3 . 6 4} \\
(1374.86)\end{array}$ & $\begin{array}{c}\mathbf{2 6 3 6 . 7 3} \\
(1305.89)\end{array}$ & 31 & $\begin{array}{c}\mathbf{2 7 6 7 . 1 8} \\
(1185.42)\end{array}$ & $\begin{array}{c}\mathbf{2 1 9 2 . 0 3} \\
(1028.39)\end{array}$ & 1.51 & 0.22 & 744768 & nein & $(0,40)$ \\
\hline $\begin{array}{l}\text { Anzahl Fehler } \\
\text { (kritische } \\
\text { Trials) } \\
\end{array}$ & 15 & $\begin{array}{c}2.33 \\
(5.23)\end{array}$ & $\begin{array}{c}1.47 \\
(2.92)\end{array}$ & 31 & $\begin{array}{c}7.68 \\
(5.95)\end{array}$ & $\begin{array}{c}4.32 \\
(4.10)\end{array}$ & 3.49 & 0.06 & 8.96 & nein & $(0,42)$ \\
\hline $\begin{array}{l}\text { Anzahl } \\
\text { Auslassungen } \\
\text { (nicht-kritische } \\
\text { Trials) } \\
\end{array}$ & 15 & $\begin{array}{c}5.80 \\
(4.50)\end{array}$ & $\begin{array}{c}6.20 \\
(4.95)\end{array}$ & 31 & $\begin{array}{c}\mathbf{5 . 3 2} \\
(5.96)\end{array}$ & $\begin{array}{c}\mathbf{2 . 2 6} \\
(3.26)\end{array}$ & 4.38 & 0.04 & 13.83 & ja & 0,70 \\
\hline \multicolumn{12}{|l|}{ Test d2 } \\
\hline $\begin{array}{l}\text { Konzentrations- } \\
\text { leistungswert }\end{array}$ & 13 & $\begin{array}{r}77.00 \\
(26.59) \\
\end{array}$ & $\begin{array}{r}\mathbf{9 5 . 8 5} \\
(25.78) \\
\end{array}$ & 15 & $\begin{array}{r}\mathbf{9 3 . 9 3} \\
(25.92) \\
\end{array}$ & $\begin{array}{r}\mathbf{1 2 3 . 2 0} \\
(25.27) \\
\end{array}$ & 5.04 & 0.03 & 75.01 & ja & 0,41 \\
\hline Anzahl Fehler & 13 & $\begin{array}{c}\mathbf{3 4 . 8 5} \\
(34.98)\end{array}$ & $\begin{array}{c}\mathbf{1 2 . 1 5} \\
(14.67)\end{array}$ & 15 & $\begin{array}{c}\mathbf{2 6 . 2 7} \\
(37.59)\end{array}$ & $\begin{array}{c}9.27 \\
(6.30)\end{array}$ & 0.20 & 0.65 & 560.24 & nein & $(0,17)$ \\
\hline
\end{tabular}




\begin{tabular}{|c|c|c|c|c|c|c|c|c|c|c|c|}
\hline \multirow{2}{*}{$\begin{array}{c}\text { Abhängige } \\
\text { Variable }\end{array}$} & \multicolumn{3}{|c|}{ Wartegruppe (WG) } & \multicolumn{3}{|c|}{ Trainingsgruppe THOP } & \multicolumn{4}{|c|}{ Interaktionseffekt } & \multirow{2}{*}{$\begin{array}{c}\text { Effekt } \\
\text {-größe } \\
D\end{array}$} \\
\hline & $n$ & Vortest & Nachtest & $N$ & Vortest & Nachtest & $F$ & $p$ & $M Q F$ & $\begin{array}{c}\text { hypo- } \\
\text { thesen- } \\
\text { konform } \\
?\end{array}$ & \\
\hline \multicolumn{12}{|l|}{ Mottier-Test } \\
\hline $\begin{array}{l}\text { Nachsprechleist } \\
\text { ung Gesamt } \\
(\max .30)\end{array}$ & 15 & $\begin{array}{l}20.80 \\
(3.97)\end{array}$ & $\begin{array}{l}20.40 \\
(5.01)\end{array}$ & 30 & $\begin{array}{l}\mathbf{2 2 . 2 0} \\
(4.00)\end{array}$ & $\begin{array}{l}23.57 \\
(3.79)\end{array}$ & 3.31 & 0.07 & 4.70 & nein & $(0,41)$ \\
\hline $\begin{array}{l}\text { Nachsprechleist } \\
\text { ung für 2- und } \\
\text { 3-silbige } \\
\text { Kunstwörter } \\
\text { (max. 12) }\end{array}$ & 15 & $\begin{array}{l}11.07 \\
(1.53)\end{array}$ & $\begin{array}{l}10.20 \\
(2.21)\end{array}$ & 30 & $\begin{array}{l}\mathbf{1 1 . 1 7} \\
(1.02)\end{array}$ & $\begin{array}{l}\mathbf{1 1 . 7 0} \\
(.60)\end{array}$ & 6.14 & 0.01 & 1.59 & ja & 1,10 \\
\hline $\begin{array}{l}\text { Nachsprechleist } \\
\text { ung für 4-, 5- } \\
\text { und 6-silbige } \\
\text { Kunstwörter } \\
\text { (max. 18) }\end{array}$ & 15 & $\begin{array}{c}9.73 \\
(3.17)\end{array}$ & $\begin{array}{l}10.20 \\
(3.86)\end{array}$ & 30 & $\begin{array}{l}\mathbf{1 1 . 0 3} \\
(3.40)\end{array}$ & $\begin{array}{l}11.87 \\
(3.46)\end{array}$ & 0.25 & 0.61 & 2.65 & nein & $(0,10)$ \\
\hline \multicolumn{12}{|c|}{ Fremdbeurteilungsbogen FBB-HKS (Eltern) } \\
\hline $\begin{array}{l}\text { Unaufmerksam- } \\
\text { keit: Anzahl an } \\
\text { Kriterien }\end{array}$ & 11 & $\begin{array}{c}4.91 \\
(3.11)\end{array}$ & $\begin{array}{l}4.45 \\
(4.06)\end{array}$ & 30 & $\begin{array}{l}\mathbf{5 . 9 3} \\
(2.58)\end{array}$ & $\begin{array}{l}4.50 \\
(2.56)\end{array}$ & 0.74 & 0.39 & 5.15 & nein & $(0,33)$ \\
\hline $\begin{array}{l}\text { Unaufmerksam- } \\
\text { keit: Kennwert }\end{array}$ & 11 & $\begin{array}{c}1.64 \\
(0.62) \\
\end{array}$ & $\begin{array}{c}1.58 \\
(0.87) \\
\end{array}$ & 30 & $\begin{array}{c}1.85 \\
(0.58) \\
\end{array}$ & $\begin{array}{c}1.48 \\
(0.57) \\
\end{array}$ & 1.60 & 0.21 & 0.23 & nein & $(0,49)$ \\
\hline $\begin{array}{l}\text { Hyperaktivität: } \\
\text { Anzahl an } \\
\text { Kriterien } \\
\end{array}$ & 11 & $\begin{array}{c}2.72 \\
(1.68)\end{array}$ & $\begin{array}{l}2.36 \\
(2.38)\end{array}$ & 30 & $\begin{array}{c}\mathbf{2 . 4 3} \\
(1.79)\end{array}$ & $\begin{array}{c}\mathbf{1 . 7 0} \\
(1.58)\end{array}$ & 0.33 & 0.56 & 1.62 & nein & $(0,21)$ \\
\hline $\begin{array}{l}\text { Hyperaktivität: } \\
\text { Kennwert }\end{array}$ & 11 & $\begin{array}{c}1.63 \\
(0.70) \\
\end{array}$ & $\begin{array}{c}1.29 \\
(0.76) \\
\end{array}$ & 30 & $\begin{array}{c}1.48 \\
(0.85)\end{array}$ & $\begin{array}{c}1.14 \\
(0.66) \\
\end{array}$ & 0.00 & 0.97 & 0.27 & nein & $(0,00)$ \\
\hline $\begin{array}{l}\text { Impulsivität: } \\
\text { Anzahl an } \\
\text { Kriterien } \\
\end{array}$ & 11 & $\begin{array}{l}1.45 \\
(1.75)\end{array}$ & $\begin{array}{l}1.27 \\
(1.79)\end{array}$ & 30 & $\begin{array}{c}\mathbf{2 . 4 0} \\
(1.61)\end{array}$ & $\begin{array}{c}\mathbf{2 . 0 0} \\
(1.43)\end{array}$ & 0.14 & 0.70 & 1.34 & nein & $(0,14)$ \\
\hline $\begin{array}{l}\text { Impulsivität: } \\
\text { Kennwert }\end{array}$ & 11 & $\begin{array}{c}1.18 \\
(0.69)\end{array}$ & $\begin{array}{c}1.15 \\
(0.78)\end{array}$ & 30 & $\begin{array}{l}1.61 \\
(0.93)\end{array}$ & $\begin{array}{c}1.50 \\
(0.81)\end{array}$ & - & - & - & - & - \\
\hline \multicolumn{12}{|c|}{ Child Behavior Checklist (CBCL) } \\
\hline $\begin{array}{l}\text { T-Wert } \\
\text { Aufmerksam- } \\
\text { keitsstörungen }\end{array}$ & 10 & $\begin{array}{l}64.50 \\
(6.89)\end{array}$ & $\begin{array}{l}61.60 \\
(8.63)\end{array}$ & 30 & $\begin{array}{l}66.27 \\
(6.70)\end{array}$ & $\begin{array}{l}\mathbf{6 0 . 5 7} \\
(6.67)\end{array}$ & 1.02 & 0.31 & 28.56 & nein & $(0,37)$ \\
\hline $\begin{array}{l}\text { T-Wert } \\
\text { Externalisieren- } \\
\text { de Störungen }\end{array}$ & 10 & $\begin{array}{c}59.50 \\
(12.04)\end{array}$ & $\begin{array}{c}59.00 \\
(12.18)\end{array}$ & 30 & $\begin{array}{c}\mathbf{6 5 . 4 7} \\
(11.47)\end{array}$ & $\begin{array}{l}\mathbf{5 9 . 9 0} \\
(9.95)\end{array}$ & 2.61 & 0.11 & 36.86 & nein & $(0,47)$ \\
\hline $\begin{array}{l}\text { T-Wert } \\
\text { Gesamtskala }\end{array}$ & 10 & $\begin{array}{l}61.00 \\
(9.46)\end{array}$ & $\begin{array}{c}57.70 \\
(10.87)\end{array}$ & 30 & $\begin{array}{l}\mathbf{6 5 . 9 3} \\
(7.93)\end{array}$ & $\begin{array}{l}\mathbf{5 9 . 9 0} \\
(8.24)\end{array}$ & 1.04 & 0.31 & 26.80 & nein & $(0,28)$ \\
\hline \multicolumn{12}{|c|}{ Fremdbeurteilungsbogen FBB-HKS (Lehrer) } \\
\hline $\begin{array}{l}\text { Unaufmerksam- } \\
\text { keit: Anzahl an } \\
\text { Kriterien }\end{array}$ & 12 & $\begin{array}{l}4.75 \\
(2.83)\end{array}$ & $\begin{array}{c}4.92 \\
(3.09)\end{array}$ & 23 & $\begin{array}{l}4.86 \\
(2.51)\end{array}$ & $\begin{array}{c}3.91 \\
(2.29)\end{array}$ & 0.88 & 0.35 & 5.64 & nein & $(0,44)$ \\
\hline $\begin{array}{l}\text { Unaufmerksam- } \\
\text { keit: Kennwert }\end{array}$ & 12 & $\begin{array}{l}1.62 \\
(.58) \\
\end{array}$ & $\begin{array}{l}1.58 \\
(.69) \\
\end{array}$ & 23 & $\begin{array}{c}1.71 \\
(0.63) \\
\end{array}$ & $\begin{array}{c}1.44 \\
(0.48) \\
\end{array}$ & 0.72 & 0.40 & 0.27 & nein & $(0,38)$ \\
\hline $\begin{array}{l}\text { Hyperaktivität: } \\
\text { Anzahl an } \\
\text { Kriterien } \\
\end{array}$ & 12 & $\begin{array}{l}2.75 \\
(2.01)\end{array}$ & $\begin{array}{l}1.67 \\
(2.19)\end{array}$ & 23 & $\begin{array}{l}2.08 \\
(1.90)\end{array}$ & $\begin{array}{c}1.47 \\
(1.59)\end{array}$ & - & - & - & - & - \\
\hline $\begin{array}{l}\text { Hyperaktivität: } \\
\text { Kennwert }\end{array}$ & 12 & $\begin{array}{c}1.63 \\
(0.79)\end{array}$ & $\begin{array}{c}1.10 \\
(0.88)\end{array}$ & 23 & $\begin{array}{l}1.26 \\
(0.94)\end{array}$ & $\begin{array}{c}1.04 \\
(0.79)\end{array}$ & - & - & - & - & - \\
\hline
\end{tabular}




\begin{tabular}{|c|c|c|c|c|c|c|c|c|c|c|c|}
\hline \multirow[t]{2}{*}{$\begin{array}{c}\text { Abhängige } \\
\text { Variable } \\
\end{array}$} & \multicolumn{3}{|c|}{ Wartegruppe (WG) } & \multicolumn{3}{|c|}{ Trainingsgruppe THOP } & \multicolumn{4}{|c|}{ Interaktionseffekt } & \multirow{2}{*}{$\begin{array}{c}\begin{array}{c}\text { Effekt } \\
\text {-größe }\end{array} \\
D\end{array}$} \\
\hline & $n$ & Vortest & Nachtest & $N$ & Vortest & Nachtest & $F$ & $p$ & $M Q F$ & $\begin{array}{c}\text { hypo- } \\
\text { thesen- } \\
\text { konform } \\
?\end{array}$ & \\
\hline $\begin{array}{l}\text { Impulsivität: } \\
\text { Anzahl an } \\
\text { Kriterien }\end{array}$ & 12 & $\begin{array}{c}2.42 \\
(1.73)\end{array}$ & $\begin{array}{c}1.67 \\
(1.83)\end{array}$ & 23 & $\begin{array}{l}1.86 \\
(1.63)\end{array}$ & $\begin{array}{c}1.56 \\
(1.70)\end{array}$ & - & - & - & - & - \\
\hline $\begin{array}{l}\text { Impulsivität: } \\
\text { Kennwert }\end{array}$ & 12 & $\begin{array}{c}\mathbf{1 . 7 0} \\
(0.94)\end{array}$ & $\begin{array}{c}\mathbf{1 . 2 0} \\
(0.89)\end{array}$ & 23 & $\begin{array}{l}1.34 \\
(0.81)\end{array}$ & $\begin{array}{c}1.28 \\
(0.92)\end{array}$ & - & - & - & - & - \\
\hline \multicolumn{12}{|c|}{ Teacher's Report Form (TRF) } \\
\hline $\begin{array}{l}\text { T-Wert } \\
\text { Aufmerksam- } \\
\text { keitsstörungen }\end{array}$ & 7 & $\begin{array}{l}61.86 \\
(4.38)\end{array}$ & $\begin{array}{l}59.86 \\
(5.61)\end{array}$ & 22 & $\begin{array}{l}\mathbf{6 2 . 1 8} \\
(6.61)\end{array}$ & $\begin{array}{l}\mathbf{5 8 . 6 4} \\
(5.74)\end{array}$ & 0.56 & 0.46 & 11.32 & nein & $(0,26)$ \\
\hline $\begin{array}{l}\text { T-Wert } \\
\text { externalisieren- } \\
\text { de Störungen }\end{array}$ & 7 & $\begin{array}{l}65.00 \\
(8.15)\end{array}$ & $\begin{array}{l}65.14 \\
(8.75)\end{array}$ & 22 & $\begin{array}{l}66.36 \\
(8.68)\end{array}$ & $\begin{array}{l}63.64 \\
(8.78)\end{array}$ & - & - & - & - & - \\
\hline $\begin{array}{l}\text { T-Wert } \\
\text { Gesamtskala }\end{array}$ & 7 & $\begin{array}{l}60.71 \\
(7.16)\end{array}$ & $\begin{array}{l}61.43 \\
(5.29)\end{array}$ & 22 & $\begin{array}{l}\mathbf{6 5 . 6 4} \\
(7.92)\end{array}$ & $\begin{array}{l}\mathbf{6 2 . 0 9} \\
(7.52)\end{array}$ & 2.77 & 0.10 & 17.35 & nein & $(0,51)$ \\
\hline
\end{tabular}

Anmerkung: p: einseitige Irrtumswahrscheinlichkeit des F-Wertes; Signifikanzniveau $\alpha=0.05$; MQF: Mittleres Fehlerquadrat

n: Anzahl der Messwertpaare (Die Anzahl umfasst nur diejenigen Kinder, von denen sowohl Vor- als auch Nachtestwert der jeweiligen Variablen vorhanden waren. Auf dieser Anzahl basieren die inferenzstatistischen Auswertungen).

TAP: Testbatterie zur Aufmerksamkeitsprüfung

In Anlehnung an Cohen (1988) wird ein Effekt von 0.20 als klein, von 0.50 als mittel und von 0.80 als groß betrachtet. 


\section{Anhang A}

Tabelle A-4

Mittelwerte und Standardabweichungen (in Klammern) im Vor- und Nachtest für die AVn und Kennwerte $(F, p, M Q F)$ des Interaktionseffekts zweifaktorieller Varianzanalysen mit Messwiederholung getrennt für das Pseudotraining „Petterson und Findus“ (PF) und das Marburger Konzentrationstraining (MK)

\begin{tabular}{|c|c|c|c|c|c|c|c|c|c|c|c|}
\hline \multirow[t]{2}{*}{$\begin{array}{c}\text { Abhängige } \\
\text { Variable }\end{array}$} & \multicolumn{3}{|c|}{$\begin{array}{c}\text { Kontrollgruppe } \\
\text { („Petterson und } \\
\text { Findus } 6\end{array}$} & \multicolumn{3}{|c|}{$\begin{array}{c}\text { Marburger } \\
\text { Konzentrationstraining } \\
\text { (MK) } \\
\end{array}$} & \multicolumn{4}{|c|}{ Interaktionseffekt } & \multirow{2}{*}{$\begin{array}{c}\text { Effekt } \\
\text {-größe }\end{array}$} \\
\hline & $n$ & Vortest & Nachtest & $N$ & Vortest & Nachtest & $F$ & $p$ & $M Q F$ & $\begin{array}{l}\text { hypo- } \\
\text { thesen- } \\
\text { konform } \\
?\end{array}$ & \\
\hline \multicolumn{12}{|c|}{ TAP: Untertest „Geteilte Aufmerksamkeit ${ }^{66}$} \\
\hline $\begin{array}{l}\text { Standard- } \\
\text { abweichung }\end{array}$ & 12 & $\begin{array}{l}324.51 \\
(86.63)\end{array}$ & $\begin{array}{l}269.44 \\
(86.32)\end{array}$ & 15 & $\begin{array}{c}275.59 \\
(129.44)\end{array}$ & $\begin{array}{l}241.21 \\
(73.63)\end{array}$ & - & - & - & - & - \\
\hline Anzahl Fehler & 12 & $\begin{array}{c}7.08 \\
(5.99)\end{array}$ & $\begin{array}{c}5.00 \\
(10.46)\end{array}$ & 15 & $\begin{array}{c}3.80 \\
(6.04)\end{array}$ & $\begin{array}{c}4.27 \\
(4.22)\end{array}$ & - & - & - & - & - \\
\hline $\begin{array}{l}\text { Anzahl } \\
\text { Auslassungen }\end{array}$ & 12 & $\begin{array}{c}6.33 \\
(4.81)\end{array}$ & $\begin{array}{c}6.25 \\
(4.83)\end{array}$ & 15 & $\begin{array}{c}7.73 \\
(5.12)\end{array}$ & $\begin{array}{c}6.80 \\
(4.49)\end{array}$ & - & - & - & - & - \\
\hline \multicolumn{12}{|c|}{ TAP: Untertest „Go/Nogo“ } \\
\hline $\begin{array}{l}\text { Standard- } \\
\text { abweichung }\end{array}$ & 14 & $\begin{array}{c}\mathbf{3 2 4 . 9 7} \\
(272.30)\end{array}$ & $\begin{array}{l}\mathbf{1 2 0 . 1 3} \\
(47.75)\end{array}$ & 15 & $\begin{array}{c}208.69 \\
(160.59)\end{array}$ & $\begin{array}{l}143.85 \\
(43.75)\end{array}$ & - & - & - & - & - \\
\hline Anzahl Fehler & 14 & $\begin{array}{l}11.29 \\
(5.76) \\
\end{array}$ & $\begin{array}{c}9.86 \\
(6.64) \\
\end{array}$ & 15 & $\begin{array}{c}9.53 \\
(4.79) \\
\end{array}$ & $\begin{array}{l}10.00 \\
(6.23)\end{array}$ & - & - & - & - & - \\
\hline $\begin{array}{l}\text { Anzahl } \\
\text { Auslassungen }\end{array}$ & 14 & $\begin{array}{c}3.86 \\
(3.76)\end{array}$ & $\begin{array}{c}2.71 \\
(3.52)\end{array}$ & 15 & $\begin{array}{c}2.80 \\
(3.10)\end{array}$ & $\begin{array}{c}1.80 \\
(1.90)\end{array}$ & - & - & - & - & - \\
\hline \multicolumn{12}{|c|}{ TAP: Untertest „Inkompatibilität““ } \\
\hline $\begin{array}{l}\text { Standard- } \\
\text { abweichung }\end{array}$ & 13 & \begin{tabular}{|c|}
209.69 \\
$(138.82)$
\end{tabular} & $\begin{array}{c}223.80 \\
(208.39)\end{array}$ & 15 & $\begin{array}{l}179.11 \\
(80.37)\end{array}$ & $\begin{array}{c}210.81 \\
(230.13)\end{array}$ & - & - & - & - & - \\
\hline Anzahl Fehler & 13 & $\begin{array}{c}16.38 \\
(14.09) \\
\end{array}$ & $\begin{array}{c}14.77 \\
(15.08) \\
\end{array}$ & 15 & $\begin{array}{c}16.27 \\
(10.37) \\
\end{array}$ & $\begin{array}{l}12.80 \\
(9.53)\end{array}$ & - & - & - & - & - \\
\hline \multicolumn{12}{|c|}{ TAP: Untertest „,Visuelles Scanning } \\
\hline $\begin{array}{l}\text { Standard- } \\
\text { abweichung } \\
\text { kritische Trials } \\
\end{array}$ & 10 & $\begin{array}{l}2187.58 \\
(765.10)\end{array}$ & $\begin{array}{c}1456.13 \\
(1118.98)\end{array}$ & 11 & $\begin{array}{c}\mathbf{3 1 4 2 . 8 0} \\
(2312.28)\end{array}$ & $\begin{array}{l}\mathbf{1 7 7 5 . 0 0} \\
(603.20)\end{array}$ & 0.66 & 0.42 & 1783861 & nein & $(0.48)$ \\
\hline $\begin{array}{l}\text { Standard- } \\
\text { abweichung } \\
\text { nicht-kritische } \\
\text { Trials }\end{array}$ & 10 & $\begin{array}{l}\mathbf{2 1 2 5 . 9 2} \\
(767.85)\end{array}$ & $\begin{array}{c}\mathbf{1 4 4 1 . 8 5} \\
(1106.18)\end{array}$ & 11 & $\begin{array}{l}\mathbf{2 1 1 8 . 4 6} \\
(831.97)\end{array}$ & $\begin{array}{l}\mathbf{1 4 8 4 . 1 5} \\
(419.35)\end{array}$ & 0.02 & 0.88 & 321460 & nein & $(0.06)$ \\
\hline $\begin{array}{l}\text { Anzahl Fehler } \\
\text { (kritische } \\
\text { Trials) } \\
\end{array}$ & 10 & $\begin{array}{c}4.80 \\
(2.20)\end{array}$ & $\begin{array}{c}7.80 \\
(5.07)\end{array}$ & 11 & $\begin{array}{c}6.75 \\
(3.47)\end{array}$ & $\begin{array}{c}4.83 \\
(5.24)\end{array}$ & - & - & - & - & - \\
\hline $\begin{array}{l}\text { Anzahl } \\
\text { Auslassungen } \\
\text { (nicht-kritische } \\
\text { Trials) }\end{array}$ & 10 & $\begin{array}{l}1.90 \\
(1.79)\end{array}$ & $\begin{array}{l}1.40 \\
(2.17)\end{array}$ & 11 & $\begin{array}{c}1.55 \\
(1.13)\end{array}$ & $\begin{array}{c}0.45 \\
(0.69)\end{array}$ & - & - & - & - & - \\
\hline \multicolumn{12}{|l|}{ Test d2 } \\
\hline $\begin{array}{l}\text { Konzentrations- } \\
\text { leistungswert }\end{array}$ & 14 & $\begin{array}{c}\mathbf{9 2 . 7 9} \\
(13.74) \\
\end{array}$ & $\begin{array}{l}118.29 \\
(16.89) \\
\end{array}$ & 15 & $\begin{array}{l}\mathbf{1 0 7 . 8 0} \\
(26.56)\end{array}$ & $\begin{array}{l}137,93 \\
(33.38) \\
\end{array}$ & 0.08 & 0.76 & 113.67 & nein & $(0.07)$ \\
\hline Anzahl Fehler & 14 & $\begin{array}{l}11.43 \\
(1.07) \\
\end{array}$ & $\begin{array}{l}10.41 \\
(7.14) \\
\end{array}$ & 15 & $\begin{array}{c}\mathbf{1 4 . 4 0} \\
(12.11) \\
\end{array}$ & $\begin{array}{c}\mathbf{5 . 6 7} \\
(4.10) \\
\end{array}$ & 1.36 & 0.25 & 50.96 & nein & $(0.42)$ \\
\hline
\end{tabular}




\begin{tabular}{|c|c|c|c|c|c|c|c|c|c|c|c|}
\hline \multirow[t]{2}{*}{$\begin{array}{l}\text { Abhängige } \\
\text { Variable }\end{array}$} & \multicolumn{3}{|c|}{$\begin{array}{c}\text { Kontrollgruppe } \\
\text { (,Petterson und } \\
\text { Findus }{ }^{66}\end{array}$} & \multicolumn{3}{|c|}{$\begin{array}{c}\text { Marburger } \\
\text { Konzentrationstraining } \\
\text { (MK) }\end{array}$} & \multicolumn{4}{|c|}{ Interaktionseffekt } & \multirow{2}{*}{\begin{tabular}{|c} 
Effekt \\
-größe \\
$D$
\end{tabular}} \\
\hline & $n$ & Vortest & Nachtest & $N$ & Vortest & Nachtest & $F$ & $p$ & $M Q F$ & $\begin{array}{c}\text { hypo- } \\
\text { thesen- } \\
\text { konform } \\
?\end{array}$ & \\
\hline \multicolumn{12}{|c|}{ Fremdbeurteilungsbogen FBB-HKS (Eltern) } \\
\hline $\begin{array}{l}\text { Unaufmerksam- } \\
\text { keit: Kennwert }\end{array}$ & 4 & $\begin{array}{c}\mathbf{2 . 0 2} \\
(0.50)\end{array}$ & $\begin{array}{c}\mathbf{1 . 3 0} \\
(0.54)\end{array}$ & 2 & $\begin{array}{c}1.45 \\
(1.76)\end{array}$ & $\begin{array}{c}1.40 \\
(1.83)\end{array}$ & - & - & - & - & - \\
\hline $\begin{array}{l}\text { Hyperaktivität: } \\
\text { Kennwert }\end{array}$ & 4 & $\begin{array}{c}1.45 \\
(1.03) \\
\end{array}$ & $\begin{array}{l}0.85 \\
(.41)\end{array}$ & 2 & $\begin{array}{l}2.10 \\
(1.27)\end{array}$ & $\begin{array}{c}2.10 \\
(0.70)\end{array}$ & - & - & - & - & - \\
\hline $\begin{array}{l}\text { Impulsivität: } \\
\text { Kennwert }\end{array}$ & 4 & $\begin{array}{c}1.47 \\
(0.96)\end{array}$ & $\begin{array}{c}1.10 \\
(0.62)\end{array}$ & 2 & $\begin{array}{l}1.50 \\
(2.12)\end{array}$ & $\begin{array}{c}1.40 \\
(1.97)\end{array}$ & - & - & - & - & - \\
\hline \multicolumn{12}{|c|}{ Child Behavior Checklist (CBCL) } \\
\hline $\begin{array}{l}\text { T-Wert } \\
\text { Aufmerksam- } \\
\text { keitsstörungen }\end{array}$ & 5 & $\begin{array}{l}69.00 \\
(8.75)\end{array}$ & $\begin{array}{l}64.80 \\
(6.53)\end{array}$ & 3 & $\begin{array}{l}59.50 \\
(7.78)\end{array}$ & $\begin{array}{l}54.00 \\
(4.24)\end{array}$ & - & - & - & - & - \\
\hline $\begin{array}{l}\text { T-Wert } \\
\text { Externalisieren- } \\
\text { de Störungen }\end{array}$ & 5 & $\begin{array}{l}60.60 \\
(4.67)\end{array}$ & $\begin{array}{l}56.20 \\
(6.72)\end{array}$ & 3 & $\begin{array}{c}48.50 \\
(14.85)\end{array}$ & $\begin{array}{c}47.00 \\
(16.97)\end{array}$ & - & - & - & - & - \\
\hline $\begin{array}{l}\text { T-Wert } \\
\text { Gesamtskala }\end{array}$ & 5 & $\begin{array}{l}64.20 \\
(8.35)\end{array}$ & $\begin{array}{l}59.60 \\
(8.05)\end{array}$ & 3 & $\begin{array}{c}53.00 \\
(11.31)\end{array}$ & $\begin{array}{l}53.00 \\
(0.00)\end{array}$ & - & - & - & - & - \\
\hline \multicolumn{12}{|l|}{ CFT } \\
\hline IQ Teil 1 & 14 & $\begin{array}{l}\mathbf{9 7 . 6 4} \\
(4.36) \\
\end{array}$ & $\begin{array}{l}\mathbf{1 0 7 . 5 7} \\
8.95() \\
\end{array}$ & 15 & $\begin{array}{l}\mathbf{1 0 7 . 1 3} \\
(12.69) \\
\end{array}$ & $\begin{array}{l}112.93 \\
(10.40) \\
\end{array}$ & 2.38 & 0.13 & 25.87 & nein & $(0.43)$ \\
\hline IQ Teil 2 & 14 & $\begin{array}{c}\mathbf{9 8 . 4 3} \\
(12.16)\end{array}$ & $\begin{array}{l}\mathbf{1 0 2 . 1 4} \\
(11.27)\end{array}$ & 15 & $\begin{array}{l}\mathbf{1 0 3 . 4 0} \\
(9.68) \\
\end{array}$ & $\begin{array}{l}\mathbf{1 1 2 . 0 0} \\
(11.92) \\
\end{array}$ & 2.30 & 0.14 & 37.56 & nein & $(0.42)$ \\
\hline IQ Gesamt & 14 & $\begin{array}{l}96.43 \\
(7.67) \\
\end{array}$ & $\begin{array}{l}\mathbf{1 0 4 . 9 3} \\
(10.80) \\
\end{array}$ & 15 & $\begin{array}{l}\mathbf{1 0 5 . 2 0} \\
(11.12) \\
\end{array}$ & $\begin{array}{l}\mathbf{1 1 3 . 3 3} \\
(11.49) \\
\end{array}$ & 0.02 & 0.88 & 24.69 & nein & $(0.03)$ \\
\hline $\begin{array}{l}\text { Differenz Teil } 1 \\
\text { minus Teil } 2\end{array}$ & 14 & $\begin{array}{c}\mathbf{- 0 . 7 8} \\
(11.43)\end{array}$ & $\begin{array}{c}\mathbf{5 . 4 2} \\
(7.83)\end{array}$ & 15 & $\begin{array}{c}3.73 \\
(10.72)\end{array}$ & $\begin{array}{c}0.93 \\
(8.40)\end{array}$ & - & - & - & - & - \\
\hline \multicolumn{12}{|c|}{ HAWIK-III (Zahlensymboltest) } \\
\hline $\begin{array}{l}\text { Bearbeitete } \\
\text { Zeichen }\end{array}$ & 14 & $\begin{array}{l}\mathbf{3 5 . 8 6} \\
(8.98)\end{array}$ & $\begin{array}{l}38.64 \\
(9.63)\end{array}$ & 15 & $\begin{array}{c}\mathbf{4 1 . 1 3} \\
(11.06)\end{array}$ & $\begin{array}{l}\mathbf{4 6 . 8 0} \\
(9.84)\end{array}$ & 1.38 & 0.25 & 21.69 & nein & $(0.28)$ \\
\hline \multicolumn{12}{|l|}{ TPK } \\
\hline Silbenzahl & 14 & $\begin{array}{c}95.21 \\
(40.32)\end{array}$ & $\begin{array}{l}105.64 \\
(46.52) \\
\end{array}$ & 15 & $\begin{array}{l}\mathbf{1 1 4 . 4 7} \\
(39.45) \\
\end{array}$ & $\begin{array}{l}\mathbf{1 4 5 . 4 0} \\
(50.34) \\
\end{array}$ & 7.17 & 0.01 & 212.30 & ja & 0.41 \\
\hline $\begin{array}{l}\text { Silbenzahl } \\
\text { Fehler }\end{array}$ & 14 & $\begin{array}{c}6.39 \\
(7.25)\end{array}$ & $\begin{array}{l}12.76 \\
(28.84)\end{array}$ & 15 & $\begin{array}{c}6.38 \\
(5.75)\end{array}$ & $\begin{array}{c}6.20 \\
(6.02)\end{array}$ & - & - & - & - & - \\
\hline Tiere & 14 & $\begin{array}{l}\mathbf{1 2 . 7 9} \\
(4.39) \\
\end{array}$ & $\begin{array}{l}\mathbf{1 6 . 1 4} \\
(3.92) \\
\end{array}$ & 15 & $\begin{array}{l}\mathbf{1 4 . 0 0} \\
(3.48) \\
\end{array}$ & $\begin{array}{l}\mathbf{1 7 . 6 0} \\
(4.00) \\
\end{array}$ & 0.03 & 0.85 & 5.83 & nein & $(0.05)$ \\
\hline Rechenleistung & 14 & $\begin{array}{c}31.64 \\
(15.97) \\
\end{array}$ & $\begin{array}{c}33.79 \\
(14.86) \\
\end{array}$ & 15 & $\begin{array}{c}44.80 \\
(23.33) \\
\end{array}$ & $\begin{array}{c}\mathbf{5 1 . 8 7} \\
(29.76) \\
\end{array}$ & 1.88 & 0.18 & 46.49 & nein & $(0.22)$ \\
\hline Rechnen Fehler & 14 & $\begin{array}{c}23.00 \\
(19.94)\end{array}$ & $\begin{array}{c}19.05 \\
(18.66) \\
\end{array}$ & 15 & $\begin{array}{c}16.58 \\
(17.48)\end{array}$ & $\begin{array}{c}16.69 \\
(15.39) \\
\end{array}$ & - & 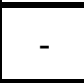 & - & - & - \\
\hline $\begin{array}{l}\text { Konzentrations- } \\
\text { wert }\end{array}$ & 14 & $\begin{array}{c}79.57 \\
(14.52)\end{array}$ & $\begin{array}{c}\mathbf{8 7 . 3 6} \\
(13.25)\end{array}$ & 15 & $\begin{array}{c}\mathbf{8 3 . 9 3} \\
(13.72)\end{array}$ & $\begin{array}{c}\mathbf{9 4 . 4 7} \\
(15.11)\end{array}$ & 0.47 & 0.49 & 57.81 & nein & $(0.19)$ \\
\hline
\end{tabular}

Anmerkung: p: einseitige Irrtumswahrscheinlichkeit des F-Wertes;

Signifikanzniveau $\alpha=0.05$;

MQF: Mittleres Fehlerquadrat

n: Anzahl der Messwertpaare (Die Anzahl umfasst nur diejenigen Kinder, von denen sowohl Vor- als auch

Nachtestwert der jeweiligen Variablen vorhanden waren. Auf dieser Anzahl basieren die inferenzstatistischen

Auswertungen).

TAP: Testbatterie zur Aufmerksamkeitsprüfung

TPK: Testbatterie zur Prüfung der Konzentrationsfähigkeit

n.b.: nicht berechnet

In Anlehnung an Cohen (1988) wird ein Effekt von 0.20 als klein, von 0.50 als mittel und von 0.80 als groß betrachtet. 


\section{Anhang A}

Tabelle A-5

Mittelwerte und Standardabweichungen (in Klammern) im Vor- und Nachtest für die AVn und Kennwerte $(F, p, M Q F)$ des Interaktionseffekts zweifaktorieller Varianzanalysen mit Messwiederholung getrennt für das Lauth- und Schlottke-Basistraining (10 Sitzungen) und LS-Basis- und Strategietraining (15 Sitzungen)

\begin{tabular}{|c|c|c|c|c|c|c|c|c|c|c|c|}
\hline \multirow[t]{2}{*}{$\begin{array}{c}\text { Abhängige } \\
\text { Variable }\end{array}$} & \multicolumn{3}{|c|}{$\begin{array}{l}\text { Basistraining nach } \\
\text { Lauth und Schlottke } \\
10 \text { Sitzungen }\end{array}$} & \multicolumn{3}{|c|}{$\begin{array}{c}\text { Basis- und } \\
\text { Strategietraining } \\
\text { Lauth und Schlottke } \\
15 \text { Sitzungen }\end{array}$} & \multicolumn{4}{|c|}{ Interaktionseffekt } & \multirow{2}{*}{$\begin{array}{c}\begin{array}{c}\text { Effekt } \\
\text {-größe }\end{array} \\
D\end{array}$} \\
\hline & $n$ & Vortest & Nachtest & $n$ & Vortest & Nachtest & $F$ & $p$ & $M Q F$ & $\begin{array}{c}\text { hypo- } \\
\text { thesen- } \\
\text { konform } \\
?\end{array}$ & \\
\hline \multicolumn{12}{|c|}{ TAP: Untertest „Geteilte Aufmerksamkeit" } \\
\hline $\begin{array}{l}\text { Standard- } \\
\text { abweichung }\end{array}$ & 14 & $\begin{array}{l}331.74 \\
(74.95)\end{array}$ & $\begin{array}{l}316.96 \\
(75.04)\end{array}$ & 29 & $\begin{array}{c}\mathbf{3 4 8 . 0 1} \\
(103.47)\end{array}$ & $\begin{array}{l}295.91 \\
(88.70)\end{array}$ & 1.02 & 0.31 & 6419.5 & nein & $(0,40)$ \\
\hline Anzahl Fehler & 14 & $\begin{array}{c}8.43 \\
(8.38)\end{array}$ & $\begin{array}{c}\mathbf{2 . 5 0} \\
(1.70)\end{array}$ & 29 & $\begin{array}{c}\mathbf{9 . 0 3} \\
(13.53) \\
\end{array}$ & $\begin{array}{c}2.97 \\
(3.46) \\
\end{array}$ & 0.00 & 0.97 & 67.18 & nein & $(0,00)$ \\
\hline $\begin{array}{l}\text { Anzahl } \\
\text { Auslassungen }\end{array}$ & 14 & $\begin{array}{c}6.79 \\
(3.93) \\
\end{array}$ & $\begin{array}{c}4.29 \\
(2.52) \\
\end{array}$ & 29 & $\begin{array}{c}8.21 \\
(6.28) \\
\end{array}$ & $\begin{array}{c}6.34 \\
(4.59) \\
\end{array}$ & 0.13 & 0.71 & 14.28 & nein & $(0,14)$ \\
\hline \multicolumn{12}{|c|}{ TAP: Untertest ,Go/Nogo“6 } \\
\hline $\begin{array}{l}\text { Standard- } \\
\text { abweichung }\end{array}$ & 14 & $\begin{array}{l}259.37 \\
291.49\end{array}$ & $\begin{array}{c}136.74 \\
24.59 \\
\end{array}$ & 31 & $\begin{array}{l}149.25 \\
(37.62) \\
\end{array}$ & $\begin{array}{l}133.18 \\
(44.62) \\
\end{array}$ & 4.06 & 0.05 & 13471 & nein & $(0,71)$ \\
\hline Anzahl Fehler & 14 & $\begin{array}{l}9.21 \\
4.85 \\
\end{array}$ & $\begin{array}{l}4.71 \\
3.97 \\
\end{array}$ & 31 & $\begin{array}{c}7.84 \\
(6.02) \\
\end{array}$ & $\begin{array}{c}4.68 \\
(5.18) \\
\end{array}$ & 0.39 & 0.53 & 21.71 & nein & $(0,24)$ \\
\hline $\begin{array}{l}\text { Anzahl } \\
\text { Auslassungen }\end{array}$ & 14 & $\begin{array}{l}4.93 \\
6.24\end{array}$ & $\begin{array}{l}\mathbf{1 . 5 0} \\
1,83\end{array}$ & 31 & $\begin{array}{c}1.87 \\
(2.13)\end{array}$ & $\begin{array}{c}3.77 \\
(4.36)\end{array}$ & 10.48 & 0.00 & 13.07 & nein & $(1,46)$ \\
\hline \multicolumn{12}{|c|}{ TAP: Untertest ,Inkompatibilität"6 } \\
\hline $\begin{array}{l}\text { Standard- } \\
\text { abweichung }\end{array}$ & 14 & $\begin{array}{c}274.24 \\
(273.95)\end{array}$ & $\begin{array}{l}189.13 \\
(41.07)\end{array}$ & 29 & $\begin{array}{c}227.93 \\
(163.70)\end{array}$ & $\begin{array}{l}187.49 \\
(80.30)\end{array}$ & - & - & - & - & - \\
\hline Anzahl Fehler & 14 & $\begin{array}{c}\mathbf{1 9 . 7 1} \\
(13.10) \\
\end{array}$ & $\begin{array}{c}8.93 \\
(8.65) \\
\end{array}$ & 29 & $\begin{array}{c}\mathbf{1 4 . 7 2} \\
(10.56) \\
\end{array}$ & $\begin{array}{c}8.52 \\
(6.76) \\
\end{array}$ & 1.57 & 0.21 & 62.72 & nein & $(0,43)$ \\
\hline \multicolumn{12}{|c|}{ TAP: Untertest „Visuelles Scanning } \\
\hline $\begin{array}{l}\text { Standard- } \\
\text { abweichung } \\
\text { kritische Trials }\end{array}$ & 14 & $\begin{array}{c}\mathbf{2 8 3 3 . 1 1} \\
(1265.76)\end{array}$ & $\begin{array}{c}\mathbf{1 9 9 9 . 7 1} \\
(1174.35)\end{array}$ & 23 & $\begin{array}{c}3577.49 \\
(1875.05)\end{array}$ & $\begin{array}{c}2887.19 \\
(1434.07)\end{array}$ & 0.05 & 0.81 & 1528355 & nein & $(0,08)$ \\
\hline $\begin{array}{l}\text { Standard- } \\
\text { abweichung } \\
\text { nicht-kritische } \\
\text { Trials }\end{array}$ & 14 & $\begin{array}{c}3332.51 \\
(1761.55)\end{array}$ & $\begin{array}{c}2637.14 \\
(1509.64)\end{array}$ & 23 & $\begin{array}{c}\mathbf{3 3 3 7 . 7 4} \\
(1680.22)\end{array}$ & $\begin{array}{l}\mathbf{2 5 1 0 . 1 5} \\
(905.79)\end{array}$ & 0.04 & 0.83 & 1786843 & nein & $(0,08)$ \\
\hline $\begin{array}{l}\text { Anzahl Fehler } \\
\text { (kritische } \\
\text { Trials) } \\
\end{array}$ & 14 & $\begin{array}{c}1.21 \\
(1.12)\end{array}$ & $\begin{array}{l}1.00 \\
(2.15)\end{array}$ & 23 & $\begin{array}{c}7.78 \\
(4.59)\end{array}$ & $\begin{array}{l}3.35 \\
(2.84)\end{array}$ & 11.07 & 0.00 & 7.00 & ja & 0,96 \\
\hline $\begin{array}{l}\text { Anzahl } \\
\text { Auslassungen } \\
\text { (nicht-kritische } \\
\text { Trials) }\end{array}$ & 14 & $\begin{array}{c}5.14 \\
(3.21)\end{array}$ & $\begin{array}{c}5.36 \\
(3.95)\end{array}$ & 23 & $\begin{array}{l}1.22 \\
(2.17)\end{array}$ & $\begin{array}{c}0.52 \\
(0.73)\end{array}$ & - & - & - & - & - \\
\hline \multicolumn{12}{|l|}{ Test d2 } \\
\hline $\begin{array}{l}\text { Konzentrations- } \\
\text { leistungswert }\end{array}$ & 14 & $\begin{array}{c}\mathbf{7 2 . 2 1} \\
(27.26) \\
\end{array}$ & $\begin{array}{l}96.50 \\
(19.07) \\
\end{array}$ & 21 & $\begin{array}{c}\mathbf{9 5 . 2 4} \\
(21.93)\end{array}$ & $\begin{array}{l}111.52 \\
(20.07)\end{array}$ & 3.40 & 0.07 & 79.04 & nein & $(0,27)$ \\
\hline Anzahl Fehler & 14 & $\begin{array}{c}\mathbf{3 4 . 0 7} \\
(41.29) \\
\end{array}$ & $\begin{array}{l}\mathbf{1 1 . 0 7} \\
(9.93) \\
\end{array}$ & 21 & $\begin{array}{l}11.19 \\
(8.69) \\
\end{array}$ & $\begin{array}{c}8.52 \\
(4.98) \\
\end{array}$ & 7.45 & 0.01 & 241.16 & nein & $(0,94)$ \\
\hline
\end{tabular}




\begin{tabular}{|c|c|c|c|c|c|c|c|c|c|c|c|}
\hline \multirow[t]{2}{*}{$\begin{array}{l}\text { Abhängige } \\
\text { Variable }\end{array}$} & \multicolumn{3}{|c|}{$\begin{array}{l}\text { Basistraining nach } \\
\text { Lauth und Schlottke } \\
10 \text { Sitzungen }\end{array}$} & \multicolumn{3}{|c|}{$\begin{array}{c}\text { Basis- und } \\
\text { Strategietraining } \\
\text { Lauth und Schlottke } \\
15 \text { Sitzungen }\end{array}$} & \multicolumn{4}{|c|}{ Interaktionseffekt } & \multirow{2}{*}{$\begin{array}{c}\begin{array}{c}\text { Effekt } \\
\text {-größe }\end{array} \\
D\end{array}$} \\
\hline & $n$ & Vortest & Nachtest & $n$ & Vortest & Nachtest & $F$ & $p$ & $M Q F$ & \begin{tabular}{|c|} 
hypo- \\
thesen- \\
konform \\
$?$
\end{tabular} & \\
\hline \multicolumn{12}{|l|}{ Mottier-Test } \\
\hline $\begin{array}{l}\begin{array}{l}\text { Nachsprechleist } \\
\text { ung Gesamt } \\
\text { (max. 30) }\end{array} \\
\end{array}$ & 14 & $\begin{array}{l}19.07 \\
(4.01)\end{array}$ & $\begin{array}{l}22.43 \\
(3.11)\end{array}$ & 32 & $\begin{array}{l}\mathbf{2 1 . 2 2} \\
(4.96)\end{array}$ & $\begin{array}{l}\mathbf{2 3 . 8 1} \\
(3.89)\end{array}$ & 0.59 & 0.44 & 4.73 & nein & $(0,17)$ \\
\hline $\begin{array}{l}\text { Nachsprechleist } \\
\text { ung für 2- und } \\
\text { 3-silbige } \\
\text { Kunstwörter } \\
\text { (max. 12) }\end{array}$ & 14 & $\begin{array}{l}11.00 \\
(1.24)\end{array}$ & $\begin{array}{l}11.29 \\
(1.14)\end{array}$ & 32 & $\begin{array}{l}\mathbf{1 1 . 2 8} \\
(1.02)\end{array}$ & $\begin{array}{c}\mathbf{1 1 . 6 3} \\
.79)\end{array}$ & 0.01 & 0.89 & 0.91 & nein & $(0,04)$ \\
\hline $\begin{array}{l}\text { Nachsprechleist } \\
\text { ung für 4-, 5- } \\
\text { und 6-silbige } \\
\text { Kunstwörter } \\
\text { (max. 18) }\end{array}$ & 14 & $\begin{array}{c}8.07 \\
(3.41)\end{array}$ & $\begin{array}{l}\mathbf{1 1 . 1 4} \\
(3.13)\end{array}$ & 32 & $\begin{array}{c}\mathbf{9 . 9 4} \\
(4.44)\end{array}$ & $\begin{array}{l}\mathbf{1 2 . 1 9} \\
(3.41)\end{array}$ & 1.17 & 0.28 & 2.80 & nein & $(0,10)$ \\
\hline \multicolumn{12}{|c|}{ Fremdbeurteilungsbogen FBB-HKS (Eltern) } \\
\hline $\begin{array}{l}\text { Unaufmerksam- } \\
\text { keit: Anzahl an } \\
\text { Kriterien } \\
\end{array}$ & 13 & $\begin{array}{c}\mathbf{4 . 6 9} \\
(1.75)\end{array}$ & $\begin{array}{c}3.23 \\
(2.31)\end{array}$ & 28 & $\begin{array}{c}5.78 \\
(2.48)\end{array}$ & $\begin{array}{c}5.57 \\
(3.07)\end{array}$ & 1.23 & 0.27 & 5.61 & nein & $(0,45)$ \\
\hline $\begin{array}{l}\text { Unaufmerksam- } \\
\text { keit: Kennwert }\end{array}$ & 13 & $\begin{array}{l}\mathbf{1 . 5 4} \\
(.37)\end{array}$ & $\begin{array}{l}1.18 \\
(.43)\end{array}$ & 28 & $\begin{array}{c}1.78 \\
(0.50)\end{array}$ & $\begin{array}{c}1.69 \\
(0.60)\end{array}$ & 1.52 & 0.22 & 0.21 & nein & $(0,50)$ \\
\hline $\begin{array}{l}\text { Hyperaktivität: } \\
\text { Anzahl an } \\
\text { Kriterien }\end{array}$ & 13 & $\begin{array}{c}\mathbf{1 . 7 6} \\
(1.48)\end{array}$ & $\begin{array}{l}\mathbf{0 . 4 6} \\
(.87)\end{array}$ & 28 & $\begin{array}{c}1.32 \\
(1.49)\end{array}$ & $\begin{array}{c}1.07 \\
(1.56)\end{array}$ & 4.96 & 0.06 & 1.35 & nein & $(0,89)$ \\
\hline $\begin{array}{l}\text { Hyperaktivität: } \\
\text { Kennwert }\end{array}$ & 13 & $\begin{array}{c}\mathbf{1 . 2 9} \\
(0.71)\end{array}$ & $\begin{array}{c}\mathbf{0 . 6 6} \\
(0.45)\end{array}$ & 28 & $\begin{array}{c}1.01 \\
(0.61)\end{array}$ & $\begin{array}{c}0.82 \\
(0.79)\end{array}$ & 3.54 & 0.06 & 0.23 & nein & $(0,71)$ \\
\hline $\begin{array}{l}\text { Impulsivität: } \\
\text { Anzahl an } \\
\text { Kriterien }\end{array}$ & 13 & $\begin{array}{c}\mathbf{1 . 3 0} \\
(1.70)\end{array}$ & $\begin{array}{c}\mathbf{0 . 0 7} \\
(0.27)\end{array}$ & 28 & $\begin{array}{c}1.03 \\
(1.45)\end{array}$ & $\begin{array}{c}1.28 \\
(1.56)\end{array}$ & 8.11 & 0.00 & 1.19 & nein & $(1,18)$ \\
\hline $\begin{array}{l}\text { Impulsivität: } \\
\text { Kennwert }\end{array}$ & 13 & $\begin{array}{c}1.12 \\
(0.79) \\
\end{array}$ & $\begin{array}{c}\mathbf{0 . 5 9} \\
(0.37) \\
\end{array}$ & 28 & $\begin{array}{c}0.82 \\
(0.75) \\
\end{array}$ & $\begin{array}{c}1.09 \\
(0.87)\end{array}$ & 8.84 & 0.00 & 0.31 & nein & $(1,25)$ \\
\hline \multicolumn{12}{|c|}{ Child Behavior Checklist (CBCL) } \\
\hline $\begin{array}{l}\text { T-Wert } \\
\text { Aufmerksam- } \\
\text { keitsstörungen }\end{array}$ & 12 & $\begin{array}{l}\mathbf{6 8 . 3 3} \\
(8.74)\end{array}$ & $\begin{array}{l}\mathbf{6 3 . 5 8} \\
(10.95)\end{array}$ & 30 & $\begin{array}{l}\mathbf{6 5 . 4 7} \\
(8.38)\end{array}$ & $\begin{array}{l}\mathbf{6 2 . 1 7} \\
(7.28)\end{array}$ & 0.30 & 0.58 & 29.38 & nein & $(0,17)$ \\
\hline $\begin{array}{l}\text { T-Wert } \\
\text { Externalisieren- } \\
\text { de Störungen } \\
\end{array}$ & 12 & $\begin{array}{l}\mathbf{6 3 . 1 7} \\
(9.23)\end{array}$ & $\begin{array}{l}\mathbf{5 8 . 6 7} \\
(8.07)\end{array}$ & 30 & $\begin{array}{c}\mathbf{5 5 . 0 0} \\
(11.90)\end{array}$ & $\begin{array}{c}\mathbf{5 1 . 7 7} \\
(11.04)\end{array}$ & 0.26 & 0.61 & 26.43 & nein & $(0,13)$ \\
\hline $\begin{array}{l}\text { T-Wert } \\
\text { Gesamtskala }\end{array}$ & 12 & $\begin{array}{l}\mathbf{6 4 . 1 7} \\
(7.07)\end{array}$ & $\begin{array}{l}\mathbf{5 9 . 2 5} \\
(7.33)\end{array}$ & 30 & $\begin{array}{l}\mathbf{5 9 . 3 0} \\
(8.55)\end{array}$ & $\begin{array}{l}\mathbf{5 5 . 7 0} \\
(9.06)\end{array}$ & 0.42 & 0.52 & 17.60 & nein & $(0,14)$ \\
\hline \multicolumn{12}{|c|}{ Fremdbeurteilungsbogen FBB-HKS (Lehrer) } \\
\hline $\begin{array}{l}\text { Unaufmerksam- } \\
\text { keit: Anzahl an } \\
\text { Kriterien }\end{array}$ & 9 & $\begin{array}{c}3.33 \\
(2.17)\end{array}$ & $\begin{array}{c}3.00 \\
(2.44)\end{array}$ & 26 & $\begin{array}{c}\mathbf{6 . 0 3} \\
(2.44)\end{array}$ & $\begin{array}{c}\mathbf{4 . 0 3} \\
(2.58)\end{array}$ & 1.58 & 0.21 & 5.84 & nein & $(0,73)$ \\
\hline $\begin{array}{l}\text { Unaufmerksam- } \\
\text { keit: Kennwert }\end{array}$ & 9 & $\begin{array}{c}1.28 \\
(0.44)\end{array}$ & $\begin{array}{c}1.13 \\
(0.52)\end{array}$ & 26 & $\begin{array}{c}\mathbf{1 . 8 3} \\
(0.55)\end{array}$ & $\begin{array}{c}\mathbf{1 . 4 3} \\
(0.61)\end{array}$ & 0.64 & 0.42 & 0.29 & nein & $(0,44)$ \\
\hline $\begin{array}{l}\text { Hyperaktivität: } \\
\text { Anzahl an } \\
\text { Kriterien }\end{array}$ & 9 & $\begin{array}{c}\mathbf{1 . 7 7} \\
(1.56)\end{array}$ & $\begin{array}{l}\mathbf{0 . 6 6} \\
(.70)\end{array}$ & 26 & $\begin{array}{c}\mathbf{1 . 5 0} \\
(1.47)\end{array}$ & $\begin{array}{c}\mathbf{0 . 6 9} \\
(1.15)\end{array}$ & 0.16 & 0.68 & 1.89 & nein & $(0,22)$ \\
\hline $\begin{array}{l}\text { Hyperaktivität: } \\
\text { Kennwert }\end{array}$ & 9 & $\begin{array}{c}1.14 \\
(0.56)\end{array}$ & $\begin{array}{c}0.77 \\
(0.52)\end{array}$ & 26 & $\begin{array}{c}\mathbf{0 . 9 7} \\
(0.71)\end{array}$ & $\begin{array}{c}\mathbf{0 . 5 4} \\
(0.60)\end{array}$ & 0.04 & 0.84 & 0.39 & nein & $(0,11)$ \\
\hline
\end{tabular}




\begin{tabular}{|c|c|c|c|c|c|c|c|c|c|c|c|}
\hline \multirow[t]{2}{*}{$\begin{array}{c}\text { Abhängige } \\
\text { Variable }\end{array}$} & \multicolumn{3}{|c|}{$\begin{array}{l}\text { Basistraining nach } \\
\text { Lauth und Schlottke } \\
10 \text { Sitzungen }\end{array}$} & \multicolumn{3}{|c|}{$\begin{array}{c}\text { Basis- und } \\
\text { Strategietraining } \\
\text { Lauth und Schlottke } \\
15 \text { Sitzungen }\end{array}$} & \multicolumn{4}{|c|}{ Interaktionseffekt } & \multirow{2}{*}{$\begin{array}{c}\text { Effekt } \\
\text {-größe }\end{array}$} \\
\hline & $n$ & Vortest & Nachtest & $n$ & Vortest & Nachtest & $F$ & $p$ & $M Q F$ & $\begin{array}{l}\text { hypo- } \\
\text { thesen- } \\
\text { konform } \\
\text { ? }\end{array}$ & \\
\hline $\begin{array}{l}\text { Impulsivität: } \\
\text { Anzahl an } \\
\text { Kriterien }\end{array}$ & 9 & $\begin{array}{c}1.00 \\
(1.58)\end{array}$ & $\begin{array}{c}0.66 \\
(1.00)\end{array}$ & 26 & $\begin{array}{c}0.80 \\
(1.29)\end{array}$ & $\begin{array}{c}0.69 \\
(1.37)\end{array}$ & - & - & - & - & - \\
\hline $\begin{array}{l}\text { Impulsivität: } \\
\text { Kennwert }\end{array}$ & 9 & $\begin{array}{c}1.06 \\
(0.70) \\
\end{array}$ & $\begin{array}{c}0.86 \\
(0.51) \\
\end{array}$ & 26 & $\begin{array}{c}0.72 \\
(0.67) \\
\end{array}$ & $\begin{array}{c}0.65 \\
(0.85) \\
\end{array}$ & - & - & - & - & - \\
\hline \multicolumn{12}{|c|}{ Teacher's Report Form (TRF) } \\
\hline $\begin{array}{l}\text { T-Wert } \\
\text { Aufmerksam- } \\
\text { keitsstörungen } \\
\end{array}$ & 11 & $\begin{array}{l}59.36 \\
(5.30)\end{array}$ & $\begin{array}{l}58.00 \\
(5.46)\end{array}$ & 25 & $\begin{array}{l}\mathbf{5 9 . 2 1} \\
(5.49)\end{array}$ & $\begin{array}{l}\mathbf{5 7 . 0 8} \\
(4.32)\end{array}$ & 0.18 & 0.67 & 11.98 & nein & $(0,14)$ \\
\hline $\begin{array}{l}\text { T-Wert } \\
\text { externalisieren- } \\
\text { de Störungen } \\
\end{array}$ & 11 & $\begin{array}{l}59.09 \\
(8.94)\end{array}$ & $\begin{array}{l}59.64 \\
(8.56)\end{array}$ & 25 & $\begin{array}{c}55.04 \\
(10.67)\end{array}$ & $\begin{array}{l}53.42 \\
(9.60)\end{array}$ & - & - & - & - & - \\
\hline $\begin{array}{l}\text { T-Wert } \\
\text { Gesamtskala }\end{array}$ & 11 & $\begin{array}{l}58.73 \\
(5.52)\end{array}$ & $\begin{array}{l}59.09 \\
(6.19)\end{array}$ & 25 & $\begin{array}{l}\mathbf{5 8 . 7 6} \\
(6.80)\end{array}$ & $\begin{array}{l}\mathbf{5 5 . 9 2} \\
(5.77)\end{array}$ & 2.63 & 0.11 & 14.85 & nein & $(0,43)$ \\
\hline
\end{tabular}

Anmerkung: p: einseitige Irrtumswahrscheinlichkeit des F-Wertes; Signifikanzniveau $\alpha=0.05$; MQF: Mittleres Fehlerquadrat

n: Anzahl der Messwertpaare (Die Anzahl umfasst nur diejenigen Kinder, von denen sowohl Vor- als auch Nachtestwert der jeweiligen Variablen vorhanden waren. Auf dieser Anzahl basieren die inferenzstatistischen Auswertungen).

TAP: Testbatterie zur Aufmerksamkeitsprüfung

In Anlehnung an Cohen (1988) wird ein Effekt von 0.20 als klein, von 0.50 als mittel und von 0.80 als groß betrachtet. 


\section{Anhang A}

Tabelle A-6

Mittelwerte und Standardabweichungen (in Klammern) im Vor- und Nachtest für die AVn und Kennwerte $(F, p, M Q F)$ des Interaktionseffekts zweifaktorieller Varianzanalysen mit Messwiederholung getrennt für das kombinierte Basis- und Stratgietraining nach Lauth und Schlottke und die Gruppe THOP als Gruppentraining

\begin{tabular}{|c|c|c|c|c|c|c|c|c|c|c|c|}
\hline \multirow[t]{2}{*}{$\begin{array}{c}\text { Abhängige } \\
\text { Variable }\end{array}$} & \multicolumn{3}{|c|}{$\begin{array}{c}\text { Basis- und } \\
\text { Strategietraining } \\
\text { Lauth und Schlottke } \\
15 \text { Sitzungen }\end{array}$} & \multicolumn{3}{|c|}{ Trainingsgruppe THOP } & \multicolumn{4}{|c|}{ Interaktionseffekt } & \multirow{2}{*}{$\begin{array}{c}\text { Effekt } \\
\text {-größe }\end{array}$} \\
\hline & $n$ & Vortest & Nachtest & $n$ & Vortest & Nachtest & $F$ & $p$ & $M Q F$ & $\begin{array}{l}\text { Hypo- } \\
\text { thesen- } \\
\text { konform } \\
?\end{array}$ & \\
\hline \multicolumn{12}{|c|}{ TAP: Untertest, ,Geteilte Aufmerksamkeit"“ } \\
\hline $\begin{array}{l}\text { Standard- } \\
\text { abweichung }\end{array}$ & 29 & $\begin{array}{c}\mathbf{3 4 8 . 0 1} \\
(103.47)\end{array}$ & $\begin{array}{r}295.91 \\
(88.70)\end{array}$ & 29 & $\begin{array}{c}\mathbf{3 3 7 . 8 2} \\
(116.48)\end{array}$ & $\begin{array}{r}\mathbf{2 9 5 . 8 6} \\
(95.81)\end{array}$ & 0.10 & 0.75 & 7350.9 & nein & $(0,10)$ \\
\hline Anzahl Fehler & 29 & $\begin{array}{c}\mathbf{9 . 0 3} \\
(13.53) \\
\end{array}$ & $\begin{array}{c}2.97 \\
(3.46) \\
\end{array}$ & 29 & $\begin{array}{c}\mathbf{9 . 3 8} \\
(13.44) \\
\end{array}$ & $\begin{array}{c}3.07 \\
(4.03) \\
\end{array}$ & 0.00 & 0.94 & 85.48 & nein & $(0,00)$ \\
\hline $\begin{array}{l}\text { Anzahl } \\
\text { Auslassungen }\end{array}$ & 29 & $\begin{array}{c}8.21 \\
(6.28)\end{array}$ & $\begin{array}{c}6.34 \\
(4.59)\end{array}$ & 29 & $\begin{array}{c}7.10 \\
(5.02)\end{array}$ & $\begin{array}{c}5.28 \\
(4.71)\end{array}$ & - & - & - & - & - \\
\hline \multicolumn{12}{|c|}{ TAP: Untertest ,Go/Nogo“6 } \\
\hline $\begin{array}{l}\text { Standard- } \\
\text { abweichung }\end{array}$ & 31 & $\begin{array}{l}\mathbf{1 4 9 . 2 5} \\
(37.62)\end{array}$ & $\begin{array}{l}\mathbf{1 3 3 . 1 8} \\
(44.62)\end{array}$ & 31 & $\begin{array}{l}140.54 \\
(42.28)\end{array}$ & $\begin{array}{l}125.59 \\
(42.09)\end{array}$ & 0.00 & 0.92 & 1102.3 & nein & $(0,00)$ \\
\hline Anzahl Fehler & 31 & $\begin{array}{c}7.84 \\
(6.02)\end{array}$ & $\begin{array}{c}4.68 \\
(5.18)\end{array}$ & 31 & $\begin{array}{c}9.81 \\
(5.50)\end{array}$ & $\begin{array}{c}6.97 \\
(5.33)\end{array}$ & 0.03 & 0.84 & 21.50 & nein & $(0,05)$ \\
\hline $\begin{array}{l}\text { Anzahl } \\
\text { Auslassungen }\end{array}$ & 31 & $\begin{array}{c}1.87 \\
(2.13)\end{array}$ & $\begin{array}{c}3.77 \\
(4.36)\end{array}$ & 31 & $\begin{array}{c}\mathbf{2 . 1 0} \\
(2.70)\end{array}$ & $\begin{array}{c}\mathbf{0 . 8 4} \\
(1.44)\end{array}$ & 9.32 & 0.00 & 8.30 & ja & 1,07 \\
\hline \multicolumn{12}{|c|}{ TAP: Untertest ,Inkompatibilität““ } \\
\hline $\begin{array}{l}\text { Standard- } \\
\text { abweichung }\end{array}$ & 29 & $\begin{array}{c}227.93 \\
(163.70)\end{array}$ & $\begin{array}{l}187.49 \\
(80.30)\end{array}$ & 31 & $\begin{array}{c}\mathbf{2 4 2 . 4 6} \\
(123.11)\end{array}$ & $\begin{array}{l}\mathbf{1 7 6 . 6 3} \\
(64.19)\end{array}$ & 0.49 & 0.48 & 9699.4 & nein & $(0,21)$ \\
\hline Anzahl Fehler & 29 & $\begin{array}{c}\mathbf{1 4 . 7 2} \\
(10.56) \\
\end{array}$ & $\begin{array}{c}8.52 \\
(6.76) \\
\end{array}$ & 31 & $\begin{array}{r}\mathbf{2 0 . 4 2} \\
(12.05) \\
\end{array}$ & $\begin{array}{l}\mathbf{1 3 . 5 5} \\
(10.00) \\
\end{array}$ & 0.05 & 0.81 & 61.86 & nein & $(0,06)$ \\
\hline \multicolumn{12}{|c|}{ TAP: Untertest ,Visuelles Scanning“ } \\
\hline $\begin{array}{l}\text { Standard- } \\
\text { abweichung } \\
\text { kritische Trials }\end{array}$ & 23 & $\begin{array}{c}3577.49 \\
(1875.05)\end{array}$ & $\begin{array}{c}2887.19 \\
(1434.07)\end{array}$ & 31 & $\begin{array}{c}\mathbf{2 4 7 2 . 9 8} \\
(1048.18)\end{array}$ & $\begin{array}{l}\mathbf{2 0 4 9 . 0 0} \\
(995.37)\end{array}$ & 0.40 & 0.52 & 1167609 & nein & $(0,20)$ \\
\hline $\begin{array}{l}\text { Standard- } \\
\text { abweichung } \\
\text { nicht-kritische } \\
\text { Trials }\end{array}$ & 23 & $\begin{array}{c}\mathbf{3 3 3 7 . 7 4} \\
(1680.22)\end{array}$ & $\begin{array}{l}\mathbf{2 5 1 0 . 1 5} \\
(905.79)\end{array}$ & 31 & $\begin{array}{c}\mathbf{2 7 6 7 . 1 8} \\
(1185.42)\end{array}$ & $\begin{array}{c}\mathbf{2 1 9 2 . 0 3} \\
(1028.39)\end{array}$ & 0.42 & 0.52 & 1002710 & nein & $(0,20)$ \\
\hline $\begin{array}{l}\text { Anzahl Fehler } \\
\text { (kritische } \\
\text { Trials) } \\
\end{array}$ & 23 & $\begin{array}{c}7.78 \\
(4.59)\end{array}$ & $\begin{array}{l}3.35 \\
(2.84)\end{array}$ & 31 & $\begin{array}{c}7.68 \\
(5.95)\end{array}$ & $\begin{array}{c}4.32 \\
(4.10)\end{array}$ & 0.72 & 0.39 & 10.58 & nein & $(0,24)$ \\
\hline $\begin{array}{l}\text { Anzahl } \\
\text { Auslassungen } \\
\text { (nicht-kritische } \\
\text { Trials) }\end{array}$ & 23 & $\begin{array}{c}1.22 \\
(2.17)\end{array}$ & $\begin{array}{c}0.52 \\
(0.73)\end{array}$ & 31 & $\begin{array}{c}\mathbf{5 . 3 2} \\
(5.96)\end{array}$ & $\begin{array}{c}2.26 \\
(3.26)\end{array}$ & 4.17 & 0.04 & 8.87 & ja & 0,61 \\
\hline \multicolumn{12}{|l|}{ Test d2 } \\
\hline $\begin{array}{l}\text { Konzentrations- } \\
\text { leistungswert }\end{array}$ & 21 & $\begin{array}{c}\mathbf{9 5 . 2 4} \\
(21.93)\end{array}$ & $\begin{array}{l}111.52 \\
(20.07)\end{array}$ & 15 & $\begin{array}{c}\mathbf{9 3 . 9 3} \\
(25.92)\end{array}$ & $\begin{array}{l}\mathbf{1 2 3 . 2 0} \\
(25.27)\end{array}$ & 14.16 & 0.00 & 52.04 & ja & 0,55 \\
\hline Anzahl Fehler & 21 & $\begin{array}{l}11.19 \\
(8.69)\end{array}$ & $\begin{array}{c}8.52 \\
(4.98)\end{array}$ & 15 & $\begin{array}{c}\mathbf{2 6 . 2 7} \\
(37.59)\end{array}$ & $\begin{array}{c}9.27 \\
(6.30)\end{array}$ & 3.74 & 0.06 & 250.13 & nein & $(0,59)$ \\
\hline
\end{tabular}




\begin{tabular}{|c|c|c|c|c|c|c|c|c|c|c|c|}
\hline \multirow[t]{2}{*}{$\begin{array}{l}\text { Abhängige } \\
\text { Variable }\end{array}$} & \multicolumn{3}{|c|}{$\begin{array}{c}\text { Basis- und } \\
\text { Strategietraining } \\
\text { Lauth und Schlottke } \\
15 \text { Sitzungen }\end{array}$} & \multicolumn{3}{|c|}{ Trainingsgruppe THOP } & \multicolumn{4}{|c|}{ Interaktionseffekt } & \multirow{2}{*}{$\begin{array}{c}\text { Effekt } \\
\text {-größe } \\
D\end{array}$} \\
\hline & $n$ & Vortest & Nachtest & $n$ & Vortest & Nachtest & $F$ & $p$ & $M Q F$ & 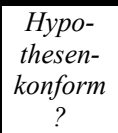 & \\
\hline \multicolumn{12}{|l|}{ Mottier-Test } \\
\hline $\begin{array}{l}\text { Nachsprechleist } \\
\text { ung Gesamt } \\
\text { (max. 30) }\end{array}$ & 32 & $\begin{array}{l}\mathbf{2 1 . 2 2} \\
(4.96)\end{array}$ & $\begin{array}{l}\mathbf{2 3 . 8 1} \\
(3.89)\end{array}$ & 30 & $\begin{array}{l}\mathbf{2 2 . 2 0} \\
(4.00)\end{array}$ & $\begin{array}{l}\mathbf{2 3 . 5 7} \\
(3.79)\end{array}$ & 2.41 & 0.12 & 4.85 & nein & $(0,29)$ \\
\hline $\begin{array}{l}\text { Nachsprechleist } \\
\text { ung für 2- und } \\
\text { 3-silbige } \\
\text { Kunstwörter } \\
\text { (max. 12) }\end{array}$ & 32 & $\begin{array}{l}\mathbf{1 1 . 2 8} \\
(1.02)\end{array}$ & $\begin{array}{l}\mathbf{1 1 . 6 3} \\
(0.79)\end{array}$ & 30 & $\begin{array}{l}\mathbf{1 1 . 1 7} \\
(1.02)\end{array}$ & $\begin{array}{c}\mathbf{1 1 . 7 0} \\
.60)\end{array}$ & 0.43 & 0.51 & 0.63 & nein & $(0,22)$ \\
\hline $\begin{array}{l}\text { Nachsprechleist } \\
\text { ung für 4-, 5- } \\
\text { und 6-silbige } \\
\text { Kunstwörter } \\
\text { (max. 18) }\end{array}$ & 32 & $\begin{array}{c}\mathbf{9 . 9 4} \\
(4.44)\end{array}$ & $\begin{array}{l}\mathbf{1 2 . 1 9} \\
(3.41)\end{array}$ & 30 & $\begin{array}{l}\mathbf{1 1 . 0 3} \\
(3.40)\end{array}$ & $\begin{array}{l}\mathbf{1 1 . 8 7} \\
(3.46)\end{array}$ & 4.90 & 0.03 & 3.16 & ja & 0,37 \\
\hline \multicolumn{12}{|c|}{ Fremdbeurteilungsbogen FBB-HKS (Eltern) } \\
\hline $\begin{array}{l}\text { Unaufmerksam- } \\
\text { keit: Anzahl an } \\
\text { Kriterien }\end{array}$ & 28 & $\begin{array}{c}5.78 \\
(2.48)\end{array}$ & $\begin{array}{c}5.57 \\
(3.07)\end{array}$ & 30 & $\begin{array}{c}\mathbf{5 . 9 3} \\
(2.58)\end{array}$ & $\begin{array}{c}\mathbf{4 . 5 0} \\
(2.56)\end{array}$ & 1.97 & 0.16 & 5.44 & nein & $(0,45)$ \\
\hline $\begin{array}{l}\text { Unaufmerksam- } \\
\text { keit: Kennwert }\end{array}$ & 28 & $\begin{array}{c}1.78 \\
(0.50)\end{array}$ & $\begin{array}{c}1.69 \\
(0.60)\end{array}$ & 30 & $\begin{array}{c}\mathbf{1 . 8 5} \\
(0.58) \\
\end{array}$ & $\begin{array}{c}\mathbf{1 . 4 8} \\
(0.57) \\
\end{array}$ & 2.62 & 0.11 & 0.21 & nein & $(0,49)$ \\
\hline $\begin{array}{l}\text { Hyperaktivität: } \\
\text { Anzahl an } \\
\text { Kriterien } \\
\end{array}$ & 28 & $\begin{array}{c}1.32 \\
(1.49)\end{array}$ & $\begin{array}{l}1.07 \\
(1.56)\end{array}$ & 30 & $\begin{array}{c}\mathbf{2 . 4 3} \\
(1.79)\end{array}$ & $\begin{array}{c}\mathbf{1 . 7 0} \\
(1.58)\end{array}$ & 1.20 & 0.27 & 1.40 & nein & $(0,30)$ \\
\hline $\begin{array}{l}\text { Hyperaktivität: } \\
\text { Kennwert }\end{array}$ & 28 & $\begin{array}{c}1.01 \\
(0.61)\end{array}$ & $\begin{array}{c}0.82 \\
(0.79)\end{array}$ & 30 & $\begin{array}{l}\mathbf{1 . 4 8} \\
(0.85)\end{array}$ & $\begin{array}{c}1.14 \\
(0.66)\end{array}$ & 0.54 & 0.46 & 0.26 & nein & $(0,19)$ \\
\hline $\begin{array}{l}\text { Impulsivität: } \\
\text { Anzahl an } \\
\text { Kriterien }\end{array}$ & 28 & $\begin{array}{c}1.03 \\
(1.45)\end{array}$ & $\begin{array}{c}1.28 \\
(1.56)\end{array}$ & 30 & $\begin{array}{c}\mathbf{2 . 4 0} \\
(1.61)\end{array}$ & $\begin{array}{c}\mathbf{2 . 0 0} \\
(1.43)\end{array}$ & 3.34 & 0.07 & 0.91 & nein & $(0,42)$ \\
\hline $\begin{array}{l}\text { Impulsivität: } \\
\text { Kennwert }\end{array}$ & 28 & $\begin{array}{c}0.82 \\
(0.75)\end{array}$ & $\begin{array}{c}1.09 \\
(0.87)\end{array}$ & 30 & $\begin{array}{l}1.61 \\
(0.93)\end{array}$ & $\begin{array}{c}1.50 \\
(0.81)\end{array}$ & - & - & - & - & - \\
\hline \multicolumn{12}{|c|}{ Child Behavior Checklist (CBCL) } \\
\hline $\begin{array}{l}\text { T-Wert } \\
\text { Aufmerksam- } \\
\text { keitsstörungen }\end{array}$ & 30 & $\begin{array}{l}\mathbf{6 5 . 4 7} \\
(8.38)\end{array}$ & $\begin{array}{c}\mathbf{6 2 . 1 7} \\
(7.28)\end{array}$ & 30 & $\begin{array}{l}\mathbf{6 6 . 2 7} \\
(6.70)\end{array}$ & $\begin{array}{l}\mathbf{6 0 . 5 7} \\
(6.67)\end{array}$ & 1.47 & 0.22 & 29.22 & nein & $(0,33)$ \\
\hline $\begin{array}{l}\text { T-Wert } \\
\text { Externalisieren- } \\
\text { de Störungen }\end{array}$ & 30 & $\begin{array}{c}\mathbf{5 5 . 0 0} \\
(11.90)\end{array}$ & $\begin{array}{c}\mathbf{5 1 . 7 7} \\
(11.04)\end{array}$ & 30 & $\begin{array}{c}\mathbf{6 5 . 4 7} \\
(11.47)\end{array}$ & $\begin{array}{l}\mathbf{5 9 . 9 0} \\
(9.95)\end{array}$ & 1.32 & 0.25 & 30.76 & nein & $(0,20)$ \\
\hline $\begin{array}{l}\text { T-Wert } \\
\text { Gesamtskala }\end{array}$ & 30 & $\begin{array}{l}\mathbf{5 9 . 3 0} \\
(8.55)\end{array}$ & $\begin{array}{l}\mathbf{5 5 . 7 0} \\
(9.06)\end{array}$ & 30 & $\begin{array}{l}\mathbf{6 5 . 9 3} \\
(7.93)\end{array}$ & $\begin{array}{l}\mathbf{5 9 . 9 0} \\
(8.24)\end{array}$ & 1.79 & 0.18 & 24.81 & nein & $(0,29)$ \\
\hline \multicolumn{12}{|c|}{ Fremdbeurteilungsbogen FBB-HKS (Lehrer) } \\
\hline $\begin{array}{l}\text { Unaufmerksam- } \\
\text { keit: Anzahl an } \\
\text { Kriterien }\end{array}$ & 26 & $\begin{array}{c}\mathbf{6 . 0 3} \\
(2.44)\end{array}$ & $\begin{array}{c}\mathbf{4 . 0 3} \\
(2.58)\end{array}$ & 23 & $\begin{array}{c}\mathbf{4 . 8 6} \\
(2.51)\end{array}$ & $\begin{array}{c}3.91 \\
(2.29)\end{array}$ & 1.36 & 0.24 & 4.86 & nein & $(0,41)$ \\
\hline $\begin{array}{l}\text { Unaufmerksam- } \\
\text { keit: Kennwert }\end{array}$ & 26 & $\begin{array}{c}\mathbf{1 . 8 3} \\
(0.55) \\
\end{array}$ & $\begin{array}{c}\mathbf{1 . 4 3} \\
(0.61) \\
\end{array}$ & 23 & $\begin{array}{c}1.71 \\
(0.63) \\
\end{array}$ & $\begin{array}{c}\mathbf{1 . 4 4} \\
(0.48) \\
\end{array}$ & 0.39 & 0.53 & 0.25 & nein & $(0,22)$ \\
\hline $\begin{array}{l}\text { Hyperaktivität: } \\
\text { Anzahl an } \\
\text { Kriterien } \\
\end{array}$ & 26 & $\begin{array}{c}\mathbf{1 . 5 0} \\
(1.47)\end{array}$ & $\begin{array}{c}\mathbf{0 . 6 9} \\
(1.15)\end{array}$ & 23 & $\begin{array}{c}2.08 \\
(1.90)\end{array}$ & $\begin{array}{c}1.47 \\
(1.59)\end{array}$ & 0.13 & 0.71 & 1.78 & nein & $(0,13)$ \\
\hline $\begin{array}{l}\text { Hyperaktivität: } \\
\text { Kennwert }\end{array}$ & 26 & $\begin{array}{c}\mathbf{0 . 9 7} \\
(0.71)\end{array}$ & $\begin{array}{c}\mathbf{0 . 5 4} \\
(0.60)\end{array}$ & 23 & $\begin{array}{c}1.26 \\
(0.94)\end{array}$ & $\begin{array}{c}1.04 \\
(0.79)\end{array}$ & 0.66 & 0.42 & 0.43 & nein & $(0,29)$ \\
\hline
\end{tabular}




\begin{tabular}{|c|c|c|c|c|c|c|c|c|c|c|c|}
\hline \multirow[t]{2}{*}{$\begin{array}{c}\text { Abhängige } \\
\text { Variable }\end{array}$} & \multicolumn{3}{|c|}{$\begin{array}{c}\text { Basis- und } \\
\text { Strategietraining } \\
\text { Lauth und Schlottke } \\
\text { 15 Sitzungen }\end{array}$} & \multicolumn{3}{|c|}{ Trainingsgruppe THOP } & \multicolumn{4}{|c|}{ Interaktionseffekt } & \multirow{2}{*}{$\begin{array}{c}\begin{array}{c}\text { Effekt } \\
\text {-größe }\end{array} \\
D\end{array}$} \\
\hline & $n$ & Vortest & Nachtest & $n$ & Vortest & Nachtest & $F$ & $p$ & $M Q F$ & $\begin{array}{c}\text { Hypo- } \\
\text { thesen- } \\
\text { konform } \\
\text { ? }\end{array}$ & \\
\hline $\begin{array}{l}\text { Impulsivität: } \\
\text { Anzahl an } \\
\text { Kriterien }\end{array}$ & 26 & $\begin{array}{c}0.80 \\
(1.29)\end{array}$ & $\begin{array}{c}0.69 \\
(1.37)\end{array}$ & 23 & $\begin{array}{l}1.86 \\
(1.63)\end{array}$ & $\begin{array}{l}1.56 \\
(1.70)\end{array}$ & - & - & - & - & - \\
\hline $\begin{array}{l}\text { Impulsivität: } \\
\text { Kennwert }\end{array}$ & 26 & $\begin{array}{c}0.72 \\
(0.67)\end{array}$ & $\begin{array}{c}0.65 \\
(0.85)\end{array}$ & 23 & $\begin{array}{c}1.34 \\
(0.81)\end{array}$ & $\begin{array}{l}1.28 \\
(0.92)\end{array}$ & - & - & - & - & - \\
\hline \multicolumn{12}{|c|}{ Teacher's Report Form (TRF) } \\
\hline $\begin{array}{l}\text { T-Wert } \\
\text { Aufmerksam- } \\
\text { keitsstörungen } \\
\end{array}$ & 25 & $\begin{array}{l}\mathbf{5 9 . 2 1} \\
(5.49)\end{array}$ & $\begin{array}{l}\mathbf{5 7 . 0 8} \\
(4.32)\end{array}$ & 22 & $\begin{array}{l}\mathbf{6 2 . 1 8} \\
(6.61)\end{array}$ & $\begin{array}{l}\mathbf{5 8 . 6 4} \\
(5.74)\end{array}$ & 0.95 & 0.33 & 12.09 & nein & $(0,25)$ \\
\hline $\begin{array}{l}\text { T-Wert } \\
\text { externalisieren- } \\
\text { de Störungen } \\
\end{array}$ & 25 & $\begin{array}{c}55.04 \\
(10.67)\end{array}$ & $\begin{array}{l}53.42 \\
(9.60)\end{array}$ & 22 & $\begin{array}{l}66.36 \\
(8.68)\end{array}$ & $\begin{array}{l}63.64 \\
(8.78)\end{array}$ & - & - & - & - & - \\
\hline $\begin{array}{l}\text { T-Wert } \\
\text { Gesamtskala }\end{array}$ & 25 & $\begin{array}{l}\mathbf{5 8 . 7 6} \\
(6.80)\end{array}$ & $\begin{array}{l}\mathbf{5 5 . 9 2} \\
(5.77)\end{array}$ & 22 & $\begin{array}{l}\mathbf{6 5 . 6 4} \\
(7.92)\end{array}$ & $\begin{array}{l}\mathbf{6 2 . 0 9} \\
(7.52)\end{array}$ & 0.16 & 0.68 & 17.29 & nein & $(0,10)$ \\
\hline
\end{tabular}

Anmerkung: p: einseitige Irrtumswahrscheinlichkeit des F-Wertes; Signifikanzniveau $\alpha=0.05$; MQF: Mittleres Fehlerquadrat

$\mathrm{n}$ : Anzahl der Messwertpaare (Die Anzahl umfasst nur diejenigen Kinder, von denen sowohl Vor- als auch Nachtestwert der jeweiligen Variablen vorhanden waren. Auf dieser Anzahl basieren die inferenzstatistischen Auswertungen).

TAP: Testbatterie zur Aufmerksamkeitsprüfung

In Anlehnung an Cohen (1988) wird ein Effekt von 0.20 als klein, von 0.50 als mittel und von 0.80 als groß betrachtet. 


\section{Anhang A}

Tabelle A-7

Mittelwerte und Standardabweichungen (in Klammern) im Vor- und Nachtest für die AVn und Kennwerte $(F, p, M Q F)$ des Interaktionseffekts zweifaktorieller Varianzanalysen mit Messwiederholung getrennt für die LS-Gruppe 15 Sitzungen und das Marburger Konzentrationstraining

\begin{tabular}{|c|c|c|c|c|c|c|c|c|c|c|c|}
\hline \multirow[t]{2}{*}{$\begin{array}{c}\text { Abhängige } \\
\text { Variable }\end{array}$} & \multicolumn{3}{|c|}{$\begin{array}{c}\text { Basis- und } \\
\text { Strategietraining } \\
\text { Lauth und Schlottke } \\
15 \text { Sitzungen }\end{array}$} & \multicolumn{3}{|c|}{$\begin{array}{c}\text { Marburger } \\
\text { Konzentrationstraining } \\
\text { (MK) }\end{array}$} & \multicolumn{4}{|c|}{ Interaktionseffekt } & \multirow{2}{*}{$\begin{array}{c}\begin{array}{c}\text { Effekt } \\
\text {-größe }\end{array} \\
D\end{array}$} \\
\hline & $n$ & Vortest & Nachtest & $n$ & Vortest & Nachtest & $F$ & $p$ & $M Q F$ & $\begin{array}{c}\text { Hypo- } \\
\text { thesen- } \\
\text { konform } \\
?\end{array}$ & \\
\hline \multicolumn{11}{|c|}{ TAP: Untertest „Geteilte Aufmerksamkeit } & \\
\hline $\begin{array}{l}\text { Standard- } \\
\text { abweichung }\end{array}$ & 29 & $\begin{array}{c}\mathbf{3 4 8 . 0 1} \\
(103.47)\end{array}$ & $\begin{array}{l}295.91 \\
(88.70)\end{array}$ & 15 & $\begin{array}{c}275.59 \\
(129.44)\end{array}$ & $\begin{array}{l}241.21 \\
(73.63)\end{array}$ & 0.19 & 0.66 & 8171.0 & ja & $(0,18)$ \\
\hline Anzahl Fehler & 29 & $\begin{array}{c}\mathbf{9 . 0 3} \\
(13.53) \\
\end{array}$ & $\begin{array}{c}2.97 \\
(3.46) \\
\end{array}$ & 15 & $\begin{array}{c}3.80 \\
(6.04) \\
\end{array}$ & $\begin{array}{c}4.27 \\
(4.22) \\
\end{array}$ & 3.38 & 0.07 & 62.42 & ja & $(0,68)$ \\
\hline $\begin{array}{l}\text { Anzahl } \\
\text { Auslassungen }\end{array}$ & 29 & $\begin{array}{c}8.21 \\
(6.28)\end{array}$ & $\begin{array}{c}6.34 \\
(4.59)\end{array}$ & 15 & $\begin{array}{c}7.73 \\
(5.12)\end{array}$ & $\begin{array}{c}6.80 \\
(4.49)\end{array}$ & - & - & - & - & - \\
\hline \multicolumn{11}{|c|}{ TAP: Untertest ,Go/Nogo ${ }^{66}$} & \\
\hline $\begin{array}{l}\text { Standard- } \\
\text { abweichung }\end{array}$ & 31 & $\begin{array}{l}\mathbf{1 4 9 . 2 5} \\
(37.62)\end{array}$ & $\begin{array}{l}\mathbf{1 3 3 . 1 8} \\
(44.62)\end{array}$ & 15 & $\begin{array}{c}208.69 \\
(160.59)\end{array}$ & $\begin{array}{l}143.85 \\
(43.75)\end{array}$ & 2.24 & 0.14 & 5352.6 & ja & $(0,60)$ \\
\hline Anzahl Fehler & 31 & $\begin{array}{c}7.84 \\
(6.02) \\
\end{array}$ & $\begin{array}{c}4.68 \\
(5.18) \\
\end{array}$ & 15 & $\begin{array}{c}9.53 \\
(4.79) \\
\end{array}$ & $\begin{array}{l}10.00 \\
(6.23) \\
\end{array}$ & 2.60 & 0.11 & 25.54 & ja & $(0,64)$ \\
\hline $\begin{array}{l}\text { Anzahl } \\
\text { Auslassungen }\end{array}$ & 31 & $\begin{array}{c}1.87 \\
(2.13)\end{array}$ & $\begin{array}{c}3.77 \\
(4.36)\end{array}$ & 15 & $\begin{array}{c}2.80 \\
(3.10)\end{array}$ & $\begin{array}{c}1.80 \\
(1.90)\end{array}$ & - & - & - & - & - \\
\hline \multicolumn{11}{|c|}{ TAP: Untertest „Inkompatibilität"“ } & \\
\hline $\begin{array}{l}\text { Standard- } \\
\text { abweichung }\end{array}$ & 29 & $\begin{array}{c}227.93 \\
(163.70)\end{array}$ & $\begin{array}{l}187.49 \\
(80.30)\end{array}$ & 15 & $\begin{array}{l}179.11 \\
(80.37)\end{array}$ & $\begin{array}{c}210.81 \\
(230.13)\end{array}$ & - & - & - & - & - \\
\hline Anzahl Fehler & 29 & $\begin{array}{c}\mathbf{1 4 . 7 2} \\
(10.56) \\
\end{array}$ & $\begin{array}{c}8.52 \\
(6.76) \\
\end{array}$ & 15 & $\begin{array}{c}16.27 \\
(10.37) \\
\end{array}$ & $\begin{array}{l}12.80 \\
(9.53) \\
\end{array}$ & 0.61 & 0.43 & 60.43 & ja & $(0,29)$ \\
\hline \multicolumn{11}{|c|}{ TAP: Untertest „,Visuelles Scanning“6 } & \\
\hline $\begin{array}{l}\text { Standard- } \\
\text { abweichung } \\
\text { kritische Trials } \\
\end{array}$ & 23 & $\begin{array}{c}3577.49 \\
(1875.05)\end{array}$ & $\begin{array}{c}2887.19 \\
(1434.07)\end{array}$ & 11 & $\begin{array}{c}\mathbf{3 1 4 2 . 8 0} \\
(2312.28)\end{array}$ & $\begin{array}{l}\mathbf{1 7 7 5 . 0 0} \\
(603.20)\end{array}$ & 0.91 & 0.34 & 2093658 & ja & $(0,44)$ \\
\hline $\begin{array}{l}\text { Standard- } \\
\text { abweichung } \\
\text { nicht-kritische } \\
\text { Trials }\end{array}$ & 23 & $\begin{array}{c}\mathbf{3 3 3 7 . 7 4} \\
(1680.22)\end{array}$ & $\begin{array}{r}\mathbf{2 5 1 0 . 1 5} \\
(905.79)\end{array}$ & 11 & $\begin{array}{r}\mathbf{2 1 1 8 . 4 6} \\
(831.97)\end{array}$ & $\begin{array}{l}\mathbf{1 4 8 4 . 1 5} \\
(419.35)\end{array}$ & 0.12 & 0.73 & 1149194 & ja & $(0,15)$ \\
\hline $\begin{array}{l}\text { Anzahl Fehler } \\
\text { (kritische } \\
\text { Trials) } \\
\end{array}$ & 23 & $\begin{array}{c}7.78 \\
(4.59)\end{array}$ & $\begin{array}{c}3.35 \\
(2.84)\end{array}$ & 11 & $\begin{array}{c}6.45 \\
(3.47)\end{array}$ & $\begin{array}{c}3.45 \\
(2.25)\end{array}$ & 2.49 & 0.12 & 10.03 & ja & $(0,60)$ \\
\hline $\begin{array}{l}\text { Anzahl } \\
\text { Auslassungen } \\
\text { (nicht-kritische } \\
\text { Trials) }\end{array}$ & 23 & $\begin{array}{c}1.22 \\
(2.17)\end{array}$ & $\begin{array}{l}.52 \\
(.73)\end{array}$ & 11 & $\begin{array}{l}1.55 \\
(1.13)\end{array}$ & $\begin{array}{c}0.45 \\
(0.69)\end{array}$ & - & - & - & - & - \\
\hline
\end{tabular}




\begin{tabular}{|c|c|c|c|c|c|c|c|c|c|c|c|}
\hline \multirow[t]{2}{*}{$\begin{array}{c}\text { Abhängige } \\
\text { Variable }\end{array}$} & \multicolumn{3}{|c|}{$\begin{array}{c}\text { Basis- und } \\
\text { Strategietraining } \\
\text { Lauth und Schlottke } \\
15 \text { Sitzungen } \\
\end{array}$} & \multicolumn{3}{|c|}{$\begin{array}{c}\text { Marburger } \\
\text { Konzentrationstraining } \\
\text { (MK) }\end{array}$} & \multicolumn{4}{|c|}{ Interaktionseffekt } & \multirow{2}{*}{$\begin{array}{c}\begin{array}{c}\text { Effekt } \\
\text {-größe }\end{array} \\
D\end{array}$} \\
\hline & $n$ & Vortest & Nachtest & $n$ & Vortest & Nachtest & $F$ & $p$ & $M Q F$ & $\begin{array}{l}\text { Hypo- } \\
\text { thesen- } \\
\text { konform } \\
?\end{array}$ & \\
\hline \multicolumn{7}{|l|}{ Test d2 } & & & & & \\
\hline $\begin{array}{l}\text { Konzentrations- } \\
\text { leistungswert }\end{array}$ & 21 & $\begin{array}{c}\mathbf{9 5 . 2 4} \\
(21.93)\end{array}$ & $\begin{array}{l}\mathbf{1 1 1 . 5 2} \\
(20.07)\end{array}$ & 15 & $\begin{array}{l}\mathbf{1 0 7 . 8 0} \\
(26.56)\end{array}$ & $\begin{array}{l}\mathbf{1 3 7 , 9 3} \\
(33.38)\end{array}$ & 8.01 & 0.00 & 69.91 & nein & 0,39 \\
\hline Anzahl Fehler & 21 & $\begin{array}{l}11.19 \\
(8.69)\end{array}$ & $\begin{array}{c}8.52 \\
(4.98)\end{array}$ & 15 & $\begin{array}{c}\mathbf{1 4 . 4 0} \\
(12.11)\end{array}$ & $\begin{array}{c}\mathbf{5 . 6 7} \\
(4.10)\end{array}$ & 5.13 & 0.03 & 33.72 & nein & 0,65 \\
\hline
\end{tabular}

Anmerkung: p: einseitige Irrtumswahrscheinlichkeit des F-Wertes; Signifikanzniveau $\alpha=0.05$; MQF: Mittleres Fehlerquadrat

n: Anzahl der Messwertpaare (Die Anzahl umfasst nur diejenigen Kinder, von denen sowohl Vor- als auch Nachtestwert der jeweiligen Variablen vorhanden waren. Auf dieser Anzahl basieren die inferenzstatistischen Auswertungen).

TAP: Testbatterie zur Aufmerksamkeitsprüfung

In Anlehnung an Cohen (1988) wird ein Effekt von 0.20 als klein, von 0.50 als mittel und von 0.80 als groß betrachtet.

Hinweis: Die Psychologischen Hypothesen gehen beim Vergleich des Marburger Konzentrationstrainings mit der Aufmerksamkeitstraining nach Lauth und Schlottke davon aus, dass sich die Trainings nicht unterscheiden. Die Antwort ja bedeutet also die Bestätigung der Nullhypothese. 


\section{Anhang A}

Tabelle A-8

Mittelwerte und Standardabweichungen (in Klammern) im Vor- und Nachtest für die AVn und Kennwerte $(F, p, M Q F)$ des Interaktionseffekts zweifaktorieller Varianzanalysen mit Messwiederholung getrennt für die THOP und das Marburger Konzentrationstraining

\begin{tabular}{|c|c|c|c|c|c|c|c|c|c|c|c|}
\hline \multirow[t]{2}{*}{$\begin{array}{l}\text { Abhängige } \\
\text { Variable }\end{array}$} & \multicolumn{3}{|c|}{$\begin{array}{c}\text { Trainingsgruppe } \\
\text { THOP }\end{array}$} & \multicolumn{3}{|c|}{$\begin{array}{c}\text { Marburger } \\
\text { Konzentrationstraining } \\
\text { (MK) } \\
\end{array}$} & \multicolumn{4}{|c|}{ Interaktionseffekt } & \multirow{2}{*}{$\begin{array}{c}\begin{array}{c}\text { Effekt } \\
\text {-größe }\end{array} \\
D\end{array}$} \\
\hline & $n$ & Vortest & Nachtest & $n$ & Vortest & Nachtest & $F$ & $p$ & $M Q F$ & $\begin{array}{l}\text { Hypo- } \\
\text { thesen- } \\
\text { konform } \\
?\end{array}$ & \\
\hline \multicolumn{12}{|c|}{ TAP: Untertest „Geteilte Aufmerksamkeit"“ } \\
\hline $\begin{array}{l}\text { Standard- } \\
\text { abweichung }\end{array}$ & 29 & $\begin{array}{r}\mathbf{3 3 7 . 8 2} \\
(116.48) \\
\end{array}$ & $\begin{array}{r}\mathbf{2 9 5 . 8 6} \\
(95.81) \\
\end{array}$ & 15 & $\begin{array}{r}275.59 \\
(129.44) \\
\end{array}$ & $\begin{array}{l}241.21 \\
(73.63) \\
\end{array}$ & 0.03 & 0.86 & 9088.4 & nein & $(0,07)$ \\
\hline Anzahl Fehler & 29 & $\begin{array}{c}\mathbf{9 . 3 8} \\
(13.44)\end{array}$ & $\begin{array}{c}3.07 \\
(4.03)\end{array}$ & 15 & $\begin{array}{c}3.80 \\
(6.04)\end{array}$ & $\begin{array}{c}4.27 \\
(4.22)\end{array}$ & 3.60 & 0.06 & 62.92 & nein & $(0,71)$ \\
\hline $\begin{array}{l}\text { Anzahl } \\
\text { Auslassungen }\end{array}$ & 29 & $\begin{array}{c}7.10 \\
(5.02) \\
\end{array}$ & $\begin{array}{c}5.28 \\
(4.71) \\
\end{array}$ & 15 & $\begin{array}{c}7.73 \\
(5.12) \\
\end{array}$ & $\begin{array}{c}6.80 \\
(4.49) \\
\end{array}$ & - & - & - & - & - \\
\hline \multicolumn{12}{|c|}{ TAP: Untertest ,Go/Nogo ${ }^{66}$} \\
\hline $\begin{array}{l}\text { Standard- } \\
\text { abweichung }\end{array}$ & 31 & $\begin{array}{l}140.54 \\
(42.28) \\
\end{array}$ & $\begin{array}{l}125.59 \\
(42.09) \\
\end{array}$ & 15 & $\begin{array}{c}208.69 \\
(160.59) \\
\end{array}$ & $\begin{array}{l}143.85 \\
(43.75) \\
\end{array}$ & - & - & - & - & - \\
\hline Anzahl Fehler & 31 & $\begin{array}{c}9.81 \\
(5.50) \\
\end{array}$ & $\begin{array}{r}\mathbf{6 . 9 7} \\
(5.33) \\
\end{array}$ & 15 & $\begin{array}{c}9.53 \\
(4.79) \\
\end{array}$ & $\begin{array}{l}10.00 \\
(6.23) \\
\end{array}$ & 2.84 & 0.09 & 19.40 & nein & $(0,62)$ \\
\hline $\begin{array}{l}\text { Anzahl } \\
\text { Auslassungen }\end{array}$ & 31 & $\begin{array}{c}2.10 \\
(2.70) \\
\end{array}$ & $\begin{array}{c}\mathbf{0 . 8 4} \\
(1.44) \\
\end{array}$ & 15 & $\begin{array}{c}2.80 \\
(3.10) \\
\end{array}$ & $\begin{array}{c}1.80 \\
(1.90) \\
\end{array}$ & 0.07 & 0.78 & 4.59 & nein & $(0,11)$ \\
\hline \multicolumn{12}{|c|}{ TAP: Untertest „Inkompatibilität ${ }^{66}$} \\
\hline $\begin{array}{l}\text { Standard- } \\
\text { abweichung }\end{array}$ & 31 & \begin{tabular}{|c|}
$\mathbf{2 4 2 . 4 6}$ \\
$(123.11)$ \\
\end{tabular} & \begin{tabular}{r|}
$\mathbf{1 7 6 . 6 3}$ \\
$(64.19)$ \\
\end{tabular} & 15 & $\begin{array}{l}179.11 \\
(80.37) \\
\end{array}$ & $\begin{array}{c}210.81 \\
(230.13) \\
\end{array}$ & 3.65 & 0.06 & 13148 & nein & $(0,73)$ \\
\hline Anzahl Fehler & 31 & $\begin{array}{c}\mathbf{2 0 . 4 2} \\
(12.05) \\
\end{array}$ & $\begin{array}{r}\mathbf{1 3 . 5 5} \\
(10.00) \\
\end{array}$ & 15 & $\begin{array}{c}16.27 \\
(10.37) \\
\end{array}$ & $\begin{array}{l}12.80 \\
(9.53) \\
\end{array}$ & 1.01 & 0.32 & 57.99 & nein & $(0,32)$ \\
\hline \multicolumn{12}{|c|}{ TAP: Untertest ,Visuelles Scanning“6 } \\
\hline $\begin{array}{l}\text { Standard- } \\
\text { abweichung } \\
\text { kritische Trials } \\
\end{array}$ & 31 & $\begin{array}{c}\mathbf{2 4 7 2 . 9 8} \\
(1048.18)\end{array}$ & $\begin{array}{l}\mathbf{2 0 4 9 . 0 0} \\
(995.37)\end{array}$ & 11 & $\begin{array}{c}\mathbf{3 1 4 2 . 8 0} \\
(2312.28)\end{array}$ & $\begin{array}{l}\mathbf{1 7 7 5 . 0 0} \\
(603.20)\end{array}$ & 3.46 & 0.07 & 1162602 & nein & $(0,80)$ \\
\hline $\begin{array}{l}\text { Standard- } \\
\text { abweichung } \\
\text { nicht-kritische } \\
\text { Trials }\end{array}$ & 31 & $\begin{array}{c}\mathbf{2 7 6 7 . 1 8} \\
(1185.42)\end{array}$ & $\begin{array}{c}\mathbf{2 1 9 2 . 0 3} \\
(1028.39)\end{array}$ & 11 & $\begin{array}{r}\mathbf{2 1 1 8 . 4 6} \\
(831.97)\end{array}$ & $\begin{array}{l}\mathbf{1 4 8 4 . 1 5} \\
(419.35)\end{array}$ & 0.02 & 0.86 & 514162 & nein & $(0,05)$ \\
\hline $\begin{array}{l}\text { Anzahl Fehler } \\
\text { (kritische } \\
\text { Trials) } \\
\end{array}$ & 31 & $\begin{array}{c}7.68 \\
(5.95)\end{array}$ & $\begin{array}{c}4.32 \\
(4.10)\end{array}$ & 11 & $\begin{array}{c}6.45 \\
(3.47)\end{array}$ & $\begin{array}{l}3.45 \\
(2.25)\end{array}$ & 0.86 & 0.35 & 10.29 & nein & $(0,29)$ \\
\hline $\begin{array}{l}\text { Anzahl } \\
\text { Auslassungen } \\
\text { (nicht-kritische } \\
\text { Trials) } \\
\end{array}$ & 31 & $\begin{array}{c}\mathbf{5 . 3 2} \\
(5.96)\end{array}$ & $\begin{array}{c}2.26 \\
(3.26)\end{array}$ & 11 & $\begin{array}{l}1.55 \\
(1.13)\end{array}$ & $\begin{array}{l}0.45 \\
(0.69)\end{array}$ & 4.08 & 0.05 & 16.76 & ja & $\mathbf{0 , 8 2}$ \\
\hline
\end{tabular}




\begin{tabular}{|l|c|c|c|c|c|c|c|c|c|c|c|}
\hline $\begin{array}{c}\text { Abhängige } \\
\text { Variable }\end{array}$ & \multicolumn{3}{|c|}{$\begin{array}{c}\text { Trainingsgruppe } \\
\text { THOP }\end{array}$} & \multicolumn{3}{c|}{$\begin{array}{c}\text { Marburger } \\
\text { Konzentrationstraining } \\
\text { (MK) }\end{array}$} & \multicolumn{3}{c|}{ Interaktionseffekt } & $\begin{array}{c}\text { Effekt } \\
\text {-größe }\end{array}$ \\
\hline & $n$ & Vortest & Nachtest & $n$ & Vortest & Nachtest & $F$ & $p$ & $\begin{array}{c}\text { Hypo- } \\
\text { thesen- } \\
\text { konform } \\
?\end{array}$ \\
$D$ \\
\hline Test d2
\end{tabular}

Anmerkung: p: einseitige Irrtumswahrscheinlichkeit des F-Wertes; Signifikanzniveau $\alpha=0.05$; MQF: Mittleres Fehlerquadrat

n: Anzahl der Messwertpaare (Die Anzahl umfasst nur diejenigen Kinder, von denen sowohl Vor- als auch Nachtestwert der jeweiligen Variablen vorhanden waren. Auf dieser Anzahl basieren die inferenzstatistischen Auswertungen).

TAP: Testbatterie zur Aufmerksamkeitsprüfung

In Anlehnung an Cohen (1988) wird ein Effekt von 0.20 als klein, von 0.50 als mittel und von 0.80 als groß betrachtet. 


\section{Anhang B}

Tabelle B-1

Mittelwerte und Standardabweichungen (in Klammern) im Vor- und Nachtest für die AVn und Kennwerte $(F, p)$ sowie Haupteffekte Zeit und Gruppe zweifaktorieller Varianzanalysen mit Messwiederholung getrennt für die Wartegruppe und die Lauth- und Schlottke-

Trainingsgruppe(10 Sitzungen)

\begin{tabular}{|c|c|c|c|c|c|c|c|c|c|c|}
\hline \multirow[t]{2}{*}{$\begin{array}{l}\text { Abhängige } \\
\text { Variable }\end{array}$} & \multicolumn{3}{|c|}{ Wartegruppe (WG) } & \multicolumn{3}{|c|}{$\begin{array}{c}\text { Trainingsgruppe (TG) } \\
\text { Lauth und Schlottke } \\
10 \text { Sitzungen }\end{array}$} & \multicolumn{2}{|c|}{$\begin{array}{c}\text { Haupteffekt } \\
\text { Zeit }\end{array}$} & \multicolumn{2}{|c|}{$\begin{array}{c}\text { Haupteffekt } \\
\text { Gruppe }\end{array}$} \\
\hline & $n$ & Vortest & Nachtest & $n$ & Vortest & Nachtest & $F$ & $p$ & $F$ & $\bar{p}$ \\
\hline \multicolumn{11}{|c|}{ TAP: Untertest „,Geteilte Aufmerksamkeit ${ }^{66}$} \\
\hline $\begin{array}{l}\text { Standard- } \\
\text { abweichung }\end{array}$ & 15 & $\begin{array}{c}364.05 \\
(100.99)\end{array}$ & $\begin{array}{l}293.60 \\
(83.94)\end{array}$ & 14 & $\begin{array}{l}331.74 \\
(74.95)\end{array}$ & $\begin{array}{l}316.96 \\
(75.04)\end{array}$ & 4.08 & 0.053 & 0.03 & 0.85 \\
\hline Anzahl Fehler & 15 & $\begin{array}{c}8.80 \\
(7.06) \\
\end{array}$ & $\begin{array}{c}2.60 \\
(1.45)\end{array}$ & 14 & $\begin{array}{c}8.43 \\
(8.38) \\
\end{array}$ & $\begin{array}{c}2.50 \\
(1.70) \\
\end{array}$ & 19.73 & 0.000 & 0.02 & 0.88 \\
\hline $\begin{array}{l}\text { Anzahl } \\
\text { Auslassungen }\end{array}$ & 15 & $\begin{array}{c}8.73 \\
(6.77)\end{array}$ & $\begin{array}{c}6.80 \\
(3.36)\end{array}$ & 14 & $\begin{array}{c}6.79 \\
(3.93)\end{array}$ & $\begin{array}{c}4.29 \\
(2.52)\end{array}$ & 7.13 & 0.013 & 2.39 & 0.13 \\
\hline \multicolumn{11}{|c|}{ TAP: Untertest „Go/Nogo“6 } \\
\hline $\begin{array}{l}\text { Standard- } \\
\text { abweichung }\end{array}$ & 16 & $\begin{array}{l}146.28 \\
(61.36)\end{array}$ & $\begin{array}{l}148.38 \\
(52.80)\end{array}$ & 14 & $\begin{array}{l}259.37 \\
291.49\end{array}$ & $\begin{array}{c}136.74 \\
24.59\end{array}$ & 2.59 & 0.118 & 1.72 & 0.20 \\
\hline Anzahl Fehler & 16 & $\begin{array}{c}8.19 \\
(4.79) \\
\end{array}$ & $\begin{array}{c}5.75 \\
(3.13) \\
\end{array}$ & 14 & $\begin{array}{l}9.21 \\
4.85 \\
\end{array}$ & $\begin{array}{l}4.71 \\
3.97 \\
\end{array}$ & 15.73 & 0.000 & 0.00 & 0.99 \\
\hline $\begin{array}{l}\text { Anzahl } \\
\text { Auslassungen }\end{array}$ & 16 & $\begin{array}{c}5.00 \\
(5.40) \\
\end{array}$ & $\begin{array}{c}2.38 \\
(3.12) \\
\end{array}$ & 14 & $\begin{array}{l}4.93 \\
6.24 \\
\end{array}$ & $\begin{array}{l}1.50 \\
1,83 \\
\end{array}$ & 8.81 & 0.006 & 0.13 & 0.71 \\
\hline \multicolumn{11}{|c|}{ TAP: Untertest „Inkompatibilität"“ } \\
\hline $\begin{array}{l}\text { Standard- } \\
\text { abweichung }\end{array}$ & 15 & $\begin{array}{c}323.82 \\
(259.98)\end{array}$ & $\begin{array}{l}196.69 \\
(56.45)\end{array}$ & 14 & $\begin{array}{c}274.24 \\
(273.95) \\
\end{array}$ & $\begin{array}{l}189.13 \\
(41.07) \\
\end{array}$ & 4.88 & 0.036 & 0.29 & 0.59 \\
\hline Anzahl Fehler & 15 & $\begin{array}{l}15.53 \\
(9.12) \\
\end{array}$ & $\begin{array}{l}12.80 \\
(8.03) \\
\end{array}$ & 14 & $\begin{array}{c}19.71 \\
(13.10) \\
\end{array}$ & $\begin{array}{c}8.93 \\
(8.65) \\
\end{array}$ & 15.47 & 0.001 & 0.00 & 0.93 \\
\hline \multicolumn{11}{|c|}{ TAP: Untertest „Visuelles Scanning“ } \\
\hline $\begin{array}{l}\text { Standard- } \\
\text { abweichung } \\
\text { kritische Trials }\end{array}$ & 15 & $\begin{array}{c}3952.57 \\
(1864.41)\end{array}$ & $\begin{array}{c}2386.72 \\
(1274.29)\end{array}$ & 14 & $\begin{array}{c}2833.11 \\
(1265.76)\end{array}$ & $\begin{array}{c}1999.71 \\
(1174.35)\end{array}$ & 20.42 & 0.000 & 2.68 & 0.11 \\
\hline $\begin{array}{l}\text { Standard- } \\
\text { abweichung } \\
\text { nicht-kritische } \\
\text { Trials }\end{array}$ & 15 & $\begin{array}{c}3683.64 \\
(1374.86)\end{array}$ & $\begin{array}{c}2636.73 \\
(1305.89)\end{array}$ & 14 & $\begin{array}{c}3332.51 \\
(1761.55)\end{array}$ & $\begin{array}{c}2637.14 \\
(1509.64)\end{array}$ & 6.87 & 0.014 & 0.15 & 0.69 \\
\hline $\begin{array}{l}\text { Anzahl Fehler } \\
\text { (kritische } \\
\text { Trials) } \\
\end{array}$ & 15 & $\begin{array}{c}2.33 \\
(5.23)\end{array}$ & $\begin{array}{l}1.47 \\
(2.92)\end{array}$ & 14 & $\begin{array}{c}1.21 \\
(1.12)\end{array}$ & $\begin{array}{l}1.00 \\
(2.15)\end{array}$ & 1.28 & 0.267 & 0.50 & 0.48 \\
\hline $\begin{array}{l}\text { Anzahl } \\
\text { Auslassungen } \\
\text { (nicht-kritische } \\
\text { Trials) }\end{array}$ & 15 & $\begin{array}{l}5.80 \\
(4.50)\end{array}$ & $\begin{array}{c}6.20 \\
(4.95)\end{array}$ & 14 & $\begin{array}{c}5.14 \\
(3.21)\end{array}$ & $\begin{array}{l}5.36 \\
(3.95)\end{array}$ & 0.16 & 0.687 & 0.29 & 0.59 \\
\hline \multicolumn{11}{|l|}{ Test d2 } \\
\hline $\begin{array}{l}\text { Konzentrations- } \\
\text { leistungswert }\end{array}$ & 13 & $\begin{array}{c}77.00 \\
(26.59)\end{array}$ & $\begin{array}{c}95.85 \\
(25.78)\end{array}$ & 14 & $\begin{array}{c}72.21 \\
(27.26)\end{array}$ & $\begin{array}{l}96.50 \\
(19.07) \\
\end{array}$ & 56.19 & 0.000 & 0.05 & 0.82 \\
\hline Anzahl Fehler & 13 & $\begin{array}{c}34.85 \\
(34.98)\end{array}$ & $\begin{array}{c}12.15 \\
(14.67)\end{array}$ & 14 & $\begin{array}{c}34.07 \\
(41.29)\end{array}$ & $\begin{array}{l}11.07 \\
(9.93)\end{array}$ & 12.55 & 0.002 & 0.01 & 0.91 \\
\hline
\end{tabular}




\begin{tabular}{|c|c|c|c|c|c|c|c|c|c|c|}
\hline \multirow[t]{2}{*}{$\begin{array}{l}\text { Abhängige } \\
\text { Variable }\end{array}$} & \multicolumn{3}{|c|}{ Wartegruppe (WG) } & \multicolumn{3}{|c|}{$\begin{array}{l}\text { Trainingsgruppe (TG) } \\
\text { Lauth und Schlottke } \\
10 \text { Sitzungen }\end{array}$} & \multicolumn{2}{|c|}{$\begin{array}{c}\text { Haupteffekt } \\
\text { Zeit }\end{array}$} & \multicolumn{2}{|c|}{$\begin{array}{c}\text { Haupteffekt } \\
\text { Gruppe }\end{array}$} \\
\hline & $n$ & Vortest & Nachtest & $n$ & Vortest & Nachtest & $F$ & $p$ & $F$ & $p$ \\
\hline \multicolumn{11}{|l|}{ Mottier-Test } \\
\hline $\begin{array}{l}\text { Nachsprechleist } \\
\text { ung Gesamt } \\
\text { (max. 30) }\end{array}$ & 15 & $\begin{array}{l}20.80 \\
(3.97)\end{array}$ & $\begin{array}{l}20.40 \\
(5.01)\end{array}$ & 14 & $\begin{array}{l}19.07 \\
(4.01)\end{array}$ & $\begin{array}{l}22.43 \\
(3.11)\end{array}$ & 7.15 & 0.013 & 0.01 & 0.91 \\
\hline $\begin{array}{l}\text { Nachsprechleist } \\
\text { ung für 2- und } \\
\text { 3-silbige } \\
\text { Kunstwörter } \\
\text { (max. 12) }\end{array}$ & 15 & $\begin{array}{l}11.07 \\
(1.53)\end{array}$ & $\begin{array}{l}10.20 \\
(2.21)\end{array}$ & 14 & $\begin{array}{l}11.00 \\
(1.24)\end{array}$ & $\begin{array}{l}11.29 \\
(1.14)\end{array}$ & 0.46 & 0.499 & 1.49 & 0.23 \\
\hline $\begin{array}{l}\text { Nachsprechleist } \\
\text { ung für 4-, 5- } \\
\text { und 6-silbige } \\
\text { Kunstwörter } \\
\text { (max. 18) }\end{array}$ & 15 & $\begin{array}{c}9.73 \\
(3.17)\end{array}$ & $\begin{array}{l}10.20 \\
(3.86)\end{array}$ & 14 & $\begin{array}{c}8.07 \\
(3.41)\end{array}$ & $\begin{array}{l}11.14 \\
(3.13)\end{array}$ & 25.85 & 0.000 & 0.08 & 0.77 \\
\hline \multicolumn{11}{|c|}{ Fremdbeurteilungsbogen FBB-HKS (Eltern) } \\
\hline $\begin{array}{l}\text { Unaufmerksam- } \\
\text { keit: Anzahl an } \\
\text { Kriterien } \\
\end{array}$ & 11 & $\begin{array}{c}4.91 \\
(3.11)\end{array}$ & $\begin{array}{c}4.45 \\
(4.06)\end{array}$ & 13 & $\begin{array}{c}4.69 \\
(1.75)\end{array}$ & $\begin{array}{c}3.23 \\
(2.31)\end{array}$ & 2.09 & 0.162 & 0.54 & 0.46 \\
\hline $\begin{array}{l}\text { Unaufmerksam- } \\
\text { keit: Kennwert }\end{array}$ & 11 & $\begin{array}{l}1.64 \\
(.62)\end{array}$ & $\begin{array}{l}1.58 \\
(.87)\end{array}$ & 13 & $\begin{array}{l}1.54 \\
(.37)\end{array}$ & $\begin{array}{l}1.18 \\
(.43)\end{array}$ & 2.14 & 0.157 & 1.66 & 0.21 \\
\hline $\begin{array}{l}\text { Hyperaktivität: } \\
\text { Anzahl an } \\
\text { Kriterien } \\
\end{array}$ & 11 & $\begin{array}{c}2.72 \\
(1.68)\end{array}$ & $\begin{array}{c}2.36 \\
(2.38)\end{array}$ & 13 & $\begin{array}{l}1.76 \\
(1.48)\end{array}$ & $\begin{array}{l}.46 \\
(.87)\end{array}$ & 4.86 & 0.038 & 6.49 & 0.01 \\
\hline $\begin{array}{l}\text { Hyperaktivität: } \\
\text { Kennwert }\end{array}$ & 11 & $\begin{array}{l}1.63 \\
(.70)\end{array}$ & $\begin{array}{l}1.29 \\
(.76)\end{array}$ & 13 & $\begin{array}{l}1.29 \\
(.71)\end{array}$ & $\begin{array}{l}.66 \\
(.45)\end{array}$ & 12.30 & 0.002 & 4.30 & 0.05 \\
\hline $\begin{array}{l}\text { Impulsivität: } \\
\text { Anzahl an } \\
\text { Kriterien } \\
\end{array}$ & 11 & $\begin{array}{c}1.45 \\
(1.75)\end{array}$ & $\begin{array}{c}1.27 \\
(1.79)\end{array}$ & 13 & $\begin{array}{c}1.30 \\
(1.70)\end{array}$ & $\begin{array}{c}0.07 \\
(0.27)\end{array}$ & 2.72 & 0.113 & 2.33 & 0.14 \\
\hline $\begin{array}{l}\text { Impulsivität: } \\
\text { Kennwert }\end{array}$ & 11 & $\begin{array}{l}1.18 \\
(.69) \\
\end{array}$ & $\begin{array}{l}1.15 \\
(.78)\end{array}$ & 13 & $\begin{array}{l}1.12 \\
(.79)\end{array}$ & $\begin{array}{l}.59 \\
(.37)\end{array}$ & 2.39 & 0.13 & 2.08 & 0.16 \\
\hline \multicolumn{11}{|c|}{ Child Behavior Checklist (CBCL) } \\
\hline $\begin{array}{l}\text { T-Wert } \\
\text { Aufmerksam- } \\
\text { keitsstörungen }\end{array}$ & 10 & $\begin{array}{l}64.50 \\
(6.89)\end{array}$ & $\begin{array}{l}61.60 \\
(8.63)\end{array}$ & 12 & $\begin{array}{l}68.33 \\
(8.74)\end{array}$ & $\begin{array}{c}63.58 \\
(10.95)\end{array}$ & 5.64 & 0.02 & 0.68 & 0.41 \\
\hline \begin{tabular}{|l|} 
T-Wert \\
externalisieren- \\
de Störungen \\
\end{tabular} & 10 & $\begin{array}{c}59.50 \\
(12.04)\end{array}$ & $\begin{array}{c}59.00 \\
(12.18)\end{array}$ & 12 & $\begin{array}{l}63.17 \\
(9.23)\end{array}$ & $\begin{array}{l}58.67 \\
(8.07)\end{array}$ & 2.02 & 0.17 & 0.16 & 0.68 \\
\hline $\begin{array}{l}\text { T-Wert } \\
\text { Gesamtskala }\end{array}$ & 10 & $\begin{array}{l}61.00 \\
(9.46)\end{array}$ & $\begin{array}{c}57.70 \\
(10.87)\end{array}$ & 12 & $\begin{array}{l}64.17 \\
(7.07)\end{array}$ & $\begin{array}{l}59.25 \\
(7.33)\end{array}$ & 12.98 & 0.00 & 0.44 & 0.51 \\
\hline \multicolumn{11}{|c|}{ Fremdbeurteilungsbogen FBB-HKS (Lehrer) } \\
\hline $\begin{array}{l}\text { Unaufmerksam- } \\
\text { keit: Anzahl an } \\
\text { Kriterien } \\
\end{array}$ & 12 & $\begin{array}{c}4.75 \\
(2.83)\end{array}$ & $\begin{array}{c}4.92 \\
(3.09)\end{array}$ & 9 & $\begin{array}{c}3.33 \\
(2.17)\end{array}$ & $\begin{array}{c}3.00 \\
(2.44)\end{array}$ & 0.00 & 0.92 & 4.23 & 0.05 \\
\hline $\begin{array}{l}\text { Unaufmerksam- } \\
\text { keit: Kennwert }\end{array}$ & 12 & $\begin{array}{l}1.62 \\
(.58)\end{array}$ & $\begin{array}{l}1.58 \\
(.69)\end{array}$ & 9 & $\begin{array}{l}1.28 \\
(.44)\end{array}$ & $\begin{array}{l}1.13 \\
(.52)\end{array}$ & 0.29 & 0.59 & 4.82 & 0.04 \\
\hline $\begin{array}{l}\text { Hyperaktivität: } \\
\text { Anzahl an } \\
\text { Kriterien } \\
\end{array}$ & 12 & $\begin{array}{c}2.75 \\
(2.01)\end{array}$ & $\begin{array}{c}1.67 \\
(2.19)\end{array}$ & 9 & $\begin{array}{l}1.77 \\
(1.56)\end{array}$ & $\begin{array}{c}.66 \\
(.70)\end{array}$ & 4.44 & 0.04 & 2.81 & 0.11 \\
\hline $\begin{array}{l}\text { Hyperaktivität: } \\
\text { Kennwert }\end{array}$ & 12 & $\begin{array}{l}1.63 \\
(.79)\end{array}$ & $\begin{array}{l}1.10 \\
(.88)\end{array}$ & 9 & $\begin{array}{l}1.14 \\
(.56)\end{array}$ & $\begin{array}{l}.77 \\
(.52)\end{array}$ & 4.83 & 0.04 & 2.67 & 0.11 \\
\hline $\begin{array}{l}\text { Impulsivität: } \\
\text { Anzahl an } \\
\text { Kriterien } \\
\end{array}$ & 12 & $\begin{array}{c}2.42 \\
(1.73)\end{array}$ & $\begin{array}{c}1.67 \\
(1.83)\end{array}$ & 9 & $\begin{array}{c}1.00 \\
(1.58)\end{array}$ & $\begin{array}{c}.66 \\
(1.00)\end{array}$ & 0.24 & 0.69 & 4.56 & 0.04 \\
\hline
\end{tabular}




\begin{tabular}{|c|c|c|c|c|c|c|c|c|c|c|}
\hline \multirow[t]{2}{*}{$\begin{array}{l}\text { Abhängige } \\
\text { Variable }\end{array}$} & \multicolumn{3}{|c|}{ Wartegruppe (WG) } & \multicolumn{3}{|c|}{$\begin{array}{c}\text { Trainingsgruppe (TG) } \\
\text { Lauth und Schlottke } \\
10 \text { Sitzungen }\end{array}$} & \multicolumn{2}{|c|}{$\begin{array}{c}\text { Haupteffekt } \\
\text { Zeit }\end{array}$} & \multicolumn{2}{|c|}{$\begin{array}{c}\text { Haupteffekt } \\
\text { Gruppe }\end{array}$} \\
\hline & $n$ & Vortest & Nachtest & $n$ & Vortest & Nachtest & $F$ & $\bar{p}$ & $F$ & $\bar{p}$ \\
\hline $\begin{array}{l}\text { Impulsivität: } \\
\text { Kennwert }\end{array}$ & 12 & $\begin{array}{l}1.70 \\
(.94)\end{array}$ & $\begin{array}{l}1.20 \\
(.89)\end{array}$ & 9 & $\begin{array}{l}1.06 \\
(.70)\end{array}$ & $\begin{array}{c}.86 \\
(.51)\end{array}$ & 3.11 & 0.09 & 2.73 & 0.11 \\
\hline \multicolumn{11}{|c|}{ Teacher's Report Form (TRF) } \\
\hline $\begin{array}{l}\text { T-Wert } \\
\text { Aufmerksam- } \\
\text { keitsstörungen } \\
\end{array}$ & 7 & $\begin{array}{l}61.86 \\
(4.38)\end{array}$ & $\begin{array}{l}59.86 \\
(5.61)\end{array}$ & 11 & $\begin{array}{l}59.36 \\
(5.30)\end{array}$ & $\begin{array}{l}58.00 \\
(5.46)\end{array}$ & 2.28 & 0.15 & 0.90 & 0.35 \\
\hline $\begin{array}{l}\text { T-Wert } \\
\text { externalisieren- } \\
\text { de Störungen }\end{array}$ & 7 & $\begin{array}{l}65.00 \\
(8.15)\end{array}$ & $\begin{array}{l}65.14 \\
(8.75)\end{array}$ & 11 & $\begin{array}{l}59.09 \\
(8.94)\end{array}$ & $\begin{array}{l}59.64 \\
(8.56)\end{array}$ & 0.02 & 0.88 & 2.08 & 0.16 \\
\hline $\begin{array}{l}\text { T-Wert } \\
\text { Gesamtskala }\end{array}$ & 7 & $\begin{array}{l}60.71 \\
(7.16)\end{array}$ & $\begin{array}{l}61.43 \\
(5.29)\end{array}$ & 11 & $\begin{array}{l}58.73 \\
(5.52)\end{array}$ & $\begin{array}{l}59.09 \\
(6.19)\end{array}$ & 0.20 & 0.65 & 0.66 & 0.42 \\
\hline
\end{tabular}

Anmerkung: p: einseitige Irrtumswahrscheinlichkeit des F-Wertes;

Signifikanzniveau $\alpha=0.05$;

n: Anzahl der Messwertpaare (Die Anzahl umfasst nur diejenigen Kinder, von denen sowohl Vor- als auch Nachtestwert der jeweiligen Variablen vorhanden waren. Auf dieser Anzahl basieren die inferenzstatistischen Auswertungen).

TAP: Testbatterie zur Aufmerksamkeitsprüfung 


\section{Anhang B}

Tabelle B-2

Mittelwerte und Standardabweichungen (in Klammern) im Vor- und Nachtest für die AVn und Kennwerte $(F, p)$ der Haupteffekte Zeit und Gruppe zweifaktorieller Varianzanalysen mit Messwiederholung getrennt für die Wartegruppe und die Lauth- und SchlottkeTrainingsgruppe(15 Sitzungen)

\begin{tabular}{|c|c|c|c|c|c|c|c|c|c|c|}
\hline \multirow[t]{2}{*}{$\begin{array}{l}\text { Abhängige } \\
\text { Variable }\end{array}$} & \multicolumn{3}{|c|}{ Wartegruppe (WG) } & \multicolumn{3}{|c|}{$\begin{array}{c}\text { Lauth und Schlottke } 15 \\
\text { Sitzungen (Basis- und } \\
\text { Strategietraining) }\end{array}$} & \multicolumn{2}{|c|}{$\begin{array}{c}\text { Haupteffekt } \\
\text { Zeit }\end{array}$} & \multicolumn{2}{|c|}{$\begin{array}{c}\text { Haupteffekt } \\
\text { Gruppe }\end{array}$} \\
\hline & $n$ & Vortest & Nachtest & $n$ & Vortest & Nachtest & $F$ & $p$ & $F$ & $p$ \\
\hline \multicolumn{11}{|c|}{ TAP: Untertest ,Geteilte Aufmerksamkeit" } \\
\hline $\begin{array}{l}\text { Standard- } \\
\text { abweichung }\end{array}$ & 15 & $\begin{array}{c}\mathbf{3 6 4 . 0 5} \\
(100.99)\end{array}$ & $\begin{array}{l}\mathbf{2 9 3 . 6 0} \\
(83.94)\end{array}$ & 29 & $\begin{array}{c}\mathbf{3 4 8 . 0 1} \\
(103.47)\end{array}$ & $\begin{array}{l}\mathbf{2 9 5 . 9 1} \\
(88.70)\end{array}$ & 10.99 & 0.00 & 0.08 & 0.77 \\
\hline Anzahl Fehler & 15 & $\begin{array}{c}\mathbf{8 . 8 0} \\
(7.06) \\
\end{array}$ & $\begin{array}{c}\mathbf{2 . 6 0} \\
(1.45) \\
\end{array}$ & 29 & $\begin{array}{c}\mathbf{9 . 0 3} \\
(13.53) \\
\end{array}$ & $\begin{array}{c}\mathbf{2 . 9 7} \\
(3.46) \\
\end{array}$ & 11.40 & 0.00 & 0.02 & 0.88 \\
\hline $\begin{array}{l}\text { Anzahl } \\
\text { Auslassungen }\end{array}$ & 15 & $\begin{array}{c}8.73 \\
(6.77)\end{array}$ & $\begin{array}{c}6.80 \\
(3.36)\end{array}$ & 29 & $\begin{array}{c}8.21 \\
(6.28)\end{array}$ & $\begin{array}{c}6.34 \\
(4.59)\end{array}$ & 4.41 & 0.04 & 0.11 & 0.74 \\
\hline \multicolumn{11}{|c|}{ TAP: Untertest ,Go/Nogo“" } \\
\hline $\begin{array}{l}\text { Standard- } \\
\text { abweichung }\end{array}$ & 16 & $\begin{array}{l}146.28 \\
(61.36)\end{array}$ & $\begin{array}{r}148.38 \\
(52.80)\end{array}$ & 31 & $\begin{array}{l}\mathbf{1 4 9 . 2 5} \\
(37.62) \\
\end{array}$ & $\begin{array}{l}\mathbf{1 3 3 . 1 8} \\
(44.62) \\
\end{array}$ & 0.76 & 0.38 & 0.25 & 0.61 \\
\hline Anzahl Fehler & 16 & $\begin{array}{c}\mathbf{8 . 1 9} \\
(4.79)\end{array}$ & $\begin{array}{c}\mathbf{5 . 7 5} \\
(3.13)\end{array}$ & 31 & $\begin{array}{c}7.84 \\
(6.02)\end{array}$ & $\begin{array}{c}\mathbf{4 . 6 8} \\
(5.18)\end{array}$ & 7.86 & 0.00 & 0.33 & 0.56 \\
\hline $\begin{array}{l}\text { Anzahl } \\
\text { Auslassungen }\end{array}$ & 16 & $\begin{array}{c}\mathbf{5 . 0 0} \\
(5.40) \\
\end{array}$ & $\begin{array}{c}\mathbf{2 . 3 8} \\
(3.12) \\
\end{array}$ & 31 & $\begin{array}{c}1.87 \\
(2.13) \\
\end{array}$ & $\begin{array}{c}3.77 \\
(4.36) \\
\end{array}$ & 0.19 & 0.66 & 1.09 & 0.30 \\
\hline \multicolumn{11}{|c|}{ TAP: Untertest ,Inkompatibilität“6 } \\
\hline $\begin{array}{l}\text { Standard- } \\
\text { abweichung }\end{array}$ & 15 & $\begin{array}{c}\mathbf{3 2 3 . 8 2} \\
(259.98)\end{array}$ & $\begin{array}{l}196.69 \\
(56.45)\end{array}$ & 29 & $\begin{array}{c}227.93 \\
(163.70) \\
\end{array}$ & $\begin{array}{l}187.49 \\
(80.30) \\
\end{array}$ & 8.29 & 0.00 & 1.88 & 0.17 \\
\hline Anzahl Fehler & 15 & $\begin{array}{l}15.53 \\
(9.12) \\
\end{array}$ & $\begin{array}{l}12.80 \\
(8.03) \\
\end{array}$ & 29 & $\begin{array}{c}\mathbf{1 4 . 7 2} \\
(10.56) \\
\end{array}$ & $\begin{array}{c}\mathbf{8 . 5 2} \\
(6.76) \\
\end{array}$ & 7.69 & 0.00 & 1.24 & 0.27 \\
\hline \multicolumn{11}{|c|}{ TAP: Untertest ,Visuelles Scanning } \\
\hline $\begin{array}{l}\text { Standard- } \\
\text { abweichung } \\
\text { kritische Trials }\end{array}$ & 15 & $\begin{array}{c}\mathbf{3 9 5 2 . 5 7} \\
(1864.41)\end{array}$ & $\begin{array}{c}\mathbf{2 3 8 6 . 7 2} \\
(1274.29)\end{array}$ & 23 & $\begin{array}{c}3577.49 \\
(1875.05)\end{array}$ & $\begin{array}{c}2887.19 \\
(1434.07)\end{array}$ & 14.80 & 0.00 & 0.02 & 0.89 \\
\hline $\begin{array}{l}\text { Standard- } \\
\text { abweichung } \\
\text { nicht-kritische } \\
\text { Trials }\end{array}$ & 15 & $\begin{array}{c}\mathbf{3 6 8 3 . 6 4} \\
(1374.86)\end{array}$ & $\begin{array}{c}\mathbf{2 6 3 6 . 7 3} \\
(1305.89)\end{array}$ & 23 & $\begin{array}{c}\mathbf{3 3 3 7 . 7 4} \\
(1680.22)\end{array}$ & $\begin{array}{l}\mathbf{2 5 1 0 . 1 5} \\
(905.79)\end{array}$ & 11.72 & 0.00 & 0.44 & 0.50 \\
\hline $\begin{array}{l}\text { Anzahl Fehler } \\
\text { (kritische } \\
\text { Trials) } \\
\end{array}$ & 15 & $\begin{array}{c}2.33 \\
(5.23)\end{array}$ & $\begin{array}{c}1.47 \\
(2.92)\end{array}$ & 23 & $\begin{array}{c}7.78 \\
(4.59)\end{array}$ & $\begin{array}{c}3.35 \\
(2.84)\end{array}$ & 15.12 & 0.00 & 10.44 & 0.00 \\
\hline $\begin{array}{l}\text { Anzahl } \\
\text { Auslassungen } \\
\text { (nicht-kritische } \\
\text { Trials) }\end{array}$ & 15 & $\begin{array}{c}5.80 \\
(4.50)\end{array}$ & $\begin{array}{c}6.20 \\
(4.95)\end{array}$ & 23 & $\begin{array}{c}1.22 \\
(2.17)\end{array}$ & $\begin{array}{c}0.52 \\
(0.73)\end{array}$ & 0.06 & 0.80 & 33.98 & 0.00 \\
\hline \multicolumn{11}{|l|}{\begin{tabular}{|l} 
Test d2 \\
\end{tabular}} \\
\hline $\begin{array}{l}\text { Konzentrations- } \\
\text { leistungswert }\end{array}$ & 13 & $\begin{array}{l}\mathbf{7 7 . 0 0} \\
(26.59)\end{array}$ & $\begin{array}{l}\mathbf{9 5 . 8 5} \\
(25.78)\end{array}$ & 21 & $\begin{array}{l}\mathbf{9 5 . 2 4} \\
(21.93)\end{array}$ & $\begin{array}{l}\text { 111.52 } \\
(20.07)\end{array}$ & 99.91 & 0.00 & 4.54 & 0.04 \\
\hline Anzahl Fehler & 13 & $\begin{array}{c}\mathbf{3 4 . 8 5} \\
(34.98)\end{array}$ & $\begin{array}{c}\mathbf{1 2 . 1 5} \\
(14.67)\end{array}$ & 21 & $\begin{array}{l}11.19 \\
(8.69)\end{array}$ & $\begin{array}{c}8.52 \\
(4.98)\end{array}$ & 12.55 & 0.00 & 8.38 & 0.00 \\
\hline
\end{tabular}




\begin{tabular}{|c|c|c|c|c|c|c|c|c|c|c|}
\hline \multirow[t]{2}{*}{$\begin{array}{l}\text { Abhängige } \\
\text { Variable }\end{array}$} & \multicolumn{3}{|c|}{ Wartegruppe (WG) } & \multicolumn{3}{|c|}{$\begin{array}{c}\text { Lauth und Schlottke } 15 \\
\text { Sitzungen (Basis- und } \\
\text { Strategietraining) } \\
\end{array}$} & \multicolumn{2}{|c|}{$\begin{array}{c}\text { Haupteffekt } \\
\text { Zeit }\end{array}$} & \multicolumn{2}{|c|}{$\begin{array}{c}\text { Haupteffekt } \\
\text { Gruppe }\end{array}$} \\
\hline & $n$ & Vortest & Nachtest & $n$ & Vortest & Nachtest & $F$ & $p$ & $F$ & $p$ \\
\hline \multicolumn{11}{|l|}{ Mottier-Test } \\
\hline $\begin{array}{l}\text { Nachsprechleist } \\
\text { ung Gesamt } \\
\text { (max. 30) }\end{array}$ & 15 & $\begin{array}{l}20.80 \\
(3.97)\end{array}$ & $\begin{array}{l}20.40 \\
(5.01)\end{array}$ & 32 & $\begin{array}{l}\mathbf{2 1 . 2 2} \\
(4.96)\end{array}$ & $\begin{array}{l}\mathbf{2 3 . 8 1} \\
(3.89)\end{array}$ & 4.92 & 0.32 & 2.13 & 015 \\
\hline $\begin{array}{l}\text { Nachsprechleist } \\
\text { ung für 2- und } \\
\text { 3-silbige } \\
\text { Kunstwörter } \\
\text { (max. 12) }\end{array}$ & 15 & $\begin{array}{l}11.07 \\
(1.53)\end{array}$ & $\begin{array}{l}10.20 \\
(2.21)\end{array}$ & 32 & $\begin{array}{l}\mathbf{1 1 . 2 8} \\
(1.02)\end{array}$ & $\begin{array}{l}\mathbf{1 1 . 6 3} \\
(0.79)\end{array}$ & 0.96 & 0.33 & 7.03 & 0.01 \\
\hline $\begin{array}{l}\text { Nachsprechleist } \\
\text { ung für 4-, 5- } \\
\text { und 6-silbige } \\
\text { Kunstwörter } \\
\text { (max. 18) }\end{array}$ & 15 & $\begin{array}{c}9.73 \\
(3.17)\end{array}$ & $\begin{array}{l}10.20 \\
(3.86)\end{array}$ & 32 & $\begin{array}{c}\mathbf{9 . 9 4} \\
(4.44)\end{array}$ & $\begin{array}{l}\mathbf{1 2 . 1 9} \\
(3.41)\end{array}$ & 11.95 & 0.00 & 5.15 & 0.03 \\
\hline \multicolumn{11}{|c|}{ Fremdbeurteilungsbogen FBB-HKS (Eltern) } \\
\hline $\begin{array}{l}\text { Unaufmerksam- } \\
\text { keit: Anzahl an } \\
\text { Kriterien } \\
\end{array}$ & 11 & $\begin{array}{c}4.91 \\
(3.11)\end{array}$ & $\begin{array}{c}4.45 \\
(4.06)\end{array}$ & 28 & $\begin{array}{c}5.78 \\
(2.48)\end{array}$ & $\begin{array}{c}5.57 \\
(3.07)\end{array}$ & 1.76 & 0.19 & 1.38 & 0.24 \\
\hline $\begin{array}{l}\text { Unaufmerksam- } \\
\text { keit: Kennwert }\end{array}$ & 11 & $\begin{array}{c}1.64 \\
(0.62)\end{array}$ & $\begin{array}{c}1.58 \\
(0.87)\end{array}$ & 28 & $\begin{array}{c}1.78 \\
(0.50)\end{array}$ & $\begin{array}{l}1.69 \\
(0.60)\end{array}$ & 2.18 & 0.14 & 0.53 & 0.46 \\
\hline $\begin{array}{l}\text { Hyperaktivität: } \\
\text { Anzahl an } \\
\text { Kriterien } \\
\end{array}$ & 11 & $\begin{array}{c}2.72 \\
(1.68)\end{array}$ & $\begin{array}{c}2.36 \\
(2.38)\end{array}$ & 28 & $\begin{array}{c}1.32 \\
(1.49)\end{array}$ & $\begin{array}{l}1.07 \\
(1.56)\end{array}$ & 5.84 & 0.02 & 7.89 & 0.00 \\
\hline $\begin{array}{l}\text { Hyperaktivität: } \\
\text { Kennwert }\end{array}$ & 11 & $\begin{array}{c}1.63 \\
(0.70) \\
\end{array}$ & $\begin{array}{c}1.29 \\
(0.76)\end{array}$ & 28 & $\begin{array}{c}1.01 \\
(0.61)\end{array}$ & $\begin{array}{c}0.82 \\
(0.79) \\
\end{array}$ & 7.77 & 0.00 & 6.58 & 0.01 \\
\hline $\begin{array}{l}\text { Impulsivität: } \\
\text { Anzahl an } \\
\text { Kriterien } \\
\end{array}$ & 11 & $\begin{array}{c}1.45 \\
(1.75)\end{array}$ & $\begin{array}{c}1.27 \\
(1.79)\end{array}$ & 28 & $\begin{array}{l}1.03 \\
(1.45)\end{array}$ & $\begin{array}{l}1.28 \\
(1.56)\end{array}$ & 1.57 & 0.21 & 0.19 & 0.65 \\
\hline $\begin{array}{l}\text { Impulsivität: } \\
\text { Kennwert }\end{array}$ & 11 & $\begin{array}{c}1.18 \\
(0.69)\end{array}$ & $\begin{array}{c}1.15 \\
(0.78)\end{array}$ & 28 & $\begin{array}{c}0.82 \\
(0.75)\end{array}$ & $\begin{array}{l}1.09 \\
(0.87)\end{array}$ & 2.78 & 0.10 & 0.73 & 0.39 \\
\hline \multicolumn{11}{|c|}{ Child Behavior Checklist (CBCL) } \\
\hline $\begin{array}{l}\text { T-Wert } \\
\text { Aufmerksam- } \\
\text { keitsstörungen }\end{array}$ & 10 & $\begin{array}{l}64.50 \\
(6.89)\end{array}$ & $\begin{array}{l}61.60 \\
(8.63)\end{array}$ & 30 & $\begin{array}{l}\mathbf{6 5 . 4 7} \\
(8.38)\end{array}$ & $\begin{array}{l}\mathbf{6 2 . 1 7} \\
(7.28)\end{array}$ & 5.36 & 0.26 & 0.09 & 0.76 \\
\hline \begin{tabular}{|l|} 
T-Wert \\
externalisieren- \\
de Störungen \\
\end{tabular} & 10 & $\begin{array}{c}59.50 \\
(12.04)\end{array}$ & $\begin{array}{c}59.00 \\
(12.18)\end{array}$ & 30 & $\begin{array}{l}\mathbf{5 5 . 0 0} \\
(11.90)\end{array}$ & $\begin{array}{l}\mathbf{5 1 . 7 7} \\
(11.04)\end{array}$ & 1.50 & 0.22 & 2.19 & 0.14 \\
\hline $\begin{array}{l}\text { T-Wert } \\
\text { Gesamtskala }\end{array}$ & 10 & $\begin{array}{l}61.00 \\
(9.46)\end{array}$ & $\begin{array}{c}57.70 \\
(10.87)\end{array}$ & 30 & $\begin{array}{l}\mathbf{5 9 . 3 0} \\
(8.55)\end{array}$ & $\begin{array}{l}\mathbf{5 5 . 7 0} \\
(9.06)\end{array}$ & 9.36 & 0.00 & 0.34 & 0.56 \\
\hline \multicolumn{11}{|c|}{ Fremdbeurteilungsbogen FBB-HKS (Lehrer) } \\
\hline $\begin{array}{l}\text { Unaufmerksam- } \\
\text { keit: Anzahl an } \\
\text { Kriterien } \\
\end{array}$ & 12 & $\begin{array}{c}4.75 \\
(2.83)\end{array}$ & $\begin{array}{c}4.92 \\
(3.09)\end{array}$ & 26 & $\begin{array}{c}\mathbf{6 . 0 3} \\
(2.44)\end{array}$ & $\begin{array}{c}\mathbf{4 . 0 3} \\
(2.58)\end{array}$ & 1.76 & 0.19 & 0.10 & 0.74 \\
\hline $\begin{array}{l}\text { Unaufmerksam- } \\
\text { keit: Kennwert }\end{array}$ & 12 & $\begin{array}{c}1.62 \\
(0.58)\end{array}$ & $\begin{array}{c}1.58 \\
(0.69)\end{array}$ & 26 & $\begin{array}{c}\mathbf{1 . 8 3} \\
(0.55)\end{array}$ & $\begin{array}{l}\mathbf{1 . 4 3} \\
(0.61)\end{array}$ & 2.18 & 0.14 & 0.05 & 0.81 \\
\hline $\begin{array}{l}\text { Hyperaktivität: } \\
\text { Anzahl an } \\
\text { Kriterien } \\
\end{array}$ & 12 & $\begin{array}{l}2.75 \\
(2.01)\end{array}$ & $\begin{array}{c}1.67 \\
(2.19)\end{array}$ & 26 & $\begin{array}{l}\mathbf{1 . 5 0} \\
(1.47)\end{array}$ & $\begin{array}{c}\mathbf{0 . 6 9} \\
(1.15)\end{array}$ & 5.84 & 0.02 & 7.74 & 0.00 \\
\hline $\begin{array}{l}\text { Hyperaktivität: } \\
\text { Kennwert }\end{array}$ & 12 & $\begin{array}{c}1.63 \\
(0.79) \\
\end{array}$ & $\begin{array}{c}1.10 \\
(0.88)\end{array}$ & 26 & $\begin{array}{c}\mathbf{0 . 9 7} \\
(0.71) \\
\end{array}$ & $\begin{array}{c}\mathbf{0 . 5 4} \\
(0.60)\end{array}$ & 7.77 & 0.00 & 10.98 & 0.00 \\
\hline $\begin{array}{l}\text { Impulsivität: } \\
\text { Anzahl an } \\
\text { Kriterien } \\
\end{array}$ & 12 & $\begin{array}{c}2.42 \\
(1.73)\end{array}$ & $\begin{array}{c}1.67 \\
(1.83)\end{array}$ & 26 & $\begin{array}{c}0.80 \\
(1.29)\end{array}$ & $\begin{array}{c}0.69 \\
(1.37)\end{array}$ & 1.57 & 0.21 & 11.11 & 0.00 \\
\hline $\begin{array}{l}\text { Impulsivität: } \\
\text { Kennwert }\end{array}$ & 12 & $\begin{array}{c}\mathbf{1 . 7 0} \\
(0.94)\end{array}$ & $\begin{array}{c}\mathbf{1 . 2 0} \\
(0.89)\end{array}$ & 26 & $\begin{array}{c}0.72 \\
(0.67)\end{array}$ & $\begin{array}{c}0.65 \\
(0.85) \\
\end{array}$ & 2.78 & 0.10 & 10.98 & 0.00 \\
\hline
\end{tabular}




\begin{tabular}{|c|c|c|c|c|c|c|c|c|c|c|}
\hline \multirow[t]{2}{*}{$\begin{array}{l}\text { Abhängige } \\
\text { Variable }\end{array}$} & \multicolumn{3}{|c|}{ Wartegruppe (WG) } & \multicolumn{3}{|c|}{$\begin{array}{l}\text { Lauth und Schlottke } 15 \\
\text { Sitzungen (Basis- und } \\
\text { Strategietraining) } \\
\end{array}$} & \multicolumn{2}{|c|}{$\begin{array}{c}\text { Haupteffekt } \\
\text { Zeit }\end{array}$} & \multicolumn{2}{|c|}{$\begin{array}{c}\text { Haupteffekt } \\
\text { Gruppe }\end{array}$} \\
\hline & $n$ & Vortest & Nachtest & $n$ & Vortest & Nachtest & $F$ & $\bar{p}$ & $F$ & $\bar{p}$ \\
\hline \multicolumn{11}{|c|}{ Teacher's Report Form (TRF) } \\
\hline $\begin{array}{l}\text { T-Wert } \\
\text { Aufmerksam- } \\
\text { keitsstörungen }\end{array}$ & 7 & $\begin{array}{l}61.86 \\
(4.38)\end{array}$ & $\begin{array}{l}59.86 \\
(5.61)\end{array}$ & 25 & $\begin{array}{l}\mathbf{5 9 . 2 1} \\
(5.49)\end{array}$ & $\begin{array}{l}\mathbf{5 7 . 0 8} \\
(4.32)\end{array}$ & 4.07 & 0.05 & 2.32 & 0.13 \\
\hline $\begin{array}{l}\text { T-Wert } \\
\text { externalisieren- } \\
\text { de Störungen }\end{array}$ & 7 & $\begin{array}{l}65.00 \\
(8.15)\end{array}$ & $\begin{array}{l}65.14 \\
(8.75)\end{array}$ & 25 & $\begin{array}{c}55.04 \\
(10.67)\end{array}$ & $\begin{array}{l}53.42 \\
(9.60)\end{array}$ & 0.28 & 0.59 & 7.40 & 0.01 \\
\hline $\begin{array}{l}\text { T-Wert } \\
\text { Gesamtskala }\end{array}$ & 7 & $\begin{array}{l}60.71 \\
(7.16)\end{array}$ & $\begin{array}{l}61.43 \\
(5.29)\end{array}$ & 25 & $\begin{array}{l}\mathbf{5 8 . 7 6} \\
(6.80)\end{array}$ & $\begin{array}{l}\mathbf{5 5 . 9 2} \\
(5.77)\end{array}$ & 0.88 & 0.35 & 2.48 & 0.12 \\
\hline
\end{tabular}

Anmerkung: p: einseitige Irrtumswahrscheinlichkeit des F-Wertes;

Signifikanzniveau $\alpha=0.05$;

n: Anzahl der Messwertpaare (Die Anzahl umfasst nur diejenigen Kinder, von denen sowohl Vor- als auch Nachtestwert der jeweiligen Variablen vorhanden waren. Auf dieser Anzahl basieren die inferenzstatistischen Auswertungen).

TAP: Testbatterie zur Aufmerksamkeitsprüfung 


\section{Anhang B}

Tabelle B-3

Mittelwerte und Standardabweichungen (in Klammern) im Vor- und Nachtest für die AVn und Kennwerte $(F, p)$ der Haupteffekte Zeit und Gruppe zweifaktorieller Varianzanalysen mit Messwiederholung getrennt für die Wartegruppe und die THOP-Trainingsgruppe

\begin{tabular}{|c|c|c|c|c|c|c|c|c|c|c|}
\hline \multirow[t]{2}{*}{$\begin{array}{l}\text { Abhängige } \\
\text { Variable }\end{array}$} & \multicolumn{3}{|c|}{ Wartegruppe (WG) } & \multicolumn{3}{|c|}{$\begin{array}{c}\text { Trainingsgruppe (TG) } \\
\text { THOP }\end{array}$} & \multicolumn{2}{|c|}{$\begin{array}{c}\text { Haupteffekt } \\
\text { Zeit } \\
\end{array}$} & \multicolumn{2}{|c|}{$\begin{array}{c}\text { Haupteffekt } \\
\text { Gruppe }\end{array}$} \\
\hline & $n$ & Vortest & Nachtest & $n$ & Vortest & Nachtest & $F$ & $p$ & $F$ & $P$ \\
\hline \multicolumn{11}{|c|}{ TAP: Untertest „Geteilte Aufmerksamkeit““ } \\
\hline $\begin{array}{l}\text { Standard- } \\
\text { abweichung }\end{array}$ & 15 & $\begin{array}{c}\mathbf{3 6 4 . 0 5} \\
(100.99) \\
\end{array}$ & $\begin{array}{l}\mathbf{2 9 3 . 6 0} \\
(83.94) \\
\end{array}$ & 29 & $\begin{array}{c}\mathbf{3 3 7 . 8 2} \\
(116.48) \\
\end{array}$ & $\begin{array}{l}\mathbf{2 9 5 . 8 6} \\
(95.81) \\
\end{array}$ & 8.14 & 0.00 & 0.21 & 0.64 \\
\hline Anzahl Fehler & 15 & $\begin{array}{c}\mathbf{8 . 8 0} \\
(7.06) \\
\end{array}$ & $\begin{array}{c}\mathbf{2 . 6 0} \\
(1.45) \\
\end{array}$ & 29 & $\begin{array}{c}\mathbf{9 . 3 8} \\
(13.44) \\
\end{array}$ & $\begin{array}{c}\mathbf{3 . 0 7} \\
(4.03) \\
\end{array}$ & 11.76 & 0.00 & 0.06 & 0.79 \\
\hline $\begin{array}{l}\text { Anzahl } \\
\text { Auslassungen }\end{array}$ & 15 & $\begin{array}{c}8.73 \\
(6.77)\end{array}$ & $\begin{array}{c}6.80 \\
(3.36)\end{array}$ & 29 & $\begin{array}{c}7.10 \\
(5.02)\end{array}$ & $\begin{array}{c}5.28 \\
(4.71)\end{array}$ & 4.07 & 0.05 & 1.47 & 0.23 \\
\hline \multicolumn{11}{|c|}{ TAP: Untertest ,Go/Nogo“6 } \\
\hline $\begin{array}{l}\text { Standard- } \\
\text { abweichung }\end{array}$ & 16 & $\begin{array}{l}146.28 \\
(61.36)\end{array}$ & $\begin{array}{l}148.38 \\
(52.80)\end{array}$ & 31 & $\begin{array}{l}140.54 \\
(42.28)\end{array}$ & $\begin{array}{l}125.59 \\
(42.09)\end{array}$ & 0.54 & 0.46 & 1.45 & 0.23 \\
\hline Anzahl Fehler & 16 & $\begin{array}{c}8.19 \\
(4.79)\end{array}$ & $\begin{array}{c}\mathbf{5 . 7 5} \\
(3.13)\end{array}$ & 31 & $\begin{array}{c}9.81 \\
(5.50)\end{array}$ & $\begin{array}{c}6.97 \\
(5.33)\end{array}$ & 9.77 & 0.00 & 1.21 & 0.27 \\
\hline $\begin{array}{l}\text { Anzahl } \\
\text { Auslassungen }\end{array}$ & 16 & $\begin{array}{c}\mathbf{5 . 0 0} \\
(5.40)\end{array}$ & $\begin{array}{c}\mathbf{2 . 3 8} \\
(3.12)\end{array}$ & 31 & $\begin{array}{l}\mathbf{2 . 1 0} \\
(2.70)\end{array}$ & $\begin{array}{c}\mathbf{0 . 8 4} \\
(1.44)\end{array}$ & 9.65 & 0.00 & 9.47 & 0.00 \\
\hline \multicolumn{11}{|c|}{ TAP: Untertest „Inkompatibilität" } \\
\hline $\begin{array}{l}\text { Standard- } \\
\text { abweichung }\end{array}$ & 15 & $\begin{array}{c}\mathbf{3 2 3 . 8 2} \\
(259.98)\end{array}$ & $\begin{array}{l}\mathbf{1 9 6 . 6 9} \\
(56.45)\end{array}$ & 31 & $\begin{array}{c}\mathbf{2 4 2 . 4 6} \\
(123.11)\end{array}$ & $\begin{array}{l}\mathbf{1 7 6 . 6 3} \\
(64.19)\end{array}$ & 13.90 & 0.00 & 2.34 & 0.13 \\
\hline Anzahl Fehler & 15 & $\begin{array}{l}15.53 \\
(9.12) \\
\end{array}$ & $\begin{array}{l}12.80 \\
(8.03) \\
\end{array}$ & 31 & $\begin{array}{c}\mathbf{2 0 . 4 2} \\
(12.05) \\
\end{array}$ & $\begin{array}{l}\mathbf{1 3 . 5 5} \\
(10.00) \\
\end{array}$ & 9.44 & 0.00 & 0.97 & 0.32 \\
\hline \multicolumn{11}{|c|}{ TAP: Untertest ,Visuelles Scanning“6 } \\
\hline $\begin{array}{l}\text { Standard- } \\
\text { abweichung } \\
\text { kritische Trials }\end{array}$ & 15 & $\begin{array}{c}\mathbf{3 9 5 2 . 5 7} \\
(1864.41)\end{array}$ & $\begin{array}{c}\mathbf{2 3 8 6 . 7 2} \\
(1274.29)\end{array}$ & 31 & $\begin{array}{c}\mathbf{2 4 7 2 . 9 8} \\
(1048.18)\end{array}$ & $\begin{array}{l}\mathbf{2 0 4 9 . 0 0} \\
(995.37)\end{array}$ & 25.31 & 0.00 & 7.39 & 0.00 \\
\hline $\begin{array}{l}\text { Standard- } \\
\text { abweichung } \\
\text { nicht-kritische } \\
\text { Trials }\end{array}$ & 15 & $\begin{array}{c}\mathbf{3 6 8 3 . 6 4} \\
(1374.86)\end{array}$ & $\begin{array}{c}\mathbf{2 6 3 6 . 7 3} \\
(1305.89)\end{array}$ & 31 & $\begin{array}{c}\mathbf{2 7 6 7 . 1 8} \\
(1185.42)\end{array}$ & $\begin{array}{l}\mathbf{2 1 9 2 . 0 3} \\
(1028.39)\end{array}$ & 17.85 & 0.00 & 4.50 & 0.04 \\
\hline $\begin{array}{l}\text { Anzahl Fehler } \\
\text { (kritische } \\
\text { Trials) } \\
\end{array}$ & 15 & $\begin{array}{c}2.33 \\
(5.23)\end{array}$ & $\begin{array}{c}1.47 \\
(2.92)\end{array}$ & 31 & $\begin{array}{c}7.68 \\
(5.95)\end{array}$ & $\begin{array}{c}4.32 \\
(4.10)\end{array}$ & 10.04 & 0.00 & 8.92 & 0.00 \\
\hline $\begin{array}{l}\text { Anzahl } \\
\text { Auslassungen } \\
\text { (nicht-kritische } \\
\text { Trials) }\end{array}$ & 15 & $\begin{array}{c}5.80 \\
(4.50)\end{array}$ & $\begin{array}{c}6.20 \\
(4.95)\end{array}$ & 31 & $\begin{array}{c}\mathbf{5 . 3 2} \\
(5.96)\end{array}$ & $\begin{array}{c}\mathbf{2 . 2 6} \\
(3.26)\end{array}$ & 2.59 & 0.11 & 3.09 & 0.08 \\
\hline \multicolumn{11}{|l|}{ Test d2 } \\
\hline $\begin{array}{l}\text { Konzentrations- } \\
\text { leistungswert }\end{array}$ & 13 & $\begin{array}{c}\mathbf{7 7 . 0 0} \\
(26.59) \\
\end{array}$ & $\begin{array}{c}\mathbf{9 5 . 8 5} \\
(25.78) \\
\end{array}$ & 15 & $\begin{array}{c}\mathbf{9 3 . 9 3} \\
(25.92) \\
\end{array}$ & $\begin{array}{l}\mathbf{1 2 3 . 2 0} \\
(25.27) \\
\end{array}$ & 107.45 & 0.00 & 5.40 & 0.02 \\
\hline Anzahl Fehler & 13 & $\begin{array}{c}\mathbf{3 4 . 8 5} \\
(34.98)\end{array}$ & $\begin{array}{c}\mathbf{1 2 . 1 5} \\
(14.67)\end{array}$ & 15 & $\begin{array}{c}\mathbf{2 6 . 2 7} \\
(37.59)\end{array}$ & $\begin{array}{c}9.27 \\
(6.30)\end{array}$ & 9.79 & 0.00 & 0.51 & 0.47 \\
\hline
\end{tabular}




\begin{tabular}{|c|c|c|c|c|c|c|c|c|c|c|}
\hline \multirow{2}{*}{\begin{tabular}{|l|}
$\begin{array}{l}\text { Abhängige } \\
\text { Variable }\end{array}$ \\
\\
\end{tabular}} & \multicolumn{3}{|c|}{ Wartegruppe (WG) } & \multicolumn{3}{|c|}{$\begin{array}{c}\text { Trainingsgruppe (TG) } \\
\text { THOP }\end{array}$} & \multicolumn{2}{|c|}{$\begin{array}{c}\text { Haupteffekt } \\
\text { Zeit }\end{array}$} & \multicolumn{2}{|c|}{$\begin{array}{c}\text { Haupteffekt } \\
\text { Gruppe }\end{array}$} \\
\hline & $n$ & Vortest & Nachtest & $n$ & Vortest & Nachtest & $F$ & $p$ & $F$ & $P$ \\
\hline \multicolumn{11}{|l|}{ Mottier-Test } \\
\hline $\begin{array}{l}\text { Nachsprechleist } \\
\text { ung Gesamt } \\
\text { (max. 30) } \\
\end{array}$ & 15 & $\begin{array}{l}20.80 \\
(3.97)\end{array}$ & $\begin{array}{l}20.40 \\
(5.01)\end{array}$ & 30 & $\begin{array}{l}22.20 \\
(4.00)\end{array}$ & $\begin{array}{l}\mathbf{2 3 . 5 7} \\
(3.79)\end{array}$ & 0.99 & 0.32 & 3.58 & 0.06 \\
\hline $\begin{array}{l}\text { Nachsprechleist } \\
\text { ung für 2- und } \\
\text { 3-silbige } \\
\text { Kunstwörter } \\
\text { (max. 12) }\end{array}$ & 15 & $\begin{array}{l}11.07 \\
(1.53)\end{array}$ & $\begin{array}{l}10.20 \\
(2.21)\end{array}$ & 30 & $\begin{array}{l}\mathbf{1 1 . 1 7} \\
(1.02)\end{array}$ & $\begin{array}{c}\mathbf{1 1 . 7 0} \\
(.60)\end{array}$ & 0.34 & 0.55 & 7.51 & 0.01 \\
\hline $\begin{array}{l}\text { Nachsprechleist } \\
\text { ung für 4-, 5- } \\
\text { und 6-silbige } \\
\text { Kunstwörter } \\
\text { (max. 18) }\end{array}$ & 15 & $\begin{array}{c}9.73 \\
(3.17)\end{array}$ & $\begin{array}{l}10.20 \\
(3.86)\end{array}$ & 30 & $\begin{array}{l}\mathbf{1 1 . 0 3} \\
(3.40)\end{array}$ & $\begin{array}{l}\mathbf{1 1 . 8 7} \\
(3.46)\end{array}$ & 3.18 & 0.08 & 2.06 & 0.15 \\
\hline \multicolumn{11}{|c|}{ Fremdbeurteilungsbogen FBB-HKS (Eltern) } \\
\hline $\begin{array}{l}\text { Unaufmerksam- } \\
\text { keit: Anzahl an } \\
\text { Kriterien } \\
\end{array}$ & 11 & $\begin{array}{l}4.91 \\
(3.11)\end{array}$ & $\begin{array}{c}4.45 \\
(4.06)\end{array}$ & 30 & $\begin{array}{c}\mathbf{5 . 9 3} \\
(2.58)\end{array}$ & $\begin{array}{l}\mathbf{4 . 5 0} \\
(2.56)\end{array}$ & 2.78 & 0.10 & 0.40 & 0.52 \\
\hline $\begin{array}{l}\text { Unaufmerksam- } \\
\text { keit: Kennwert }\end{array}$ & 11 & $\begin{array}{c}1.64 \\
(0.62)\end{array}$ & $\begin{array}{l}1.58 \\
(0.87)\end{array}$ & 30 & $\begin{array}{l}\mathbf{1 . 8 5} \\
(0.58) \\
\end{array}$ & $\begin{array}{l}\mathbf{1 . 4 8} \\
(0.57)\end{array}$ & 3.16 & 0.21 & 0.10 & 0.75 \\
\hline $\begin{array}{l}\text { Hyperaktivität: } \\
\text { Anzahl an } \\
\text { Kriterien } \\
\end{array}$ & 11 & $\begin{array}{c}2.72 \\
(1.68)\end{array}$ & $\begin{array}{c}2.36 \\
(2.38)\end{array}$ & 30 & $\begin{array}{c}\mathbf{2 . 4 3} \\
(1.79)\end{array}$ & $\begin{array}{c}\mathbf{1 . 7 0} \\
(1.58)\end{array}$ & 2.98 & 0.09 & 0.76 & 0.38 \\
\hline $\begin{array}{l}\text { Hyperaktivität: } \\
\text { Kennwert }\end{array}$ & 11 & $\begin{array}{c}1.63 \\
(0.70) \\
\end{array}$ & $\begin{array}{c}1.29 \\
(0.76) \\
\end{array}$ & 30 & $\begin{array}{c}\mathbf{1 . 4 8} \\
(0.85) \\
\end{array}$ & $\begin{array}{c}1.14 \\
(0.66) \\
\end{array}$ & 6.73 & 0.01 & 0.41 & 0.52 \\
\hline $\begin{array}{l}\text { Impulsivität: } \\
\text { Anzahl an } \\
\text { Kriterien }\end{array}$ & 11 & $\begin{array}{c}1.45 \\
(1.75)\end{array}$ & $\begin{array}{c}1.27 \\
(1.79)\end{array}$ & 30 & $\begin{array}{c}\mathbf{2 . 4 0} \\
(1.61)\end{array}$ & $\begin{array}{c}\mathbf{2 . 0 0} \\
(1.43)\end{array}$ & 1.01 & 0.32 & 3.01 & 0.09 \\
\hline $\begin{array}{l}\text { Impulsivität: } \\
\text { Kennwert }\end{array}$ & 11 & $\begin{array}{c}1.18 \\
(0.69) \\
\end{array}$ & $\begin{array}{c}1.15 \\
(0.78) \\
\end{array}$ & 30 & $\begin{array}{c}1.61 \\
(0.93) \\
\end{array}$ & $\begin{array}{c}1.50 \\
(0.81) \\
\end{array}$ & 0.31 & 0.57 & 2.18 & 0.14 \\
\hline \multicolumn{11}{|c|}{ Child Behavior Checklist (CBCL) } \\
\hline $\begin{array}{l}\text { T-Wert } \\
\text { Aufmerksam- } \\
\text { keitsstörungen } \\
\end{array}$ & 10 & $\begin{array}{l}64.50 \\
(6.89)\end{array}$ & $\begin{array}{l}61.60 \\
(8.63)\end{array}$ & 30 & $\begin{array}{l}\mathbf{6 6 . 2 7} \\
(6.70)\end{array}$ & $\begin{array}{l}\mathbf{6 0 . 5 7} \\
(6.67)\end{array}$ & 9.70 & 0.00 & 0.02 & 0.86 \\
\hline $\begin{array}{l}\text { T-Wert } \\
\text { externalisieren- } \\
\text { de Störungen }\end{array}$ & 10 & $\begin{array}{c}59.50 \\
(12.04)\end{array}$ & $\begin{array}{c}59.00 \\
(12.18)\end{array}$ & 30 & $\begin{array}{c}\mathbf{6 5 . 4 7} \\
(11.47)\end{array}$ & $\begin{array}{l}\mathbf{5 9 . 9 0} \\
(9.95)\end{array}$ & 3.74 & 0.06 & 0.84 & 0.36 \\
\hline $\begin{array}{l}\text { T-Wert } \\
\text { Gesamtskala }\end{array}$ & 10 & $\begin{array}{l}61.00 \\
(9.46)\end{array}$ & $\begin{array}{c}57.70 \\
(10.87)\end{array}$ & 30 & $\begin{array}{l}\mathbf{6 5 . 9 3} \\
(7.93)\end{array}$ & $\begin{array}{l}\mathbf{5 9 . 9 0} \\
(8.24)\end{array}$ & 12.18 & 0.00 & 1.51 & 0.21 \\
\hline \multicolumn{11}{|c|}{ Fremdbeurteilungsbogen FBB-HKS (Lehrer) } \\
\hline $\begin{array}{l}\text { Unaufmerksam- } \\
\text { keit: Anzahl an } \\
\text { Kriterien } \\
\end{array}$ & 12 & $\begin{array}{c}4.75 \\
(2.83)\end{array}$ & $\begin{array}{c}4.92 \\
(3.09)\end{array}$ & 23 & $\begin{array}{l}\mathbf{4 . 8 6} \\
(2.51)\end{array}$ & $\begin{array}{c}3.91 \\
(2.29)\end{array}$ & 0.43 & 0.51 & 0.38 & 0.53 \\
\hline $\begin{array}{l}\text { Unaufmerksam- } \\
\text { keit: Kennwert }\end{array}$ & 12 & $\begin{array}{c}1.62 \\
(0.58)\end{array}$ & $\begin{array}{c}1.58 \\
(0.69) \\
\end{array}$ & 23 & $\begin{array}{c}\mathbf{1 . 7 1} \\
(0.63) \\
\end{array}$ & $\begin{array}{l}\mathbf{1 . 4 4} \\
(0.48) \\
\end{array}$ & 1.40 & 0.24 & 0.01 & 0.89 \\
\hline $\begin{array}{l}\text { Hyperaktivität: } \\
\text { Anzahl an } \\
\text { Kriterien }\end{array}$ & 12 & $\begin{array}{l}2.75 \\
(2.01)\end{array}$ & $\begin{array}{c}1.67 \\
(2.19)\end{array}$ & 23 & $\begin{array}{c}2.08 \\
(1.90)\end{array}$ & $\begin{array}{l}1.47 \\
(1.59)\end{array}$ & 5.02 & 0.03 & 0.59 & 0.44 \\
\hline $\begin{array}{l}\text { Hyperaktivität: } \\
\text { Kennwert }\end{array}$ & 12 & $\begin{array}{c}1.63 \\
(0.79) \\
\end{array}$ & $\begin{array}{c}1.10 \\
(0.88) \\
\end{array}$ & 23 & $\begin{array}{c}1.26 \\
(0.94) \\
\end{array}$ & $\begin{array}{c}1.04 \\
(0.79) \\
\end{array}$ & 4.79 & 0.03 & 0.70 & 0.40 \\
\hline $\begin{array}{l}\text { Impulsivität: } \\
\text { Anzahl an } \\
\text { Kriterien }\end{array}$ & 12 & $\begin{array}{c}2.42 \\
(1.73)\end{array}$ & $\begin{array}{c}1.67 \\
(1.83)\end{array}$ & 23 & $\begin{array}{l}1.86 \\
(1.63)\end{array}$ & $\begin{array}{c}1.56 \\
(1.70)\end{array}$ & 1.84 & 0.18 & 0.48 & 0.49 \\
\hline $\begin{array}{l}\text { Impulsivität: } \\
\text { Kennwert }\end{array}$ & 12 & $\begin{array}{c}1.70 \\
(0.94) \\
\end{array}$ & $\begin{array}{l}\mathbf{1 . 2 0} \\
(0.89) \\
\end{array}$ & 23 & $\begin{array}{c}1.34 \\
(0.81)\end{array}$ & $\begin{array}{c}1.28 \\
(0.92) \\
\end{array}$ & 2.37 & 0.13 & 0.26 & 0.61 \\
\hline
\end{tabular}




\begin{tabular}{|c|c|c|c|c|c|c|c|c|c|c|}
\hline \multirow{2}{*}{\begin{tabular}{|l|}
$\begin{array}{l}\text { Abhängige } \\
\text { Variable }\end{array}$ \\
\end{tabular}} & \multicolumn{3}{|c|}{ Wartegruppe (WG) } & \multicolumn{3}{|c|}{$\begin{array}{c}\text { Trainingsgruppe (TG) } \\
\text { THOP }\end{array}$} & \multicolumn{2}{|c|}{$\begin{array}{c}\text { Haupteffekt } \\
\text { Zeit }\end{array}$} & \multicolumn{2}{|c|}{$\begin{array}{c}\text { Haupteffekt } \\
\text { Gruppe }\end{array}$} \\
\hline & $n$ & Vortest & Nachtest & $n$ & Vortest & Nachtest & $F$ & $p$ & $F$ & $P$ \\
\hline \multicolumn{11}{|c|}{ Teacher's Report Form (TRF) } \\
\hline $\begin{array}{l}\text { T-Wert } \\
\text { Aufmerksam- } \\
\text { keitsstörungen }\end{array}$ & 7 & $\begin{array}{l}61.86 \\
(4.38)\end{array}$ & $\begin{array}{l}59.86 \\
(5.61)\end{array}$ & 22 & $\begin{array}{l}\mathbf{6 2 . 1 8} \\
(6.61)\end{array}$ & $\begin{array}{l}\mathbf{5 8 . 6 4} \\
(5.74)\end{array}$ & 7.21 & 0.12 & 0.03 & 0.85 \\
\hline $\begin{array}{l}\text { T-Wert } \\
\text { externalisieren- } \\
\text { de Störungen }\end{array}$ & 7 & $\begin{array}{l}65.00 \\
(8.15)\end{array}$ & $\begin{array}{l}65.14 \\
(8.75)\end{array}$ & 22 & $\begin{array}{l}66.36 \\
(8.68)\end{array}$ & $\begin{array}{l}63.64 \\
(8.78)\end{array}$ & 0.48 & 0.49 & 0.00 & 0.98 \\
\hline $\begin{array}{l}\text { T-Wert } \\
\text { Gesamtskala }\end{array}$ & 7 & $\begin{array}{l}60.71 \\
(7.16)\end{array}$ & $\begin{array}{l}61.43 \\
(5.29)\end{array}$ & 22 & $\begin{array}{l}\mathbf{6 5 . 6 4} \\
(7.92)\end{array}$ & $\begin{array}{l}\mathbf{6 2 . 0 9} \\
(7.52)\end{array}$ & 1.22 & 0.20 & 0.89 & 0.35 \\
\hline
\end{tabular}

Anmerkung: p: einseitige Irrtumswahrscheinlichkeit des F-Wertes;

Signifikanzniveau $\alpha=0.05$;

n: Anzahl der Messwertpaare (Die Anzahl umfasst nur diejenigen Kinder, von denen sowohl Vor- als auch Nachtestwert der jeweiligen Variablen vorhanden waren. Auf dieser Anzahl basieren die inferenzstatistischen Auswertungen).

TAP: Testbatterie zur Aufmerksamkeitsprüfung 


\section{Anhang B}

Tabelle B-4

Mittelwerte und Standardabweichungen (in Klammern) im Vor- und Nachtest für die AVn und Kennwerte $(F, p)$ der Haupteffekte Zeit und Gruppe zweifaktorieller Varianzanalysen mit Messwiederholung getrennt für das Pseudotraining „Petterson und Findus “ und das Marburger Konzentrationstraining

\begin{tabular}{|c|c|c|c|c|c|c|c|c|c|c|}
\hline \multirow[t]{2}{*}{$\begin{array}{l}\text { Abhängige } \\
\text { Variable }\end{array}$} & \multicolumn{3}{|c|}{$\begin{array}{l}\text { Petterson und Findus } \\
\text { (PF) }\end{array}$} & \multicolumn{3}{|c|}{$\begin{array}{c}\text { Marburger } \\
\text { Konzentrationstraining } \\
\text { (MK) } \\
\end{array}$} & \multicolumn{2}{|c|}{$\begin{array}{c}\text { Haupteffekt } \\
\text { Zeit }\end{array}$} & \multicolumn{2}{|c|}{$\begin{array}{c}\text { Haupteffekt } \\
\text { Gruppe }\end{array}$} \\
\hline & $n$ & Vortest & Nachtest & $n$ & Vortest & Nachtest & $F$ & $p$ & $F$ & $p$ \\
\hline \multicolumn{11}{|c|}{ TAP: Untertest „Geteilte Aufmerksamkeit" } \\
\hline $\begin{array}{l}\text { Standard- } \\
\text { abweichung }\end{array}$ & 12 & $\begin{array}{l}324.51 \\
(86.63)\end{array}$ & $\begin{array}{l}269.44 \\
(86.32)\end{array}$ & 15 & $\begin{array}{c}275.59 \\
(129.44)\end{array}$ & $\begin{array}{l}241.21 \\
(73.63)\end{array}$ & 2.99 & 0.96 & 1.96 & 0.17 \\
\hline Anzahl Fehler & 12 & $\begin{array}{c}7.08 \\
(5.99) \\
\end{array}$ & $\begin{array}{c}5.00 \\
(10.46) \\
\end{array}$ & 15 & $\begin{array}{r}3.80 \\
(6.04) \\
\end{array}$ & $\begin{array}{c}4.27 \\
(4.22) \\
\end{array}$ & 0.44 & 0.51 & 0.72 & 0.40 \\
\hline $\begin{array}{l}\text { Anzahl } \\
\text { Auslassungen }\end{array}$ & 12 & $\begin{array}{r}6.33 \\
(4.81) \\
\end{array}$ & $\begin{array}{r}6.25 \\
(4.83) \\
\end{array}$ & 15 & $\begin{array}{r}7.73 \\
(5.12) \\
\end{array}$ & $\begin{array}{r}6.80 \\
(4.49) \\
\end{array}$ & 0.39 & 0.53 & 0.33 & 0.56 \\
\hline \multicolumn{11}{|c|}{ TAP: Untertest ,Go/Nogo“6 } \\
\hline $\begin{array}{l}\text { Standard- } \\
\text { abweichung }\end{array}$ & 14 & $\begin{array}{r}\mathbf{3 2 4 . 9 7} \\
(272.30) \\
\end{array}$ & $\begin{array}{r}\mathbf{1 2 0 . 1 3} \\
(47.75) \\
\end{array}$ & 15 & $\begin{array}{c}208.69 \\
(160.59) \\
\end{array}$ & $\begin{array}{l}143.85 \\
(43.75) \\
\end{array}$ & 8.93 & 0.00 & 1.43 & 0.24 \\
\hline Anzahl Fehler & 14 & $\begin{array}{l}11.29 \\
(5.76) \\
\end{array}$ & $\begin{array}{r}9.86 \\
(6.64) \\
\end{array}$ & 15 & $\begin{array}{r}9.53 \\
(4.79) \\
\end{array}$ & $\begin{array}{l}10.00 \\
(6.23) \\
\end{array}$ & 0.15 & 0.69 & 0.19 & 0.66 \\
\hline $\begin{array}{l}\text { Anzahl } \\
\text { Auslassungen }\end{array}$ & 14 & $\begin{array}{c}3.86 \\
(3.76) \\
\end{array}$ & $\begin{array}{c}2.71 \\
(3.52) \\
\end{array}$ & 15 & $\begin{array}{c}2.80 \\
(3.10) \\
\end{array}$ & $\begin{array}{c}1.80 \\
(1.90) \\
\end{array}$ & 2.88 & 0.10 & 1.01 & 0.32 \\
\hline \multicolumn{11}{|c|}{ TAP: Untertest „Inkompatibilität" } \\
\hline $\begin{array}{l}\text { Standard- } \\
\text { abweichung }\end{array}$ & 13 & $\begin{array}{c}209.69 \\
(138.82) \\
\end{array}$ & $\begin{array}{c}223.80 \\
(208.39) \\
\end{array}$ & 15 & $\begin{array}{l}179.11 \\
(80.37) \\
\end{array}$ & $\begin{array}{c}210.81 \\
(230.13) \\
\end{array}$ & 0.27 & 0.60 & 0.19 & 0.66 \\
\hline Anzahl Fehler & 13 & $\begin{array}{c}16.38 \\
(14.09) \\
\end{array}$ & $\begin{array}{c}14.77 \\
(15.08) \\
\end{array}$ & 15 & $\begin{array}{c}16.27 \\
(10.37) \\
\end{array}$ & $\begin{array}{l}12.80 \\
(9.53) \\
\end{array}$ & 0.95 & 0.33 & 0.07 & 0.79 \\
\hline \multicolumn{11}{|c|}{ TAP: Untertest „,Visuelles Scanning } \\
\hline $\begin{array}{l}\text { Standard- } \\
\text { abweichung } \\
\text { kritische Trials }\end{array}$ & 10 & $\begin{array}{l}2187.58 \\
(765.10)\end{array}$ & $\begin{array}{c}1456.13 \\
(1118.98)\end{array}$ & 11 & $\begin{array}{c}\mathbf{3 1 4 2 . 8 0} \\
(2312.28)\end{array}$ & $\begin{array}{l}\mathbf{1 7 7 5 . 0 0} \\
(603.20)\end{array}$ & 6.87 & 0.16 & 1.60 & 0.21 \\
\hline $\begin{array}{l}\text { Standard- } \\
\text { abweichung } \\
\text { nicht-kritische } \\
\text { Trials }\end{array}$ & 10 & $\begin{array}{l}\mathbf{2 1 2 5 . 9 2} \\
(767.85)\end{array}$ & $\begin{array}{l}\mathbf{1 4 4 1 . 8 5} \\
(1106.18)\end{array}$ & 11 & $\begin{array}{l}\mathbf{2 1 1 8 . 4 6} \\
(831.97)\end{array}$ & $\begin{array}{l}\mathbf{1 4 8 4 . 1 5} \\
(419.35)\end{array}$ & 14.16 & 0.00 & 0.00 & 0.95 \\
\hline $\begin{array}{l}\text { Anzahl Fehler } \\
\text { (kritische } \\
\text { Trials) } \\
\end{array}$ & 10 & $\begin{array}{c}4.80 \\
(2.20)\end{array}$ & $\begin{array}{c}7.80 \\
(5.07)\end{array}$ & 11 & $\begin{array}{c}6.45 \\
(3.47)\end{array}$ & $\begin{array}{c}3.45 \\
(2.25)\end{array}$ & 0.25 & 0.62 & 0.12 & 0.72 \\
\hline $\begin{array}{l}\text { Anzahl } \\
\text { Auslassungen } \\
\text { (nicht-kritische } \\
\text { Trials) }\end{array}$ & 10 & $\begin{array}{c}1.90 \\
(1.79)\end{array}$ & $\begin{array}{l}1.40 \\
(2.17)\end{array}$ & 11 & $\begin{array}{c}1.55 \\
(1.13)\end{array}$ & $\begin{array}{c}0.45 \\
(0.69)\end{array}$ & 0.03 & 0.86 & 0.10 & 0.75 \\
\hline \multicolumn{11}{|l|}{ Test d2 } \\
\hline $\begin{array}{l}\text { Konzentrations- } \\
\text { leistungswert }\end{array}$ & 14 & $\begin{array}{c}92.79 \\
(13.74) \\
\end{array}$ & $\begin{array}{l}\mathbf{1 1 8 . 2 9} \\
(16.89) \\
\end{array}$ & 15 & $\begin{array}{l}\mathbf{1 0 7 . 8 0} \\
(26.56) \\
\end{array}$ & $\begin{array}{l}\mathbf{1 3 7 , 9 3} \\
(33.38) \\
\end{array}$ & 91.26 & 0.00 & 3.86 & 0.06 \\
\hline Anzahl Fehler & 14 & $\begin{array}{l}11.43 \\
(1.07) \\
\end{array}$ & $\begin{array}{l}10.41 \\
(7.14) \\
\end{array}$ & 15 & $\begin{array}{c}\mathbf{1 4 . 4 0} \\
(12.11) \\
\end{array}$ & $\begin{array}{c}\mathbf{5 . 6 7} \\
(4.10) \\
\end{array}$ & 12.17 & 0.00 & 0.08 & 0.77 \\
\hline \multicolumn{11}{|c|}{ Fremdbeurteilungsbogen FBB-HKS (Eltern) } \\
\hline $\begin{array}{l}\text { Unaufmerksam- } \\
\text { keit: Kennwert }\end{array}$ & 4 & $\begin{array}{c}\mathbf{2 . 0 2} \\
(0.50) \\
\end{array}$ & $\begin{array}{c}\mathbf{1 . 3 0} \\
(0.54) \\
\end{array}$ & 2 & $\begin{array}{c}1.45 \\
(1.76) \\
\end{array}$ & $\begin{array}{c}1.40 \\
(1.83) \\
\end{array}$ & 3.51 & 0.13 & 0.07 & 0.79 \\
\hline $\begin{array}{l}\text { Hyperaktivität: } \\
\text { Kennwert }\end{array}$ & 4 & $\begin{array}{r}1.45 \\
(1.03) \\
\end{array}$ & $\begin{array}{l}0.85 \\
(.41) \\
\end{array}$ & 2 & $\begin{array}{c}2.10 \\
(1.27) \\
\end{array}$ & $\begin{array}{l}2.10 \\
(0.70) \\
\end{array}$ & 1.00 & 0.37 & 1.96 & 0.23 \\
\hline
\end{tabular}




\begin{tabular}{|c|c|c|c|c|c|c|c|c|c|c|}
\hline \multirow[t]{2}{*}{$\begin{array}{l}\text { Abhängige } \\
\text { Variable }\end{array}$} & \multicolumn{3}{|c|}{$\begin{array}{l}\text { Petterson und Findus } \\
\text { (PF) }\end{array}$} & \multicolumn{3}{|c|}{$\begin{array}{c}\text { Marburger } \\
\text { Konzentrationstraining } \\
\text { (MK) }\end{array}$} & \multicolumn{2}{|c|}{$\begin{array}{c}\text { Haupteffekt } \\
\text { Zeit }\end{array}$} & \multicolumn{2}{|c|}{$\begin{array}{c}\text { Haupteffekt } \\
\text { Gruppe }\end{array}$} \\
\hline & $n$ & Vortest & Nachtest & $n$ & Vortest & Nachtest & $F$ & $p$ & $F$ & $p$ \\
\hline $\begin{array}{l}\text { Impulsivität: } \\
\text { Kennwert }\end{array}$ & 4 & $\begin{array}{c}1.47 \\
(0.96)\end{array}$ & $\begin{array}{c}1.10 \\
(0.62)\end{array}$ & 2 & $\begin{array}{l}1.50 \\
(2.12)\end{array}$ & $\begin{array}{c}1.40 \\
(1.97)\end{array}$ & 1.52 & 0.28 & 0.02 & 0.88 \\
\hline \multicolumn{11}{|c|}{ Child Behavior Checklist (CBCL) } \\
\hline $\begin{array}{l}\text { T-Wert } \\
\text { Aufmerksam- } \\
\text { keitsstörungen } \\
\end{array}$ & 5 & $\begin{array}{l}69.00 \\
(8.75)\end{array}$ & $\begin{array}{l}64.80 \\
(6.53)\end{array}$ & 3 & $\begin{array}{l}59.50 \\
(7.78)\end{array}$ & $\begin{array}{l}54.00 \\
(4.24)\end{array}$ & 1.63 & 0.24 & 0.08 & 0.78 \\
\hline $\begin{array}{l}\text { T-Wert } \\
\text { externalisieren- } \\
\text { de Störungen }\end{array}$ & 5 & $\begin{array}{l}60.60 \\
(4.67)\end{array}$ & $\begin{array}{l}56.20 \\
(6.72)\end{array}$ & 3 & $\begin{array}{c}48.50 \\
(14.85)\end{array}$ & $\begin{array}{c}47.00 \\
(16.97)\end{array}$ & 1.10 & 0.33 & 0.01 & 0.90 \\
\hline $\begin{array}{l}\text { T-Wert } \\
\text { Gesamtskala }\end{array}$ & 5 & $\begin{array}{l}64.20 \\
(8.35)\end{array}$ & $\begin{array}{l}59.60 \\
(8.05)\end{array}$ & 3 & $\begin{array}{c}53.00 \\
(11.31)\end{array}$ & $\begin{array}{l}53.00 \\
(0.00)\end{array}$ & 0.17 & 0.69 & 4.93 & 0.07 \\
\hline \multicolumn{11}{|l|}{ CFT } \\
\hline IQ Teil 1 & 14 & $\begin{array}{l}\mathbf{9 7 . 6 4} \\
(4.36) \\
\end{array}$ & $\begin{array}{l}\mathbf{1 0 7 . 5 7} \\
8.95() \\
\end{array}$ & 15 & $\begin{array}{l}\mathbf{1 0 7 . 1 3} \\
(12.69) \\
\end{array}$ & \begin{tabular}{|l|}
$\mathbf{1 1 2 . 9 3}$ \\
$(10.40)$ \\
\end{tabular} & 34.61 & 0.00 & 4.94 & 0.03 \\
\hline IQ Teil 2 & 14 & $\begin{array}{c}\mathbf{9 8 . 4 3} \\
(12.16) \\
\end{array}$ & $\begin{array}{l}\mathbf{1 0 2 . 1 4} \\
(11.27) \\
\end{array}$ & 15 & $\begin{array}{l}\mathbf{1 0 3 . 4 0} \\
(9.68) \\
\end{array}$ & $\begin{array}{l}\mathbf{1 1 2 . 0 0} \\
(11.92) \\
\end{array}$ & 14.61 & 0.00 & 3.66 & 0.06 \\
\hline IQ Gesamt & 14 & $\begin{array}{l}\mathbf{9 6 . 4 3} \\
(7.67) \\
\end{array}$ & $\begin{array}{l}\mathbf{1 0 4 . 9 3} \\
(10.80) \\
\end{array}$ & 15 & $\begin{array}{l}\mathbf{1 0 5 . 2 0} \\
(11.12) \\
\end{array}$ & $\begin{array}{l}\mathbf{1 1 3 . 3 3} \\
(11.49) \\
\end{array}$ & 40.57 & 0.00 & 5.55 & 0.02 \\
\hline $\begin{array}{l}\text { Differenz Teil } 1 \\
\text { minus Teil } 2\end{array}$ & 14 & $\begin{array}{c}\mathbf{- 0 . 7 8} \\
(11.43) \\
\end{array}$ & $\begin{array}{c}\mathbf{5 . 4 2} \\
(7.83) \\
\end{array}$ & 15 & $\begin{array}{c}3.73 \\
(10.72) \\
\end{array}$ & $\begin{array}{c}0.93 \\
(8.40) \\
\end{array}$ & 0.74 & 0.39 & 0.00 & 0.99 \\
\hline \multicolumn{11}{|c|}{ HAWIK-III (Zahlensymboltest) } \\
\hline $\begin{array}{l}\text { Bearbeitete } \\
\text { Zeichen } \\
\end{array}$ & 14 & $\begin{array}{l}35.86 \\
(8.98) \\
\end{array}$ & $\begin{array}{l}38.64 \\
(9.63) \\
\end{array}$ & 15 & $\begin{array}{c}\mathbf{4 1 . 1 3} \\
(11.06) \\
\end{array}$ & $\begin{array}{l}46.80 \\
(9.84) \\
\end{array}$ & 11.92 & 0.00 & 3.72 & 0.06 \\
\hline \multicolumn{11}{|l|}{ TPK } \\
\hline Silbenzahl & 14 & $\begin{array}{c}95.21 \\
(40.32) \\
\end{array}$ & $\begin{array}{l}105.64 \\
(46.52) \\
\end{array}$ & 15 & $\begin{array}{l}\mathbf{1 1 4 . 4 7} \\
(39.45) \\
\end{array}$ & $\begin{array}{l}\mathbf{1 4 5 . 4 0} \\
(50.34) \\
\end{array}$ & 29.17 & 0.00 & 3.37 & 0.07 \\
\hline $\begin{array}{l}\text { Silbenzahl } \\
\text { Fehler }\end{array}$ & 14 & $\begin{array}{c}6.39 \\
(7.25)\end{array}$ & $\begin{array}{l}12.76 \\
(28.84)\end{array}$ & 15 & $\begin{array}{c}6.38 \\
(5.75)\end{array}$ & $\begin{array}{c}6.20 \\
(6.02)\end{array}$ & 0.62 & 0.43 & 0.65 & 0.42 \\
\hline Tiere & 14 & $\begin{array}{l}\mathbf{1 2 . 7 9} \\
(4.39) \\
\end{array}$ & $\begin{array}{l}\mathbf{1 6 . 1 4} \\
(3.92) \\
\end{array}$ & 15 & $\begin{array}{l}14.00 \\
(3.48) \\
\end{array}$ & $\begin{array}{l}\mathbf{1 7 . 6 0} \\
(4.00) \\
\end{array}$ & 30.06 & 0.00 & 1.01 & 0.32 \\
\hline Rechenleistung & 14 & $\begin{array}{c}31.64 \\
(15.97)\end{array}$ & $\begin{array}{c}33.79 \\
(14.86)\end{array}$ & 15 & $\begin{array}{c}\mathbf{4 4 . 8 0} \\
(23.33)\end{array}$ & $\begin{array}{c}\mathbf{5 1 . 8 7} \\
(29.76)\end{array}$ & 6.60 & 0.16 & 3.82 & 0.06 \\
\hline Rechnen Fehler & 14 & $\begin{array}{c}23.00 \\
(19.94) \\
\end{array}$ & $\begin{array}{c}19.05 \\
(18.66) \\
\end{array}$ & 15 & $\begin{array}{c}16.58 \\
(17.48) \\
\end{array}$ & $\begin{array}{c}16.69 \\
(15.39) \\
\end{array}$ & 0.33 & 0.56 & 0.57 & 0.45 \\
\hline $\begin{array}{l}\text { Konzentrations- } \\
\text { wert }\end{array}$ & 14 & $\begin{array}{c}79.57 \\
(14.52)\end{array}$ & $\begin{array}{c}\mathbf{8 7 . 3 6} \\
(13.25)\end{array}$ & 15 & $\begin{array}{c}\mathbf{8 3 . 9 3} \\
(13.72)\end{array}$ & $\begin{array}{c}\mathbf{9 4 . 4 7} \\
(15.11)\end{array}$ & 21.01 & 0.00 & 1.38 & 0.25 \\
\hline
\end{tabular}

Anmerkung: p: einseitige Irrtumswahrscheinlichkeit des F-Wertes;

Signifikanzniveau $\alpha=0.05$;

n: Anzahl der Messwertpaare (Die Anzahl umfasst nur diejenigen Kinder, von denen sowohl Vor- als auch Nachtestwert der jeweiligen Variablen vorhanden waren. Auf dieser Anzahl basieren die inferenzstatistischen Auswertungen).

TAP: Testbatterie zur Aufmerksamkeitsprüfung 


\section{Anhang B}

Tabelle B-5

Mittelwerte und Standardabweichungen (in Klammern) im Vor- und Nachtest für die AVn und Kennwerte $(F, p)$ der Haupteffekte Zeit und Gruppe zweifaktorieller Varianzanalysen mit Messwiederholung getrennt für das Basistraining nach Lauth- und Schlottke (10 Sitzungen) und das kombinierte Basis- und Strategietraining nach Lauth und Schlottke (15 Sitzungen)

\begin{tabular}{|c|c|c|c|c|c|c|c|c|c|c|}
\hline \multirow[t]{2}{*}{$\begin{array}{c}\text { Abhängige } \\
\text { Variable }\end{array}$} & \multicolumn{3}{|c|}{$\begin{array}{l}\text { Basistraining nach } \\
\text { Lauth und Schlottke } \\
10 \text { Sitzungen }\end{array}$} & \multicolumn{3}{|c|}{$\begin{array}{c}\text { Basis- und } \\
\text { Strategietraining } \\
\text { Lauth und Schlottke } \\
15 \text { Sitzungen }\end{array}$} & \multicolumn{2}{|c|}{$\begin{array}{c}\text { Haupteffekt } \\
\text { Zeit }\end{array}$} & \multicolumn{2}{|c|}{$\begin{array}{l}\text { Haupteffekt } \\
\text { Gruppe }\end{array}$} \\
\hline & $n$ & Vortest & Nachtest & $n$ & Vortest & Nachtest & $F$ & $p$ & $F$ & $p$ \\
\hline \multicolumn{11}{|c|}{ TAP: Untertest „Geteilte Aufmerksamkeit““ } \\
\hline $\begin{array}{l}\text { Standard- } \\
\text { abweichung }\end{array}$ & 14 & $\begin{array}{l}331.74 \\
(74.95)\end{array}$ & $\begin{array}{l}316.96 \\
(75.04)\end{array}$ & 29 & $\begin{array}{c}\mathbf{3 4 8 . 0 1} \\
(103.47)\end{array}$ & $\begin{array}{l}\mathbf{2 9 5 . 9 1} \\
(88.70)\end{array}$ & 3.29 & 0.07 & 0.01 & 0.91 \\
\hline Anzahl Fehler & 14 & $\begin{array}{c}\mathbf{8 . 4 3} \\
(8.38)\end{array}$ & $\begin{array}{c}\mathbf{2 . 5 0} \\
(1.70)\end{array}$ & 29 & $\begin{array}{c}\mathbf{9 . 0 3} \\
(13.53)\end{array}$ & $\begin{array}{c}\mathbf{2 . 9 7} \\
(3.46)\end{array}$ & 10.11 & 0.00 & 0.06 & 0.80 \\
\hline $\begin{array}{l}\text { Anzahl } \\
\text { Auslassungen }\end{array}$ & 14 & $\begin{array}{c}\mathbf{6 . 7 9} \\
(3.93)\end{array}$ & $\begin{array}{c}4.29 \\
(2.52)\end{array}$ & 29 & $\begin{array}{c}8.21 \\
(6.28)\end{array}$ & $\begin{array}{c}6.34 \\
(4.59)\end{array}$ & 6.29 & 0.01 & 1.68 & 0.20 \\
\hline \multicolumn{11}{|c|}{ TAP: Untertest „Go/Nogo“6 } \\
\hline $\begin{array}{l}\text { Standard- } \\
\text { abweichung }\end{array}$ & 14 & $\begin{array}{l}259.37 \\
291.49\end{array}$ & $\begin{array}{c}136.74 \\
24.59\end{array}$ & 31 & $\begin{array}{l}\mathbf{1 4 9 . 2 5} \\
(37.62)\end{array}$ & $\begin{array}{l}\mathbf{1 3 3 . 1 8} \\
(44.62)\end{array}$ & 6.88 & 0.01 & 4.21 & 0.04 \\
\hline Anzahl Fehler & 14 & $\begin{array}{l}9.21 \\
4.85 \\
\end{array}$ & $\begin{array}{l}4.71 \\
3.97 \\
\end{array}$ & 31 & $\begin{array}{c}7.84 \\
(6.02) \\
\end{array}$ & $\begin{array}{c}4.68 \\
(5.18)\end{array}$ & 13.03 & 0.00 & 0.28 & 0.59 \\
\hline $\begin{array}{l}\text { Anzahl } \\
\text { Auslassungen }\end{array}$ & 14 & $\begin{array}{l}4.93 \\
6.24 \\
\end{array}$ & $\begin{array}{l}\mathbf{1 . 5 0} \\
1,83\end{array}$ & 31 & $\begin{array}{l}1.87 \\
(2.13)\end{array}$ & $\begin{array}{c}3.77 \\
(4.36)\end{array}$ & 0.85 & 0.35 & 0.18 & 0.67 \\
\hline \multicolumn{11}{|c|}{ TAP: Untertest „Inkompatibilität““ } \\
\hline $\begin{array}{l}\text { Standard- } \\
\text { abweichung }\end{array}$ & 14 & $\begin{array}{c}274.24 \\
(273.95) \\
\end{array}$ & $\begin{array}{l}189.13 \\
(41.07) \\
\end{array}$ & 29 & $\begin{array}{c}227.93 \\
(163.70) \\
\end{array}$ & $\begin{array}{r}187.49 \\
(80.30) \\
\end{array}$ & 3.50 & 0.06 & 0.42 & 0.52 \\
\hline Anzahl Fehler & 14 & $\begin{array}{c}\mathbf{1 9 . 7 1} \\
(13.10) \\
\end{array}$ & $\begin{array}{c}\mathbf{8 . 9 3} \\
(8.65) \\
\end{array}$ & 29 & $\begin{array}{c}\mathbf{1 4 . 7 2} \\
(10.56) \\
\end{array}$ & $\begin{array}{c}8.52 \\
(6.76)\end{array}$ & 21.73 & 0.00 & 1.12 & 0.29 \\
\hline \multicolumn{11}{|c|}{ TAP: Untertest „Visuelles Scanning“6 } \\
\hline $\begin{array}{l}\text { Standard- } \\
\text { abweichung } \\
\text { kritische Trials }\end{array}$ & 14 & $\begin{array}{c}\mathbf{2 8 3 3 . 1 1} \\
(1265.76)\end{array}$ & $\begin{array}{c}\mathbf{1 9 9 9 . 7 1} \\
(1174.35)\end{array}$ & 23 & $\begin{array}{c}3577.49 \\
(1875.05)\end{array}$ & $\begin{array}{c}2887.19 \\
(1434.07)\end{array}$ & 6.61 & 0.01 & 3.76 & 0.06 \\
\hline $\begin{array}{l}\text { Standard- } \\
\text { abweichung } \\
\text { nicht-kritische } \\
\text { Trials }\end{array}$ & 14 & $\begin{array}{c}3332.51 \\
(1761.55)\end{array}$ & $\begin{array}{c}2637.14 \\
(1509.64)\end{array}$ & 23 & $\begin{array}{c}\mathbf{3 3 3 7 . 7 4} \\
(1680.22)\end{array}$ & $\begin{array}{l}\mathbf{2 5 1 0 . 1 5} \\
(905.79)\end{array}$ & 5.64 & 0.02 & 0.02 & 0.87 \\
\hline $\begin{array}{l}\text { Anzahl Fehler } \\
\text { (kritische } \\
\text { Trials) } \\
\end{array}$ & 14 & $\begin{array}{c}1.21 \\
(1.12)\end{array}$ & $\begin{array}{c}1.00 \\
(2.15)\end{array}$ & 23 & $\begin{array}{c}7.78 \\
(4.59)\end{array}$ & $\begin{array}{l}\mathbf{3 . 3 5} \\
(2.84)\end{array}$ & 13.43 & 0.00 & 25.62 & 0.00 \\
\hline $\begin{array}{l}\text { Anzahl } \\
\text { Auslassungen } \\
\text { (nicht-kritische } \\
\text { Trials) }\end{array}$ & 14 & $\begin{array}{l}5.14 \\
(3.21)\end{array}$ & $\begin{array}{c}5.36 \\
(3.95)\end{array}$ & 23 & $\begin{array}{l}1.22 \\
(2.17)\end{array}$ & $\begin{array}{l}0.52 \\
(.73)\end{array}$ & 0.47 & 0.49 & 30.92 & 0.00 \\
\hline \multicolumn{11}{|l|}{ Test d2 } \\
\hline $\begin{array}{l}\text { Konzentrations- } \\
\text { leistungswert }\end{array}$ & 14 & $\begin{array}{c}72.21 \\
(27.26)\end{array}$ & $\begin{array}{c}\mathbf{9 6 . 5 0} \\
(19.07) \\
\end{array}$ & 21 & $\begin{array}{l}\mathbf{9 5 . 2 4} \\
(21.93) \\
\end{array}$ & $\begin{array}{l}\mathbf{1 1 1 . 5 2} \\
(20.07)\end{array}$ & 87.45 & 0.00 & 6.81 & 0.01 \\
\hline Anzahl Fehler & 14 & $\begin{array}{c}\mathbf{3 4 . 0 7} \\
(41.29)\end{array}$ & $\begin{array}{l}\mathbf{1 1 . 0 7} \\
(9.93)\end{array}$ & 21 & $\begin{array}{l}11.19 \\
(8.69)\end{array}$ & $\begin{array}{c}8.52 \\
(4.98)\end{array}$ & 11.53 & 0.00 & 5.70 & 0.02 \\
\hline
\end{tabular}




\begin{tabular}{|c|c|c|c|c|c|c|c|c|c|c|}
\hline \multirow[t]{2}{*}{$\begin{array}{l}\text { Abhängige } \\
\text { Variable }\end{array}$} & \multicolumn{3}{|c|}{$\begin{array}{l}\text { Basistraining nach } \\
\text { Lauth und Schlottke } \\
10 \text { Sitzungen }\end{array}$} & \multicolumn{3}{|c|}{$\begin{array}{c}\text { Basis- und } \\
\text { Strategietraining } \\
\text { Lauth und Schlottke } \\
\text { 15 Sitzungen }\end{array}$} & \multicolumn{2}{|c|}{$\begin{array}{c}\text { Haupteffekt } \\
\text { Zeit }\end{array}$} & \multicolumn{2}{|c|}{$\begin{array}{l}\text { Haupteffekt } \\
\text { Gruppe }\end{array}$} \\
\hline & $n$ & Vortest & Nachtest & $n$ & Vortest & Nachtest & $F$ & $p$ & $F$ & $p$ \\
\hline \multicolumn{11}{|l|}{ Mottier-Test } \\
\hline $\begin{array}{l}\text { Nachsprechleist } \\
\text { ung Gesamt } \\
\text { (max. 30) } \\
\end{array}$ & 14 & $\begin{array}{l}\mathbf{1 9 . 0 7} \\
(4.01)\end{array}$ & $\begin{array}{l}\mathbf{2 2 . 4 3} \\
(3.11)\end{array}$ & 32 & $\begin{array}{l}\mathbf{2 1 . 2 2} \\
(4.96)\end{array}$ & $\begin{array}{l}\mathbf{2 3 . 8 1} \\
(3.89)\end{array}$ & 36.39 & 0.00 & 1.97 & 0.16 \\
\hline $\begin{array}{l}\text { Nachsprechleist } \\
\text { ung für 2- und } \\
\text { 3-silbige } \\
\text { Kunstwörter } \\
\text { (max. 12) }\end{array}$ & 14 & $\begin{array}{l}11.00 \\
(1.24)\end{array}$ & $\begin{array}{l}11.29 \\
(1.14)\end{array}$ & 32 & $\begin{array}{l}\mathbf{1 1 . 2 8} \\
(1.02)\end{array}$ & $\begin{array}{c}\mathbf{1 1 . 6 3} \\
(.79)\end{array}$ & 2.12 & 0.15 & 1.69 & 0.20 \\
\hline $\begin{array}{l}\text { Nachsprechleist } \\
\text { ung für 4-, 5- } \\
\text { und 6-silbige } \\
\text { Kunstwörter } \\
\text { (max. 18) }\end{array}$ & 14 & $\begin{array}{c}\mathbf{8 . 0 7} \\
(3.41)\end{array}$ & $\begin{array}{l}\mathbf{1 1 . 1 4} \\
(3.13)\end{array}$ & 32 & $\begin{array}{c}\mathbf{9 . 9 4} \\
(4.44)\end{array}$ & $\begin{array}{l}\mathbf{1 2 . 1 9} \\
(3.41)\end{array}$ & 49.14 & 0.00 & 1.60 & 0.21 \\
\hline \multicolumn{11}{|c|}{ Fremdbeurteilungsbogen FBB-HKS (Eltern) } \\
\hline $\begin{array}{l}\text { Unaufmerksam- } \\
\text { keit: Anzahl an } \\
\text { Kriterien }\end{array}$ & 13 & $\begin{array}{c}\mathbf{4 . 6 9} \\
(1.75)\end{array}$ & $\begin{array}{c}\mathbf{3 . 2 3} \\
(2.31)\end{array}$ & 28 & $\begin{array}{c}5.78 \\
(2.48)\end{array}$ & $\begin{array}{c}5.57 \\
(3.07)\end{array}$ & 2.22 & 0.14 & 6.72 & 0.01 \\
\hline $\begin{array}{l}\text { Unaufmerksam- } \\
\text { keit: Kennwert }\end{array}$ & 13 & $\begin{array}{l}1.54 \\
(.37) \\
\end{array}$ & $\begin{array}{l}1.18 \\
(.43) \\
\end{array}$ & 28 & $\begin{array}{c}1.78 \\
(0.50) \\
\end{array}$ & $\begin{array}{c}1.69 \\
(0.60) \\
\end{array}$ & 4.18 & 0.04 & 7.91 & 0.00 \\
\hline $\begin{array}{l}\text { Hyperaktivität: } \\
\text { Anzahl an } \\
\text { Kriterien } \\
\end{array}$ & 13 & $\begin{array}{c}\mathbf{1 . 7 6} \\
(1.48)\end{array}$ & $\begin{array}{l}\mathbf{0 . 4 6} \\
(.87)\end{array}$ & 28 & $\begin{array}{c}1.32 \\
(1.49)\end{array}$ & $\begin{array}{c}1.07 \\
(1.56)\end{array}$ & 7.92 & 0.00 & 0.04 & 0.83 \\
\hline $\begin{array}{l}\text { Hyperaktivität: } \\
\text { Kennwert }\end{array}$ & 13 & $\begin{array}{l}\mathbf{1 . 2 9} \\
(.71) \\
\end{array}$ & $\begin{array}{l}\mathbf{0 . 6 6} \\
(.45) \\
\end{array}$ & 28 & $\begin{array}{c}1.01 \\
(0.61) \\
\end{array}$ & $\begin{array}{c}0.82 \\
(0.79) \\
\end{array}$ & 12.84 & 0.00 & 0.09 & 0.75 \\
\hline $\begin{array}{l}\text { Impulsivität: } \\
\text { Anzahl an } \\
\text { Kriterien }\end{array}$ & 13 & $\begin{array}{c}\mathbf{1 . 3 0} \\
(1.70)\end{array}$ & $\begin{array}{c}\mathbf{0 . 0 7} \\
(0.27)\end{array}$ & 28 & $\begin{array}{c}1.03 \\
(1.45)\end{array}$ & $\begin{array}{c}1.28 \\
(1.56)\end{array}$ & 3.56 & 0.06 & 1.36 & 0.25 \\
\hline $\begin{array}{l}\text { Impulsivität: } \\
\text { Kennwert }\end{array}$ & 13 & $\begin{array}{l}\mathbf{1 . 1 2} \\
(.79) \\
\end{array}$ & $\begin{array}{l}\mathbf{. 5 9} \\
(.37) \\
\end{array}$ & 28 & $\begin{array}{c}0.82 \\
(0.75) \\
\end{array}$ & $\begin{array}{c}1.09 \\
(0.87) \\
\end{array}$ & 0.97 & 0.33 & 0.23 & 0.63 \\
\hline \multicolumn{11}{|c|}{ Child Behavior Checklist (CBCL) } \\
\hline $\begin{array}{l}\text { T-Wert } \\
\text { Aufmerksam- } \\
\text { keitsstörungen }\end{array}$ & 12 & $\begin{array}{l}\mathbf{6 8 . 3 3} \\
(8.74)\end{array}$ & $\begin{array}{c}\mathbf{6 3 . 5 8} \\
(10.95)\end{array}$ & 30 & $\begin{array}{l}\mathbf{6 5 . 4 7} \\
(8.38)\end{array}$ & $\begin{array}{l}\mathbf{6 2 . 1 7} \\
(7.28)\end{array}$ & 9.45 & 0.00 & 0.69 & 0.41 \\
\hline $\begin{array}{l}\text { T-Wert } \\
\text { externalisieren- } \\
\text { de Störungen }\end{array}$ & 12 & $\begin{array}{l}\mathbf{6 3 . 1 7} \\
(9.23)\end{array}$ & $\begin{array}{l}\mathbf{5 8 . 6 7} \\
(8.07)\end{array}$ & 30 & $\begin{array}{c}\mathbf{5 5 . 0 0} \\
(11.90)\end{array}$ & $\begin{array}{c}\mathbf{5 1 . 7 7} \\
(11.04)\end{array}$ & 9.69 & 0.00 & 4.72 & 0.03 \\
\hline $\begin{array}{l}\text { T-Wert } \\
\text { Gesamtskala }\end{array}$ & 12 & $\begin{array}{l}\mathbf{6 4 . 1 7} \\
(7.07)\end{array}$ & $\begin{array}{l}\mathbf{5 9 . 2 5} \\
(7.33)\end{array}$ & 30 & $\begin{array}{l}\mathbf{5 9 . 3 0} \\
(8.55)\end{array}$ & $\begin{array}{l}\mathbf{5 5 . 7 0} \\
(9.06)\end{array}$ & 17.66 & 0.00 & 2.46 & 0.12 \\
\hline \multicolumn{11}{|c|}{ Fremdbeurteilungsbogen FBB-HKS (Lehrer) } \\
\hline $\begin{array}{l}\text { Unaufmerksam- } \\
\text { keit: Anzahl an } \\
\text { Kriterien } \\
\end{array}$ & 9 & $\begin{array}{c}3.33 \\
(2.17)\end{array}$ & $\begin{array}{c}3.00 \\
(2.44)\end{array}$ & 26 & $\begin{array}{c}\mathbf{6 . 0 3} \\
(2.44)\end{array}$ & $\begin{array}{c}\mathbf{4 . 0 3} \\
(2.58)\end{array}$ & 3.11 & 0.08 & 7.40 & 0.01 \\
\hline $\begin{array}{l}\text { Unaufmerksam- } \\
\text { keit: Kennwert }\end{array}$ & 9 & $\begin{array}{l}1.28 \\
(.44) \\
\end{array}$ & $\begin{array}{l}1.13 \\
(.52) \\
\end{array}$ & 26 & $\begin{array}{c}\mathbf{1 . 8 3} \\
(0.55) \\
\end{array}$ & $\begin{array}{c}\mathbf{1 . 4 3} \\
(0.61) \\
\end{array}$ & 3.43 & 0.73 & 7.22 & 0.01 \\
\hline $\begin{array}{l}\text { Hyperaktivität: } \\
\text { Anzahl an } \\
\text { Kriterien } \\
\end{array}$ & 9 & $\begin{array}{c}\mathbf{1 . 7 7} \\
(1.56)\end{array}$ & $\begin{array}{c}.66 \\
(.70)\end{array}$ & 26 & $\begin{array}{c}\mathbf{1 . 5 0} \\
(1.47)\end{array}$ & $\begin{array}{c}\mathbf{0 . 6 9} \\
(1.15)\end{array}$ & 6.50 & 0.01 & 0.14 & 0.70 \\
\hline $\begin{array}{l}\text { Hyperaktivität: } \\
\text { Kennwert }\end{array}$ & 9 & $\begin{array}{l}1.14 \\
(.56) \\
\end{array}$ & $\begin{array}{l}.77 \\
(.52) \\
\end{array}$ & 26 & $\begin{array}{c}\mathbf{0 . 9 7} \\
(0.71) \\
\end{array}$ & $\begin{array}{c}\mathbf{0 . 5 4} \\
(0.60) \\
\end{array}$ & 5.41 & 0.02 & 1.31 & 0.26 \\
\hline $\begin{array}{l}\text { Impulsivität: } \\
\text { Anzahl an } \\
\text { Kriterien }\end{array}$ & 9 & $\begin{array}{c}1.00 \\
(1.58)\end{array}$ & $\begin{array}{c}.66 \\
(1.00)\end{array}$ & 26 & $\begin{array}{c}0.80 \\
(1.29)\end{array}$ & $\begin{array}{c}0.69 \\
(1.37)\end{array}$ & 0.40 & 0.53 & 0.04 & 0.82 \\
\hline
\end{tabular}




\begin{tabular}{|c|c|c|c|c|c|c|c|c|c|c|}
\hline \multirow[t]{2}{*}{$\begin{array}{c}\text { Abhängige } \\
\text { Variable }\end{array}$} & \multicolumn{3}{|c|}{$\begin{array}{l}\text { Basistraining nach } \\
\text { Lauth und Schlottke } \\
10 \text { Sitzungen }\end{array}$} & \multicolumn{3}{|c|}{$\begin{array}{c}\text { Basis- und } \\
\text { Strategietraining } \\
\text { Lauth und Schlottke } \\
15 \text { Sitzungen }\end{array}$} & \multicolumn{2}{|c|}{$\begin{array}{c}\text { Haupteffekt } \\
\text { Zeit }\end{array}$} & \multicolumn{2}{|c|}{$\begin{array}{l}\text { Haupteffekt } \\
\text { Gruppe }\end{array}$} \\
\hline & $n$ & Vortest & Nachtest & $n$ & Vortest & Nachtest & $F$ & $p$ & $F$ & $p$ \\
\hline $\begin{array}{l}\text { Impulsivität: } \\
\text { Kennwert }\end{array}$ & 9 & $\begin{array}{l}1.06 \\
(.70) \\
\end{array}$ & $\begin{array}{l}.86 \\
(.51)\end{array}$ & 26 & $\begin{array}{c}0.72 \\
(0.67) \\
\end{array}$ & $\begin{array}{c}0.65 \\
(0.85) \\
\end{array}$ & 0.53 & 0.47 & 1.59 & 0.21 \\
\hline \multicolumn{11}{|c|}{ Teacher's Report Form (TRF) } \\
\hline $\begin{array}{l}\text { T-Wert } \\
\text { Aufmerksam- } \\
\text { keitsstörungen }\end{array}$ & 11 & $\begin{array}{l}59.36 \\
(5.30)\end{array}$ & $\begin{array}{l}58.00 \\
(5.46)\end{array}$ & 25 & $\begin{array}{l}\mathbf{5 9 . 2 1} \\
(5.49)\end{array}$ & $\begin{array}{l}\mathbf{5 7 . 0 8} \\
(4.32)\end{array}$ & 3.82 & 0.05 & 0.10 & 0.74 \\
\hline $\begin{array}{l}\text { T-Wert } \\
\text { externalisieren- } \\
\text { de Störungen }\end{array}$ & 11 & $\begin{array}{l}59.09 \\
(8.94)\end{array}$ & $\begin{array}{l}59.64 \\
(8.56)\end{array}$ & 25 & $\begin{array}{c}55.04 \\
(10.67)\end{array}$ & $\begin{array}{l}53.42 \\
(9.60)\end{array}$ & 0.22 & 0.64 & 2.33 & 0.13 \\
\hline $\begin{array}{l}\text { T-Wert } \\
\text { Gesamtskala }\end{array}$ & 11 & $\begin{array}{l}58.73 \\
(5.52)\end{array}$ & $\begin{array}{l}59.09 \\
(6.19)\end{array}$ & 25 & $\begin{array}{l}\mathbf{5 8 . 7 6} \\
(6.80)\end{array}$ & $\begin{array}{l}\mathbf{5 5 . 9 2} \\
(5.77)\end{array}$ & 1.57 & 0.21 & 0.61 & 0.44 \\
\hline
\end{tabular}

Anmerkung: p: einseitige Irrtumswahrscheinlichkeit des F-Wertes; Signifikanzniveau $\alpha=0.05$;

n: Anzahl der Messwertpaare (Die Anzahl umfasst nur diejenigen Kinder, von denen sowohl Vor- als auch Nachtestwert der jeweiligen Variablen vorhanden waren. Auf dieser Anzahl basieren die inferenzstatistischen Auswertungen).

TAP: Testbatterie zur Aufmerksamkeitsprüfung 


\section{Anhang B}

Tabelle B-6

Mittelwerte und Standardabweichungen (in Klammern) im Vor- und Nachtest für die AVn und Kennwerte $(F, p)$ der Haupteffekte Zeit und Gruppe zweifaktorieller Varianzanalysen mit Messwiederholung getrennt für das kombinierte Basis- und Strategietraining nach Lauth und Schlottke (15 Sitzungen) und das THOP

\begin{tabular}{|c|c|c|c|c|c|c|c|c|c|c|}
\hline \multirow[t]{2}{*}{$\begin{array}{c}\text { Abhängige } \\
\text { Variable }\end{array}$} & \multicolumn{3}{|c|}{$\begin{array}{c}\text { Basis- und } \\
\text { Strategietraining } \\
\text { Lauth und Schlottke } \\
15 \text { Sitzungen }\end{array}$} & \multicolumn{3}{|c|}{ Trainingsgruppe THOP } & \multicolumn{2}{|c|}{$\begin{array}{c}\text { Haupteffekt } \\
\text { Zeit }\end{array}$} & \multicolumn{2}{|c|}{$\begin{array}{c}\text { Haupteffekt } \\
\text { Gruppe }\end{array}$} \\
\hline & $n$ & Vortest & Nachtest & $n$ & Vortest & Nachtest & $F$ & $P$ & $F$ & $P$ \\
\hline \multicolumn{11}{|c|}{ TAP: Untertest ,Geteilte Aufmerksamkeit" } \\
\hline $\begin{array}{l}\text { Standard- } \\
\text { abweichung }\end{array}$ & 29 & $\begin{array}{c}\mathbf{3 4 8 . 0 1} \\
(103.47) \\
\end{array}$ & $\begin{array}{l}295.91 \\
(88.70) \\
\end{array}$ & 29 & $\begin{array}{c}\mathbf{3 3 7 . 8 2} \\
(116.48) \\
\end{array}$ & $\begin{array}{r}\mathbf{2 9 5 . 8 6} \\
(95.81) \\
\end{array}$ & 8.72 & 0.00 & 0.05 & 0.81 \\
\hline Anzahl Fehler & 29 & $\begin{array}{c}\mathbf{9 . 0 3} \\
(13.53) \\
\end{array}$ & $\begin{array}{r}\mathbf{2 . 9 7} \\
(3.46) \\
\end{array}$ & 29 & $\begin{array}{c}\mathbf{9 . 3 8} \\
(13.44) \\
\end{array}$ & $\begin{array}{c}\mathbf{3 . 0 7} \\
(4.03) \\
\end{array}$ & 12.99 & 0.00 & 0.01 & 0.90 \\
\hline $\begin{array}{l}\text { Anzahl } \\
\text { Auslassungen }\end{array}$ & 29 & $\begin{array}{c}8.21 \\
(6.28)\end{array}$ & $\begin{array}{c}6.34 \\
(4.59)\end{array}$ & 29 & $\begin{array}{c}7.10 \\
(5.02)\end{array}$ & $\begin{array}{c}5.28 \\
(4.71)\end{array}$ & 5.33 & 0.02 & 0.96 & 0.33 \\
\hline \multicolumn{11}{|c|}{ TAP: Untertest ,Go/Nogo“6 } \\
\hline $\begin{array}{l}\text { Standard- } \\
\text { abweichung }\end{array}$ & 31 & $\begin{array}{l}\mathbf{1 4 9 . 2 5} \\
(37.62) \\
\end{array}$ & $\begin{array}{r}\mathbf{1 3 3 . 1 8} \\
(44.62) \\
\end{array}$ & 31 & $\begin{array}{l}140.54 \\
(42.28)\end{array}$ & $\begin{array}{l}125.59 \\
(42.09)\end{array}$ & 6.76 & 0.01 & 0.86 & 0.35 \\
\hline Anzahl Fehler & 31 & $\begin{array}{c}7.84 \\
(6.02) \\
\end{array}$ & $\begin{array}{c}\mathbf{4 . 6 8} \\
(5.18) \\
\end{array}$ & 31 & $\begin{array}{c}\mathbf{9 . 8 1} \\
(5.50) \\
\end{array}$ & $\begin{array}{r}\mathbf{6 . 9 7} \\
(5.33) \\
\end{array}$ & 12.97 & 0.00 & 3.55 & 0.06 \\
\hline $\begin{array}{l}\text { Anzahl } \\
\text { Auslassungen }\end{array}$ & 31 & $\begin{array}{c}1.87 \\
(2.13)\end{array}$ & $\begin{array}{c}3.77 \\
(4.36)\end{array}$ & 31 & $\begin{array}{c}\mathbf{2 . 1 0} \\
(2.70)\end{array}$ & $\begin{array}{c}\mathbf{0 . 8 4} \\
(1.44)\end{array}$ & 0.38 & 0.53 & 6.97 & 0.01 \\
\hline \multicolumn{11}{|c|}{ TAP: Untertest, „Inkompatibilität" } \\
\hline $\begin{array}{l}\text { Standard- } \\
\text { abweichung }\end{array}$ & 29 & $\begin{array}{c}227.93 \\
(163.70)\end{array}$ & $\begin{array}{l}187.49 \\
(80.30)\end{array}$ & 31 & $\begin{array}{c}\mathbf{2 4 2 . 4 6} \\
(123.11)\end{array}$ & $\begin{array}{r}\mathbf{1 7 6 . 6 3} \\
(64.19) \\
\end{array}$ & 8.72 & 0.00 & 0.00 & 0.93 \\
\hline Anzahl Fehler & 29 & $\begin{array}{r}\mathbf{1 4 . 7 2} \\
(10.56) \\
\end{array}$ & $\begin{array}{r}\mathbf{8 . 5 2} \\
(6.76) \\
\end{array}$ & 31 & $\begin{array}{r}\mathbf{2 0 . 4 2} \\
(12.05) \\
\end{array}$ & $\begin{array}{c}\mathbf{1 3 . 5 5} \\
(10.00) \\
\end{array}$ & 20.71 & 0.00 & 6.11 & 0.01 \\
\hline \multicolumn{11}{|c|}{ TAP: Untertest „,Visuelles Scanning“6 } \\
\hline $\begin{array}{l}\text { Standard- } \\
\text { abweichung } \\
\text { kritische Trials } \\
\end{array}$ & 23 & $\begin{array}{c}3577.49 \\
(1875.05)\end{array}$ & $\begin{array}{c}2887.19 \\
(1434.07)\end{array}$ & 31 & $\begin{array}{c}\mathbf{2 4 7 2 . 9 8} \\
(1048.18)\end{array}$ & $\begin{array}{l}\mathbf{2 0 4 9 . 0 0} \\
(995.37)\end{array}$ & 7.02 & 0.01 & 10.40 & 0.00 \\
\hline $\begin{array}{l}\text { Standard- } \\
\text { abweichung } \\
\text { nicht-kritische } \\
\text { Trials }\end{array}$ & 23 & $\begin{array}{c}\mathbf{3 3 3 7 . 7 4} \\
(1680.22)\end{array}$ & $\begin{array}{l}\mathbf{2 5 1 0 . 1 5} \\
(905.79)\end{array}$ & 31 & $\begin{array}{c}\mathbf{2 7 6 7 . 1 8} \\
(1185.42)\end{array}$ & $\begin{array}{c}\mathbf{2 1 9 2 . 0 3} \\
(1028.39)\end{array}$ & 12.95 & 0.00 & 2.66 & 0.10 \\
\hline $\begin{array}{l}\text { Anzahl Fehler } \\
\text { (kritische } \\
\text { Trials) } \\
\end{array}$ & 23 & $\begin{array}{c}7.78 \\
(4.59)\end{array}$ & $\begin{array}{c}\mathbf{3 . 3 5} \\
(2.84)\end{array}$ & 31 & $\begin{array}{c}7.68 \\
(5.95)\end{array}$ & $\begin{array}{c}4.32 \\
(4.10)\end{array}$ & 37.84 & 0.00 & 0.15 & 0.69 \\
\hline $\begin{array}{l}\text { Anzahl } \\
\text { Auslassungen } \\
\text { (nicht-kritische } \\
\text { Trials) }\end{array}$ & 23 & $\begin{array}{l}1.22 \\
(2.17)\end{array}$ & $\begin{array}{l}.52 \\
(.73)\end{array}$ & 31 & $\begin{array}{c}\mathbf{5 . 3 2} \\
(5.96)\end{array}$ & $\begin{array}{c}\mathbf{2 . 2 6} \\
(3.26)\end{array}$ & 10.52 & 0.00 & 11.29 & 0.00 \\
\hline \multicolumn{11}{|l|}{ Test d2 } \\
\hline $\begin{array}{l}\text { Konzentrations- } \\
\text { leistungswert }\end{array}$ & 21 & $\begin{array}{c}\mathbf{9 5 . 2 4} \\
(21.93) \\
\end{array}$ & $\begin{array}{l}\mathbf{1 1 1 . 5 2} \\
(20.07) \\
\end{array}$ & 15 & $\begin{array}{c}93.93 \\
(25.92) \\
\end{array}$ & $\begin{array}{l}\mathbf{1 2 3 . 2 0} \\
(25.27) \\
\end{array}$ & 174.42 & 0.00 & 0.46 & 0.49 \\
\hline Anzahl Fehler & 21 & $\begin{array}{l}11.19 \\
(8.69) \\
\end{array}$ & $\begin{array}{r}8.52 \\
(4.98) \\
\end{array}$ & 15 & $\begin{array}{c}\mathbf{2 6 . 2 7} \\
(37.59) \\
\end{array}$ & $\begin{array}{r}9.27 \\
(6.30) \\
\end{array}$ & 6.77 & 0.01 & 3.09 & 0.08 \\
\hline
\end{tabular}




\begin{tabular}{|c|c|c|c|c|c|c|c|c|c|c|}
\hline \multirow[t]{2}{*}{$\begin{array}{l}\text { Abhängige } \\
\text { Variable }\end{array}$} & \multicolumn{3}{|c|}{$\begin{array}{c}\text { Basis- und } \\
\text { Strategietraining } \\
\text { Lauth und Schlottke } \\
15 \text { Sitzungen }\end{array}$} & \multicolumn{3}{|c|}{ Trainingsgruppe THOP } & \multicolumn{2}{|c|}{$\begin{array}{l}\text { Haupteffekt } \\
\text { Zeit }\end{array}$} & \multicolumn{2}{|c|}{$\begin{array}{l}\text { Haupteffekt } \\
\text { Gruppe }\end{array}$} \\
\hline & $n$ & Vortest & Nachtest & $n$ & Vortest & Nachtest & $F$ & $P$ & $F$ & $P$ \\
\hline \multicolumn{11}{|l|}{ Mottier-Test } \\
\hline $\begin{array}{l}\text { Nachsprechleist } \\
\text { ung Gesamt } \\
\text { (max. 30) }\end{array}$ & 32 & $\begin{array}{l}\mathbf{2 1 . 2 2} \\
(4.96)\end{array}$ & $\begin{array}{l}\mathbf{2 3 . 8 1} \\
(3.89)\end{array}$ & 30 & $\begin{array}{l}\mathbf{2 2 . 2 0} \\
(4.00)\end{array}$ & $\begin{array}{l}\mathbf{2 3 . 5 7} \\
(3.79)\end{array}$ & 25.00 & 0.00 & 0.13 & 0.71 \\
\hline $\begin{array}{l}\text { Nachsprechleist } \\
\text { ung für 2- und } \\
\text { 3-silbige } \\
\text { Kunstwörter } \\
\text { (max. 12) }\end{array}$ & 32 & $\begin{array}{l}\mathbf{1 1 . 2 8} \\
(1.02)\end{array}$ & $\begin{array}{c}\mathbf{1 1 . 6 3} \\
(.79)\end{array}$ & 30 & $\begin{array}{l}\mathbf{1 1 . 1 7} \\
(1.02)\end{array}$ & $\begin{array}{c}\mathbf{1 1 . 7 0} \\
(.60)\end{array}$ & 9.32 & 0.00 & 0.01 & 0.91 \\
\hline $\begin{array}{l}\text { Nachsprechleist } \\
\text { ung für 4-, 5- } \\
\text { und 6-silbige } \\
\text { Kunstwörter } \\
\text { (max. 18) } \\
\end{array}$ & 32 & $\begin{array}{c}\mathbf{9 . 9 4} \\
(4.44)\end{array}$ & $\begin{array}{l}\mathbf{1 2 . 1 9} \\
(3.41)\end{array}$ & 30 & $\begin{array}{l}\mathbf{1 1 . 0 3} \\
(3.40)\end{array}$ & $\begin{array}{l}\mathbf{1 1 . 8 7} \\
(3.46)\end{array}$ & 23.23 & 0.00 & 0.19 & 0.66 \\
\hline \multicolumn{11}{|c|}{ Fremdbeurteilungsbogen FBB-HKS (Eltern) } \\
\hline $\begin{array}{l}\text { Unaufmerksam- } \\
\text { keit: Anzahl an } \\
\text { Kriterien }\end{array}$ & 28 & $\begin{array}{c}5.78 \\
(2.48)\end{array}$ & $\begin{array}{c}5.57 \\
(3.07)\end{array}$ & 30 & $\begin{array}{c}\mathbf{5 . 9 3} \\
(2.58)\end{array}$ & $\begin{array}{l}\mathbf{4 . 5 0} \\
(2.56)\end{array}$ & 3.60 & 0.06 & 0.69 & 0.41 \\
\hline $\begin{array}{l}\text { Unaufmerksam- } \\
\text { keit: Kennwert }\end{array}$ & 28 & $\begin{array}{c}1.78 \\
(0.50) \\
\end{array}$ & $\begin{array}{c}1.69 \\
(0.60) \\
\end{array}$ & 30 & $\begin{array}{c}\mathbf{1 . 8 5} \\
(0.58) \\
\end{array}$ & $\begin{array}{c}1.48 \\
(0.57) \\
\end{array}$ & 7.02 & 0.01 & 0.30 & 0.58 \\
\hline $\begin{array}{l}\text { Hyperaktivität: } \\
\text { Anzahl an } \\
\text { Kriterien }\end{array}$ & 28 & $\begin{array}{c}1.32 \\
(1.49)\end{array}$ & $\begin{array}{c}1.07 \\
(1.56)\end{array}$ & 30 & $\begin{array}{c}\mathbf{2 . 4 3} \\
(1.79)\end{array}$ & $\begin{array}{c}\mathbf{1 . 7 0} \\
(1.58)\end{array}$ & 4.99 & 0.02 & 5.76 & 0.02 \\
\hline $\begin{array}{l}\text { Hyperaktivität: } \\
\text { Kennwert }\end{array}$ & 28 & $\begin{array}{c}1.01 \\
(0.61) \\
\end{array}$ & $\begin{array}{c}0.82 \\
(0.79) \\
\end{array}$ & 30 & $\begin{array}{c}\mathbf{1 . 4 8} \\
(0.85) \\
\end{array}$ & $\begin{array}{c}1.14 \\
(0.66) \\
\end{array}$ & 7.69 & 0.00 & 5.42 & 0.02 \\
\hline $\begin{array}{l}\text { Impulsivität: } \\
\text { Anzahl an } \\
\text { Kriterien } \\
\end{array}$ & 28 & $\begin{array}{c}1.03 \\
(1.45)\end{array}$ & $\begin{array}{c}1.28 \\
(1.56)\end{array}$ & 30 & $\begin{array}{c}\mathbf{2 . 4 0} \\
(1.61)\end{array}$ & $\begin{array}{c}\mathbf{2 . 0 0} \\
(1.43)\end{array}$ & 0.17 & 0.67 & 8.47 & 0.00 \\
\hline $\begin{array}{l}\text { Impulsivität: } \\
\text { Kennwert }\end{array}$ & 28 & $\begin{array}{c}0.82 \\
(0.75)\end{array}$ & $\begin{array}{c}1.09 \\
(0.87)\end{array}$ & 30 & $\begin{array}{c}1.61 \\
(0.93)\end{array}$ & $\begin{array}{c}1.50 \\
(0.81)\end{array}$ & 0.69 & 0.40 & 8.65 & 0.00 \\
\hline \multicolumn{11}{|c|}{ Child Behavior Checklist (CBCL) } \\
\hline $\begin{array}{l}\text { T-Wert } \\
\text { Aufmerksam- } \\
\text { keitsstörungen } \\
\end{array}$ & 30 & $\begin{array}{l}\mathbf{6 5 . 4 7} \\
(8.38)\end{array}$ & $\begin{array}{l}\mathbf{6 2 . 1 7} \\
(7.28)\end{array}$ & 30 & $\begin{array}{l}\mathbf{6 6 . 2 7} \\
(6.70)\end{array}$ & $\begin{array}{l}\mathbf{6 0 . 5 7} \\
(6.67)\end{array}$ & 20.78 & 0.00 & 0.06 & 0.80 \\
\hline $\begin{array}{l}\text { T-Wert } \\
\text { externalisieren- } \\
\text { de Störungen }\end{array}$ & 30 & $\begin{array}{c}\mathbf{5 5 . 0 0} \\
(11.90)\end{array}$ & $\begin{array}{c}\mathbf{5 1 . 7 7} \\
(11.04)\end{array}$ & 30 & $\begin{array}{c}\mathbf{6 5 . 4 7} \\
(11.47)\end{array}$ & $\begin{array}{l}\mathbf{5 9 . 9 0} \\
(9.95)\end{array}$ & 18.87 & 0.00 & 11.99 & 0.00 \\
\hline $\begin{array}{l}\text { T-Wert } \\
\text { Gesamtskala }\end{array}$ & 30 & $\begin{array}{l}\mathbf{5 9 . 3 0} \\
(8.55)\end{array}$ & $\begin{array}{l}\mathbf{5 5 . 7 0} \\
(9.06) \\
\end{array}$ & 30 & $\begin{array}{l}\mathbf{6 5 . 9 3} \\
(7.93) \\
\end{array}$ & $\begin{array}{l}\mathbf{5 9 . 9 0} \\
(8.24) \\
\end{array}$ & 28.05 & 0.00 & 7.45 & 0.00 \\
\hline \multicolumn{11}{|c|}{ Fremdbeurteilungsbogen FBB-HKS (Lehrer) } \\
\hline $\begin{array}{l}\text { Unaufmerksam- } \\
\text { keit: Anzahl an } \\
\text { Kriterien }\end{array}$ & 26 & $\begin{array}{c}\mathbf{6 . 0 3} \\
(2.44)\end{array}$ & $\begin{array}{c}\mathbf{4 . 0 3} \\
(2.58)\end{array}$ & 23 & $\begin{array}{c}4.86 \\
(2.51)\end{array}$ & $\begin{array}{c}3.91 \\
(2.29)\end{array}$ & 10.97 & 0.00 & 1.40 & 0.24 \\
\hline $\begin{array}{l}\text { Unaufmerksam- } \\
\text { keit: Kennwert }\end{array}$ & 26 & $\begin{array}{c}\mathbf{1 . 8 3} \\
(0.55) \\
\end{array}$ & $\begin{array}{c}1.43 \\
(0.61) \\
\end{array}$ & 23 & $\begin{array}{c}1.71 \\
(0.63) \\
\end{array}$ & $\begin{array}{c}\mathbf{1 . 4 4} \\
(0.48) \\
\end{array}$ & 10.33 & 0.00 & 0.18 & 0.66 \\
\hline $\begin{array}{l}\text { Hyperaktivität: } \\
\text { Anzahl an } \\
\text { Kriterien } \\
\end{array}$ & 26 & $\begin{array}{c}\mathbf{1 . 5 0} \\
(1.47)\end{array}$ & $\begin{array}{c}\mathbf{0 . 6 9} \\
(1.15)\end{array}$ & 23 & $\begin{array}{c}2.08 \\
(1.90)\end{array}$ & $\begin{array}{c}1.47 \\
(1.59)\end{array}$ & 6.86 & 0.01 & 3.86 & 0.05 \\
\hline $\begin{array}{l}\text { Hyperaktivität: } \\
\text { Kennwert }\end{array}$ & 26 & $\begin{array}{c}\mathbf{0 . 9 7} \\
(0.71) \\
\end{array}$ & $\begin{array}{c}\mathbf{0 . 5 4} \\
(0.60) \\
\end{array}$ & 23 & $\begin{array}{c}1.26 \\
(0.94) \\
\end{array}$ & $\begin{array}{c}1.04 \\
(0.79) \\
\end{array}$ & 6.13 & 0.01 & 5.16 & 0.03 \\
\hline $\begin{array}{l}\text { Impulsivität: } \\
\text { Anzahl an } \\
\text { Kriterien } \\
\end{array}$ & 26 & $\begin{array}{c}0.80 \\
(1.29)\end{array}$ & $\begin{array}{c}0.69 \\
(1.37)\end{array}$ & 23 & $\begin{array}{c}1.86 \\
(1.63)\end{array}$ & $\begin{array}{c}1.56 \\
(1.70)\end{array}$ & 0.51 & 0.47 & 9.49 & 0.00 \\
\hline
\end{tabular}




\begin{tabular}{|c|c|c|c|c|c|c|c|c|c|c|}
\hline \multirow[t]{2}{*}{$\begin{array}{c}\text { Abhängige } \\
\text { Variable }\end{array}$} & \multicolumn{3}{|c|}{$\begin{array}{c}\text { Basis- und } \\
\text { Strategietraining } \\
\text { Lauth und Schlottke } \\
15 \text { Sitzungen }\end{array}$} & \multicolumn{3}{|c|}{ Trainingsgruppe THOP } & \multicolumn{2}{|c|}{$\begin{array}{c}\text { Haupteffekt } \\
\text { Zeit }\end{array}$} & \multicolumn{2}{|c|}{$\begin{array}{c}\text { Haupteffekt } \\
\text { Gruppe }\end{array}$} \\
\hline & $n$ & Vortest & Nachtest & $n$ & Vortest & Nachtest & $F$ & $P$ & $F$ & $P$ \\
\hline $\begin{array}{l}\text { Impulsivität: } \\
\text { Kennwert }\end{array}$ & 26 & $\begin{array}{c}0.72 \\
(0.67)\end{array}$ & $\begin{array}{c}0.65 \\
(0.85)\end{array}$ & 23 & $\begin{array}{c}1.34 \\
(0.81)\end{array}$ & $\begin{array}{c}1.28 \\
(0.92)\end{array}$ & 0.20 & 0.65 & 11.81 & 0.00 \\
\hline \multicolumn{11}{|c|}{ Teacher's Report Form (TRF) } \\
\hline $\begin{array}{l}\text { T-Wert } \\
\text { Aufmerksam- } \\
\text { keitsstörungen } \\
\end{array}$ & 25 & $\begin{array}{l}\mathbf{5 9 . 2 1} \\
(5.49)\end{array}$ & $\begin{array}{l}\mathbf{5 7 . 0 8} \\
(4.32)\end{array}$ & 22 & $\begin{array}{l}\mathbf{6 2 . 1 8} \\
(6.61)\end{array}$ & $\begin{array}{l}\mathbf{5 8 . 6 4} \\
(5.74)\end{array}$ & 15.26 & 0.00 & 2.35 & 0.13 \\
\hline $\begin{array}{l}\text { T-Wert } \\
\text { externalisieren- } \\
\text { de Störungen }\end{array}$ & 25 & $\begin{array}{c}55.04 \\
(10.67)\end{array}$ & $\begin{array}{l}53.42 \\
(9.60)\end{array}$ & 22 & $\begin{array}{l}66.36 \\
(8.68)\end{array}$ & $\begin{array}{l}63.64 \\
(8.78)\end{array}$ & 3.43 & 0.07 & 17.90 & 0.00 \\
\hline $\begin{array}{l}\text { T-Wert } \\
\text { Gesamtskala }\end{array}$ & 25 & $\begin{array}{l}\mathbf{5 8 . 7 6} \\
(6.80)\end{array}$ & $\begin{array}{l}\mathbf{5 5 . 9 2} \\
(5.77)\end{array}$ & 22 & $\begin{array}{l}\mathbf{6 5 . 6 4} \\
(7.92)\end{array}$ & $\begin{array}{l}\mathbf{6 2 . 0 9} \\
(7.52)\end{array}$ & 13.79 & 0.00 & 12.33 & 0.00 \\
\hline
\end{tabular}

Anmerkung: p: einseitige Irrtumswahrscheinlichkeit des F-Wertes; Signifikanzniveau $\alpha=0.05$;

n: Anzahl der Messwertpaare (Die Anzahl umfasst nur diejenigen Kinder, von denen sowohl Vor- als auch Nachtestwert der jeweiligen Variablen vorhanden waren. Auf dieser Anzahl basieren die inferenzstatistischen Auswertungen).

TAP: Testbatterie zur Aufmerksamkeitsprüfung 


\section{Anhang B}

Tabelle B-7

Mittelwerte und Standardabweichungen (in Klammern) im Vor- und Nachtest für die AVn und Kennwerte $(F, p)$, der Haupteffekte Zeit und Gruppe zweifaktorieller Varianzanalysen mit Messwiederholung getrennt für das kombinierte Basis- und Strategietraining nach Lauth und Schlottke (15 Sitzungen) und das Marburger Konzentratiosntraining

\begin{tabular}{|c|c|c|c|c|c|c|c|c|c|c|}
\hline \multirow[t]{2}{*}{$\begin{array}{c}\text { Abhängige } \\
\text { Variable }\end{array}$} & \multicolumn{3}{|c|}{$\begin{array}{c}\text { Basis- und } \\
\text { Strategietraining } \\
\text { Lauth und Schlottke } \\
15 \text { Sitzungen }\end{array}$} & \multicolumn{3}{|c|}{$\begin{array}{c}\text { Marburger } \\
\text { Konzentrationstraining } \\
\text { (MK) }\end{array}$} & \multicolumn{2}{|c|}{$\begin{array}{c}\text { Haupteffekt } \\
\text { Zeit }\end{array}$} & \multicolumn{2}{|c|}{$\begin{array}{l}\text { Haupteffekt } \\
\text { Gruppe }\end{array}$} \\
\hline & $n$ & Vortest & Nachtest & $n$ & Vortest & Nachtest & $F$ & $P$ & $F$ & $P$ \\
\hline \multicolumn{11}{|c|}{ TAP: Untertest „Geteilte Aufmerksamkeit““ } \\
\hline $\begin{array}{l}\text { Standard- } \\
\text { abweichung }\end{array}$ & 29 & $\begin{array}{c}\mathbf{3 4 8 . 0 1} \\
(103.47)\end{array}$ & $\begin{array}{r}\mathbf{2 9 5 . 9 1} \\
(88.70)\end{array}$ & 15 & $\begin{array}{c}275.59 \\
(129.44)\end{array}$ & $\begin{array}{l}241.21 \\
(73.63)\end{array}$ & 4.52 & 0.03 & 6.88 & 0.01 \\
\hline Anzahl Fehler & 29 & $\begin{array}{c}\mathbf{9 . 0 3} \\
(13.53) \\
\end{array}$ & $\begin{array}{c}2.97 \\
(3.46)\end{array}$ & 15 & $\begin{array}{c}3.80 \\
(6.04) \\
\end{array}$ & $\begin{array}{c}4.27 \\
(4.22) \\
\end{array}$ & 2.48 & 0.12 & 0.89 & 0.35 \\
\hline $\begin{array}{l}\text { Anzahl } \\
\text { Auslassungen }\end{array}$ & 29 & $\begin{array}{c}8.21 \\
(6.28)\end{array}$ & $\begin{array}{c}6.34 \\
(4.59)\end{array}$ & 15 & $\begin{array}{c}7.73 \\
(5.12)\end{array}$ & $\begin{array}{c}6.80 \\
(4.49)\end{array}$ & 2.44 & 0.12 & 0.00 & 0.99 \\
\hline \multicolumn{11}{|c|}{ TAP: Untertest ,Go/Nogo“6 } \\
\hline $\begin{array}{l}\text { Standard- } \\
\text { abweichung }\end{array}$ & 31 & $\begin{array}{l}\mathbf{1 4 9 . 2 5} \\
(37.62)\end{array}$ & $\begin{array}{l}\mathbf{1 3 3 . 1 8} \\
(44.62)\end{array}$ & 15 & $\begin{array}{c}208.69 \\
(160.59)\end{array}$ & $\begin{array}{l}143.85 \\
(43.75)\end{array}$ & 6.18 & 0.01 & 4.29 & 0.04 \\
\hline Anzahl Fehler & 31 & $\begin{array}{c}7.84 \\
(6.02) \\
\end{array}$ & $\begin{array}{c}4.68 \\
(5.18) \\
\end{array}$ & 15 & $\begin{array}{c}9.53 \\
(4.79) \\
\end{array}$ & $\begin{array}{l}10.00 \\
(6.23) \\
\end{array}$ & 1.43 & 0.23 & 6.69 & 0.01 \\
\hline $\begin{array}{l}\text { Anzahl } \\
\text { Auslassungen }\end{array}$ & 31 & $\begin{array}{l}1.87 \\
(2.13)\end{array}$ & $\begin{array}{c}3.77 \\
(4.36) \\
\end{array}$ & 15 & $\begin{array}{c}2.80 \\
(3.10)\end{array}$ & $\begin{array}{c}1.80 \\
(1.90)\end{array}$ & 0.38 & 0.53 & 0.57 & 0.45 \\
\hline \multicolumn{11}{|c|}{ TAP: Untertest „Inkompatibilität"“ } \\
\hline $\begin{array}{l}\text { Standard- } \\
\text { abweichung }\end{array}$ & 29 & $\begin{array}{c}227.93 \\
(163.70) \\
\end{array}$ & $\begin{array}{r}187.49 \\
(80.30)\end{array}$ & 15 & $\begin{array}{l}179.11 \\
(80.37)\end{array}$ & $\begin{array}{c}210.81 \\
(230.13)\end{array}$ & 0.02 & 0.88 & 0.12 & 0.72 \\
\hline Anzahl Fehler & 29 & $\begin{array}{c}\mathbf{1 4 . 7 2} \\
(10.56) \\
\end{array}$ & $\begin{array}{c}\mathbf{8 . 5 2} \\
(6.76)\end{array}$ & 15 & $\begin{array}{c}16.27 \\
(10.37) \\
\end{array}$ & $\begin{array}{l}12.80 \\
(9.53)\end{array}$ & 7.65 & 0.00 & 1.51 & 0.22 \\
\hline \multicolumn{11}{|c|}{ TAP: Untertest „Visuelles Scanning“6 } \\
\hline $\begin{array}{l}\text { Standard- } \\
\text { abweichung } \\
\text { kritische Trials }\end{array}$ & 23 & $\begin{array}{c}3577.49 \\
(1875.05)\end{array}$ & $\begin{array}{c}2887.19 \\
(1434.07)\end{array}$ & 11 & $\begin{array}{c}\mathbf{3 1 4 2 . 8 0} \\
(2312.28)\end{array}$ & $\begin{array}{l}\mathbf{1 7 7 5 . 0 0} \\
(603.20)\end{array}$ & 8.13 & 0.00 & 3.45 & 0.07 \\
\hline $\begin{array}{l}\text { Standard- } \\
\text { abweichung } \\
\text { nicht-kritische } \\
\text { Trials }\end{array}$ & 23 & $\begin{array}{c}\mathbf{3 3 3 7 . 7 4} \\
(1680.22)\end{array}$ & $\begin{array}{l}\mathbf{2 5 1 0 . 1 5} \\
(905.79)\end{array}$ & 11 & $\begin{array}{l}\mathbf{2 1 1 8 . 4 6} \\
(831.97)\end{array}$ & $\begin{array}{l}\mathbf{1 4 8 4 . 1 5} \\
(419.35)\end{array}$ & 6.91 & 0.01 & 11.52 & 0.00 \\
\hline $\begin{array}{l}\text { Anzahl Fehler } \\
\text { (kritische } \\
\text { Trials) }\end{array}$ & 23 & $\begin{array}{c}7.78 \\
(4.59)\end{array}$ & $\begin{array}{l}\mathbf{3 . 3 5} \\
(2.84)\end{array}$ & 11 & $\begin{array}{c}6.45 \\
(3.47)\end{array}$ & $\begin{array}{l}3.45 \\
(2.25)\end{array}$ & 15.84 & 0.00 & 0.03 & 0.85 \\
\hline $\begin{array}{l}\text { Anzahl } \\
\text { Auslassungen } \\
\text { (nicht-kritische } \\
\text { Trials) }\end{array}$ & 23 & $\begin{array}{l}1.22 \\
(2.17)\end{array}$ & $\begin{array}{l}.52 \\
(.73)\end{array}$ & 11 & $\begin{array}{l}1.55 \\
(1.13)\end{array}$ & $\begin{array}{c}0.45 \\
(0.69)\end{array}$ & 0.02 & 0.88 & 1.93 & 0.17 \\
\hline \multicolumn{11}{|l|}{ Test d2 } \\
\hline $\begin{array}{l}\text { Konzentrations- } \\
\text { leistungswert }\end{array}$ & 21 & $\begin{array}{l}\mathbf{9 5 . 2 4} \\
(21.93)\end{array}$ & $\begin{array}{l}\mathbf{1 1 1 . 5 2} \\
(20.07)\end{array}$ & 15 & $\begin{array}{l}\mathbf{1 0 7 . 8 0} \\
(26.56)\end{array}$ & $\begin{array}{l}\mathbf{1 3 7 , 9 3} \\
(33.38)\end{array}$ & 120.52 & 0.00 & 3.26 & 0.08 \\
\hline Anzahl Fehler & 21 & $\begin{array}{l}11.19 \\
(8.69)\end{array}$ & $\begin{array}{c}8.52 \\
(4.98)\end{array}$ & 15 & $\begin{array}{l}\mathbf{1 4 . 4 0} \\
(12.11)\end{array}$ & $\begin{array}{c}\mathbf{5 . 6 7} \\
(4.10)\end{array}$ & 16.68 & 0.00 & 0.05 & 0.82 \\
\hline
\end{tabular}




\section{Anhang B}

Tabelle B- 8

Mittelwerte und Standardabweichungen (in Klammern) im Vor- und Nachtest für die AVn und Kennwerte $(F, p)$, der Haupteffekte Zeit und Gruppe des Interaktionseffekts zweifaktorieller Varianzanalysen mit Messwiederholung getrennt für die THOP und das Marburger Konzentrationstraining

\begin{tabular}{|c|c|c|c|c|c|c|c|c|c|c|}
\hline \multirow[t]{2}{*}{$\begin{array}{c}\text { Abhängige } \\
\text { Variable }\end{array}$} & \multicolumn{3}{|c|}{ Trainingsgruppe THOP } & \multicolumn{3}{|c|}{$\begin{array}{c}\text { Marburger } \\
\text { Konzentrationstraining } \\
\text { (MK) }\end{array}$} & \multicolumn{2}{|c|}{ Haupteffekt Zeit } & \multicolumn{2}{|c|}{$\begin{array}{c}\text { Haupteffekt } \\
\text { Gruppe }\end{array}$} \\
\hline & $n$ & Vortest & Nachtest & $n$ & Vortest & Nachtest & $F$ & $\bar{p}$ & $F$ & $\bar{p}$ \\
\hline \multicolumn{11}{|c|}{ TAP: Untertest „Geteilte Aufmerksamkeit““ } \\
\hline $\begin{array}{l}\text { Standard- } \\
\text { abweichung }\end{array}$ & 29 & $\begin{array}{c}\mathbf{3 3 7 . 8 2} \\
(116.48)\end{array}$ & $\begin{array}{l}\mathbf{2 9 5 . 8 6} \\
(95.81)\end{array}$ & 15 & $\begin{array}{c}275.59 \\
(129.44)\end{array}$ & $\begin{array}{l}241.21 \\
(73.63)\end{array}$ & 3.17 & 0.08 & 5.01 & 0.03 \\
\hline Anzahl Fehler & 29 & $\begin{array}{c}\mathbf{9 . 3 8} \\
(13.44) \\
\end{array}$ & $\begin{array}{c}\mathbf{3 . 0 7} \\
(4.03)\end{array}$ & 15 & $\begin{array}{c}3.80 \\
(6.04)\end{array}$ & $\begin{array}{c}4.27 \\
(4.22)\end{array}$ & 2.68 & 0.10 & 1.09 & 0.30 \\
\hline $\begin{array}{l}\text { Anzahl } \\
\text { Auslassungen }\end{array}$ & 29 & $\begin{array}{c}7.10 \\
(5.02)\end{array}$ & $\begin{array}{c}5.28 \\
(4.71)\end{array}$ & 15 & $\begin{array}{c}7.73 \\
(5.12)\end{array}$ & $\begin{array}{c}6.80 \\
(4.49)\end{array}$ & 2.23 & 0.14 & 0.76 & 0.38 \\
\hline \multicolumn{11}{|c|}{ TAP: Untertest „Go/Nogo“ } \\
\hline $\begin{array}{l}\text { Standard- } \\
\text { abweichung }\end{array}$ & 31 & $\begin{array}{l}140.54 \\
(42.28)\end{array}$ & $\begin{array}{l}125.59 \\
(42.09)\end{array}$ & 15 & $\begin{array}{c}208.69 \\
(160.59)\end{array}$ & $\begin{array}{l}143.85 \\
(43.75)\end{array}$ & 5.73 & 0.02 & 6.70 & 0.01 \\
\hline Anzahl Fehler & 31 & $\begin{array}{c}\mathbf{9 . 8 1} \\
(5.50)\end{array}$ & $\begin{array}{c}\mathbf{6 . 9 7} \\
(5.33)\end{array}$ & 15 & $\begin{array}{c}9.53 \\
(4.79)\end{array}$ & $\begin{array}{l}10.00 \\
(6.23)\end{array}$ & 1.46 & 0.23 & 0.95 & 0.33 \\
\hline $\begin{array}{l}\text { Anzahl } \\
\text { Auslassungen }\end{array}$ & 31 & $\begin{array}{c}\mathbf{2 . 1 0} \\
(2.70)\end{array}$ & $\begin{array}{c}\mathbf{0 . 8 4} \\
(1.44)\end{array}$ & 15 & $\begin{array}{c}2.80 \\
(3.10)\end{array}$ & $\begin{array}{c}1.80 \\
(1.90)\end{array}$ & 5.61 & 0.78 & 2.33 & 0.13 \\
\hline \multicolumn{11}{|c|}{ TAP: Untertest „Inkompatibilität"6 } \\
\hline $\begin{array}{l}\text { Standard- } \\
\text { abweichung }\end{array}$ & 31 & $\begin{array}{c}\mathbf{2 4 2 . 4 6} \\
(123.11) \\
\end{array}$ & $\begin{array}{r}\mathbf{1 7 6 . 6 3} \\
(64.19) \\
\end{array}$ & 15 & $\begin{array}{l}179.11 \\
(80.37) \\
\end{array}$ & $\begin{array}{c}210.81 \\
(230.13) \\
\end{array}$ & 0.44 & 0.50 & 0.22 & 0.63 \\
\hline Anzahl Fehler & 31 & $\begin{array}{c}\mathbf{2 0 . 4 2} \\
(12.05)\end{array}$ & $\begin{array}{l}\mathbf{1 3 . 5 5} \\
(10.00)\end{array}$ & 15 & $\begin{array}{c}16.27 \\
(10.37)\end{array}$ & $\begin{array}{l}12.80 \\
(9.53)\end{array}$ & 9.31 & 0.00 & 0.70 & 0.40 \\
\hline \multicolumn{11}{|c|}{ TAP: Untertest ,Visuelles Scanning“ } \\
\hline $\begin{array}{l}\text { Standard- } \\
\text { abweichung } \\
\text { kritische Trials }\end{array}$ & 31 & $\begin{array}{c}\mathbf{2 4 7 2 . 9 8} \\
(1048.18)\end{array}$ & $\begin{array}{l}\mathbf{2 0 4 9 . 0 0} \\
(995.37)\end{array}$ & 11 & $\begin{array}{c}\text { 3142.80 } \\
(2312.28)\end{array}$ & $\begin{array}{l}\mathbf{1 7 7 5 . 0 0} \\
(603.20)\end{array}$ & 12.22 & 0.00 & 0.10 & 0.74 \\
\hline $\begin{array}{l}\text { Standard- } \\
\text { abweichung } \\
\text { nicht-kritische } \\
\text { Trials }\end{array}$ & 31 & $\begin{array}{c}\mathbf{2 7 6 7 . 1 8} \\
(1185.42)\end{array}$ & $\begin{array}{c}\mathbf{2 1 9 2 . 0 3} \\
(1028.39)\end{array}$ & 11 & $\begin{array}{l}\mathbf{2 1 1 8 . 4 6} \\
(831.97)\end{array}$ & $\begin{array}{l}\mathbf{1 4 8 4 . 1 5} \\
(419.35)\end{array}$ & 11.54 & 0.00 & 4.82 & 0.03 \\
\hline $\begin{array}{l}\text { Anzahl Fehler } \\
\text { (kritische } \\
\text { Trials) } \\
\end{array}$ & 31 & $\begin{array}{c}7.68 \\
(5.95)\end{array}$ & $\begin{array}{c}4.32 \\
(4.10)\end{array}$ & 11 & $\begin{array}{c}6.45 \\
(3.47)\end{array}$ & $\begin{array}{l}3.45 \\
(2.25)\end{array}$ & 11.67 & 0.00 & 0.01 & 0.89 \\
\hline $\begin{array}{l}\text { Anzahl } \\
\text { Auslassungen } \\
\text { (nicht-kritische } \\
\text { Trials) }\end{array}$ & 31 & $\begin{array}{c}\mathbf{5 . 3 2} \\
(5.96)\end{array}$ & $\begin{array}{c}\mathbf{2 . 2 6} \\
(3.26)\end{array}$ & 11 & $\begin{array}{c}1.55 \\
(1.13)\end{array}$ & $\begin{array}{c}0.45 \\
(0.69)\end{array}$ & 1.19 & 0.28 & 1.71 & 0.19 \\
\hline \multicolumn{11}{|l|}{ Test d2 } \\
\hline $\begin{array}{l}\text { Konzentrations- } \\
\text { leistungswert }\end{array}$ & 15 & $\begin{array}{c}\mathbf{9 3 . 9 3} \\
(25.92)\end{array}$ & $\begin{array}{l}\mathbf{1 2 3 . 2 0} \\
(25.27)\end{array}$ & 15 & $\begin{array}{l}\mathbf{1 0 7 . 8 0} \\
(26.56)\end{array}$ & $\begin{array}{l}\mathbf{1 3 7 , 9 3} \\
(33.38)\end{array}$ & 123.91 & 0.00 & 0.96 & 0.33 \\
\hline Anzahl Fehler & 15 & $\begin{array}{c}\mathbf{2 6 . 2 7} \\
(37.59) \\
\end{array}$ & $\begin{array}{c}9.27 \\
(6.30) \\
\end{array}$ & 15 & $\begin{array}{c}\mathbf{1 4 . 4 0} \\
(12.11) \\
\end{array}$ & $\begin{array}{c}\mathbf{5 . 6 7} \\
(4.10) \\
\end{array}$ & 7.78 & 0.00 & 1.83 & 0.18 \\
\hline
\end{tabular}


Anhang C.1

Psychologischen Vorhersagen (PVn), Statistische Hypothesen (SHn) und Testplanung zu Studie 2: Wirksamkeit der Kombination von Basistraining und Strategietraining nach Lauth und Schlottke

\section{PVn und SVn zu Studie 2}

Die bei Studie 1 genannten Hypothesen 1.1 bis $3.1 \mathrm{~b}$ wurden sinngemäß auf Studie 2 übertragen. Gleiches gilt für die Operationalisierungen, die Psychologischen Vorhersagen und Statistischen Hypothesen. Da das Training mehr Stunden umfasst und qualitativ umfangreicher ist, sollten die Effekte jeweils etwas stärker zu Tage treten. Der Vergleich der beiden Interventionsgruppen aus Studie 1 und 2 (PH 2.4) wird bei der vergleichenden Evaluation der verschiedenen Interventionen dargestellt (s.u.).

\section{Testplanung zu Studie 2}

Die Gruppengröße der Trainingsgruppe ist in dieser Studie höher. Das $n$ beträgt gemittelt maximal 23, woraus sich bei Berechnung der o.g. Formel für die Fehlerwahrscheinlichkeit $\beta$ ein Wert von .41. Die Teststärke beträgt somit .59.

Der durch drop-out bedingte Minimalwert des $n$ beträgt gemittelt 16, woraus sich eine Fehlerwahrscheinlichkeit $\beta$ von .53 ergibt. 


\section{Anhang C.2}

\section{PVn und SVn zu Studie 3}

Auch die Autoren des THOP konnten in eigenen Evaluationsuntersuchungen positive Effekte ihres Trainings auf die Störungssymptomatik nachweisen. Es werden auch hier in der Trainingsgruppe in allen AVn Verbesserungen vom Vor- zum Nachtest erwartet. In der Wartegruppe sollten sich hingegen keine (bzw. lediglich geringfügige) Verbesserungen zeigen. Je nach Definition der AV äußern sich positive Veränderungen vom Vor- zum Nachtest in einem Absinken oder einem Ansteigen der entsprechenden Kennwerte.

Der Vergleich des THOP mit den beiden Interventionsgruppen nach Lauth und Schlottke aus Studie 1 und 2 wird bei der vergleichenden Evaluation der verschiedenen Interventionen dargestellt (s.u.).

Tabelle C.2-1

Operationalisierungen der psychologischen Hypothesen

PH 3.1: Das THOP führt zu einer Verminderung der Störungssymptomatik, wenn es in Form einer Gruppenbehandlung im Rahmen einer kinder- und jugendpsychiatrischen Ambulanz durchgeführt wird.

\begin{tabular}{|c|c|}
\hline Untersuchungsverfahren & Kriteriumsmaße (AV) \\
\hline $\begin{array}{l}\text { TAP } \\
\text { Geteilte Aufmerksamkeit }\end{array}$ & $\begin{array}{ll}- & \text { Standardabweichung } \\
- & \text { Anzahl Fehler } \\
\text { - } & \text { Anzahl Auslassungen }\end{array}$ \\
\hline Go/ Nogo & $\begin{array}{ll}- & \text { Standardabweichung } \\
- & \text { Anzahl Fehler } \\
- & \text { Anzahl Auslassungen }\end{array}$ \\
\hline Inkompatibilität & $\begin{array}{ll}- & \text { Standardabweichung } \\
- & \text { Anzahl Fehler }\end{array}$ \\
\hline Visuelles Scanning & $\begin{array}{ll}- & \text { Standardabweichung kritische Trials } \\
- & \text { Standardabweichung nicht- kritische Trials } \\
- & \text { Anzahl Fehler } \\
- & \text { Anzahl Auslassungen } \\
\end{array}$ \\
\hline Test d2 & $\begin{array}{ll}- & \text { Konzentrationsleistungswert } \\
- & \text { Fehler }\end{array}$ \\
\hline $\begin{array}{l}\text { FBB-HKS: Elternurteil } \\
\text { FBB-HKS: Lehrerurteil }\end{array}$ & $\begin{array}{l}\text { Jeweils für die Bereiche Unaufmerksamkeit, Hyperaktivität und } \\
\text { Impulsivität: } \\
-\quad \text { Anzahl erfüllter Kriterien } \\
-\quad \text { Kennwert }\end{array}$ \\
\hline $\begin{array}{l}\text { CBCL } \\
\text { TRF }\end{array}$ & - Jeweils T-Wert der Skala Aufmerksamkeitsstörungen \\
\hline
\end{tabular}


PH 3.2: Das THOP als Gruppenprogramm führt zu einer Verminderung von oppositionellen bzw. aggressiven Verhaltensweisen.

\begin{tabular}{|c|c|}
\hline Untersuchungsverfahren & Kriteriumsmaße (AV) \\
\hline $\begin{array}{l}\text { CBCL } \\
\text { TRF }\end{array}$ & - Jeweils T-Wert der Skala Externalisierende Störungen \\
\hline
\end{tabular}

PH 3.3: Das THOP als Gruppenprogramm führt zu einer Verminderung emotionaler Beeinträchtigungen.

\begin{tabular}{l|l}
\hline \multicolumn{1}{c|}{ Untersuchungsverfahren } & \multicolumn{1}{c}{ Kriteriumsmaße (AV) } \\
\hline CBCL & $-\quad$ Jeweils T-Wert der Gesamtskala ${ }_{(\mathrm{INT})}$ \\
TRF & \\
\hline $\begin{array}{l}\text { Anmerkungen. PH: psychologische Hypothese, AV: abhängige Variable, FBB-HKS: Fremdbeurteilungsbogen } \\
\text { Hyperkinetische Störungen, CBCL: Child Behavior Checklist, TRF: Teacher's Report Form, TAP: Testbatterie zur } \\
\text { Aufmerksamkeitsprüfung. }\end{array}$ \\
\hline
\end{tabular}

Exemplarisch wird im Folgenden für die isolierte Evaluation die psychologische Vorhersage (PV) der Variablen „T-Wert der Skala Aufmerksamkeitsstörungen in der CBCL“ dargestellt, sowie die daraus abgeleitete statistische Vorhersage (SV), die sich aus den einzelnen testbaren statistischen Hypothesen zusammensetzt.

PV 3.1: $\quad$ In der THOP-Gruppe (THOP) verringert sich im Mittel der T-Wert der Skala Aufmerksamkeitsstörungen der Child Behavior Checklist (CBCL-A) vom Vorzum Nachtest und in der Wartegruppe (WG) verringert sich im Mittel der TWert der Skala Aufmerksamkeitsstörungen der Child Behavior Checklist vom Vor- zum Nachtest oder er bleibt gleich.

SV 3.1: $\quad\left\{\left[\mathrm{H}_{1,1}: \mu_{(\mathrm{THOP})(\mathrm{N})}(\mathrm{CBCL}-\mathrm{A})-\mu_{(\mathrm{THOP})(\mathrm{V})}(\mathrm{CBCL}-\mathrm{A})<0\right] \wedge\right.$ $\left.\left[\mathrm{H}_{0,2}: \quad \mu_{(\mathrm{WG})(\mathrm{N})}(\mathrm{CBCL}-\mathrm{A})-\mu_{(\mathrm{WG})(\mathrm{V})}(\mathrm{CBCL}-\mathrm{A}) \leq 0\right]\right\}$

Zur Überprüfung der PH 3.2 wird der T-Wert der Skala Externalisierende Störungen im TRF und CBCL als abhängige Maße eingesetzt, für die wieder jeweils eine PV formuliert wird. Wenn das THOP die theoretisch angenommenen Wirkungen zeigt, wird für jede dieser AVn ein Absinken der Kennwerte vom Vor- zum Nachtest erwartet. Es wird beispielhaft die Skala Externalisierende Störungen der CBCL dargestellt. 
PV 3.2: $\quad$ In der THOP-Gruppe (THOP) verringert sich im Mittel der T-Wert der Skala Externalisierende Störungen der Child Behavior Checklist (CBCL-E) vom Vor- zum Nachtest und in der Wartegruppe (WG) verringert sich im Mittel der T-Wert der Skala Externalisierende Störungen der Child Behavior Checklist vom Vor- zum Nachtest oder er bleibt gleich.

SV 3.2: $\left\{\left[\mathrm{H}_{1,1}: \mu_{(\mathrm{THOP})(\mathrm{N})}(\mathrm{CBCL}-\mathrm{E})-\mu_{(\mathrm{THOP})(\mathrm{V})}(\mathrm{CBCL}-\mathrm{E})<0\right] \wedge\right.$ $\left.\left[\mathrm{H}_{0,2}: \quad \mu_{(\mathrm{WG})(\mathrm{N})}(\mathrm{CBCL}-\mathrm{E})-\mu_{(\mathrm{WG})(\mathrm{V})}(\mathrm{CBCL}-\mathrm{E}) \leq 0\right]\right\}$

Zur Überprüfung der PH 3.3 werden jeweils der T-Wert des Gesamtwertes im TRF und CBCL als abhängige Maße eingesetzt, für die wieder jeweils eine PV formuliert wird. Wenn das THOP die theoretisch angenommenen Wirkungen zeigt, wird für jede dieser AVn ein Absinken der Kennwerte vom Vor- zum Nachtest erwartet. Die PV 3.3 und SH 3.3 entspricht sinngemäß der Darstellung PV 3.2 und SH 3.2.

\section{Testplanung zu Studie 3}

Bei einer Stichprobengröße von 16 (Wartegruppe) und $31^{3}$ (THOP) errechnet sich für den Interaktionskontrast (unter Verwendung der Verteilungsfunktion der Standardnormalverteilung in Bortz, 1993) in der isolierten Evaluation für die Fehlerwahrscheinlichkeit $\beta$ ein Wert von .41. Über den Ausdruck 1- $\beta$ kann damit auch die Teststärke eines Signifikanztests bestimmt werden. Für den Interaktionskontrast liegt sie bei einem Wert von .59.

\footnotetext{
${ }^{3}$ Auf eine Feinadjustierung der Testplanungsformel für den Fall ungleichgroßer Stichproben wurde verzichtet, da der Unterschied in der Stichprobengröße relativ gering ist. Als Berechnungsgrundlage für den Interaktionskontrast der isolierten Evaluation wurde der Mittelwert der Stichprobengrößen der Wartegruppe $(n=16)$ und der THOP-Gruppe $(n=31)$ gebildet.
} 


\section{Anhang C.3}

\section{PVn und SVn zu Studie 4}

Auch für das Marburger Konzentrationstraining werden positive Effekte auf die Störungssymptomatik erwartet, da dies in einigen wenigen Untersuchungen gezeigt werden konnte. Es werden auch hier in der Trainingsgruppe in allen AVn Verbesserungen vom Vorzum Nachtest erwartet. In der Wartegruppe sollten sich hingegen keine (bzw. lediglich geringfügige) Verbesserungen zeigen, was sich wiederum je nach Definition der AV in positiven Veränderungen vom Vor- zum Nachtest bzw. in einem Absinken oder einem Ansteigen der entsprechenden Kennwerte äußert.

Tabelle C.3-1

Operationalisierungen der psychologischen Hypothesen

PH 4.1: Das Marburger Konzentrationsprogramm führt bei Kindern mit Aufmerksamkeitsstörungen zu einer verbesserten Konzentrationsleistung, wenn es im Rahmen eines Gruppentrainings in einer Kinder- und Jugendpsychiatrischen Praxis durchgeführt wird

\begin{tabular}{|c|c|}
\hline Untersuchungsverfahren & Kriteriumsmaße (AV) \\
\hline \multicolumn{2}{|l|}{ TAP } \\
\hline Geteilte Aufmerksamkeit & $\begin{array}{ll}- & \text { Standardabweichung } \\
- & \text { Anzahl Fehler } \\
- & \text { Anzahl Auslassungen }\end{array}$ \\
\hline Go/ Nogo & $\begin{array}{ll}- & \text { Standardabweichung } \\
- & \text { Anzahl Fehler } \\
- & \text { Anzahl Auslassungen }\end{array}$ \\
\hline Inkompatibilität & $\begin{array}{ll}- & \text { Standardabweichung } \\
- & \text { Anzahl Fehler }\end{array}$ \\
\hline Visuelles Scanning & $\begin{array}{ll}- & \text { Standardabweichung kritische Trials } \\
- & \text { Standardabweichung nicht- kritische Trials } \\
- & \text { Anzahl Fehler } \\
- & \text { Anzahl Auslassungen }\end{array}$ \\
\hline Test d2 & $\begin{array}{ll}- & \text { Konzentrationsleistungswert } \\
- & \text { Fehler }\end{array}$ \\
\hline ZST aus HAWIK-III & - $\quad$ Anzahl richtiger Items \\
\hline CFT 20 & $\begin{array}{ll}- & \text { IQ Gesamt } \\
- & \text { IQ Differenz Teil 1 - Teil } 2 \\
\end{array}$ \\
\hline $\begin{array}{l}\text { FBB-HKS: Elternurteil } \\
\text { FBB-HKS: Lehrerurteil }\end{array}$ & $\begin{array}{l}\text { Jeweils für die Bereiche Unaufmerksamkeit, Hyperaktivität und } \\
\text { Impulsivität: } \\
-\quad \text { Anzahl erfüllter Kriterien } \\
-\quad \text { Kennwert }\end{array}$ \\
\hline $\begin{array}{l}\text { CBCL } \\
\text { TRF }\end{array}$ & - Jeweils T-Wert der Skala Aufmerksamkeitsstörungen \\
\hline
\end{tabular}


PH 4.2: Durch das Marburger Konzentrationstraining übertragen die trainierten Kinder die erarbeiteten Selbstanweisungen auch auf schulische Belange.

\begin{tabular}{|c|c|}
\hline Untersuchungsverfahren & Kriteriumsmaße (AV) \\
\hline $\begin{array}{l}\text { Testbatterie zur Prüfung der } \\
\text { Konzentrationsfähigkeit (TPK) }\end{array}$ & $\begin{array}{ll}- & \text { TSL } \\
- & \text { TF\% } \\
- & \text { Tiere } \\
- & \text { RL } \\
- & \text { RF\% } \\
- & \text { KW }\end{array}$ \\
\hline
\end{tabular}

PH 4.3: Das Marburger Konzentrationstraining führt zu einer positiven Veränderung der Eltern-Kind- und LehrerKind-Interaktion

\begin{tabular}{|c|c|}
\hline Untersuchungsverfahren & Kriteriumsmaße (AV) \\
\hline $\begin{array}{l}\text { CBCL } \\
\text { TRF }\end{array}$ & - Jeweils T-Wert der Gesamtskala \\
\hline
\end{tabular}

Anmerkungen. PH: psychologische Hypothese, AV: abhängige Variable, FBB-HKS: Fremdbeurteilungsbogen Hyperkinetische Störungen, CBCL: Child Behavior Checklist, TRF: Teacher's Report Form, TAP: Testbatterie zur Aufmerksamkeitsprüfung, ZST: Zahlen-Symbol-Test, HAWIK-III: Hamburg Wechsler Intelligenztest für Kinder, TSL: Anzahl geschriebener Silben in 10 Minuten, TF\%: Fehler in Relation zur Gesamtmenge geschriebener Silben, RL: Rechenleistung, Anzahl in 10 Minuten, RF\%: Fehlerprozent bei Rechenaufgaben, KW: Konzentrationswert

Aus diesen psychologischen Hypothesen werden für jede abhängige Variable (AV) psychologische Vorhersagen (PV) abgeleitet. Es wird erwartet, dass sich bei den Kindern der Trainingsgruppe Verbesserungen hinsichtlich der Konzentrationsleistung vom Vor- zum Nachtest zeigen. Diese Erwartung wird durch bisherige Untersuchungen zum Marburger Konzentrationstraining gestützt. Bei den Kindern der Kontrollgruppe wird davon ausgegangen, dass sich keine oder nur minimale Veränderungen zeigen. Hierbei können die positiven Veränderungen sowohl als negative Werte (z.B. Verringerung der Fehlerzahl) wie auch durch positive Werte (z.B. schnellere Bearbeitung) deutlich werden.

Für die PH1 wird angenommen, dass sich die Kennwerte bei den AVn Zahlensymboltest (ZST), IQ gesamt im CFT 20 (CFT) und Konzentrationsleistungswert (KL) im d2 erhöhen und bei allen anderen Kennwerten verringern. Somit ergibt sich, exemplarisch für die Variable Zahlensymboltest, folgende empirische Vorhersage:

PV 4.1: In der Trainingsgruppe steigt die Anzahl der richtig bearbeiteten Zeichen im Zahlensymboltest deutlicher an als in der Kontrollgruppe, d.h. die Differenz der ZST Werte Nachtest minus Vortest ist in der TG größer als in der KG.

Aus dieser Empirischen Vorhersage wird die statistische Interaktionshypothese abgeleitet: 
SH 4.1: $\left[\mu_{\mathrm{TG}, \mathrm{N}}(\mathrm{ZST})-\mu_{\mathrm{TG}, \mathrm{V}}(\mathrm{ZST})\right]-\left[\mu_{\mathrm{KG}, \mathrm{N}}(\mathrm{ZST})-\mu_{\mathrm{KG}, \mathrm{v}}(\mathrm{ZST})\right]>0$

Anmerkung: ZST steht für die Anzahl richtig reproduzierter Zeichen im Zahlensymboltest des HAWIK- III, $\mu_{\text {TG }}$ und $\mu_{\text {KG }}$ bezeichnen die Mittelwerte der Trainingsgruppe (TG) bzw. der Kontrollgruppe (KG), V und N beziehen sich auf die Erhebungszeitpunkte Vor- (V) und Nachtest (N).

Zur Überprüfung der PH 4.2 (Transferhypothese) wurden als AVn die Kennwerte der Testbatterie zur Prüfung der Konzentrationsfähigkeit (TPK) eingesetzt. Hierbei wird für die AVn TSL, Tiere, RL und KW eine Steigerung und für die AVn TF\% und RF\% ein Absinken der Kennwerte in der Trainingsgruppe erwartet, welche sich von den Ergebnissen der Kontrollgruppe signifikant abheben. Demzufolge sind die empirischen Vorhersagen und statistischen Hypothesen analog aufzustellen.

Für die Prüfung der PH 4.3 (Transferhypothese) werden analog die empirischen Vorhersagen und statistischen Hypothesen aufgestellt. Hierbei wird erwartet, dass sich die TWerte der Gesamtskala in der Trainingsgruppe bedeutsam verringern.

\section{Testplanung zu Studie 4}

Bei einer gemittelten Stichprobengröße von 15 errechnet sich für den Interaktionskontrast (unter Verwendung der Verteilungsfunktion der Standardnormalverteilung in Bortz, 1993) in der isolierten Evaluation für die Fehlerwahrscheinlichkeit $\beta$ ein Wert von .55. Über den Ausdruck 1- $\beta$ kann damit auch die Teststärke eines Signifikanztests bestimmt werden. Für den Interaktionskontrast liegt sie bei einem Wert von .45. 


\section{Anhang C.4}

\section{PVn und SVn zu den Studien 1-4}

Neben einer Verbesserung der Störungssymptomatik über die Zeit (Vergleich Vor- zu Nachtest) für die verschiedenen Trainings im Vergleich zu einer Wartegruppe interessiert wie die verschiedenen Trainings im Vergleich zueinander abschneiden.

Dabei werden die beiden Interventionen nach Lauth und Schlottke (10 vs. 15 Sitzungen), das THOP und das Marburger Konzentrationstraining miteinander verglichen.

Analog der zu den isolierten Studien dargestellten Vorgehensweise der Ableitung der Psychologischen Vorhersagen und der statistischen Hypothesen aus den Psychologischen Hypothesen wird auch hier exemplarisch vorgegangen.

Die Operationalisierungen zur PH 5.1 entsprechen der Tabelle 11 (s.o.). Es wird erwartet, dass die Ergebnisse durch das Training nach Lauth und Schlottke, bei dem in 15 Sitzungen mit den Kindern gearbeitet wurde zu besseren Ergebnissen in allen AVn führt als das Training nach Lauth und Schlottke, bei dem nur das Basistraining durchgeführt wurde.

Die Psychologische Hypothese:

PH 5.1: Das Training nach Lauth und Schlottke führt zu einer stärkeren Reduzierung der Symptomatik, wenn die Kinder sowohl mit dem Basis- als auch dem Strategietraining behandelt werden als wenn sie nur das Basistraining erhalten

wird in folgende Psychologische Vorhersagen und Statistische Hypothesen überführt.

Exemplarisch wird dies für den Fehlerwert der TAP (Untertest Go/Nogo) dargestellt:

PV 5.1a: In der Trainingsgruppe (LS-15) nimmt die Anzahl der Fehler im TAP-Untertest „Go/Nogo“ vom Vor- zum Nachtest deutlicher ab als in der Trainingsgruppe (LS-10), d.h. die Differenz der Fehler vom Vor- vs. Nachtest ist in der LS-15 größer als in der LS-10.

(Anmerkung. LS-15 / LS-10 steht für Training nach Lauth und Schlottke 15 Sitzungen bzw. 10 Sitzungen).

SH 5.1a: $\quad\left[\mu_{\mathrm{LS}-15, \mathrm{v}}(\mathrm{F}-\mathrm{Go} / \mathrm{Nogo})-\mu_{\mathrm{LS}-15, \mathrm{~N}}(\mathrm{~F}-\mathrm{Go} / \mathrm{Nogo})\right]-\left[\mu_{\mathrm{LS}-10, \mathrm{~V}}(\mathrm{~F}-\mathrm{Go} / \mathrm{Nogo})-\mu_{\mathrm{LS}-10,}\right.$ $\left.{ }_{\mathrm{N}}(\mathrm{F}-\mathrm{Go} / \mathrm{Nogo})\right]>0$ 
Anmerkung: F-Go/Nogo steht für die AV: Anzahl der Fehler im TAP-Untertest „Go/Nogo“, $\mu_{\mathrm{LS}-15}$ bzw. $\mu_{\mathrm{LS}-10}$ zeigt an, dass der Mittelwert für die Trainingsgruppen nach Lauth und Schlottke mit je 15 bzw. 10 Sitzungen betrachtet wird, V steht für Vortest und $\mathrm{N}$ für Nachtest

In den Studien 1 und 2 wurden zum Teil andere AVn eingesetzt als in Studie 4. In der folgenden Tabelle sind alle jene AVn aufgelistet, die in allen Studien eingesetzt wurden.

Tabelle C.4-1

Operationalisierungen der psychologischen Hypothesen

PH 5.2: Das Training nach Lauth und Schlottke führt in gleichem Maße zu einer Reduzierung der Symptomatik wie das Marburger Konzentrationstraining.

PH 5.3: Das Training nach Lauth und Schlottke sowie das Marburger Konzentrationstraining führen zu einer stärkeren Verminderung von Aufmerksamkeitsproblemen als das THOP als Gruppenprogramm.

\begin{tabular}{|c|c|}
\hline Untersuchungsverfahren & Kriteriumsmaße (AV) \\
\hline \multicolumn{2}{|l|}{ TAP } \\
\hline Geteilte Aufmerksamkeit & $\begin{array}{ll}- & \text { Standardabweichung } \\
- & \text { Anzahl Fehler } \\
- & \text { Anzahl Auslassungen } \\
\end{array}$ \\
\hline Go/ Nogo & $\begin{array}{ll}- & \text { Standardabweichung } \\
- & \text { Anzahl Fehler } \\
- & \text { Anzahl Auslassungen } \\
\end{array}$ \\
\hline Inkompatibilität & $\begin{array}{ll}- & \text { Standardabweichung } \\
- & \text { Anzahl Fehler } \\
\end{array}$ \\
\hline Visuelles Scanning & $\begin{array}{ll}- & \text { Standardabweichung kritische Trials } \\
- & \text { Standardabweichung nicht- kritische Trials } \\
- & \text { Anzahl Fehler } \\
- & \text { Anzahl Auslassungen }\end{array}$ \\
\hline Test d2 & $\begin{array}{ll}- & \text { Konzentrationsleistungswert } \\
- & \text { Fehler } \\
\end{array}$ \\
\hline FBB-HKS: Elternurteil & $\begin{array}{l}\text { Jeweils für den Bereich Unaufmerksamkeit: } \\
-\quad \text { Anzahl erfüllter Kriterien }\end{array}$ \\
\hline FBB-HKS: Lehrerurteil & $-\quad$ Kennwert \\
\hline $\begin{array}{l}\text { CBCL } \\
\text { TRF }\end{array}$ & - Jeweils T-Wert der Skala Aufmerksamkeitsstörungen \\
\hline
\end{tabular}


PH 5.4a: Das THOP als Gruppenprogramm führt zu einer stärkeren Verminderung von oppositionellen und aggressiven Verhaltensweisen im häuslichen Bereich als das Vergleichsprogramm nach Lauth und Schlottke sowie dem Marburger Konzentrationstraining und in mindestens dem gleichen Maße wie die Vergleichsprogramme zu einer Verminderung von oppositionellen und aggressiven Verhaltensweisen im schulischen Bereich und

PH 5.4b: Das THOP als Gruppenprogramm führt zu einer stärkeren Verminderung von hyperaktivem und impulsivem Verhalten im häuslichen Bereich als das Vergleichsprogramm nach Lauth und Schlottke sowie dem Marburger Konzentrationstraining und in mindestens dem gleichen Maße wie die Vergleichsprogramme zu einer Verminderung von hyperaktivem und impulsivem Verhalten im schulischen Bereich.

\begin{tabular}{l|l}
\hline CBCL & $-\quad$ Jeweils T-Wert der Skala Externalisierende Störungen \\
TRF & $\begin{array}{l}\text { Jeweils für die Bereiche Hyperaktivität und Impulsivität: } \\
-\quad \text { Anzahl erfüllter Kriterien }\end{array}$ \\
\hline FBB-HKS: Elternurteil & $-\quad$ Kennwert \\
\hline FBB-HKS: Lehrerurteil & \\
\hline
\end{tabular}

PH 5.5: Das THOP als Gruppenprogramm führt in mindestens dem gleichen Maße wie die Vergleichsprogramme zu einer Verminderung emotionaler Beeinträchtigungen.

\begin{tabular}{l|c}
\hline \multicolumn{1}{c|}{ Untersuchungsverfahren } & Kriteriumsmaße (AV) \\
\hline CBCL & - Jeweils T-Wert der Gesamtskala \\
TRF & \\
\hline
\end{tabular}

Anmerkungen. PH: psychologische Hypothese, AV: abhängige Variable, FBB-HKS: Fremdbeurteilungsbogen

Hyperkinetische Störungen, CBCL: Child Behavior Checklist, TRF: Teacher's Report Form, TAP: Testbatterie zur Aufmerksamkeitsprüfung

Zur Überprüfung der PH 5.2 wird der T-Wert der Skala Aufmerksamkeitsstörungen im TRF und CBCL als abhängige Maße eingesetzt, für die wieder jeweils eine PV formuliert wird. Wenn sowohl das Training nach Lauth und Schlottke als auch das Marburger Konzentrationstraining die theoretisch angenommenen Wirkungen zeigen, wird für die AV ein Absinken der Kennwerte vom Vor- zum Nachtest in beiden Trainingsgruppen erwartet. Sie sollten sich nicht signifikant voneinander unterscheiden.

PV 5.2: $\quad$ In der Trainingsgruppe nach Lauth und Schlottke (LS) sowie in der Trainingsgruppe, die das Marburger Konzentrationstraining (MK) absolviert hat, verringert sich im Mittel der T-Wert der Skala Aufmerksamkeitsstörungen der Child Behavior Checklist (CBCL-A) vom Vor- zum Nachtest. Der Differenzwert des Marburger Trainings sollte nicht schlechter sein als jener für die Lauth und Schlottke-Gruppe 
SH 5.2: $\quad\left\{\left[\mathrm{H}_{1,1}: \mu_{(\mathrm{LS})(\mathrm{N})}(\mathrm{CBCL}-\mathrm{A})-\mu_{(\mathrm{LS})(\mathrm{V})}(\mathrm{CBCL}-\mathrm{A})>0\right] \wedge\right.$

$\left[\mathrm{H}_{1,2}: \mu \mu_{(\mathrm{MK})(\mathrm{N})}(\mathrm{CBCL}-\mathrm{A})-\mu_{(\mathrm{MK})(\mathrm{V})}(\mathrm{CBCL}-\mathrm{A})>0\right] \wedge$

$\left[\mathrm{H}_{0,3}: \quad\left[\mu_{(\mathrm{LS})(\mathrm{N})}(\mathrm{CBCL}-\mathrm{A})-\mu_{(\mathrm{LS})(\mathrm{V})}(\mathrm{CBCL}-\mathrm{A})\right]-\left[\mu_{(\mathrm{MK})(\mathrm{N})}(\mathrm{CBCL}-\mathrm{A})-\mu_{(\mathrm{MK})}\right.\right.$ (V) $(\mathrm{CBCL}-\mathrm{A})] \leq 0]\}$

Die PH 5.3 besagt, dass das Training nach Lauth und Schlottke sowie das Marburger Konzentrationstraining dem THOP hinsichtlich der Verbesserung von Aufmerksamkeitsparametern überlegen sind. Exemplarisch sei dies ebenfalls als psychologische Vorhersage (PV) für die Variable „T-Wert der Skala Aufmerksamkeitsstörungen in der $\mathrm{CBCL}^{\text {“ }}$ sowie die daraus abgeleitete statistische Vorhersage (SH) dargestellt.

PV 5.3: $\quad$ In der THOP-Gruppe (THOP) verringert sich im Mittel der T-Wert der Skala Aufmerksamkeitsstörungen der Child Behavior Checklist (CBCL-A) vom Vorzum Nachtest und in der Lauth-und-Schlottke-Gruppe (LS) verringert sich im Mittel der T-Wert der Skala Aufmerksamkeitsstörungen der Child Behavior Checklist vom Vor- zum Nachtest. Gleiches gilt für das Marburger Konzentrationstraining. Der T-Wert der Skala Aufmerksamkeitsstörungen der Child Behavior Checklist nimmt im Mittel in der Lauth-und-Schlottke-Gruppe sowie der Gruppe mit dem Marburger Konzentrationstraining vom Vor- zum Nachtest stärker ab als in der THOP-Gruppe.

SH-5.3: $\left\{\left[\mathrm{H}_{1,1}: \mu_{(\mathrm{THOP})(\mathrm{N})}(\mathrm{CBCL}-\mathrm{A})-\mu_{(\mathrm{THOP})(\mathrm{V})}(\mathrm{CBCL}-\mathrm{A})<0\right] \wedge\right.$

$$
\begin{array}{ll}
{\left[\mathrm{H}_{1,2}:\right.} & \left.\mu_{(\mathrm{LS})(\mathrm{N})}(\mathrm{CBCL}-\mathrm{A})-\mu_{(\mathrm{LS})(\mathrm{V})}(\mathrm{CBCL}-\mathrm{A})<0\right] \wedge \\
{\left[\mathrm{H}_{1,3}:\right.} & \left.\mu_{(\mathrm{MK})(\mathrm{N})}(\mathrm{CBCL}-\mathrm{A})-\mu_{(\mathrm{MK})(\mathrm{V})}(\mathrm{CBCL}-\mathrm{A})<0\right] \wedge \\
{\left[\mathrm{H}_{1,4}:\right.} & {\left[\mu_{(\mathrm{LS})(\mathrm{N})}(\mathrm{CBCL}-\mathrm{A})-\mu_{(\mathrm{LS})(\mathrm{V})}(\mathrm{CBCL}-\mathrm{A})\right]-} \\
& \left.\left[\mu_{(\mathrm{THOP})(\mathrm{N})}(\mathrm{CBCL}-\mathrm{A})-\mu_{(\mathrm{THOP})(\mathrm{V})}(\mathrm{CBCL}-\mathrm{A})\right]>0\right] \wedge \\
{\left[\mathrm{H}_{1,5}:\right.} & {\left[\mu_{(\mathrm{MK})(\mathrm{N})}(\mathrm{CBCL}-\mathrm{A})-\mu_{(\mathrm{MK})(\mathrm{V})}(\mathrm{CBCL}-\mathrm{A})\right]-} \\
& \left.\left.\left[\mu_{(\mathrm{THOP})(\mathrm{N})}(\mathrm{CBCL}-\mathrm{A})-\mu_{(\mathrm{THOP})(\mathrm{V})}(\mathrm{CBCL}-\mathrm{A})\right]>0\right]\right\}
\end{array}
$$

Sinngemäß würden die Psychologischen Vorhersagen sowie die Statistischen Hypothesen für die PH 5.4 ausfallen. Hier würde jedoch erwartet werden, dass es z.B. im TWert der Skala Externalisierende Störungen in der CBCL in der THOP-Gruppe zu einer 
deutlicheren Verbesserung kommt als in den anderen Interventionsgruppen. Im schulischen Bereich würde eine Nichtunterlegenheit erwartet werden

Auch hinsichtlich des emotionalen Befindens (PH 5.5) wird aus Sicht des THOP eine Nicht-Unterlegenheitshypothese formuliert, welche in entsprechende Psychologische Vorhersagen und Statistische Hypothesen hinsichtlich z.B. des T-Wertes der Gesamtskala der CBCL überführt wurde.

\section{Testplanung zu den Studien 1- 4}

Für die vergleichende Evaluation (Kombiniertes Basis- und Strategietraining nach Lauth und Schlottke mit dem THOP) ergibt sich bei einem gemittelten Stichprobenumfang von $32^{4}$ und gleichen Festlegungen bzgl. der anderen Kennwerte (s.o. Testplanung zur Isolierten Evaluation Studie 1) ein Wert für $\beta$ von .28 und damit eine Teststärke von .72.

Für den Vergleich des LS-Trainings mit dem Marburger Konzentrationstraining ergibt sich bei einem gemittelten Stichprobenumfang von 24 ein Wert für $\beta$ von .24 und damit eine Teststärke von .76.

Für den Vergleich des THOP mit dem Marburger Konzentrationstraining ergibt sich bei einem gemittelten Stichprobenumfang von 23 ein Wert für $\beta$ von .23 und damit eine Teststärke von .77 .

\footnotetext{
${ }^{4}$ Als Berechnungsgrundlage für den Interaktionskontrast der vergleichenden Evaluation wurde analog dem Vorgehen in der isolierten Evaluation der Mittelwert der Stichprobengrößen der THOP-Gruppe $(n=31)$ und der Lauth-Schlottke-Gruppe $(\mathrm{n}=34)$ gebildet.
} 
Anhang D

\section{Fragebogen zur Einschätzung hyperkinetischen und aufmerksamkeitsgestörten Verhaltens}

Name:

geb.:
Vorname:

ausgefüllt von:

\section{Anleitung}

Dieser Fragebogen beschreibt typische Verhaltensweisen bzw. -auffälligkeiten, die häufig bei Kindern mit hyperaktivem Syndrom beobachtet werden können. Sie können uns wesentlich dabei unterstützen, das betreffende Kind zutreffend zu untersuchen und zu behandeln, indem Sie die nachfolgenden Fragen sorgfältig durchlesen und beantworten.

Sie haben insbesondere die Möglichkeit, die Ausprägung bzw. Stärke der jeweiligen Verhaltensweisen genauer einzuschätzen. Außerdem möchten wir Sie bitten zu überlegen, seit wann (Lebensjahr) das Kind dieses Verhalten zeigt und dies neben bzw. unter der entsprechenden Frage anzugeben, sofern dies möglich ist.

Das Vorgehen soll am folgenden Beispiel erläutert werden:

\section{Bsp. „Das Kind fuchtelt häufig mit Händen und Füßen oder rutscht auf dem} Stuhl umher. (seit dem Lbj.)“

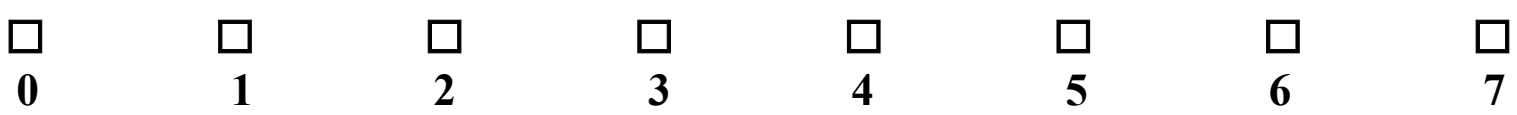

Wenn Sie der Meinung sind, dass Ihr Kind (bzw. das von Ihnen zu beurteilende Kind) überhaupt nicht bzw. nie mit Händen und Füßen zappelt oder auf dem Sitz umherrutscht, dann kreuzen Sie bitte das Kästchen über der „,“ an.

Wenn Sie der Meinung sind, dass das Kind mehr oder weniger mit Händen und Füßen zappelt, können Sie auf der Skala von 1 bis 7 einschätzen, wie stark dieses Verhalten üblicherweise zu beobachten ist. Dabei bedeutet „,“", dass das Kind nur sehr wenig bzw.

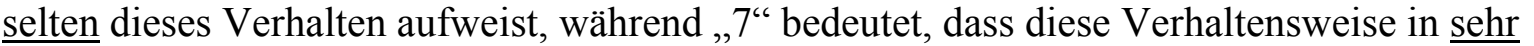
starkem Maße (extrem) bzw. immer oder fast immer auftritt.

Nun bitten wir Sie, auf der folgenden Seite mit der Einschätzung zu beginnen. Wichtig ist, dass Sie sorgfältig überlegen, wobei Sie sich auch mit Ihrem Partner, Kollegen o.ä. beraten können. 
1. Das Kind ist häufig unaufmerksam gegenüber Kleinigkeiten bzw. Einzelheiten oder macht Sorgfaltsfehler (,,Schusselfehler"6). (seit dem Lbj.)



2. Das Kind ist häufig nicht in der Lage, die Aufmerksamkeit (Konzentration) bei Aufgaben (z.B. Hausaufgaben) und beim Spielen aufrechtzuerhalten. (seit dem Lbj.)

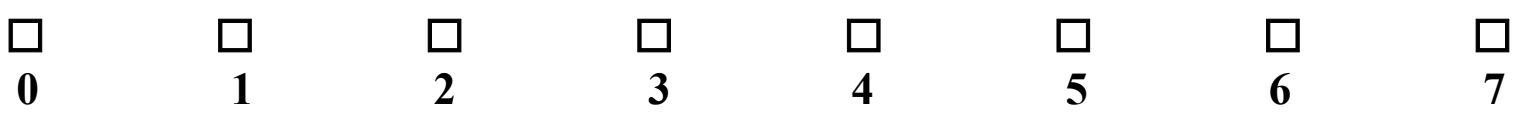

3. Das Kind hört scheinbar nicht, was ihm gesagt wird (gemeint ist aufmerksam zuhören). (seit dem Lbj.)

0

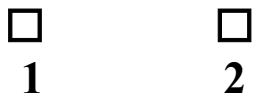

$\begin{array}{ll}\square & \square \\ 3 & 4\end{array}$

5

$\square$

4. Das Kind kann oft Erklärungen nicht folgen oder seine Schularbeiten, Aufgaben und Pflichten zu Hause oder in der Schule (bzw. Kindereinrichtung) nicht erfüllen, obwohl es geistig in der Lage ist, die Erklärung zu verstehen und nicht bockt bzw. trotzt. (seit dem Lbj.)

0


4

5

6

5. Das Kind kann seine Aufgaben oder Aktivitäten selbst schlecht planen und organisieren (z.B. für das notwendige Material sorgen usw.). (seit dem Lbj.)

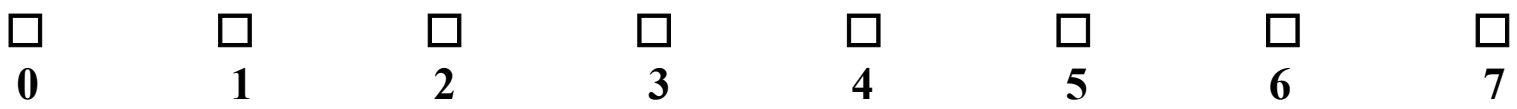

6. Das Kind vermeidet unangenehme Aufgaben, wie z.B. das Anfertigen von Hausaufgaben, die Ausdauer und Konzentration (geistiges Durchhaltevermögen) erfordern. (seit dem Lbj.)

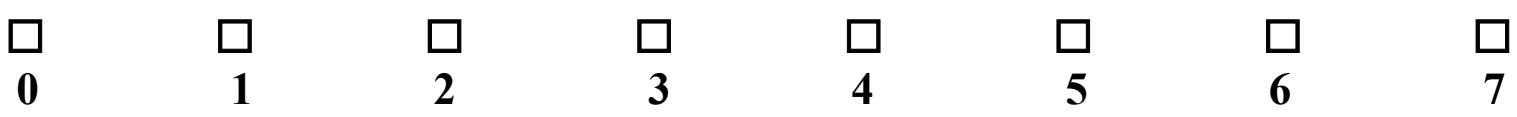

7. Das Kind verliert häufig Gegenstände, die für bestimmte Aufgaben, z.B. Schularbeiten wichtig sind (Bleistifte, Bücher, Spielsachen, Werkzeuge, Schlüssel). (seit dem Lbj.)

$\begin{array}{rrrrrrrr}\square & \square & \square & \square & \square & \square & \square & \square \\ \mathbf{0} & \mathbf{1} & \mathbf{2} & \mathbf{3} & \mathbf{4} & \mathbf{5} & \mathbf{6} & \mathbf{7}\end{array}$


8. Das Kind wird häufig von Dingen oder Vorgängen in seiner Umgebung von seiner momentanen Aufgabe abgelenkt (z.B. Geräusche, plötzliche Bewegungen usw.) (seit dem Lbj.)

0

$\begin{array}{ll}\square & \square \\ \mathbf{1} & \mathbf{2}\end{array}$

$\begin{array}{ll}\square & \square \\ 3 & 4\end{array}$

$\square$

$\square$

9. Das Kind ist in alltäglich wiederkehrenden Verrichtungen oft vergesslich oder muss immer wieder daran erinnert werden (z.B. anziehen, waschen, aufräumen, Ranzen packen usw.). (seit dem Lbj.)

$\begin{array}{rrrrrrrr}\square & \square & \square & \square & \square & \square & \square & \square \\ \mathbf{0} & \mathbf{1} & \mathbf{2} & \mathbf{3} & \mathbf{4} & \mathbf{5} & \mathbf{6} & \mathbf{7}\end{array}$

10. Das Kind fuchtelt häufig mit den Händen oder Füßen oder rutscht auf dem Stuhl umher. (seit dem Lbj.)

$\mathbf{0}$
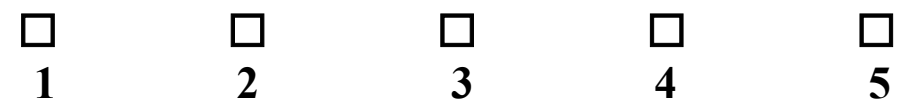

$$
6
$$

11. Das Kind verlässt seinen Platz im Klassenzimmer oder in anderen Situationen, in denen Sitzen bleiben erwartet wird. (seit dem Lbj.)

$\begin{array}{rrrrrrrr}\square & \square & \square & \square & \square & \square & \square & \square \\ \mathbf{0} & \mathbf{1} & \mathbf{2} & \mathbf{3} & \mathbf{4} & \mathbf{5} & \mathbf{6} & \mathbf{7}\end{array}$

12. Das Kind läuft häufig umher oder klettert ständig in Situationen herum, in denen dies unpassend ist, z.B. im Klassenraum, Verkehrsmitteln (Bus), oder wenn das Kind abwarten soll, während sich Erwachsene unterhalten. (seit dem Lbj.)

$\begin{array}{rrrrrrrr}\square & \square & \square & \square & \square & \square & \square & \square \\ \mathbf{0} & \mathbf{1} & \mathbf{2} & \mathbf{3} & \mathbf{4} & \mathbf{5} & \mathbf{6} & 7\end{array}$

13. Das Kind ist häufig unnötig laut beim Spielen oder hat Schwierigkeiten bei leisen Freizeitbeschäftigungen, z.B. selbständiges ,stilles“6 Arbeiten im Unterricht. (seit dem Lbj.)

$\begin{array}{rrrrrrrr}\square & \square & \square & \square & \square & \square & \square & \square \\ \mathbf{0} & \mathbf{1} & \mathbf{2} & \mathbf{3} & \mathbf{4} & \mathbf{5} & \mathbf{6} & \mathbf{7}\end{array}$

14. Das Kind ist häufig unruhig und zappelig, wobei es nicht gelingt, durch Aufforderungen, Verbote, Ermahnungen oder Körperkontakt (z.B. liebevolles Festhalten) dieses unruhige Verhalten dauerhaft zu unterbinden, d.h. das Kind zappelt nach kurzer Pause unvermindert weiter. (seit dem Lbj.)

$\begin{array}{cccccccc}\square & \square & \square & \square & \square & \square & \square & \square \\ \mathbf{0} & \mathbf{1} & \mathbf{2} & \mathbf{3} & \mathbf{4} & \mathbf{5} & \mathbf{6} & \mathbf{7}\end{array}$


15. Das Kind redet häufig laut und „ohne Punkt und Komma“ auch wenn die anwesenden Personen dies gar nicht möchten. (seit dem Lbj.)

$\begin{array}{rrrrrrrr}\square & \square & \square & \square & \square & \square & \square & \square \\ \mathbf{0} & \mathbf{1} & \mathbf{2} & \mathbf{3} & \mathbf{4} & \mathbf{5} & \mathbf{6} & 7\end{array}$

16. Das Kind platzt häufig mit einer Antwort heraus, bevor die Frage beendet wurde, z.B. im Schulunterricht. (seit dem Lbj.)

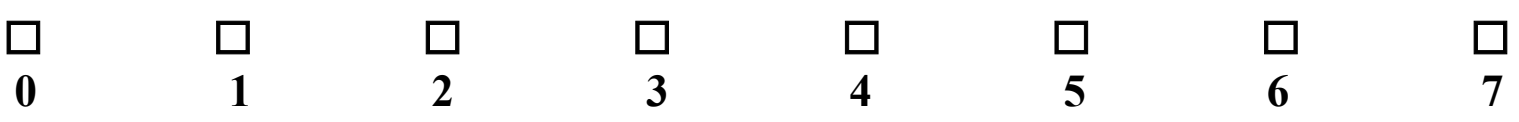

17. Das Kind kann häufig nicht in einer Reihe warten. (seit dem Lbj.)

$\begin{array}{cccccccc}\square & \square & \square & \square & \square & \square & \square & \square \\ \mathbf{0} & \mathbf{1} & \mathbf{2} & \mathbf{3} & \mathbf{4} & \mathbf{5} & \mathbf{6} & \mathbf{7}\end{array}$

18. Das Kind unterbricht und stört andere häufig, z.B. mischt sich in das Spiel oder die Gespräche anderer ein. (seit dem Lbj.)

$\begin{array}{rrrrrrrr}\square & \square & \square & \square & \square & \square & \square & \square \\ \mathbf{0} & \mathbf{1} & \mathbf{2} & \mathbf{3} & \mathbf{4} & \mathbf{5} & \mathbf{6} & \mathbf{7}\end{array}$


Bitte schätzen Sie nachfolgend noch ein, ob das Kind in folgenden „Lebensbereiche“ durch die oben beschriebenen Verhaltensweisen beeinträchtigt ist, und wenn ja, in welchem Maße:

1. im familiären Zusammenleben (bzw. Heimgruppe o. ä.)

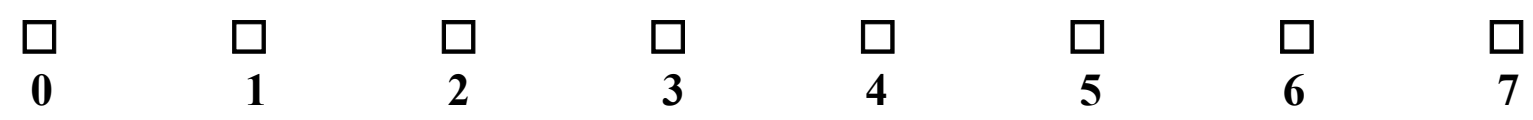

2. in der Schule und bei den damit verbundenen Lernanforderungen in der Klasse

$\begin{array}{cccccccc}\square & \square & \square & \square & \square & \square & \square & \square \\ \mathbf{0} & \mathbf{1} & \mathbf{2} & \mathbf{3} & \mathbf{4} & \mathbf{5} & \mathbf{6} & \mathbf{7}\end{array}$

3. beim Umgang mit Gleichaltrigen

\begin{tabular}{cccccccc}
$\square$ & $\square$ & $\square$ & $\square$ & $\square$ & $\square$ & $\square$ & $\square$ \\
$\mathbf{0}$ & $\mathbf{1}$ & $\mathbf{2}$ & $\mathbf{3}$ & $\mathbf{4}$ & $\mathbf{5}$ & $\mathbf{6}$ & $\mathbf{7}$ \\
& & & & & & & \\
\hline
\end{tabular}

Wir möchten uns für Ihre Mitarbeit herzlich bedanken! 
Anhang E.1: Interaktionsfragebogen

Liebe Eltern,

Name des Kindes

wir haben einige allgemeine Fragen zu den Verhaltensproblemen Ihres Kindes und wie Sie damit umgehen. Kreuzen sie bitte jeweils an, was am besten für Sie zutriff.

1a) Wie stark sahen Sie Ihr Kind vor der Behandlung durch die Verhaltensprobleme belastet?

$\begin{array}{lllllllllll}\square & \square & \square & \square & \square & \square & \square & \square & \square & \square & \square \\ 0 & 10 & 20 & 30 & 40 & 50 & 60 & 70 & 80 & 90 & 100\end{array}$

gar nicht belastet

sehr stark belastet

1b) Wie stark sehen Sie Ihr Kind derzeit durch die Verhaltensprobleme belastet?



gar nicht belastet

sehr stark belastet

1c) Wie hat sich die Belastung Ihres Kindes durch die Verhaltensprobleme im Vergleich zur Zeit vor der Behandlung verändert?

Mein Kind ist im Vergleich zu vorher folgendermaßen belastet:

Sehr viel weniger weniger gleich mehr sehr viel mehr

2a) Wie stark sahen Sie Sich als Mutter/Vater vor der Behandlung durch die Verhaltensprobleme Ihres Kindes belastet?

$\begin{array}{lllllllllll}\square & \square & \square & \square & \square & \square & \square & \square & \square & \square & \square \\ 0 & 10 & 20 & 30 & 40 & 50 & 60 & 70 & 80 & 90 & 100\end{array}$

gar nicht belastet

sehr stark belastet

2b) Wie stark fühlen Sie sich als Mutter/Vater derzeit durch die Verhaltensprobleme Ihres Kindes belastet?

$\begin{array}{lllllllllll}\square & \square & \square & \square & \square & \square & \square & \square & \square & \square & \square \\ 0 & 10 & 20 & 30 & 40 & 50 & 60 & 70 & 80 & 90 & 100\end{array}$

gar nicht belastet

sehr stark belastet 
2c) Wie hat sich Ihres Belastung als Eltern durch die Verhaltensprobleme im Vergleich zur Zeit vor der Behandlung verändert?

Meine Belastung hat sich im Vergleich zu vorher folgendermaßen verändert:

Viel abgenommen abgenommen gleich etwas zugenommen viel zugenommen

3a) Wie gut gelang es Ihnen vor der Behandlung, die Verhaltensauffälligkeiten Ihres Kind positiv zu beeinflussen?

$\begin{array}{lllllllllll}\square & \square & \square & \square & \square & \square & \square & \square & \square & \square & \square \\ 0 & 10 & 20 & 30 & 40 & 50 & 60 & 70 & 80 & 90 & 100\end{array}$

gelang gar nicht

gelang sehr gut

3b) Wie gut gelingt es Ihnen derzeit, die Verhaltensauffälligkeiten Ihres Kind positiv zu beeinflussen?

$\begin{array}{lllllllllll}\square & \square & \square & \square & \square & \square & \square & \square & \square & \square & \square \\ 0 & 10 & 20 & 30 & 40 & 50 & 60 & 70 & 80 & 90 & 100\end{array}$

gelingt gar nicht

gelingt sehr gut

3c) Wie hat sich durch die Behandlung Ihre Fähigkeit verändert, die Verhaltensauffälligkeiten Ihres Kind positiv zu beeinflussen?

$\begin{array}{ccccc}\square & \square & \square & \square & \square \\ \text { gelingt viel besser } & \text { gelingt besser } & \text { gleich } & \text { gelingt schlechter } & \text { gelingt viel schlechter }\end{array}$

4a) Wenn mein Kind verhaltensauffällig war, wusste ich vor der Behandlung nicht was ich tun soll!

$\begin{array}{lllllllllll}\square & \square & \square & \square & \square & \square & \square & \square & \square & \square & \square \\ 0 & 10 & 20 & 30 & 40 & 50 & 60 & 70 & 80 & 90 & 100\end{array}$

stimmt gar nicht

stimmt völlig

4b) Wenn mein Kind jetzt verhaltensauffällig ist, weiß ich nicht was ich tun soll!

$\begin{array}{lllllllllll}\square & \square & \square & \square & \square & \square & \square & \square & \square & \square & \square \\ 0 & 10 & 20 & 30 & 40 & 50 & 60 & 70 & 80 & 90 & 100\end{array}$

stimmt gar nicht

stimmt völlig 
4c) Durch die Behandlung hat sich mein Wissen verändert, was ich tun soll, wenn mein Kind verhaltensauffällig ist. Ich weiß jetzt, was zu tun ist:<smiles>[Hg]</smiles>

sehr verbessert etwas verbessert gleich geblieben etwas viel abgenommen abgenommen

5a) Wenn mein Kind vor der Behandlung nicht das tat, was ich von ihm erwartete, setzte ich Konsequenzen ein!



stimmt gar nicht

stimmt völlig

5b) Wenn mein Kind jetzt nicht das tut, was ich von ihm erwarte, setze ich Konsequenzen ein!

$\begin{array}{lllllllllll}\square & \square & \square & \square & \square & \square & \square & \square & \square & \square & \square \\ 0 & 10 & 20 & 30 & 40 & 50 & 60 & 70 & 80 & 90 & 100\end{array}$

stimmt gar nicht

stimmt völlig

5c) Wie hat sich durch die Behandlung Ihre Fähigkeit verändert, Konsequenzen einzusetzen, wenn Ihr Kind nicht das tut, was Sie von ihm erwarten?

$\begin{array}{ccccc}\square & \square & \square & \square & \square \\ \text { gelingt viel besser } & \text { gelingt besser } & \text { gleich } & \text { gelingt schlechter } & \text { gelingt viel schlechter }\end{array}$

6a) Durch meine Erziehungsmaßnahmen erreichte ich vor der Behandlung bei meinem Kind, was ich will!

$\begin{array}{lllllllllll}\square & \square & \square & \square & \square & \square & \square & \square & \square & \square & \square \\ 0 & 10 & 20 & 30 & 40 & 50 & 60 & 70 & 80 & 90 & 100\end{array}$

stimmt gar nicht

stimmt völlig 
6b) Durch meine Erziehungsmaßnahmen erreiche ich jetzt bei meinem Kind, was ich will!

$\begin{array}{lllllllllll}\square & \square & \square & \square & \square & \square & \square & \square & \square & \square & \square \\ 0 & 10 & 20 & 30 & 40 & 50 & 60 & 70 & 80 & 90 & 100\end{array}$

stimmt gar nicht

stimmt völlig

6c) Wie hat sich durch die Behandlung Ihre Fähigkeit verändert, durch Erziehungsmaßnahmen das zu erreichen, was sie von Ihrem Kind wollen?

$\square$

gelingt viel besser gelingt besser gleich gelingt schlechter gelingt viel schlechter

7a) Ich sah vor der Behandlung viele positive Dinge an meinem Kind!



stimmt gar nicht

stimmt völlig

7b) Ich sehe jetzt viele positive Dinge an meinem Kind!

$\begin{array}{lllllllllll}\square & \square & \square & \square & \square & \square & \square & \square & \square & \square & \square \\ 0 & 10 & 20 & 30 & 40 & 50 & 60 & 70 & 80 & 90 & 100\end{array}$

stimmt gar nicht

stimmt völlig

7c) Wie hat sich die Sicht von Ihrem Kind durch die Behandlung verändert? Ich sehe mein Kind:

viel positiver etwas positiver gleich etwas negativer viel negativer

8a) Mein Kind hielt sich vor der Behandlung an Familienregeln!

$\begin{array}{lllllllllll}\square & \square & \square & \square & \square & \square & \square & \square & \square & \square & \square \\ 0 & 10 & 20 & 30 & 40 & 50 & 60 & 70 & 80 & 90 & 100\end{array}$

stimmt gar nicht

stimmt völlig 
8b) Mein Kind hält sich jetzt an Familienregeln!

$\begin{array}{lllllllllll}\square & \square & \square & \square & \square & \square & \square & \square & \square & \square & \square \\ 0 & 10 & 20 & 30 & 40 & 50 & 60 & 70 & 80 & 90 & 100\end{array}$

stimmt gar nicht

stimmt völlig

8c) Wie hat sich das Befolgen von Familienregeln durch die Behandlung verändert? Mein Kind hält sich an Familienregeln:

viel besser etwas besser gleich etwas schlechter viel schlechter

9a) Bei Verhaltensproblemen meines Kindes wusste ich vor der Behandlung, was ich tun konnte, um sie zu bewältigen!

$\begin{array}{lllllllllll}\square & \square & \square & \square & \square & \square & \square & \square & \square & \square & \square \\ 0 & 10 & 20 & 30 & 40 & 50 & 60 & 70 & 80 & 90 & 100\end{array}$

stimmt gar nicht

stimmt völlig

9b) Bei Verhaltensproblemen meines Kindes weiß ich jetzt, was ich tun kann, um sie zu bewältigen!

$\begin{array}{lcccccccccc}\square & \square & \square & \square & \square & \square & \square & \square & \square & \square & \square \\ 0 & 10 & 20 & 30 & 40 & 50 & 60 & 70 & 80 & 90 & 100 \\ \text { stimmt gar nicht } & & & & & & & & & \text { stimmt völlig }\end{array}$

9c) Wie hat sich durch die Behandlung Ihre Fähigkeit verändert, bei Verhaltensproblemen etwas zu tun, um diese zu bewältigen?

gelingt viel besser gelingt besser gleich gelingt schlechter gelingt viel schlechter

Kontrollieren Sie bitte noch einmal, ob Sie alle Fragen beantworten haben.

Vielen Dank für Ihre Mithilfe! 
Anhang E.2: Kodierung für die Auswertung des Interaktionsfragebogens

\begin{tabular}{|c|c|c|}
\hline Veränderungsitems & Beschriftung & Zugeordneter Wert \\
\hline \multirow{5}{*}{$1 \mathrm{c}$} & Sehr viel weniger & 5 \\
\hline & Weniger & 4 \\
\hline & Gleich & 3 \\
\hline & Mehr & 2 \\
\hline & Sehr viel mehr & 1 \\
\hline \multirow{5}{*}{$2 \mathrm{c}$} & Viel abgenommen & 5 \\
\hline & Abgenommen & 4 \\
\hline & Gleich & 3 \\
\hline & Etwas zugenommen & 2 \\
\hline & Viel zugenommen & 1 \\
\hline \multirow{5}{*}{$\begin{array}{l}3 c \\
5 c \\
6 c \\
9 c\end{array}$} & Gelingt viel besser & 5 \\
\hline & Gelingt besser & 4 \\
\hline & Gleich & 3 \\
\hline & Gelingt schlechter & 2 \\
\hline & Gelingt viel schlechter & 1 \\
\hline \multirow{5}{*}{$4 c$} & Sehr verbessert & 5 \\
\hline & Etwas verbessert & 4 \\
\hline & Gleich geblieben & 3 \\
\hline & Etwas abgenommen & 2 \\
\hline & Viel abgenommen & 1 \\
\hline \multirow{5}{*}{$7 \mathrm{c}$} & Viel positiver & 5 \\
\hline & Etwas positiver & 4 \\
\hline & Gleich & 3 \\
\hline & Etwas negativer & 2 \\
\hline & Viel negativer & 1 \\
\hline \multirow{5}{*}{$8 c$} & Viel besser & 5 \\
\hline & Etwas besser & 4 \\
\hline & Gleich & 3 \\
\hline & Etwas schlechter & 2 \\
\hline & Viel schlechter & 1 \\
\hline
\end{tabular}




\section{Lebenslauf}

Persönliche Daten:

Schulausbildung:

$1971-1975$

$1975-1984$

Hochschulausbildung:

10/1990 - 10/1996

10/1992

$10 / 1996$

seit $10 / 1998$

Zivildienst:

09/1984 - 12/1985

Berufsausbildung:

10/1986 - 09/1989

\section{Berufsweiterbildung:}

12/1998 - 07/2003

\section{Berufstätigkeit:}

10/1989 - 09/1990

$12 / 1996-12 / 1997$

01/1998 bis heute
Thomas Dreisörner

Am Brauksweg 3

37083 Göttingen

geb. am 8.7.1964 in Verl (Kreis Gütersloh)

verheiratet, vier Kinder, deutsch

Grundschule Gütersloh-Spexard

Städtisches Gymnasium Gütersloh

Abitur: Mai 1984, Gesamtnote: 2,4

Studium der Psychologie an der Georg-August-Universität Göttingen

Diplom-Vorprüfung bestanden, Gesamtnote: gut

Diplom-Hauptprüfung bestanden, Gesamtnote sehr gut

Beginn einer Dissertation zum Thema „Zur Wirksamkeit von Trainings bei Kindern mit Aufmerksamkeitsstörungen“ an der Georg-August-Universität Göttingen

Berufspraktikum in der Klinik und Poliklinik für Psychiatrie der Universitätsklinik Göttingen; Abteilung Psychopathologie und Neuropsychologie

Berufspraktikum in der Frauenklinik der Universitätsklinik

Göttingen; Schwerpunkt Psychoonkologie

Zivildienst im Altenpflegeheim St. Johannes, Rietberg

Ausbildung zum Krankenpfleger im Evangelischen

Krankenhaus in Lippstadt

Teilzeitweiterbildung zum Psychologischen Psychotherapeuten mit der Qualifikation Verhaltenstherapie bei der Weiterbildungseinrichtung für Klinische Verhaltenstherapie e.V. (AWKV)

Krankenpfleger im Evangelischen Krankenhaus GöttingenWeende e. V. (Internistische Station für Blutgefäßerkrankungen)

Krankenpfleger im Evangelischen Krankenhaus GöttingenWeende e. V. (Station für Allgemeinchirurgie)

Beschäftigung als Diplom-Psychologe im Ökumenischen Hainich Klinikum Mühlhausen, Klinik für Kinder- und 
$01 / 1998-09 / 2000$

$10 / 2000$ bis heute
Jugendpsychiatrie

Wissenschaftliche Hilfskraft in der Abteilung für Pädagogische Psychologie und Entwicklungspsychologie, Institut für Psychologie in Göttingen

Wissenschaftlicher Mitarbeiter in der Abteilung für Pädagogische Psychologie und Entwicklungspsychologie, Institut für Psychologie in Göttingen 